\title{
Radiation length measurements with high-resolution telescopes
}

\author{
Dissertation
}

zur Erlangung des mathematisch-naturwissenschaftlichen Doktorgrades "Doctor rerum naturalium“

der Georg-August-Universität Göttingen

im Promotionsprogramm ProPhys

der Georg-August University School of Science (GAUSS)

vorgelegt von

Ulf Hagen Stolzenberg

aus Clausthal-Zellerfeld

Göttingen, 2019 
Betreuungsausschuss

Prof. Dr. Ariane Frey

PD Dr. Jörn Große-Knetter

\section{Mitglieder der Prüfungskommission:}

$\begin{array}{ll}\text { Referent: } & \text { Prof. Dr. Ariane Frey } \\ & \text { II. Physikalisches Institut, Georg-August-Universität Göttingen } \\ \text { Koreferent: } & \text { PD Dr. Jörn Große-Knetter } \\ & \text { II. Physikalisches Institut, Georg-August-Universität Göttingen }\end{array}$

Weitere Mitglieder der Prüfungskommission:

Prof. Dr. Stan Lai

II. Physikalisches Institut, Georg-August-Universität Göttingen

Prof. Dr. Hans-Christian Hofsäss

II. Physikalisches Institut, Georg-August-Universität Göttingen

Prof. Dr. Wolfram Kollatschny

Institut für Astrophysik, Georg-August-Universität Göttingen

Prof. Dr. Ansgar Reiners

Institut für Astrophysik, Georg-August-Universität Göttingen

Tag der mündlichen Prüfung: 05.07.2019

Referenz: II.Physik-UniGö-Diss-2019/06 


\title{
Georg-August-Universität Göttingen
}

\section{Radiation length measurements with high-resolution telescopes}

\begin{abstract}
The radiation length imaging method presented in this work can be used to conduct spatially resolved measurements of scattering angles of particles traversing a object under study. For the measurements a planar object is centred in a high-resolution telescope and the whole setup is positioned in a multi-GeV particle beam. The scattering angle distributions depend on the radiation length $X / X_{0}$ of the traversed object. Combining the radiation length information with the reconstructed intersections provides the opportunity to generate 2D images of the measurement objects material profile. Measured radiation length images of detector planes can be compared to existing detector models for validation and, when necessary, improvement of the detector simulation.
\end{abstract}

In order to demonstrate the capabilities and scope of the X/X0 imaging method, several beam tests have been conducted in the last years. The imaged objects varied from a ultra thin vertex detector ladder designed for the Belle II experiment to a prototype module for the ATLAS ITk upgrade. Additionally a series of measurements were conducted to determine the radiation length constant $X_{0}$ of hardened conductive glues. To test systematic effects, such as energy loss due to bremsstrahlung, measurements on copper and aluminium wedges with a continuously increasing radiation length were conducted. 

2. The Standard Model of Particle Physics and the Belle II experiment 5

2.1. The Standard Model and an overview of the foundations of particle physics 5

2.2. Belle II at SuperKEKB . . . . . . . . . . . . . . . . . . . . . 9

2.3. Overview of research topics . . . . . . . . . . . . . 20

3. Interactions between charged particles and matter 23

3.1. Ionisation and energy loss straggling . . . . . . . . . . . . . 23

3.2. Bremsstrahlung and the radiation length constant $X_{0} \ldots \ldots \ldots$. . . 24

3.3. Coulomb scattering . . . . . . . . . . . . . . . . 27

4. Beam test experiments: Experimental setup and analysis of data 39

4.1. Generation of high energy particle beams . . . . . . . . . . . . . . 40

4.2. Reference telescopes . . . . . . . . . . . . . . . . . . 42

4.3. The Test Beam Software Framework $(T B S W)$. . . . . . . . . . . . . 44

4.4. Measurements of intrinsic M26 cluster resolutions . . . . . . . . . . . . . . 69

4.5. The beam test simulation algorithm . . . . . . . . . . . . 76

5. Scattering angle reconstruction and radiation length imaging $\mathbf{7 9}$

5.1. Scattering angle reconstruction . . . . . . . . . . . . . 80

5.2 . Telescope angle resolution . . . . . . . . . . . . . . . 85

5.3. Radiation length imaging . . . . . . . . . . . . . . . . . 87

5.4. Radiation length calibration measurements . . . . . . . . . . . . . 90

5.5. Telescope angle resolution and signal-to-noise ratio of $X / X_{0}$ measurements 93

6. Systematic influences and simulation studies $\quad 97$

6.1. Validation of the scattering angle fitting procedure with toy studies . . . . 98

6.2. Angle reconstruction with binary pixel sensors . . . . . . . . . . . . 110 
6.3. Validation of the radiation length calibration in simulation studies . . . 119

7. Radiation length measurements at DESY 129

7.1. Aluminium wedge measurements in June 2017 . . . . . . . . . . . . . . . 130

7.2. Copper wedge measurements in November 2017 . . . . . . . . . . . . . . 152

7.3. Glue measurements . . . . . . . . . . . . . . . . . . . . . 156

7.4. Chip measurements . . . . . . . . . . . . . . . . . . . 160

7.5. ATLAS ITk prototype measurements . . . . . . . . . . . . . . . 162

7.6. Measurements on a Belle II PXD mechanical sample . . . . . . . . . . . 167

$\begin{array}{lr}\text { 8. Conclusion and outlook } & 183\end{array}$

8.1. Conclusion . . . . . . . . . . . . . . . . . . . 183

8.2. Outlook . . . . . . . . . . . . . . . . . . 185

$\begin{array}{lr}\text { Bibliography } & 187\end{array}$

$\begin{array}{ll}\text { Appendices } & 193\end{array}$

$\begin{array}{lr}\text { A. TBSW example files } & \mathbf{1 9 5}\end{array}$

A.1. Masking path example . . . . . . . . . . . . . . . . . . . 195

A.2. Geometry file example . . . . . . . . . . . . . . . . . . . 196

A.3. Efficiency measurements . . . . . . . . . . . . . . . . . 198

$\begin{array}{ll}\text { B. Calculation of angle reconstruction errors with an unscented transform } & 199\end{array}$

C. Systematical studies of the radiation length calibration procedure 201

C.1. Target thickness measurements of beam energy uncertainty simulations . 201

C.2. Target thickness measurements of beam energy variation simulations . . . 204

D. Telescope and $X / X_{0}$ calibration of several beam test measurements 207

D.1. Aluminium wedge measurements in June 2017 . . . . . . . . . . . . . . 207

D.2. Copper wedge measurements in November 2017 . . . . . . . . . . . . . . 211

D.3. Glue measurements . . . . . . . . . . . . . . . . . . . . . . . . . 219

D.4. Chip measurements in October 2016 . . . . . . . . . . . . . . 227

D.5. ATLAS ITk prototype measurements . . . . . . . . . . . . . . . 236

D.6. Measurements on Belle II PXD mechanical samples, March 2015 . . . . . 244 
CHAPTER 1

\section{Motivation and overview}

The Standard Model of Particle Physics (SM) is one of the most successful and most thoroughly tested theories in physics describing fundamental interactions between elementary particles. The three fundamental interactions described by the Standard Model are the strong force mediated by gluons, the electromagnetic force mediated by photons and the weak force mediated by $W^{ \pm}$and $Z^{0}$ bosons. The gravitational force is not included in the Standard Model.

Predictions from the Standard Model are tested in particle physics experiments at high-energy colliders. Particle colliders accelerate charged particles such as electrons, positrons or protons and collide two high energy particle beams head-on. For example, at the KEKB [1] accelerator in Japan, electrons and positrons are collided at centre of mass energies of more than $10 \mathrm{GeV}$.

The highest centre of mass energies in particle collisions are reached at the Large Hadron collider [2] (LHC) at CERN, where protons are collided at centre of mass energies of $13 \mathrm{TeV}$ to produce unstable massive particles. These unstable particles decay nearly instantaneously into lighter particles, so that information about them can only be gathered by reconstructing the decay products. In order to gather information about particles produced in beam collisions, hermetic particle detectors surround the interaction point. An example of such a detector is the ATLAS detector [3] at the LHC . These detectors typically consist of multiple sub-detector systems with different functionalities: The inner part of the detector consists of several layers of position sensitive sensors in a magnetic field used to reconstruct trajectories of the decay products and measure their momenta. Further outside calorimeters for energy measurement and particle identification systems are located. 


\section{Motivation and overview}

The basic constituents of the Standard Model are elementary particles and mediators of the three fundamental forces. There are a total of six leptons and quarks, each divided into three families or generations. Leptons and quarks are fermions with half-integer spin which obey Fermi-Dirac statistics. Each fermion is paired with an associated antiparticle that has the same mass but opposite additive quantum numbers such as the electric charge. There are four types of bosons, namely gluons, the photon, the $Z^{0}$ and $W^{ \pm}$bosons with integer spin, which are mediating the fundamental forces with the exception of gravity. They behave according to Bose-Einstein statistics. The last part of the Standard Model is the Higgs mechanism [4,5] which causes the electro-weak symmetry breaking and gives mass to the mediators of the weak interaction, called $W^{ \pm}$and $Z$ bosons, as well as the charged fermions. The Higgs mechanism requires at least one additional boson, called Higgs boson. The discovery of a SM-Higgs-like particle at the ATLAS [6] and CMS [7] experiments at the LHC in 2012 was a great success for the Standard Model.

The weak interaction has a special role in the Standard model because, unlike strong and electromagnetic interactions, it allows direct coupling between different generations of quarks. This mixing of all three generations in the weak interaction was described by Cabibbo, Kobayashi and Maskawa in the form of the CKM formalism [8]. Small violations of the combined CP symmetry, where $\mathrm{C}$ corresponds to charge-conjugation and $\mathrm{P}$ to parity symmetry, in weak interactions can be explained within the frame of the CKM theory. However, the observed CP violation for example in precision measurements on systems of neutral mesons ${ }^{1}[9]$ is too small to explain the excess of matter over anti-matter in the universe [10].

Studies of CP violations were mainly conducted at B-factories such as the BaBar [11] experiment at the SLAC National Accelerator Laboratory (US) and the Belle [12] experiment at KEK (Japan). B-factories are collider experiments designed to provide boosted pairs of $B$ mesons $^{2}$ at the centre of mass energy of the $\Upsilon(4 \mathrm{~S})$ resonance which corresponds to $10.58 \mathrm{GeV}$. The Belle experiment at the asymmetric $e^{+} e^{-} \mathrm{KEKB}$ collider finished its operation after collecting a data sample with an integrated luminosity of $1000 \mathrm{fb}^{-1}$ (corresponding to a final sample of 772 million $\mathrm{B} \overline{\mathrm{B}}$ pairs) which led to important insights into elementary particle physics and the nature of CP violation [13]. These measurements ultimately led to the confirmation of the CKM mechanism, which was rewarded with the Nobel prize in 2008. The upgrade of KEKB, SuperKEKB, started its operation in 2019. Its instantaneous luminosity of $8 \times 10^{35} \mathrm{~cm}^{-2} \mathrm{~s}^{-1}$ will be 40 times larger than KEKB [14]. In order to cope with the increased particle rates, a new detector, Belle II $[15,16]$, was designed. The commissioning of Belle II took place in 2018 and first physics runs were recorded in 2019. Belle II is a $4 \pi$ detector that is used to reconstruct particle tracks of products from $B$ decays and measure the position of the decays, called interaction vertices.

\footnotetext{
${ }^{1}$ Bound states of a quark and an anti-quark

${ }^{2}$ Mesons containing $\mathrm{a} b$ or $\overline{\mathrm{b}}$
} 
The physics program at B-factories depends crucially on resolving the time difference between the decays of the two $B$ mesons. As the $B$ pair is boosted ${ }^{3}$, this time difference can be measured by determining the difference between vertex positions of both $B$ decays along the direction of the boost. The mean flight length of a $B$ meson produced at SuperKEKB is approximately $100 \mu \mathrm{m}$. In order to resolve the spatial distance between the two decay vertices from the $B$ meson pair, the Belle II detector is required to have a vertex resolution below $50 \mu \mathrm{m}$. To achieve this resolution, a lightweight high-resolution inner Vertex Detector (VXD) is installed close to the beam pipe and the interaction point. The planned setup of the VXD consisted of two layers of Pixel Vertex Detectors (PXD) based on the Depleted Field Effect Transistor (DEPFET) technology [17] very close $^{4}$ to the beam pipe and four layers of Silicon Strip Vertex Detectors [18] (SVD) further outside ${ }^{5}$. Due to the proximity to the interaction point the PXD is needed to be radiation hard and able to distinguish between individual particle tracks in an environment with a high track density.

The tracking and vertex resolution of the VXD is on one hand determined by the intrinsic resolution of its sensors and the distance between them. On the other hand, the resolution is reduced by multiple scattering on the beam pipe, sensor planes and in the air in between. Multiple scattering affects charged particles traversing matter. The charged particle is scattered repeatedly due to Coulomb interactions with atomic nuclei of the traversed material. The net effect of many scattering processes is called multiple scattering and causes a direction change of the particle.

Multiple scattering angle distributions can be approximated by a Gaussian with a standard deviation that depends on the radiation length $X / X_{0} . X$ is the thickness of the material and $X_{0}$ is a material dependent quantity called the radiation length constant. Due to the unwanted but unavoidable uncertainty introduced by multiple scattering the sensors are required to be as lightweight as possible to keep the multiple scattering angles small. However, a certain thickness of silicon is required to generate a sufficiently large signal from a charged particle transition. Therefore, a delicate balance between a thin detector design and a sufficient signal yield has to be found.

Each layer of the PXD is required to have an average radiation length of below $0.2 \%$ [15] which corresponds to approximately $190 \mu \mathrm{m}$ of silicon. The sensitive area of the PXD has a thickness of $75 \mu \mathrm{m}$ which is sufficient to produce measurable signal due to the large signal-to-noise ratio of DEPFET pixels. However, the thin and consequently fragile sensitive area has to be supported by a silicon balcony with a thickness up to $525 \mu \mathrm{m}$. The complex design and high level of integration of data acquisition, support structures and other services on the PXD lead to a complicated material profile. As the vertex

\footnotetext{
${ }^{3}$ i.e. has a momentum in the laboratory/detector frame

${ }^{4}$ The beam pipe has a radius of $10 \mathrm{~mm}$ and the first PXD is located at a radius of $14 \mathrm{~mm}$

${ }^{5}$ Radii between 38 and $140 \mathrm{~mm}$
} 


\section{Motivation and overview}

resolution depends on the material actually traversed by a particle and not the average radiation length $X / X_{0}$, a correct assessment of the vertex resolution is highly dependent on the accurate description of the radiation length profile of the PXD.

In order to validate and, when necessary, update existing detector models, a method has been developed to perform spatially resolved measurements of the radiation length. This method requires a high energy beam of charged particles and a reference telescope, consisting of two arrays of position-sensitive charged particle detectors with a good intrinsic resolution. The particle beam traverses the reference telescope and a target under investigation, which is placed at the centre of the telescope between the two arrays. The method is based on accurate measurements of multiple scattering angles and corresponding particle intersections on the scattering target. By dividing the plane of the centred scattering target into small image pixels and reconstructing scattering angle distributions in each image pixel, a local radiation length value can be calculated. The resulting image shows the radiation length distribution projected onto the scattering target plane.

The method has been tested on various measurement objects. It was found that in order to perform accurate measurements, the beam energy and telescope angle resolution must be known very well. In order to determine these quantities, calibration measurements on well known materials must be conducted. Spatially resolved radiation length measurements can then be used to cross-check and improve existing detector models.

In this thesis these measurements and comparisons have been performed for a PXD module. Additionally, the method can be used to determine the radiation length constant $X_{0}$, which is presented here for a set of adhesives investigated to be used for the construction of the future ATLAS Inner Tracker [19] (ITk).

This thesis is structured as follows. In chapter 2, a general overview of the Standard Model, the Belle II detector and in particular the inner detector are given. The measurements performed in this thesis are motivated by the dependency of the vertex resolution on multiple scattering effects which depend on the local radiation length. Chapter 3 provides an introduction to particle-matter interactions with a main focus on single and multiple Coulomb scattering. Beam test experiments, reference telescopes as the required measurement tool, the relevant analysis steps and software framework are explained in chapter 4 . In chapter 5 , the radiation length measurement method is described in detail. This entails multiple scattering angle reconstruction as well as the imaging and calibration steps. Chapter 6 summarises results from Monte Carlo simulation experiments that were used to identify and evaluate systematic effects of radiation length measurements. In chapter 7 , measurement results from various beam tests are presented. These results include the measurements of PXD prototype modules and comparisons to the detector model. Chapter 8 summarises the presented measurements and provides an outlook for future plans. 
CHAPTER 2

\section{The Standard Model of Particle Physics and the Belle II experiment}

This chapter gives an overview over the Standard Model and CP violation. In order to motivate the radiation length measurements presented in this work, the dependence of the Belle II vertex detector on the radiation length distribution of the detector layers is explained. The chapter is structured as follows: First, an overview over the Standard Model is given in section 2.1. This includes the theoretical description of the CKM formalism as the Standard Model source of CP violation. Section 2.2 presents the $B$ factory SuperKEKB and the Belle II detector. In that section, the focus lies on the vertex detector of Belle II and the vertex resolution. Section 2.3 gives an overview over the studies performed in this thesis and places them in context.

\subsection{The Standard Model and an overview of the foundations of particle physics}

The overview over the Standard Model given in this section follows the explanations in $[20,21]$. More detailed descriptions of the concepts of the Standard Model can be found there.

\subsubsection{The Standard Model}

The Standard Model describes interactions between all known elementary particles. An overview of the elementary particles described by the Standard Model is depicted in fig. 2.1 .

All elementary particles have a quantum mechanical angular momentum called spin that is measured in units of the Planck constant $\hbar$. The fermions with spin $1 / 2$ on the left side of fig. 2.1 are divided into leptons and quarks. The electron $e$ and electron neutrino $\nu_{e}$ 


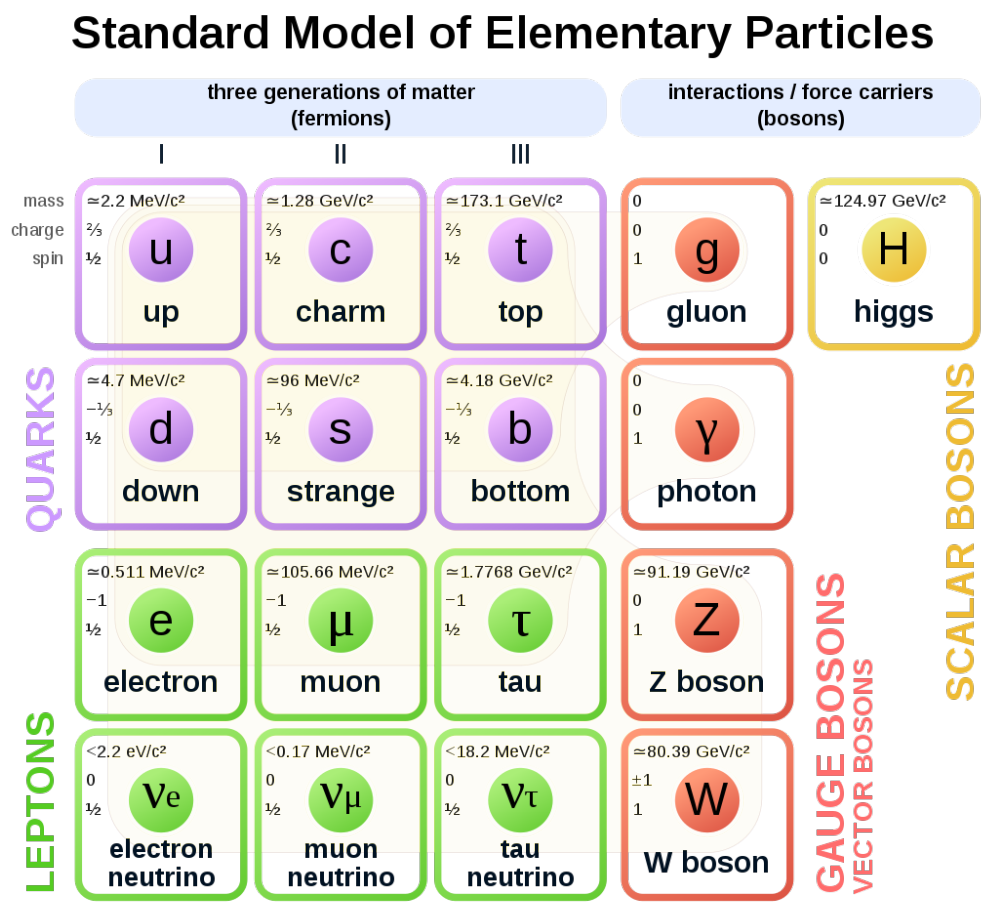

Figure 2.1.: All known particles described by the Standard Model. The fermions are separated into three generations indicated by the three columns. There are four bosons with a spin of 1 that correspond to the carriers of the interactions.

form the first generation. Electrons have an electric charge of $-e$, called the elementary charge, while neutrinos are electrically neutral. The second generation is formed by the muon $\mu$ and the muon neutrino $\nu_{\mu}$, while the third generation consists of the tau $\tau$ and the tau neutrino $\nu_{\tau}$. The charged leptons interact via the electromagnetic and weak forces, while the neutrinos only interact weakly. As will be explained later the weak force violates parity and left-handed ${ }^{1}$ electrons and left-handed electron neutrinos form a doublet with respect to the weak isospin $T_{3}$. The left-handed electron has a weak isospin of $+1 / 2$, while the left-handed electron neutrino has $T_{3}=-1 / 2$. The weak isospins of the left handed particles in generation 2 and 3 are analogously defined. Right-handed charged leptons have a weak isospin of zero and form singlets, while right-handed neutrinos do not exist in the SM.

The first generation of quarks is formed by the up- $(u)$ and down $(d)$ quark. Generation 2 has the charm $(c)$ and strange $(s)$ quark and generation 3 consists of the top $(t)$ and bottom $(b)$ quark. Up-type quarks $(u, c, t)$ have an electric charge of $+2 / 3$, while

\footnotetext{
${ }^{1}$ The concept of left- and right-handedness in this context and the differences between chirality and helicity will not be explained here, but can be found in the text books [20,21].
} 
down-type quarks have a charge of $-1 / 3$. In analogy to leptons, quarks also form lefthanded doublets and right-handed singlets. Moreover, quarks have one of three different colours, the charge of the strong interaction. Quarks interact via the strong, electromagnetic and weak forces. All fermions have an anti-particle that is identical to the particle but has opposite additive quantum numbers such as electric charge, colour charge and weak isospin.

The fundamental interactions are mediated by four types of bosons with spin 1 depicted in fig. 2.1. The massless photon $(\gamma)$ is the mediator for the electromagnetic force, massless gluons $(g)$ carry the strong force and the massive bosons $Z$ and $W^{ \pm}$carry the weak force. In mathematical terms the Standard Model is a relativistic local gauge invariant quantum field theory. The gauge group of the Standard Model corresponds to $S U_{C}(3) \times S U_{L}(2) \times U_{Y}(1) . S U_{C}(3)$ is the special unitary group of degree three, which is the gauge group of Quantum Chromodynamics and describes strong interactions [22]. The index $C$ refers to the colour charge. $S U_{L}(2)$ x $U_{Y}(1)$ is the gauge group for the unified electroweak interaction [23,24]. The index $L$ refers to the left-handed doublets ${ }^{2}$ and $Y$ to the hypercharge which depends on the weak isospin and the electric charge. The Higgs mechanism $[4,5]$ leads to the spontaneous symmetry breaking of the electroweak group. This electroweak symmetry breaking is the cause for the non-zero masses of the $Z$ and $W^{ \pm}$bosons. The Higgs mechanism introduces at least one additional boson with spin 0, the Higgs boson. The particle discovered in 2012 at ATLAS and CMS [6,7] has all properties of the SM Higgs boson.

The Standard Model as it is known today emerged in the 1960s to 1970s as a collection of theories that included all known elementary particles and all interactions with the exception of gravity. It has been thoroughly tested over the last decades and the theoretical expectations of the model have been confirmed every time. However, observations in astrophysics and cosmology show that the Standard Model fails to answer several fundamental questions. One of these open questions is the nature of dark matter and dark energy. Dark matter is required to correctly describe the movement of stars in galactic disks. Dark energy is connected to the acceleration of the expansion of the universe. Only $5 \%$ of all energy in the universe comes from known particles described by the Standard Model, mostly bound states of quarks called hadrons. The remaining $95 \%$ consist of $27 \%$ dark matter and $68 \%$ dark energy. Both dark energy and dark matter cannot be explained with particles in the Standard Model.

Neutrino oscillations have been observed $[25,26]$ which are not predicted by the Standard Model. The observation of neutrino oscillations implies that neutrinos have different masses contrary to the expectation of the Standard Model where neutrinos are considered to be massless. Another open question is the dominance of hadronic matter over anti-matter in the observable universe. The magnitude of $\mathrm{CP}$ violation present in the Standard Model does not suffice to explain the imbalance between matter and

\footnotetext{
${ }^{2} S U_{L}(2)$ is the gauge group of the weak isospin, which is only non-zero for left-handed particles
} 


\section{The Standard Model of Particle Physics and the Belle II experiment}

anti-matter [10]. This second question motivated the construction of the $B$-factory SuperKEKB which provides the opportunity to study $\mathrm{CP}$ violation and reveal new sources of it in high precision measurements of $B$ meson decays.

\subsubsection{CP violation in the Standard Model}

Parity transformations $\mathrm{P}$ change space coordinates $\mathbf{x}$ into $-\mathbf{x}$. In $1957 \mathrm{Wu}$ [27] found the first evidence for parity violation in $\beta$ decays of magnetised cobalt $\left({ }^{60} \mathrm{Co}\right)$

$$
{ }^{60} \mathrm{Co} \longrightarrow{ }^{60} \mathrm{Ni}^{*}+e^{-}+\overline{\nu_{e}}
$$

She found that the produced electrons were nearly always emitted in the direction opposite to the magnetisation. The magnetisation is an axial vector, therefore it is invariant under parity transformations. Accordingly, only the momentum of the emitted electrons is affected by parity transformations. If parity was conserved, the rate of emitted electrons in the direction of the magnetisation would be identical to the rate in the opposite direction. However, Wus measurements clearly shows that there is a preferred direction. Accordingly, parity is violated in weak interactions such as the $\beta$ decay. Today we know that parity is maximally violated in weak interactions. The charge conjugation transformation $\mathrm{C}$ changes the sign of all additive quantum numbers of a particle. It replaces particles with their anti-particles. As explained in [20] the weak interaction also violates the charge conjugation symmetry.

The combined symmetry, CP, was observed to be violated in weak decays involving $W^{ \pm}$in the quark sector. This was first observed by Cronin and Fitch [9] in the neutral kaon $^{3}$ system. In 1963 the concept of quark mixing was formulated by Cabibbo [28]. The weak eigenstates that couple to the $W$ boson correspond to superpositions of the quark mass states. Kobayashi and Maskawa extended the formalism and predicted the existence of a third quark generation that had not been discovered at the time [8]. The $3 \times 3$ matrix that connects the weak eigenstates $\left(d^{\prime}, s^{\prime}, b^{\prime}\right)^{\mathrm{T}}$ to the mass eigenstates $(d, s, b)^{\mathrm{T}}$ is called the Cabibbo-Kobayashi-Maskawa (CKM) matrix and is defined as

$$
\left(\begin{array}{c}
d^{\prime} \\
s^{\prime} \\
b^{\prime}
\end{array}\right)=\left(\begin{array}{ccc}
V_{u d} & V_{u s} & V_{u b} \\
V_{c d} & V_{c s} & V_{c b} \\
V_{t d} & V_{t s} & V_{t b}
\end{array}\right)\left(\begin{array}{c}
d \\
s \\
b
\end{array}\right)=V_{\mathrm{CKM}}\left(\begin{array}{c}
d \\
s \\
b
\end{array}\right)
$$

As of 2019 according to [29] the magnitudes of the matrix elements are

$$
\left(\begin{array}{ccc}
\left|V_{u d}\right| & \left|V_{u s}\right| & \left|V_{u b}\right| \\
\left|V_{c d}\right| & \left|V_{c s}\right| & \left|V_{c b}\right| \\
\left|V_{t d}\right| & \left|V_{t s}\right| & \left|V_{t b}\right|
\end{array}\right)=\left(\begin{array}{ccc}
0.9742 \pm 0.00021 & 0.2243 \pm 0.0005 & 0.00394 \pm 0.00036 \\
0.218 \pm 0.004 & 0.997 \pm 0.017 & 0.0422 \pm 0.0008 \\
0.0081 \pm 0.0005 & 0.0394 \pm 0.0023 & 1.019 \pm 0.025
\end{array}\right) .
$$

\footnotetext{
${ }^{3}$ Mesons which contain a $u / d$ quark or anti-quark and a strange quark or anti-quark
} 
A clear hierarchical pattern in the magnitudes of the matrix entries is visible: The diagonal elements are close to one, while the off-diagonal entries are small. As a consequence weak interactions between quarks of the same generation are much more likely than cross-generation interactions. The CKM matrix is a unitary $3 \times 3$ matrix and can, alternatively, be parametrised by three real parameters and one complex phase that is responsible for $\mathrm{CP}$ violation. Since $V_{\mathrm{CKM}}$ is a unitary matrix, it must fulfil constraints given by

$$
\begin{aligned}
& 0=\sum_{\alpha=u, c, t} V_{\alpha i} V_{\alpha j}^{*}, i \neq j \\
& 0=\sum_{i=d, s, b} V_{\alpha i} V_{\beta i}^{*}, \alpha \neq \beta .
\end{aligned}
$$

These equations can be visualised by six unitarity triangles in the complex plane, where each term in the sum corresponds to a side of the triangle. In case the complex phase is non-zero the area of the triangles is non-vanishing and can serve as a measure for the magnitude of CP violation in the Standard Model. One unitarity triangle can be found in the mixing of the $b$ quark into the three down-type quarks. Consequently, measurements of mesons containing $b$ quarks such as $B^{ \pm}, B^{0}$ and $\bar{B}^{0}$ provide an opportunity to study $\mathrm{CP}$ violation. Experiments at $B$-factories such as BaBar at the PEP-II collider [11] and Belle at the KEKB collider [12] were constructed to measure $B$ decays and determine the parameters of the unitarity triangle. The goal is to over-constrain the triangle by conducting independent measurements of all angles and side lengths. The constraints from the measurements together with the triangle reconstructed from a combined fit [30] is depicted in fig. 2.2. The parametrisation of the triangle here is defined in such a way that the base of the triangle has a length of exactly one, starting at $(0,0)$ and ending at $(0,1)$. As can be seen the independent measurements of all sides and angles agree with a triangle within the systematic and statistical uncertainties.

As mentioned before, the magnitude of CP violation in the Standard Model is not sufficient to explain the observed excess of hadronic matter in the universe. Inconsistencies of parameters of the unitarity triangle would indicate physics beyond the Standard model and potentially point to additional sources of $\mathrm{CP}$ violation which might explain the observed excess of hadronic matter over anti-matter. In order to expand the knowledge of $\mathrm{CP}$ violations in nature and further constrain the unitarity triangle, the $B$-factory SuperKEKB has been constructed.

\subsection{Belle II at SuperKEKB}

SuperKEKB is an asymmetric ring accelerator that collides $7 \mathrm{GeV}$ electrons and $4 \mathrm{GeV}$ positrons at a centre of mass energy which corresponds to the mass resonance of the 


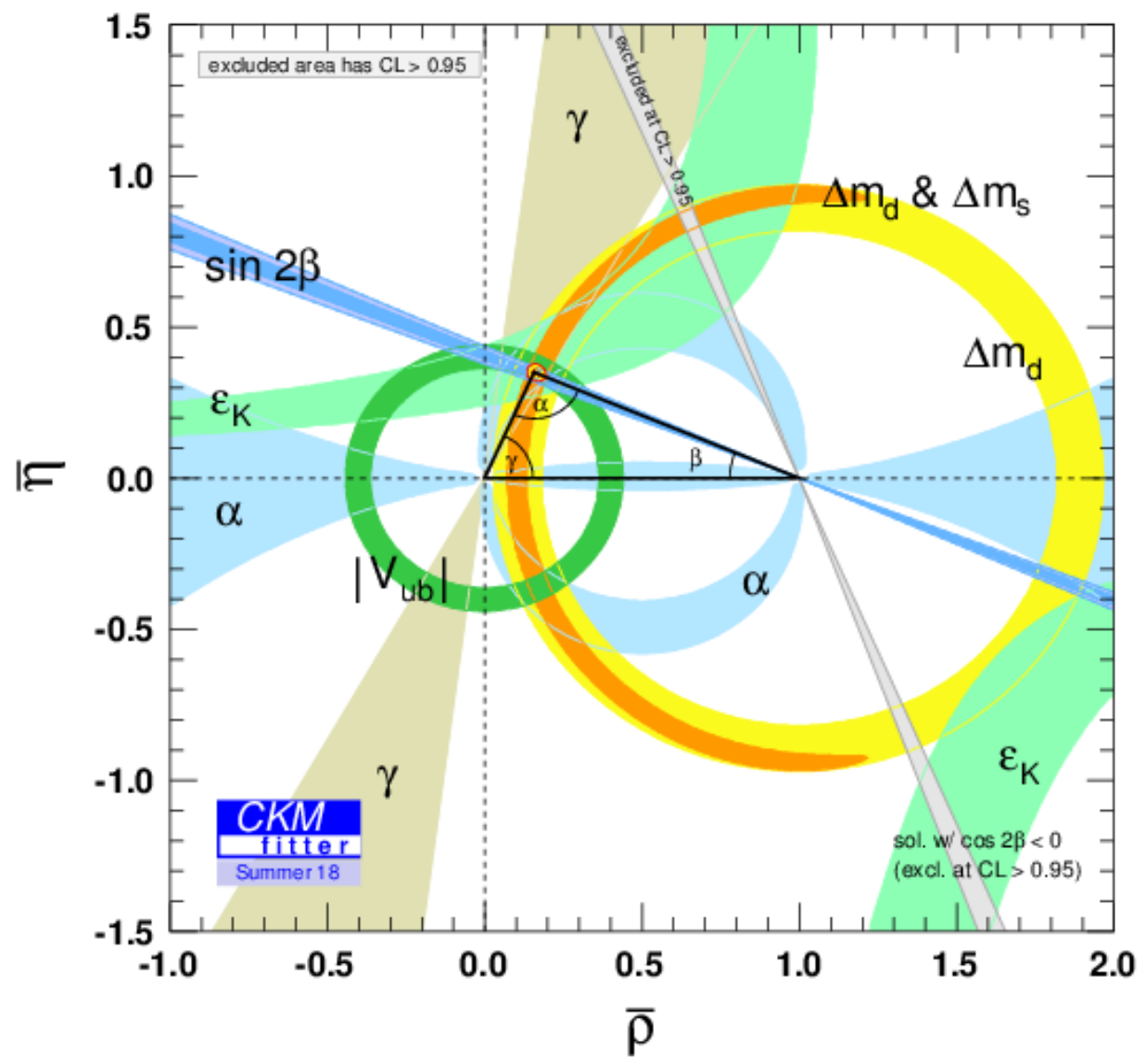

Figure 2.2.: Constraints due to independent measurements of various parameters and the corresponding best fit unitarity triangle (from [30]).

$\Upsilon(4 \mathrm{~S})$ meson $^{4}$. The upgrade of the accelerator yields a luminosity increase to 40 times the level of KEKB. Accordingly, the acquisition of $B$ decay data will increase and the statistical uncertainty of the measurements of the unitarity triangle is expected to decrease rapidly. The luminosity upgrade is accompanied by an upgrade of the detector system. The inner detector of Belle II is closer to the interaction point, has a larger radiation hardness and a better vertex resolution.

\subsubsection{The B-factory SuperKEKB}

A schematic overview of the SuperKEKB accelerator is depicted in fig. 2.3. SuperKEKB consists of two storage rings, the low energy ring (LER) which accelerates positrons to

\footnotetext{
${ }^{4}$ consisting of a $b \bar{b}$ quark pair
} 


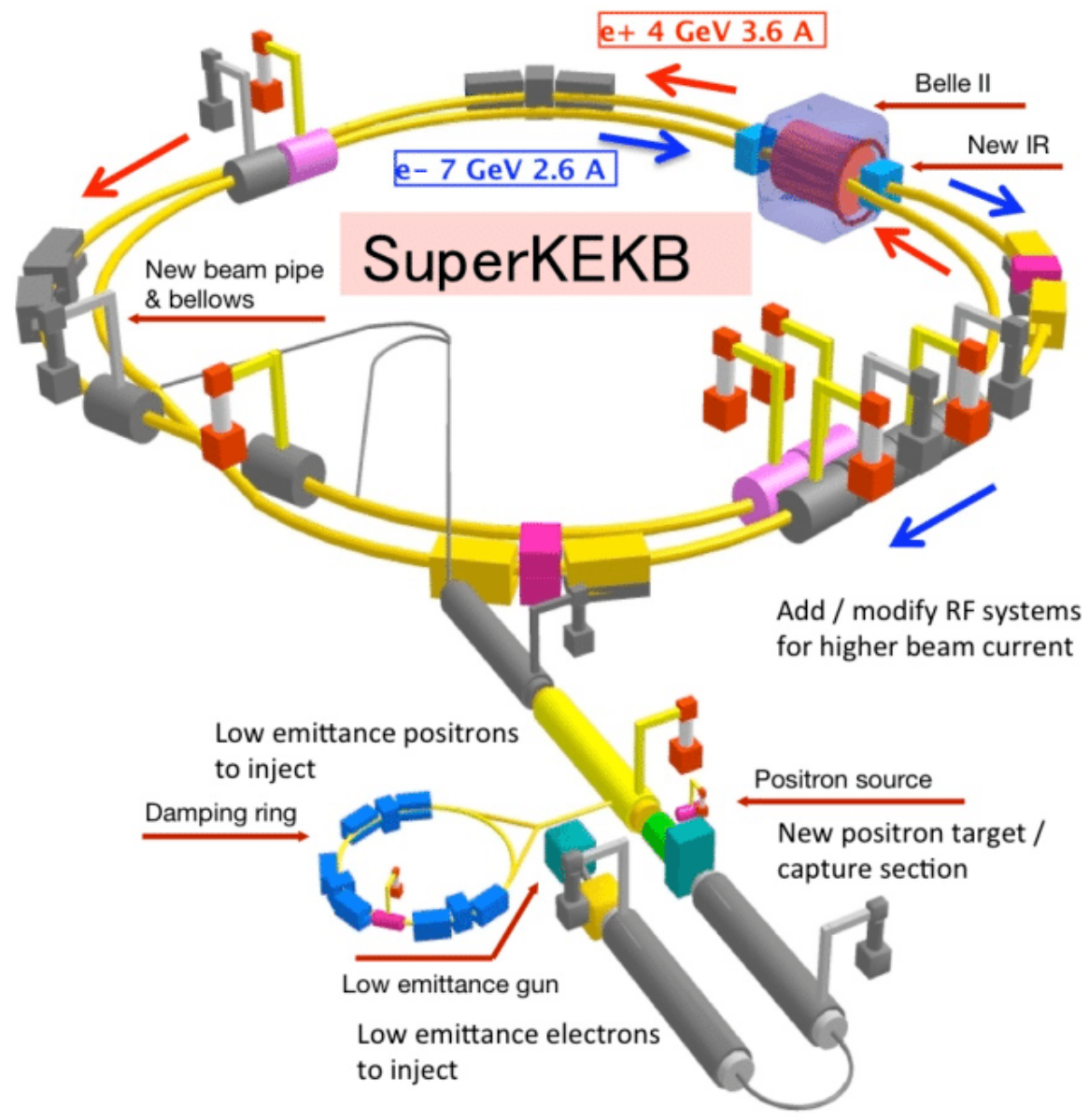

Figure 2.3.: Schematic drawing of the SuperKEKB accelerator (from [31]). The most prominent changes with respect to KEKB are indicated in the drawing.

an energy of $4 \mathrm{GeV}$ and the high energy ring (HER) accelerating electrons to an energy of $7 \mathrm{GeV}$. The target luminosity of SuperKEKB $8 \times 10^{35} \frac{1}{\mathrm{~cm}^{2} \mathrm{~s}}$ is a factor of 40 larger than the corresponding KEKB value [16]. This luminosity increase is achieved on one hand by an increase of the HER and LER currents by a factor of approximately two. On the other hand, a nano-beam scheme is employed. The beams are squeezed to a vertical size of approximately $50 \mathrm{~nm}$, which corresponds to a decrease by a factor of 20 compared to the vertical beam size of $1 \mu \mathrm{m}$ at KEKB [16]. The beams collide at an angle of $83 \mathrm{mrad}$ and due to the asymmetric beam energies, the produced $\Upsilon(4 \mathrm{~S})$ mesons are boosted approximately in the direction of the electron beam. The average particle boost $\langle\gamma \beta\rangle$ relative to the Belle II detector is roughly 0.284 , where $\beta$ is the particle velocity $v$ relative to the speed of light $c$ and $\gamma$ is the Lorentz factor. The $\Upsilon(4 \mathrm{~S})$ meson has a very short lifetime and decays nearly instantaneously at the interaction point into two $B$ mesons. The $B$ meson pair consists either of two neutral mesons $B^{0} \bar{B}^{0}$ or two charged mesons $B^{+} B^{-}$. The $B$ meson decay involves a cross-generation weak interaction and 
the lifetime of $B$ mesons is rather large, in the order of 1.5 ps [29]. Accordingly, the boosted $B$ meson pair travels a short distance before decaying. The average flight path length is in the order of $130 \mu \mathrm{m}$. An example of $B$ decays is depicted in fig. 2.4.

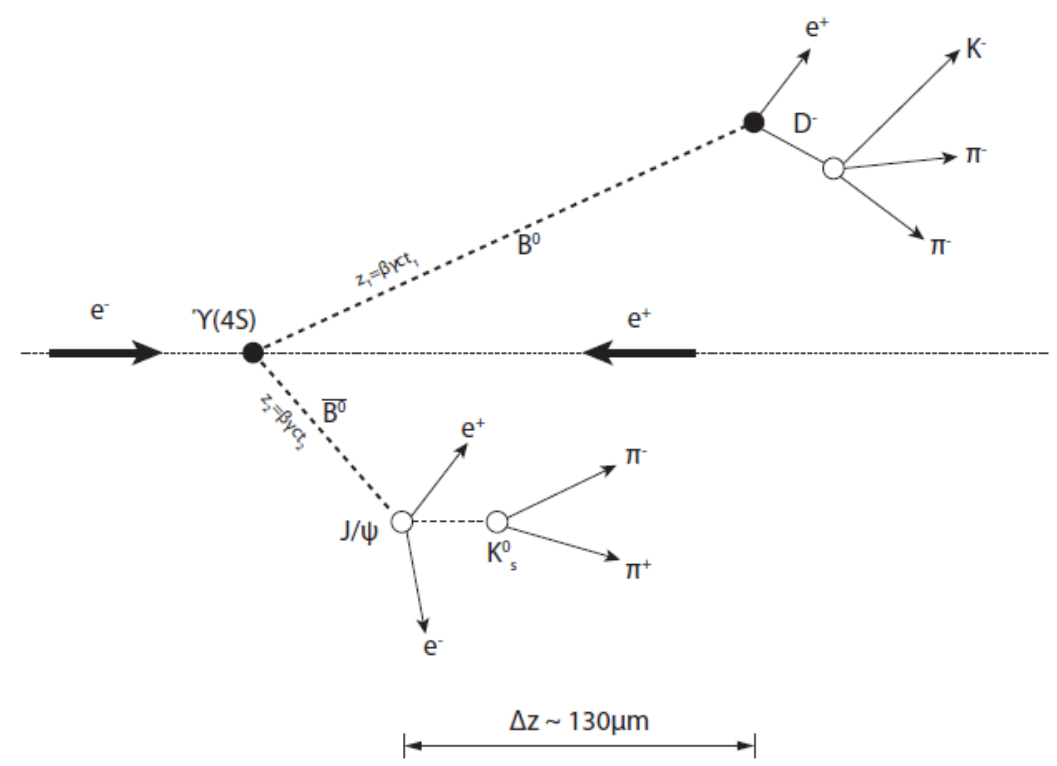

Figure 2.4.: Example decay of a pair of $B$ mesons generated at the $\Upsilon(4 \mathrm{~S})$ resonance (from [32]). The distance between the decay vertices of the $B$ mesons $\Delta z$ differs from event to event.

The vertex resolution along the boost direction $z$ of the Belle II detector has to be well below this value to be able to distinguish the decay vertices of the $B$ meson pair. The distance between the decay vertices $\Delta z$ can also be expressed by a time difference between the decays $\Delta t$. The time difference can than be used for measurements on time-dependent $\mathrm{CP}$ violation in the mixed $B$ meson state. A good vertex resolution is therefore crucial for the success of the Belle II experiment. According to [16], the required vertex resolution lies in the order of $50 \mu \mathrm{m}$. However, the vertex resolution with a full Belle II VXD is expected to be $20 \mu \mathrm{m}$ for particles with $2 \mathrm{GeV}$ [15].

\subsubsection{The Belle II detector}

The Belle II detector was designed to deal with a 40 times higher event rate, as well as 10 to 20 times larger background rates compared to Belle [16]. The boost of produced $B$ meson pairs is slightly smaller than at KEKB and therefore the vertex resolution had to be improved. The following section gives an overview over the individual systems of the Belle II detector. The focus lies on the inner vertex system, the VXD consisting of the pixel detector PXD and the silicon strip vertex detector SVD. 
The Belle II detector is depicted in fig. 2.5. Belle II is a hermetic detector surrounding the interaction point. It consists of the following subdetector systems $[15,16]$ :

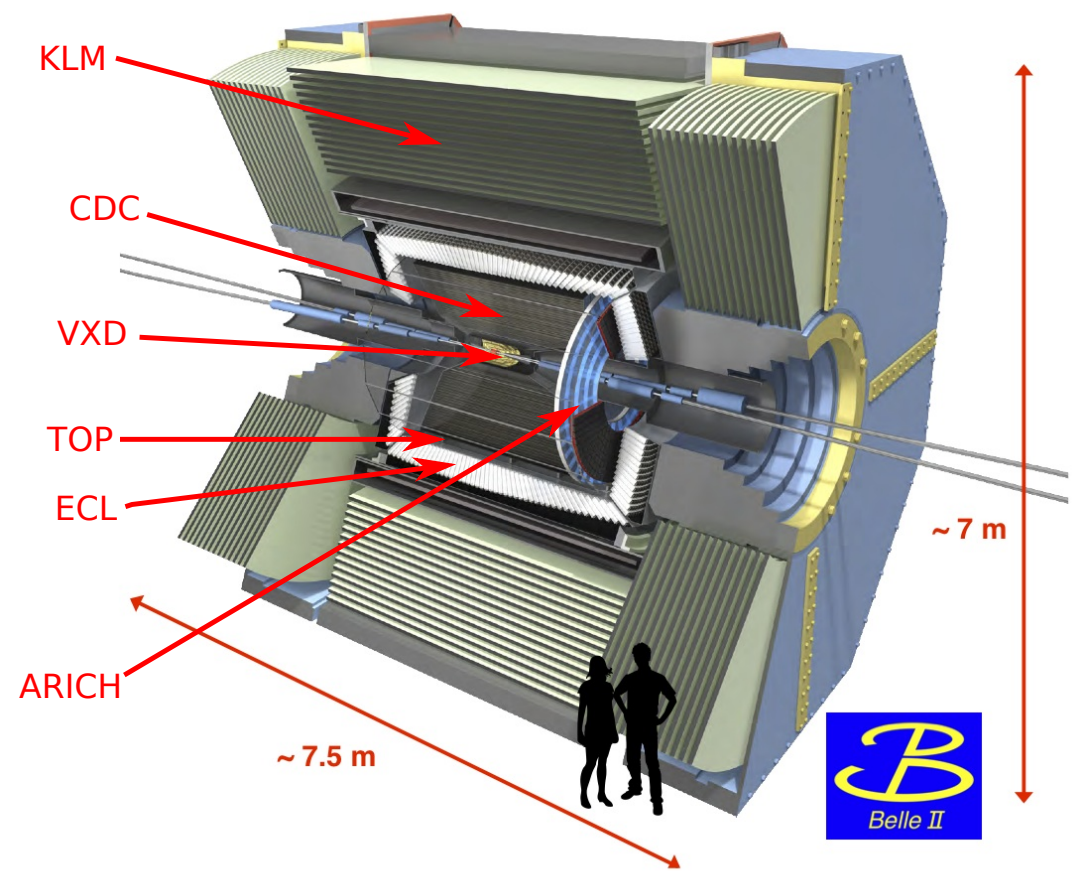

Figure 2.5.: Schematic view of the Belle II detector. The vertex detector VXD is located close to the beam pipe. Tracking, particle identification and calorimeter systems are can be found further away from the interaction region.

- Vertex Detector (VXD): The VXD consist of two subsystems, the pixel detector (PXD) and the silicon vertex detector (SVD). The PXD consists of two layers located at a radius $r=14 \mathrm{~mm}$ and $r=22 \mathrm{~mm}$ from the interaction point. The pixel sensors are based on the Depleted Field Effect Transistor (DEPFET) technology [17]. The SVD consists of four layers located at $r=38,80,115$ and $140 \mathrm{~mm}$. SVD layers consists of double-sided silicon strip sensors. Compared to Belle, the innermost layer is much closer to the interaction point which improves the vertex resolution. The VXD volume lies within the volume of a solenoidal magnetic field.

- Central Drift Chamber (CDC): As the VXD, the CDC also lies in the volume of the magnetic field. It is used to measure particle momenta and perform basic particle identification via energy loss measurements in its gas volume. The chamber gas consists of an equal mixture of $\mathrm{He}$ and $\mathrm{C}_{2} \mathrm{H}_{6}$ and contains approximately 14000 wires arranged in 56 layers. 
- Particle Identification systems (TOP and ARICH): The time-of-propation counter (TOP) is located in the barrel region of the Belle II detector. The propagation time of Cherenkov photons in a Quartz volume is measured. This includes internal reflections inside the Quartz bar. Using the time information and the measurement position of the photon, a Cherenkov image can be reconstructed and the Cherenkov angle can be determined. In the forward end-cap region the Aerogel Ring-Imaging Cherenkov detector (ARICH) is located. As the name suggests, aerogel is used as an Cherenkov radiator. The Cherenkov photons traverse an expansion volume of approximately $20 \mathrm{~cm}$ and are reconstructed on a single photon sensitive highgranularity photon detector to determine the Cherenkov angle which depends on the particle velocity. The velocity information can be used for particle identification when combining it with an independent measurement of the particle momentum.

- Electromagnetic calorimeter (ECL): The ECL consists of thallium-doped caesiumiodide ( $\mathrm{CsI}(\mathrm{Tl}))$ crystals. The barrel, forward and backward end-caps are equipped with a total number of approximately 8800 crystals. The ECL is used to identify electrons and distinguish them from pions. Additionally it is used to measure and identify photon signals and determine their energy precisely.

- $K_{L}$ and $\mu$ detection (KLM): The KLM consists of an alternating sandwich of $4.7 \mathrm{~cm}$ iron plates and active detector elements. The task of the KLM is to identify muons which traverse the detector volume in an approximately straight line. Long lived kaons $K_{L}$ can be identified in the detector because they are expected to form a hadronic shower.

Additional information on the individual subsystems of the detector can be found in $[15,16]$.

\subsubsection{Belle II pixel detector and vertex resolution}

The PXD is the innermost detector system of Belle II. It is located outside of the beam pipe close to the interaction point. The first cylindrical PXD layer consists of eight ladders arranged in a windmill structure. The second layer has the same windmill structure, but was planned to consist of twelve ladders. However, due to technical difficulties only two ladders for layer 2 have been installed for the early Belle II operation. Each PXD ladder is constructed by glueing together two individual PXD modules, called half ladders. An overview of a single PXD half ladder and the geometric arrangement of the $\mathrm{PXD}$ as a whole is depicted in fig. 2.6.

The sensitive area, also called active area, of the PXD is based on the DEPFET technology. Each sensor pixel consists of a MOS-type field effect transistor combined with a sidewards depleted silicon detector substrate. The structure of a DEPFET pixel is depicted in fig. 2.7. The silicon is thinned down to a thickness of $75 \mu \mathrm{m}$. The sidewards depletion generates a potential minimum, the internal gate, which is positioned $1 \mu \mathrm{m}$ beneath the transistor structure by an additional deep n-implantation. Electrons 


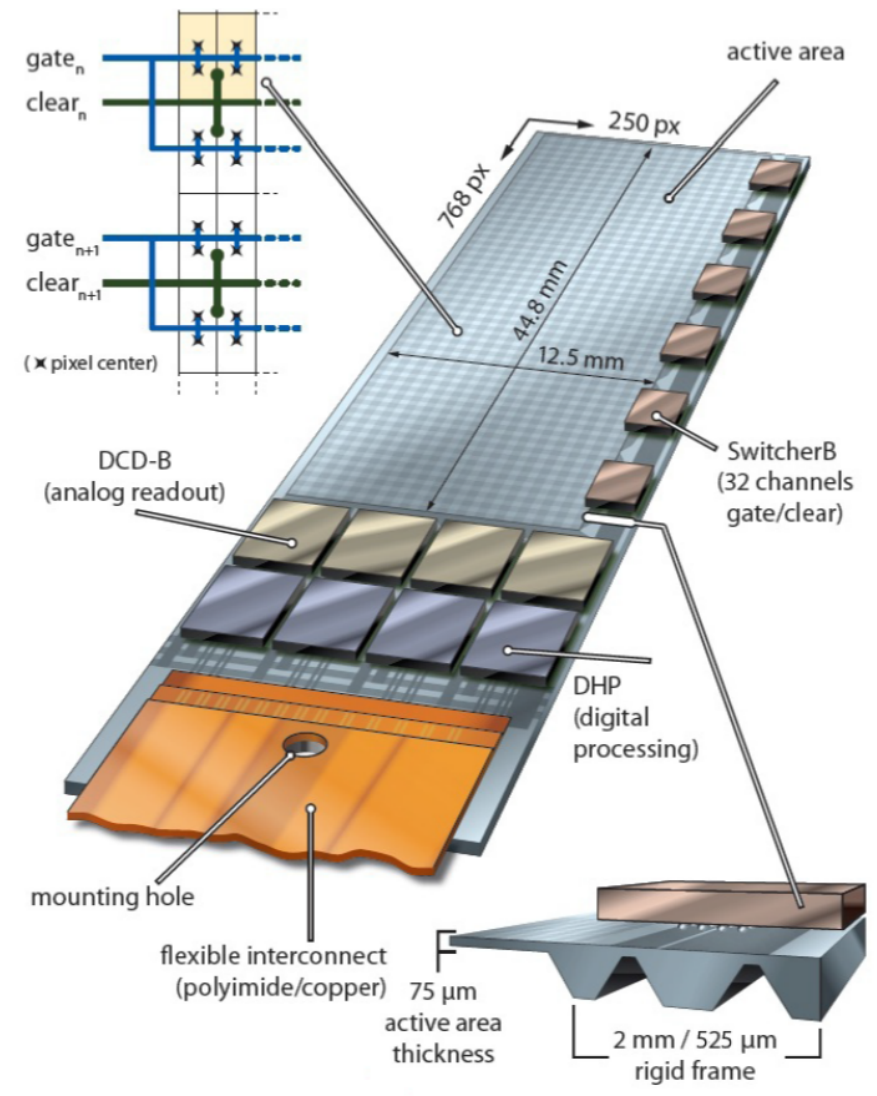

(a)

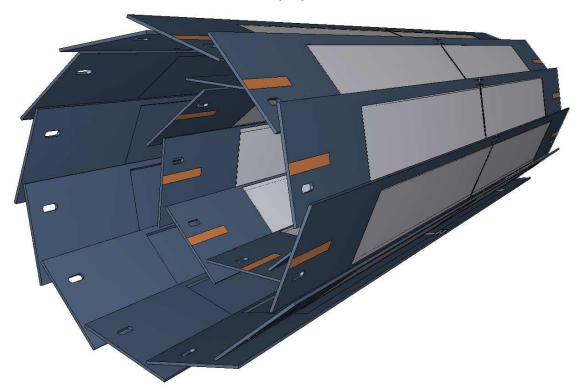

(b)

Figure 2.6.: (a) Schematic drawing of a PXD half ladder. The most important structures such as the active area and the switcherB are marked. (b) Depiction of the windmill arrangement of the PXD system. A ladder consists of two half ladders glued together. The windmill arrangement of the PXD leads to an overlap of neighbouring ladders, so that particles crossing the non-active balcony region of a PXD also traverse the active area of a neighbouring ladder. 


\section{The Standard Model of Particle Physics and the Belle II experiment}

generated by a charged particle traversing the substrate are collected in the internal gate, while holes drift towards the backside contact of the sensor. When the transistor is switched on the accumulated charge in the internal gate leads to a modulation of the transistor current. Accordingly, the current holds information on the signal stored in the internal gate. Signal charges can be removed from the internal gate by applying a large positive voltage at the clear contact.

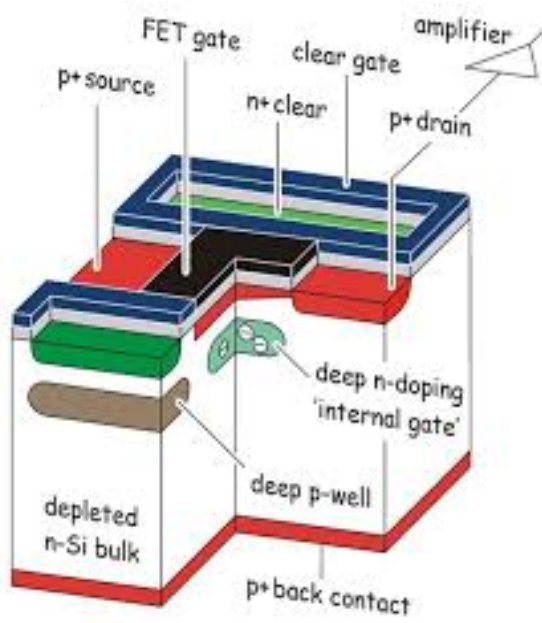

Figure 2.7.: Schematic depiction of a DEPFET pixel (from [15]).

In dependence of the distance from the interaction point in $z$ direction, the size of the DEPFET pixels along the $z$ direction are variable to produce an approximately equal intrinsic resolution everywhere on the matrix. There are four different types of PXD sensors. They can be categorised as inner and outer modules as well as forward and backward modules. Each type of PXD has two different pixel sizes. The pixel sizes range from $50 \mu \mathrm{m} \times 55 \mu \mathrm{m}$ very close to the interaction point to $50 \mu \mathrm{m} \times 85 \mu \mathrm{m}$ far away. Each PXD half ladder has $768 \times 250$ pixels that are read-out within $20 \mu \mathrm{s}$. As each pixel provides an 8 bit digital signal the required data transfer rate to read out the PXD raw signal is very large and a reduction of the data rate of measured data is mandatory. A part of the data reduction takes place on the PXD half ladders themselves, another part must be provided by the data acquisition system. Only PXD hits within a region of interest defined by tracks in the outer tracking systems of Belle II are read out. All other hits are discarded.

Outside of the sensitive area, silicon balconies with a maximum thickness of $525 \mu \mathrm{m}$ can be found. These balconies are required to mechanically stabilise the PXD half ladders. On one of the balconies next to the long side of the matrix six switcherB chips are located. They consist of $300 \mu \mathrm{m}$ thick silicon. An array of bump bonds is located beneath the switchers. Two other types of chips, the drain current digitizer $(D C D)$ and the data handling processor ( $D H P)$, are located next to the small side of the matrix not 
glued to another PXD half ladder. This area is called the end-of-staves (EOS) region of the PXD. The $D C D$ and the $D H P$ consist of silicon with an overall thickness of approximately $350 \mu \mathrm{m}$. In order to reduce the average radiation length of the PXD half ladders, a groove pattern is etched into the balconies next to the long side of the sensitive area. Examples of these groove patterns are depicted in fig. 2.8.
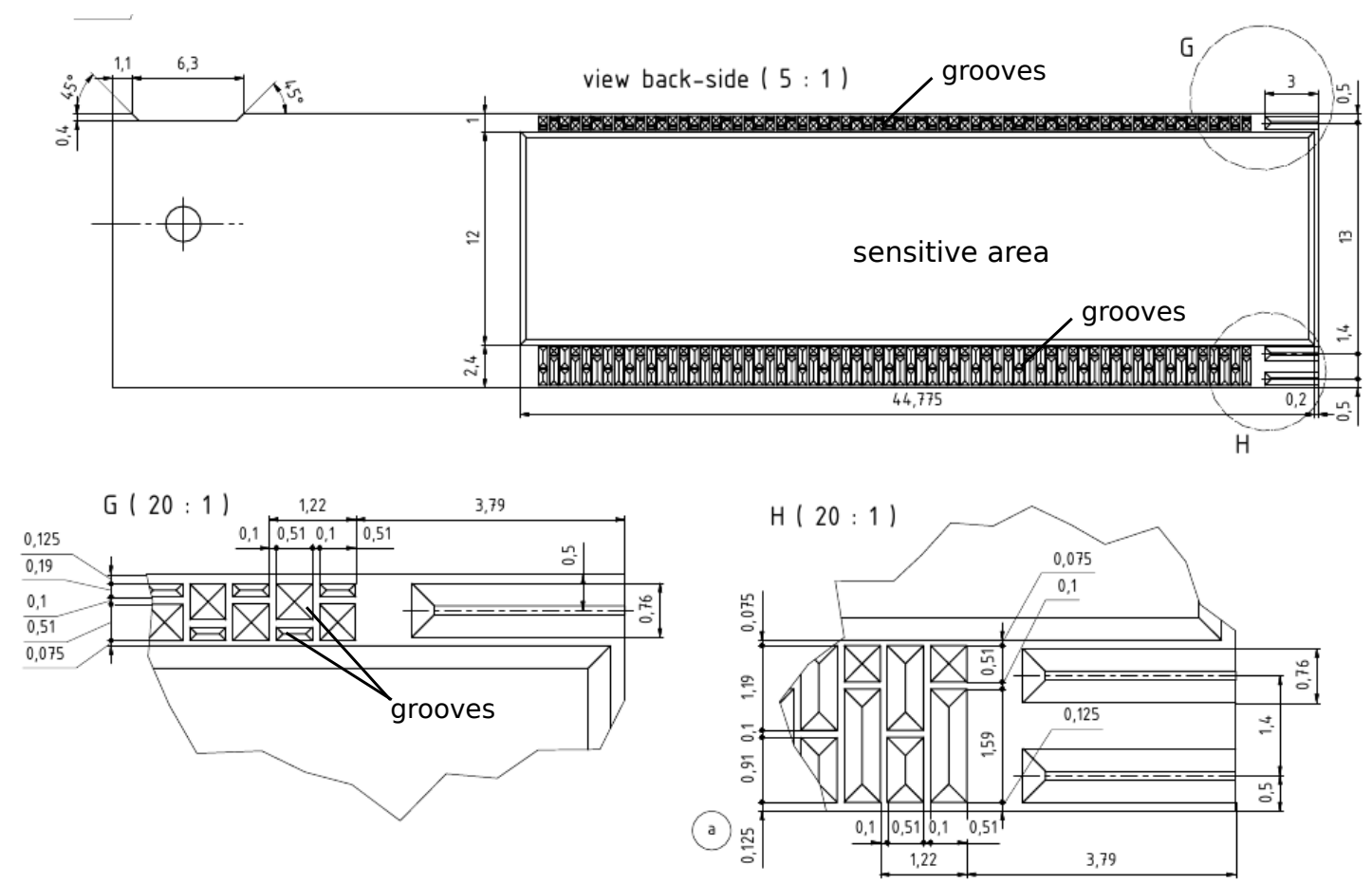

Figure 2.8.: Technical design drawing of the backside of a PXD half ladder. The groove patterns on the balcony are visible and enlargements of both pattern types arranged on opposite sides of the senstive area are shown (from [33]).

The larger groove pattern ( $\mathrm{H}$ in the picture) is located on the balcony with the switchers. The smaller groove pattern ( $\mathrm{G}$ in the picture) lies on the opposite side of the sensitive area. The grooves are added to the silicon via deep anisotropic etching [34]. The lightweight concept of the PXD design leads to a small material budget which is crucial to achieve a good vertex resolution for the PXD.

The vertex resolution in $z$ is dependent on the radii of the PXD layers with respect to the interaction point, the material of the PXD layers and the beam pipe and the particle momentum. Determining the vertex resolution is a non-trivial task. A simple approach to modelling the vertex resolution in $z$ direction is given by a quadratic sum as explained in $[35,36]$ 


$$
\sigma_{z}=a \oplus \frac{b}{p \sin ^{\frac{5}{2}}(\theta)}
$$

where the first term $a$ is purely geometrical and the second term $b / p \sin ^{\frac{5}{2}}(\theta)$ comes from uncertainties introduced by multiple scattering. The second term depends on the momentum of the particle $p$ and material quantities described by $b$. Multiple scattering corresponds to the combined effect of a large amount of individual Coulomb scatterings on nuclei in the detector material. The uncertainty due to multiple scattering must be considered for every material layer between the interaction decay vertex and the outermost VXD layer.

The geometrical term depends on the intrinsic resolution of the sensors and the distances from the interaction point. For example, for two identical sensor layers with uniform intrinsic resolution of $\sigma_{m}$ at $r_{1}$ and $r_{2}$ with $r_{1}<r_{2}$ the first term can be expressed as

$$
a=\sqrt{\frac{r_{1}^{2}+r_{2}^{2}}{\left(r_{2}-r_{1}\right)^{2}}} \sigma_{m} .
$$

This formula neglects the magnetic field and assumes straight lines as particle trajectories. The multiple scattering term depends on the momentum $p$ of the particle, the polar angle of its trajectory $\theta$ as well as the radiation length of the traversed material planes. The quantity $b$ can be expressed by the sum over the material surfaces along the particle track. In case of two detector layers at $r_{1}$ and $r_{2}$ the uncertainty due to scattering on the first layer is given by

$$
\frac{b}{p \sin ^{\frac{5}{2}}(\theta)} \approx \frac{r_{1} 0.0136 \mathrm{GeV}}{p[\mathrm{GeV}] \sin ^{\frac{5}{2}}(\theta)} \sqrt{\frac{X_{1}}{X_{0}}}
$$

The quantity $X_{1} / X_{0}$ is called the radiation length of the first detector layer. It corresponds to the thickness of the layer $X_{1}$ divided by a material dependent constant $X_{0}$, called the radiation length constant.

In case of more than two detector layers with different intrinsic resolutions and nonequidistant detector layers, as is the case for the Belle II VXD, the formula for the vertex resolution is more complicated. However, the vertex resolution of the detector system can still be computed with eq. 2.6. According to simulations [37], for the Belle II VXD with two PXD and four SVD layers the constants are given by $a=18.2 \mu \mathrm{m}$ and $b=10.8 \mu \mathrm{m} \mathrm{GeV}$. The corresponding vertex resolution according to eq. 2.6 in dependence of $p \sin ^{5 / 2}(\vartheta)$ is depicted in fig. 2.9 . 


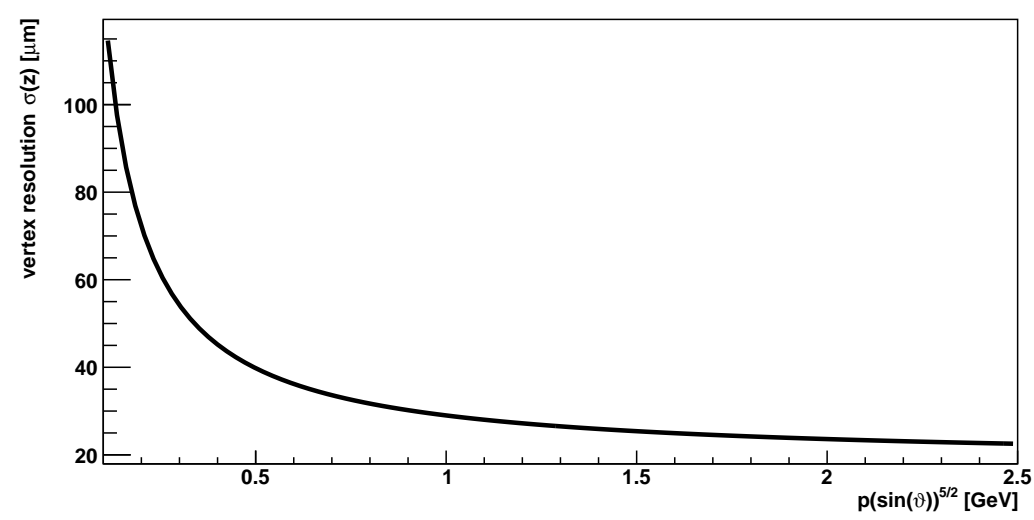

Figure 2.9.: Vertex resolution as a function of $p \sin ^{5 / 2}(\vartheta)$ according to eq. 2.6. The parameters $a=18.2 \mu \mathrm{m}$ and $b=10.8 \mu \mathrm{m} \mathrm{GeV}$ were determined from simulations [37].

The multiple scattering term is very important in the Belle II case, because the particle momenta can be very small, in the order of $500 \mathrm{MeV}$. As can be seen from fig. 2.9 and from the multiple scattering term in eq. 2.6 the vertex resolution increases with decreasing momentum $p$. It has a value of approximately $30 \mu \mathrm{m}$ at $1 \mathrm{GeV}$ and a value of approximately $40 \mu \mathrm{m}$ at $500 \mathrm{MeV}$. In addition to the material of the detector layers, particles also have to traverse the beam pipe with a $6 \mu \mathrm{m}$ thick gold foil to shield the detectors from synchrotron radiation. This is an unavoidable contribution to the multiple scattering term in eq. 2.6. Consequently, minimising the average radiation length of the VXD layers is crucial to ensure a sufficiently small vertex resolution. PXD modules, for example, are required to have an average radiation length $X / X_{0}$ below $0.2 \%$ per layer.

However, as explained on the last pages, the PXD does not consist of a homogeneously thick layer of silicon, but has a more complex material profile consisting of $75 \mu \mathrm{m}$ thick silicon in the sensitive area and of $525 \mu \mathrm{m}$ silicon in the balcony regions. When determining the uncertainty of the decay vertex position on the level of individual events and tracks, the local radiation length may therefore differ widely from the average radiation length. This has to be considered in track and vertex reconstruction. For example a particle crossing the sensitive area of a PXD at perpendicular incidence will be affected by multiple scattering on a layer with a radiation length of $X / X_{0}=75 \mu \mathrm{m} / 93.66 \mathrm{~mm}=$ $0.08 \%$. Alternatively, due to the windmill arrangement and the consequential overlap of ladders (see fig. 2.6b) it is possible for a particle to first cross the balcony of a PXD in layer 1 and afterwards generate a hit on the sensitive area on a neighbouring plane in layer 1 . In this case, the particle could traverse the balcony with $525 \mu \mathrm{m}$ silicon at its thickest point and additionally traverse a switcher volume and possibly bump bonds. Admittedly, this scenario is rather unlikely, however in this case the actually traversed material would have a radiation length of approximately $1.6 \%{ }^{5}$. This value widely dif-

\footnotetext{
${ }^{5}$ Assuming a bump bond diameter of $80 \mu \mathrm{m}$ and the length constant $X_{0}$ of tin as $12 \mathrm{~mm}$ [29]
} 


\section{The Standard Model of Particle Physics and the Belle II experiment}

fers from the expected average radiation length of a PXD layer of below $0.2 \%$. On the level of individual tracks the radiation length of the traversed material in the VXD is expected to vary strongly. It is therefore important to have a precise description of the detector material to ensure reliable uncertainty estimations of individual tracks.

\subsection{Overview of research topics}

The precise knowledge of material distributions in vertex detectors is important to ensure a reliable estimation of the vertex resolution. In the frame of this thesis a spatially resolved radiation length measurement was developed and validated. This included developing $\mathrm{C}++$ code, conducting beam test measurements and analysing the recorded beam test data. The presented method requires a scattering target centred in a highresolution reference telescope, i.e. an array of at least six charged particle pixel sensors with a good intrinsic resolution, and a particle beam with an energy of several GeV. These requirements are fulfilled at beam test facilities such as DESY. The radiation length measurement method is based on the precise measurement of scattering angles and intersection positions on the target plane (see fig. 2.10). The reconstructed scattering angle distributions can be fitted by a model function based on theoretical descriptions of multiple scattering distributions to determine the local radiation length. The scope of this section is to give an overview of the research presented in this thesis. There are five distinct chapters in which the research conducted in this thesis will be presented.

In chapter 3 the theoretical description of multiple Coulomb scattering processes will introduced. Multiple scattering corresponds to a large number of single Coulomb scatterings on atomic nuclei. Even in comparably thin material layers such as $50 \mu \mathrm{m}$ of aluminium well above 300 scatterings take place. Each scattering leads to a small direction change. Due to the large number of independent scatterings the net effect after traversing the material, called multiple scattering, leads to an approximately Gaussian distributed scattering angle distribution. There are, however, non-Gaussian tails located at large scattering angles caused by rare, large-angle single scattering events. Several different multiple scattering models are introduced in this chapter. The Highland model for example can be used to describe the Gaussian core of the distribution. The Moliere model additionally describes the non-Gaussian tails. For materials with a large radiation length and in case of an electron beam, energy losses due to bremsstrahlung have to be considered. Two different models which take energy losses into account will be presented. Typically, the standard deviation of the Gaussian core of multiple scattering angle distributions is larger than $50 \mu \mathrm{rad}$ and smaller than ten mrad. All of these angles are therefore very small ${ }^{6}$ and precision measurements are needed to determine them accurately.

\footnotetext{
${ }^{6} 1^{\circ}=17.4 \mathrm{mrad}$
} 
In chapter 4 beam test experiments and the analysis and calibration of beam test data will be explained. In this work the Test Beam Software Framework (TBSW) is employed to process the beam test data. The analysis starts with a collection of digitised measurements of the sensor pixels, called digits. The analysis combines these digits to clusters and subsequently yields an estimate of the intersection of a charged particle. Afterwards hits on multiple telescope layers are combined with a Kalman filter tracking approach to precisely predict the intersections and slopes of the particle trajectory on the telescope planes. As multiple scattering angles are very small, trajectory slopes and their uncertainties have to be determined very accurately. This is only possible, when the intrinsic resolution of the telescope sensors and the position and orientation of the telescope planes is well known. Consequently the beam test analysis also includes a telescope calibration step in which these quantities are determined precisely by exploiting the resolution of the reference telescope. In preparation to this thesis, a new scheme to measure the spatial resolution of binary detectors was developed and validated ${ }^{7}$. This measurement scheme is now a core functionality of $T B S W$.

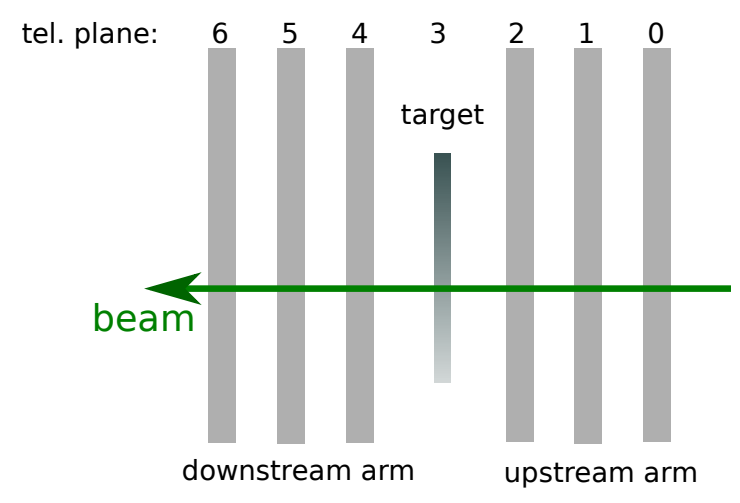

Figure 2.10.: Schematic drawing of a reference telescope with two arrays of three positionsensitive sensor planes, called up- and downstream telescope arm. The scattering target is placed between the upstream and the downstream telescope arm.

Chapter 5 gives a detailed summary of the functionality of spatially resolved radiation length measurements. The $\mathrm{C}++$ code required to extend $T B S W$ to also cover the radiation length measurements was developed and tested in the scope of this thesis. A typical telescope setup for radiation length measurements is depicted in fig. 2.10. The method uses measured tracks in the upstream and downstream telescope arms to reconstruct multiple scattering angles. After matching up- and downstream tracks which are likely coming from the same particle transition according to a distance criterium, the multiple scattering angles are reconstructed from the corresponding track states on the central scattering plane. Due to the finite resolution of the track states, the angle reconstruction broadens the reconstructed scattering distribution. The telescope angle

\footnotetext{
${ }^{7}$ in cooperation with Benjamin Schwenker
} 


\section{The Standard Model of Particle Physics and the Belle II experiment}

resolution, a measure for this broadening, must be determined via error propagation. Wrong assumptions of the beam energy and the telescope angle resolution can have a large impact on the quality of radiation length measurements. Consequently, these parameters must be determined in a radiation length calibration measurement performed on a well known reference target.

In chapter 6 results from Monte Carlo simulation experiments are presented. Simulations provide the opportunity to study, evaluate and disentangle individual systematic effects of the radiation length measurements. Radiation length values are determined by performing a binned fit of scattering angle distributions. In order to find the optimal range and binning of scattering angle histograms, simulations of angle distributions are used. Fits are performed with ROOT [38], which provides two different fitting procedures: Binned $\chi^{2}$ and binned log-likelihood fit. These two different fitting options are tested and compared. Additionally, the influence of position measurements with binary pixel sensors on the reconstructed angle distributions are examined. The chapter ends with the validation of the radiation length calibration measurements.

In chapter 7 several radiation length measurements are presented. The versatility of the method is demonstrated on different scattering targets such as wedges with a continuous material profile and high radiation length values, PXD prototype modules, hardened conductive glues and an ATLAS ITk prototype module. The wedge measurements were conducted to test the upper limit of radiation length values that can be determined with the method introduced in this thesis. For large $X / X_{0}$ values, energy losses due to bremsstrahlung have to be considered and two different approaches to include the energy losses in the fit models are explored. Results from measurements on a PXD prototype were compared to the detector model of the PXD in the Basf2 framework [39]. The comparison revealed flaws in the detector model which have been corrected. The detailed changes are summarised at the end of chapter 7 . 
CHAPTER 3

\section{Interactions between charged particles and matter}

On the following pages, an overview of particle interactions with surrounding matter will be given. The emphasis is on interactions which are especially important for the radiation length measurement method described in chapter 5 and applied in chapters 6 and 7. As all measurements presented in this work were conducted with an electron beam of several $\mathrm{GeV}$, the focus of this chapter will be on the interactions of high-energy electrons. For such large energies, the electron mass can be neglected so that energy $E$ and momentum $p$ are interchangeable

$$
1 \approx \beta=\frac{p c}{E},
$$

where $\beta=v / c$ is the particle velocity relative to the speed of light $c$. Energy loss due to ionisation of the traversed material will be explained in the first section of this chapter. Section two gives an overview of bremsstrahlung. The final section of this chapter concerns Coulomb scattering on atomic nuclei. As the method presented in this work is based on measurements of multiple scattering distributions due to Coulomb scattering, three different multiple scattering models will be explained in detail. Afterwards, a short overview of possible corrections of multiple scattering models in extremely large radiation length materials will be given.

\subsection{Ionisation and energy loss straggling}

A relativistic charged particle traversing matter undergoes a continuous loss of its kinetic energy due to collisions with electrons on the outer shell of atoms. These collisions cause excitations of electrons and thereby ionisations of individual atoms in the traversed 
material. The average energy loss per unit length of a homogeneous material due to ionisation is given by [40] as

$$
-\frac{\mathrm{d} E}{\mathrm{~d} x}=K \frac{Z}{A \beta^{2}}\left(\ln \left(\frac{\gamma m_{e} c^{2} \beta \sqrt{\gamma-1}}{\sqrt{2} I}\right)+\frac{1}{2}\left(1-\beta^{2}\right)-\frac{2 \gamma-1}{2 \gamma^{2}}+\frac{1}{16}\left(\frac{\gamma-1}{\gamma}\right)^{2}\right)
$$

where $\gamma$ is the Lorentz factor, $K$ is a constant with a value of $0.3071 \mathrm{MeV} /\left(\mathrm{g} / \mathrm{cm}^{2}\right)$ and $m_{e}$ is the electron mass. $I$ is the ionisation constant, $Z$ is the atomic charge and $A$ is the number of nucleons of the material. The calculation of ionisation energy losses in mixtures and compounds is more complicated because typically the ionisation energy is larger in composites, where the electrons are more tightly bound. Some strategies for calculating the energy loss in mixtures are explained in [29]. Eq. 3.2 takes screening effects and special kinematics due to electron-electron scattering processes into account.

Energy losses of individual particles due to ionisation, especially in thin materials, are fluctuating. $\mathrm{d} E / \mathrm{d} x$ has a broad distribution with large tails towards large energy losses. This effect is called energy loss straggling and it is best described by a Landau function [41] with the most probable energy loss per unit length given by eq. 3.2.

\subsection{Bremsstrahlung and the radiation length constant $X_{0}$}

Another important contribution to the energy loss of relativistic electrons traversing matter is bremsstrahlung. When moving through matter, electrons are affected by Coulomb fields of nuclei in the proximity of their path. The most common result of these interactions is a direction change of the electron trajectory at a constant energy as described in the next section. However, in some cases the interaction with a nucleus leads to a deceleration of the electron. As a consequence, it loses part of its kinetic energy in the form of an emitted photon. As can be seen from fig. 3.1, this process, called bremsstrahlung, is the predominant source of energy loss for high-energy electrons.

The average energy loss due to bremsstrahlung for high-energy electrons is given by [40] as

$$
-\frac{\mathrm{d} E}{\rho \mathrm{d} x}=\frac{E}{\tilde{X}_{0}}
$$

where $\rho$ is the density of the traversed material. This equation defines the radiation length constant $\tilde{X}_{0}$. According to $[29], \tilde{X}_{0}$ can be expressed via

$$
\frac{1}{\tilde{X}_{0}}=4 \alpha r_{e}^{2} \frac{N_{A}}{A}\left(Z^{2}\left[L_{\mathrm{rad}}-f(Z)\right]+Z L_{\mathrm{rad}}^{\prime}\right)
$$




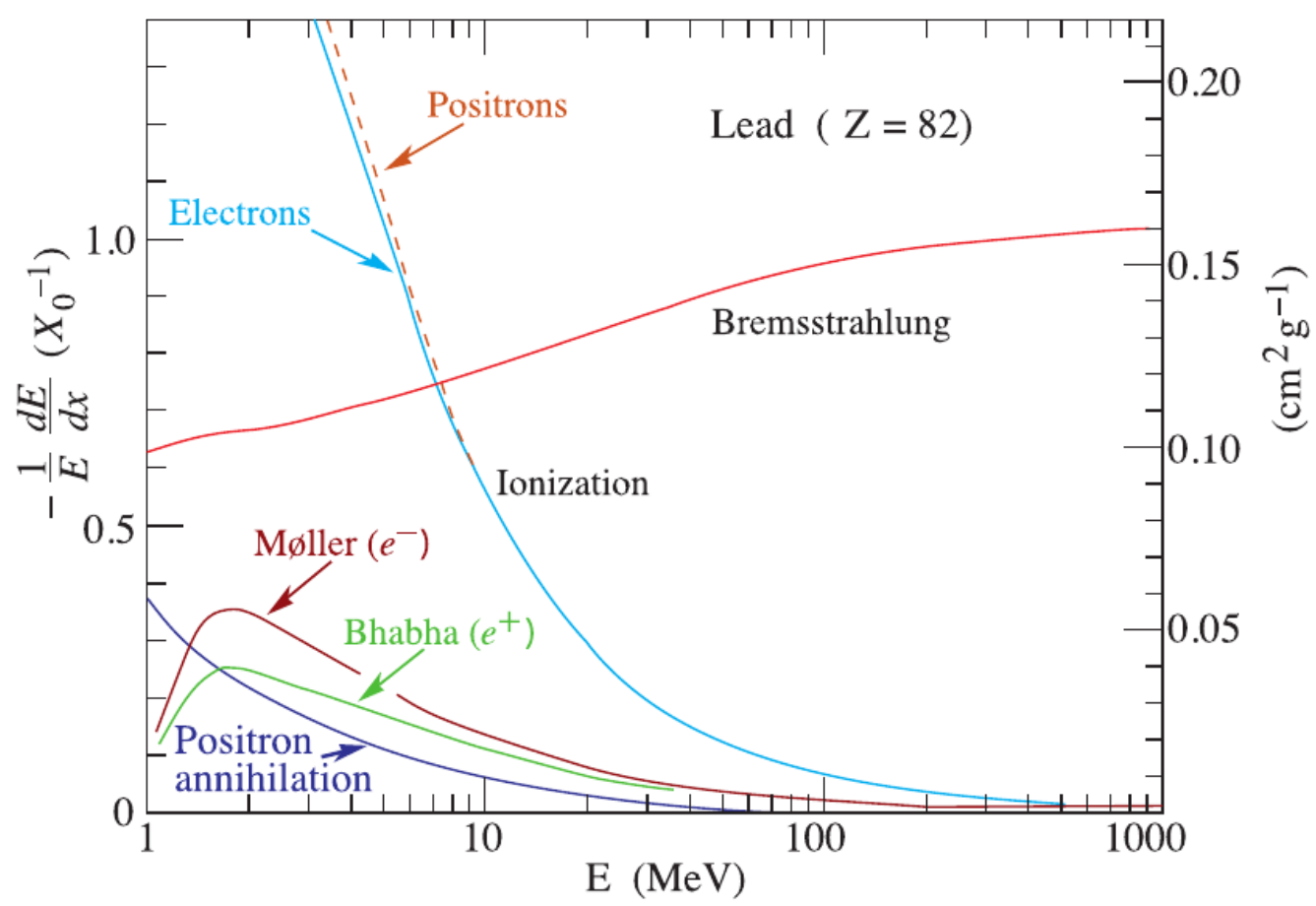

Figure 3.1.: Fractional energy loss in lead as a function of the particle energy for electrons and positrons (from [29]).

Here $\alpha$ is the fine-structure constant, $r_{e}$ is the classical electron radius and $N_{A}$ is Avogadro's number. $L_{\mathrm{rad}}=\ln \left(184.15 Z^{-\frac{1}{3}}\right)$ and $L_{\mathrm{rad}}^{\prime}=\ln \left(1194 Z^{-\frac{2}{3}}\right)$ for elements with $Z$ larger than four. For all elements up to uranium $f(Z)$ can be represented by

$$
f(Z)=\tilde{a}^{2}\left(\left(1+\tilde{a}^{2}\right)^{-1}+0.20206-0.0369 \tilde{a}^{2}+0.0083 \tilde{a}^{4}-0.002 \tilde{a}^{6}\right) \quad .
$$

Here $\tilde{a}=\alpha Z$. A simpler approximative way of calculating the radiation length constant, which is frequently used in the literature [40,42,43], is

$$
\tilde{X}_{0}=\frac{716.4\left[\mathrm{~g} / \mathrm{cm}^{2}\right] A}{Z(Z+1) \ln (287 / \sqrt{Z})}
$$

The radiation length constants $\tilde{X}_{0}$ in eq. 3.4 and 3.6 are given in units of $\mathrm{g} / \mathrm{cm}^{2}$, but they can also be expressed in length units by dividing by the density $\rho$

$$
X_{0}=\frac{\tilde{X}_{0}}{\rho}
$$




\section{Interactions between charged particles and matter}

In this work the dimensionless quantity $X / X_{0}$ is of interest, where the thickness of the material $X$ is given in $\mathrm{cm}, \mathrm{mm}$ or $\mu \mathrm{m}$ and the radiation length constant in eq. 3.7 is used. $X / X_{0}$ is called the radiation length.

The approximation in eq. 3.6 deviates from the exact values in eq. 3.4 by less than $2.5 \%$ for all elements but helium. A comparison between the correct radiation length constants and the constants determined from eq. 3.6 is summarised in table 3.1. A list of radiation length constants for all elements can also be found in [44]. The densities necessary to convert the radiation length constants were taken from [29].

\begin{tabular}{|c|c|c|c|c|c|}
\hline Element & $\boldsymbol{Z}$ & $\boldsymbol{A}$ & $\boldsymbol{\rho}\left[\mathrm{g} / \mathbf{c m}^{\mathbf{3}}\right]$ & $\boldsymbol{X}_{\mathbf{0}}\left[\mathbf{m m}^{\mathbf{2}}\right]$ (from $\left.[\mathbf{2 9}]\right)$ & $\left.\boldsymbol{X}_{\mathbf{0}}\left[\mathbf{m m}^{\mathbf{2}}\right] \mathbf{( e q . ~ 3 . 6}\right)$ \\
\hline \hline $\mathrm{Al}$ & 13 & 26.9898 & 2.699 & 88.97 & 89.92 \\
\hline $\mathrm{Si}$ & 14 & 28.086 & 2.329 & 93.70 & 94.79 \\
\hline $\mathrm{Cu}$ & 29 & 63.5400 & 8.96 & 14.35 & 14.68 \\
\hline $\mathrm{Ag}$ & 47 & 107.87 & 10.50 & 8.54 & 8.74 \\
\hline
\end{tabular}

Table 3.1.: Literature value and approximation of the radiation length constant for different materials relevant for this work. The literature values were used for all relevant calculations in chapter 6 and 7 .

In this work, the approximation in eq. 3.6 is not used, because for most materials a literature value of $X_{0}$ can be found in [29]. However, eq. 3.6 can be used in case no literature value of the radiation length constant is available. According to [29], the radiation length $X_{0}$ of a compound material can be calculated as

$$
\frac{1}{X_{0}}=\sum_{i} \frac{w_{i}}{X_{0 i}}
$$

where $w_{i}$ is the fraction of element $i$ by weight and $X_{0 i}$ is its radiation length. Using eq. 3.3 the resulting average energy of particles after transition of a material with the radiation length constant $X_{0}$ and a thickness $X$ with an electron energy $E_{0}$ at $X=0 \mathrm{~mm}$ is

$$
E(X)=E_{0} \exp \left(-\frac{X}{X_{0}}\right)
$$

Energy loss probabilities due to bremsstrahlung can be described by the Bethe-Heitler model $[45,46]$.

If $E_{i}$ denotes the initial energy of a particle before an energy loss due to bremsstrahlung and $E_{f}$ is the energy of the particle after transition, the energy loss due to bremsstrahlung is given by $\Delta E=E_{i}-E_{f}$. The probability density function $f$ of the ratio of the final energy over the initial energy $g=E_{f} / E_{i}=\left(E_{i}-\Delta E\right) / E_{i}$ is given by 


$$
f(g)=\frac{[-\ln g]^{b-1}}{\Gamma(b)},
$$

where $b$ is

$$
b=X /\left(X_{0} \ln 2\right)
$$

and $\Gamma(b)$ is the Gamma function. Using the cumulative distribution function $F$, one can calculate the probability to have a relative energy loss $\Delta E / E_{i}$ larger than $\xi / E_{i}$ as

$$
p\left(g \leq \frac{E_{i}-\xi}{E_{i}}\right)=F\left(\frac{E_{i}-\xi}{E_{i}}\right)=\int_{-\infty}^{\frac{E_{i}-\xi}{E_{i}}} f(g) d g \stackrel{g>0}{=} \int_{0}^{\frac{E_{i}-\xi}{E_{i}}} f(g) d g .
$$

Table 3.2 gives an overview of the probability of bremsstrahlung processes with resulting ratios $g$ smaller than $80 \%, 90 \%$ and $95 \%$ depending on the material thickness. The numbers were extracted from a simulation, which employs the Bethe-Heitler model of bremsstrahlung. Note that a ratio $g<1-x$ is equal to a relative energy loss $\Delta E / E_{i} \geq x$. Fig. 3.2 depicts the energy distribution $E_{f}$ after energy losses due to the Bethe-Heitler model of a charged particle beam with an initial energy $E_{i}$ of $3 \mathrm{GeV}$.

\begin{tabular}{|c||c|c|c|c|}
\hline Thickness & $1.0 \mathrm{~mm}$ & $2.0 \mathrm{~mm}$ & $6.0 \mathrm{~mm}$ & $30 \mathrm{~mm}$ \\
\hline $\mathbf{p}(\boldsymbol{g} \leq \mathbf{8 0} \%)$ & $(1.836 \pm 0.004) \%$ & $(3.686 \pm 0.006) \%$ & $(10.98 \pm 0.01) \%$ & $(49.28 \pm 0.02) \%$ \\
\hline $\mathbf{p}(\mathbf{g} \leq \mathbf{9 0} \%)$ & $(2.862 \pm 0.005) \%$ & $(5.671 \pm 0.008) \%$ & $(16.42 \pm 0.01) \%$ & $(63.49 \pm 0.03) \%$ \\
\hline $\mathbf{p}(\boldsymbol{g} \leq \mathbf{9 5} \%)$ & $(3.890 \pm 0.006) \%$ & $(7.693 \pm 0.009) \%$ & $(21.73 \pm 0.01) \%$ & $(73.82 \pm 0.03) \%$ \\
\hline
\end{tabular}

Table 3.2.: Probabilities for fractional energy losses due to bremsstrahlung in aluminium. Bremsstrahlung energy spectra are simulated from $10^{7}$ bremsstrahlung events using eq. 3.10. The given probabilities are calculated from the number of events with the corresponding energy losses, stated uncertainties are therefore Poisson uncertainties.

As can be seen from the energy distributions and table entries, for thin materials most particle transitions are not affected by bremsstrahlung at all. There are, however, a few events, where the particle loses a large fraction of its initial energy. The probability for high energy losses increases with the thickness of the traversed material. For example in case of a $30 \mathrm{~mm}$ aluminium layer almost $50 \%$ of traversing particles will lose at least $20 \%$ of their energy due to bremsstrahlung.

\subsection{Coulomb scattering}

The last interaction of interest between electrons and surrounding matter is Coulomb scattering on atomic nuclei. 


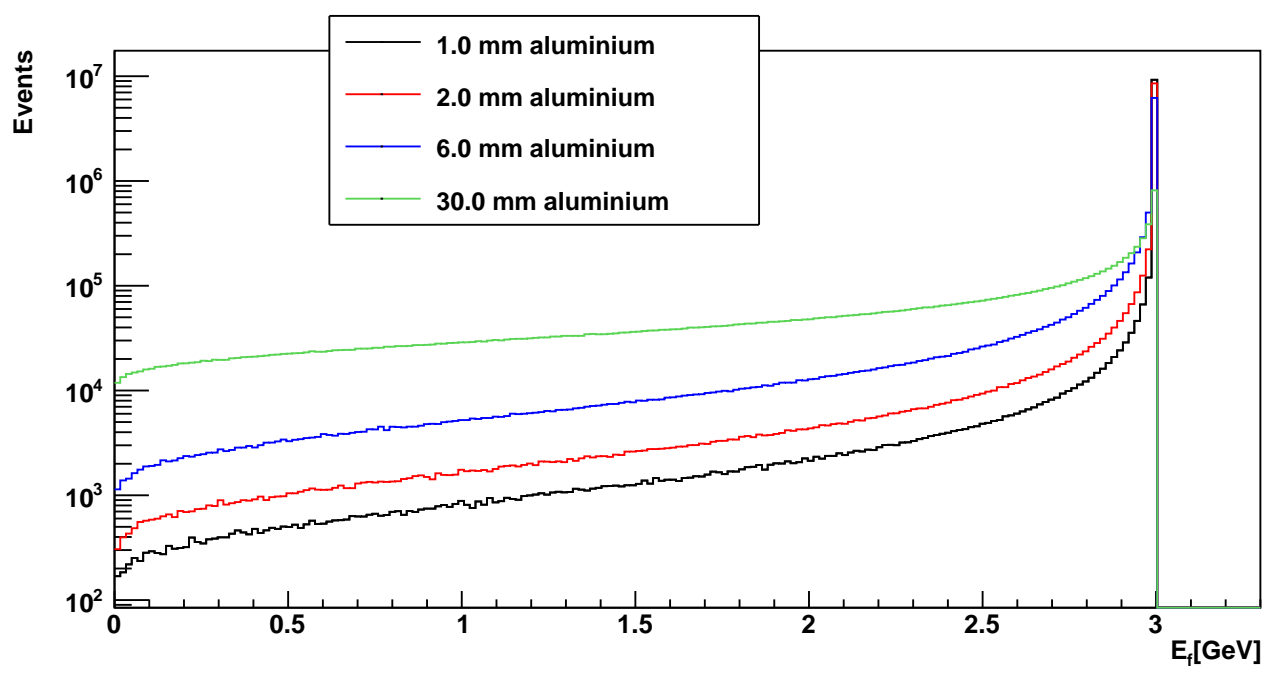

Figure 3.2.: Energy spectra of $10^{7}$ electrons with an initial energy of $3 \mathrm{GeV}$ after traversing an aluminium block with different thicknesses. The distribution peaks at the initial energy. The number of events, which lie in the low energy tail region depends strongly on the target thickness.

\subsubsection{The single Coulomb scattering process}

The following explanations follow the theoretical description of single scattering processes described in detail in [47]. In case of small scattering angles with $\theta \approx \sin (\theta)$, the Coulomb scattering process can be expressed by the differential cross section $\frac{\mathrm{d} \sigma}{\mathrm{d} \Omega}$ from the Rutherford scattering formula

$$
\frac{\mathrm{d} \sigma}{\mathrm{d} \Omega} \approx\left(\frac{2 Z e^{2}}{p v}\right)^{2} \frac{1}{\theta^{4}}
$$

Here, $v$ is the particle velocity and $\mathrm{d} \Omega=\sin \theta \mathrm{d} \theta \mathrm{d} \phi$, where $\theta$ is the polar angle and $\phi$ is the azimuth angle. The initial momentum of the scattered particle is parallel to the $z$ direction. The Rutherford formula has a singularity for $\theta=0$, which is caused by the infinite range of the Coulomb potential of the nucleus. However, the partial shielding of the nucleus charge by shell electrons, called screening effect, causes the cross section to be finite at $\theta=0$. The simplest solution for including this effect is cutting off the Coulomb force at an unspecified radius $r=a$. Considering a scattering process using a classical impact parameter approach yields the following differential cross section

$$
\frac{\mathrm{d} \sigma}{\mathrm{d} \Omega} \approx\left(\frac{2 Z e^{2}}{p v}\right)^{2} \frac{1}{\left(\theta^{2}+\theta_{\min }^{2}\right)^{2}},
$$


where the classical cut-angle $\theta_{\min }=\theta_{\min }^{\mathrm{c}}$ is given by

$$
\theta_{\min }^{\mathrm{c}}=\frac{2 Z e^{2}}{p v a}
$$

However, the approximation is more accurate when a screened potential like

$$
V(r)=\left(\frac{Z e^{2}}{r}\right) \exp \left(-\frac{r}{a}\right)
$$

is used instead of the undisturbed Coulomb potential or the Coulomb potential with a cut-off. Here $a$ is given by $a=1.4 a_{0} Z^{-\frac{1}{3}}$, where $a_{0}$ is the Bohr radius. Using the Born approximation [48] on the potential in eq. 3.16 yields the differential cross section in eq. 3.14 , but with a modified quantum mechanical cut-angle $\theta_{\min }^{\mathrm{q}}[47] . \theta_{\min }^{\mathrm{q}}$ is given by

$$
\theta_{\min }^{\mathrm{q}}=\frac{Z^{\frac{1}{3}}}{192} \frac{m_{e} c}{p}
$$

Note that the modified differential cross section in eq. 3.14 with $\theta_{\min }=\theta_{\min }^{\mathrm{q}}$ does not diverge for $\theta \rightarrow 0$. Due to the finite size of the nucleus, electron trajectories in close proximity to the nucleus can not be assumed to be scattered on a point-like charge distribution like trajectories farther away. In this case, form factors of the nucleus have to be considered. The inclusion of form factors yields an upper boundary of the scattering angles which is given according to [47] by

$$
\theta_{\max }=\frac{274}{A^{\frac{1}{3}}} \frac{m_{e} c}{p}
$$

In case of relativistic particles, such as electrons with energies of several $\mathrm{GeV}$, the maximum scattering angle $\theta_{\max }$ of individual scattering processes is much smaller than $1 \mathrm{rad}$. Accordingly, single scattering angles are generally quite small. However $\theta_{\max }$, is still much larger than $\theta_{\min }$ as can be seen from the ratio

$$
\frac{\theta_{\max }}{\theta_{\min }^{\mathrm{q}}}=\frac{5.26 \cdot 10^{4}}{A^{\frac{1}{3}} Z^{\frac{1}{3}}}
$$

According to [43], the differential cross section can be expressed by the product of the total cross section $\sigma$ and the normalised probability density function $f(\theta)$ of a single scattering process

$$
\frac{\mathrm{d} \sigma}{\mathrm{d} \Omega}=\sigma f(\theta)
$$


The total cross section can be calculated using $\theta_{\max } \gg \theta_{\min }^{q}$, so that

$$
\begin{aligned}
\sigma & =\int \frac{\mathrm{d} \sigma}{\mathrm{d} \Omega} \sin \theta \mathrm{d} \theta \mathrm{d} \phi \approx 2 \pi\left(\frac{2 Z e^{2}}{p v}\right)^{2} \int_{0}^{\theta_{\max }} \frac{\theta \mathrm{d} \theta}{\left(\theta^{2}+\left(\theta_{\min }^{q}\right)^{2}\right)^{2}} \\
& \approx \pi a^{2}\left(\frac{2 Z e^{2}}{h v}\right)^{2} .
\end{aligned}
$$

The normalised probability density function is given by

$$
f(\theta)=\frac{k \theta}{\left(\theta^{2}+\left(\theta_{\min }^{q}\right)^{2}\right)^{2}} I_{\left[0, \theta_{\max }\right]}(\theta),
$$

where $I_{\left[0, \theta_{\max }\right]}(\theta)$ is 1 in the range between zero and $\theta_{\max }$ and zero outside of this range and $k=2 \theta_{\min }^{q}\left(1+\left(\theta_{\min }^{q}\right)^{2} / \theta_{\max }^{2}\right)$ is the normalisation factor. $\theta_{\max }, \theta_{\min }^{\mathrm{q}}$, the mean and the standard deviation of $f(\theta)$ are summarised in table 3.3 for four different materials at a particle energy of $4 \mathrm{GeV}$.

\begin{tabular}{|c|c|c|c|c|c|}
\hline Element & $\boldsymbol{\theta}_{\min }^{\mathbf{q}}[\boldsymbol{\mu r a d}]$ & $\boldsymbol{\theta}_{\max }[\mathbf{r a d}]$ & mean $[\boldsymbol{\mu r a d}]$ & std.dev. $[\boldsymbol{\mu r a d}]$ & $\left(\boldsymbol{\theta}_{\max } /\right.$ std.dev. $)$ \\
\hline \hline $\mathrm{Al}$ & 1.6 & 0.012 & 2.5 & 5.9 & 2000 \\
\hline $\mathrm{Si}$ & 1.6 & 0.012 & 2.5 & 6.1 & 1900 \\
\hline $\mathrm{Cu}$ & 2.0 & 0.009 & 3.2 & 7.4 & 1200 \\
\hline $\mathrm{Ag}$ & 2.4 & 0.007 & 3.8 & 8.5 & 900 \\
\hline
\end{tabular}

Table 3.3.: Important parameters of the single scattering distribution $f(\theta)$ for $4 \mathrm{GeV}$ electrons and nuclei of different materials. The range of the distribution is very large compared to its standard deviation.

As can be seen $f(\theta)$ has an extremely long range, evaluated by $\theta_{\max }$, compared to its standard deviation. The ratios of range to standard deviation are typically in the order of $10^{3}$.

In the scope of this work Cartesian coordinates are used. $\theta$ can be expressed by two projected scattering angles via

$$
\begin{aligned}
& \vartheta_{u}=\theta \cos \phi \\
& \vartheta_{v}=\theta \sin \phi .
\end{aligned}
$$

A simulation of single scattering angles $\theta$ can be achieved by utilising the following expression [43]:

$$
\theta=\theta_{\min } \theta_{\max } \sqrt{\frac{1-t}{t \theta_{\max }^{2}+\theta_{\min }^{2}}}
$$


Here, $t$ is a uniformly distributed random number between zero and one. The projected angles are calculated according to eq. 3.23 or 3.24 and another uniformly distributed random number $\phi$ between 0 and $2 \pi$.

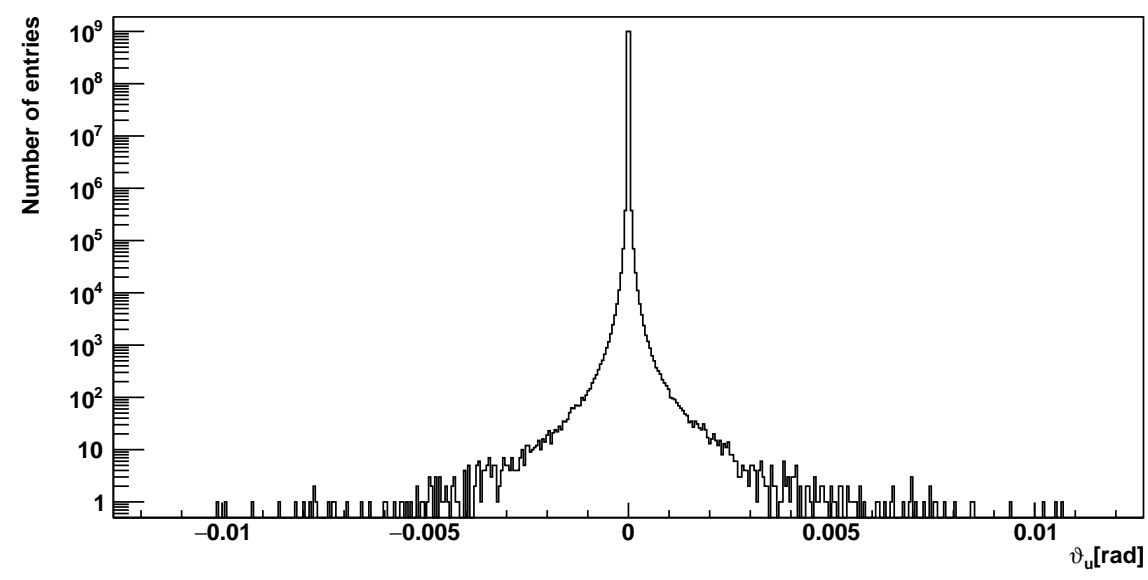

Figure 3.3.: Single scattering distribution for $\vartheta_{u}$ consisting of 2 billion simulated angles of one $4 \mathrm{GeV}$ particle scattering on one silicon nucleus. The probability for small scattering angles is very large, but there are long tails with rarely occurring large scattering angles. The maximum scattering angle is $\theta_{\max }=0.0115 \mathrm{rad}$.

Fig. 3.3 depicts a simulated distribution of $\vartheta_{u}$ for $4 \mathrm{GeV}$ electrons scattering on a silicon nucleus. The projected scattering angle distribution is symmetric around $\vartheta_{u}=0$. Accordingly, the mean of the distribution is equal to zero. As already mentioned, the range of the distribution is large compared to the standard deviation. However, large angles are extremely rare in single scattering events.

\subsubsection{Multiple Coulomb scattering}

An electron traversing material undergoes many single scatterings on individual atomic nuclei. The mean number of single scatterings in a material with thickness $X$ is given by [43]

$$
N_{\text {scatt. }}=\sigma \frac{X N_{A} \rho}{A}
$$

Where $\sigma$ is given by eq. 3.21 and $N_{A}$ is Avogadro's number. According to [43], $N_{\text {scatt. }}$ can also be expressed as 


$$
N_{\text {scatt. }}=\frac{1}{\beta^{2}} \frac{X}{X_{0}}\left(\frac{1.587 \cdot 10^{7} Z}{Z^{\frac{2}{3}}(Z+1) \ln \left(287 Z^{-\frac{1}{2}}\right)}\right),
$$

where the radiation length constant $X_{0}$ is given by eq. 3.7 and $Z$ is the atomic charge. As can be seen, the average number of scatterings depends on the radiation length $X / X_{0}$. The average number of scatterings of a high-energy electron $(\beta \approx 1)$ for different scattering materials and thicknesses are summarised in table 3.4. The selected quantities are representative of the materials and thicknesses measured in chapter 7 of this work.

\begin{tabular}{|c|c|c|c|}
\hline Element & $\mathbf{X}[\mathbf{m m}]$ & $\boldsymbol{X} / \boldsymbol{X}_{\mathbf{0}}[\mathbf{\%}]$ & $\boldsymbol{N}_{\text {scatt. }}$ \\
\hline \hline $\mathrm{Al}$ & 0.05 & 0.06 & 343 \\
\hline $\mathrm{Al}$ & 1.0 & 1.12 & 6857 \\
\hline $\mathrm{Al}$ & 20.0 & 22.48 & 137000 \\
\hline $\mathrm{Cu}$ & 0.05 & 0.35 & 1590 \\
\hline $\mathrm{Cu}$ & 1.0 & 6.97 & 31900 \\
\hline $\mathrm{Cu}$ & 20.0 & 139.37 & 637000 \\
\hline
\end{tabular}

Table 3.4.: Average number of single scattering processes for three different thicknesses of aluminium and copper for particles with $\beta \approx 1$

Even for extremely thin materials, such as $50 \mu \mathrm{m}$ of aluminium, on average well above 100 single scatterings take place. The overall effect of a multitude of single scatterings is called multiple scattering.

\subsubsection{Modelling multiple scattering}

Large numbers of single scatterings lead, according to the central limit theorem, to a Gaussian scattering angle distribution. However, due to the large range of the single scattering distribution, the convergence to a Gaussian distribution is very slow. Multiple scattering distributions consist of a Gaussian core and non-Gaussian tails which are caused by individual rare single scattering events with large scattering angles.

Fig 3.4 depicts a multiple scattering process in the $u$ - $w$ plane of a Cartesian coordinate system $(u, v, w)$, where $w$ points in the direction of the initial moment of the particle and $u$ and $v$ are arranged in the scattering plane. Multiple scattering leads to a direction change evaluated by a solid angle $\theta$ and a spatial shift evaluated by a vector $Y$. The $u$ and $v$ axes can be defined in analogy to the single scattering case in eq. 3.23 and 3.24 and as already mentioned the solid angle corresponds to two projected angles $\vartheta_{u}$ and $\vartheta_{v}$. The spatial shift vector can be expressed by $Y=\left(y_{u} y_{v}\right)^{\mathrm{T}}$. Fig. 3.4 depicts a schematic drawing of a multiple scattering process in one of the two projected planes. Multiple scattering processes are symmetric with respect to rotations around the axis defined by the initial trajectory of the incoming particle. The choice of the $u$ and $v$ 
axis is therefore arbitrary and distributions of $\vartheta_{u}$ and $\vartheta_{v}$ can be described by the same probability density function. Therefore, instead of $\vartheta_{u}$ and $\vartheta_{v}$, on the following pages the generalised quantity of the projected angle $\vartheta_{p}$ and projected spatial shift $y_{p}$ will be used.

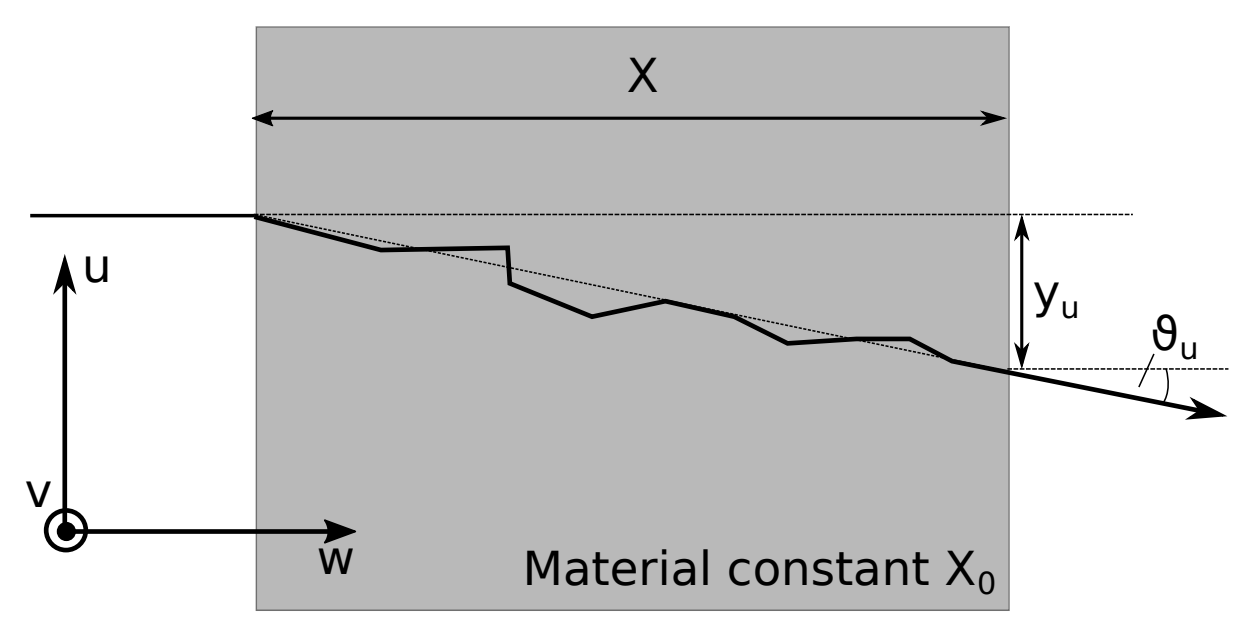

Figure 3.4.: Schematic drawing of a multiple scattering process in a projected plane. The multiple scattering process leads to a direction change and a spatial shift.

On the following pages three basic approaches to modelling multiple scattering will be explained. The first possibility to model multiple scattering distributions of a given material is a simulation of the single scattering processes:

- The average number of scatterings for the given radiation length has to be computed. The number of single scatterings for individual particles is determined from a Poisson distribution with a mean given by eq. 3.27 .

- For each single scattering process single scattering angles are determined according to the procedure described by eq. $3.25,3.23$ and 3.24 .

- Single scattering angles are quite small $(\mathcal{O}(\mu \mathrm{rad}))$. Accordingly, it can be assumed that the net scattering angle is still small (well below $1^{\circ}$ ) after a large number of single scatterings. Therefore, $\sin \vartheta_{p} \approx \vartheta_{p}$ and the multiple scattering projected angle is given by the sum of the projected single scattering angles.

The second possible multiple scattering model was introduced by Highland [29, 49]. It consists of a Gaussian function $f_{\mathrm{HL}}$ with a standard deviation $\sigma_{\mathrm{HL}}$ and describes the central $98 \%$ of a multiple scattering angle distribution 


$$
\begin{aligned}
\sigma_{\mathrm{HL}} & =\frac{0.0136}{\beta p[\mathrm{GeV}]} \sqrt{\frac{X}{X_{0}}}\left(1+0.038 \ln \left(\frac{X}{X_{0}}\right)\right) \\
f_{\mathrm{HL}}\left(\vartheta_{p}, p, \frac{X}{X_{0}}\right) & =\frac{1}{\sigma_{\mathrm{HL}} \sqrt{2 \pi}} \exp \left(-\frac{1}{2}\left(\frac{\vartheta_{p}}{\sigma_{\mathrm{HL}}}\right)^{2}\right) .
\end{aligned}
$$

Lynch [50] determined the two numerical values (0.0136 and 0.038) in eq. 3.28 so that eq. 3.29 is a valid model of the core width of scattering angle distributions for all elements. According to [50], the Highland model in eq. 3.29 describes multiple scattering angle distributions at an uncertainty below $11 \%$ for all $Z$. As mentioned before, multiple scattering can not only cause a direction change but also a spatial shift in the plane of the scattering material (see fig. 3.4). The rooted mean square (RMS) of the projected spatial shift due to multiple scattering is proportional to the thickness $X$ of the material and the width of the angle distribution $\sigma_{\mathrm{HL}}$ (given in eq. 3.28)

$$
y_{p}^{\mathrm{RMS}}=X \cdot \sigma_{\mathrm{HL}} .
$$

The RMS of the projected spatial shift is typically quite small. Some examples of Highland widths and spatial shift RMS values for different materials, thicknesses and particle energies, that will be encountered in this work are summarised, in table 3.5.

\begin{tabular}{|c|c|c|c|c|c|}
\hline Element & $\boldsymbol{p}[\mathbf{G e V}]$ & $\mathbf{X}[\mathbf{m m}]$ & $\boldsymbol{X} / \boldsymbol{X}_{\mathbf{0}}[\boldsymbol{\%}]$ & $\boldsymbol{\sigma}_{\mathbf{H L}}[\mathbf{\mu r a d}]$ & $\boldsymbol{y}_{\boldsymbol{p}}^{\mathrm{RMS}}[\mathbf{\mu m}]$ \\
\hline $\mathrm{Al}$ & 2 & 0.05 & 0.056 & 115 & $\mathcal{O}\left(10^{-3}\right)$ \\
\hline $\mathrm{Al}$ & 4 & 0.05 & 0.056 & 58 & $\mathcal{O}\left(10^{-3}\right)$ \\
\hline $\mathrm{Al}$ & 2 & 1.0 & 1.124 & 598 & 1 \\
\hline $\mathrm{Al}$ & 4 & 1.0 & 1.124 & 299 & $\mathcal{O}\left(10^{-1}\right)$ \\
\hline $\mathrm{Al}$ & 2 & 20.0 & 22.482 & 3041 & 60 \\
\hline $\mathrm{Al}$ & 4 & 20.0 & 22.482 & 1521 & 30 \\
\hline $\mathrm{Cu}$ & 2 & 0.05 & 0.341 & 311 & $\mathcal{O}\left(10^{-2}\right)$ \\
\hline $\mathrm{Cu}$ & 4 & 0.05 & 0.341 & 156 & $\mathcal{O}\left(10^{-2}\right)$ \\
\hline $\mathrm{Cu}$ & 2 & 1.0 & 6.812 & 1594 & 2 \\
\hline $\mathrm{Cu}$ & 4 & 1.0 & 6.812 & 797 & 1 \\
\hline $\mathrm{Cu}$ & 2 & 20.0 & 136.24 & 8030 & 160 \\
\hline $\mathrm{Cu}$ & 4 & 20.0 & 136.24 & 4015 & 80 \\
\hline
\end{tabular}

Table 3.5.: Highland width and RMS of the projected shifts for different materials, thicknesses and particle energies, that are representative of the radiation lengths and energies used in this work.

The RMS of the spatial shift is in the order of several $\mu \mathrm{m}$ in most cases. As will be shown in chapter 4 , this is smaller than the best possible track resolution on the central plane of a typical telescope and can therefore be neglected. However, for materials with a large thickness $X$ or small $X_{0}$ the shifts may be in the order of $100 \mu \mathrm{m}$ and can be 
resolved. The Highland width $\sigma_{\mathrm{HL}}$ ranges from tens of $\mu$ rad for low-radiation-length materials to a few mrad for high-radiation-length materials.

A third possibility to model multiple scattering was introduced by Moliere [51]. A detailed explanation of the Moliere model can be found in [52]. The fully analytical Moliere model describes both the core and the tails of a multiple scattering angle distribution. The probability density function $f_{\mathrm{M}}$ of multiple scattering angles is given by

$$
f_{\mathrm{M}}\left(\vartheta_{p}\right) \mathrm{d} \vartheta_{p}=\frac{1}{\chi_{c} \sqrt{B}}\left(\frac{2}{\sqrt{\pi}} e^{-\frac{\theta}{\chi_{c} \sqrt{B}}}+\frac{f_{1}\left(\vartheta_{p}\right)}{B}+\frac{f_{2}\left(\vartheta_{p}\right)}{B^{2}}+\ldots\right) \mathrm{d} \vartheta_{p},
$$

where (for $p c$ in $\mathrm{GeV}) \chi_{c}$ is given as

$$
\chi_{c}=\frac{0.0229 Z}{p c \beta} \cdot \sqrt{\frac{\rho X}{A}}
$$

and $B$ is given by the transcendental equation

$$
\ln B-B=b=\left(8.215+\log _{10}\left[Z^{-\frac{2}{3}} \frac{\rho X}{A} a^{2}\left(1.13+3.76 a^{2}\right)\right]\right)
$$

with $a=\frac{Z}{137 \beta^{2}}$. Function $f_{i}$ can either be determined analytically via

$$
f_{i}\left(\vartheta_{p}\right)=(i !)^{-1} \int_{0}^{\infty} u J_{0}\left(\vartheta_{p} u\right) \exp \left(\frac{1}{4} u^{2}\right)\left[\frac{1}{4} u^{2} \ln \left(\frac{1}{4} u^{2}\right)\right]^{i} \mathrm{~d} u
$$

where $J_{0}$ is the Bessel function. Alternatively, $f_{i}$ function values can be taken from from table II in [52]. Here the tabulated values of $f_{1}$ and $f_{2}$ up to angles of $4 \chi_{c} \sqrt{B}$ are used, as this range is typically sufficient for our purpose. For a $4 \mathrm{GeV}$ electron beam traversing $0.5 \mathrm{~mm}$ of silicon, $\chi_{c}$ has a value of approximately $90 \mu \mathrm{rad}$ and $\sqrt{B}$ is approximately 3 . Accordingly, with function values $f_{1}$ and $f_{2}$ from the table an projected angle range of $\vartheta_{p} \in[-1080 \mu \mathrm{rad},+1080 \mu \mathrm{rad}]$ is covered.

A comparison between the scattering angle distributions from the simulation of the single scatterings, the Highland and the Moliere model are depicted in fig. 3.5 for $4 \mathrm{GeV}$ electrons traversing a $0.5 \mathrm{~mm}$ silicon layer. The process of simulating single scattering angles and computing their sum is time-consuming due to the large average number of single scatterings $(\approx 3100)$. However, simulating the single scattering angles is the best way to describe a real multiple scattering distribution. 


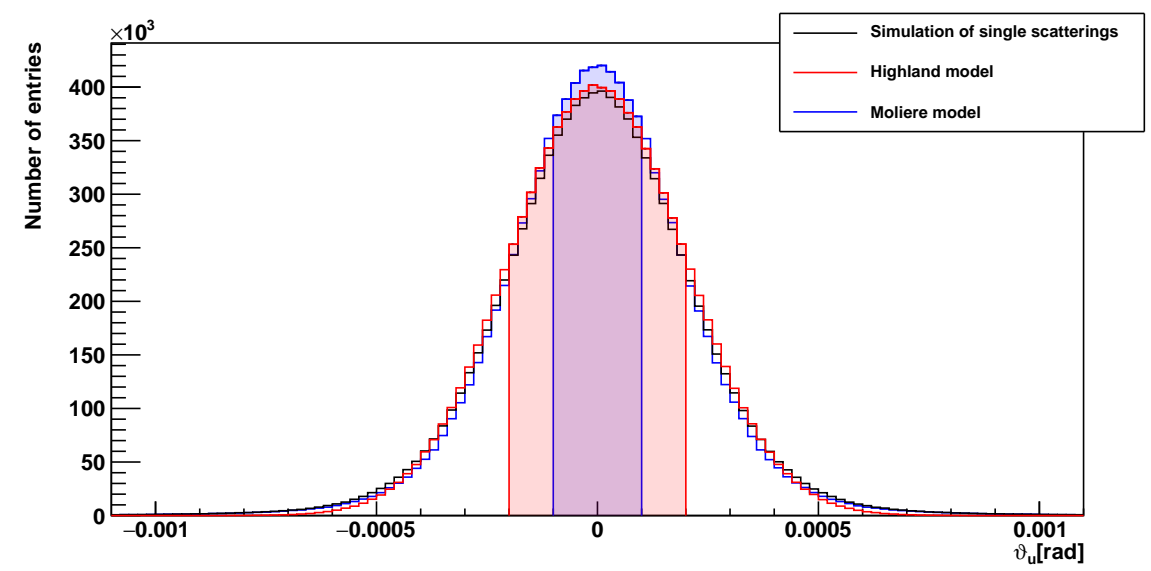

(a) zoomed-in,linear distributions

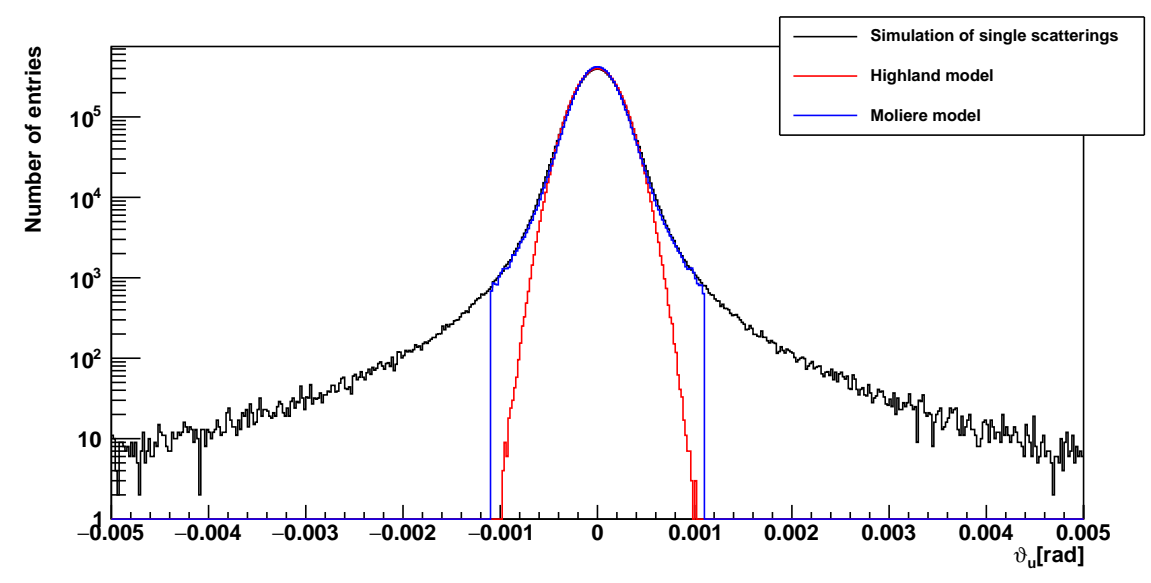

(b) logarithmic distributions

Figure 3.5.: (a) Linear and (b) logarithmic comparison between three different multiple scattering models. 10 billion angles have been simulated according to the Moliere (blue) and Highland (red) model as well as simulations of the single scattering processes (black) for $4 \mathrm{GeV}$ electrons traversing $0.5 \mathrm{~mm}$ of silicon. The highlighted red area in (a) corresponds to the interval between $\pm \sigma_{\mathrm{HL}}$ of the Highland distribution. The highlighted blue area in (a) corresponds to interval between $\pm \chi_{c}$ of the Moliere distribution. The Moliere model in (b) falls to zero abruptly at $\pm 4 \chi_{c} \sqrt{B}$ because a limited number of tabulated $f_{1}$ and $f_{2}$ was used.

As can be seen in fig. 3.5, the multiple scattering angle distribution has large nonGaussian tails, even though the average number of scatterings is quite large. As explained earlier, this is ultimately caused by the large tails of the single scattering angle distribution (see fig. 3.3). 
The Moliere model describes the tail region well in the investigated region of $\vartheta_{p}$ up to $\pm 4 \chi_{c} \sqrt{B}$, where it abruptly falls to zero. This drop is not part of the Moliere model, but caused by using the table entries for $f_{1}$ and $f_{2}$. Additionally, small deviations from the angle distribution according to simulations of single scatterings can be observed. The Highland model describes the multiple scattering distribution very well in the core region, but as expected fails to describe the long non-Gaussian tails. As the tails are caused by individual scattering processes, the form of the tails contains no information about the thickness $X$ of the scattering material. Information about the radiation length $X / X_{0}$ can be gained from the width of the core of the multiple scattering distribution, which depends on the average number of single scatterings, which in turn depends on $X / X_{0}$ (see eq. 3.27). For all measurements in chapter 6 and 7 the Highland model is employed.

\subsubsection{Multiple scattering on high radiation length materials}

In case of multiple scattering on materials with radiation length values $\left(>10 \% X / X_{0}\right)$ the Highland width $\sigma_{\mathrm{HL}}$ in eq. 3.28 is inadequate to describe the core of the scattering distribution due to additional energy losses. In this case the approximation that the particle energy stays constant during the transition and can be expressed by the energy before the scattering is flawed: Depending on the radiation length, individual particles are likely to lose energy by emitting a bremsstrahlung photon. The average particle energy during the material transition is described by eq. 3.9: It decreases exponentially with the radiation length. In the last part of this section, two possibilities to include this energy loss due to bremsstrahlung in the Highland model of multiple scattering are discussed. Applications of both are shown in chapter 7 .

The first possibility to take additional energy losses into account is to replace the constant particle energy $p$ in eq. 3.28 by a weighted mean of the average energy before and after the material transition. The average particle energy after the transition of a material with the radiation length $X / X_{0}$ (expressed by the symbol $p_{\mathrm{f}}$ ) can be calculated from the energy before the transition $p_{\mathrm{i}}$ and the radiation length $X / X_{0}$ via eq. 3.9

$$
p_{\mathrm{f}}\left(\frac{X}{X_{0}}\right)=p_{\mathrm{i}} \exp \left(-\frac{X}{X_{0}}\right)
$$

The weighted mean of the energy before $\left(p_{\mathrm{i}}\right)$ and after $\left(p_{\mathrm{f}}\right)$ the scattering $p_{\mathrm{wm}}$ is then given by

$$
p_{\mathrm{wm}}=p_{\mathrm{i}} \cdot\left((1-\epsilon)+\epsilon \cdot \exp \left(-\frac{X}{X_{0}}\right)\right)
$$

The weight factor $\epsilon$ has to be determined experimentally. Using the corrected energy $p_{\mathrm{wm}}$ instead of the constant energy $p$ in eq. 3.28 yields 


$$
\sigma_{\text {weight }}=\frac{0.0136}{\beta \cdot p_{\mathrm{wm}}[\mathrm{GeV}]} \cdot \sqrt{\frac{X}{X_{0}}}\left(1+0.038 \ln \left(\frac{X}{X_{0}}\right)\right)
$$

This modification of the Highland width will be called the weight model in chapter 7 .

Another possibility is to use the modification of the Highland model introduced by Gottschalk [53]. For thick scattering materials an integral over the material thickness is introduced

$$
\sigma_{\mathrm{GS}}=0.0136\left(1+0.038 \ln \left(\frac{X}{X_{0}}\right)\right) \sqrt{\int_{0}^{X} \frac{1}{\left(p_{\mathrm{f}} \beta\right)^{2}} \frac{\mathrm{d} X}{X_{0}}}
$$

By inserting eq. 3.35 into eq. 3.38 the following expressions are obtained

$$
\begin{aligned}
\sigma_{\mathrm{GS}} & =\frac{0.0136}{p_{\mathrm{i}}[G e V] \beta}\left(1+0.038 \ln \left(\frac{X}{X_{0}}\right)\right) \sqrt{\frac{1}{2}\left(\exp \left(2 \frac{X}{X_{0}}\right)-1\right)} \\
& \approx \frac{0.0136}{p_{\mathrm{i}}[G e V] \beta}\left(1+0.038 \ln \left(\frac{X}{X_{0}}\right)\right) \sqrt{\frac{X}{X_{0}}+\left(\frac{X}{X_{0}}\right)^{2}+\ldots}
\end{aligned}
$$

The parametrisation of the Highland formula from Lynch [50] (see eq. 3.28) is slightly different from the parametrisation in Gottschalks publication [53]. Instead of $0.0136 \mathrm{GeV}$ Gottschalk uses $14.1 \mathrm{MeV}$ and the logarithm term is $1 / 9 \log _{10}\left(X / X_{0}\right)$ instead of 0.038 $\ln \left(X / X_{0}\right)$. In this work, the original parametrisation of Lynch will be used, because it is valid for a larger range of materials. Comparisons between the two modified multiple scattering models, the weight model in eq. 3.37 and the Gottschalk model in 3.40, can be found in sections 7.1.2 and 7.2. 


\section{CHAPTER 4}

\section{Beam test experiments: Experimental setup and analysis of data}

Beam tests with monoenergetic particle beams provide a good opportunity to test the performance of new detector systems. The performance is tested by triggering individual charged particles and reconstructing their trajectory in an array of pixel sensors with high granularity, called a high-resolution reference telescope. The reconstructed trajectory, called track, is then extrapolated to a device under test (DUT) and can be used as an external probe to measure the DUT response. In order to conduct these conventional beam test measurements with a DUT, a common trigger and data acquisition system is required.

The novel concept presented in this thesis is based on the idea of installing a passive scattering target as a DUT in between the telescope arms and measuring scattering angles and positions. Scattering angle distributions depend on the radiation length of the traversed material. Therefore, radiation length profiles of the scattering plane can be measured. Details on the radiation length measurements are described in chapter 5 . In this chapter the basic workflow of ordinary beam tests, consisting of of clustering, position reconstruction, tracking and alignment, is explained instead.

The structure of this chapter is as follows: First, the beam generation and experimental environment at DESY and CERN beam test sites will be described in section 4.1. Facilities at DESY in Hamburg and CERN near Geneva are the two most convenient locations for beam test campaigns in Europe. The focus lies on the DESY beam, because all results in this work are based on data recorded at the DESY beam test facilities. However, in principle measurements at CERN SPS are possible as well and for measurements on high radiation length materials the higher beam energy compared to DESY might be advantageous. In section 4.2 the setup, readout and functionality of reference beam telescopes will be explained. The most common reference telescope type is the 
EUDET telescope which is used at DESY and CERN. In section 4.3 an overview over the sequential analysis steps in the test beam software framework (TBSW) will be given. $T B S W$ was used to perform calibration and reconstruction steps on beam test data and produce the results in chapter 6 and 7 . The calibration of the reference telescope and reconstruction of particle tracks from digitised measurements of the deposited energy of charged particles, or short digits, on the telescope sensors will be explained. Section 4.4 summarises the results of a beam test conducted in October 2016. The whole chain of analysis steps explained earlier was used to conduct measurements of the efficiency and the intrinsic resolution of M26 sensors at different comparator thresholds. Finally, in section 4.5 an overview of beam test simulations in $T B S W$ is given.

\subsection{Generation of high energy particle beams}

The two most easily accessible beam test facilities from Europe are located at the CERN and DESY. After a number of intermediate steps the storage ring at DESY, called DESY II, supplies collimated electron or positron beams with mean energies between 1 and $6 \mathrm{GeV}$. The particle flux, which corresponds to the number of particles per second and unit area, depends on the beam energy. The particle rate given by the flux in the full area of the beam can reach values of $40 \mathrm{kHz}$ with fully open collimators [54]. The CERN SPS accelerator provides a large range of particles such as hadrons and electrons. The particles used in the beam tests are often charged pions with energies in the order of $100 \mathrm{GeV}$. Alternatively, by selecting appropriate secondary targets an electron beam can be generated [55]. The following section gives an overview over the beam generation at DESY, where the measurements presented in this work were performed.

The particle beam at the DESY facilities is ultimately generated by the DESY II accelerator. DESY II is a synchrotron with a circumference of $293 \mathrm{~m}$ that accelerates electrons. The primary purpose of DESY II is to provide the synchrotron source PETRA III with pre-accelerated electrons. Four beam test areas T21, T22 and the combined area T24 and T24/1 are parasitic users of DESY II electrons. Fig. 4.1 depicts a schematic overview over the beam generation at DESY beam tests. $7 \mathrm{GeV}$ electrons in the synchrotron hit a primary carbon fibre target and produce a beam of bremsstrahlung-photons [56]. A secondary target is located further downstream. It consists, depending on the choice of the user, of several millimetres of copper or aluminium. During the transition of the secondary target photons are converted into electron-positron pairs. A dipole magnet separates the electrons from the positrons and leads to a horizontal fan-out of the particle beam according to their energy. The current of the magnet can be steered by the beam test user and a collimator opening located further downstream functions as a selector of the particle type and energy. Electrons or positrons with the selected energy arrive in the area, where the reference telescope is located, and can be used for measurements.

The rate of the particle flux in the beam areas depends on the selected beam energy and secondary target material and thickness. As can be seen in fig. 4.2, the rate of 


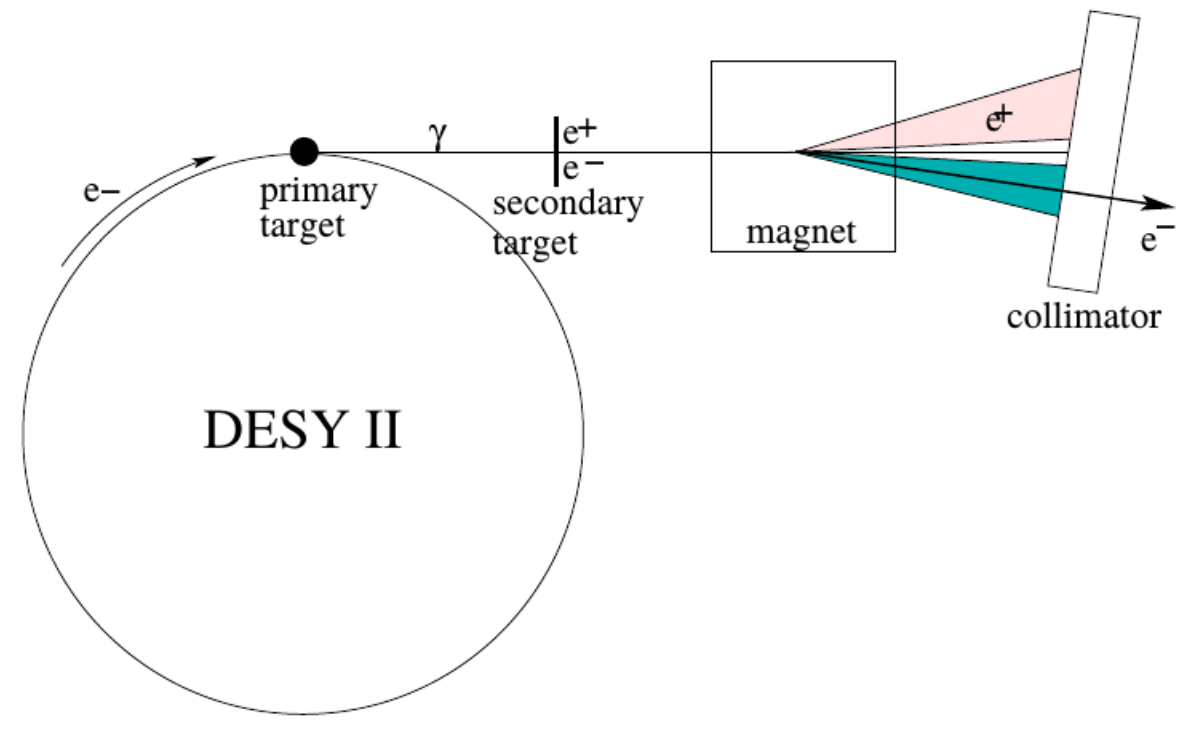

Figure 4.1.: Beam generation in a beam test area at the DESY beam test facilities (from [56]). $7 \mathrm{GeV}$ electrons hit a primary carbon fibre target and produce bremsstrahlung photons. The photons hit a conversion target to produce $e^{+}-e^{-}$pairs. The particle beam traverses a magnetic field which leads to a horizontal fan-out of the particles. The configuration of the magnet and the collimator determine the energy and flux of the particle beam.

incoming particles lies between $100 \mathrm{~Hz}$ to $5 \mathrm{kHz}$. As a larger continuous particle rate increases the number of measured particle tracks, it is generally beneficial to use copper as the secondary target material. The particle rate decreases rapidly at the fringe of the particle energy range. It is therefore advantageous for a fast data collection to select beam energies between 2 and $4 \mathrm{GeV}$.

The horizontal fan-out of the particle beam downstream of the magnet leads to a small energy dependence on the horizontal position. The spread is limited due to a collimator system downstream of the magnet which reduces the maximum beam spot area to approximately $10 \times 15 \mathrm{~mm}^{2}$. Simulation results in [57] give a good overview of the beam energy spread that can be expected for different energies. During the measurements in preparation for this work, horizontal energy gradients of several $\mathrm{MeV} / \mathrm{mm}$ have been observed. The relative uncertainty of the beam energy at DESY is expected to be $6 \%$ for a beam energy of $2 \mathrm{GeV}$ and below $5 \%$ for larger beam energies [54]. 


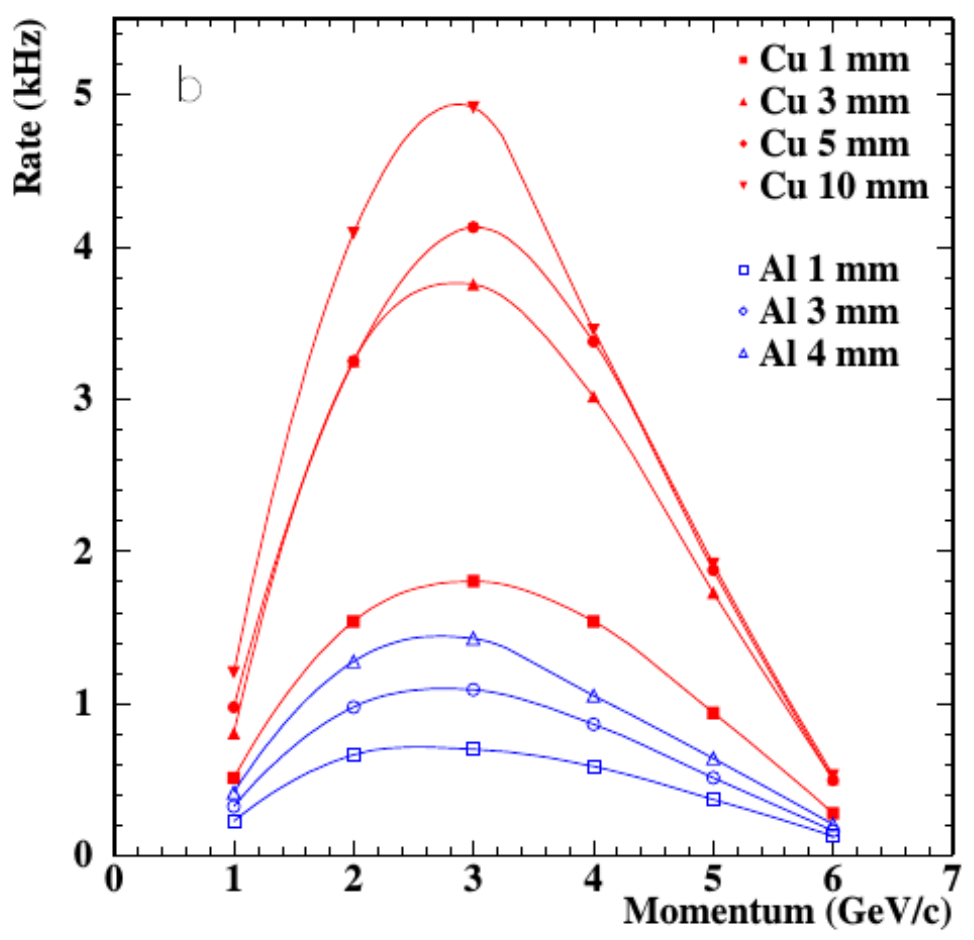

Figure 4.2.: Measured particle rate as a function of the selected beam energy for different secondary targets in T22. Picture taken from [56].

\subsection{Reference telescopes}

Performance tests of pixel detector prototypes are often conducted by operating the DUT in a reference telescope with a common trigger and readout system. There are a number of requirements for a reference telescope employed in beam tests. First of all, the telescope must consist of two telescope arms with three sensors per telescope arm. The sensors should have a good efficiency and intrinsic resolution so that the spatial resolution of the telescope between the two telescope arms is in the order of $5 \mu \mathrm{m}$. Additionally, the telescope should have lightweight mechanics to limit the amount of material traversed by the beam particles which is beneficial to the spatial resolution of the telescope.

In order to maximise the data taking efficiency, the sensitive area of telescope sensors is required to be at least as large as the beam spot. If this is not the case a non-negligible fraction of potential particle transitions is lost and cannot be used in measurements. Finally, a trigger, run control and data acquisition system is needed that steers the operation of the telescope, the sensor readout and saves the collected data to disk. Any 
telescope that suffices these requirements can be used for beam test measurements. Here, however, EUDET telescopes and their infrastructure will be described, as the EUDETtype telescopes called DATURA and DURANTA [54] were used for all measurements presented in chapter 7 .

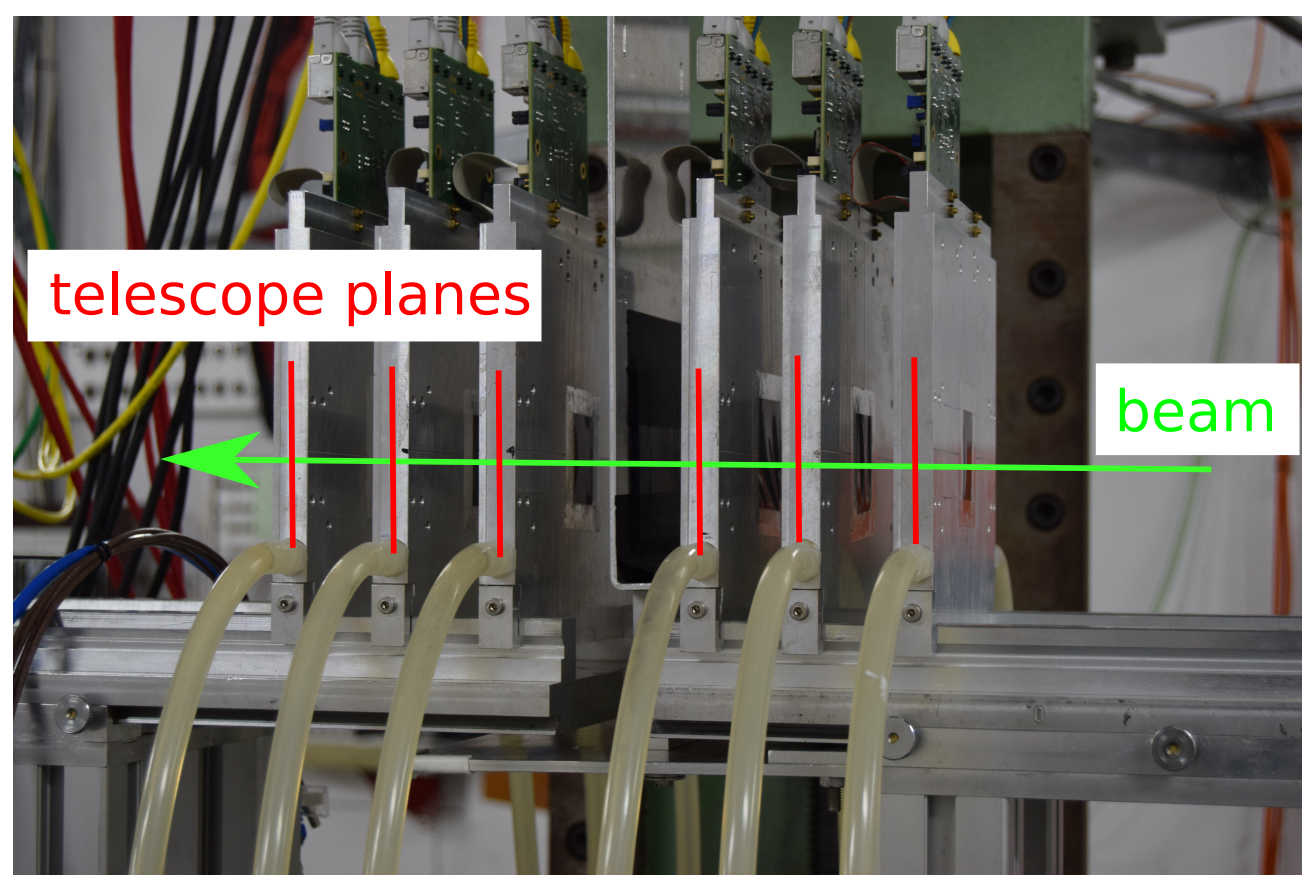

Figure 4.3.: Photograph of an EUDET beam telescope during a measurement in March 2015. The path of the particle beam is indicated in green and the telescope planes are indicated in red.

The European project EUDET provided a high-resolution telescope for position measurements of charged particles, a fast trigger and a data acquisition system with the aim to support research and development of International linear collider detector prototypes [58]. A photograph of an EUDET telescope installed in a beam area at DESY is depicted in fig. 4.3. The telescope consists of two telescope arms with three planes of high-resolution Mimosa26 (M26) silicon pixel sensors [59] each. Some of the most important characteristic values of the M26 sensors are summarised in table 4.1. For the radiation length measurements described in chapter 7 a scattering plane has to be positioned between the two telescope arms. Two sets of two scintillators up- and downstream of the six telescope planes can be used to provide a trigger signal from charged particles. In case of a trigger signal, the M26 pixel data is read out and sent to a data acquisition system called EUDAQ running on a designated PC. The data from all six M26 sensors for a single trigger is merged, labelled with an event number and saved to disk. 
4. Beam test experiments: Experimental setup and analysis of data

\begin{tabular}{|c|c|c|c|}
\hline Sensor & Pitch $[\mathbf{\mu m}]$ & Size $\left[\mathbf{m m}^{\mathbf{2}}\right]$ & Thickness $[\mathbf{\mu m}]$ \\
\hline \hline Mimosa 26 & 18.4 & $21.2 \times 10.6$ & 50 (silicon) +25 (carbon) \\
\hline
\end{tabular}

Table 4.1.: Characteristic values of Mimosa 26 pixel sensors

Mimosa26 sensors are the first large scale sensor from the MIMOSA (Minimum Ionizing MOS Active pixel sensor) series. They are based on the complementary metal-oxide semiconductor (CMOS) technology [60]. The sensitive area consists of a $50 \mu \mathrm{m}$ silicon layer. However, M26 sensors are located inside a box, which protects the sensors from light. In the region of the sensitive area, two carbon windows, one up- and one downstream of the sensitive area, with a combined thickness of $25 \mu \mathrm{m}$ are placed. The material of the windows has to be added to the silicon thickness in order to model the material budget of M26 sensors correctly. The resulting radiation length per M26 layer corresponds to $0.07 \% X / X_{0}$. M26 sensors have a pixel matrix consisting of 576 rows and 1152 columns of sensor pixels that cover an area of approximately $2.2 \mathrm{~cm}^{2}$. M26 pixels have a symmetric pitch of $18.4 \mathrm{\mu m}$ [59], which leads to a very good inherent spatial resolution of the M26 sensors and consequently a very good spatial resolution of the telescope.

M26 sensors provide a binary output due to signal discrimination and zero suppression steps, which take place on the chip. Accordingly, only pixels with a signal larger than the selected comparator threshold are read out and the coordinates of these firing pixels are send to EUDAQ. The comparator threshold is defined as an integer value multiplied with the average sensor pixel noise level $\sigma_{\text {noise }}$. The integer value is selected by the beam test user. In practice, the M26 sensor is divided into four vertical segments. For each segment the average noise $\sigma_{\text {noise }}$ is determined and the local threshold of the M26 segment is set accordingly [61]. In [59] these segments are mentioned as quarters of the M26 sensor in chapter 3. A good threshold setting provides maximum hit efficiency, while minimising fake hits from noisy pixels.

The measured position information from M26 sensors can be used to model particle trajectories with tracks and determine the intersection and incidence angle of the charged particle on planes within the telescope volume. The resolution of the determined track intersection depends on the geometrical setup of the telescope planes, especially the distance between neighbouring planes, the beam energy and the selected M26 sensors read-out threshold. Some typical resolution values can be found in [62] and in chapter 7.

\subsection{The Test Beam Software Framework (TBSW)}

All beam test measurements presented in this work have been analysed with the Test Beam Software Framework (TBSW) [63]. Data analysis in TBSW is organised using a modular application framework called Marlin (Modular Analysis \& Reconstruction for 
the Linear Collider) [64]. Marlin processors are customised C++ classes that provide functionalities to carry out all different reconstruction and calibration steps in a beam test data analysis. The processors are grouped in a path to form a processing pipeline. The data is passed event by event through the pipeline. A processor can read data from events and add new data to the event as output. For example, a track finding processor requires a hit collection as input and adds a track collection as output. Data persistence needed to pass data from processor to processor is achieved with LCIO (Linear Collider I/O) files [65]. The EUDAQ library is embedded in $T B S W$ to sequentially read raw data events and start the analysis pipeline. The paths and configurations of the processors in the path are handled by a python script. The python module xml.tree [66] is used to construct XML steering files for Marlin on the fly. An example of such a xml steering file of a simple clustering path can be found in the appendix A.1.

\subsubsection{Telescope geometry model in TBSW}

An important preparation for the actual analysis of beam test data is to describe positions and rotations of individual sensors in the global coordinate system of the telescope. In $T B S W$, the nominal geometry information of the telescope is stored in XML files. This information includes material information such as the radiation length. The parsing of the XML files is handled by a Marlin library. An example geometry XML file can be found in the appendix A.2.

A schematic side view of a typical telescope and the definition of local coordinates on a telescope plane is depicted in fig. 4.4. The telescope planes are approximately perpendicular to and arranged along the beam axis. Small tilts in the order of a few degrees between the different telescope planes are expected. Each telescope plane has an associated coordinate system which is defined in the following way: The origin of the coordinate system lies in the centre of the sensitive area of the telescope sensor. The $u$ and $v$ coordinate axes are arranged in the telescope plane. The coordinate $u$ increases with the readout columns, while $v$ increases with readout rows of the telescope sensor ${ }^{1}$. The third coordinate $w$, which is perpendicular to the sensor surface, completes the right handed coordinate system. The global telescope coordinate system $(x, y, z)$ used in this work corresponds to the local coordinate system of the first telescope plane to be crossed by the particle beam. In the case of perpendicular incidence the $z$ axis can be identified as the beam axis ${ }^{2}$. The transformation of global $\left(\vec{r}=(x, y, z)^{\mathrm{T}}\right)$ to local $\left(\vec{q}^{k}=\left(u^{k}, v^{k}, w^{k}\right)^{\mathrm{T}}\right)$ coordinates of sensor $k$ can be expressed via

$$
\vec{q}^{k}=R_{0}^{k}\left(\vec{r}-{\overrightarrow{r_{0}}}^{k}\right),
$$

where $\overrightarrow{r_{0}}=\left(x_{0}, y_{0}, z_{0}\right)^{\mathrm{T}}$ is the translation vector from the origin of the global to the origin of the local coordinate system of telescope sensor $k$ and $R_{0}$ is the corresponding

\footnotetext{
${ }^{1}$ For M26 sensors with 1152 columns and 576 rows and a symmetric pixel pitch of $18.4 \mathrm{\mu m}, u$ ranges between $\pm 10.6 \mathrm{~mm}$ and $v$ ranges between $\pm 5.3 \mathrm{~mm}$.

${ }^{2}$ This is the most natural arrangement for a hit correlation based alignment
} 


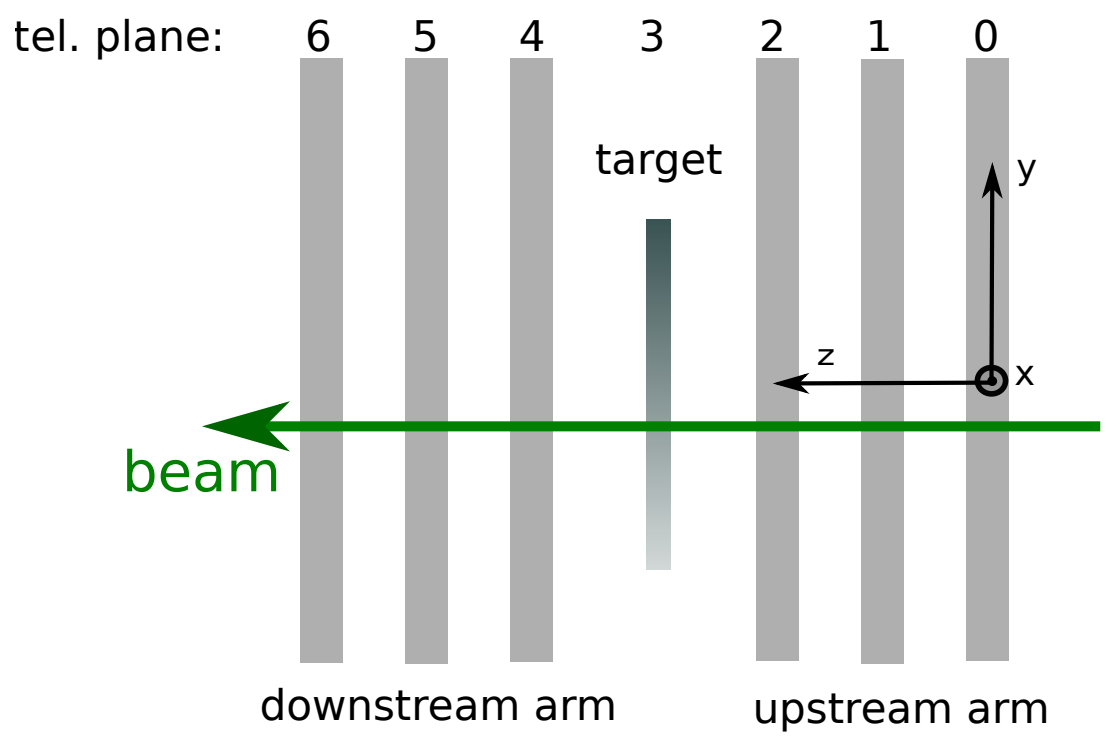

(a)

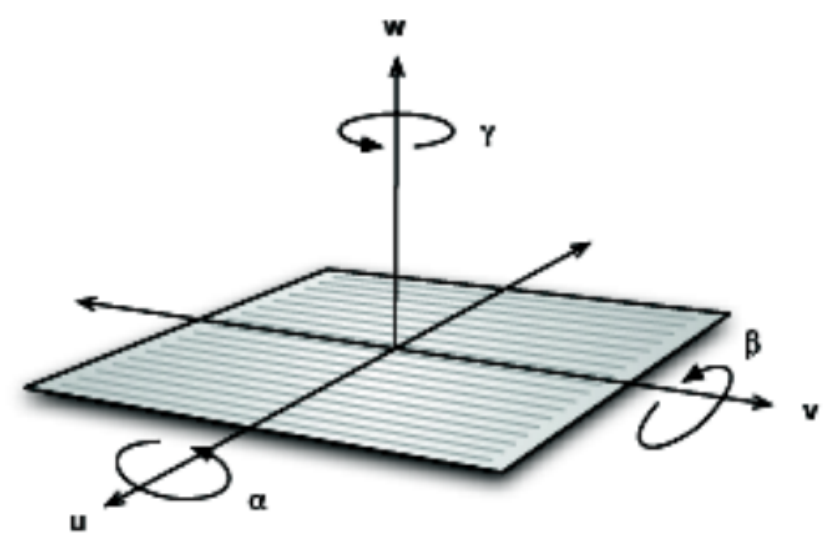

(b)

Figure 4.4.: Typical telescope setup for beam test measurements. The telescope consists of an upstream and a downstream telescope arm with three sensors each. The global coordinate system $(x, y, z)$ corresponds to the local coordinate system of the most upstream plane and is indicated in sub-figure (a). Local coordinates $(u, v, w)$ and the associated rotation angles $\alpha, \beta$ and $\gamma$ of an individual telescope plane are depicted in subfigure (b) (from [67]).

rotation matrix. For the sake of simplicity the index $k$ is left out from now on for $\vec{q}^{k}, R_{0}^{k}$ and ${\overrightarrow{r_{0}}}^{k}$. In $T B S W, R_{0}$ is expressed by the product of three rotation matrices 
$R_{1}\left(\alpha_{0}\right), R_{2}\left(\beta_{0}\right)$ and $R_{3}\left(\gamma_{0}\right)$ describing the continuous rotation on the axis $u, v$ and $w$ and a discrete rotation matrix $R_{\mathrm{D}}$. The parametrisation of the telescope planes is selected in such a way that the continuous rotations are small, typically in the order of a few degrees. There are eight possibilities to embed the local coordinate system $u, v, w$ into the global coordinate system when assuming that they are perpendicular to the beam axis which coincides with the global $z$ axis. Accordingly, the discrete rotations can be expressed as

$$
R_{\mathrm{D}}=\left(\begin{array}{ccc}
d_{1} & d_{2} & 0 \\
d_{3} & d_{4} & 0 \\
0 & 0 & d_{5}
\end{array}\right)
$$

where $d_{i}=( \pm 1,0)$ for $i=1,2,3,4$. As $R_{\mathrm{D}}$ is a rotation matrix, only a eight combinations of values for $d_{1}$ to $d_{4}$ are valid and $d_{5}$ is not selected but determined from the rotation matrix condition. The three continuous angles are indicated in the schematic drawing $4.4 \mathrm{~b}$ and correspond to the three Euler angles. The total rotation $R_{0}$ can be expressed via

$$
R_{0}=R_{3}\left(\gamma_{0}\right) R_{2}\left(\beta_{0}\right) R_{1}\left(\alpha_{0}\right) R_{\mathrm{D}} \quad .
$$

For all measurements in chapter 7, telescope planes are arranged so that the local coordinate axes approximately point in the same direction in the global coordinate system. In other words the angles $\alpha_{0}, \beta_{0}$ and $\gamma_{0}$ are expected to be close to $0^{\circ}$.

Since during the beam test analysis requires translation and rotations between the global and individual local coordinates systems, the XML file describing the telescope geometry must contain values for the $d_{1}, d_{2}, d_{3}, d_{4}, x_{0}, y_{0}, z_{0}, \alpha_{0}, \beta_{0}$ and $\gamma_{0}$ of each telescope plane. The six parameters for each plane documented in the XML file are called the nominal geometry. This geometry corresponds to the best estimation of the real telescope geometry based on manual measurements. It is expected that the real telescope geometry, the misaligned geometry, deviates from the nominal geometry. The goal of the track based alignment explained later is to determine small shifts and rotation between the nominal and the misaligned geometry. The result of the alignment is the aligned geometry.

\subsubsection{Overview of the telescope calibration steps}

Fig. 4.5 depicts a schematic overview of paths and collection of paths that are required to conduct telescope calibration measurements. Typically, each analysis step is performed by a single path which is represented by a rectangle in the figure. However, some analysis steps either require multiple different paths or iterations of the same path. This is indicated by stacks of rectangles as can be seen for example for the alignment and cluster calibration steps. The goal of the telescope calibration measurement is to generate a set of calibration data bases (DB), the hotpixelDB, alignmentDB and clusterDB, that 


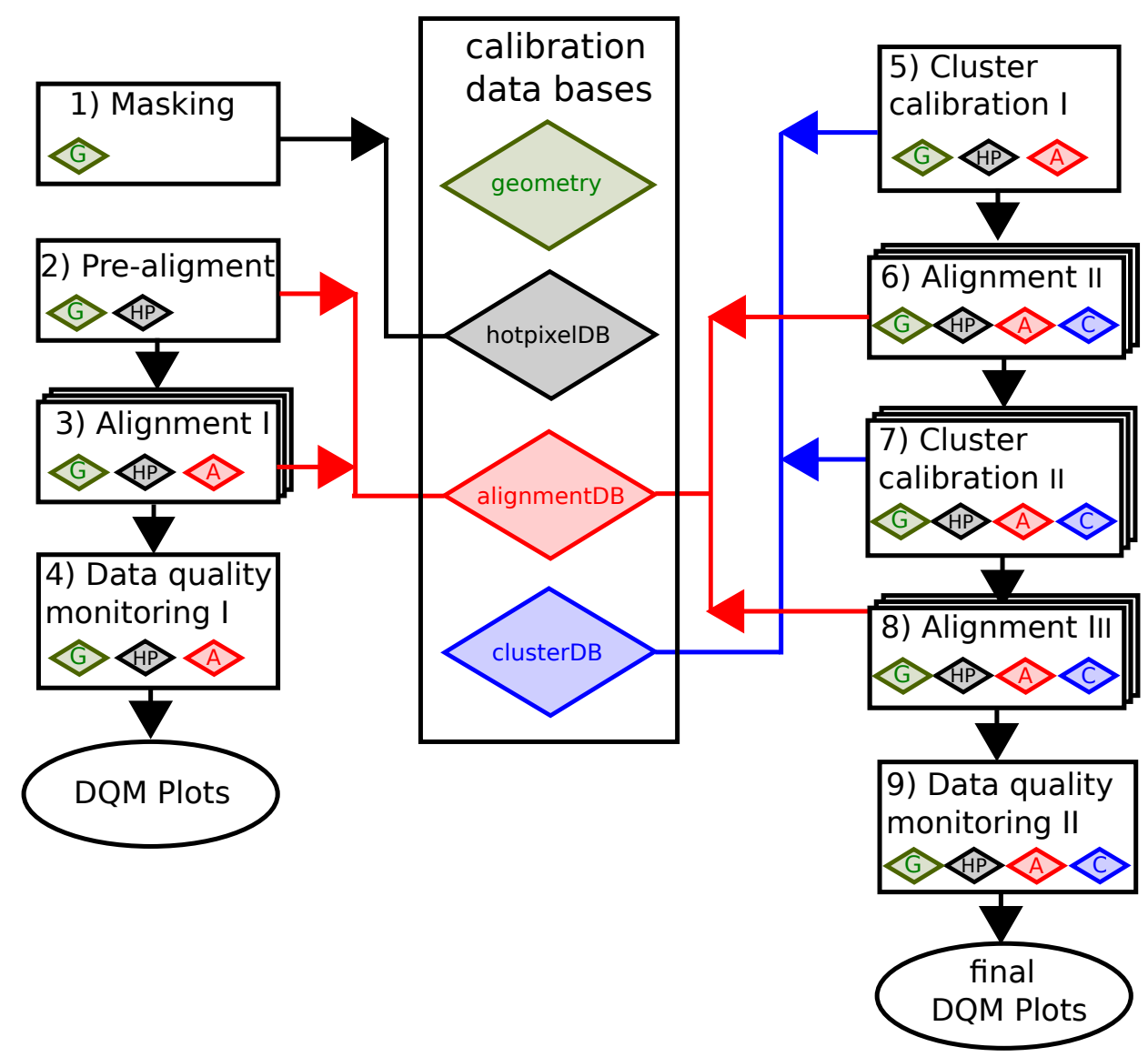

Figure 4.5.: Flow chart that depicts the sequence of paths required to perform telescope calibration measurements. Rectangles represent a single path. The telescope calibration starts with the masking step and proceeds with clustering. Alignment and cluster calibration tasks are typically performed by multiple paths which are indicated by a stack of rectangles. The goal of the telescope calibration is to generate data bases, the hotpixelDB, alignment $D B$ and cluster $D B$ that can be used for the subsequent analysis of the data.

are stored in a calibration database folder and can be used by subsequent calibration and analysis steps. In fig. 4.5 the data bases are represented by diamonds of different colours. The presence of such a diamond inside a rectangle, representing a path, means that the path uses the information contained in the DB file. Arrows that start at paths and end at a data base indicate that the corresponding path updates the information in the data base file.

The analysis starts with the masking path which is used to identify noisy pixels and stores them in the hotpixelDB. This data base is used in all subsequent paths and the pixels marked as noisy pixels are ignored in the analysis steps afterwards. In the pre- 
alignment path, a hit based pre-alignment of the $x$ and $y$ positions of the telescope is conducted. The determined offsets are stored in the alignmentDB. In the alignment steps reconstructed tracks are used to determine $x, y, z$ and $\gamma$ on all inner detector planes and update the alignment $D B$ accordingly. In the cluster calibration steps a track based measurement of particle intersections for different cluster shapes are used to conduct a measurement of cluster resolutions. The results, estimated particle intersections and their covariance matrices for different cluster shapes, are stored in the clusterDB. As can be seen from the figure, alignment and cluster calibration steps are performed multiple times. The reasoning behind this is explained in section 4.3.11. Finally, there are data quality monitoring paths which are used to generate histograms that are useful to determine whether the telescope calibration worked.

All paths in fig. 4.5 are required to read and process data from the EUDAQ raw files. The Marlin processor used for this purpose is called EUDAQInputProcessor. This processor employs the EUDAQ file reader to sequentially read EUDAQ raw files and create an event collection. The EUDAQ data is stored in a format that can be read by Marlin. The next processor is the NIUnpacker which decodes the data format and produces a collection of digits that can be used by subsequent processors. An example of a single event with digits can be seen on the left side of fig. 4.6. Here, it can be assumed that the digits are zero suppressed. Accordingly, all digits in the collection come from pixels with a signal larger than a predefined comparator threshold. The digits themselves are considered to have a signal of one.

Additional information and details of individual processors and analysis steps can be found in [67]. All initial work, in particular Kalman filter based track fitting and telescope alignment algorithm which forms the basis of $T B S W$, was started with [67].

\subsubsection{Hot pixel masking and reconstruction of clusters from digits}

The masking path in fig.4.5 is the first step of the telescope calibration. The masking path tests each telescope sensor for pixels which produce signals too frequently. These pixels, called hot pixels, measure hit signals in absence of real particle transitions and provide no useful information. Hot pixels are collected in the hotpixelDB. Digits originating from hot pixels listed in the data base are ignored in the subsequent analysis of telescope data. In order to determine hot pixels a number of $M$ events are processed. For each sensor pixel on every plane of the telescope the total number $N$ of signals over threshold in these $M$ events is determined. A pixel is considered a hot pixel in case the signal rate $f=N / M$ is larger than a predefined rate $f_{0}{ }^{3}$.

After the generation of the hotpixelDB the next step is to combine digits to clusters. This is done by the PixelClusterizer processor. A simple example of a clustering process is depicted in fig. 4.6. Clusters are a collection of digits, which are likely to be caused

\footnotetext{
${ }^{3}$ Typically in the order of $1 \%$
} 

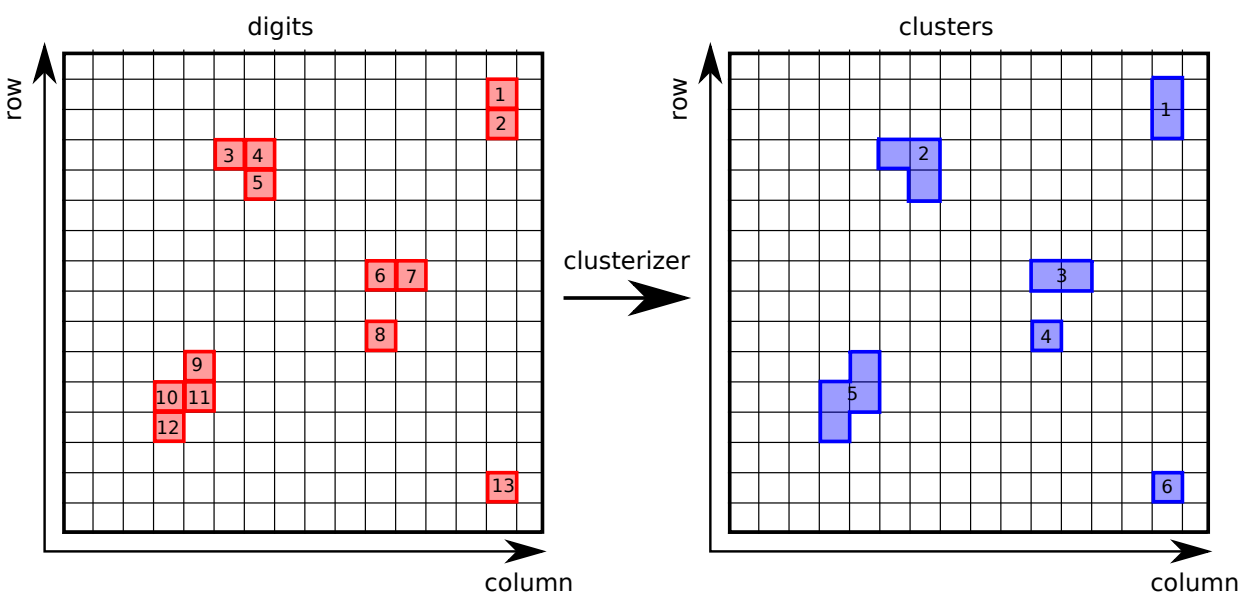

Figure 4.6.: Schematic visualisation of the PixelClusterizer processor. Digits with a neighbour are combined to form a multi-digit cluster, while isolated digits form single-digit clusters. The 13 digits on the left are combined to six clusters on the right.

by the same charged particle transition. In case the particle transition takes place in the vicinity of pixel edges multiple neighbouring pixels may collect enough charge to produce a signal over threshold. In this case it is important to combine all digits into a cluster.

$T B S W$ uses the sparse clustering approach. The clustering algorithm starts with a list of all digits. Another list that will be used to store clusters is empty at the beginning. The first digit is added to the first cluster object. The next digit is added to the first cluster, if it is coming from a neighbour pixel of the digit which is already assigned to the first cluster. Digits are considered to be neighbours, when the corresponding sensor pixels either share a common edge or corner. In case it is not coming from a neighbouring pixel the second digit is assigned to the second cluster. This procedure is repeated until all digits are assigned to a cluster. In case a digit is neighbour to two digits already associated with different clusters these clusters are merged and the digit is added to the combined cluster. After the clustering process, a list of clusters for every sensor in every event is stored to disk. Clusters consist of a mixture of single isolated digits and multiple adjacent digits.

\subsubsection{Definition of particle hits and centre-of-gravity approach to hit reconstruction}

In the next step clusters are used to reconstruct intersection positions of particle transitions and their associated hit position covariance matrix. The objects describing these quantities are called hits. Per definition they consist of a vector corresponding to the intersection position on detector plane $k$ 


$$
m_{k}=\left(\begin{array}{c}
u^{\mathrm{H}} \\
v^{\mathrm{H}}
\end{array}\right)
$$

and an associated covariance matrix $V_{k}$

$$
V_{k}=\left(\begin{array}{cc}
\sigma_{u^{\mathrm{H}}}^{2} & \sigma_{u^{\mathrm{H}} v^{\mathrm{H}}}^{2} \\
\sigma_{u^{\mathrm{H}} v^{\mathrm{H}}}^{2} & \sigma_{v^{\mathrm{H}}}^{2}
\end{array}\right) .
$$

A simple approach to reconstruct a list of hits from the list of clusters is the centreof-gravity algorithm. In $T B S W$ this algorithm is implemented in the CogHitmaker processor. In order to determine the hit position, a weighted mean of the centre position of the individual digits is computed for each cluster. In the case of M26 sensors with binary readout, all digits of a cluster are weighted equally.

The centre-of-gravity approach assumes that the off-diagonal entries of the covariance matrix are zero. The uncertainty $\sigma_{u^{\mathrm{H}}}$ depends on the cluster size $S_{u}$ and the pixel pitch $P_{u}$ in $u$ direction [68]. Analogously, $\sigma_{v^{\mathrm{H}}}$ depends on the size and pitch in $v$ direction. In the worst case only the pixel that was traversed by the charged particle measures a signal. Then the hit resolution is given by the binary hit resolution as [68]

$$
\sigma_{u^{\mathrm{H}}, v^{\mathrm{H}}}=\frac{P u, v}{\sqrt{12}} .
$$

In the case of the optimal resolution, particle transitions close to edges of pixels and subsequent charge sharing with the closest neighbouring pixel leads to measurements of two-digit-clusters. Only for particle transitions near the centre of the pixel singledigit-clusters are measured. Accordingly, the pixel can be separated into two areas: One where a charged particle transition is likely to produce one-digit-clusters and one where two-digit-clusters are likely to be measured. In case of an equal number of one and two-digit-clusters this leads to the binary resolution (eq. 4.6) divided by 2

$$
\sigma_{u^{\mathrm{H}}, v^{\mathrm{H}}}=\frac{P_{u, v}}{2 \sqrt{12}} .
$$

The centre-of-gravity approach calculates the variances in both direction and for all cluster sizes via

$$
\sigma_{u^{\mathrm{H}}, v^{\mathrm{H}}}^{2}=\left(\frac{C\left(S_{u, v}\right) S_{u, v} P_{u, v}}{\sqrt{12}}\right)^{2} .
$$

The correction factors $C\left(S_{u, v}\right)$ of the variances must be selected beforehand. $S_{u, v}$ is the size of the cluster in $u$ or $v$ direction. The pixel pitches are read from the geometry 


\section{Beam test experiments: Experimental setup and analysis of data}

XML file. The correctness of the hit position covariance matrix entries depends on good input constants $C$. Too large or small covariance entries can lead to a bias during the track fit. In order to circumvent this issue, another more sophisticated hit making algorithm can be used. It employs track-based measurements of particle intersections and their resolutions for the most common cluster types to calculate the hit intersection and covariance matrix. These measurements are called cluster calibration and will be explained later in subsection 4.3.10.

\subsubsection{Track model and seeding}

At this point of the analysis it can be assumed that for every event multiple hits have been reconstructed on each telescope sensor. Typically the telescope planes are numbered as indicated in fig. 4.4a. Assuming a telescope with six sensor planes, the most upstream plane corresponds to $k=0$ and the most downstream plane corresponds to $k=5$. TBSW employs the following track model when no magnetic field is present, which is the case in this thesis. The particle trajectory is modelled by $3 \mathrm{D}$ straight lines with multiple scattering kinks at discrete positions at sensors planes and half way between sensors in air.

The goal of the track finding is to select a set of hits which are likely to originate from the same particle trajectory. The track finding itself is a combinatorial task: Hit combinations have to be tried out and sorted according to a quality parameter. In order to start the track finding algorithm a seed track is required. The seed track defines a straight line that is extrapolated onto telescope planes. By extrapolating the seed track from telescope plane to telescope plane and incrementally adding hits to the seed track, a track candidate is formed. There are many different possibilities to start the track finding with a seed track. A frequently used approach is to iteratively select hits on two telescope planes (typically planes $k=0$ and 1) and to connect them to form a 3D straight line between the hit positions. This straight line can be parametrised in the local coordinate system of the telescope sensor at the $k$ th position along the beam

$$
\eta_{k}=\left(\begin{array}{c}
d u / d w \\
d v / d w \\
u \\
v \\
\frac{q}{p}
\end{array}\right)
$$

where $q$ is the charge and $p$ is the momentum of the particle. $q / p$ is therefore determined by the particle beam. Because of the limited beam divergence ${ }^{4}$, nearly all particles move on trajectories parallel to the beam axis, which is approximately parallel to the global $z$ axis. Accordingly, the slopes of the seed track $d u / d w$ and $d v / d w$ should be small for all viable track candidates. It is therefore prudent to limit the slopes and discard all

\footnotetext{
${ }^{4}$ At DESY beams typically between 1 and $2 \mathrm{mrad}$
} 
seed tracks that fail the slope criterium to avoid a time consuming track extrapolation through the telescope of highly unrealistic tracks.

Another possibility to create a seed track, called single hit seeding, is used when the number of hits per plane is large and therefore trying out all combinations of hits of two planes is very slow. In this case the seed track is constructed from a single hit typically on the most upstream $(k=0)$ or most downstream $(k=5)$ telescope plane. The seed track is formed by extrapolating the hit position along the global $z$ axis. Both seeding methods are used in chapter 7 .

\subsubsection{Kalman filter tracking}

$T B S W$ uses Kalman filters for track finding and fitting. This approach to track fitting was introduced by Frühwirth [69]. In case of a linear dynamic system and Gaussian measurement and process noise the Kalman filter is the optimal recursive estimator of the state vector. In our case, i.e. track fitting, the dynamic system is the evolution of the track state through the telescope. The measurement noise corresponds to the $2 \times 2$ hit covariance matrix in eq. 4.5 and the process noise is caused by multiple scattering on the telescope planes and in the air between the planes. However, the track model is non-linear in case of tilted telescope sensors. In order to linearise the track model the Kalman filter is applied to small deviations from a 3D straight line, called the reference track. In the following calculations $\eta$ is therefore not the track state itself but a small deviation from the reference track state. Details of this linearisation can be found in [69] and additional explanations of the various matrices employed here can also be found in $[67]$.

The fundamental equation, which describes the propagation of a particle trajectory through the telescope volume, is called the system equation. The forward Kalman filter equation is based on this equation. It models the in-state propagation from plane $k-1$ to $k$ and yields a prediction of the track state $\eta_{k}{ }^{\text {pred. }}$ and its covariance matrix $C_{k}$ pred. on telescope plane $k$

$$
\begin{aligned}
\eta_{k}^{\text {pred }} & =\eta_{k}^{\text {in }}=F_{\mathrm{k} \mid k-1} \eta_{k-1}{ }^{\text {in }} \\
C_{k}^{\text {pred }} & =C_{k}^{\text {in }}=F_{\mathrm{k} \mid k-1} C_{k-1}^{\text {in }} F_{\mathrm{k} \mid k-1}^{T}+F_{k \mid k-1} G_{k-1} Q_{k-1} G_{k-1}^{\mathrm{T}} F_{k \mid k-1}{ }^{\mathrm{T}} .
\end{aligned}
$$

Here $F_{\mathrm{k} \mid k-1}$ is the propagation matrix from plane $k-1$ to plane $k$. The propagation matrix depends on the geometry, because it is calculated from the coordinate transformation between the local coordinate systems of plane $k$ and $k-1 . G_{k-1}$ is the scatter gain matrix at plane $k-1$ and $Q_{k-1}$ corresponds to the increase of the slope covariance entries due to multiple scattering on plane $k-1 . Q_{k-1}$ is given by

$$
Q_{k-1}=\left(\begin{array}{cc}
\sigma_{\mathrm{HL}}^{2} & 0 \\
0 & \sigma_{\mathrm{HL}}^{2}
\end{array}\right)
$$




\section{Beam test experiments: Experimental setup and analysis of data}

The effects due to multiple scattering are given by the Highland width $\sigma_{\mathrm{HL}}$ defined in eq. 3.28. As can be seen, eq. 4.10 and 4.11 are recursive equations. $\eta_{k-1}$ in and $C_{k-1}$ in are given by a similar equation. Accordingly, the prediction $\eta_{k-1}$ in and $C_{k-1}$ in takes multiple scattering on all planes $0, \ldots, k-1$ into account. Additionally all available measurement information on the planes $0, \ldots, k-1$ are used to predict the states and covariance matrix on plane $k$. However, the hit measurement and the scattering effects on plane $k$ are not used for the prediction. Because of this the track state $x_{k}{ }^{\text {in }}$ is called in-state. The in-state is a prediction of the particle trajectory on the plane $k$ before the scattering on plane $k$.

The updated or filtered state can be calculated from the predicted state by using the hit measurement on plane $k$. If there are multiple hits on plane $k$, the hit closest to the predicted intersection on the plane is selected for the update. The quantity describing the distance between the hit position and the extrapolated track state is the residual $r_{k}$ given by

$$
\begin{aligned}
r_{k} & =m_{k}-H_{k} \eta_{k} \\
R_{k} & =V_{k}+H C_{k} H^{\mathrm{T}} \\
\text { with } H & =\left(\begin{array}{lllll}
0 & 0 & 1 & 0 & 0 \\
0 & 0 & 0 & 1 & 0
\end{array}\right) .
\end{aligned}
$$

The uncertainties of the residuals are given by the covariance matrix $R_{k} . R_{k}$ is given by the sum of the hit covariance matrix $V_{k}$ and the telescope pointing covariance $H C_{k} H^{\mathrm{T}}$. When a suitable hit $\left(m_{k}, V_{k}\right)$ on plane $k$ has been found, the Kalman gain matrix formalism provides a way to update the predicted track state $\eta_{k}{ }^{\text {pred. }}$ and its covariance matrix $C_{k}^{\text {pred. via }}$

$$
\begin{aligned}
\eta_{k} & =\eta_{k}^{\text {pred }}+K_{k}\left(m_{k}-H \eta_{k}^{\text {pred. }}\right) \\
C_{k} & =C_{k}^{\text {pred. }}-K_{k} H C_{k}^{\text {pred. }}
\end{aligned}
$$

where the Kalman gain matrix $K_{k}$ is defined as

$$
K_{k}=C_{k}{ }^{\text {pred }} H^{\mathrm{T}}\left(V_{k}+H C_{k}^{\text {pred. }} H^{\mathrm{T}}\right)^{-1} .
$$

In case the residual of the closest hit is larger than a user defined cut-off residual, no hit is added from the current sensor plane $k$. Predicted and filtered track states on all telescope planes can be determined by iteratively applying the eqs. 4.10, 4.11, 4.16 and 4.17. When the extrapolation and update steps have been performed on all telescope planes, the track finding algorithm stops. Repeating this procedure for all valid seed tracks yields a list of track candidates with a number of associated hits on the individual telescope planes along the beam. 
An alternative to the forward Kalman filter is a time reversed backward Kalman filter. It describes the out-state propagation from plane $k+1$ to plane $k$. The predicted state and covariance matrix are given by

$$
\begin{aligned}
\eta_{k}^{\text {pred. }} & =\eta_{k}{ }^{\text {out }}=F_{\mathrm{k} \mid k+1} \eta_{k+1}{ }^{\text {out }} \\
C_{k}{ }^{\text {pred. }} & =C_{k}{ }^{\text {out }}=F_{\mathrm{k} \mid k+1} C_{k+1}^{\text {out }} F_{\mathrm{k}+1 \mid k}{ }^{T}+F_{k \mid k+1} G_{k+1} Q_{k+1} G_{k+1}{ }^{\mathrm{T}} F_{k \mid k+1}^{\mathrm{T}},
\end{aligned}
$$

where the matrices are analogously defined, but the $F_{\mathrm{k} \mid k+1}$ describes the propagation in the opposite direction from plane $k+1$ to plane $k$. Out-states describe the track state on a telescope plane $k$ after multiple scattering on plane $k$. Calculating in- and out-states on a plane provides the opportunity to calculate scattering angles and perform radiation length measurements as described in the next chapter.

Track fitting involves the usage of a forward and a backward Kalman filter at the same time. The goal is to predict the track state on layer $k$ as precisely as possible by employing all available hit measurements on all other telescope planes. The forward Kalman filter produces an in-state from all available measurements on the planes upstream of $k$. The backward Kalman filter estimates an in-state from all available measurements on the planes downstream of $k$. Note that the for the purpose of track fitting, the backward filter is not a time-reversed filter and eq. 4.19 and 4.20 do not apply as they would lead to the estimate of an out-state. Also the hit measurement on plane $k$ itself is not included in the forward and backward Kalman filter. This ensures that the track states are uncorrelated with the hit measurement $m_{k}$.

Assuming the predicted track states and covariance of the forward filter are denoted by $\eta_{k}^{\mathrm{fw}}$ and $C_{k}^{\mathrm{fw}}$ and the corresponding states of the backward filter are denoted by $\eta_{k}^{\mathrm{bw}}$ and $C_{k}^{\mathrm{bw}}$, the fitted track states and covariances are given by

$$
\begin{aligned}
C_{k}^{\mathrm{fit}} & =\left(\left(C_{k}^{\mathrm{fw}}\right)^{-1}+\left(C_{k}^{\mathrm{bw}}\right)^{-1}\right)^{-1} \\
\eta_{k}^{\mathrm{fit}} & =C_{k}^{\mathrm{fit}}\left(\left(C_{k}^{\mathrm{fw}}\right)^{-1} \eta_{k}^{\mathrm{fw}}+\left(C_{k}^{\mathrm{bw}}\right)^{-1} \eta_{k}^{\mathrm{bw}}\right)^{-1}
\end{aligned}
$$

The residuals $r_{k}^{\text {fit }}$ between measurements $m_{k}$ and fitted track states can be calculated with eq. 4.13 and 4.14 by replacing $\eta_{k}$ and $C_{k}$ by the corresponding fitted quantities. A frequently used quality parameter of track fits is the $\chi^{2}$ value. It can be calculated from the residuals $r_{k}$ and their covariances on the individual telescope planes via

$$
\chi^{2}=\sum_{k} \chi_{k}^{2}=\sum_{k} r_{k}^{\mathrm{T}}\left(R_{k}\right)^{-1} r_{k}
$$




\section{Beam test experiments: Experimental setup and analysis of data}

The number of degrees of freedom $j$ of position measurements in the telescope without a magnetic field is given by $j=n \cdot 2-4$ where $n$ is the number of telescope planes. The $\chi^{2}$ distribution of fitted tracks should have a mean value of $j$, a variance of $\sigma^{2}=2 j$ and a standard deviation of $\sigma=\sqrt{2 j}$ [70]. For a telescope with six planes the number of degrees of freedom is eight. Another connected quality parameter, the track probability value, or $p$ value, can be calculated from $m$ and $\chi^{2}$ [70]. The $p$ value distribution is supposed to be a uniform distribution between 0 and 1. Fig. 4.7 depicts a track $\chi^{2}$ and its associated $p$ value distribution in an aligned EUDET telescope with 6 sensor planes. The mean and RMS values of the $\chi^{2}$ distribution match the expectations and the $p$ value distribution is uniformly distributed between 0 and 1 .

Another quantity that can be used to test the quality of tracks is the residual and residual pull distribution on every telescope plane. The residuals of the fitted tracks are calculated as described above according to eq. 4.13 and 4.14. The pulls of the residuals in $u$ and $v$ can be calculated from the corresponding residual $r_{k}$ entries divided by the corresponding covariance $R_{k}$ diagonal entries. Pull distributions are expected to have a Gaussian shape with a mean of zero and an RMS of one.

\subsubsection{Selecting reconstructed tracks from track candidates}

In $T B S W$ the FastTracker processor is used for track finding. It employs the Kalman Filter tracking approach and one of the two different options for a seed track explained in section 4.3.5. It is possible that after the track finding step multiple track candidates share one or more hits. This is not allowed and only one of these tracks can be used for subsequent analysis steps. In order to remove any ambiguity of track candidates, several criteria can be used. Tracks that are used for alignment and cluster calibration purposes (see subsections 4.3.8 and 4.3.10) are required to have a hit on every telescope plane. The tracks must also have a small enough track $\chi^{2}$ value. Tracks that fail these criteria are discarded. Additionally all ambiguous tracks are compared according to their track $\chi^{2}$ and only the track with the smallest value is kept. All remaining track candidates are called reconstructed tracks.

Reconstructed tracks are used for alignment and cluster calibration purposes as described in the following sections 4.3.8 to 4.3.11. Also tracks with three hits on the upstream telescope planes, called upstream tracks and tracks with three hits on the downstream telescope planes called downstream tracks can be used to determine scattering angles as described in chapter 5 .

\subsubsection{Telescope alignment with Kalman filters}

The parametrisation of the sensor alignment in $T B S W$ follows the geometry introduced in [71]. There are six rigid body parameters $x, y, z, \alpha, \beta$ and $\gamma$ to describe the position of each telescope plane in the global coordinate system. The telescope mechanics enforces that telescope planes are placed almost parallel to each other along the telescope table. 


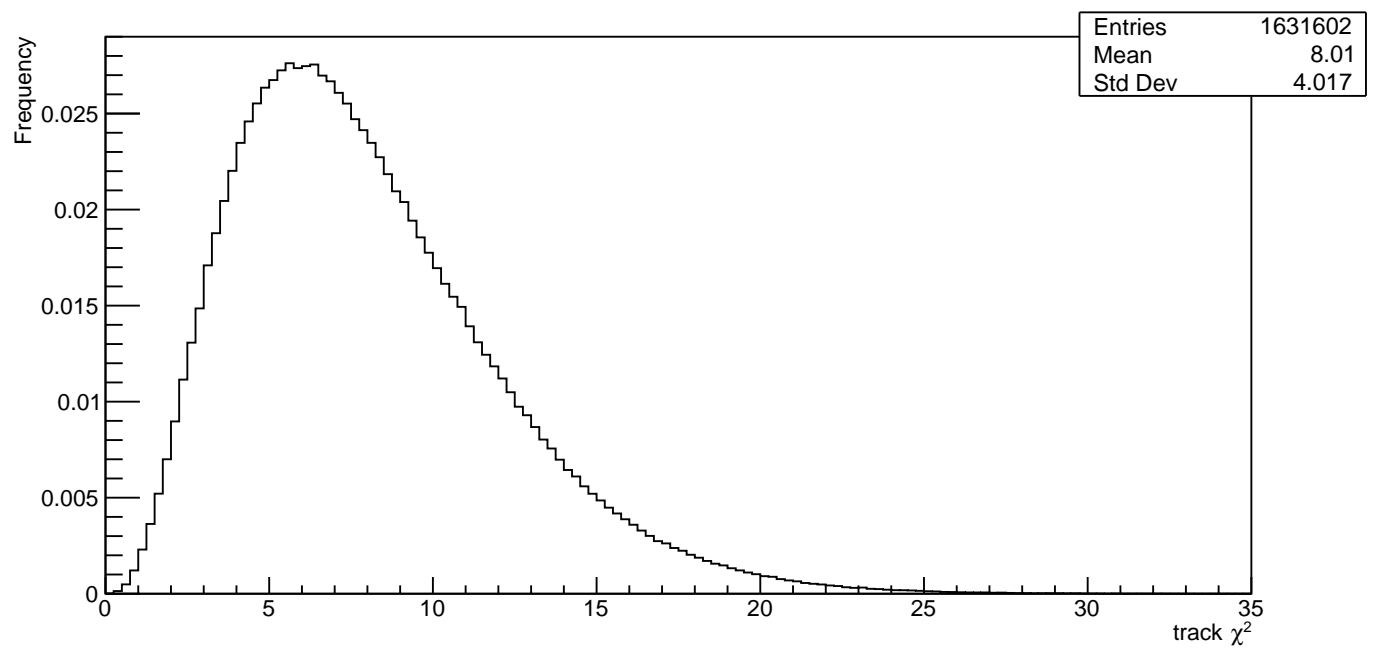

(a) $\chi^{2}$ track fit distribution

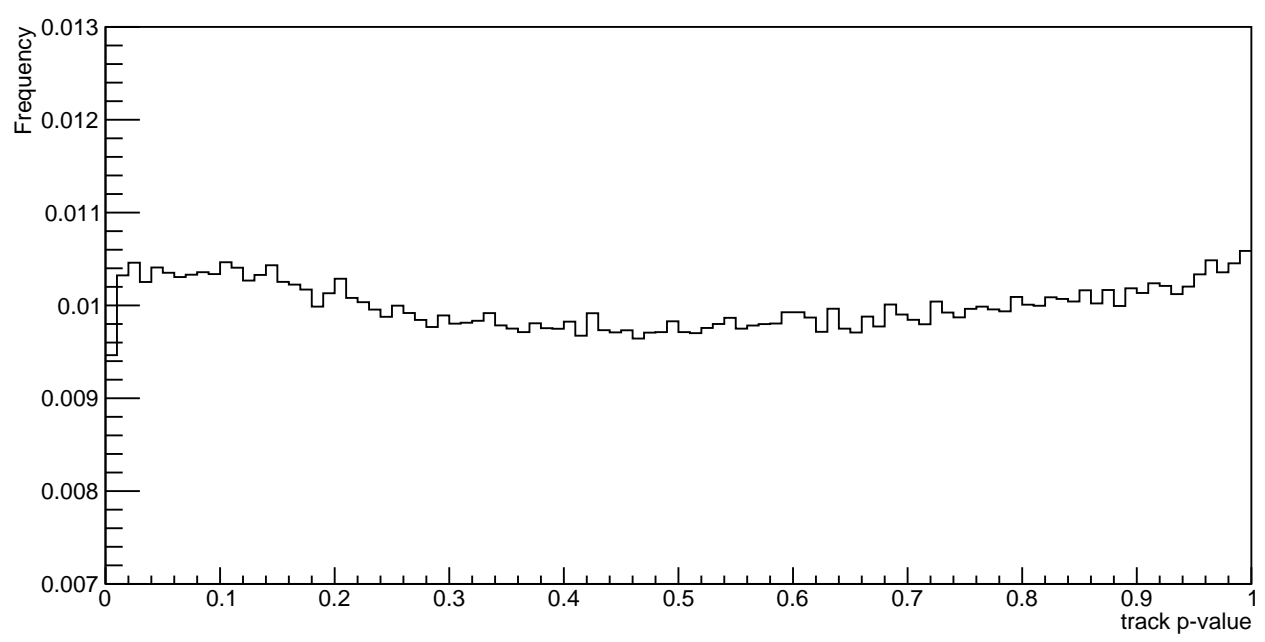

(b) $p$ value distribution

Figure 4.7.: Example $\chi^{2}$ (a) and $p$ value (b) distribution of a track sample with six associated hits per track. The beam test measurement was conducted at DESY in October 2016. The measurements took place at beam line 21 with the DURANTA telescope. The beam energy was $2 \mathrm{GeV}$ and the distance between neighbouring telescope planes was approximately $25 \mathrm{~mm}$. The mean value of the distribution is close to eight and the RMS is close to the expected value of four. The distribution was normalised so that it has an integral of 1 .

The beam traversing through the telescope has an almost perpendicular incidence on the planes. During beam tests the telescope geometry is measured manually. The un- 


\section{Beam test experiments: Experimental setup and analysis of data}

certainties of the manual measurements of the position $(x, y, z)$ of the telescope plane are expected to be in the order of several millimetres. The rotation of the telescope planes is expected to be smaller than $1^{\circ}$ which corresponds to roughly $17 \mathrm{mrad}$. Typically, only the length of the telescope and the distances between the M26 planes in $z$ direction are noted. Rotations and $x, y$ positions of the sensor planes are set to zero in the geometry XML file.

An accurate track fit requires precise knowledge of the telescope sensor positions and rotations. In case of a non-negligible misalignment the fitted track states are biased. Ideally, the sensor position must be known well enough to limit the misalignment induced biases to values that are smaller than the statistical uncertainties of the track states. These uncertainties are typically in the order of $5 \mu \mathrm{m}$ for $u$ and $v$ and $100 \mu \mathrm{rad}$ in $d u / d w$ and $d v / d w$. In order to determine sensor rotations and positions with the required accuracy, a track based alignment approach is employed. The alignment procedure is based on Kalman filter alignment with annealing as formulated in [72]. The implementation of this approach for beam test alignment in $T B S W$ is explained and validated in [67]. In practice, the alignment is managed in a data base file, the alignmentDB. The align$m e n t D B$ contains information on all alignment parameters of every telescope plane. It is updated after every successful alignment step and used, at the beginning analysis steps, to update the geometry information from the geometry XML file.

The final alignment corrections are determined using a large sample of tracks. However, in order to find tracks efficiently a hit based pre-alignment is implemented in $T B S W$. The Marlin processor is called Correlator, which can be used to give a good estimate of the $x$ and $y$ positions of each telescope plane relative to a reference plane. Starting from this reference plane, typically the plane defining the global coordinate system, hits are extrapolated along the $z$ or beam axis onto the other telescope planes. For all other telescope planes, residuals between the extrapolated intersections and all reconstructed hits are calculated. The results are $u$ and $v$ residual distributions on all telescope planes except the reference plane. In case the telescope is perfectly aligned in $x$ and $y$ the residuals should be centred at $u=0 \mathrm{~mm}$ and $v=0 \mathrm{~mm}$. If that is not the case the mean residual value is a good estimate for the relative shift between the reference plane and the corresponding telescope plane.

In $T B S W$ the KalmanAligner processor is employed to calculate alignment corrections. The track sample used as input for the alignment measurements should be of the order of 10000 tracks. For a given track sample, a global $\chi^{2}$, function that depends on the telescope alignment parameters, must be minimised. The $\chi^{2}$ function is given by

$$
\chi^{2}=\sum_{j} \sum_{i} r_{i j}^{\mathrm{T}}\left(R_{k}\right)^{-1} r_{i j}+\left(a-a_{0}\right)^{\mathrm{T}} E_{0}^{-1}\left(a-a_{0}\right),
$$

where index $j$ corresponds to the sum over the track sample and index $i$ corresponds to the sum over hits of an individual track. $r_{i j}$ is the fitted residual of hit $i$ for track $j$ 
as explained in section 4.3.6. The second term of eq. 4.24 characterises the alignment update. The quantity $a$ corresponds to the current vector of alignment parameters, while $a_{0}$ corresponds to the initial vector of alignment parameters. Therefore the second term increases with increasing difference between $a$ and $a_{0}$. The initial alignment covariance matrix $E_{0}$ determines the uncertainty of the initial alignment. Entries of $E_{0}$ should be close to the uncertainties of the manual measurements of the telescope, which were mentioned at the beginning of this subsection. Additionally individual alignment parameters can be fixed to their start values by selecting zero for the corresponding $E_{0}$ matrix entry. This freezing of individual alignment parameters is very useful when performing a step by step alignment approach. The $E_{0}$ entries are parameters of the KalmanAligner processor and can be selected beforehand.

\subsubsection{Gradual telescope alignment}

The individual alignment parameters are not all equally sensitive to the alignment procedure. The parameters $x$ and $y$ as well as the rotation around the normal vector of the telescope plane $\gamma$ are the most sensitive quantities. In practice trying to determine all alignment parameters at the same time does frequently not lead to convergence. Doing so with reconstructed tracks from a mostly misaligned telescope decreases the stability of the alignment process and leads to failed minimisation attempts. In order to circumvent these issues, a gradual determination of the alignment parameters should be used. As mentioned before the alignment steps, alignment I, II and III, in fig. 4.5 consist of multiple paths which must be processed gradually. An overview of these paths as well as the pre-alignment path and the DQM paths is depicted in fig. 4.8 and specified below:

1. Pre-alignment path: The path starts with the EUDAQInputProcessor, NIUnpacker and PixelClusterizer which have been explained in section 4.3.2. A CogHitMaker is used to form centre-of-gravity hits. Using the Correlator processor with the hit collection yields a rough $x$ and $y$ estimate of all telescope sensors except the first in the beam line which defines the global coordinates. These corrections are stored in the alignment $D B$. The sequence of processors used in the pre-alignment path is depicted on the left side of fig. 4.8. This sequence of processors is used at position 2 in the overall telescope calibration scheme displayed in fig. 4.5.

2. First alignment path: The first three processors, EUDAQ conversion, unpacking and clustering, of this path are identical to the pre-alignment path. For hit making, either the already established CogHitmaker processor or the GoeHitMaker, which will be explained in section 4.3.10, can be used. A TrackFinder processor is employed as explained in subsection 4.3.7. The TrackFinder uses the alignmentDB to update the nominal geometry before starting the track finding process. In order to find tracks in the suboptimal telescope alignment, track cuts on track $\chi^{2}$ values are very loose. Typically, cut values of $\chi^{2}=10^{7}$ are used. The tracks are used in the KalmanAligner processor to determine the three most sensitive alignment parameters $x, y$ and $\gamma$ on all sensors except the first and the last in the beam line. 

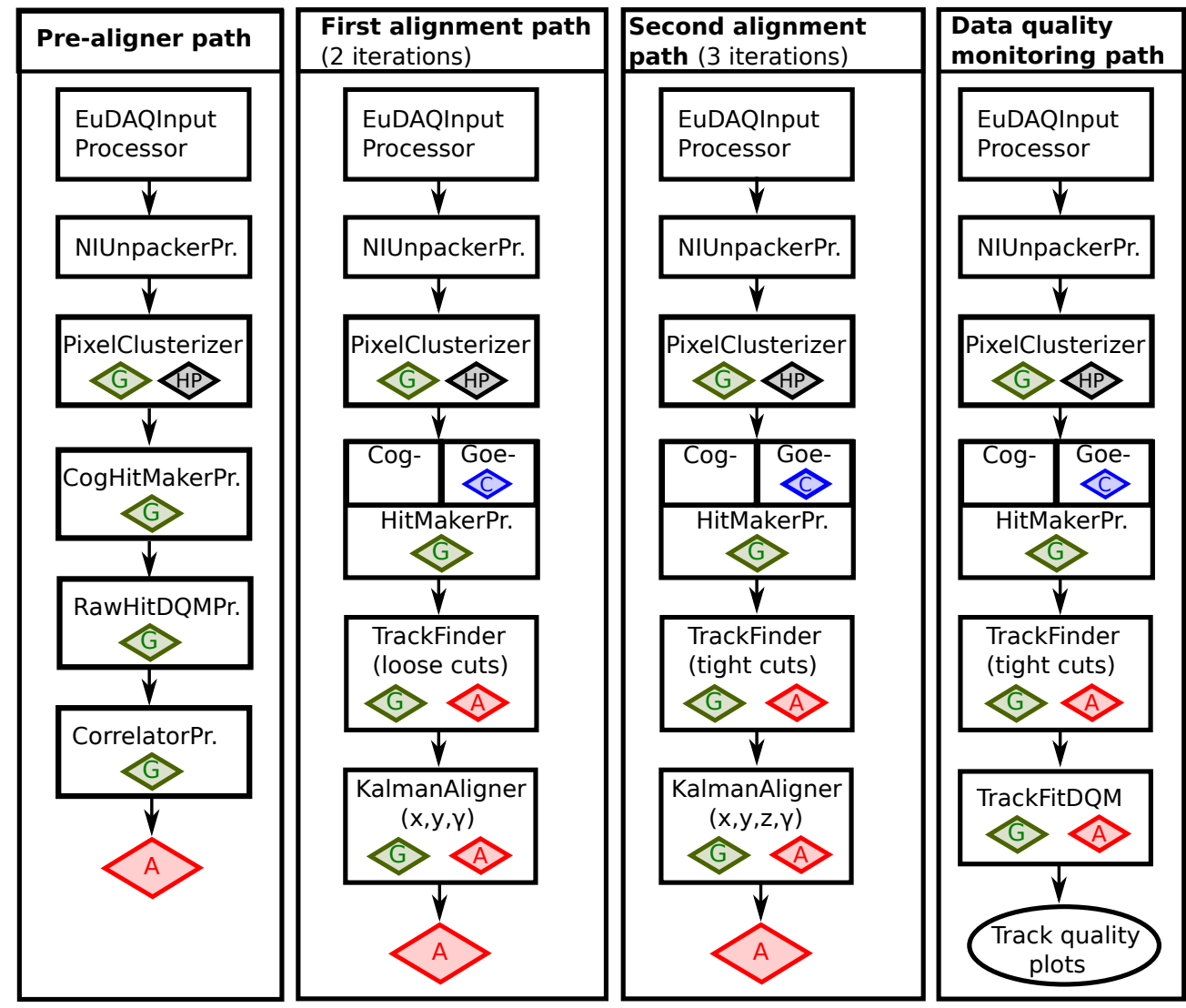

Figure 4.8.: Sequence of Marlin processors used in the pre-aligner, $x, y$ and $\gamma$ alignment, total alignment and data quality monitoring path. The rectangles correspond to individual Marlin processors, while the diamond shapes signify various DB files. In case a small diamond is visible inside of a processor box, the corresponding processor uses the associated data base.

All other alignment parameters are fixed by setting the corresponding entries in the $E_{0}$ matrix to zero. Fixing $x, y$ and $\gamma$ on the last plane avoids large shearing and torsion distortions in the telescope. Shearing and torsion are called weak modes that leave the track residuals invariant but introduce bias in the track states. Details on weak modes will not be discussed here, but are explained in detail in [67]. The result of this first alignment path is an updated set of alignment parameters, which now also includes the $\gamma$ rotations. The alignment $D B$ is updated accordingly. The sequence of Marlin processors used in the first alignment path is depicted in the second box from the left in fig. 4.8. As the name suggests this path is the first path employed in the alignment I,II and III steps displayed in the overview in fig. 4.5 and is typically iterated twice.

3. Second alignment path: This path is very similar to the previous one. However, 
the TrackFinder processor uses much stricter $\chi^{2}$ cuts. Typically the cut value is $\chi^{2}=100$. At this point the quality of the tracks will be reasonably good and due to the improved telescope alignment a larger track sample can be successfully reconstructed. This improved track sample allows the alignment of the less sensitive parameter $z$ in addition to $x, y$ and $\gamma$ of all inner telescope planes with the KalmanAligner processor. The rotation angles, $\alpha$ and $\beta$, are even less sensitive to the alignment approach than $z$. For radiation length measurements introduced in chapter $5 \alpha$ and $\beta$ rotations of the telescope planes are only of minor importance. They are therefore not determined during the alignment processes in this work. The result of the second alignment path is an updated set of alignment parameters, which now includes the $x, y, z$ and $\gamma$ on all inner telescope planes. These updated alignment parameters are stored in the alignmentDB. The sequence of Marlin processors used in the second alignment path is depicted in the third box from the left in fig. 4.8. This path is the third path employed in the alignment steps after processing the first alignment path twice. The second alignment path is typically iterated three times.

4. Data quality monitoring path: The first 5 processors of this path are identical to the corresponding processors in the second alignment path. After the TrackFinder processor a TrackFitDQM processor is used to generate control diagrams such as track $p$ value distributions and residuals on every sensor. The diagrams are used to check whether the track fitting and consequently the connected alignment procedure worked.

In case of a long telescope with large distances between the telescope sensors, a large beam divergence or a very noisy telescope the previously described simple pre-alignment approach may be problematic because of broad residual distributions. Accordingly, the TrackFinder in the first alignment path, even with very loose residual cuts, may not be able to find suitable hits on the downstream telescope arm. In this case an iterative track correlation approach is a more stable alternative. The first step of this approach is to pre-align and align the sensors in the upstream telescope arm as described in the enumeration above. This is achieved by only using hits on the upstream sensors of the telescope in the TrackFinder processors and fixing the alignment parameters of the downstream telescope planes in the KalmanAligner processors. Then, instead of employing a simplified extrapolation along the $z$ axis, like described for the default Correlator processor, the upstream tracks are extrapolated to the nearest downstream telescope sensor. Residuals between the extrapolated upstream track and the sensor hits are calculated. The expected $x$ and $y$ shifts of this newly added sensor relative to the upstream telescope arm can be determined from the residual mean values. After this tracklet correlator step the usual alignment procedure, as described above, is used to finalise the alignment of the newly added individual telescope sensor. The procedure is repeated and new sensors are aligned gradually until all inner telescope sensors are aligned in $x, y, z$ and $\gamma$. 


\subsubsection{Calibration of cluster positions and resolutions based on cluster shapes}

The centre-of-gravity hit making algorithm described in subsection 4.3.4 has a number of limitations that can be removed by employing a data driven approach described in this section. The goal of the approach is a track based estimate of the hit position and the full 2x2 covariance matrix including the of diagonal elements. The estimates of the hit position and covariance matrix yield unbiased residual pulls and the estimate of the covariances yield residual pull RMS values close to one. Deviations from a RMS of one are typically smaller than $5 \%$. Estimates of the hit position and covariance differ for different arrangements of digits in a given cluster. The arrangement of digits in a cluster is called the cluster shape.

The calibration procedure is iterative but can be robustly automatised and there is no need to tune parameters like in the centre-of-gravity approach. The result of the cluster calibration is a data base file called cluster $D B$. This DB file contains information on hit positions and covariance matrices for all cluster shapes which have been encountered at least several hundred times. The corresponding Marlin processor is called GoeClusterCalibrator. It was developed within the $T B S W$ framework in Göttingen ${ }^{5}$ and has been tested successfully as part of this thesis.

The approach requires a good track sample in an aligned telescope and works as follows. For every track in the track sample associated clusters are collected. For each cluster shape encountered, a shape ID can be calculated. To give an example three common cluster shapes are depicted in figure 4.9 with their shape ID, hit position and error ellipse. The estimated hit position is indicated by the black dot. The position and size of the error ellipse can be calculated from the hit position and the covariance matrix entries. As can be seen, in the simple case of M26 telescope sensors with binary read-out there is exactly one kind of cluster with a single digit. This cluster shape has the shape ID P0.0.0D0.0. Also there are two cluster shapes for two digit configurations that share a common edge in $u$ or in $v$ direction with the shape IDs P0.0.0D0.0D1.0 and P0.0.0D0.0D0.1. In the case of an EUDET telescope with M26 sensors a single cluster $D B$ file for all M26 sensors can be used, because the pixel pitch and threshold setting of all M26 sensors is identical.

Per definition the shape ID is identical for all clusters with the same shape regardless where on the pixel matrix it is located. In order to perform cluster calibration measurements, a translation from the local coordinate system of the telescope plane into the coordinate system of a cluster shape is required. The coordinate system of the cluster shape is defined in the following way: The smallest row and column values of digits in the cluster are determined. These values must not necessarily come from the same digit

${ }^{5}$ The development and implementation was done by Benjamin Schwenker, testing and inclusion into the existing framework was done by Benjamin and me 

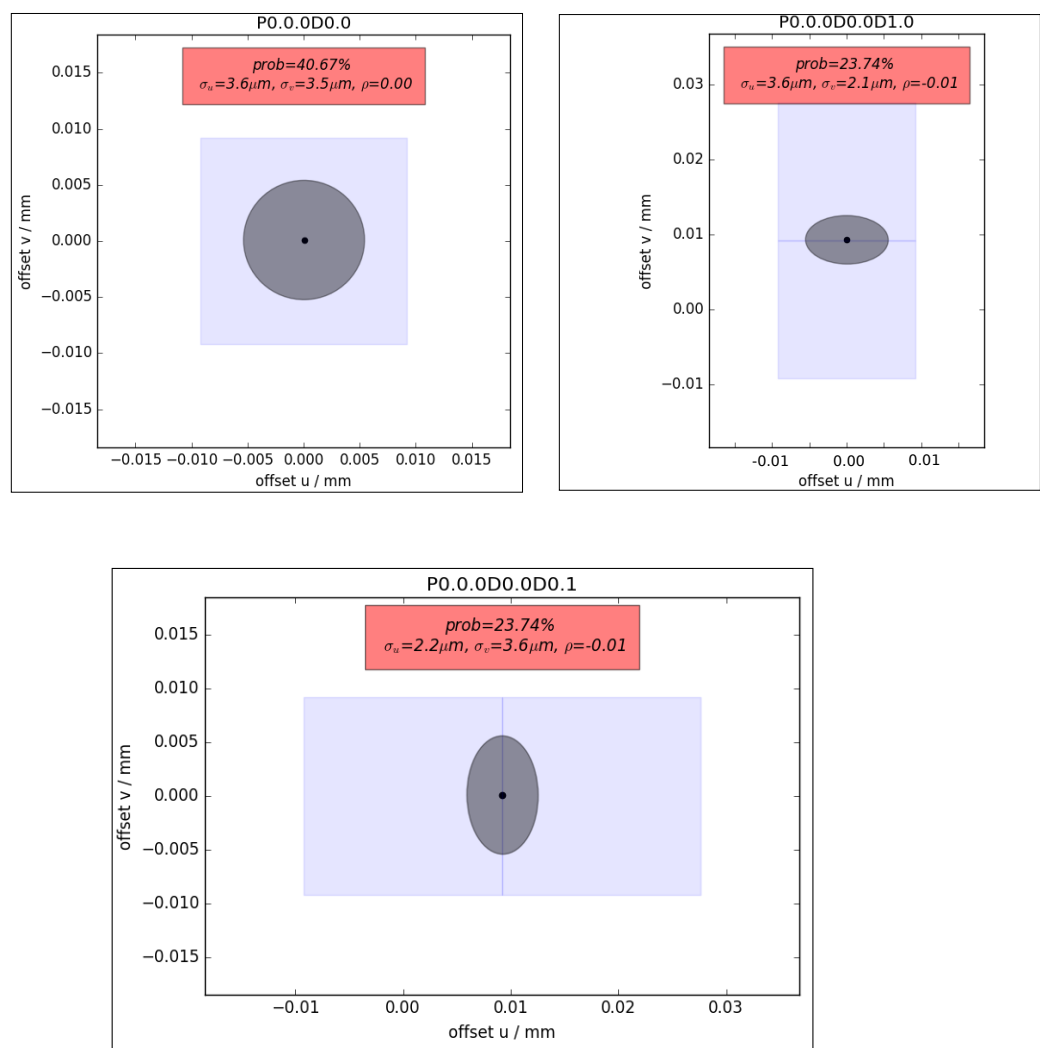

Figure 4.9.: Three common M26 cluster shapes in case of a particle beam with perpendicular incidence. The most probable particle intersection and the error ellipse is depicted for each cluster shape. The shape ID is P0.0.0D0.0 for the one-digit-cluster on the top left, P0.0.0D0.0D1.0 for the two-digit-cluster on the top right and P0.0.0D0.0D0.1 for the two-digit-cluster on the bottom. A simulation of an EUDET telescope was used to generate these shapes.

of the cluster. The origin of the cluster shape specific coordinate system $\left(u^{*}, v^{*}\right)$ lies in the center of the pixel with the smallest row and column of the cluster. In case of the cluster shapes depicted in 4.9, for example, the origin is always located in the centre of the bottom-left pixel.

For every cluster in the collection, the intersection of the associated track is transformed into the cluster type specific coordinate system and stored. This is done for all tracks and for all associated clusters on every telescope plane. Additionally an average track intersection resolution, $\sigma_{u}^{\text {trk }}$ and $\sigma_{u}^{\text {trk }}$, in both directions and their correlation $\sigma_{u, v}^{\text {trk }}$ is calculated 


$$
C^{\mathrm{trk}}=\left(\begin{array}{cc}
\left(\sigma_{u}^{\mathrm{trk}}\right)^{2} & \left(\sigma_{u, v}^{\mathrm{trk}}\right)^{2} \\
\left(\sigma_{u, v}^{\mathrm{trk}}\right)^{2} & \left(\sigma_{v}^{\text {trk }}\right)^{2}
\end{array}\right)
$$

from every used track state on every telescope plane. This covariance matrix will be used later to correct for the track resolution.

The result of this procedure is a track intersection distribution in the cluster coordinate system for every cluster type that was encountered. Example track intersection distribution for four cluster types P0.0.0D0.0, P0.0.0D0.0D0.1D1.0D1.1, P0.0.0D0.0D0.1 and P0.0.0D0.0D1.0 are depicted in figure 4.10. They were measured at a beam test in October 2016 at the DURANTA telescope. Individual shape IDs have associated track intersection distributions with different mean and RMS values. For the distributions depicted in figure 4.10, the measured average track resolution is $\sigma_{u}^{\text {trk }}=\sigma_{v}^{\text {trk }}=4.7 \mu \mathrm{m}$ in both directions. This rather large track resolution leads to broad intersection distributions with a 2D Gaussian shape. The track intersection correlation $\sigma_{u, v}^{\text {trk }}$ is compatible with zero.

In order to achieve a stable estimate of the cluster resolution, a minimum number of track intersections is required. Rare cluster types with too few track intersections cannot be reliably calibrated and must be discarded. The exact threshold for the minimum number of track intersections must be selected beforehand ${ }^{6}$. Of all remaining cluster types the mean $m^{\text {total }}$ and covariance $C^{\text {total }}$ of the $2 \mathrm{D}$ track intersection distributions are determined

$$
m^{\text {total }}=\left(\begin{array}{c}
u^{\text {total }} \\
v^{\text {total }}
\end{array}\right)
$$

and

$$
C^{\text {total }}=\left(\begin{array}{cc}
\left(\sigma_{u}^{\text {total }}\right)^{2} & \left(\sigma_{u, v}^{\text {total }}\right)^{2} \\
\left(\sigma_{u, v}^{\text {total }}\right)^{2} & \left(\sigma_{v}^{\text {total }}\right)^{2}
\end{array}\right)
$$

The mean of the cluster intersection is given by the mean of the track intersections $m^{\text {total }}$. The covariance matrix of the cluster intersections can be calculated via

$$
C^{\text {cluster }}=C^{\text {total }}-C^{\text {trk }}
$$

The cluster resolutions correspond to the square root of the diagonal elements of $C^{\text {cluster }}$. It is possible that for some cluster types the determined track resolution is larger than

\footnotetext{
${ }^{6}$ Typically at least 2000 track intersections are required
} 


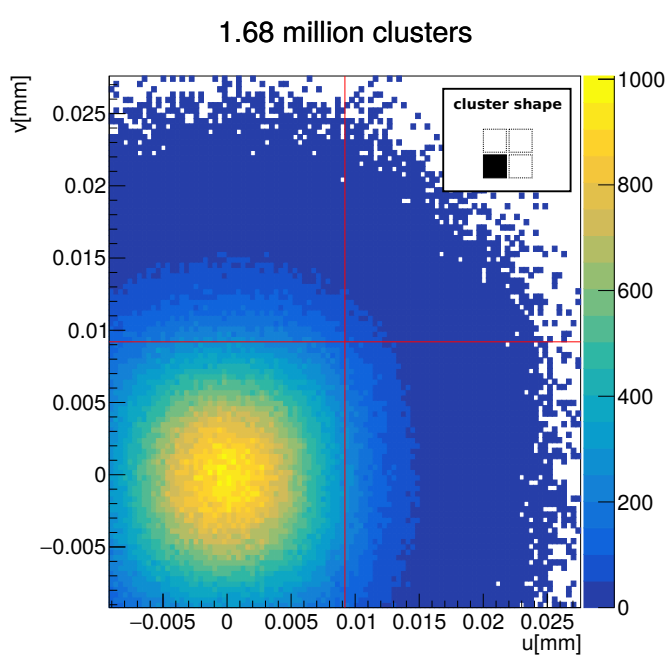

(a)

0.71 million clusters

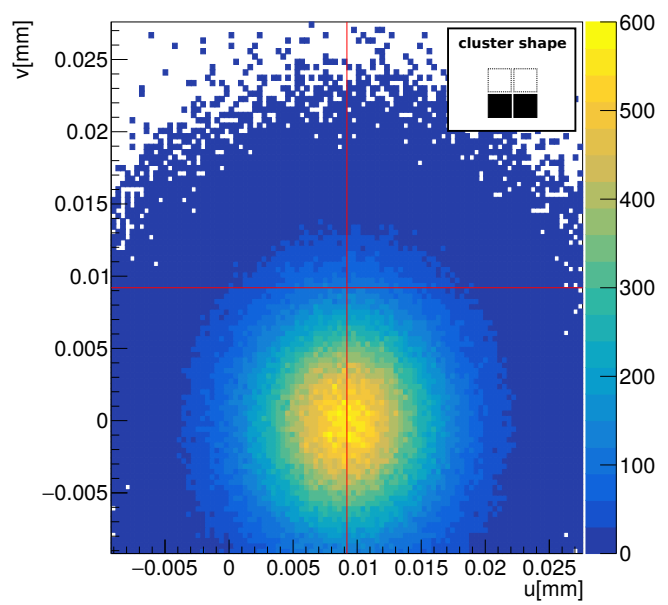

(c)
0.48 million clusters

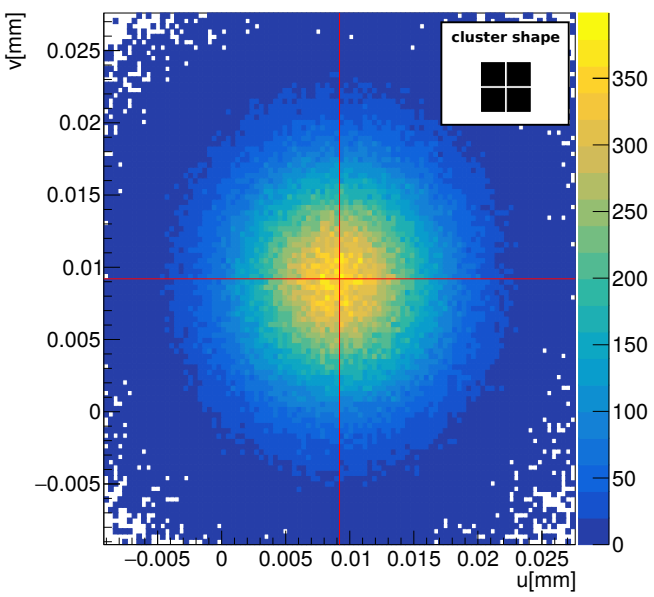

(b)

0.70 million clusters

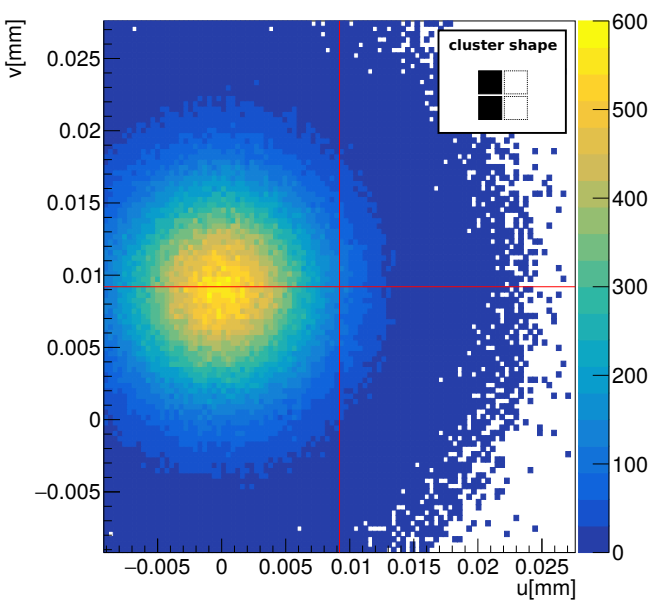

(d)

Figure 4.10.: Example track intersection distributions in the cluster shape coordinate system for four different cluster shapes: (a) P0.0.0D0.0, (b) P0.0.0D0.0D0.1D1.0D1.1 (the most compact four digit cluster), (c) P0.0.0D0.0D0.1 and (d)P0.0.0D0.0D1.0. Pixel edges are indicated by the red lines. A pictogram of the corresponding cluster shape is depicted in the top right corner of every distribution. The measurement of a specific cluster type is connected to the position in the pixel, where the particle transition took place. The small difference in numbers of clusters between the twodigit-clusters in $u$ and $v$ direction is most likely caused by slightly tilted telescope planes. The average track resolution is very large $\left(\sigma_{u, v}^{\text {trk }}=4.7 \mu \mathrm{m}\right)$ leading to broad distributions with a 2D Gaussian shape. 


\section{Beam test experiments: Experimental setup and analysis of data}

the RMS of the measured distribution. In this case the cluster variance is set to a predefined minimum value ${ }^{7}$.

The result of these measurements is a set of cluster mean positions, resolutions and covariances that can be used for hit making purposes. Measured intersections, resolutions and associated cluster shapes are stored in a database called the cluster $D B$. The hit making processor which uses the information from the clusterDB is called GoeHitmaker.

As the track resolution depends on the cluster resolution and the cluster resolution is determined from fitted tracks, the cluster calibration must be iterated. After each iteration the cluster $D B$ is updated and the hit making, track finding and fitting process must be repeated. In preparation for the measurements summarised in chapter 7 tests were performed to evaluate the convergence of the cluster calibration procedure. It was found that typically eight iterations of hit making, track finding and cluster calibration are sufficient for the cluster resolutions to converge.

The paths of the two cluster calibration steps in fig. 4.5 are displayed in detail in fig. 4.11. As can be seen the only difference between the two paths is that in the first iteration hits are reconstructed with the centre-of-gravity approach, while the subsequent cluster calibration paths use the cluster $D B$ in the hit making process.

In order to demonstrate the improvement due to cluster calibration measurements, $p$ values of two track samples are compared in fig. 4.12. One track sample is reconstructed from centre-of-gravity hits, while the other track sample is reconstructed from calibrated hits with cluster resolutions determined in a cluster calibration measurement. As can be seen the cluster calibration leads to a much flatter $p$ value distribution of the corresponding tracks.

\subsubsection{Telescope calibration: Combination of alignment and cluster calibration processes}

As explained in the last two subsections successful telescope alignment and cluster calibration measurements ultimately lead to an improved quality of the fitted tracks. However, both telescope alignment and cluster calibration need high quality tracks to achieve reliable results. A strategy for the alignment, as explained in the enumeration in subsection 4.3.9, is to start with the alignment of the most sensitive alignment parameters when using a suboptimal track sample in the misaligned telescope. Afterwards the track fit is repeated with the updated alignment and then the alignment is finalised with the improved track sample. The following subsection presents a method to also include the cluster calibration and create a sequential alignment and cluster calibration process. The combination of both calibration procedures and the masking path is called telescope calibration.

\footnotetext{
${ }^{7}$ Typically this minimum variance is $(2 \mu \mathrm{m})^{2}$
} 


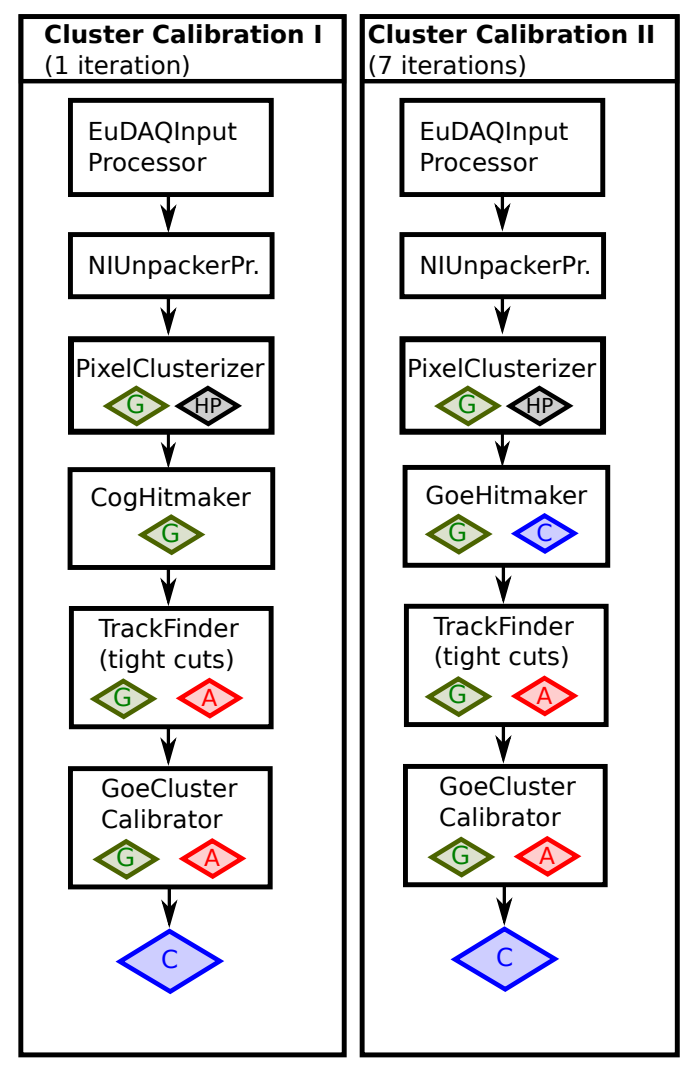

Figure 4.11.: Sequence of Marlin processors used in cluster calibration paths. The rectangles correspond to individual Marlin processors, while the diamond shapes signify various DB files. In case a small diamond is visible inside of a processor box, the corresponding processor uses the associated data base.

Different telescope calibration approaches were tested in preparation for the analysis presented in chapter 7. A stable approach which leads to successful calibration and consequently a high quality sample of final tracks is summarised below and in the flow chart in figure 4.5.

1. Masking: A hotpixelDB is created with the masking path as explained in section 4.3.3.

2. Pre-alignment: The pre-alignment path with centre-of-gravity hits is used to determine $x$ and $y$ parameters of all telescope planes except the first in the beam line. The pre-alignment path is depicted as the right box in fig. 4.8 and explained in the enumeration in section 4.3.9.

3. Alignment I: A telescope alignment step according the two alignment paths in fig. 4.8 is conducted. The two paths are also explained in the enumeration in section 


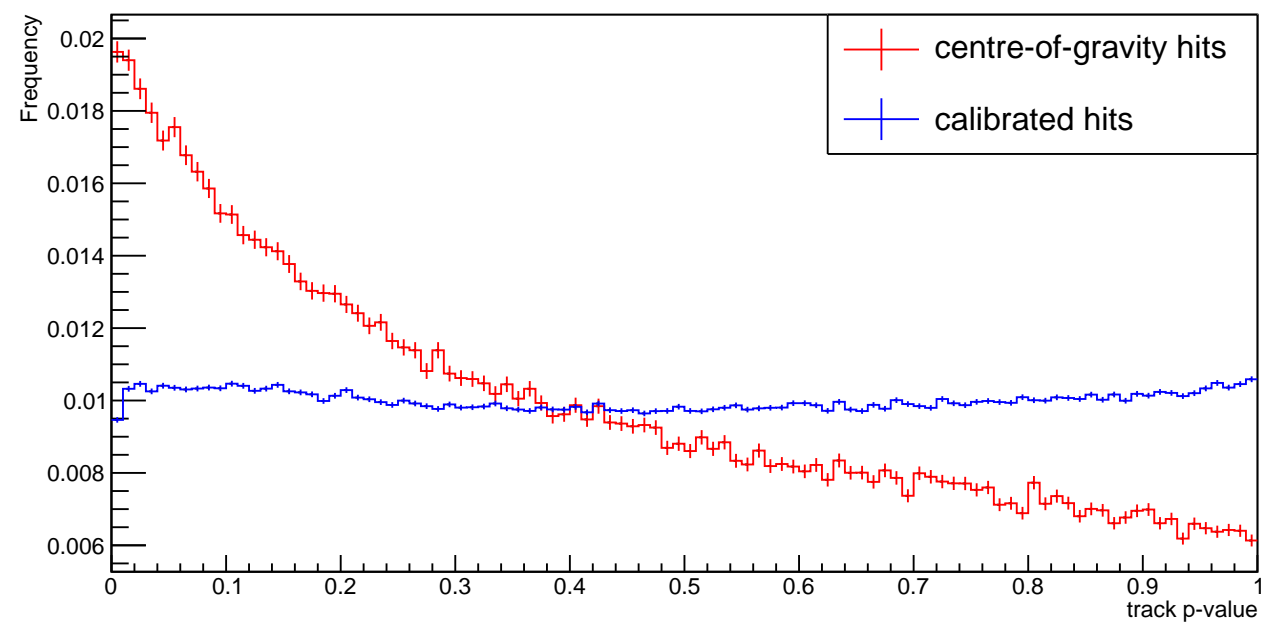

Figure 4.12.: Comparison of the track $p$ values of a track based on centre-of-gravity hits and hits reconstructed using the information in the cluster $D B$. Clearly the track fit worked much better for the track sample based on the calibrated hits: The $p$ value distribution is nearly uniform in the range between zero and one.

4.3.9. Center-of-gravity hits are used to reconstruct tracks. After this step $x, y, z$ and $\gamma$ on all inner telescope planes are stored in the alignmentDB.

4. Data quality monitoring I: A data quality monitoring path is used to generate diagrams which can be used to evaluate the quality of the center-of-gravity hit based tracks.

5. Cluster calibration I: Employing the aligned telescope geometry a single cluster calibration path (see left side of fig. 4.11) with a track sample based on centre-ofgravity hits is conducted to calculate cluster resolutions and create a clusterDB. A track sample with strict $\chi^{2}$ and residual cuts is used and it can be assumed that the tracks are coming from real particle transitions.

6. Alignment II: This alignment step is similar to the one described in 3. However, this time the GoeHitMaker and clusterDB are used to reconstruct hits. These hits are used for track finding and fitting with strict cuts. The alignmentDB is then updated with the determined $x, y, z$ and $\gamma$ values on all inner telescope sensors.

7. Cluster calibration II: Seven iterations of the cluster calibration paths on the right in fig. 4.11 are used to finalise the cluster calibration process. After this large number of iterations it can be assumed that the cluster resolutions have converged. Track finding and fitting is always performed with strict cut conditions.

8. Alignment III: The procedure is concluded with a final alignment step. The parameters $x, y, z$ and $\gamma$ on all inner telescope sensors are determined with tracks 
based on the converged calibrated hits.

9. Data quality monitoring II: The final track sample based on the converged calibrated hits in the fully aligned telescope is utilised to generate data quality diagrams and evaluate the full telescope calibration.

If the data quality diagrams indicate that the telescope calibration procedure worked (for examples see section 7.1) the angle reconstruction and subsequent radiation length measurements explained in chapter 5 can be conducted.

\subsection{Measurements of intrinsic M26 cluster resolutions}

In this section the complete calibration chain, depicted in fig. 4.5, was used to conduct measurements of the intrinsic resolution of M26 sensors for different comparator threshold settings. These measurements were conducted with the DURANTA telescope at DESY in October 2016. The tests took place at beam line 22. Several runs with different M26 comparator thresholds were recorded. The cluster calibration method explained in section 4.3.10 was used to measure the resolution of different cluster types and compare them. The goal was to determine the threshold setting which provides the optimal ratio between different cluster types and yields the optimal intrinsic sensor resolution. Additionally, the hit detection efficiency for different threshold settings was measured. Combining the results of both measurements corresponds to the characterisation of a sensor of the DURANTA reference telescope located at beam area 22 at DESY.

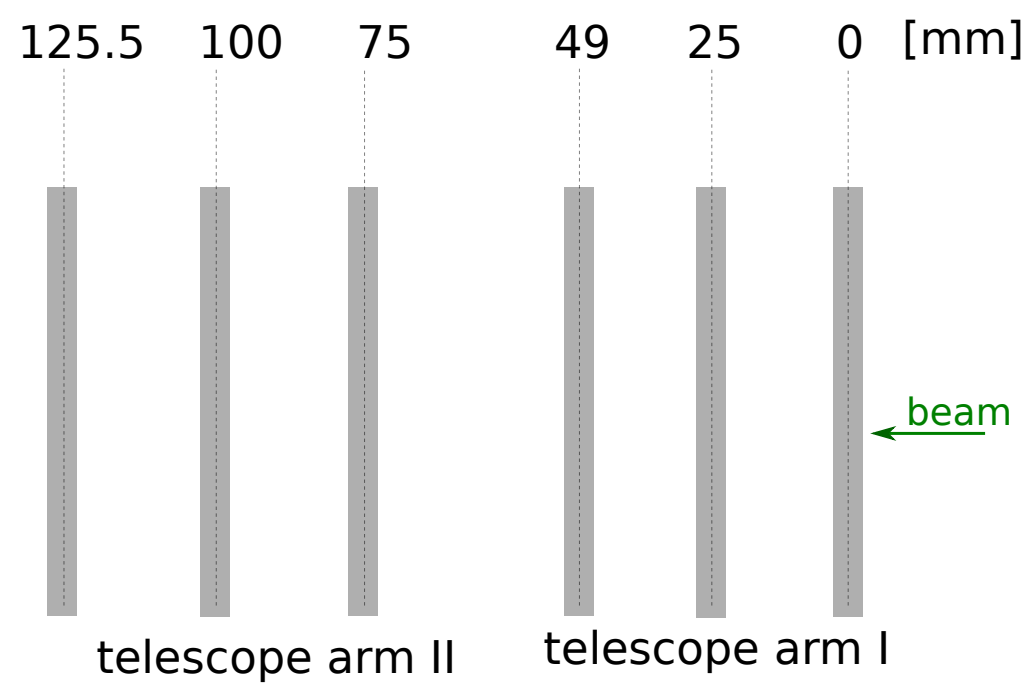

Figure 4.13.: Telescope setup during the M26 intrinsic resolution and efficiency measurements in October 2016. 


\section{Beam test experiments: Experimental setup and analysis of data}

The distance between neighbouring telescope planes was approximately $25 \mathrm{~mm}$ and there was no additional material plane centred between the two telescope arms. A schematic drawing of the telescope setup is depicted in fig. 4.13. A beam energy of $3 \mathrm{GeV}$ was selected and the comparator threshold was varied between three and twelve times the noise of the M26 sensors.

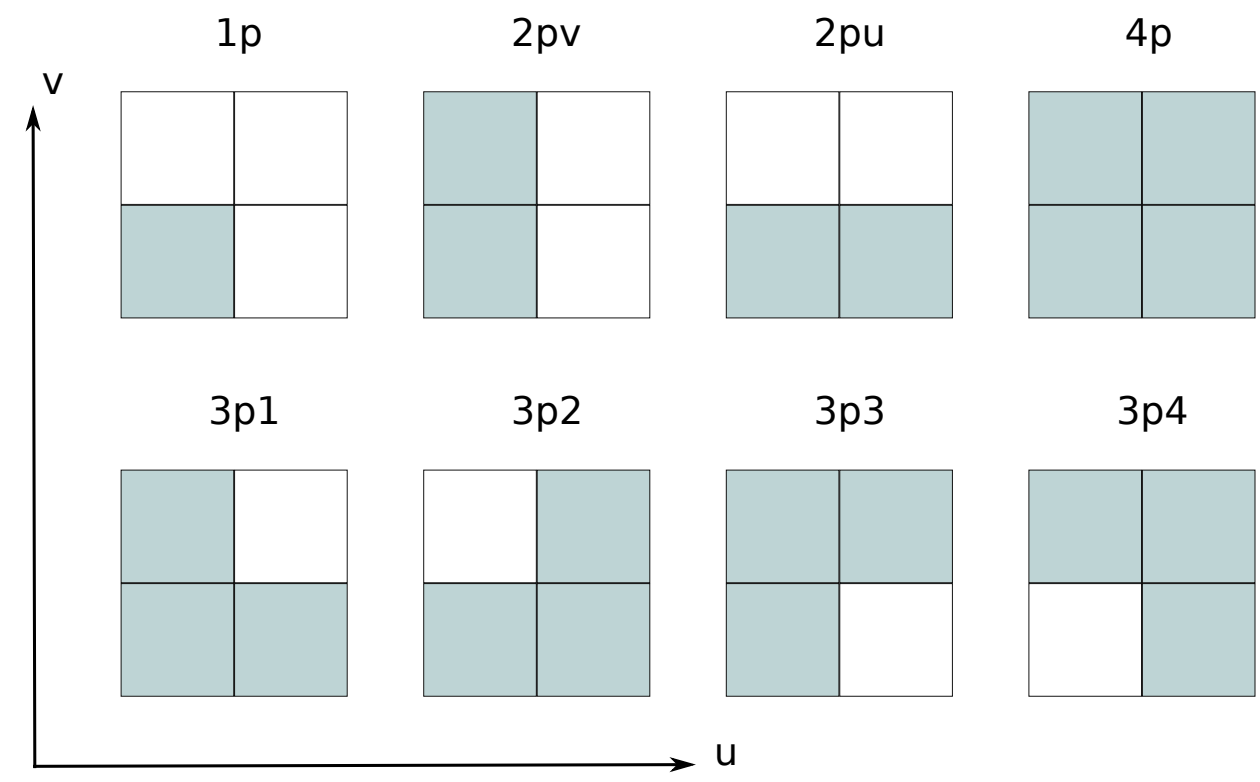

Figure 4.14.: The eight most common M26 cluster shapes with simplified names.

The cluster calibration method described in section 4.3 .10 was used to establish a clus$\operatorname{ter} D B$ with all relevant cluster shapes and their measured intersection points and their $2 \times 2$ covariance matrix. The square root of the diagonals of this matrix is called the cluster resolution in $u$ and in $v$ direction. The intersection point and the associated covariance matrix define a $1 \sigma$ error ellipse for each cluster shape. In case of digital sensors with binary readout, like the Mimosa26, and perpendicular incidence the number of important cluster shapes is rather small. The most important cluster shapes are the one-digit-cluster and the two-digit-clusters in both directions. As shown in section 4.3.10, the corresponding shape IDs are rather long. To simplify the names of the cluster shapes the one-digit-cluster will be called $1 \mathrm{p}$ here. The two-digit-cluster in $u$ direction is called $2 \mathrm{pu}$ and the two-digit-cluster in $v$ direction is called $2 \mathrm{pv}$. The frequency of larger clusters like the most compact three- and four-digit-clusters is typically below $10 \%$. The most common cluster shapes with simplified names are depicted in fig. 4.14.

The probability to measure a particular cluster shape is mostly dependent on the intersection coordinate $(u, v)$. In order to clarify this dependency fig. 4.15 depicts the number of cluster digits as a function of the track intersection for two different comparator thresholds. For a threshold of $5 \sigma_{\text {noise }}$ the average cluster size is close to one near 


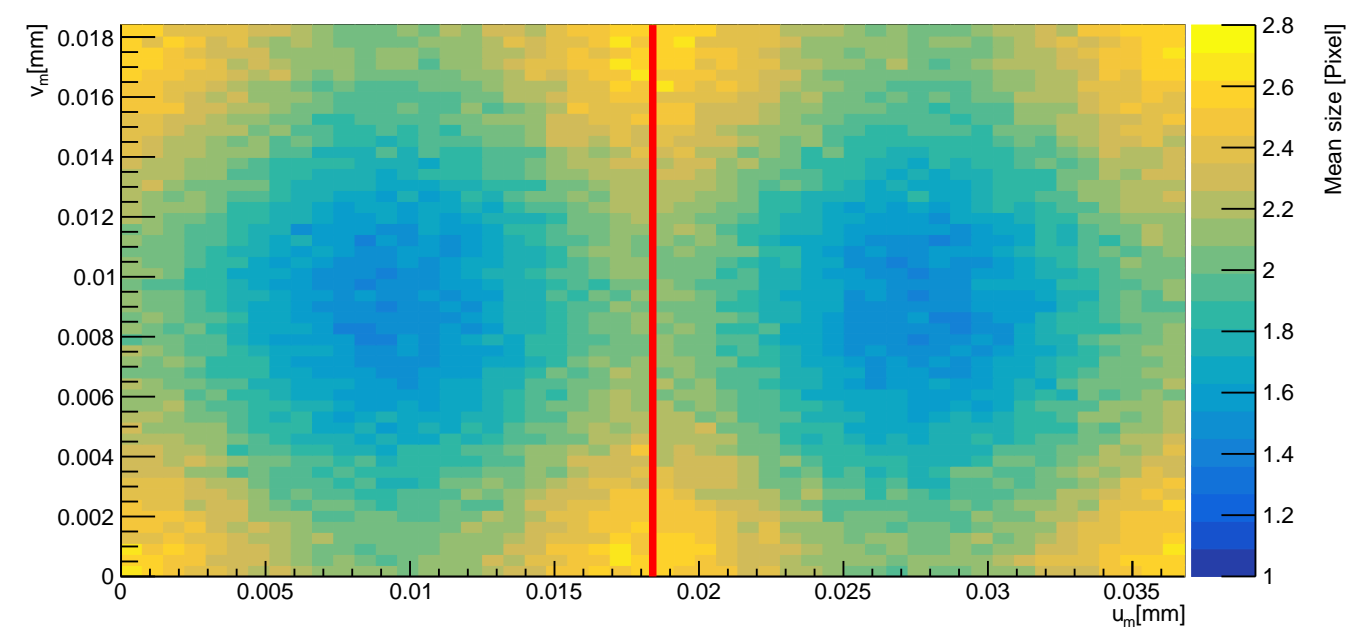

(a) Comparator threshold: 5 times $\sigma_{\text {noise }}$

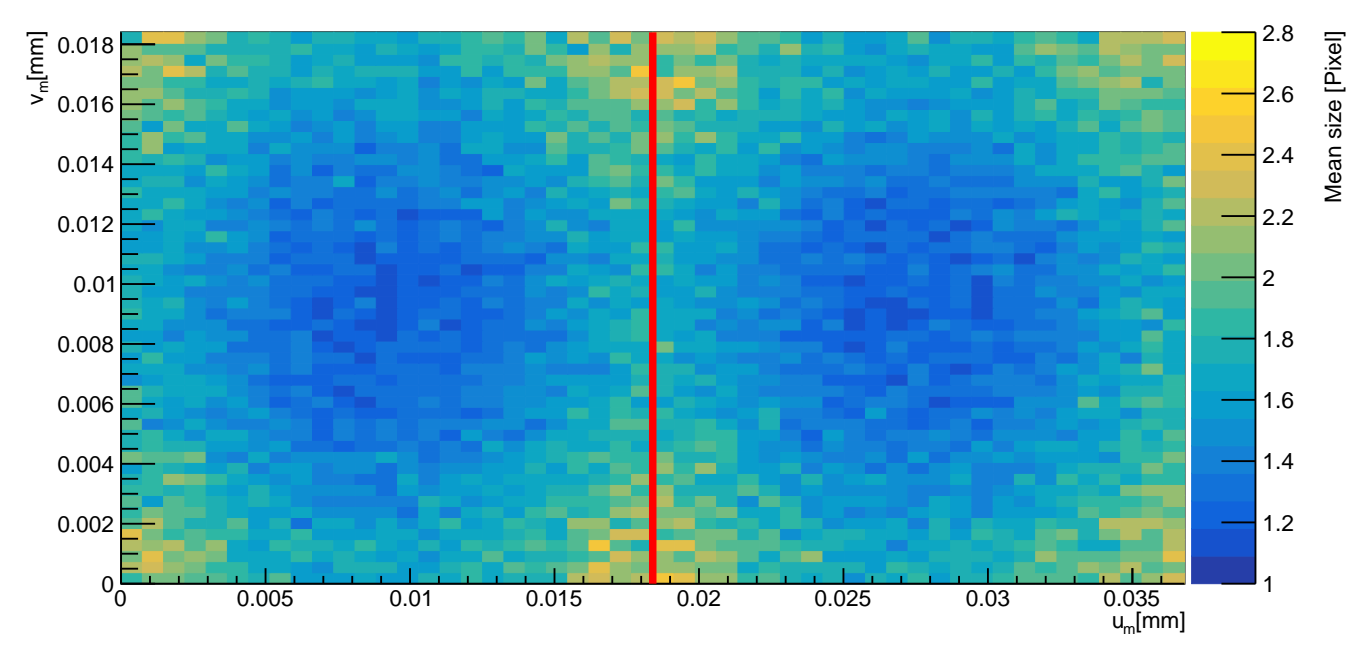

(b) Comparator threshold: 8 times $\sigma_{\text {noise }}$

Figure 4.15.: Mean size of signal clusters (projected into a 2x1 pixel grid) as a function of the measured intersection coordinates for two different M26 comparator thresholds of 5 (a) and 8 (b). The red line indicates the shared pixel border.

the centre of a pixel. Particle intersections near the pixel edges tend to produce clusters with a size of two. This can be explained through diffusion of signal charge in the sensor volume which leads to charge sharing between the two pixels, which are separated by the corresponding edge. Near the corner of the pixels another rise of the mean cluster sizes is observed because there the complete signal charge is shared between all neighbouring pixels and the probability to measure three- or four-digit-clusters gets very large. 


\section{Beam test experiments: Experimental setup and analysis of data}

The influence of the M26 threshold can be seen by comparing the two depicted average cluster size images at different thresholds in fig. 4.15a and 4.15b. The larger the threshold the larger the chance for one-digit-clusters because the probability to lose a secondary pixel with a small fraction of the signal charge increases, when the comparator threshold is raised. Accordingly, the mean cluster size is generally smaller for a larger threshold. However, for a threshold of eight the increase of cluster sizes at the edges and corners can be observed as well. The statistics in fig. $4.15 \mathrm{~b}$ is smaller than in fig. $4.15 \mathrm{a}$ due to a loss in M26 hit efficiency explained later.

As mentioned in section 4.3.10, every cluster shape has an associated 2D intersection distribution defined in the local cluster shape coordinate system. From each intersection distribution a $1 \sigma$ error ellipse can be reconstructed. The error ellipses for different cluster shapes can have an overlap and the edges are not sharp, but rather defined by the underlying 2D density. The size of the error ellipse for a certain cluster shape is proportional to the fraction of the corresponding cluster shape. The larger the ellipse the higher the probability that a particle intersection at a random location within the pixel produces the corresponding cluster shape.

Effectively, the increase of the comparator threshold mostly decreases the size of error ellipses of multi-digit-clusters. The error ellipse of single-digit-clusters remains approximately constant. Therefore, the cluster shape fraction of one-digit-clusters is expected to rise, while the other cluster fractions are expected to fall. However, increasing the threshold will ultimately also affect the one-digit-clusters: When the comparator threshold gets too large the signal charge, which remains in the pixel region, might not be sufficient to exceed the threshold and in this case the particle signal is lost altogether. When this happens, a noticeable drop in telescope efficiency can be observed.

The mean efficiency of the third M26 sensor as a function of the M26 threshold is depicted in fig. 4.16. The efficiency was calculated on the third M26 plane in the beam. This sensor can therefore be considered the device under test (DUT). The first step is to reconstruct telescope tracks without considering hits on the DUT plane. The tracks are required to have a $\chi^{2}$ value smaller than 100 and a hit on every other telescope plane. A hit on the DUT is matched to the telescope track in case the residual between the hit position and the track intersection is smaller than $200 \mu \mathrm{m}$.

The local efficiency is defined as the ratio between the number of tracks with a matched DUT hit and the overall number of tracks crossing the corresponding region. In order to calculate the mean efficiency a region of interest was defined that ensures a flat homogeneous efficiency distribution. Local efficiency variations due to triggering scintillator edges were removed by this selection. The selected area lies between column 200 and 1000 and row 100 and 500 on the DUT. Two dimensional histograms of the efficiency with indications of the selected region with homogeneous efficiency is shown in appendix 


\section{M26 efficiency}

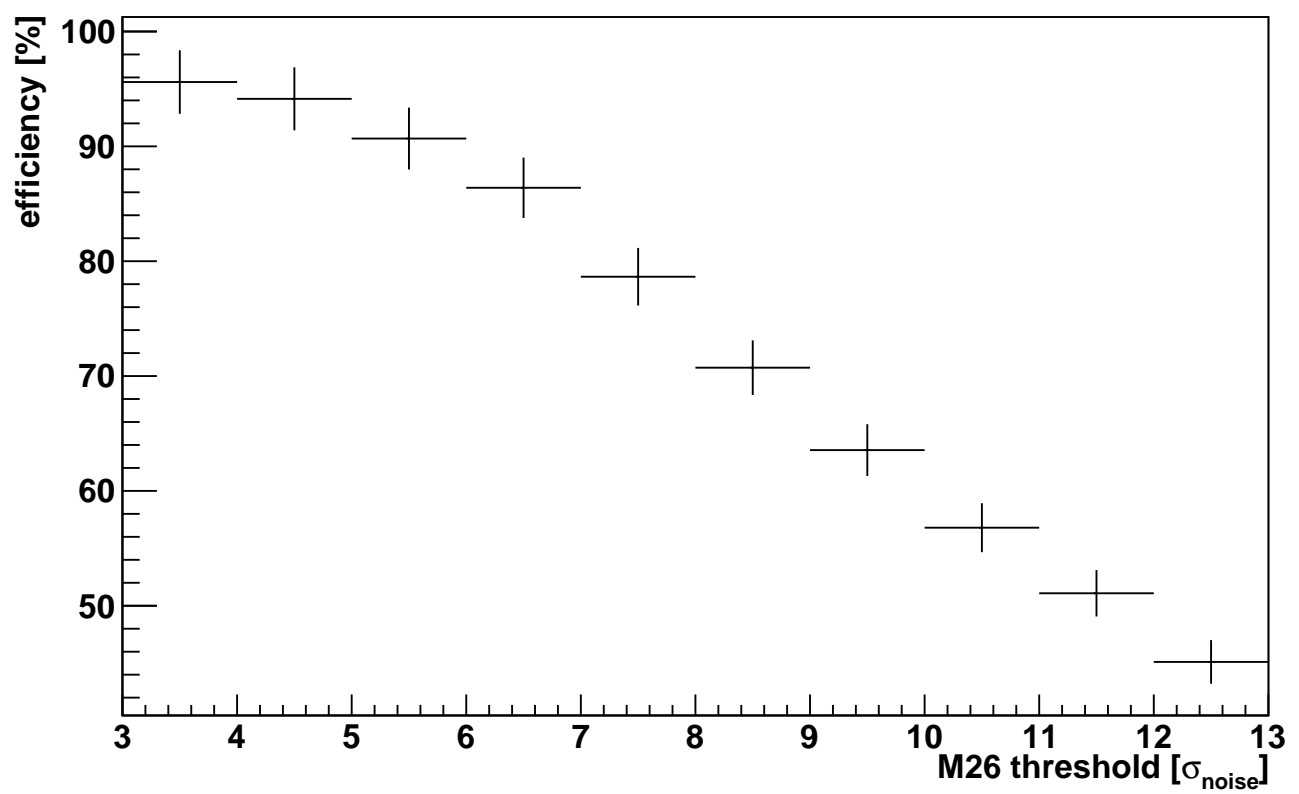

Figure 4.16.: M26 efficiency as a function of the selected comparator threshold. From a threshold of $6 \sigma_{\text {noise }}$ upwards a significant drop in efficiency is visible.

A.3. A rapid decrease in efficiency for comparator thresholds larger than six is clearly visible. For thresholds above six the efficiency drops below $90 \%$ and reaches an efficiency of approximately $50 \%$ for very large comparator thresholds of twelve. The scattering angle reconstruction and $X / X_{0}$ measurements require two tracks with three hits each in both telescope arms. A M26 efficiency below $90 \%$ would lead to a large loss of reconstructed scattering angles. In order to collect a large scattering angle sample in a reasonable amount of time, it is therefore recommended to use a M26 threshold below six.

In addition to the efficiency studies, the relative fraction and resolution of the different cluster shape types was measured. Fig. 4.17 depicts the fraction of three different cluster shapes on the M26 sensor at the third position in the beam line as a function of the comparator threshold. As can be seen the fraction of $1 \mathrm{p}$ clusters increases with larger thresholds, while the number of clusters with two digits decreases. This behaviour is not surprising as larger clusters require charge sharing between two or more pixels. This charge sharing leads to a smaller amount of signal charge per pixel. Accordingly, for large comparator threshold the measurement of multi-digit-clusters is less probable. As can be seen in figure 4.17 , the fractions of $2 \mathrm{pu}$ and $2 \mathrm{pv}$ clusters are approximately identical for a given threshold setting. This is not unexpected as the M26 pixel pitch is identical in both directions and the beam incidence is approximately perpendicular. 


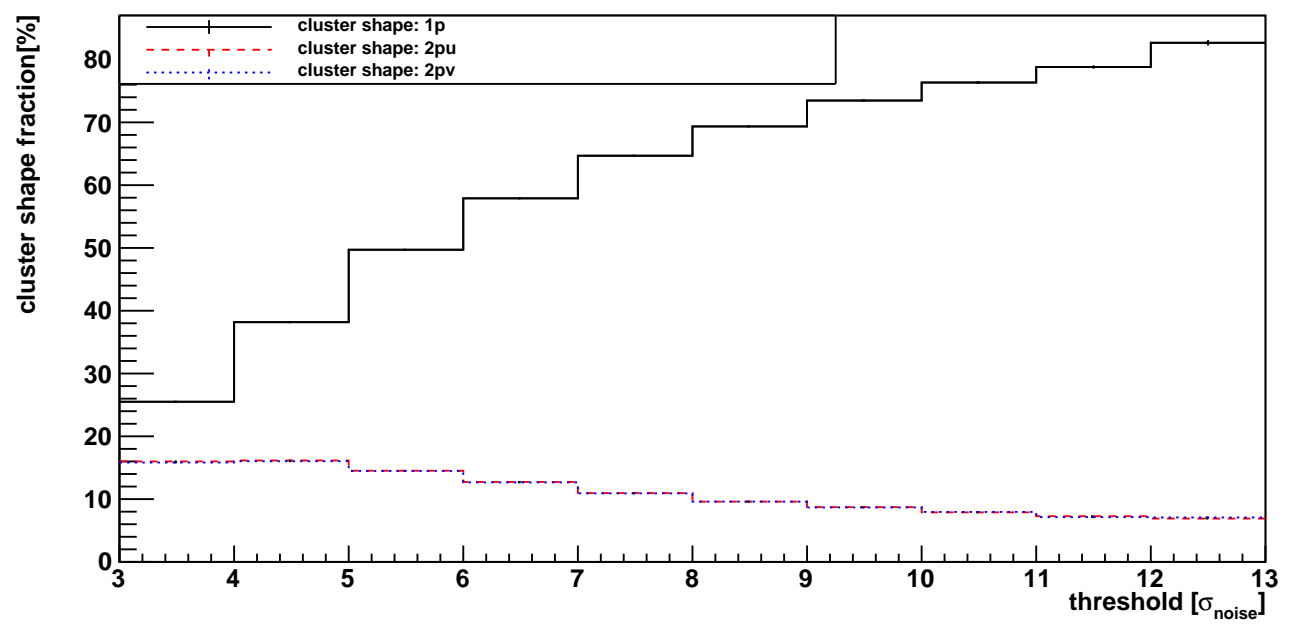

Figure 4.17.: Cluster shape fraction for the $1 \mathrm{p}, 2 \mathrm{pu}$ and $2 \mathrm{pv}$ cluster shapes as a function of the M26 threshold.

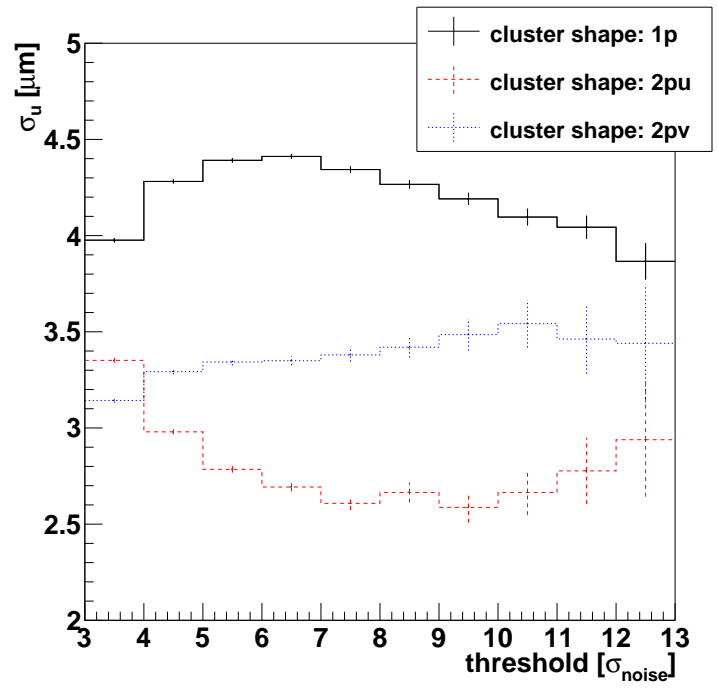

(a)

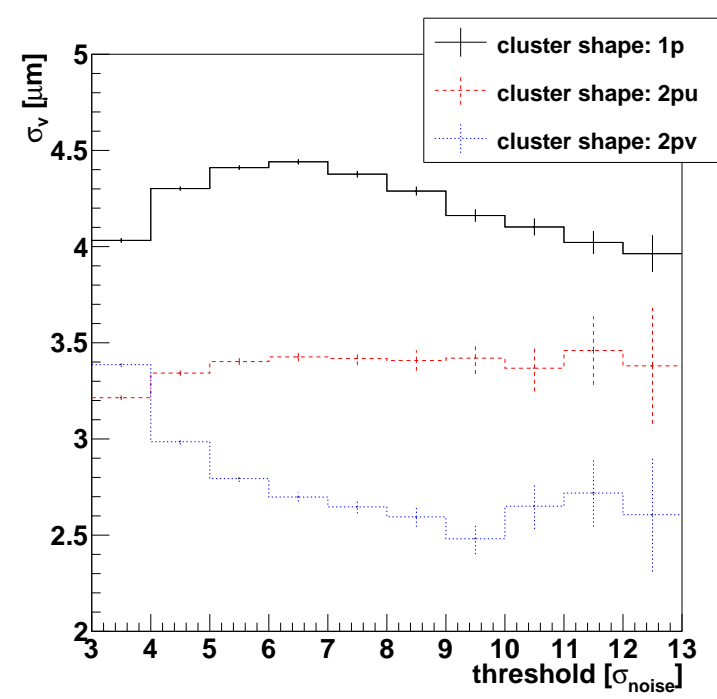

(b)

Figure 4.18.: Cluster shape errors in $u$ (a) and $v$ (b) direction for the 1p, 2pu and 2pv cluster shapes as a function of the M26 threshold.

The cluster resolution in both directions for the three most important cluster shapes as a function of the M26 threshold is depicted in fig. 4.18. As the cluster fraction is proportional to the size of the error ellipse of the corresponding cluster shape the 
resolution of single-digit-clusters worsens with a growing threshold up to a threshold of six. The resolution in $u$ direction improves for the $2 \mathrm{pu}$ cluster shape as the cluster shape fraction and therefore the size of the error ellipse (in $u$ direction) decreases. An analogous behaviour can be observed for the $v$ resolution of $2 \mathrm{pv}$ clusters. The $v$ resolution of $2 \mathrm{pu}$ clusters and the $u$ resolution of $2 \mathrm{pv}$ increases slightly with increasing comparator threshold. The $1 \mathrm{p}$ cluster resolution deteriorates up to a M26 threshold of six. For thresholds larger than six the resolution improves again. This is connected to the already mentioned efficiency loss at high comparator thresholds: The increased amount of charge sharing for particle trajectories, which are traversing the original pixel close to one of the pixels edges leads to less signal charge left in the initial pixel and therefore increases the probability to lose the signal altogether. Consequently, the size of the error ellipse is effectively limited to a smaller region around the centre of the pixel.

Fig. 4.18 depicts the resolutions for three cluster shapes. However, the goal of this section is to determine the optimal comparator threshold which leads to the optimal (i.e. smallest) average cluster resolution of all cluster shapes. The average cluster resolution is defined by a weighted mean of individual cluster resolutions

$$
\begin{aligned}
& \left\langle\sigma_{u}\right\rangle=\sum_{i} w_{i} \sigma_{u}^{i} \\
& \left\langle\sigma_{v}\right\rangle=\sum_{i} w_{i} \sigma_{v}^{i} .
\end{aligned}
$$

The sum over the index $i$ corresponds to a sum over all cluster shapes with an entry in the cluster DB. The weights $w_{i}$ are given by the fractions of the corresponding cluster shapes. The efficiency measurements in fig. 4.16 indicated that M26 threshold settings larger than six will lead to inefficient telescope sensors. The threshold range used here is therefore limited to values below six. The resulting mean cluster resolutions in both directions for thresholds below six are summarised in table 4.2. As mentioned in section 4.3.3 a minimum number of clusters is required to determine the resolution of a cluster type. Therefore some rare cluster types will not be covered by the cluster DB. The fraction of clusters covered by the cluster $D B$, called cluster coverage, is included in the table.

\begin{tabular}{|c||c|c|c|c|}
\hline Threshold $\left[\boldsymbol{\sigma}_{\text {noise }}\right]$ & 3 & 4 & 5 & 6 \\
\hline$\left\langle\boldsymbol{\sigma}_{\boldsymbol{u}}\right\rangle[\mathbf{\mu m}]$ & $3.30 \pm 0.08$ & $3.5 \pm 0.1$ & $3.7 \pm 0.1$ & $3.8 \pm 0.1$ \\
\hline$\left\langle\boldsymbol{\sigma}_{\boldsymbol{v}}\right\rangle[\mathbf{\mu m}]$ & $3.35 \pm 0.08$ & $3.5 \pm 0.1$ & $3.7 \pm 0.1$ & $3.8 \pm 0.1$ \\
\hline cluster coverage & 99.8 & 99.8 & 99.9 & 99.9 \\
\hline
\end{tabular}

Table 4.2.: Average cluster resolution at different comparator thresholds

For this measurement, it was found that a threshold of three is the best choice, as the mean cluster resolution in this configuration is the smallest. However, the selection of the threshold should be based on the individual experimental setup. Especially in the case of a noisy telescope it may be beneficial to use a slightly larger threshold. 


\subsection{The beam test simulation algorithm}

$T B S W$ also provides processors to simulate particle transitions and detector responses of beam test experiments. These simulations are used to validate the reconstruction processors against verified information from the simulations. The simulation processors are based on simplified models of the particle beam, beam particle propagation and signal generation in the sensor volume. These simplifications are mentioned in the descriptions of the simulation processors below. Beam test simulations are, for example, used in chapter 5 to determine the optimal telescope setup for radiation length measurements and in chapter 6 to validate the radiation length imaging procedure and $X / X_{0}$ calibration scheme. The simulations involve three steps that will be explained on the following pages. A much more detailed description of the simulations can be found in [67]. Fig. 4.19 depicts a schematic drawing of the simulation path which consists of three processors.

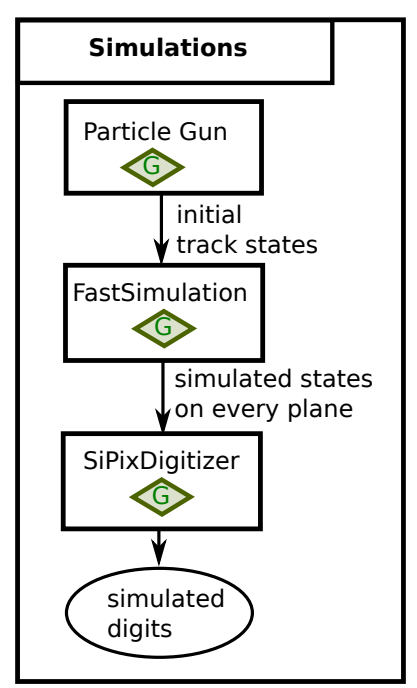

Figure 4.19.: Processor sequence for the generation of digits from a simulated particle transition through a telescope.

The ParticleGun processor generates a set of particles at a position upstream of the telescope. The particles are represented by track states, which are randomly determined from the preselected beam parameters. The track state $\eta_{\mathrm{PG}}$ of the particle is similar to the one defined in eq. 4.9, but has the particle momentum in $\mathrm{GeV} / c$ as a fifth vector component instead of $q / p$

$$
\eta_{\mathrm{PG}}=\left(\begin{array}{c}
(d u / d w) \\
(d v / d w) \\
u \\
v \\
p
\end{array}\right)
$$


The task of the particle gun processor is to generate a randomised track state for each new particle, which is in agreement with the beam parameters. The beam parameters can be described by the following two quantities corresponding to the mean track state $\eta_{\text {beam }}$ of beam particles and the covariance matrix $C_{\text {beam }}$

$$
\begin{aligned}
\eta_{\text {beam }} & =\left(\begin{array}{c}
(d u / d w)_{\text {mean }} \\
(d v / d w)_{\text {mean }} \\
u_{\text {mean }} \\
v_{\text {mean }} \\
p_{\text {mean }}
\end{array}\right) \\
C_{\text {beam }} & =\left(\begin{array}{ccccc}
\sigma_{m_{u}, m_{u}}^{2} & 0 & \sigma_{m_{u}, u}^{2} & 0 & 0 \\
0 & \sigma_{m_{v}, m_{v}}^{2} & 0 & \sigma_{m_{v}, v}^{2} & 0 \\
\sigma_{m_{u}, u}^{2} & 0 & \sigma_{u, u}^{2} & 0 & \sigma_{u, p}^{2} \\
0 & \sigma_{m_{v}, v}^{2} & 0 & \sigma_{v, v}^{2} & \sigma_{v, p}^{2} \\
0 & 0 & \sigma_{u, p}^{2} & \sigma_{v, p}^{2} & \sigma_{p, p}^{2}
\end{array}\right) .
\end{aligned}
$$

After generating initial track states with the ParticleGun processor, the simulation of particle propagations through the telescope is conducted. The FastSimulation processor is used to simulate the trajectory of each particle, which takes different material effects like multiple scattering and bremsstrahlung into account. Multiple scattering angles are randomly determined according to the Highland model. The scattering processes takes place on the telescope planes and, in air, halfway between the telescope planes. Energy loss due to bremsstrahlung of electrons is simulated by using the Bethe-Heitler model. Alternatively, the simulation of energy losses due to bremsstrahlung can be switched off. Only electron trajectories are affected by the energy loss and secondary particles are not created in the simulations.

The simulation of a trajectory works as follows: The initial track state is used to define a straight line, which is then extrapolated to the next material or artificial air plane. At these surfaces, the effective thickness, current particle momentum and material parameters like the radiation length constant $X_{0}$ are used to simulate the material effects on the track state. Multiple scattering has an impact on the incidence angles $d u / d w$ and $d v / d w$, while bremsstrahlung effects modify the particle momentum. After a material interaction, the track state entries are updated accordingly and the modified state is extrapolated towards the next material surface. A schematic trajectory of a simulated electron is depicted in fig. 4.20.

The last simulation processor is the SiPixDigitizer which is used to simulate a Pixel detector and model the detector response to the particle trajectory simulated in the previous step. The processor can be tuned to roughly model a M26 sensor. The initial step of the SiPixDigitizer is to determine a path through the sensitive sensor volume. The path is divided into small segments. For each segment the deposited energy in the 


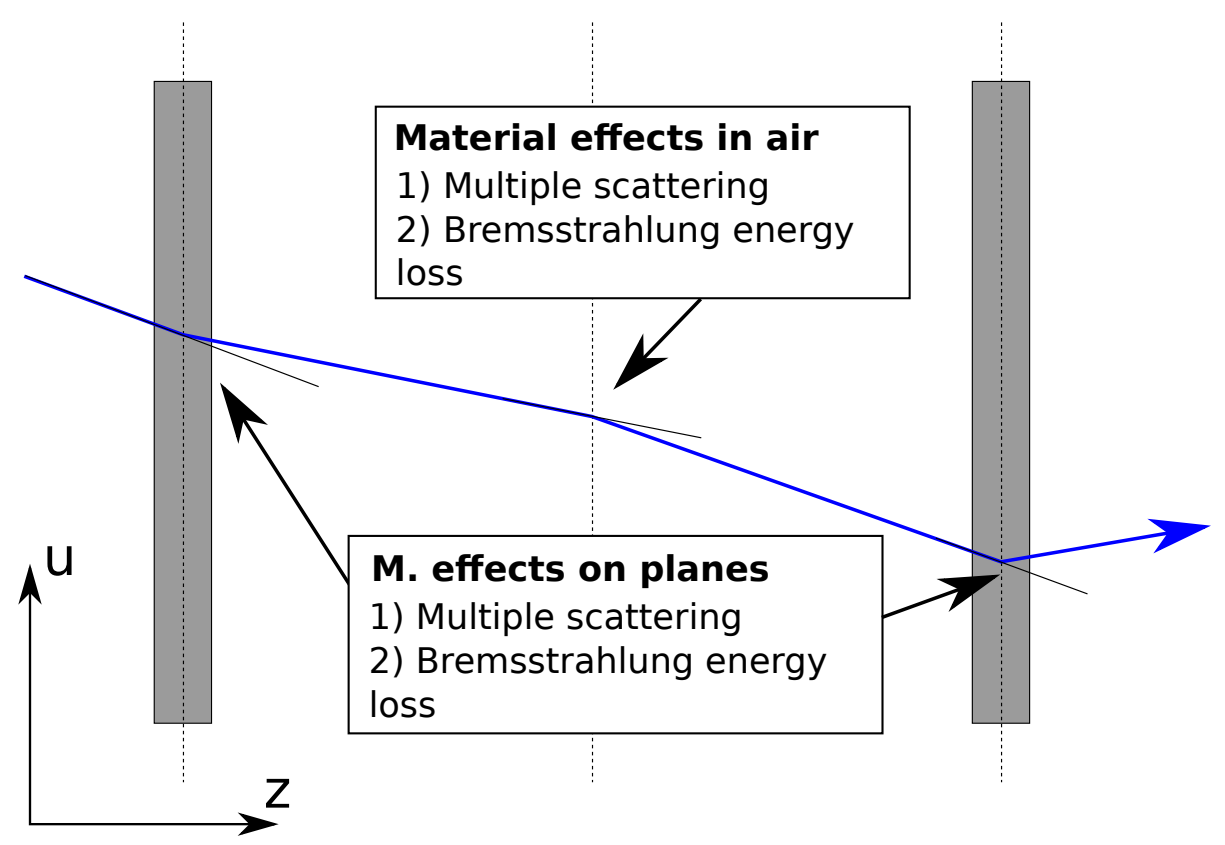

Figure 4.20.: Schematic drawing of the functionality of the FastSimulation processor. Multiple scattering, bremsstrahlung and ionisation processes are simulated on the telescope planes and in the air volume in between.

detector volumes is calculated. The deposited energy can be modelled in two ways. The first option is to calculate the mean energy loss using the Bethe-Bloch formula. The default case is to use this mean value as the deposited energy. Alternatively the mean value can be used as the parameter of a Landau distribution and the individual energy loss can be determined as a random variable from this distribution. This deposited energy is converted into electron hole pairs and then used in the last step to simulate the response of the telescope planes to the particle transition and generate sensor digits. This involves drift processes of the charge cloud, charge sharing between sensor pixels and simulation of readout noise. The digitiser model is explained in detail in [67].

The simulated digits are connected to the event number of the simulated particle and stored in a LCIO file. The LCIO file can be used for calibration, angle reconstruction and radiation length measurements. The only difference is that the EUDAQ conversion and unpacking steps are not necessary for the simulated data. 


\section{CHAPTER 5}

\section{Scattering angle reconstruction and radiation length imaging}

The scope of this chapter is to give an overview of the individual steps of the radiation length measurement method that is the subject of this work. As explained in chapter 3 , the width of multiple scattering angle distributions depends on the radiation length $X / X_{0}$ of the traversed material. Radiation length measurement require collections of in- and out-states which describe the particle trajectory on the target plane before and after the scattering, respectively. The individual steps of the radiation length imaging method are summarised in the following enumeration:

1. Matching of track states and angle reconstruction (section 5.1): In- and out-states likely related to the same particle trajectory are matched. The matching approach is based on the distance between the intersections of the track states. Slopes of matched track state pairs are used to calculate projected scattering angles on the central plane.

2. Determination of the telescope angle resolution (section 5.2): A numerical approach is used to estimate the uncertainties of the reconstructed scattering angles. The telescope angle resolution is defined from the angle uncertainty distributions.

3. Radiation length imaging (section 5.3): The scattering plane is divided into a $2 \mathrm{D}$ grid which is aligned with the local $u$ and $v$ axes. A 1D scattering angle histogram is assigned to each pixel of the grid. Track intersections of each track state pair are calculated and the projected scattering angles are added to the histogram of the appropriate grid pixel. After all available angles are filled into histograms, the scattering angle distributions are fitted successively with a model function and the radiation length value is determined from the fit. The determined $X / X_{0}$ values are assigned to the corresponding grid pixels. Then, the radiation length values are visualised in the $u-v$ plane and a radiation length image is generated. 
4. $X / X_{0}$ calibration measurements (section 5.4): The analysis of scattering angle data also includes a calibration step. This step is required to calibrate the telescope angle resolution and other parameters of the model function mentioned in the previous step.

The first two steps are performed by a TBSW processor called X0Estimator. Steps 3 and 4 describe the radiation length imaging and calibration process which is performed by ROOT macros. Sections 5.1 to 5.4 describe the details of the algorithms mentioned in the enumeration. Section 5.5 presents results from simulations that give an overview of optimised telescope setups and beam energy settings for radiation length measurements in a specific radiation length range.

\subsection{Scattering angle reconstruction}

For the reconstruction of scattering angles two separate track samples, the up-and downstream track, are reconstructed in the upstream and downstream telescope arm. A schematic depiction of the basic idea of the angle reconstruction on the central scattering plane $(k=3)$ can be seen in fig. 5.1.

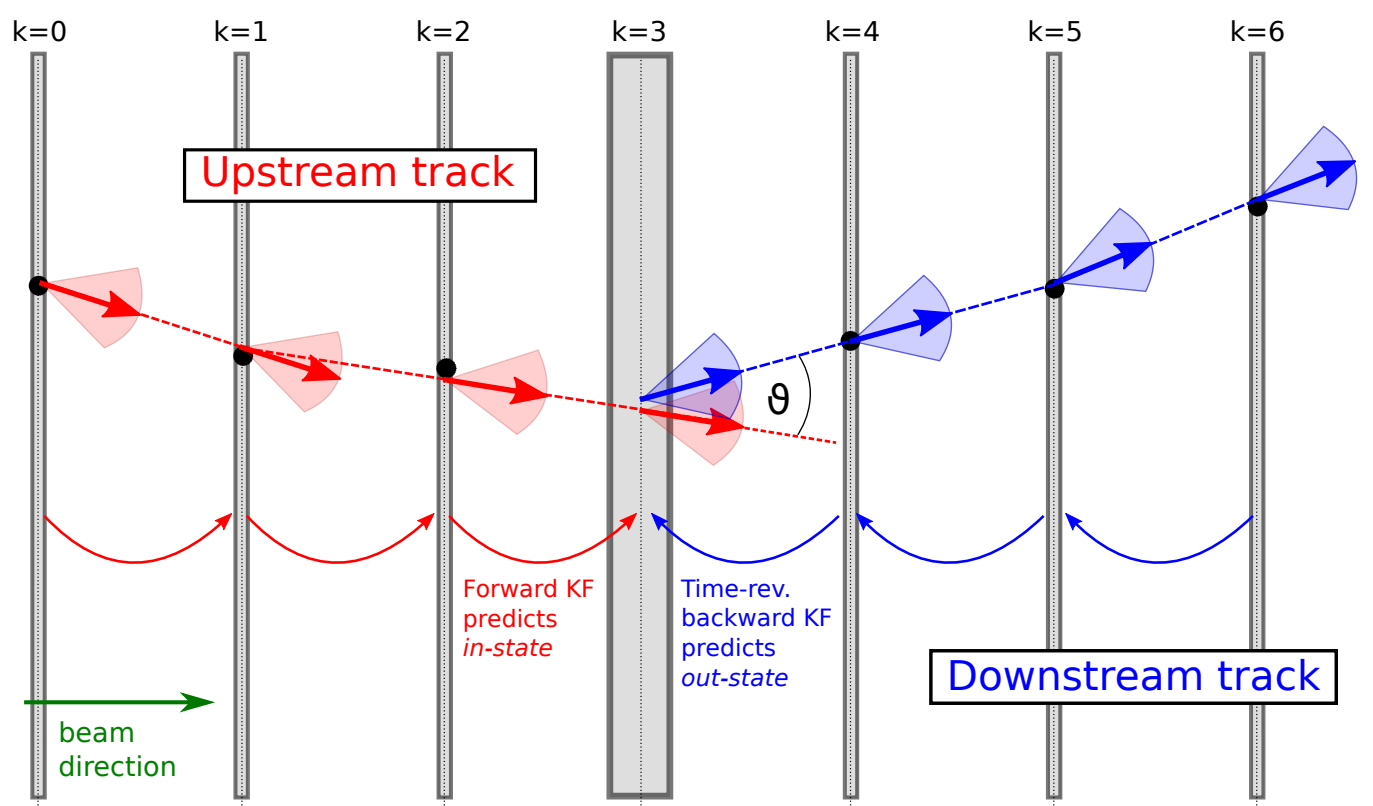

Figure 5.1.: Schematic drawing of a particle transition in a telescope with six sensor planes and a central scattering target $(k=3)$. Up- and downstream tracks and their prediction of the in- and out-state using a forward and time-reversed backward Kalman filter, respectively, are shown.

Both track samples must have at least three hits per track, as indicated by the black dots in the figure. Furthermore, the track samples undergo a strict selection process 
with respect to the track $\chi^{2}$ values. This ensures that the selected tracks are most likely coming from the transition of a real beam particle. These two collections of tracks are used by a $T B S W$ processor called XOEstimator to calculate scattering angles. The individual steps performed by the X0Estimator are shown in figure 5.2.

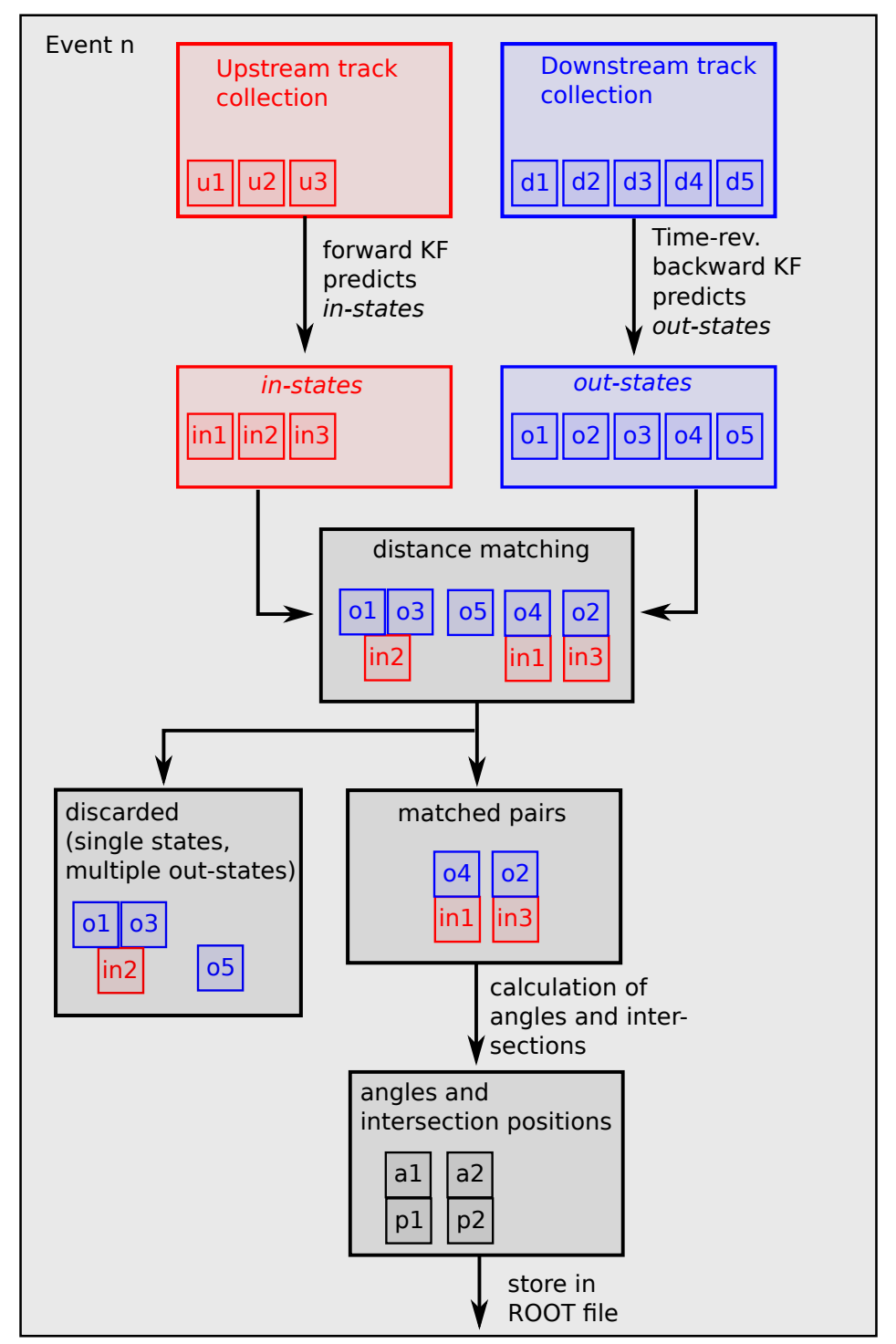

Figure 5.2.: Schematic drawing depicting the individual steps performed by the X0Estimator processor starting from a up- and a downstream track collection. It is possible that the two track collections have a different number of tracks.

In order to predict the track state on the target plane $k$ before the scattering on plane $k$, a forward Kalman filter is applied to the hits in the upstream telescope arm. As 


\section{Scattering angle reconstruction and radiation length imaging}

explained in the previous chapter, the forward Kalman filter is an incremental algorithm that predicts track states on a telescope plane, updates the track state with a hit measurement on that plane and then extrapolates the state onto the next plane. During the extrapolation step, multiple scattering effects are taken into account. The extrapolation of the track state $\eta$ and its covariance matrix $C$ from the closest upstream telescope plane $k-1$ to the target plane $k$ is given by

$$
\begin{aligned}
\eta_{k}^{\text {pred }} & =\eta_{k}^{\text {in }}=F_{\mathrm{k} \mid k-1} \eta_{k-1}^{\text {in }} \\
C_{k}^{\text {pred }} & =C_{k}^{\text {in }}=F_{\mathrm{k} \mid k-1} C_{k-1}^{\text {in }} F_{\mathrm{k} \mid k-1}{ }^{T}+F_{k \mid k-1} G_{k-1} Q_{k-1} G_{k-1}^{\mathrm{T}} F_{k \mid k-1}{ }^{\mathrm{T}} .
\end{aligned}
$$

As mentioned before, $F_{\mathrm{k} \mid k-1}$ describes the straight-line propagation of the track state from plane $k-1$ to plane $k$. $Q_{k-1}$ models the uncertainty due to multiple scattering on plane $k-1$. The important feature of the forward Kalman filter is that the extrapolation takes the scattering on plane $k-1$ into account. The scattering on plane $k$, however, is not used in the extrapolation step. Accordingly, the forward Kalman filter yields a prediction of the track state $\eta_{k}^{\text {in }}$ before the scattering on target plane $k$, called in-state:

$$
\begin{aligned}
& \eta_{k}^{\text {in }}=\left(\begin{array}{c}
(d u / d w)^{\text {in }} \\
(d v / d w)^{\text {in }} \\
u^{\text {in }} \\
v^{\text {in }}
\end{array}\right)=\left(\begin{array}{c}
m_{u}^{\text {in }} \\
m_{v}^{\text {in }} \\
u^{\text {in }} \\
v^{\text {in }}
\end{array}\right)
\end{aligned}
$$

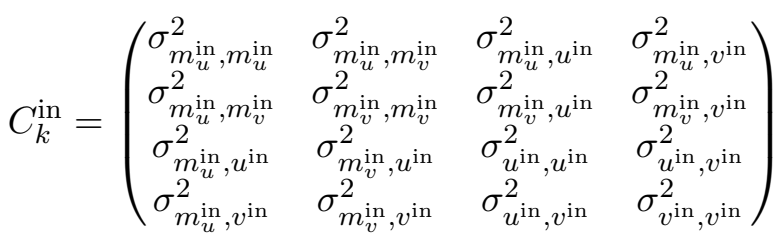

Here, $u$ and $v$ indicate intersection positions and $m_{u}$ and $m_{v}$ describe slopes of the trajectory in $u$ and $v$ direction. In order to calculate scattering angles (see fig. 5.1), the track state after scattering on the target plane is required. This track state can be estimated by using a time reversed backward Kalman filter on the hits in the downstream telescope arm. The extrapolation from the closest downstream telescope plane $k+1$ to the target plane $k$ is given by

$$
\begin{aligned}
\eta^{\text {pred }} & =\eta_{k}^{\text {out }}=F_{\mathrm{k} \mid k+1} \eta_{k+1}^{\text {out }} \\
C_{k}^{\text {pred }} & =C_{k}^{\text {out }}=F_{\mathrm{k} \mid k+1} C_{k+1}^{\text {out }} F_{\mathrm{k}+1 \mid k}{ }^{T}+F_{k \mid k+1} G_{k+1} Q_{k+1} G_{k+1}{ }^{\mathrm{T}} F_{k \mid k+1}{ }^{\mathrm{T}} .
\end{aligned}
$$

As can be seen, in this case, the straight-line propagation matrix $F_{\mathrm{k} \mid k+1}$ leads to an extrapolation in the opposite direction compared to the forward Kalman filter. During the extrapolation, the scattering effects on plane $k+1$ are taken into account, while the 
effects on plane $k$ are not used. As a consequence, the time-reversed backward Kalman filter predicts the track state after the scattering on a target plane. This state is called the out-state and is given by

$$
\begin{gathered}
\eta_{k}^{\text {out }}=\left(\begin{array}{c}
(d u / d w)^{\text {out }} \\
(d v / d w)^{\text {out }} \\
u^{\text {out }} \\
v^{\text {out }}
\end{array}\right)=\left(\begin{array}{c}
m_{u}^{\text {out }} \\
m_{v}^{\text {out }} \\
u^{\text {out }} \\
v^{\text {out }}
\end{array}\right) \\
C_{k}^{\text {out }}=\left(\begin{array}{llll}
\sigma_{m_{u}^{\text {out }}, m_{u}^{\text {out }}}^{2} & \sigma_{m_{u}^{\text {out }}, m_{v}^{\text {out }}}^{2} & \sigma_{m_{u}^{\text {out }}, u^{\text {out }}}^{2} & \sigma_{m_{u}^{\text {out }}, v^{\text {out }}}^{2} \\
\sigma_{m_{u}^{\text {out }}, m_{v}^{\text {out }}}^{2} & \sigma_{m_{v}^{\text {out }}, m_{v}^{\text {out }}}^{2} & \sigma_{m_{v}^{\text {out }}, u^{\text {out }}}^{\sigma^{2}} & \sigma_{m_{v}^{\text {out }}, v^{\text {out }}}^{2} \\
\sigma_{m_{u}^{\text {out }}, u^{\text {out }}}^{2} & \sigma_{m_{v}^{\text {out }}, u^{\text {out }}}^{2} & \sigma_{u^{\text {out }}, u^{\text {out }}}^{2} & \sigma_{u^{\text {out }}, v^{\text {out }}}^{2} \\
\sigma_{m_{u}^{\text {out }}, v^{\text {out }}}^{2} & \sigma_{m_{v}^{\text {out }}, v^{\text {out }}}^{2} & \sigma_{u^{\text {out }}, v^{\text {out }}}^{2} & \sigma_{v^{\text {out }}, v^{\text {out }}}^{2}
\end{array}\right) .
\end{gathered}
$$

For the sake of simplicity the plane index $k$ will be left out from now on. The in- and out-states used here always correspond to the states on the target plane $k=3$. The schematic drawing in fig. 5.1 depicts telescope hits from a charged particle transition, where the upstream track (and its associated instates) are shown in red and the downstream track (and its associated outstates) are shown in blue. After reconstructing all available in- and out-states from a single event, track states have to be paired according to the likelihood being associated with the same particle transition. A distance criterium on the target plane is used to determine which track states to match.

The distance matching algorithm starts with an in-state. The distance between the intersection position of the in-state and every available out-state is computed and the out-state with the minimum distance is selected. In case the minimum distance is smaller than a previously selected threshold in the order of $100 \mu \mathrm{m}$, the two track states are matched and the matched out-state is removed from the pool of available out-states. Afterwards the procedure is repeated with the same in-state in case there is a another out-state that fulfils the matching criterium. The matching loop of this particular instate will be terminated once the minimum distances between the in-state intersection and all remaining out-state intersections are larger than the distance threshold. Afterwards the matching algorithm repeats with the next in-state until all in-states have been accounted for. In few cases more than one out-state is matched with a single in-state. It can be assumed that most of them are either wrongly matched due to rare two particle transitions in close vicinity of each other or due to one of the downstream tracks being wrongly reconstructed. All multi-out-state matches are discarded and not used in the subsequent analysis steps.

Under the assumption that an in- and out-state have been matched successfully, the intersection vector of the particle transition $(\bar{u}, \bar{v})^{\mathrm{T}}$ can be calculated from the two track states: 


$$
\left(\begin{array}{c}
\bar{u} \\
\bar{v}
\end{array}\right)=\frac{1}{2}\left(\left(\begin{array}{l}
u_{\text {in }}^{\text {in }} \\
v^{\text {in }}
\end{array}\right)+\left(\begin{array}{l}
u^{\text {out }} \\
v^{\text {out }}
\end{array}\right)\right)
$$

This arithmetic mean is an approximation to the optimal weighted mean and neglects small correlation effects. The directions of a particle trajectory before and after scattering on the central scattering plane are given by

$$
\begin{gathered}
\vec{n}^{\text {in }}=\frac{1}{\sqrt{1+\left(m_{u}^{\text {in }}\right)^{2}+\left(m_{v}^{\text {in }}\right)^{2}}} \cdot\left(\begin{array}{c}
m_{u}^{\text {in }} \\
m_{v}^{\text {in }} \\
1
\end{array}\right) \\
\vec{n}^{\text {out }}=\frac{1}{\sqrt{1+\left(m_{u}^{\text {out }}\right)^{2}+\left(m_{v}^{\text {out }}\right)^{2}}} \cdot\left(\begin{array}{c}
m_{u}^{\text {out }} \\
m_{v}^{\text {out }} \\
1
\end{array}\right) .
\end{gathered}
$$

In order to simplify the calculation of the projected scattering angles, a coordinate transformation from the local coordinate system into the co-moving system of the incoming particle $\left(\overrightarrow{e_{\mathrm{a}}}, \overrightarrow{e_{\mathrm{b}}}, \overrightarrow{e_{\mathrm{c}}}\right)$ is carried out (see fig. 5.3). The co-moving frame is defined in the following way: The unit vector $\overrightarrow{e_{\mathrm{c}}}$ points into the same direction as $\overrightarrow{n_{\mathrm{in}}}, \overrightarrow{e_{\mathrm{b}}}$ is perpendicular to $\overrightarrow{e_{\mathrm{c}}}$ and the $\mathrm{u}$-axis of the local coordinate system of the scattering plane. $\overrightarrow{e_{\mathrm{a}}}$ is the last vector to complete the right-handed Cartesian system. The rotation matrix $A$ from the local coordinate system of the target plane into the co-moving frame can be computed via

$$
\left(\overrightarrow{e_{\mathrm{a}}}\right)^{\mathrm{T}}=A \cdot \overrightarrow{e_{u}}, \quad\left(\overrightarrow{e_{\mathrm{b}}}\right)^{\mathrm{T}}=A \cdot \overrightarrow{e_{v}} \quad \text { and } \quad\left(\overrightarrow{e_{\mathrm{c}}}\right)^{\mathrm{T}}=A \cdot \overrightarrow{e_{w}} .
$$

By applying the rotation $A$ to the vector $\vec{n}^{\text {out }}$, the direction of the out-state can be calculated as

$$
\vec{n}_{(\mathrm{a}, \mathrm{b}, \mathrm{c})}^{\text {out }}=\left(\begin{array}{c}
n_{1} \\
n_{2} \\
n_{3}
\end{array}\right)=\left(A \cdot \vec{n}_{(u, v, w)}^{\text {out }}\right)^{\mathrm{T}}
$$

An illustration of the track states in the co-moving frame of the incoming particle can be seen in fig. 5.3. In this rotated coordinate system, projected scattering angles $\vartheta_{u}$ and $\vartheta_{v}$ can be calculated directly from the slopes of the out-states as

$$
\begin{aligned}
& \vartheta_{u}=\arctan \left(\left(m_{u}^{\text {out }}\right)_{(\mathrm{a}, \mathrm{b}, \mathrm{c})}\right) \stackrel{\vartheta_{u} \text { small }}{\approx}\left(\frac{n_{1}}{n_{3}}\right) \\
& \vartheta_{v}=\arctan \left(\left(m_{v}^{\text {out }}\right)_{(\mathrm{a}, \mathrm{b}, \mathrm{c})}\right) \stackrel{\vartheta_{v} \text { small }}{\approx}\left(\frac{n_{2}}{n_{3}}\right) .
\end{aligned}
$$




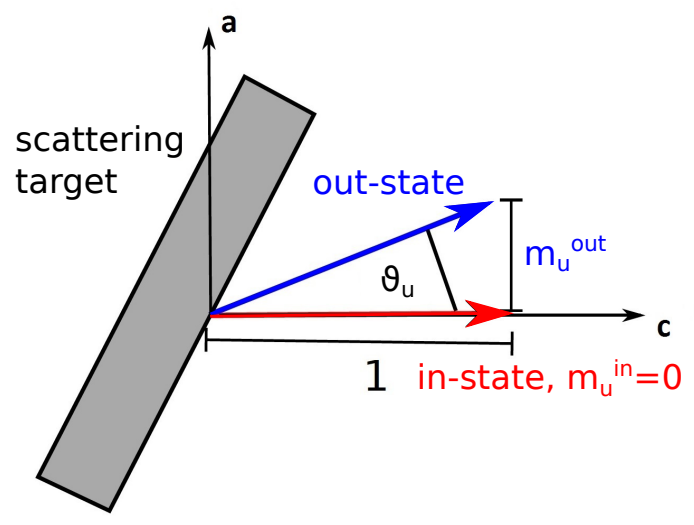

Figure 5.3.: Schematic depiction of the in- and out-state of a particle trajectory in the co-moving frame of the incoming particle. Since scattering angles can be assumed to be small, the slope of the out-state corresponds to the scattering angle $\vartheta_{u}$.

The variances $\sigma_{\vartheta_{u}}^{2}$ and $\sigma_{\vartheta_{v}}^{2}$ of the scattering angles can be computed from the slope entries and covariances of the in- and out-states via Gaussian error propagation. Correlations between the slope in $u$ and in $v$ direction such as for example $\sigma_{m_{u}^{\text {in }}, m_{v}^{\text {in }}}^{2}$ can be assumed to be small and are neglected here. However, there is no obvious analytical dependency between the scattering angles $\vartheta_{u}$ and $\vartheta_{v}$ and the in- and out-state entries. Instead of calculating the dependencies analytically, a numerical method called unscented transform is employed [73]. A detailed description of this approach can be found in appendix B. The unscented transform is used to determine numerical values for the variances $\sigma_{\vartheta_{u}}^{2}$ and $\sigma_{\vartheta_{v}}^{2}$ for every track state pair.

Afterwards all calculated quantities, the intersection coordinates, the projected scattering angles and the variances, are written into a ROOT tree. The determination of the scattering angles and the variance is repeated with the next matched in- and outstate pair. When all matched pairs have been used, the process depicted in fig. 5.2 proceeds with the next event. After all events have been processed the ROOT tree is saved and can be used for radiation length imaging (5.3) and calibration purposes (5.4).

\subsection{Telescope angle resolution}

The slope entries of the in- and out-states and their uncertainties, which are given by the corresponding entries of the covariance matrix, are used by the unscented transform to calculate the variances of the projected scattering angles. The covariance matrix $C$ of track states in turn depends on the covariance matrix $V$ of the hit measurements (see eq. 4.17 and 4.18). As was explained in section 4.3.10, the hit covariance is ultimately dependent on the size and shape of the reconstructed clusters on the telescope planes. 
Accordingly, reconstructed tracks with different cluster shape signatures on individual telescope planes will inevitably have slightly different reconstructed angle variances. However, the width of these angle reconstruction uncertainty distributions is expected to be small.

If the cluster resolution in one direction is slightly better, for example due to slightly tilted telescope planes, mean and RMS values of the $\sigma_{\vartheta_{u}}^{2}$ and $\sigma_{\vartheta_{v}}^{2}$ distributions will also have small differences. For an approximately perpendicular beam incidence this effect is expected to be smaller than the width of the uncertainty distribution.

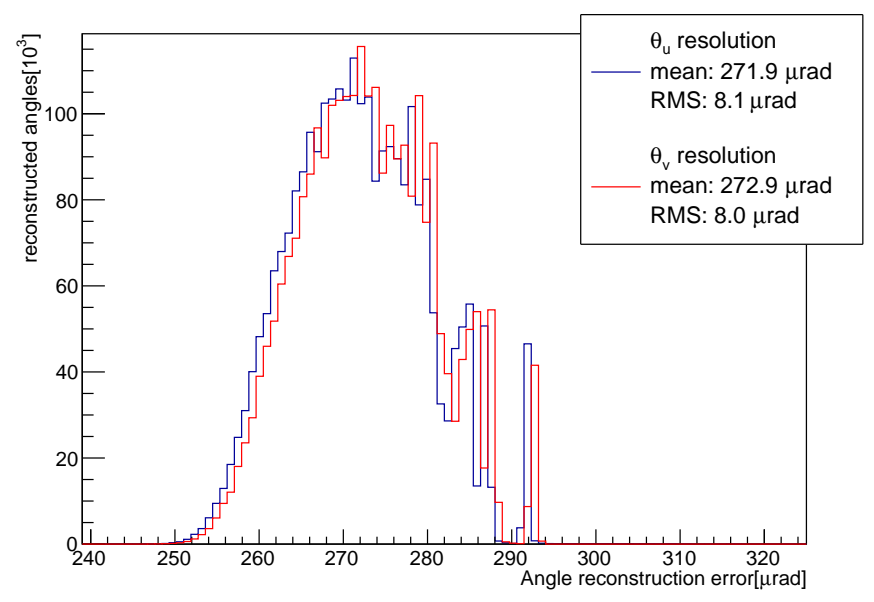

Figure 5.4.: Example distributions of measured angle reconstruction uncertainties from a beam test in October 2016 at the DURANTA telescope. The beam energy was $2 \mathrm{GeV}$. The solid red curve corresponds to $\sigma_{\vartheta_{u}}$, while the solid blue curve corresponds to $\sigma_{\vartheta_{v}}$. The difference between the two mean values is small compared to the width of the distribution. The corresponding variance values $\sigma_{\vartheta_{v}}^{2}$ were calculated via unscented transform.

All measurements analysed in this work were performed on telescopes with symmetric sensor pixels and nearly perpendicular beam incidence on all telescope planes. Additionally only very small rotations and shifts of the telescope planes are expected. Consequently, there will be no large difference between the measured distributions of the two variances $\sigma_{\vartheta_{u}}^{2}$ and $\sigma_{\vartheta_{v}}^{2}$. This notion has been confirmed in multiple beam test measurements. Fig. 5.4 depicts a comparison between the uncertainties $\sigma_{\vartheta_{u}}$ and $\sigma_{\vartheta_{v}}$ for a typical beam test measurement. The variance values determined from different inand out-state pairs are assumed to be uncorrelated. It is therefore possible to define a nominal telescope angle resolution $\sigma_{\text {err }}$ which corresponds to the square root of the mean variance of the projected angle in $u$ direction 


$$
\sigma_{\mathrm{err}} \equiv \sqrt{\left\langle\sigma_{\vartheta_{u}}^{2}\right\rangle} \approx \sqrt{\left\langle\sigma_{\vartheta_{v}}^{2}\right\rangle} .
$$

The telescope angle resolution $\sigma_{\text {err }}$, as defined in eq. 5.12, only depends on the telescope geometry, beam energy and cluster resolution and is independent of the scattering material between the telescope planes.

\subsection{Radiation length imaging}

The XOEstimator processor described in section 5.1 produced a ROOT tree with a list of scattering angles and track intersections for every matched in- and out-state. In order to perform spatially resolved measurements, image pixels have to be defined. In practice this is done in the following way: In the local coordinates of the target plane, a rectangular image area, which is aligned with the local $u$ and $v$ axes, is defined. Appropriate side lengths $l_{u}$ and $l_{v}$ of the image area must be selected. Due to the limited size of the sensitive area of individual sensors of the telescope, only a finite area on the scattering target is traversed by charged particles, which produce hits on all telescope sensors. This area is called the telescope acceptance region and is typically of the same size as the sensitive area of the telescope sensors for a well aligned telescope and perpendicular beam incidence. The telescope acceptance region is a good choice for the aforementioned image area. Image pixel pitches in both directions are selected and the area is divided into image pixels accordingly.

For each image pixel, three histograms are created which are used to store scattering angles. One histogram contains $\vartheta_{u}$, another histogram contains $\vartheta_{v}$ and the last histogram contains both angles. The combination of both angles is valid here because the projected multiple scattering angles in $u$ and $v$ direction are uncorrelated and described by the same model function (for example eq. 3.29). The symbol for projected angles in the combined angle histogram is $\vartheta_{\mathrm{p}}$. Each in- and out-state pair has an associated measured intersection that can be assigned to exactly one image pixel. The corresponding scattering angles are filled into the three histograms associated with this particular image pixel. This procedure is repeated for all available ROOT tree entries, i.e. matched inand out-state pairs. The resulting histograms contain angles that were measured from different tracks that are statistically independent. It is therefore natural to assume that the angle histograms of individual image pixels are also statistically independent.

It has been observed that sometimes reconstructed multiple scattering angle distributions are not centred at zero as expected from eq. 3.29. This effect is caused by small errors in the telescope alignment and the mean values of these shifted scattering angle distributions were observed to be in the order of $100 \mu \mathrm{rad}$. As described in section 4.3.8, $\alpha$ and $\beta$ rotations of telescope sensors are not determined in the alignment procedure. The neglected $\alpha$ and $\beta$ rotations can cause the angle distributions to have a non-zero mean. As a non-zero mean is an disturbance to subsequent analysis steps, the mean 


\section{Scattering angle reconstruction and radiation length imaging}

value has to be corrected for. The mean of the three angle distributions in each image pixel is filled into a 2D histogram and can be examined after the imaging process. Afterwards the angle histogram is centred by subtracting the corresponding mean value.

Additionally, angle histograms at the edges of the telescope acceptance region may be asymmetric because particle trajectories with large scattering angles in the direction of the acceptance edge can not be reconstructed in the downstream telescope arm. The size of this region depends on the telescope setup, the scattering material thickness and also the beam energy. The larger the width of the multiple scattering distribution the more image pixels at the edges of the acceptance region have histograms with asymmetric angle distributions.

In preparation for this work it was found that a good choice for the range of the scattering angle histograms is $[-\tilde{R}, \tilde{R}]$, where $\tilde{R}$ is given by the five times the RMS value of the angle distribution. As the histogram range therefore depends on the RMS of the corresponding angle distribution and the histogram has to be centred, the generation of the histogram is done in two steps. First an uncorrected angle distribution is plotted. The RMS and mean are determined from the uncorrected histogram. Then, with the determined mean and RMS the corrected angle histogram is created. The exact binning of the histograms will not be discussed here, instead the optimal settings for angle histograms are discussed in section 6.1.1.

Theoretically, small image pixel pitches are preferable as they will lead to a better image resolution. There are, however, limitations to the pixel pitch that must be observed. The number of entries per angle distribution must be large enough to ensure a stable fit with a reasonable uncertainty estimate. Some other issues, that perturb the fit process and arise in case of very small image pixel pitches are described in section 6.2. A reasonable choice of the image pixel pitch always depends on the statistics of the scattering angle sample at hand.

The angle distribution in each image pixel with the pixel centre at $(u, v)$ is described by the model function $f_{\text {reco }}^{u v}$. The function can be used to fit the angle distribution and determine the radiation length $X / X_{0}$. An overview of the parameters of the model function $f_{\text {reco }}^{u v}$ is given below:

- $\vartheta$ is the projected scattering angle ${ }^{1}$ and therefore the variable of the probability density function $f_{\text {reco }}^{u v}$.

- $\frac{X}{X_{0}}$ is the radiation length that is determined during the fit of the image pixel angle distribution.

- $\sigma_{\text {err }}$ is the telescope angle resolution as defined in eq. 5.12. It depends on the telescope geometry and beam energy. $\sigma_{\text {err }}$ is fixed and not determined during the

\footnotetext{
${ }^{1}$ Either $\vartheta_{u}, \vartheta_{v}$ or $\vartheta_{\mathrm{p}}$ depending on the distribution that is fitted
} 
fit.

- $p(u, v)$ is the average beam energy in the centre of the image pixel. A linear dependence on the position of the pixel center $(u, v)$ is assumed. The exact linear gradient depends on the particle beam and is determined during calibration measurements as described in section 5.4. $p(u, v)$ is fixed and not determined during the fit.

- $\lambda$ is a correction factor of the telescope angle resolution. It is determined during radiation length calibrations as described in section 5.4. It is fixed and not determined during the fit of the image pixel angle distribution.

- $\kappa$ is a correction factor of the scattering angle width. It is determined during radiation length calibrations as described in section 5.4. It is fixed and not determined during the fit.

With the parameters summarised above $f_{\text {reco }}^{u v}$ can be described by the following formula:

$$
\begin{aligned}
f_{\text {reco }}^{u v}\left(\vartheta, p(u, v), \frac{X}{X_{0}}, \lambda, \kappa, \sigma_{\text {err }}\right) & =f_{\text {msc }}^{u v}\left(\vartheta, p(u, v), \frac{X}{X_{0}}, \kappa\right) \\
& \otimes f_{\text {err }}\left(\vartheta, \lambda \sigma_{\text {err }}\right),
\end{aligned}
$$

where

$$
f_{\mathrm{err}}\left(\vartheta_{p}, \sigma_{\mathrm{err}}, \lambda\right)=\frac{1}{\lambda \sigma_{\mathrm{err}} \sqrt{2 \pi}} \exp \left(-\frac{1}{2}\left(\frac{\vartheta}{\lambda \sigma_{\mathrm{err}}}\right)^{2}\right) .
$$

$f_{\text {reco }}^{u v}$ is a normalised, continuous probability density function and can therefore be used in a fit to extract the radiation length $X / X_{0}$ from angle distributions. The first part of the convolution is the multiple scattering model $f_{\mathrm{msc}}^{u v}$. Here, the Highland model is used (eq. 3.29). $f_{\mathrm{msc}}^{u v}$ can therefore be expressed as

$$
f_{\mathrm{msc}}^{u v}\left(\vartheta, p(u, v), \frac{X}{X_{0}}, \kappa\right)=\frac{1}{\kappa \sigma_{\mathrm{HL}} \sqrt{2 \pi}} \exp \left(-\frac{1}{2}\left(\frac{\vartheta}{\kappa \sigma_{\mathrm{HL}}}\right)^{2}\right) .
$$

The Highland width $\sigma_{\mathrm{HL}}$ is given by eq. 3.28 and depends on the radiation length $X / X_{0}$ and beam energy $p(u, v)$. The second part of the convolution corresponds to the angle resolution function $f_{\text {err }}$ of the telescope. As can be seen in eq. $5.14 f_{\text {err }}$ corresponds to a Gaussian with a standard deviation of $\lambda \sigma_{\text {err }}$. The Gaussian angle resolution function must be considered here because of the finite scattering angle resolution $\lambda \sigma_{\text {err }}$ which broadens the multiple scattering distribution in eq. 3.29.

The function $f_{\text {reco }}^{u v}$ in eq. 5.13 is used to fit all three angle histograms of every image pixel using the ROOT binned fit function. ROOT provides a $\chi^{2}$ and a log-likelihood 
fit method, which are compared in section 6.1.1. The fit range must be limited to the Gaussian core of the reconstructed angle distribution, because the Highland model is only valid there (see section 3.3.3). In practice the fit range lies in the interval $[-R, R]$, where $R$ is given by

$$
R=\sqrt{2} \sigma_{\text {fit }}
$$

The quantity $\sigma_{\text {fit }}$ is the standard deviation of the core of the angle distribution and must be determined in a Gaussian fit. This Gaussian fit must be limited to the core of the angle distribution in order to not include the non-Gaussian tails of the distribution. Therefore, this fit is limited to the interval between -1 RMS to +1 RMS of the angle distribution. The determined standard deviation $\sigma_{\text {fit }}$ is then used to calculate the points at which the angle distribution has fallen to $1 / e$ of its maximum value according to eq. 5.16.

All three histograms of every image pixel are fitted and for each pixel an associated radiation length is determined. There are two possibilities to determine the radiation length of the image pixel. Either the fit value of the combined histogram is selected or an arithmetic mean of the radiation length values from the $\vartheta_{u}$ and $\vartheta_{v}$ is used. For all measurements in this work the first option was used.

\subsubsection{Fit model modification due to energy loss by bremsstrahlung}

In case of an electron beam and especially for a high $X / X_{0}$ scattering target, energy losses due to bremsstrahlung must be considered. They can be accounted for by modifying the beam energy $p$. A simple approach to include the energy loss is to calculate a weighted mean of the beam energy before and after the material transition. This approach is called the weight model in section 3.3.4. Energy losses can be included in the fit model function $f_{\mathrm{msc}}^{u v}$ by replacing the Highland width $\sigma_{\mathrm{HL}}$ in eq. 5.15 with the modified Highland width $\sigma_{\text {weight }}$ defined in eq. 3.37.

Another model that includes energy losses due to bremsstrahlung was introduced by Gottschalk [53]. Analogously to the weight model a modified Highland width is defined (see eq. 3.40). Both models, the weight and the Gottschalk model, are tested and compared in chapter 7 .

\subsection{Radiation length calibration measurements}

In order to ensure an accurate estimate of the radiation length, environmental conditions such as the telescope angle resolution and the beam energy have to be known very precisely. This is especially important, when a thin material is measured and the Highland width $\sigma_{\mathrm{HL}}$ is smaller than the angle resolution $\sigma_{\mathrm{err}}$. However, the telescope angle resolution calculated via eq. 5.12 is expected to be slightly wrong due to small errors in the alignment. Additionally, a small underestimation of the scattering effects on the 


\subsection{Radiation length calibration measurements}

telescope planes is expected because the non-Gaussian tails of the multiple scattering distributions are neglected in the track fit.

Moreover, a wrong assumption of the beam energy can lead to a global offset of the width in the first part of eq. 5.13 describing the multiple scattering distribution. This offset can also be caused by inaccuracies in measurements of the telescope length, which is not determined during the alignment procedure, but must be measured manually (see section 4.3.8). Another issue is the profile of the beam energy. Due to the beam energy selection via magnet, it can be assumed that the energy of the beam particles is not completely monochromatic, but depends on the position relative to the centre of the particle beam.

A calibration measurement allows to account for the effects listed above by determining four calibration parameters, listed below:

- $\lambda$ is used to correct for inaccuracies that lead to a global offset of the telescope angle resolution $\sigma_{\mathrm{err}}$. The calibrated telescope angle resolution is given by $\lambda \sigma_{\mathrm{err}}$. $\lambda$ must be determined experimentally from calibration measurements.

- $\kappa$ is used to correct for wrong assumptions that lead to a global offset of the scattering distribution width $\sigma_{\mathrm{HL}}$. The calibrated width of the multiple scattering angle distribution is given by $\kappa \sigma_{\mathrm{HL}} . \kappa$ must be determined experimentally from calibration measurements.

- The beam energy can be dependent on the position on the scattering plane expressed by $(u, v)$. The beam energy is modelled as a linear function in $u$ and $v$ direction

$$
p(u, v)=p_{0}+\Delta_{u} p u+\Delta_{v} p v \quad,
$$

where $p_{0}$ is the beam energy at the origin of the local coordinate system on the target plane. $p_{0}$ is the nominal beam energy that was selected during the beam test. Any additional beam energy offsets are corrected for by $\kappa$, because the multiple scattering width $\sigma_{\mathrm{HL}}$ is inversely proportional to $p$ as can be seen from eq. 3.28. $\Delta_{u} p$ and $\Delta_{v} p$ are linear beam energy gradients in $u$ and $v$ direction that have to be determined experimentally from calibration measurements.

The four calibration factors $\lambda, \kappa, \Delta_{u} p$ and $\Delta_{v} p$ must be determined in a dedicated calibration measurement on a target with a well known material profile. A schematic view of an example calibration target is depicted in fig. 5.5. The example reference target consists of a stack of nine aluminium layers with a thickness of $200 \mu \mathrm{m}$ per layer. Each layer has between one and nine cut-outs. The layers are stacked in such a way that a region with only air and 8 other measurement areas with aluminium thicknesses ranging from $0.2 \mathrm{~mm}$ to $1.6 \mathrm{~mm}$ are formed. Alternatively, a radiation length calibration 
can also be performed on the scattering angle data set of multiple homogeneously thick aluminium targets in a fixed telescope geometry and a constant nominal beam energy $p_{0}$.

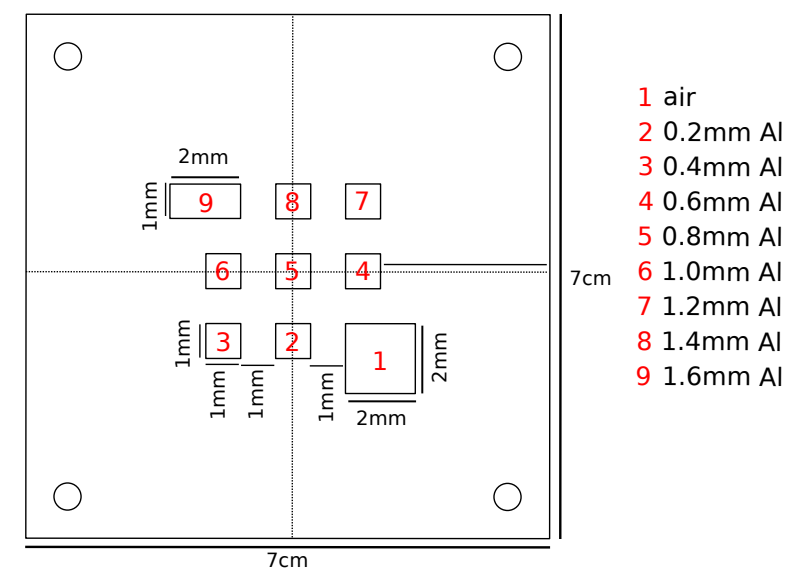

Figure 5.5.: Schematic view of a dedicated calibration target, which was used during beam tests in 2015. The target consists of nine aluminium layers with a thickness of $200 \mu \mathrm{m}$ each, which are arranged in such a way that nine measurement areas with different aluminium thicknesses are formed.

After generating a radiation length image of the calibration target with $\lambda, \kappa$ fixed to one and linear beam energy gradients fixed to zero, the exact calibration target position in the local frame is well known and measurement areas can be selected. The calibration measurement areas are rectangles with a selected centre position and side lengths parallel to the local $u$ and $v$ direction. For every selected calibration measurement area, scattering angles histograms are created and filled just like for the radiation length imaging procedure. The following steps explain the calibration procedure. Here, combined angle histograms $\left(\vartheta_{p}\right)$ are used, but the calibration can also be performed using the $\vartheta_{u}$ and $\vartheta_{v}$ distributions. For each histogram, a fit function based on eq. 5.13 is defined. As the radiation length $X / X_{0}$ of the calibration target is well known, the $X / X_{0}$ values of all fit functions are set to the expected values and fixed during the fit. Also, the nominal telescope angle resolution $\sigma_{\text {err }}$ and nominal beam energy $p_{0}$ are set to the appropriate values and fixed. The calibration factors $\lambda, \kappa$ and the two linear beam energy gradients $\Delta_{u} p$ and $\Delta_{v} p$ are shared fit parameters for all fit functions. Now a simultaneous combined fit of all calibration angle histograms is performed using the corresponding fit functions. The parameters varied and fitted during the simultaneous fit of all histograms are the four calibration parameters.

Usually the determined values of $\lambda$ and $\kappa$ should be close to 1.0. If their values differ from 1.0 by more than $20 \%$ there must be a large systematic influence, which has to be investigated before proceeding with $X / X_{0}$ measurements. Only one of the linear beam energy gradients, typically the gradient in $u$ direction, should be in the order of 
10 to $20 \mathrm{MeV} / \mathrm{mm}$. The other gradient is typically compatible with zero. Calibration parameters outside this range are typically accompanied by further irregularities which can be identified in data quality plots as described in chapter 7 .

Several considerations were found to improve the obtained calibration measurement results: First, there should always be a measurement region with air and additionally a high $X / X_{0}$ region available for $\lambda$ and $\kappa$ calibration. The scattering angle distribution in the air region is dominated by the second part of the fit function $f_{\text {err }}$ (eq. 5.14), because the radiation length constant $X_{0}$ of air is very large ${ }^{2}$ and consequently the Highland width of several millimetres of air is very small. Therefore, the angle distribution of air mainly depends on $\lambda$. On the other hand the fit function in an area with a large radiation length is dominated by the first part of the fit function $f_{\mathrm{msc}}$, which only depends on $\kappa$ and the beam energy gradients. The uncertainty of the beam energy gradient measurements can be minimised by sampling measurement areas on a high $X / X_{0}$ target along the whole $u$ and $v$ range of the telescope acceptance region.

If the determined calibration factors are reasonable, the calibration target can be exchanged for a target with an unknown material distribution and $X / X_{0}$ measurements can conducted. As mentioned before, the telescope angle resolution depends on the telescope setup, while the multiple scattering width (see eq. 3.29) depends on the beam energy. Accordingly, for every change in the telescope setup or beam energy, a new $X / X_{0}$ calibration measurement has to be performed.

\subsection{Telescope angle resolution and signal-to-noise ratio of $X / X_{0}$ measurements}

Simulations in $T B S W$ (see section 4.5) were used to find the optimal telescope geometry and beam energy settings to perform radiation length measurements. Beam energies tested here correspond to the energy range available at DESY beam test facilities and the reference telescope employed here is a EUDET beam telescope equipped with six M26 planes. Electrons are used as beam particles.

The model function $f_{\text {reco }}$, which is used to calculate the radiation length from scattering angle distributions is defined in eq. 5.13. This function is a convolution of two Gaussian functions: The multiple scattering distribution $f_{\mathrm{msc}}$ and $f_{\mathrm{err}}$, which describes the uncertainty due to the angle reconstruction in the telescope . $f_{\text {reco }}$ is also a Gaussian function with a standard deviation $\sigma_{\text {total }}$ of

$$
\sigma_{\text {total }}=\sqrt{\sigma_{\mathrm{MSC}}^{2}+\sigma_{\mathrm{err}}^{2}}
$$

\footnotetext{
${ }^{2} 303.9 \mathrm{~m}$ for dry air at $1 \mathrm{~atm}$ according to [29]
} 


\section{Scattering angle reconstruction and radiation length imaging}

Only $\sigma_{\mathrm{MSC}}$ is sensitive to variations of the radiation length. The first part of eq. 5.18 therefore corresponds to the signal of the radiation length measurement. Accordingly, the second part can be called the measurement noise. The signal-to-noise ratio $S$ of radiation length measurements can therefore be defined as

$$
S=\frac{\sigma_{\mathrm{MSC}}}{\sigma_{\mathrm{err}}} .
$$

The signal-to-noise ratio $S$ is a good indicator for the ability to extract valid information about a material from scattering distributions. When the scattering distribution is dominantly influenced by the reconstruction error distribution, the signal-to-noise ratio $S$ is small. In this case the overall width of the distribution can be rewritten as

$$
\sigma_{\text {total }}\left(\sigma_{\mathrm{MSC}}\right)=\sqrt{\sigma_{\mathrm{err}}^{2}+\sigma_{\mathrm{MSC}}^{2}} \sigma_{\mathrm{err}} \gg \sigma_{\mathrm{MSC}} \sigma_{\mathrm{err}}\left(1+\frac{\sigma_{\mathrm{MSC}}^{2}}{2 \sigma_{\mathrm{err}}^{2}}+\mathcal{O}\left(\sigma_{\mathrm{MSC}}^{4}\right)\right)
$$

For $\sigma_{\text {err }} \gg \sigma_{\mathrm{MSC}}$, the influence of the actual multiple scattering distribution on the overall reconstructed distribution is very limited. The total distribution width is mainly determined by the constant angle resolution $\sigma_{\text {err }}$, while $\sigma_{\mathrm{MSC}}$ is only a part of the quadratic term and even less important terms of higher order. On the other hand, if the signal-to-noise ratio is large, the total standard deviation of the model function is

$$
\sigma_{\text {total }}\left(\sigma_{\mathrm{MSC}}\right)=\sqrt{\sigma_{\mathrm{err}}^{2}+\sigma_{\mathrm{MSC}}^{2}} \sigma_{\mathrm{MSC} \gg \sigma_{\mathrm{err}}}^{\approx} \sigma_{\mathrm{MSC}} .
$$

For the extraction of the radiation length $X / X_{0}$ from the overall distribution, the second case is preferable, because the reconstructed angle distribution is very sensitive to $X / X_{0}$ variations. For measurements on small $X / X_{0}$ materials and consequently small signalto-noise ratios, it is of great importance to optimise the setup to achieve the largest possible signal-to-noise ratio. Fig. 5.6 depicts the dependency of $\sigma_{\mathrm{MSC}}, \sigma_{\mathrm{err}}$ and $S$ as a function of the beam energy for multiple aluminium thicknesses. A telescope setup with $40 \mathrm{~mm}$ distances between neighbouring M26 planes is used (see fig. 6.8).

The Highland width of a material with a constant radiation length $X / X_{0}$ as well as the angle resolution increase with decreasing beam energy $p$. However, the Highland width increases more rapidly than the telescope angle resolution. This leads to a small increase of the signal-to-noise ratio for smaller beam energies. Unsurprisingly, the signalto-noise ratio is larger for materials with a larger radiation length. The results indicate that, especially for low $X / X_{0}$ materials, the smallest possible beam energy should be used to achieve a reasonable signal-to-noise ratio.

As can be seen from fig. 5.7, the telescope setup has a similarly large impact on the signal-to-noise ratio. The figure depicts the telescope angle resolution and signal-tonoise ratio for $4 \mathrm{GeV}$ electrons as a function of the spacings between neighbouring M26 


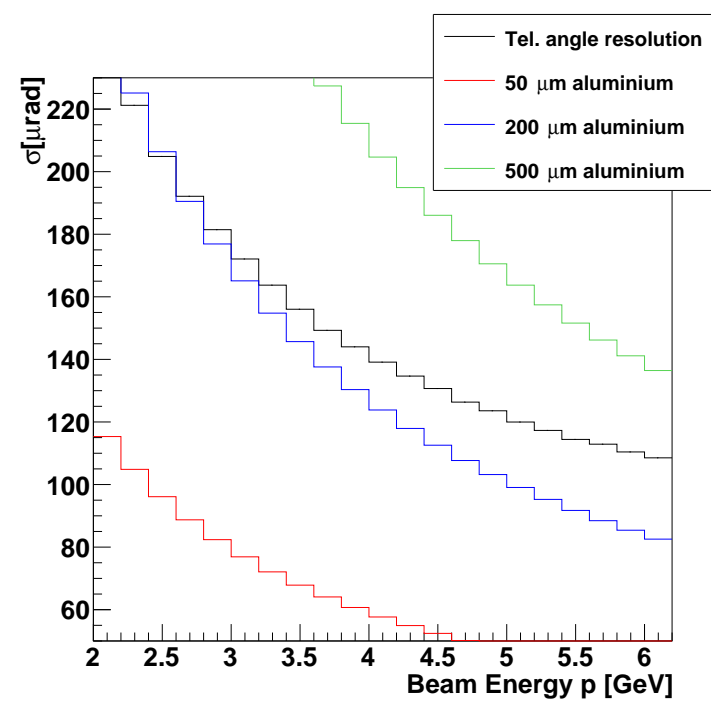

(a)

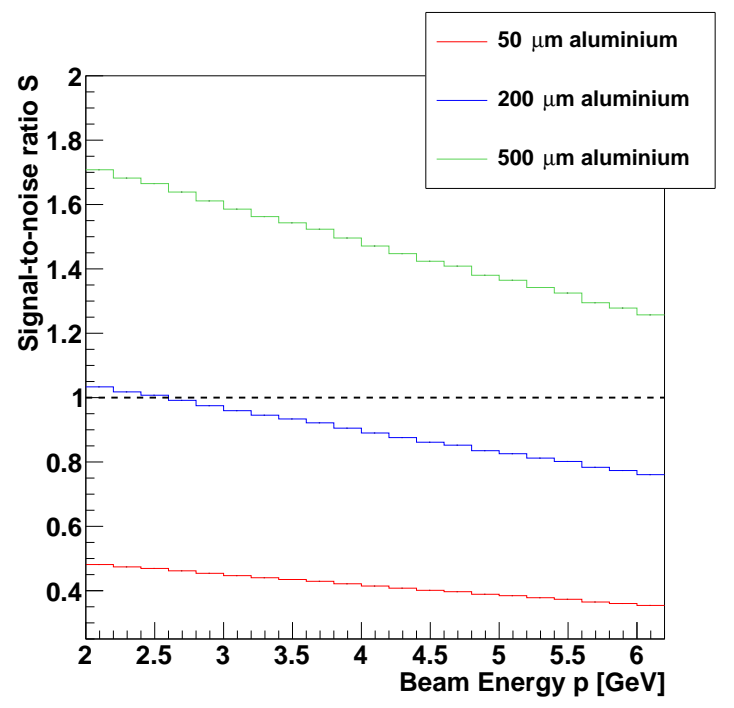

(b)

Figure 5.6.: (a) Telescope angle resolution $\sigma_{\text {err }}$ for a telescope with 40mm spacings (see fig. 6.8) as a function of the beam energy $p$. The angle resolution is shown in comparison to the Highland standard deviations of materials with various thicknesses. (b) Signalto-noise ratio for various aluminium thicknesses as a function of the beam energy $p$.

sensors in the experimental setup. During the spacing variations the distance from the innermost M26 sensors to the centred target plane remains constant at $50 \mathrm{~mm}$.

The spacings between telescope planes should always be chosen larger than $40 \mathrm{~mm}$ in order to avoid small signal-to-noise values and consequently suboptimal radiation length measurement conditions. Also, when measuring thin materials such as $100 \mu \mathrm{m}$ of silicon at DESY, using an beam energy of $2 \mathrm{GeV}$ is optimal. For materials with $X / X_{0}$ values of several percent ${ }^{3} X / X_{0}$ the beam energy choice is less important, because the signal-to-noise ratio will always be much larger than one.

\footnotetext{
${ }^{3}$ For example, a radiation length of $X / X_{0}=1 \%$ corresponds to a aluminium thickness of approximately $890 \mu \mathrm{m}$
} 
5. Scattering angle reconstruction and radiation length imaging

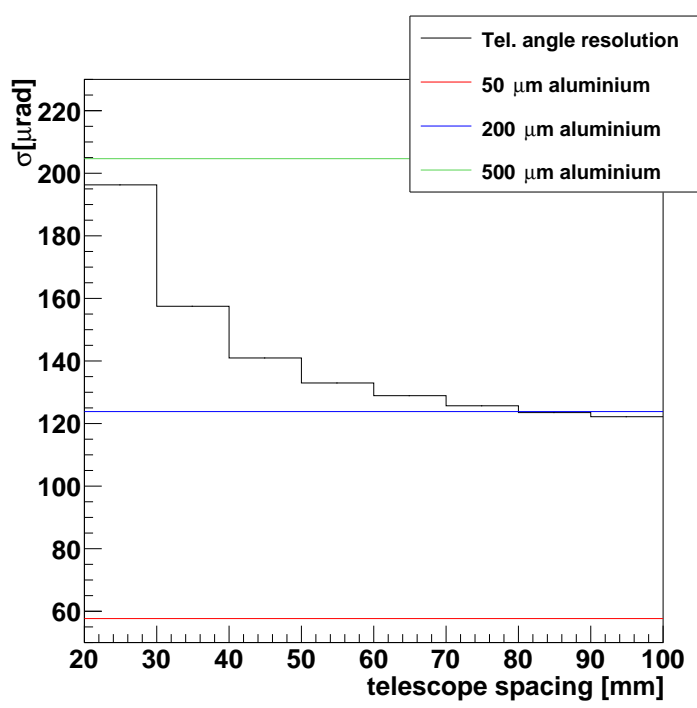

(a)

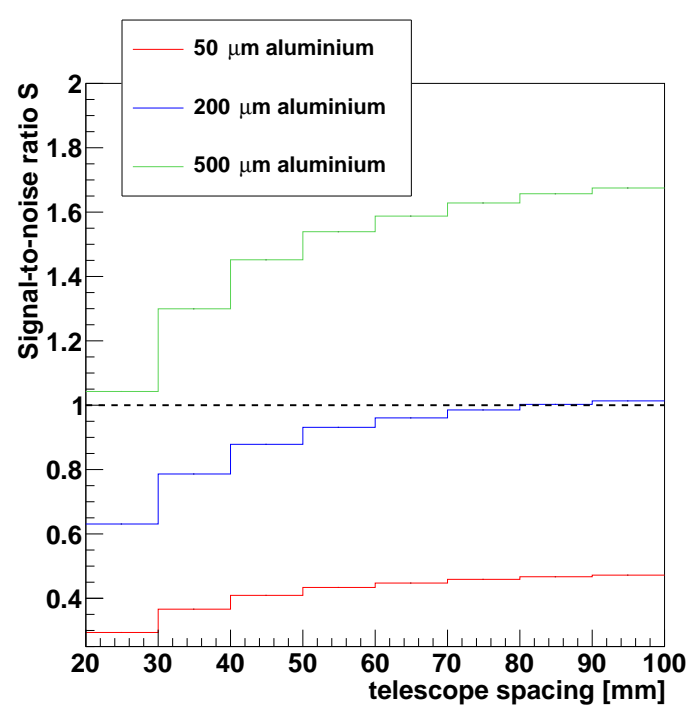

(b)

Figure 5.7.: (a) Telescope angle resolution $\sigma_{\text {err }}$ and (b) signal-to-noise ratio for a $4 \mathrm{GeV}$ electron beam as a function of the spacing between neighbouring M26 sensors. The telescope resolution is shown compared to Highland standard deviations of various aluminium target thicknesses. 


\section{CHAPTER 6}

\section{Systematic influences and simulation studies}

The scope of this chapter is to test the validity of the radiation length measurement method and study the most frequently encountered systematic influences in the well controlled environment of beam test simulations. Monte Carlo (MC) simulations provide a good opportunity to disentangle different systematic influences, that also affect the analysis of real beam test data, by subsequently adding new sources of systematic uncertainties to the simulations and evaluating them.

The radiation length method introduced in section 5 depends on the precise knowledge of the experimental conditions. Small errors in the telescope alignment, especially sensor shifts along the beam axis, have a large impact on scattering angle distributions and consequently radiation length measurements. Additionally, wrong assumptions of the beam energy and small measurement errors of the cluster resolutions can lead to wrong assumptions of the telescope angle resolution $\sigma_{\text {err }}$ and the scattering angle width $\sigma_{\mathrm{HL}}$. Most of these systematic uncertainties can be corrected for by conducting $X / X_{0}$ calibration measurements as described in section 5.4. Global offsets of the telescope angle resolution are determined directly in the form of the calibration factor $\lambda$. Beam energy offsets and the telescope length are corrected for indirectly by determining the global offset of the multiple scattering width $\kappa$.

On the following pages the results of simulation studies will be presented. A step-by-step approach is used here. With each step, additional sources of systematic uncertainties are added to the simulations. In section 6.1 a direct generation of scattering angle distributions is used. This removes systematic influences such as telescope misalignment, tracking and wrong assumptions of the beam energy. In this simple scenario, the validity of multiple scattering angle fits is tested. Additionally, tests of the optimal angle histogram settings and of the available fitting methods are conducted. Section 6.2 presents 


\section{Systematic influences and simulation studies}

results from $T B S W$ simulations of beam test experiments. These simulations are used to study systematic effects caused by binary measurements of digital pixel sensors. Especially disturbances of the angle reconstruction are examined. In section $6.3 T B S W$ simulations are used to validate the radiation length calibration measurements. In order to test the capability to correct for slightly wrong beam energies, small beam energy offsets are introduced in the simulations and compared to results from radiation length calibration measurements.

\subsection{Validation of the scattering angle fitting procedure with toy studies}

Scattering angle distributions in this section are generated directly without any intermediate steps such as tracking and angle reconstruction. The angle distributions are therefore not affected by uncertainties due to misalignment and track reconstruction. In section 6.1.1 different scattering angle histogram settings and fitting methods are tested. The quality of the fits is evaluated by calculating residuals and pulls between the fitted radiation length values and the corresponding values used in the simulations of the angle distributions. In section 6.1.2 the systematic and statistic uncertainty of the fit in dependence of the size of the underlying angle sample is studied.

\subsubsection{Optimal setup of the angle distribution histograms and tests of the fitting procedure}

The first step of the simulation tests is the generation of $M=4000$ multiple scattering angle distributions, each with a total number of $N$ angles. For a chosen particle momentum $p$ and radiation length $X / X_{0}$ the individual angles are calculated as the sum of a random multiple scattering angle $\vartheta_{\mathrm{HL}}$ generated from the Highland function $f_{\mathrm{HL}}\left(\vartheta_{\mathrm{HL}}, p, \frac{X}{X_{0}}\right)$ in eq. 3.29 and another angle $\vartheta_{\text {err }}$ distributed according to a Gaussian function $f_{\mathrm{err}}\left(\vartheta_{\mathrm{err}}, \sigma_{\mathrm{err}}, \lambda=1.0\right)$. The function $f_{\mathrm{err}}\left(\vartheta_{\mathrm{err}}, \sigma_{\mathrm{err}}, \lambda=1.0\right)$ is defined in eq. 5.14. The sum $\vartheta_{\text {total }}=\vartheta_{\mathrm{HL}}+\vartheta_{\text {err }}$ of the two simulated angles is Gaussian distributed with a mean at zero and an overall standard deviation of

$$
\sigma_{\text {truth }}=\sqrt{\sigma_{\mathrm{HL}}^{2}+\sigma_{\mathrm{err}}^{2}}
$$

The simulated angles $\vartheta_{\text {total }}$ are stored in histograms with a range that depends on $\sigma_{\text {truth }}$. Defining the histogram range in multitudes of $\sigma_{\text {truth }}$ leads to a consistent ratio between core and tail region and distribution shape, regardless of the radiation length of the scattering material $X / X_{0}$ and beam energy $p$. An abscissa ranging from $-5 \sigma_{\text {truth }}$ to $+5 \sigma_{\text {truth }}$ is a suitable choice and matches the range used for angle histograms in real beam test experiments. 
On the following pages, the radiation length will be expressed by $t=X / X_{0}$ to have more concise equations. In order to find the optimal number of histogram bins and select the best fitting procedure, different numbers of bins were tested. The number of bins was varied between 20 and 230 in steps of 10 . In order to evaluate the best fitting and binning option, the measured radiation length $t^{\mathrm{m}}$ is compared to the true ${ }^{1}$ radiation length $t^{\text {true }}$. The simulation parameters are given by the values listed in table 6.1 . The selected radiation length value corresponds to $1 \mathrm{~mm}$ of aluminium and the telescope resolution is a typical value for the EUDET telescope with six M26 planes at energies of $2 \mathrm{GeV}$. The Highland width for the selected parameters is $\sigma_{\mathrm{HL}}=598 \mu \mathrm{rad}$. Accordingly, the standard deviation of the simulated angle distribution is $\sigma_{\text {truth }}=644 \mu \mathrm{rad}$.

\begin{tabular}{|c|c|c|c|c|c|c|}
\hline $\boldsymbol{t}^{\text {true }}[\%]$ & $\boldsymbol{p}[\mathrm{GeV}]$ & $\mathbf{M}$ & $\mathbf{N}$ & $\boldsymbol{\sigma}_{\text {err }}[\mathbf{\mu r a d}]$ & $\boldsymbol{\sigma}_{\text {truth }}[\mathbf{\mu r a d}]$ & particles \\
\hline 1.124 & 2 & 4000 & 5000 & 240 & 644 & electrons \\
\hline
\end{tabular}

Table 6.1.: Simulation parameters used to generate scattering angle distributions that are used to validate the scattering angle fitting procedure.

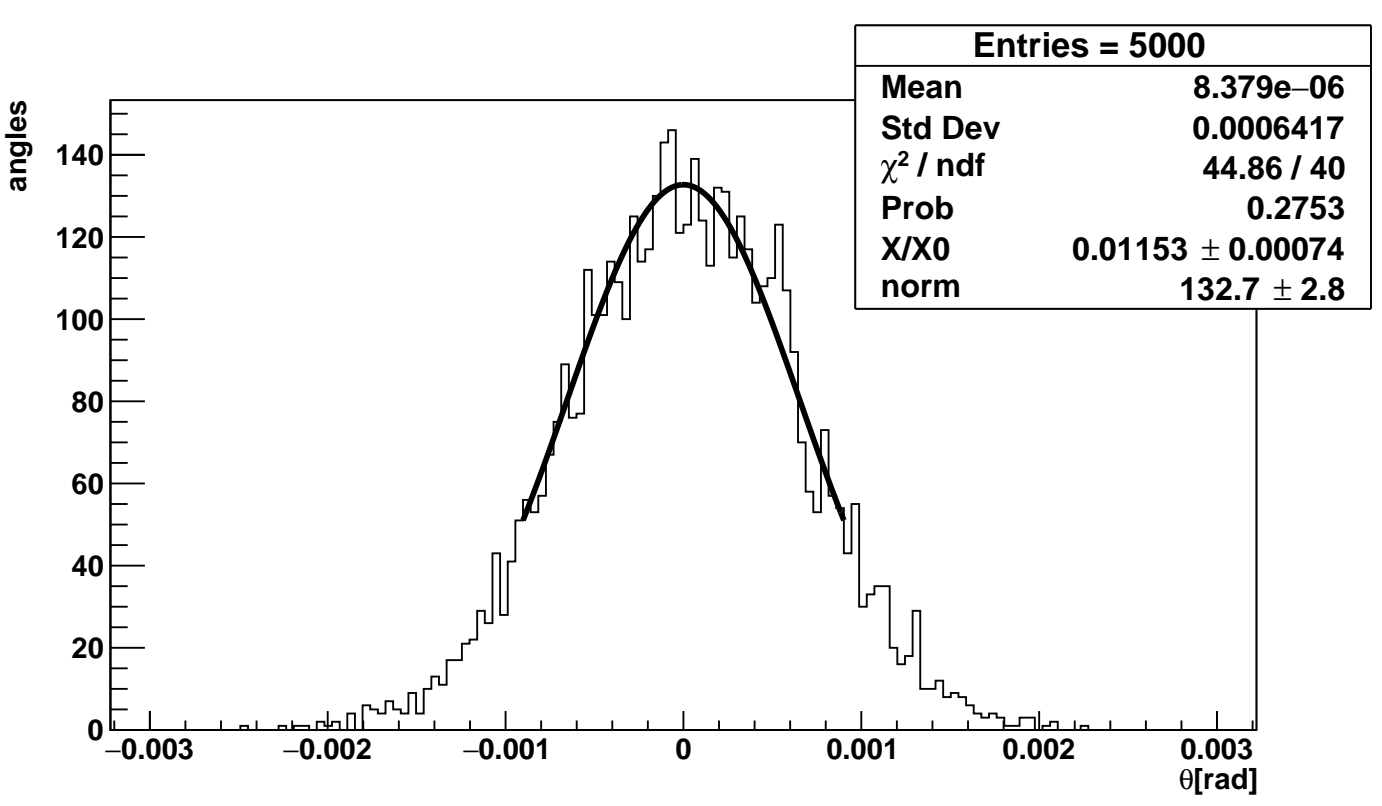

Figure 6.1.: Example scattering angle distribution with 5000 events for an electron beam of $2 \mathrm{GeV}$ traversing an aluminium layer with a thickness of $1 \mathrm{~mm}$. The angle reconstruction error $\sigma_{\text {err }}$ is $240 \mu \mathrm{rad}$ and the expected total standard deviation $\sigma_{\text {truth }}$ is 644 urad. The histograms $\mathrm{x}$ axis ranges from $-5 \sigma_{\text {truth }}$ to $+5 \sigma_{\text {truth }}$ and has 150 bins. The displayed fitting function is a Gaussian function and the fitting procedure is based on a log-likelihood fit.

${ }^{1} t^{\text {true }}$ corresponds to the radiation length used to simulate the angle distributions. 


\section{Systematic influences and simulation studies}

As mentioned in [49], the Highland multiple scattering model is only valid in the central part of the scattering angle distribution. The simulations here are based on two Gaussian distributions and the resulting angle distributions are also Gaussian distributions. Regardless, the fit range is limited to the centre of the distribution, as described in section 5.3, to be comparable to the fit procedure of non-simulated scattering angle distributions. The binned angle distributions are fitted with the fit model in eq. 5.13 and the radiation length $t^{\mathrm{m}}$ with its statistical uncertainty $\sigma_{t^{\mathrm{m}}}$ are determined. An example of a simulated angle distribution and the corresponding fit function are shown in fig. 6.1. The fit displayed is based on the log-likelihood estimator.

The fitted radiation length $t^{\mathrm{m}}$, its statistical fit uncertainty $\sigma_{t^{\mathrm{m}}}$ and the truth radiation length $t^{\text {true }}$ of the simulation are used to calculate the residual and pull values of the individual fits. The residual of the fitted radiation length is given by

$$
t^{\mathrm{res}}=t^{\mathrm{m}}-t^{\mathrm{true}} .
$$

The pull values are calculated from the residuals and the fit uncertainties via

$$
t^{\mathrm{pull}}=\frac{t^{\mathrm{res}}}{\sigma_{t^{\mathrm{m}}}} .
$$

The scattering angle histogram generation and fitting procedure is repeated $M=4000$ times for every bin configuration and both fitting techniques. The individual pulls, residuals and fit uncertainties are recorded. Examples of the resulting pull, residual and fit uncertainty distributions are depicted in fig. 6.2.

The fit procedure is called unbiased, when the mean of the pull (or residual) distribution is compatible with zero

$$
\left\langle t^{\mathrm{res}}\right\rangle=0 \quad \text {. }
$$

A non-zero bias is an indicator for a systematic problem during the measurement. The example in fig. 6.2 displays a small bias. The systematic uncertainty due to biased fits can be defined as

$$
\sigma^{\text {sys. }} \equiv\left\langle t^{\text {res }}\right\rangle \quad .
$$

In analogy to the systematic error, the statistical uncertainty of the radiation length measurements can be defined as the mean fit uncertainty of the individual measurements

$$
\sigma^{\text {stat. }} \equiv\left\langle\sigma_{t^{\mathrm{m}}}\right\rangle \quad
$$


6.1. Validation of the scattering angle fitting procedure with toy studies

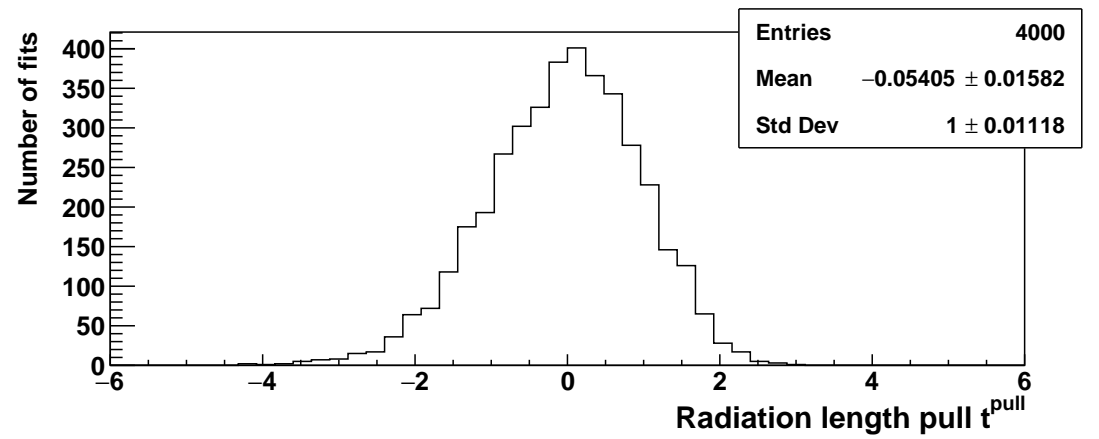

(a) Pull $t^{\text {pull }}$ distribution

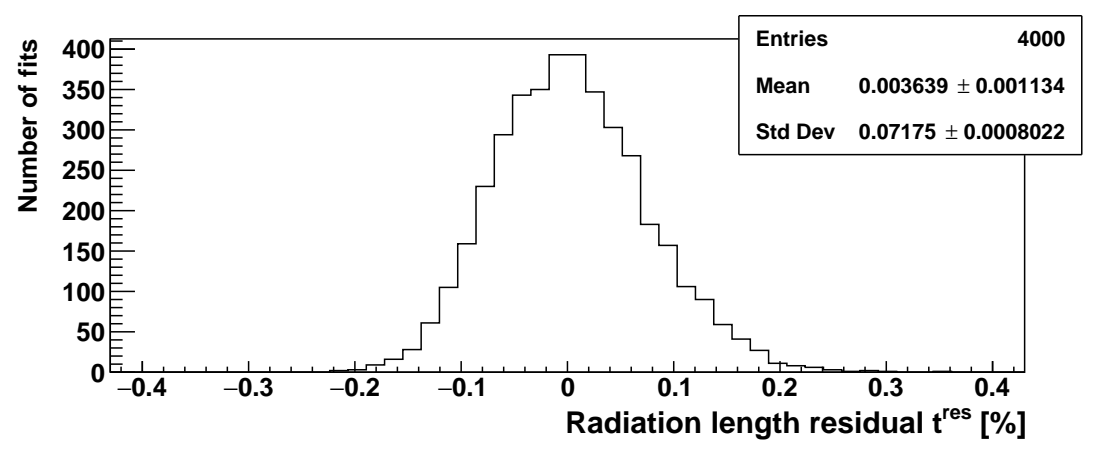

(b) Residual $t^{\text {res }}$ distribution

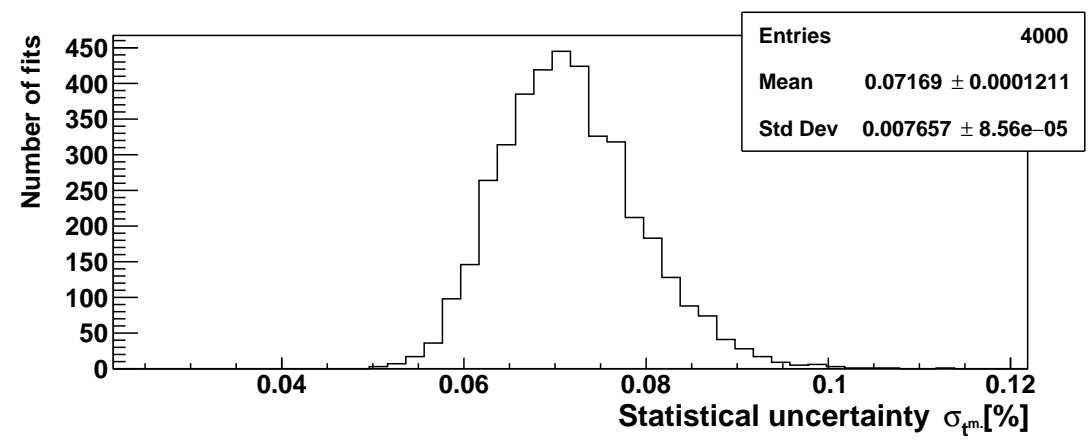

(c) Statistical fit uncertainty $\sigma_{t^{\mathrm{m}}}$ distribution

Figure 6.2.: Pull (a), residual (b) and statistical fit uncertainty distributions (c) from fits of an ensemble of 4000 simulated angle distributions with 5000 angles each. The simulated angle histograms were generated with the settings summarised in table 6.1.

Ideally, the standard deviation of the residual distribution $\sigma_{t^{\text {res }}}$ should be identical to the above definition of the statistical uncertainty 


\section{Systematic influences and simulation studies}

$$
\sigma_{t^{\mathrm{res}}}=\sigma^{\text {stat. }}
$$

If the statistical uncertainty is smaller or larger than the standard deviation of the residual the mean fit error has been overestimated or underestimated, respectively. The pull distribution is expected to have a Gaussian shape with a mean of zero, which corresponds to the condition in eq. 6.4 and a standard deviation of one, which corresponds to the condition in eq. 6.7. However, the distributions in fig. 6.2 are not completely Gaussian. The example residual and fit uncertainty distributions have non-Gaussian tails on the right part of the distributions, while a similar tail is visible in the negative region of the pull distribution.

The first step is to determine which of the two fitting techniques available in the ROOT framework [38] ( $\chi^{2}$ or log-likelihood fit) yields better, less biased results. The example in fig. 6.1 and 6.2 employed the log-likelihood fit. In order to decide, which fitting technique to use, the mean and standard deviations of the pull and residual distributions will be compared. It is useful to express the statistical and systematic uncertainty in relation to the true radiation length. Therefore, the relative statistical and the relative systematic uncertainty are defined as

$$
\begin{aligned}
\sigma^{\text {rel.stat. }} & \equiv \frac{\sigma^{\text {stat. }}}{t^{\text {true }}} \\
\sigma^{\text {rel.sys. }} & \equiv \frac{\sigma^{\text {sys. }}}{t^{\text {true }}} .
\end{aligned}
$$

The four parameters of interest, pull mean, pull RMS, relative statistical and relative systematic error, are depicted in fig. 6.3 as a function of the number of bins of the scattering angle histograms.

The mean pulls and the relative systematic error both indicate the presence of a bias for both fitting methods. This bias is an inherent issue of binned $\chi^{2}$ and log-likelihood fits in the small statistics case $[70,74]$ and manifests as the non-Gaussian tails in the pull and residual distribution in fig. 6.2. However, the log-likelihood fit is clearly the better option of the two fitting algorithms. The pull and relative systematic uncertainty curves indicate a small bias, but the magnitude of the bias is nearly independent of the histogram setting for histograms with more than 100 bins. The bias of the $\chi^{2}$ fits on the other hand is dependent on the histogram binning and is compatible with zero only if scattering angle histograms with 70 to 80 bins are used. The curve of the pull RMS values confirms the notion that the log-likelihood fit is preferable, because it agrees with one for all bin numbers larger than 50. The corresponding curve for the $\chi^{2}$ fit on the other hand is neither independent of the histogram settings nor in agreement with the expected value of one. 
6.1. Validation of the scattering angle fitting procedure with toy studies

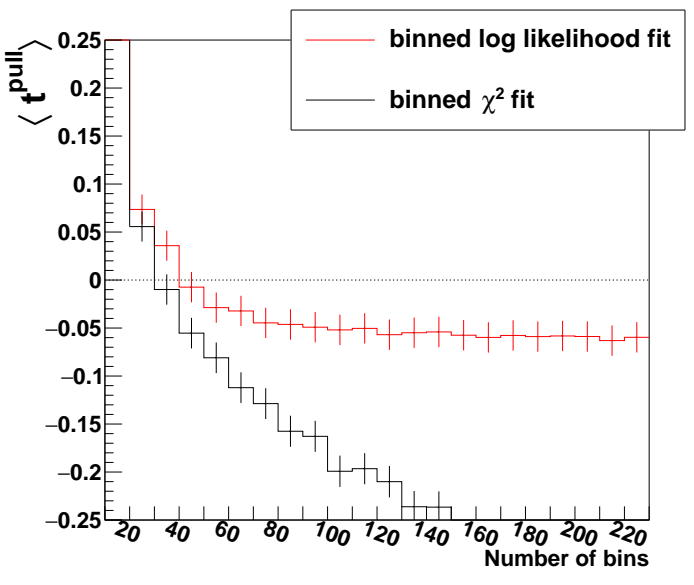

(a) Mean pull

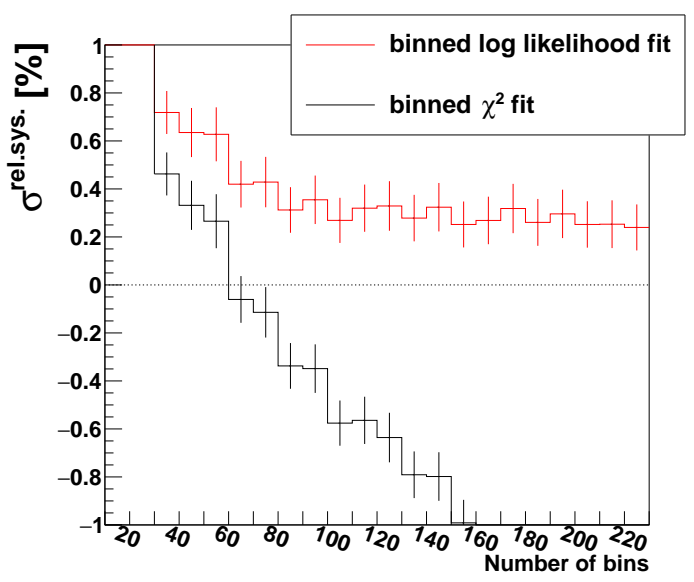

(c) Rel. systematic uncertainty

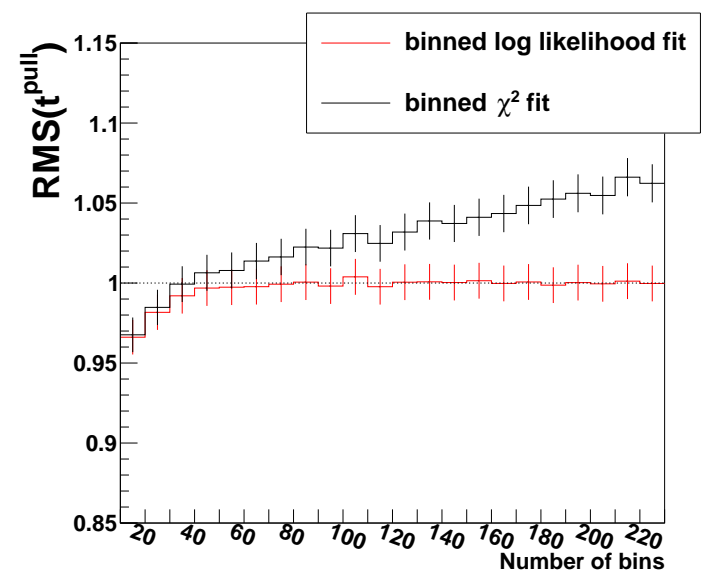

(b) Pull RMS

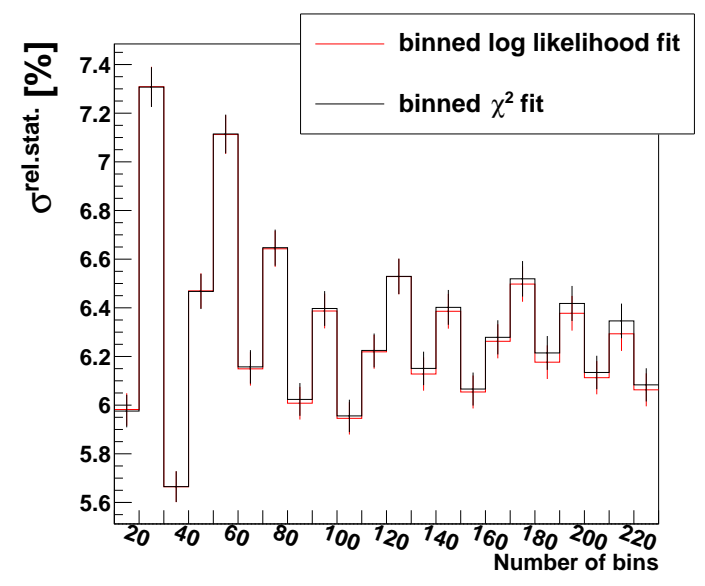

(d) Rel. statistical uncertainty

Figure 6.3.: Pull mean (a), pull RMS (b), relative residual mean (c) and relative residual RMS of the $t^{\mathrm{m}}$ measurements as a function of the number of bins of the underlying scattering angle histograms for the two different fitting methods. The black dashed line in (a),(b) and (c) indicate the expected value in the case of an ideal fit.

Due to these clear results, the log-likelihood estimator will be used for all fits on scattering angle distributions. As can be seen from fig. 6.3c the bias of the log-likelihood fit is approximately constant for all numbers of bins larger than 100 . Therefore, scattering angle histograms with 150 bins and a range from $-5 \sigma_{\text {truth }}$ and $+5 \sigma_{\text {truth }}$ will be employed for all measurements in this thesis. For a measurement setup summarised in table 6.1 and a scattering angle histograms with 150 bins the following expected relative statistical and systematic uncertainties per measurement were determined: 


\section{Systematic influences and simulation studies}

$$
\begin{aligned}
\sigma^{\text {rel.stat. }} & \approx 6.4 \% \\
\sigma^{\text {rel.sys. }} & \approx 0.3 \%
\end{aligned}
$$

The dominant source of uncertainty is statistical, at least for this specific setup. This is not surprising as the statistic is quite small and only one histogram with 5000 entries is used to determine the radiation length value. Typically, when determining radiation length values of a specific material or structure depicted in a radiation length image, the arithmetic mean of multiple radiation length measurements coming from different image pixels is calculated. This is a valid approach, as the $X / X_{0}$ measurements in the individual image pixels are statistically independent. Accordingly, a large number $\tilde{M}$ of multiple scattering histograms is used to determine the radiation length of interesting materials. When computing the arithmetic mean of all $\tilde{M}$ radiation length measurements, the statistical uncertainty of the arithmetic mean is given by $\sigma^{\text {stat. }} / \sqrt{\tilde{M}}$.

The systematic uncertainty on the other hand is expected to behave differently. Each measurement will have roughly the same small bias, that propagates also to the arithmetic mean of all $\tilde{M}$ measurements. Accordingly, the significance of the systematic uncertainty will increase when multiple radiation length measurements are combined in an arithmetic mean.

However, increasing the number of entries per scattering angle distribution and therefore improving the fit conditions, may decrease the magnitude of the bias. The relative systematic and statistical uncertainties as a function of the number of angles per histogram $N$ are shown in fig. 6.4 and 6.5.

The relative systematic uncertainty is approximately proportional to $1 / \sqrt{N}$ for values of $\mathrm{N}$ below 6000 . For values larger than $6000, \sigma^{\text {rel.sys. }}$ converges to a small positive value $c$. The numerical value of $c$ depends on the number of histogram bins. The larger the number of bins the smaller $c$.

The relative statistical error shown in fig. 6.5, on the other hand, behaves as a function proportional to $1 / \sqrt{N}$ for all numbers of histogram entries in the tested range between 500 and 12000.

As the number of scattering angles in a beam test is determined by the duration of the radiation length measurement, the goal is to minimise $N$ in such a way that the systematic and statistical error is below a certain tolerable threshold. The statistical error can typically be decreased to the desired threshold by combining multiple radiation length measurements. For the following considerations the statistical uncertainty will therefore be disregarded for the moment. As can be seen in fig. 6.4, a number of 5000 histogram entries is a good compromise between minimising the required statistics per angle distribution and a small measurement bias. The decrease of the bias is very slow 


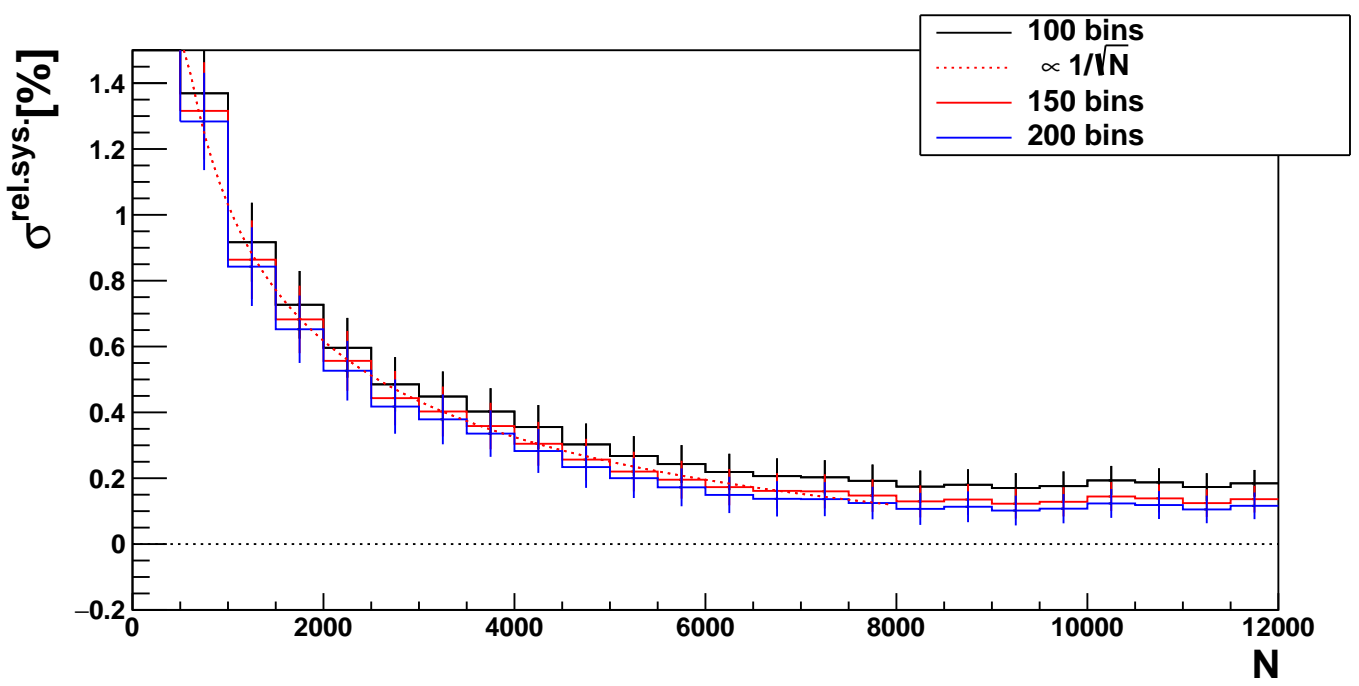

Figure 6.4.: The relative systematic uncertainty $\sigma^{\text {rel.sys. }}$ as a function of the number of scattering angles per distribution $N$. The observed bias is approximately proportional to $1 / \sqrt{N}$. For large $N$ values the residual mean converges against a small positive value, which is independent of $N$, but depends on the number of bins of the angle histograms.

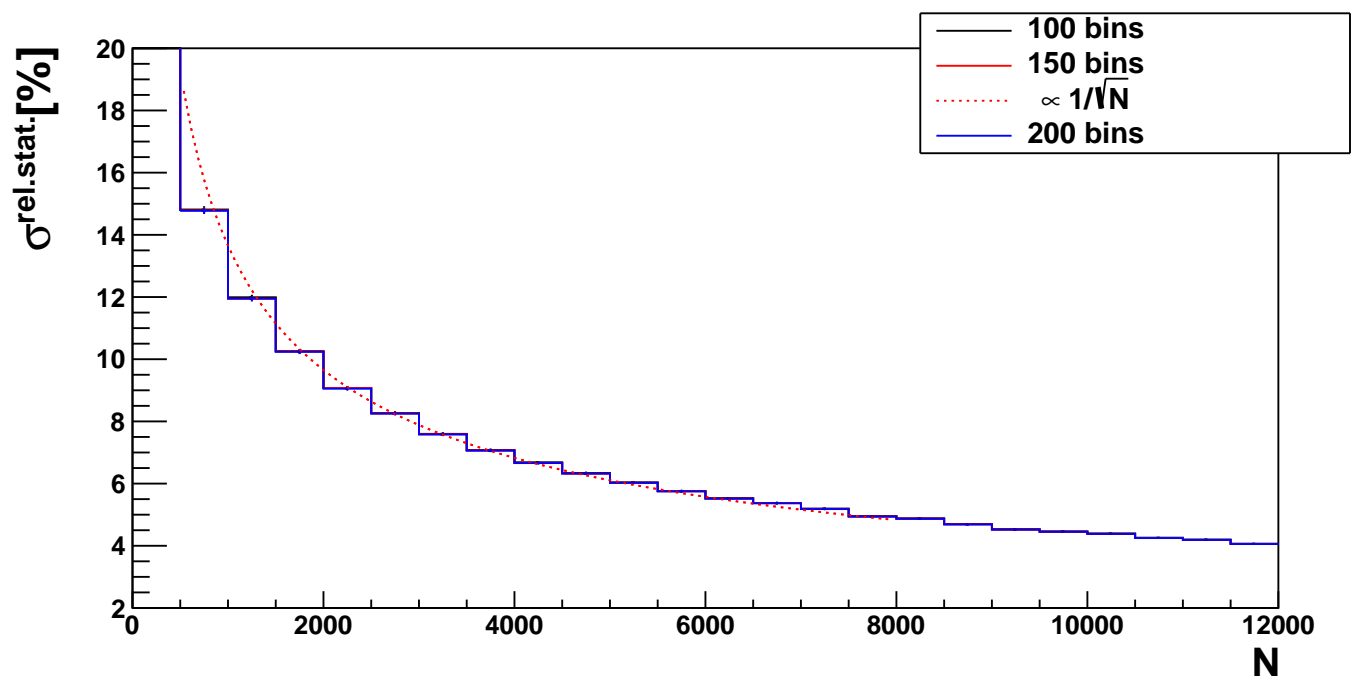

Figure 6.5.: The relative statistical uncertainty $\sigma^{\text {rel.stat. }}$ as a function of the number of scattering angles per distribution $N$. The statistical is approximately proportional to $1 / \sqrt{N}$.

for larger values of $N$. For the example measurement at the beginning of this section with the parameters given in table 6.1, this bias would correspond to less than $3 \mu \mathrm{m}$ for 


\section{Systematic influences and simulation studies}

a measurement on aluminium with a thickness of $1 \mathrm{~mm}$. As will be shown in section 6.1.2, the number of required scattering angles per distribution to keep the bias below a certain threshold depends on the beam energy and the radiation length of the scattering material.

\subsubsection{Expected statistical and systematic uncertainties}

Fig. 6.6 depicts the measured relative systematic uncertainty as a function of $N$ for several different radiation length values and beam energies. The beam particles are electrons and the beam energies studied here are 2,4 , and $6 \mathrm{GeV}$. The three values cover the range of beam energies used in chapter 7 . The target material is aluminium with thicknesses between $50 \mu \mathrm{m}$ and $20 \mathrm{~mm}$. The resulting radiation length range ${ }^{2}$ is representative of most materials measured in this thesis.

As demonstrated in section 5.5 the telescope resolution $\sigma_{\text {err }}$ depends on the beam energy and telescope setup. Therefore, when varying the beam energy in this section, the telescope angle resolution must be modified accordingly. Telescope angle resolutions for an EUDET telescope geometry depicted in fig. 6.8 for different beam energies are summarised in table 6.2 .

\begin{tabular}{|c||c|c|c|}
\hline $\mathbf{p}[\mathbf{G e V}]$ & 2 & 4 & 6 \\
\hline$\sigma_{\text {err }}[\mathbf{p r a d}]$ & 240 & 140 & 110 \\
\hline
\end{tabular}

Table 6.2.: Telescope angle resolution for different beam energies and the EUDET telescope geometry depicted in fig. 6.8.

As mentioned before, the goal of the simulation study is to determine the statistics required to limit the relative systematic uncertainty below $0.5 \%$ for each combination of material thickness and beam energy. This value was selected because most radiation length measurements in chapter 7 have a comparable statistical uncertainty. The threshold is marked by a dashed line in the diagrams in fig. 6.6.

The diagrams indicate that for measurements of extremely thin materials it is prudent to either use scattering histograms with a large statistics or to measure with a small beam energy. In general, the radiation length measurements are slightly less biased, when a smaller beam energy is used. Also, as already seen section 6.1.1, increasing the statistics reduces the bias. The magnitude of the fit bias can be kept below the threshold for all tested beam energies and most scattering material thicknesses, if a large enough number of scattering angles is used. The only problematic case is the measurement on $50 \mu \mathrm{m}$ aluminium with a beam energy of $6 \mathrm{GeV}$. ${ }^{2}$ For $20 \mathrm{~mm}$ of aluminium and an electron beam, energy losses due to bremsstrahlung have to be
considered. However, they are neglected here 
6.1. Validation of the scattering angle fitting procedure with toy studies

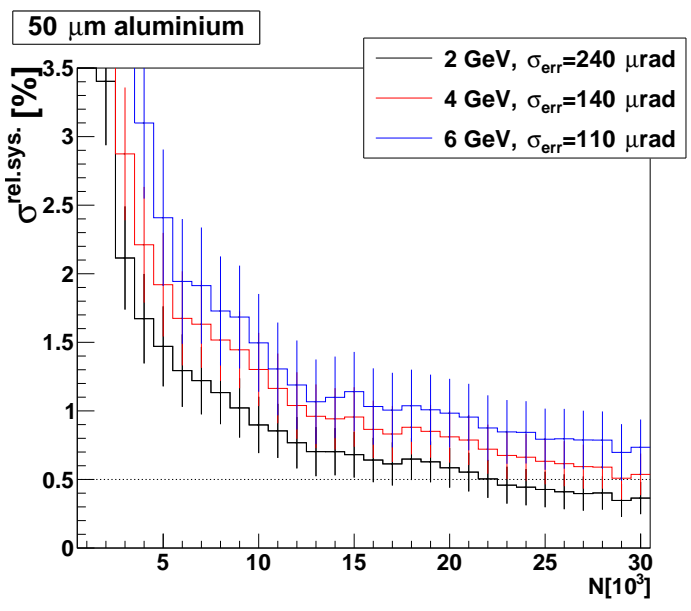

(a)

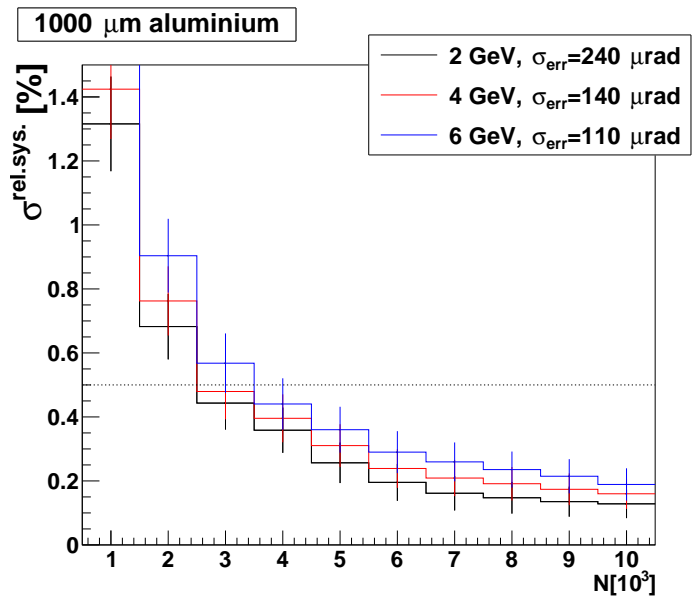

(c)

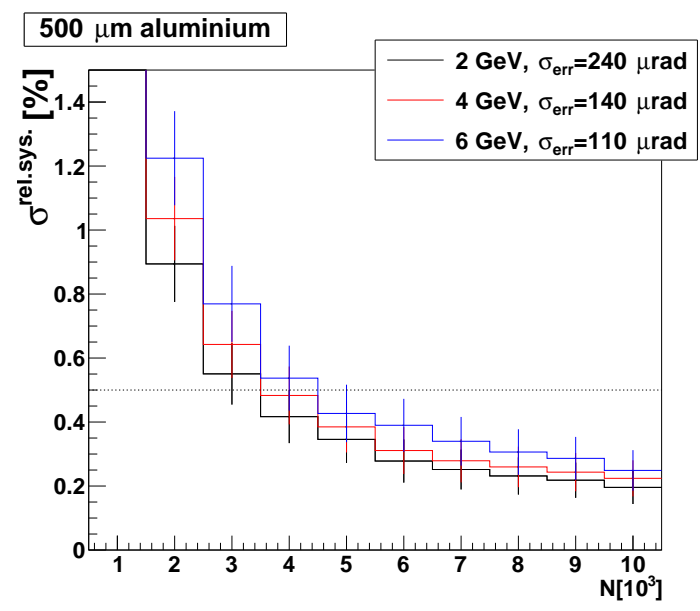

(b)

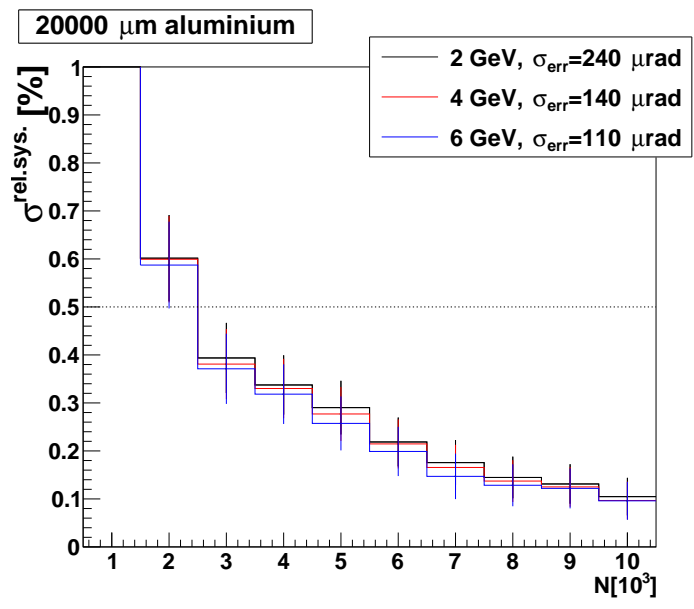

(d)

Figure 6.6.: Depiction of the relative systematic uncertainty of radiation length measurements for a 50 (a), 500 (b), 1000 (c) and 20000 (d) pm aluminium scattering target at three different beam energies. The black dashed line indicates a relative systematic uncertainty of $0.5 \%$.

A summary of these results can be found in table 6.3. For each beam energy and aluminium thickness combination, two quantities are listed: The expected relative systematic uncertainty in case of $N=5000$ scattering angles per histogram (denoted as $\left.\sigma^{\text {rel.sys. }}(N=5000)[\%]\right)$ and the required minimum number of scattering angles to achieve a relative systematic uncertainty below $0.5 \%$ of the true value $t^{\text {true }}$ (denoted as $N\left(\sigma^{\text {rel.sys. }}=0.5 \%\right)$ ). The first quantity can be seen as a rough estimate of the systematic uncertainty of individual radiation length image pixels, while the second quantity can be used to decide how many angles are needed for extremely precise radiation length measurements. 


\begin{tabular}{|c|c|c|c|c|}
\hline $\boldsymbol{X}[\mathbf{p m}]$ & $\boldsymbol{E}[\mathbf{G e V}]$ & $\left(\boldsymbol{X} / \boldsymbol{X}_{\mathbf{0}}\right)^{\text {true }}[\boldsymbol{\%}]$ & $\boldsymbol{\sigma}^{\text {rel.sys. }}(\boldsymbol{N}=\mathbf{5 ~ 0 0 0})[\%]$ & $\boldsymbol{N}\left(\boldsymbol{\sigma}^{\text {rel.sys. }}=\mathbf{0 . 5} \boldsymbol{\%}\right)$ \\
\hline \hline 50 & 2 & 0.056 & $1.5 \pm 0.3$ & 22000 \\
\hline 50 & 4 & 0.056 & $1.9 \pm 0.4$ & $\approx 30000$ \\
\hline 50 & 6 & 0.056 & $2.4 \pm 0.5$ & $>30000$ \\
\hline 500 & 2 & 0.562 & $0.35 \pm 0.07$ & 4000 \\
\hline 500 & 4 & 0.562 & $0.38 \pm 0.08$ & 5000 \\
\hline 500 & 6 & 0.562 & $0.42 \pm 0.09$ & 3000 \\
\hline 1000 & 2 & 1.124 & $0.26 \pm 0.06$ & 3000 \\
\hline 1000 & 4 & 1.124 & $0.31 \pm 0.07$ & 4000 \\
\hline 1000 & 6 & 1.124 & $0.36 \pm 0.07$ & 3000 \\
\hline 20000 & 2 & 22.472 & $0.29 \pm 0.06$ & 3000 \\
\hline 20000 & 4 & 22.472 & $0.28 \pm 0.06$ & 3000 \\
\hline 20000 & 6 & 22.472 & $0.26 \pm 0.06$ & \\
\hline
\end{tabular}

Table 6.3.: Summary of the results from the toy studies of four different aluminium thicknesses and three different energies. The table contains the expected relative residual mean for angle distributions with $N=5000$ entries and the minimum number of entries required to keep the relative systematic uncertainty below $0.5 \%$.

To fulfil the requirement of a relative systematic uncertainty smaller than $0.5 \%$ of the truth radiation length typically 3000 to 5000 scattering angles are sufficient. Only for very thin materials the number of scattering angles should be increased to more than 20000 in order to keep the bias small. This result should be considered, when measuring materials like for example the ultra-thin sensitive area of the PXD. In order to reduce the systematic uncertainty of the radiation length measurement, it might be beneficial to use a large pixel size and consequently a large number of entries per angle histogram. As increasing the number of angles per distribution decreases both the statistical and the systematic uncertainty, this is a better option than using multiple measurements with a small number of entries per histogram, which only improves the statistical error. However, one still has to consider that a relative systematic error of $0.5 \%$ of the radiation length of $50 \mu \mathrm{m}$ aluminium correspond to $0.25 \mu \mathrm{m}$, which for tracking considerations is a negligible thickness even for ultra-thin charged particle detectors.

The relative statistical uncertainties as a function of the number of scattering angles per angle histogram are depicted in fig. 6.7. Unsurprisingly, the statistical error decreases with an increasing number of scattering angles per histogram. Additionally, the relative statistical error is smaller for measurements on materials with a large radiation length. The overall width of the reconstructed angle distribution is given by

$$
\sigma_{\text {total }}=\sqrt{\sigma_{\mathrm{MSC}}^{2}+\sigma_{\mathrm{err}}^{2}} .
$$


6.1. Validation of the scattering angle fitting procedure with toy studies

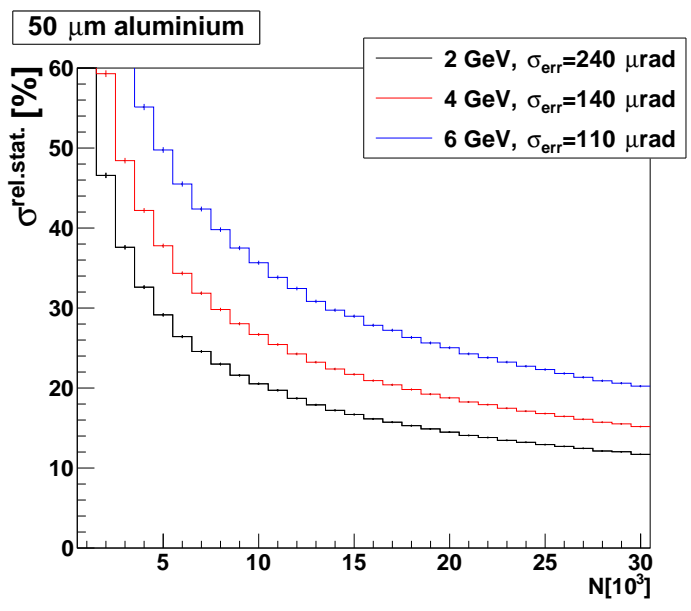

(a)

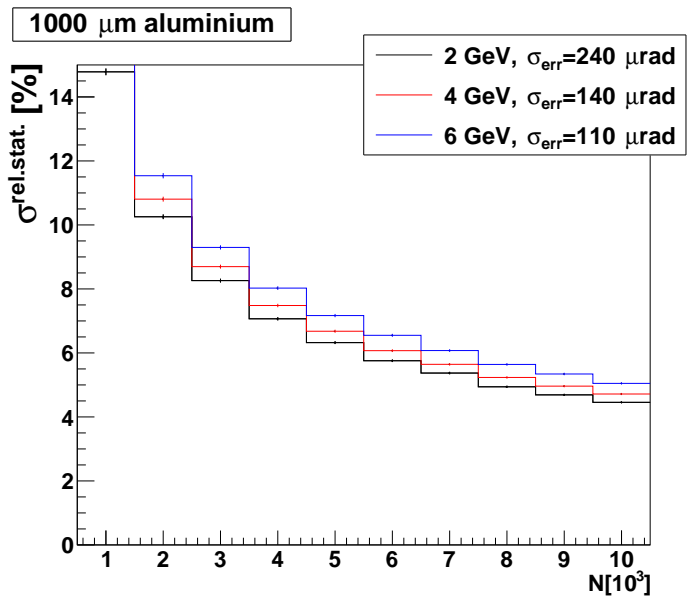

(c)

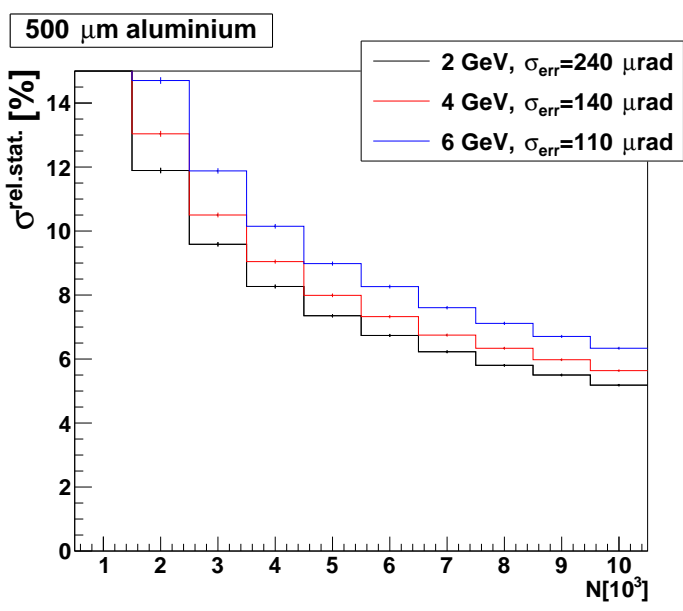

(b)

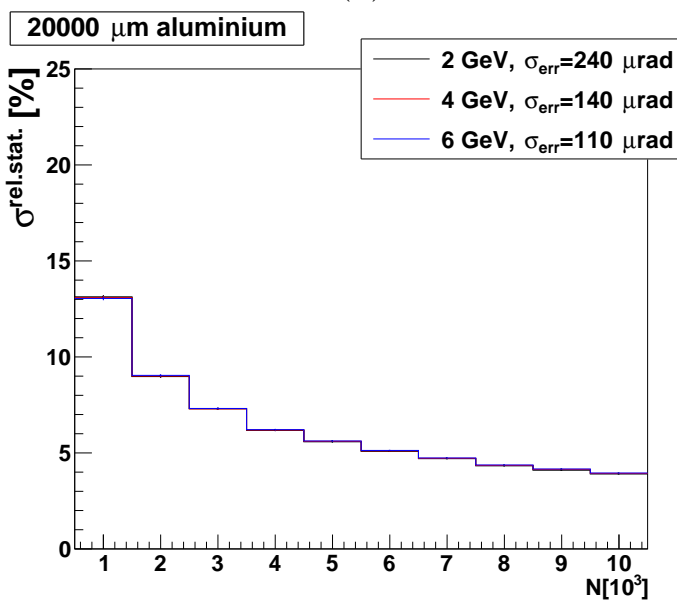

(d)

Figure 6.7.: Depiction of the relative statistical uncertainty of radiation length measurements for a 50 (a), 500 (b), 1000 (c) and $20000 \mu \mathrm{m}$ (d) aluminium scattering target at three different beam energies.

The multiple scattering distribution width $\sigma_{\mathrm{MSC}}$ can be expressed via the Highland width $\sigma_{\mathrm{HL}}$ (see eq. 3.28) and increases with an increasing radiation length $X / X_{0}$. The telescope angle resolution $\sigma_{\text {err }}$ on the other hand is independent of the radiation length. Therefore with increasing radiation length the multiple scattering part contributes more to the overall scattering angle distribution width. In other words, the signal-to-noise ratio $S=\sigma_{\mathrm{MSC}} / \sigma_{\mathrm{err}}$, which has been defined in section 5.5 , is large for high radiation length materials and small for thin materials. Consequently, the scattering angle distributions of high $X / X_{0}$ materials are more sensitive to radiation length variations and the relative statistical error is comparatively small. 


\section{Systematic influences and simulation studies}

For thin materials, lower beam energies yield a smaller relative statistical uncertainty than large beam energies. The relative statistical uncertainty for large radiation length materials on the other hand is nearly independent of the beam energy. This can be explained by the signal-to-noise ratio $S$ as well. Both $\sigma_{\mathrm{MSC}}$ and $\sigma_{\text {err }}$ depend on the beam energy. But as can be seen from fig. 5.6a $\sigma_{\mathrm{MSC}}$ increases more rapidly with decreasing beam energy than $\sigma_{\text {err }}$. As a consequence the signal-to-noise ratio $S$ increases with decreasing beam energy, which leads to smaller statistical uncertainties as the overall width has a stronger dependence on the radiation length. For large radiation length materials, where the overall angle distribution width $\sigma_{\text {total }}$ is already dominated by $\sigma_{\mathrm{MSC}}$ a beam energy variation has only a small influence on the relative statistical uncertainty.

\subsection{Angle reconstruction with binary pixel sensors}

After the fitting studies in the last section, additional systematic influences due to the scattering angle reconstruction are examined in this section. In order to test the angle reconstruction, $T B S W$ beam test simulations are conducted. The three Marlin processors, ParticleGun, FastSimulation and SiPixDigitizer, used to simulate a beam test measurement in $T B S W$ are explained in section 4.5. Here, particle beams with $p=2 \mathrm{GeV}$ electrons were simulated that traversed a EUDET telescope consisting of six M26 sensors (see fig. 6.8). Each simulation consists of approximately 15 million simulated electron trajectories. Energy losses due to bremsstrahlung were switched of in the FastSimulation processor. The beam energy uncertainty was $\sigma_{p, p}=1 \mathrm{MeV}$. The beam divergences $\sigma_{m_{u}, m_{u}}$ and $\sigma_{m_{v}, m_{v}}$ in both directions were $3.5 \mathrm{mrad}$ and the beam diameters $\sigma_{u, u}$ and $\sigma_{v, v}$ in both directions were $10 \mathrm{~mm}$.

At the beginning of the simulation process, the telescope geometry was artificially misaligned by determining random shifts and rotations from Gaussian distributions with a standard deviation depending on the alignment parameter. For the simulations here the following standard deviations were used:

$$
\begin{aligned}
& \sigma_{x}=\sigma_{y}=\sigma_{z}=0.5 \mathrm{~mm} \\
& \sigma_{\alpha}=\sigma_{\beta}=\sigma_{\gamma}=0.1^{\circ}
\end{aligned}
$$

The complete analysis chain described in chapter 4 and 5 is used to reconstruct scattering angles from the simulated M26 digits. Several simplifications were used during the analysis of the simulated beam test data sets. The simulated telescope was intentionally misaligned as explained before, but instead of performing a data-driven alignment of the telescope, the true telescope shifts and rotations were used during the scattering angle reconstruction.

The track states used during the angle reconstruction are reconstructed from position measurements on digital M26 pixel sensors. Particle trajectory intersections measured 


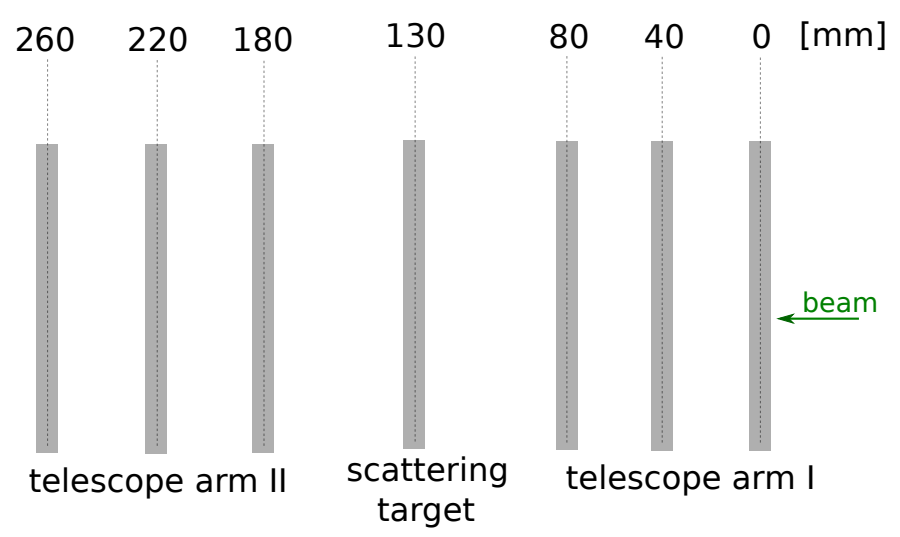

Figure 6.8.: Default telescope setup consisting of two telescope arms with 3 M26 sensors each. The distance between two neighbouring M26 sensors is $40 \mathrm{~mm}$. Additionally a scattering target is placed in the center between the two telescope arms.

by digital sensors are not expected to be Gaussian distributed but rather a convolution of a uniform distribution with a Gaussian. An example of such a intersection distribution is depicted in 6.9. The distribution corresponds to the simulated hit positions in $u$ direction of one-digit clusters. However, the Kalman filter update equation (eq. 4.17) expects hit measurements with Gaussian uncertainties. Accordingly, the reconstructed track states and their covariance matrices are expected to display non-Gaussian behaviour.

As explained in $[75,76]$, the quantisation of coordinate measurements of digital telescope sensors can lead to correlations between measurements on different telescope planes. The magnitude of these coordinate correlations between neighbouring sensors depends on the specific alignment and setup of the telescope planes. For example, in an idealised case of perpendicular incidence and a perfectly aligned telescope of identical sensors, the coordinates of each plane are fully correlated. Measurements on neighbouring planes in a realistic slightly misaligned reference telescope can also be strongly correlated.

Coordinate correlations between telescope sensors can cause biases in the track states which also propagate to the reconstructed scattering distributions. In order to visualise artefacts caused by coordinate correlations two reconstructed angle distributions are depicted in fig. 6.10. Both distributions are reconstructed from beam test simulation data with identical beam parameters. The only difference is that the distribution in fig. $6.10 \mathrm{~b}$ was reconstructed in a perfectly aligned telescope with large coordinate correlations between telescope planes, while the distribution in fig. 6.10a was reconstructed in a telescope with small artificial shifts and rotations.

The angle distribution reconstructed in a telescope with large coordinate correlations in fig. $6.10 \mathrm{~b}$ consists of a Gaussian distribution overlaid with a pattern of periodic peaks. 


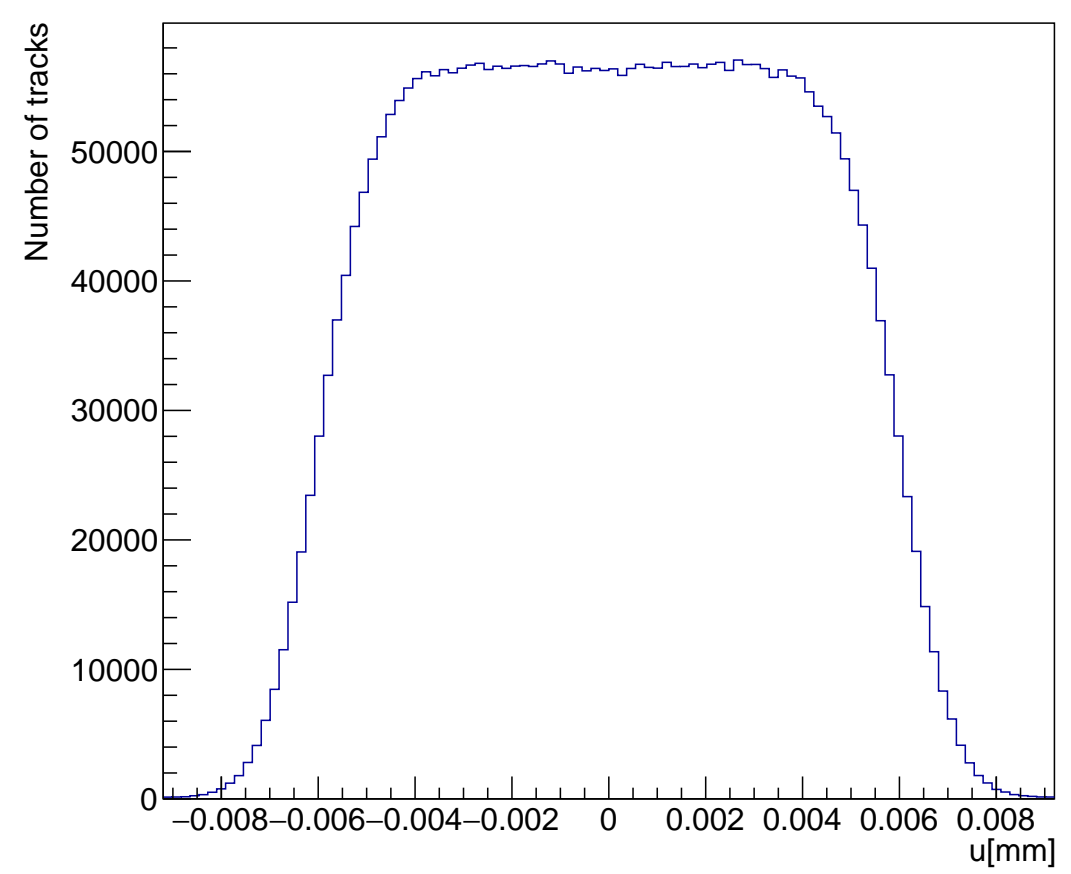

Figure 6.9.: Simulated particle intersection in $u$ direction for a single-digit cluster. As can be seen, the distribution is flat in the centre and behaves like a Gaussian on the edge of the distribution.

The distribution in fig. 6.10a has a Gaussian shape. The goal of this section is to evaluate the impact of these artefacts on the radiation length measurements. Afterwards, several strategies are presented that can be used to avoid large coordinate correlations and consequently angle distributions that are not properly described by Gaussian distributions.

In order to test the impact on the radiation length measurements, radiation length images are used. The statistics variation due to the Gaussian beam spot is excluded by limiting the number of scattering angles per angle histogram to 5000 . On the following pages measurements with different image pixel sizes are compared. A sample of measured radiation length values $t^{\mathrm{m}}$ can be generated by using the fitted radiation length values from the radiation length image pixels. In order to get $t^{\mathrm{m}}$ samples of approximately the same size, larger pixel sizes have to provide multiple radiation length measurements. For example, four $100 \times 100 \mathrm{~mm}^{2}$ image pixels with a single angle histogram with 5000 entries each provide four individual radiation length measurements. The same area is covered by a single 200x200 $\mathrm{mm}^{2}$ image pixel with an angle histogram with at least 20000 


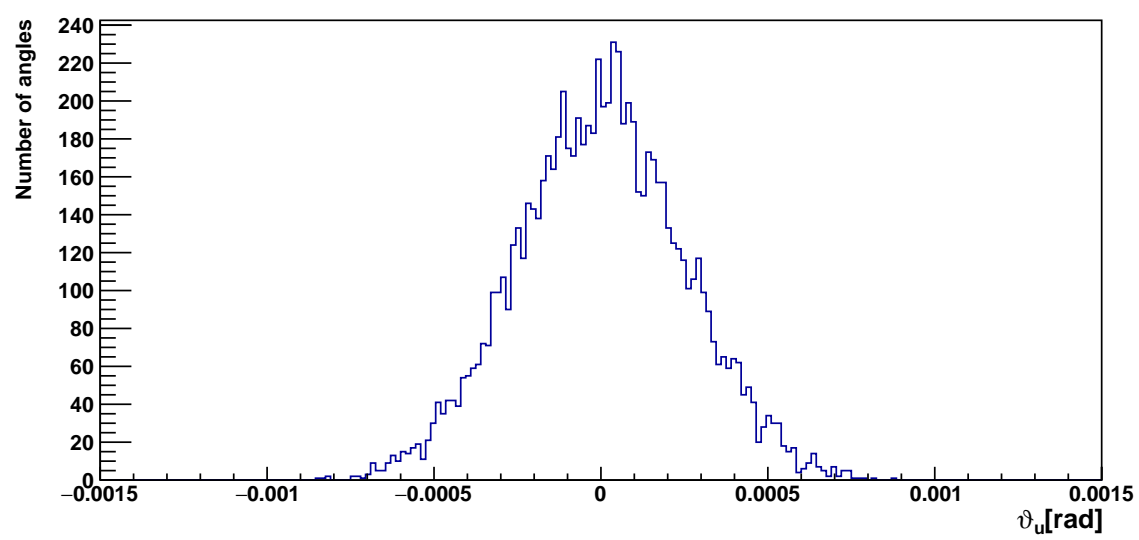

(a) Angle distribution reconstructed in realistic telescope

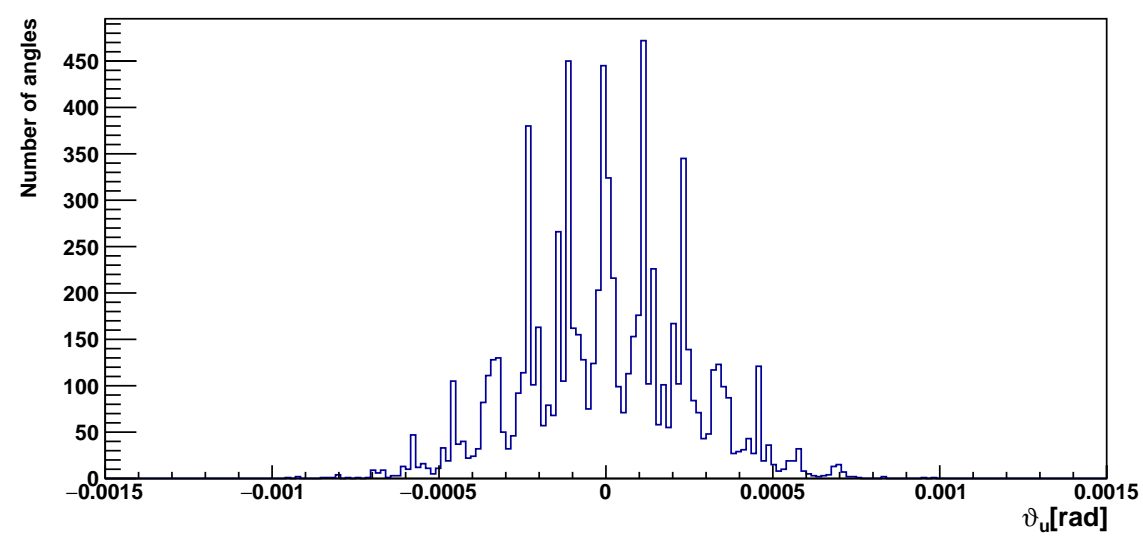

(b) Angle distribution reconstructed in perfectly aligned telescope

Figure 6.10.: Reconstructed angle distributions of two beam test simulations. The beam energy was $4 \mathrm{GeV}$. The simulated telescope geometry in (a) has small random rotations and shifts, while the telescope in (b) was perfectly aligned. The angle distribution in (a) has a Gaussian shape, while the distribution in (b) shows clear signs of measurement artefacts in form of periodic peaks.

entries. This angle histogram is split up into four histograms with 5000 angles each to also provide four radiation length measurements.

First, radiation length images with $100 \times 100 \mu \mathrm{m}^{2}$ and $200 \times 200 \mu \mathrm{m}^{2}$ pixels are tested. The radiation length image of a simulated $1 \mathrm{~mm}$ aluminium layer with image pixel sizes of $200 \times 200 \mathrm{um}^{2}$ is depicted in fig. 6.11. The black box indicates the area used to generate a radiation length samples which are used in the measurements depicted in fig. 6.13c. The area is limited to the centre of the image to avoid border effects. 


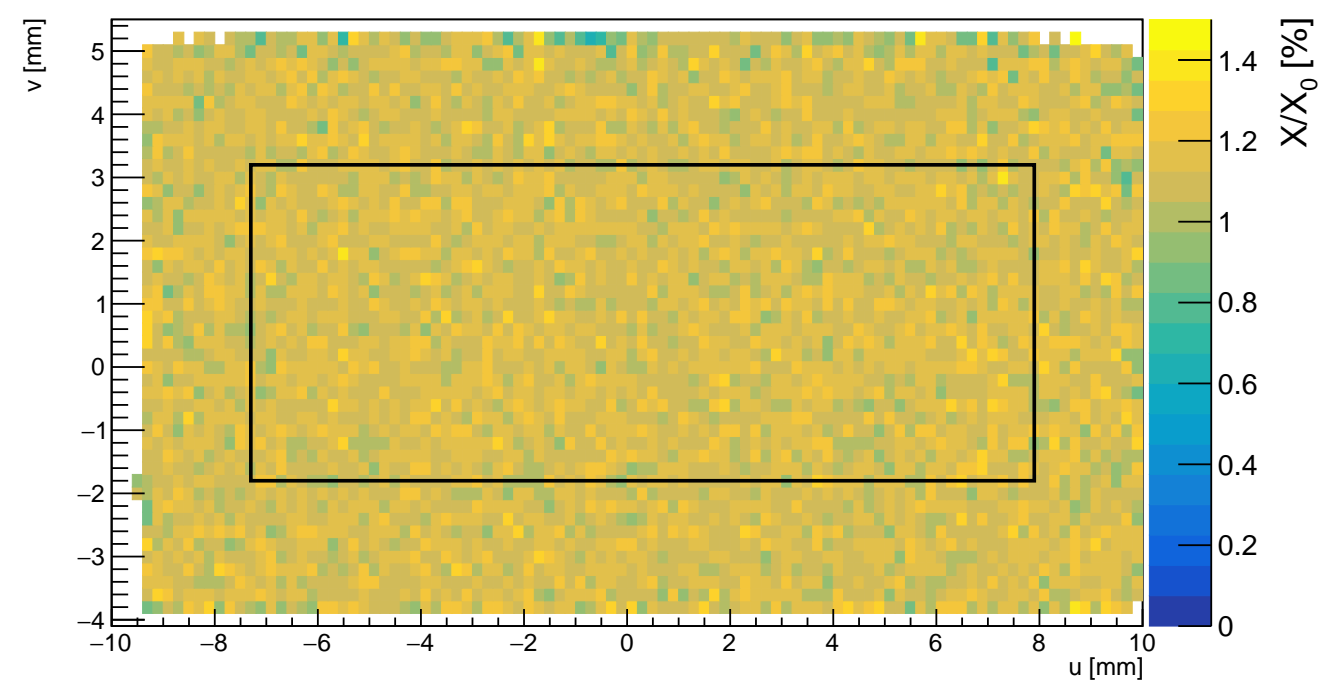

Figure 6.11.: Radiation length image of a simulated $1 \mathrm{~mm}$ aluminium layer. The black box indicates the area with image pixels used to generate the radiation length $t^{\mathrm{m}}$ sample, which is used in the measurements in fig. $6.13 \mathrm{c}$

Fig. 6.12 depicts scattering angle distributions from images of both pixel types. Both distributions come from an image pixel located near the centre of the corresponding image.

The reconstructed angle distribution for the smaller pixel pitch deviates from the expected Gaussian multiple scattering distribution (see for example fig. 6.1). Similar to the distribution in fig. $6.10 \mathrm{~b}$, the Gaussian distribution is convoluted with a periodic pattern of peaks. These peaks are caused by coordinate correlations between telescope sensors, which are in turn caused by the digital position measurements of the M26 sensor. The distance between peaks was found to be between 200 and 300 prad. The corresponding distribution for an image pixel with a larger pitch shows much smaller disturbance (see fig. 6.12b). This can be explained by an effective decrease of the coordinate correlations between the M26 sensors due to an increase of valid measurement coordinates on the telescope sensors when increasing the area of image pixels.

In order to evaluate the influence of the periodic peak pattern, residual and pull distributions of the radiation length measurements are used. The individual radiation length measurements of the image pixels are used to calculate $t^{\text {res }}$ and $t^{\text {pull }}$ distributions as explained in section 6.1. Fig. 6.13 depicts pull $t^{\text {pull }}$, residual $t^{\text {res }}$ and statistical fit uncertainty $\sigma_{t^{\mathrm{m}}}$ distributions for measurements with image pixel pitches of 100 and $200 \mu \mathrm{m}$. 


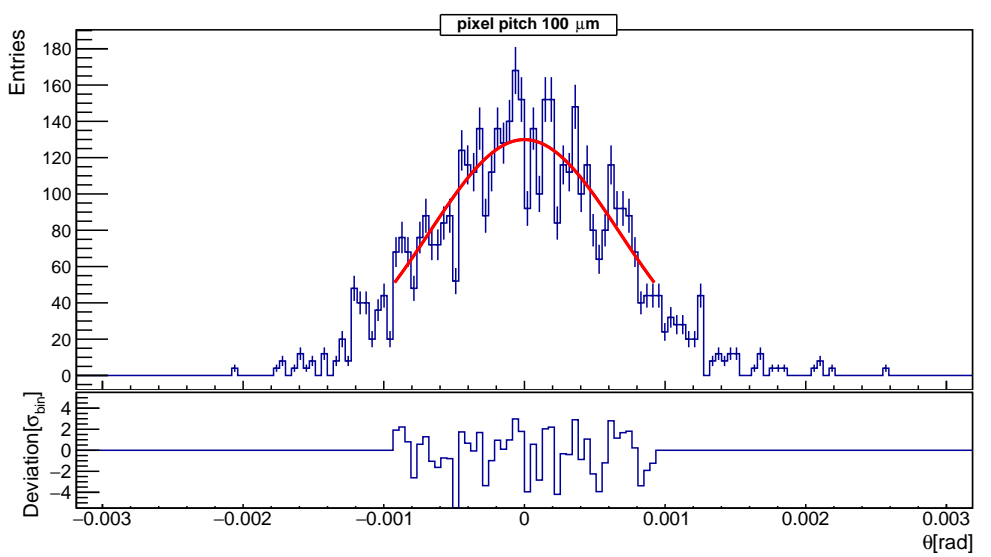

(a) $100 \times 100 \mu m^{2}$ image pixels

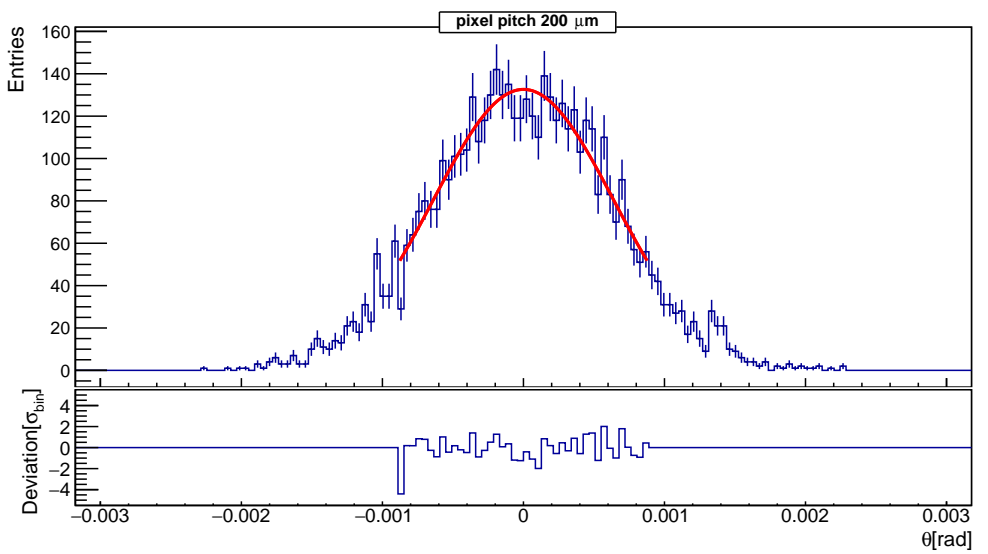

(b) $200 \times 200 \mu \mathrm{m}^{2}$ image pixels

Figure 6.12.: Reconstructed angle distribution and associated fit function for different image pixel sizes. A ratio plot between the Gaussian fit function and the angle distribution is shown beneath the angle distributions. In the angle distribution with a smaller image pixel pitch periodic peaks can be observed.

As can be seen, the radiation length measurements from images with $200 x 200 \mu^{2}$ pixels worked well. The observed systematic uncertainty due to the bias in the log-likelihood estimator is smaller than expected from the simulation studies in section 6.1.2. The mean value of the residual distribution is compatible with zero. Also the pull RMS value for this pixel size is close to one. Accordingly, when using large image pixels the scattering angle reconstruction and potential correlation effects have no negative impact on the fit of the angle distributions. However, for a small pixel size of $100 \times 100 \mu^{2}$ the pull distributions is extremely broad and the pull RMS value is $1.74 \pm 0.02$ which differs widely from the expected value of one. 


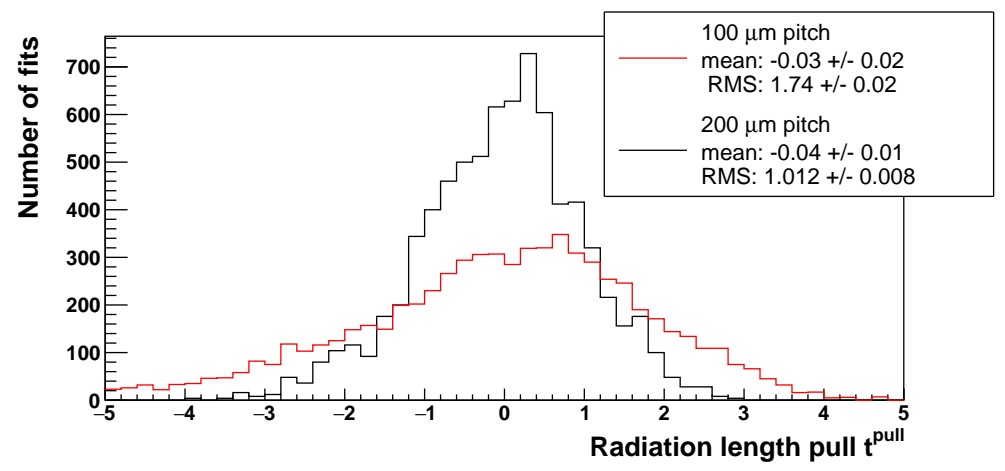

(a) Radiation length pull distribution

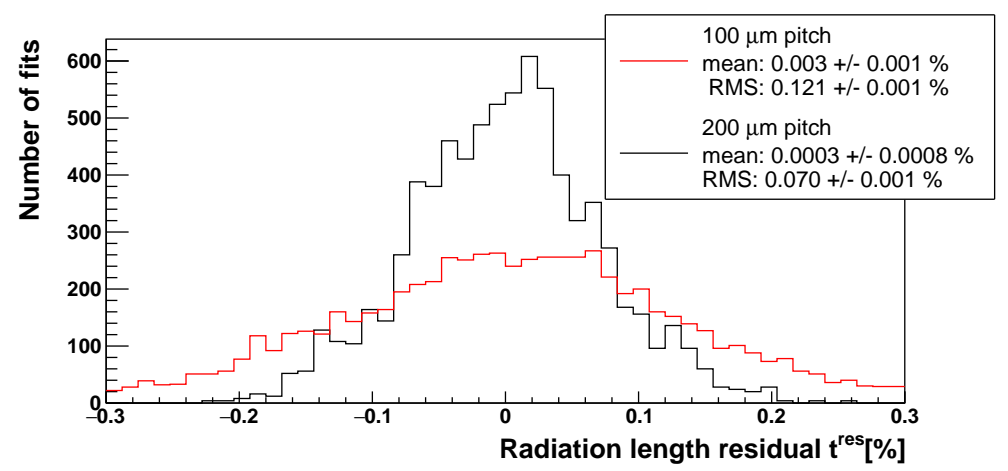

(b) Radiation length residual distribution

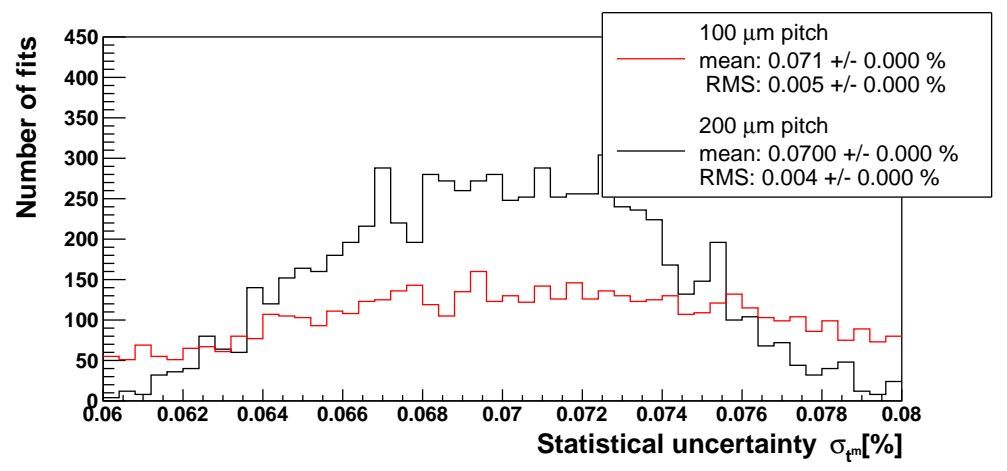

(c) Radiation length statistical fit uncertainty distribution

Figure 6.13.: Pull (a), residual (b) and statistical fit uncertainty (c) distributions for $T B S W$ simulation experiments with different image pixel sizes. The angle histograms have a range between -5 times to +5 times of the RMS value of the individual distributions and 150 bins. The pull and residual distributions are much broader in the small image pixel case. 
The increase of the RMS for small image pixels can be explained by the artefacts visible in the angle distribution in fig. 6.12a. When fitting an angle distribution similar to the one depicted in fig. 6.12a with a Gaussian function a few issues occur. The peaks will inevitably lead to a much less stable fit procedure. The fit is limited to the core region of the angle distribution and the presence of peaks in the border region of the fit might lead to an over- or underestimation of the fitted radiation length value. As this effect depends on arbitrarily distributed peaks, the mean of the fitted radiation length will not be shifted. As can be seen in the residual distribution of the small pixel size (fig. 6.13b), the measurement show only a very small bias. However, the variance of the radiation length measurements is affected by the peaks. It can therefore be expected that the spread of the measured radiation length values is larger than what would be expected from the statistical fit uncertainty. Consequently, the RMS value of the pull distribution is expected to be larger than one.

As can be seen in fig. 6.14, the increase of the pull RMS values, when going to smaller pixel pitches, is suppressed when using a telescope with larger distances between neighbouring sensor planes. The cause of the broadening of the pull distributions is connected to the angle reconstruction as explained before. The telescope setup has an impact on the aforementioned correlations between the M26 sensors and consequently on the overlaid peak structures in the angle distributions. Increasing the spacings between neighbouring M26 sensors leads to a decrease of the pull RMS values.

This can be explained with a simple example. A digital sensor with symmetrical pixel with a pitch of $l_{0}$ is employed to measure particle tracks. For the sake of simplicity, it is assumed that a traversing particle leads to a signal only in the traversed sensor pixel. The neighbouring sensor pixels are not affected by charge sharing or similar effects. In this case the best estimate for the position of the particle transition is always the centre of the firing sensor pixel. When considering all pixels of the sensor this leads to a quadratic grid of valid measurement positions. In one dimension all valid position measurements of the transition are given by

$$
x=\left(N+\frac{1}{2}\right) \cdot l_{0}, \text { where } N \in \mathbb{N}
$$

The quantity $l_{0}$ is called the quantum length in the paper by Drijard [75]. $N$ is the corresponding counter of the sensor pixels. Combining the position measurements of two sensors, which are separated by the distance $D$, we can calculate an angle. When assuming that both sensors are perfectly aligned the minimum difference between two valid angle measurements is

$$
\vartheta_{0} \stackrel{D \gg l_{0}}{\approx} \frac{l_{0}}{D}
$$




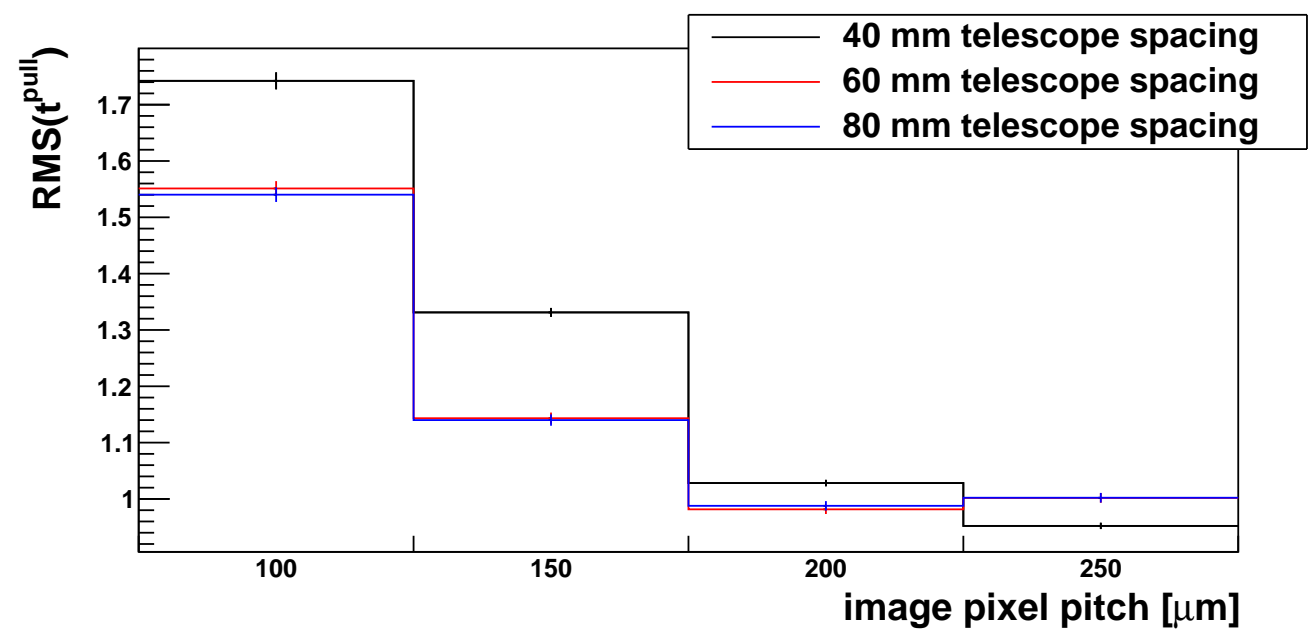

Figure 6.14.: RMS value of the pull distribution for radiation length image measurements with different image pixel pitches. The three depicted curves have different telescope spacings, i.e. distances between neighbouring telescope planes. Radiation length pull RMS values are nearly identical for 60 and $80 \mathrm{~mm}$ telescope spacings and always smaller than the corresponding value at a telescope spacing of $40 \mathrm{~mm}$.

$\vartheta_{0}$ can be called the quantum angle of the sensor duplet in analogy to the quantum length $l_{0}$ of individual sensors. This smallest angle difference depends on the distance between the two telescope sensors. In the case of a realistic telescope setup the grid structure is much more complicated, because two-digit-clusters and clusters with even more digits will introduce additional valid measurement positions. Additionally the telescope sensors are not perfectly aligned, which leads to a rotation and shift between the measurement grids of the telescope sensors. However, the basic idea remains the same. Due to a discrete set of measurement positions, there will always be a finite number of valid angle measurements.

In a realistic setup it is difficult to estimate the size of the quantum angle $\vartheta_{0}$. However, it is expected to depend on the distance between the telescope planes: The larger the distance $D$ the smaller $\vartheta_{0}$. Additionally, it is reasonable to assume that the quantisation effects of the angle measurements determine the distance between the peaks in the scattering angle distributions. Therefore by increasing the telescope spacing the distance between the peaks is decreased and some of the fluctuations are absorbed by the finite size of the scattering angle histogram bins.

The last part of this section describes different strategies to minimise the digital effects on the angle distributions. The first option is, as already demonstrated, to use larger image pixels. As can be seen in fig. 6.14, for pixel sizes of $200 \times 200 \mu^{2}$ and 
above digital effects have no measurable impact on the radiation length measurement. However, using large image pixels is not always possible or desirable, especially when local radiation length variations on a small scale have to be resolved. Alternatively, larger telescope spacings can be used. Spacings smaller than $40 \mathrm{~mm}$ should be avoided to reduce the magnitude of the artefacts in reconstructed angle distributions.

Another factor that has a significant impact on the magnitude of the peaks is the misalignment of the sensors (see fig. 6.10). Especially arbitrary rotations along the beam axis have a large impact as they reduce the correlations drastically. In order to demonstrate this, two different simulations were conducted. As explained in the beginning of this section the telescope planes are rotated and shifted artificially by random angles and translations determined from Gaussian distributions with a preselected standard deviation. One simulation used a standard deviation $\sigma_{\gamma}=0.1^{\circ}=1.7 \mathrm{mrad}$ and the other used a standard deviation of $\sigma_{\gamma}=5.0^{\circ}=87.2 \mathrm{mrad}$. Fig. 6.15 depicts the effect of random $\gamma$ rotations with a different magnitude. The larger the gamma rotation, the smaller the influence of the digital effects. A torsion of the telescope planes should not be manually introduced during beam test measurements. However, the existing misalignment of the telescope with random shifts in the order of one millimetre and random rotations in the order of one degree is typically sufficient to limit the impact of digital effects.

In conclusion, spacings between the telescope planes larger than $40 \mathrm{~mm}$ are beneficial for the estimation of the statistical uncertainty of the radiation length measurements. This result agrees with results from the signal-to-noise studies in section 5.5. Telescope spacings larger than $40 \mathrm{~mm}$ yield a much better signal-to-noise ratio than smaller spacings. If telescope spacings smaller than $40 \mathrm{~mm}$ are used, one should consider using larger image pixel pitches to avoid errors of the determined statistical uncertainties.

\subsection{Validation of the radiation length calibration in simulation studies}

Radiation length calibration measurements are an essential part of the radiation length measurement method presented in this thesis. They use well known material profiles to determine $X / X_{0}$ calibration parameters such as the global offset of telescope angle resolution $\lambda$ and the energy profile of the particle beam. A detailed description of the radiation length calibration process can be found in section 5.4. There are four different calibration factors, which have to be determined in the frame of a calibration measurement:

- The calibration factor $\lambda$ corrects for global offsets of the nominal telescope resolution $\sigma_{\text {err }}$. The calibrated telescope resolution is given by the product of $\lambda$ and the nominal telescope resolution.

- The calibration factor $\kappa$ corrects for global offsets of the multiple scattering width 


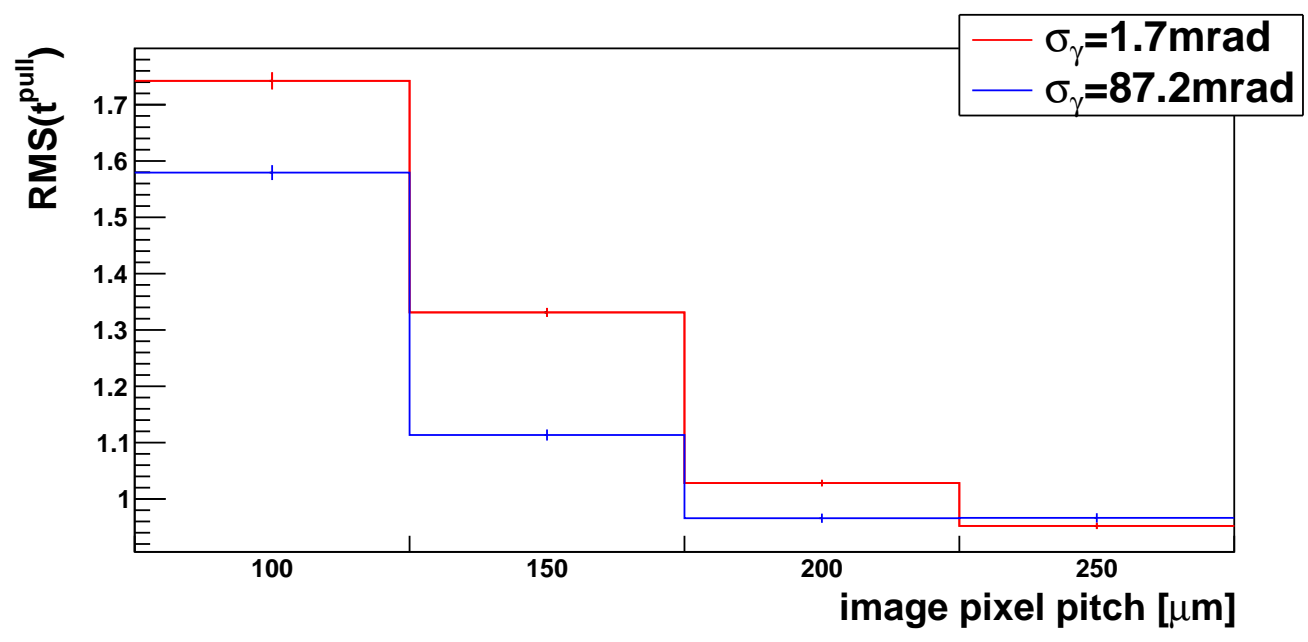

Figure 6.15.: RMS value of the pull distribution for radiation length image measurements with different image pixel pitches. The two curves are based on measurements with different magnitudes of initial $\gamma$ misalignment. The measurement in a telescope with a large $\gamma$ misalignment yields radiation length pulls with smaller RMS values.

$\sigma_{\mathrm{HL}}$. Deviations from the expected value of 1.0 indicate wrong assumptions about the beam energy or telescope length.

- $\Delta_{u} p$ and $\Delta_{v} p$ are linear beam energy gradients in $\mathrm{u}$ and $\mathrm{v}$ direction on the target plane. They are a consequence of the beam energy selection using dipole magnets.

These four calibration parameters must be known with a high precision in order to perform precise radiation length measurements on targets with a unknown material distribution. The goal of this section is to validate the radiation length calibration by conducting measurements on simulated beam test data.

\begin{tabular}{|c|c|c|c|}
\hline $\boldsymbol{\sigma}_{\boldsymbol{x}, \boldsymbol{y}}[\mathbf{\mu m}]$ & $\boldsymbol{\sigma}_{\boldsymbol{z}}[\mathbf{\mu m}]$ & $\boldsymbol{\sigma}_{\boldsymbol{\alpha}, \boldsymbol{\beta}}[\mathbf{m r a d}]$ & $\boldsymbol{\sigma}_{\boldsymbol{\gamma}}[\mathbf{m r a d}]$ \\
\hline 500 & 500 & 1.7 & 87.2 \\
\hline
\end{tabular}

Table 6.4.: Standard deviations of the random Gaussian distributed alignment offsets of the simulated telescope.

Several simulations using $T B S W$ have been conducted to validate the $X / X_{0}$ calibration. The nominal telescope setup of the simulations is depicted in fig. 6.8. Similar to the simulations in section 6.2 the telescope planes are artificially shifted and rotated according to Gaussian distributions. The standard deviation of the different alignment parameters are summarised in table 6.4. Two different studies will be conducted in this section. 
In the first study, influences due to beam energy uncertainties $\sigma_{p, p}$ are examined. For this study, the particle energy is determined from a Gaussian distribution with a mean of $p=2 \mathrm{GeV}$ and a standard deviation $\sigma_{p, p}$ varying between 1 and $10 \%$ of $p$. For the second study the mean beam energy of $p=2 \mathrm{GeV}$ is shifted by a small amount to test the capability to determine the correct $\kappa$ calibration factor. All other beam parameters are similar to the ones described in section 6.2.

As the radiation length calibration require scattering angles from multiple reference materials, several toy simulations with different scattering materials between the telescope arms are conducted. An overview of the used materials is given in table 6.5 and the three-step beam test simulations in $T B S W$ are explained in section 4.5.

\begin{tabular}{|c||c|c|c|}
\hline Material & air & aluminium & aluminium \\
\hline Thickness[mm] & $\approx 0$ & 0.5 & 1.0 \\
\hline $\boldsymbol{X} / \boldsymbol{X}_{\mathbf{0}}[\%]$ & $\approx 0$ & 0.562 & 1.124 \\
\hline
\end{tabular}

Table 6.5.: Simulated materials used in the validation of the $X / X_{0}$ calibration measurements.

The telescope calibration (see section 4.3) which includes measurements of cluster resolutions and alignment are performed using the simulated air data. Then, the angle reconstruction and radiation length calibration are performed on all three simulated data sets and the impact of the beam energy uncertainty or beam energy offset are evaluated. The last step, after the calibration of the four radiation length calibration factors, is a self consistency check. As the calibration uses a sample of scattering angle distributions with a well known radiation length value $X / X_{0}$ to determine the four calibration factors, a subsequent radiation length measurement with the determined calibration factors must yield exactly the same thickness values, which were used during the calibration procedure.

The radiation length calibration measurements are performed with a total number of 15 scattering angle distributions, five for each of the three materials. For each material, one measurement area is positioned in the centre of the beam spot and the other four are located near diagonal edges of the roughly rectangular telescope acceptance region. The side length of each area is $4 \mathrm{~mm}$ in both directions. An example scattering angle distribution (in air) together with its fit function is depicted in fig. 6.16.

The uncertainty of the selected beam energy at the DESY beam facilities is expected to be below $5 \%$ for beam energies larger than $2 \mathrm{GeV}$ and approximately $6 \%$ for an energy of $2 \mathrm{GeV}$ [54]. The first study is a test of the $\kappa$ or beam energy calibration quality, when applying a beam energy uncertainty $\sigma_{p, p} / p$ up to $10 \%$. The simulations are conducted without beam energy gradients or causes for offsets in the telescope resolution. The calibration is therefore expected to yield a telescope angle resolution parameter $\lambda$ consistent with 1.0 and beam energy gradient consistent with $0 \mathrm{MeV} / \mathrm{mm}$. As previously 


\section{Area 0: air}

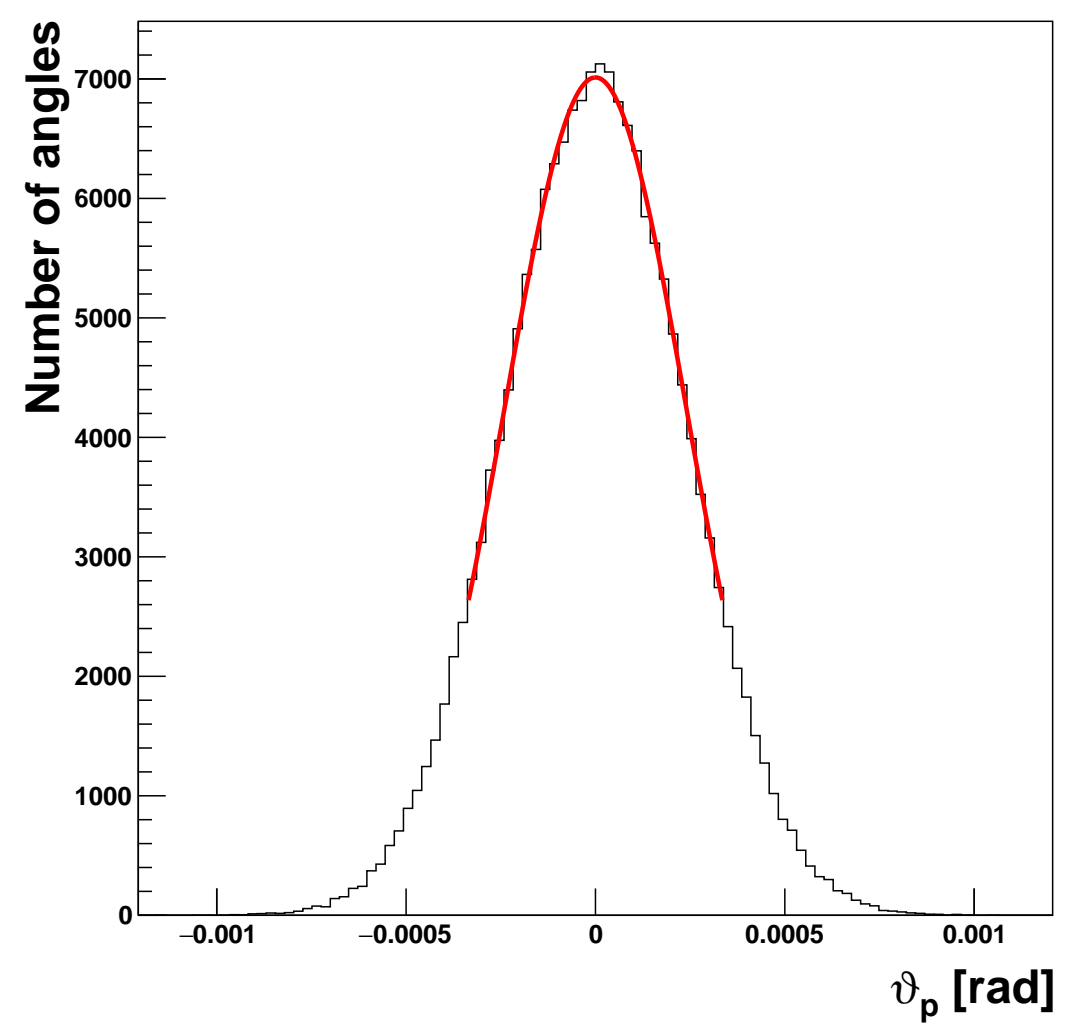

Figure 6.16.: Example scattering angle distribution used in the combined fit during the calibration measurement. The angle distribution comes from a measurement area with air at the centre of the beam spot. The beam uncertainty during the corresponding simulation was $5 \%$.

mentioned, the calibration parameter $\kappa$ deviates from 1.0 for wrong assumptions of the telescope length or the beam energy. The simulated telescope length is identical to the nominal telescope length. This is achieved by setting the artificial $z$ shifts to zero for the first and last sensor of the telescope. As the assumption about the telescope length is correct, the difference between the calibrated beam energy $p_{\text {cal }}=p_{\text {nom }} / \kappa$ and the nominal beam energy $p_{\text {nom }}$ will be examined instead of $\kappa$.

Fig. 6.17 shows the determined calibration parameters as a function of the beam energy uncertainties $\sigma_{p, p} / p$. As can be seen, $\lambda$ is consistent with 1.0 within the limits of the statistical uncertainty for all tested beam energy uncertainty values. Accordingly, the calibrated telescope angle resolution is in agreement with the nominal telescope angle resolution. Additionally, the beam energy gradients in both directions are consistent with $0 \mathrm{MeV} / \mathrm{mm}$ for the whole range of beam energy uncertainties $\sigma_{p, p} / p$. The cali- 
6.3. Validation of the radiation length calibration in simulation studies

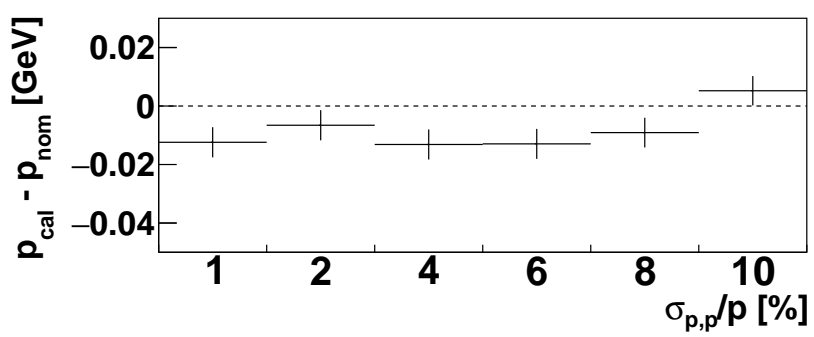

(a) $p_{\text {cal }}-p_{\text {nom }}$ as a function of $\sigma_{p, p} / p$

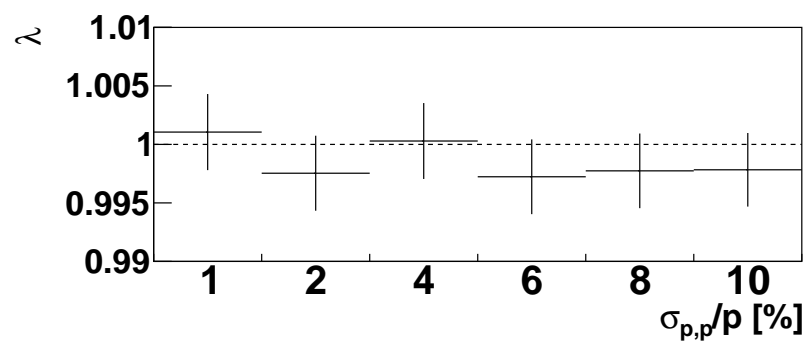

(b) $\lambda$ as a function of $\sigma_{p, p} / p$

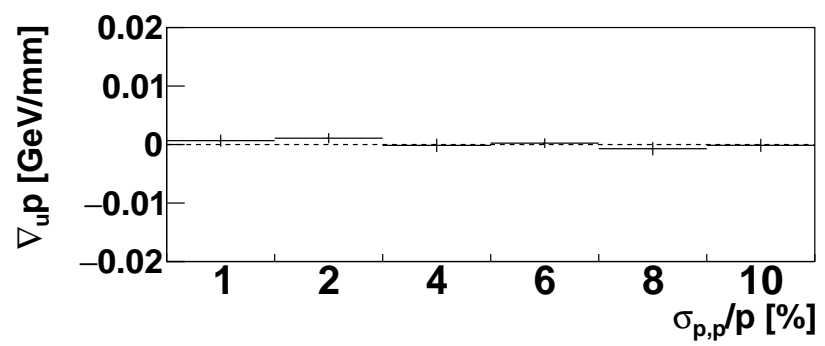

(c) $\Delta_{u} p$ as a function of $\sigma_{p, p} / p$

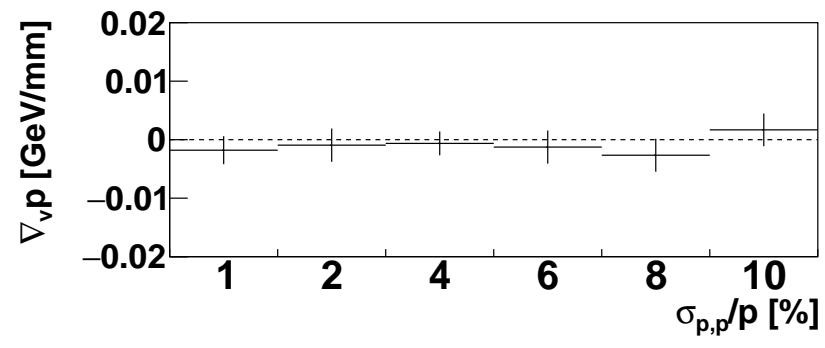

(d) $\Delta_{v} p$ as a function of $\sigma_{p, p} / p$

Figure 6.17.: Radiation length calibration parameters as a function of $\sigma_{p, p} / p$. All parameters except $p_{\text {cal }}-p_{\text {nom }}$ are compatible with their expected values (indicated by the dashed black lines). The calibrated beam energy shows a deviation of approximately $-10 \mathrm{MeV}$ from the expected value. 


\section{Systematic influences and simulation studies}

brated beam energy shows a deviation of approximately $p_{\text {cal }}-p_{\text {nom }}=-10 \mathrm{MeV}$ for all beam energy uncertainties below $10 \%$. This observed deviation is larger than the statistical error of the individual measurements, which corresponds to approximately $5 \mathrm{MeV}$. The determined beam energy for a beam energy uncertainty of $\sigma_{p, p} / p=10 \%$ is close to $0 \mathrm{MeV}$. This single measurement is most likely an outlier caused by statistical fluctuation. This outlier is of no concern here as the energy uncertainty of the electron beam at the DESY beam test facilities is expected to be smaller then $10 \%$.

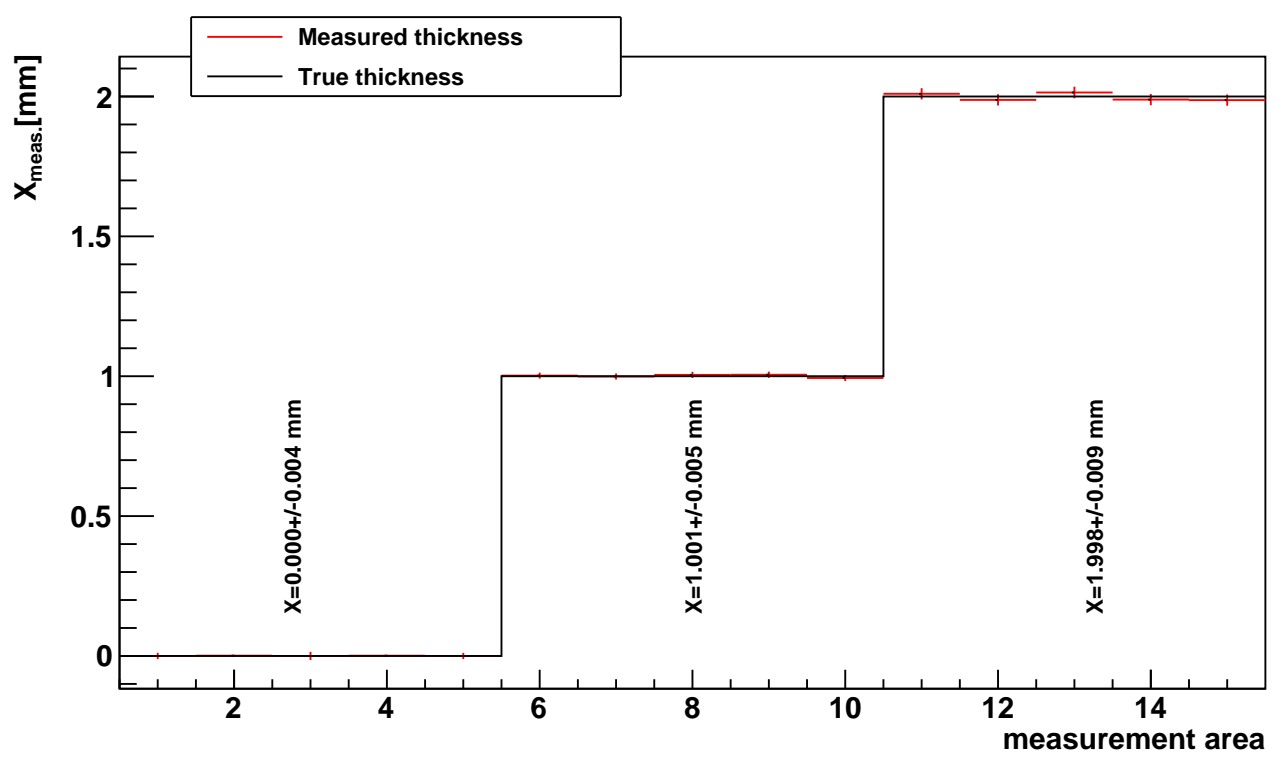

Figure 6.18.: Self consistency test measurement for a beam test simulation with an beam energy uncertainty of $6 \%$. The measured thickness values are in agreement with the expected aluminium and air thicknesses.

In order to determine whether the observed deviation is caused by a bias in the loglikelihood estimator like in section 6.1 and whether the measured deviation will correct for a corresponding bias in the subsequent radiation length measurements, a thickness measurement called the self-consistency test of the radiation length calibration is conducted. During the self-consistency test, the angle distributions used in the radiation length calibration are fitted to determine the thickness of the calibration material. The previously determined calibration parameters $\lambda, \kappa\left(p_{\text {cal }}\right), \Delta_{u} p$ and $\Delta_{u} p$ are used in this fit. If the calibration measurement is self-consistent, the determined thickness values are identical to the values assumed during the calibration measurement. Fig. 6.18 depicts the results of the self-consistency test for a beam energy uncertainty of $6 \%$. The corresponding measurements for the other five beam energy uncertainties can be found in appendix C.1. 
When using the determined calibration parameters, all thickness measurements are compatible with the original thickness assumptions ${ }^{3}$. Accordingly, the offset in the determined beam energy leads to an unbiased thickness measurement within the limits of the statistical uncertainty and the radiation length calibration procedure fulfils its intended purpose. In conclusion, the expected beam energy uncertainties $\sigma_{p, p} / p$ at the DESY beam test facilities of $6 \%$ or less have no observable impact on the radiation length calibration procedure.

The second part of this section deals with the capability of the radiation length calibration to identify and correct for wrong assumptions of the beam energy. A particle beam with an energy ranging from 92.5 to $107.5 \%$ of the nominal beam energy of $2 \mathrm{GeV}$ is simulated. The step size of the beam energy offset is $2.5 \%$. The beam energy uncertainty is selected to be $5 \%$. For each beam energy, the scattering angle reconstruction on all 3 different targets is conducted using the true beam energy. By using the true beam energy in the angle reconstruction the correct angle resolution is determined in the angle reconstruction step. Accordingly, the $\lambda$ values are expected to be distributed around 1.0 as before. Afterwards a radiation length calibration and self-consistency test are conducted. The fit function (see eq. 5.13) assumes a beam energy corresponding to the nominal energy of $p_{\text {nom }}=2.0 \mathrm{GeV}$. The measured calibration parameters as a function of the simulated beam energy $p_{\text {true }}$ are depicted in fig. 6.19.

The determined beam energy is very close to the true beam energy used in the simulation. However, similar to the previous study, the beam energy has an offset of roughly $-10 \mathrm{MeV}$ regardless of the simulated beam energy. All the other calibration parameters are consistent with the expected values within the range of their statistical uncertainties. As shown in the self-consistency diagrams (fig. 6.20) the calibration measurements lead to an unbiased thickness measurement. Fig. 6.20 depicts the thickness measurements as self-consistency test in the case of a simulation with a beam energy of $2150 \mathrm{MeV}$. The corresponding plots for the six other simulated beam energies can be found in appendix C.2.

The results in this section show that the calibration of the beam energy is reliable and self-consistent. If the nominal beam energy deviates from the real beam energy, the radiation length calibration measurement will measure calibration parameters which cause the subsequent radiation measurements to be bias free. The radiation length calibration and $X / X_{0}$ measurement are therefore confirmed to ensure unbiased results, when the angle histograms used during the calibration and the radiation length measurement have a similar number of entries. In order to minimise the statistical error of the calibration factors, the radiation length calibration measurements are typically conducted on angle histograms with at least 50000 entries. On the other hand radiation length imaging is typically conducted using angle histograms with approximately 5000 entries. However, as can be seen from fig. 6.4 the bias in radiation length measurements is expected to be approximately independent of the number of entries per angle distribution for values

\footnotetext{
${ }^{3}$ Corresponding to the true thickness used in the simulation process.
} 
6. Systematic influences and simulation studies

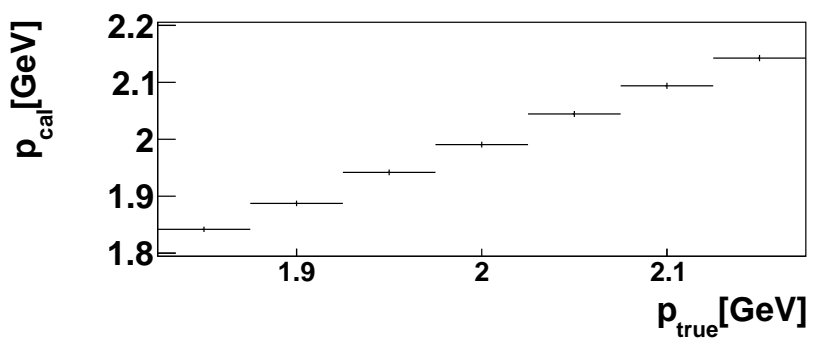

(a)

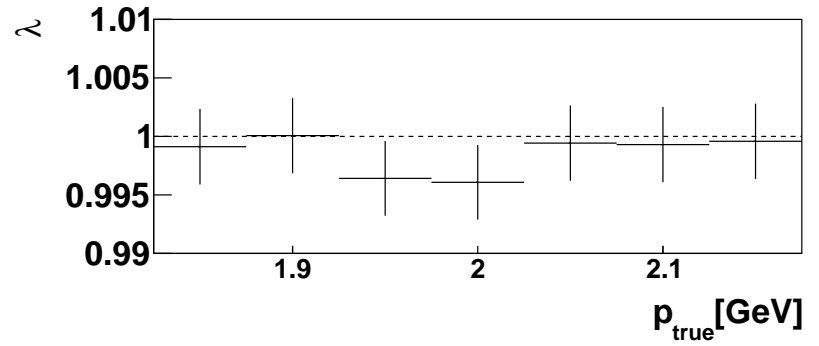

(b)

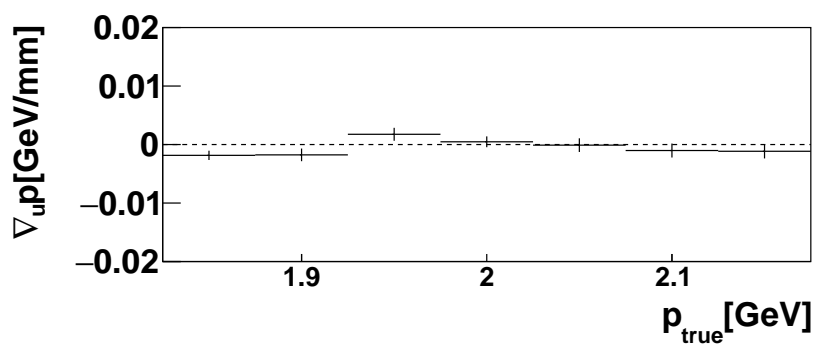

(c)

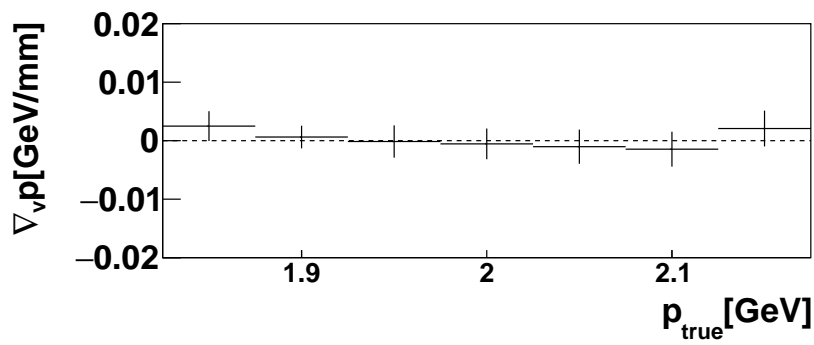

(d)

Figure 6.19.: The calibrated beam energy $p_{\text {cal }}$ (a), $\lambda(\mathrm{b}), \Delta_{u} p(\mathrm{c})$ and $\Delta_{v} p$ (d) as a function of the true beam energy $p_{\text {true. }}$. All parameters except the calibrated beam energy $p_{\text {cal }}$ are consistent with the expected values (indicated by the dashed black lines). A small deviation of $-10 \mathrm{MeV}$ from the expected values is observed for $p_{\text {cal }}$. 
6.3. Validation of the radiation length calibration in simulation studies

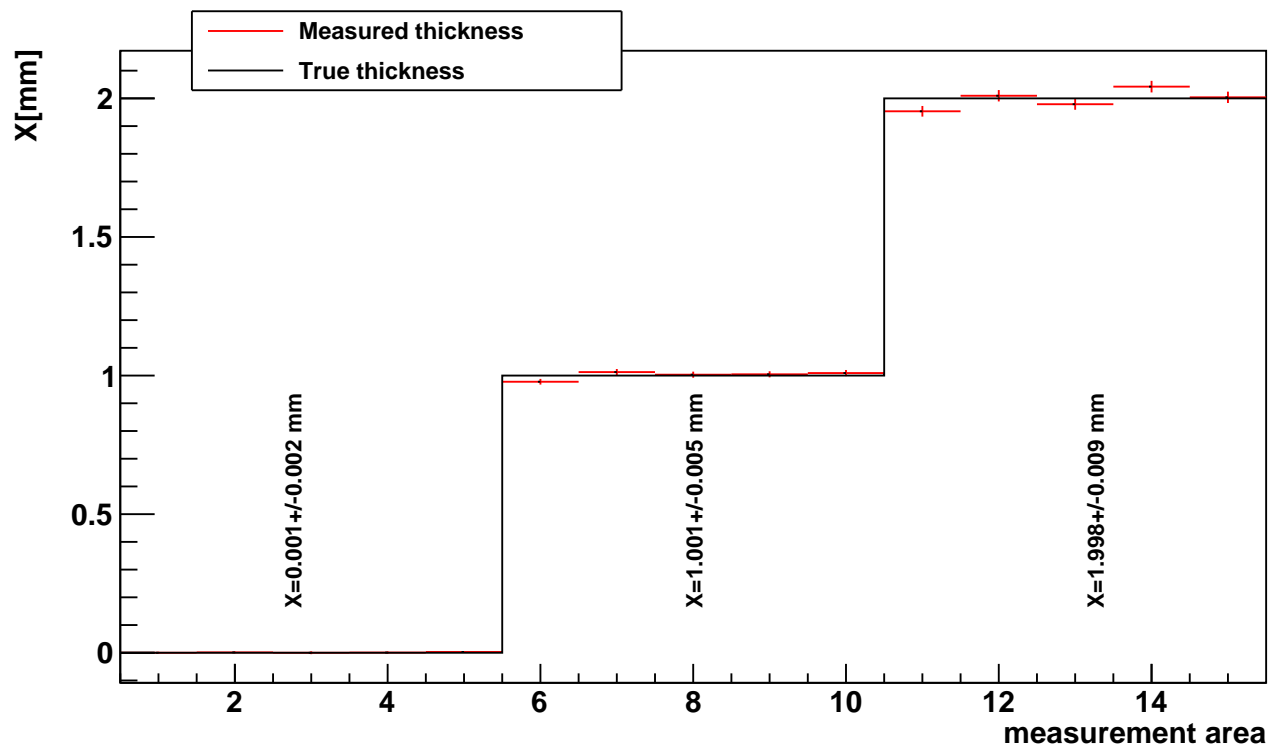

Figure 6.20.: Self consistency test plot for a beam test simulation with an beam energy of $2150 \mathrm{MeV}$. The measured thickness values are in agreement with the expected aluminium and air thicknesses.

larger than 5000 angles. Accordingly, using angle distributions with 50000 or more angles for radiation length calibrations does not have a negative impact on subsequent radiation length imaging measurements as long as the angle distributions of image pixels have approximately 5000 entries. 



\section{CHAPTER 7}

\section{Radiation length measurements at DESY}

After summarising systematic challenges and demonstrating the feasibility of radiation length measurements (see chapter 6), the next step is to analyse actual beam test data. The scope of this chapter is to give an overview of radiation length measurements conducted at several beam test campaigns. The analysis of beam test data can be separated into four analysis steps:

1. Telescope calibration: This step includes hot pixel masking, telescope alignment and the calibration of cluster resolutions as explained in section 4.3.

2. Scattering angle reconstruction: This step includes upstream-downstream track matching and the calculation of scattering angles and the angle resolution on the central telescope plane as explained in section 5.1 and 5.2.

3. $X / X_{0}$ calibration measurement: By using calibration targets with known thickness profiles the calibration factors $\lambda$ and $\kappa$ are determined as explained in section 5.4. Typically the beam energy selection during beam tests leads to the presence a beam energy gradient along the long side of the M26 sensors ( $u$ or column direction). This beam energy gradient must also be determined within the frame of the radiation length calibration. The beam gradient in $v$ direction is determined as well, but is expected to be compatible with zero.

4. $X / X_{0}$ imaging: The last step is to perform spatially resolved measurements of radiation length distributions on the scattering target as explained in section 5.3.

Fig. 7.1 depicts a flow chart, which describes radiation length analysis steps together with necessary input and output data as well as the corresponding sets of DQM diagrams. The DQM diagrams listed in the flow chart are useful tools to determine the quality of analysis results after each of the four analysis steps. In the next section which presents 


\section{Radiation length measurements at DESY}

the results from a beam test conducted in June of 2017 the DQM diagrams are explained in detail. The underlying analysis steps are explained in the chapters 4 and 5. DQM diagrams of other beam tests are very similar and in order to avoid redundancy the diagrams for all other beam test campaigns will be summarised in appendix D. All measurements presented in this chapter were analysed with $T B S W$ release version 0.7.1 [63].

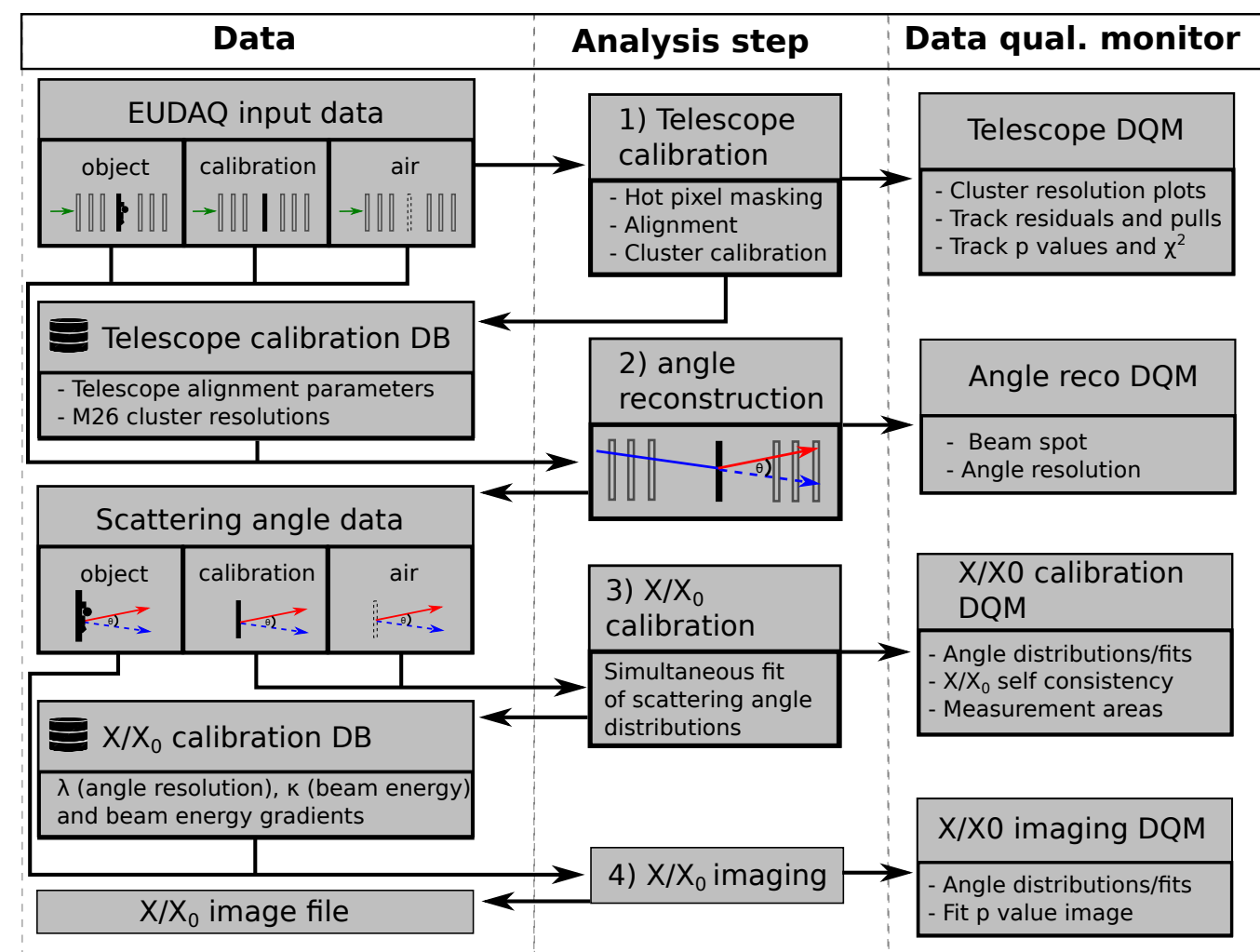

Figure 7.1.: Flow chart depicting the main radiation length analysis steps.

\subsection{Aluminium wedge measurements in June 2017}

The results summarised in this section are based on a beam test data set taken in June of 2017 at the DESY beam test facilities. The measurements took place at beam line 21 with the DATURA telescope [54]. Data taking at DESY was performed by ATLAS group members [77], while the analysis of the recorded data was conducted by me. The overall goal of the measurements was to test the validity of radiation length measurements for very high $X / X_{0}$ materials in the range between 10 and $30 \% X / X_{0}$. Electron beams traversing material with such large radiation length $X / X_{0}$ values are affected by bremsstrahlung. Therefore energy losses due to bremsstrahlung in the target must be considered. The detailed theoretical description of bremsstrahlung processes 
and their effects on the energy spectrum of a particle beam are explained in section 3.2. In order to study the influence of bremsstrahlung systematically an aluminium wedge was used as a scattering target. The wedge had a length of approximately $70 \mathrm{~mm}$ and its thickness ranged from approximately $5 \mathrm{~mm}$ to $30 \mathrm{~mm}$. As the whole length of the aluminium wedge did not fit into the acceptance area of the telescope, approximately corresponding to the size of a M26 sensor $\left(\approx 10 \times 20 \mathrm{~mm}^{2}\right)$, eleven positions along the long side of the wedge had to be measured. The distance between two neighbouring measurement positions was $7 \mathrm{~mm}$ to ensure sufficient overlap between measurements.

\subsubsection{Calibration and Reconstruction of the June 2017 beam test data}

The goal of this section is to present results of each individual analysis step for a successful beam test analysis. Using the example of the beam test in June 2017 all relevant DQM diagrams are explained.

The telescope setup of this beam test campaign is depicted in fig. 7.2. The nominal beam energy was set to $3 \mathrm{GeV}$ and the M26 threshold was selected as four times the M26 sensor noise. Table 7.1 gives an overview of the total number of runs, events and scattering angles that were recorded during the beam test. Rows of the table specify the scattering target materials and the purpose of the corresponding measurements. The $X / X_{0}$ calibration data set consists of scattering angles from 8 different calibration targets ranging from air to $6 \mathrm{~mm}$ of aluminium. For each scattering target, two runs with 2 million events and between 18 and 30 million scattering angles are used for radiation length calibration measurements. The number of angles per event for different scattering materials varies due to fluctuations in the beam intensity. At each of the eleven measurement positions along the length of the aluminium wedge four runs with four million events and approximately 40 million scattering angles were recorded. The EUDAQ raw data of these 60 runs is the input data in the top left corner of the flowchart (fig. 7.1).

\begin{tabular}{|c||c|c|c|c|}
\hline Target & \#runs & \#events $\left[10^{6}\right]$ & \#angles $\left[10^{6}\right]$ & purpose \\
\hline air & 2 & 2 & 29 & telescope $\& X / X_{0}$ calibration \\
\hline $0.5 \mathrm{~mm}$ alu & 2 & 2 & 29 & $X / X_{0}$ calibration \\
\hline $1.0 \mathrm{~mm}$ alu & 2 & 2 & 26.8 & $X / X_{0}$ calibration \\
\hline $1.5 \mathrm{~mm}$ alu & 2 & 2 & 17.7 & $X / X_{0}$ calibration \\
\hline $2.0 \mathrm{~mm}$ alu & 2 & 2 & 23.2 & $X / X_{0}$ calibration \\
\hline $3.0 \mathrm{~mm}$ alu & 2 & 2 & 23.8 & $X / X_{0}$ calibration \\
\hline $4.0 \mathrm{~mm}$ alu & 2 & 2 & 20.6 & $X / X_{0}$ calibration \\
\hline $6.0 \mathrm{~mm}$ alu & 2 & 2 & 22.7 & $X / X_{0}$ measurement $(11$ positions $)$ \\
\hline alu wedge & 44 & 44 & 955 &
\end{tabular}

Table 7.1.: Overview of the telescope data from the beam test in June 2017. The spacings between M26 sensors (see fig. 7.2), beam energy and M26 comparator threshold were not changed between measurements. 


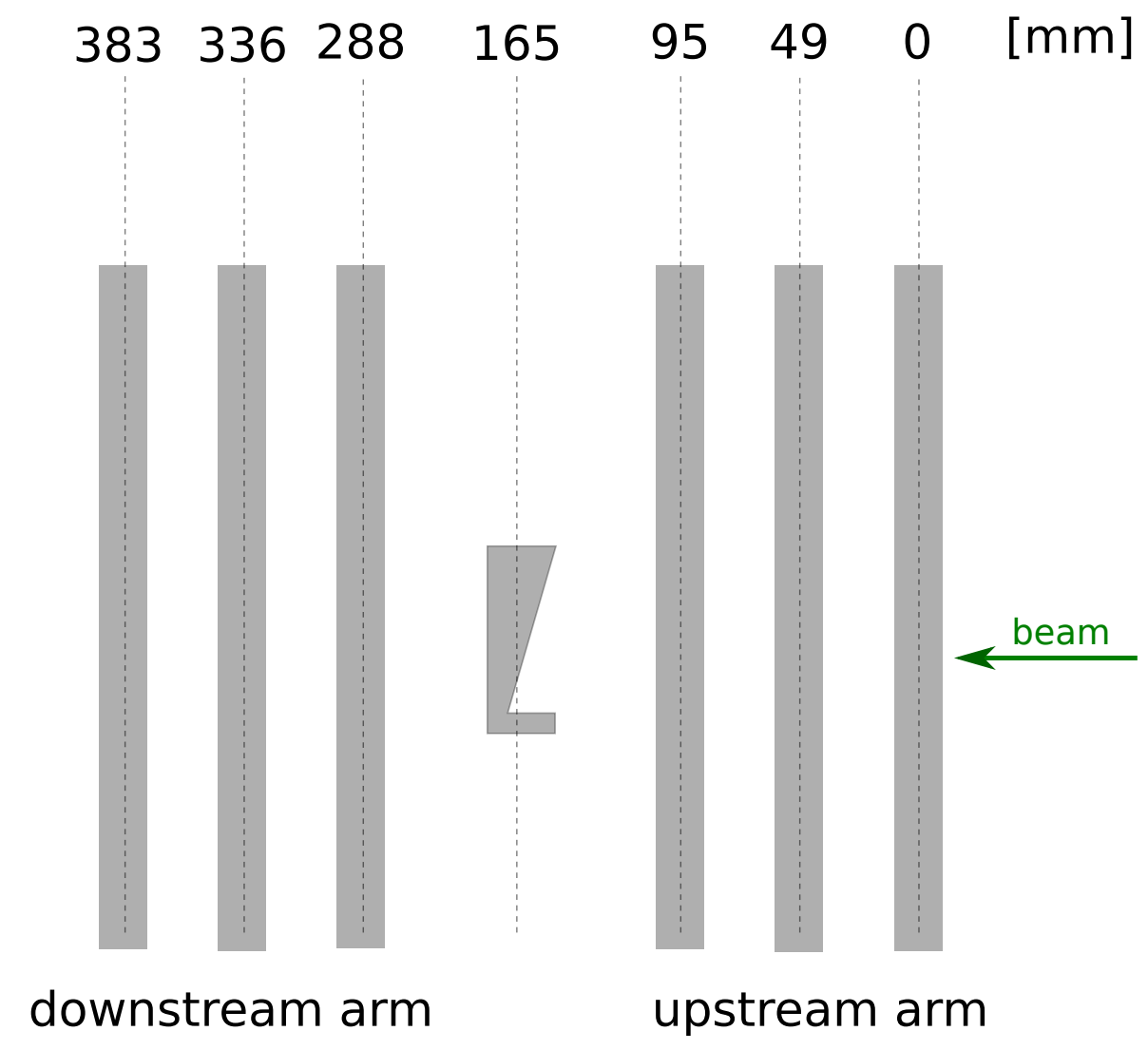

Figure 7.2.: Schematic side view of the telescope setup during the June 2017 beam test campaign. The aluminium wedge used as a scattering target is depicted as well.

\section{DQM diagrams of the telescope calibration step}

The first step of the analysis is a telescope calibration measurement, which includes hot pixel masking, telescope alignment and measurements of the M26 cluster resolution. This step is marked as 1) telescope calibration in the analysis flow chart (fig. 7.1) and is described in section 4.3. For the telescope calibration 50 thousand events with approximately 600000 tracks with a hit on each telescope plane are used. An air run was used for the telescope calibration measurement. During an air run all material between the telescope planes is moved out of the particle beam. This ensures that the track resolution is not increased by additional multiple scattering on the target plane. Using such an air run as input data for the telescope calibration is highly recommended, but using a thin homogeneous material works as well. Wrong assumptions about the target material thickness and consequently the amount of scattering in the telescope should be 
avoided. They cause wrongly estimated track covariance matrices, which inevitably lead to errors in the alignment and cluster calibration steps.

The functionality of the track based cluster calibration is explained in detail in section 4.3.10. The results from an in-depth study of the influence of the M26 threshold on the cluster resolution can be found in section 4.4. The results in that section indicated that the optimal threshold setting ${ }^{1}$ was found to be three times the noise level. During the July 2017 beam test campaign a threshold of four was selected. It should be noted that the threshold scan in section 4.4 was performed on the DURANTA telescope, while the measurements here were conducted with the DATURA telescope. Accordingly, some differences can be expected. Fig. 7.3 depicts the frequency of the eight most common M26 cluster shapes.

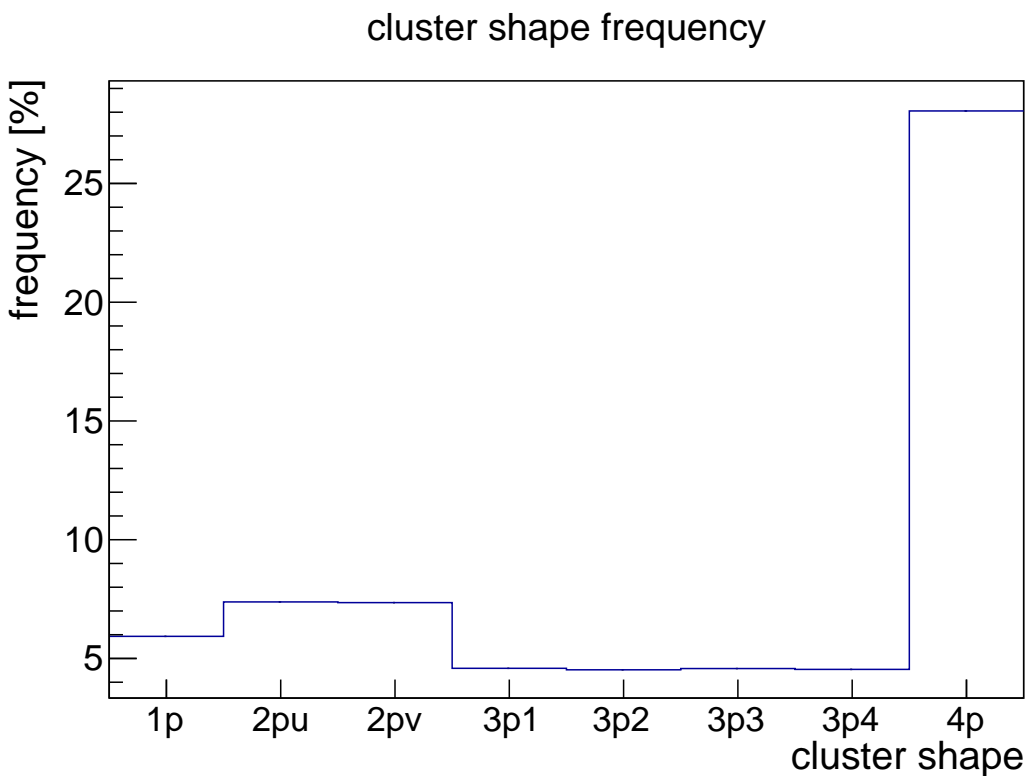

Figure 7.3.: Cluster shape frequencies during the $X / X_{0}$ measurements of the June 2017 beam test campaign. The cluster shapes, which correspond to the individual labels on the $x$ axis, are depicted in fig. 4.14 .

The observed cluster shape frequencies are quite unusual, especially when compared to the measurement with a threshold value of four in section 4.4. The optimal threshold setting which provides the optimal cluster resolution is typically indicated by having a large and approximately equal number of one- and two-digit-clusters. Here, the most common cluster shape is the most compact quadratic four-digit-cluster with a probability of approximately $28 \%$. One-digit-clusters have a frequency of approximately $6 \%$, while two-digit-clusters, which share a common pixel edge have a frequency of $7 \%$. This

\footnotetext{
${ }^{1}$ This corresponds to the threshold setting where the average cluster resolution is smallest
} 


\section{Radiation length measurements at DESY}

indicates that the M26 threshold, even though it was comparatively high, could have been selected even higher to generate a better cluster shape distribution. The difference between the cluster shape distribution here and in section 4.4 may be caused by different electronic noise levels of the two different telescopes. As explained in section 4.3.10 different cluster shapes have different cluster resolutions. The cluster resolutions in $u$ and $v$ direction of the eight most common cluster shapes are listed in fig. 7.4.

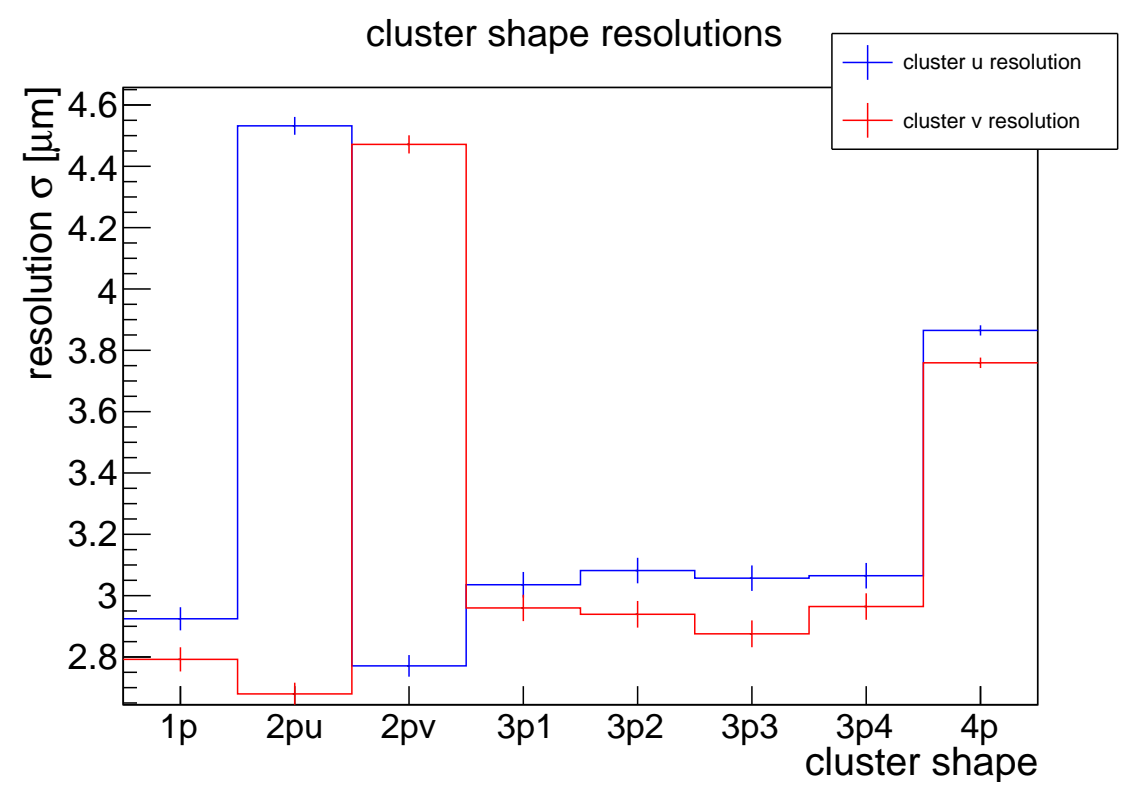

Figure 7.4.: Cluster $u$ and $v$ resolution during the $X / X_{0}$ measurements of the June 2017 beam test campaign. The cluster shapes, which correspond to the individual labels on the $x$ axis, are depicted in fig. 4.14.

One-digit-clusters have a spatial resolution of approximately 2.8 to $2.9 \mu \mathrm{m}$. This small value is expected as the one-digit-cluster frequency is very small and only a nearly perpendicular particle hit in a small region in the centre of a M26 pixel will lead to onedigit-clusters. The two- and four-digit-clusters have rather large cluster resolutions of $4.4 \mu \mathrm{m}$ and $3.8 \mu \mathrm{m}$, respectively. The four different three-digit-clusters have a resolution of approximately $3.0 \mu \mathrm{m}$. As can be seen in fig. 7.4 the cluster resolution in $u$ direction is slightly worse than the resolution in $v$ direction. This is most likely caused by a small incidence angle of the particle beam in the $v-w$ plane. Even though the distribution of cluster shapes in fig. 7.3 is rather uncommon, the determined cluster resolution values are reasonable, when compared to the expected resolution. The resolution based on the symmetric M26 pixel pitch $P$ of $18.4 \mu \mathrm{m}$ is expected be between $P / \sqrt{12}(5.3 \mu \mathrm{m})$ and $P / 2 \sqrt{12}(2.7 \mu \mathrm{m})$.

The telescope calibration also includes a Kalman filter alignment procedure, which is explained in section 4.3.8. The goal of the alignment is to determine the telescope sen- 
sor positions and rotations of the setup with an accuracy comparable to the statistic uncertainty of track states. In practice, this is done with a given set of high-resolution telescope tracks, by minimizing a global $\chi^{2}$ function which depends on the sensor alignment parameters (see eq. 4.24). In this work the free alignment parameters are the positions $x, y, z$ and the rotation along the beam axis $\gamma$ on all inner M26 sensors. For radiation length measurements the precise determination of the $z$ positions of the M26 planes is especially important as the sensor positions directly affect the scattering angles on the central measurement plane. The difference between the nominal sensor $z$ position and the aligned $z$ position is depicted in fig. 7.5.

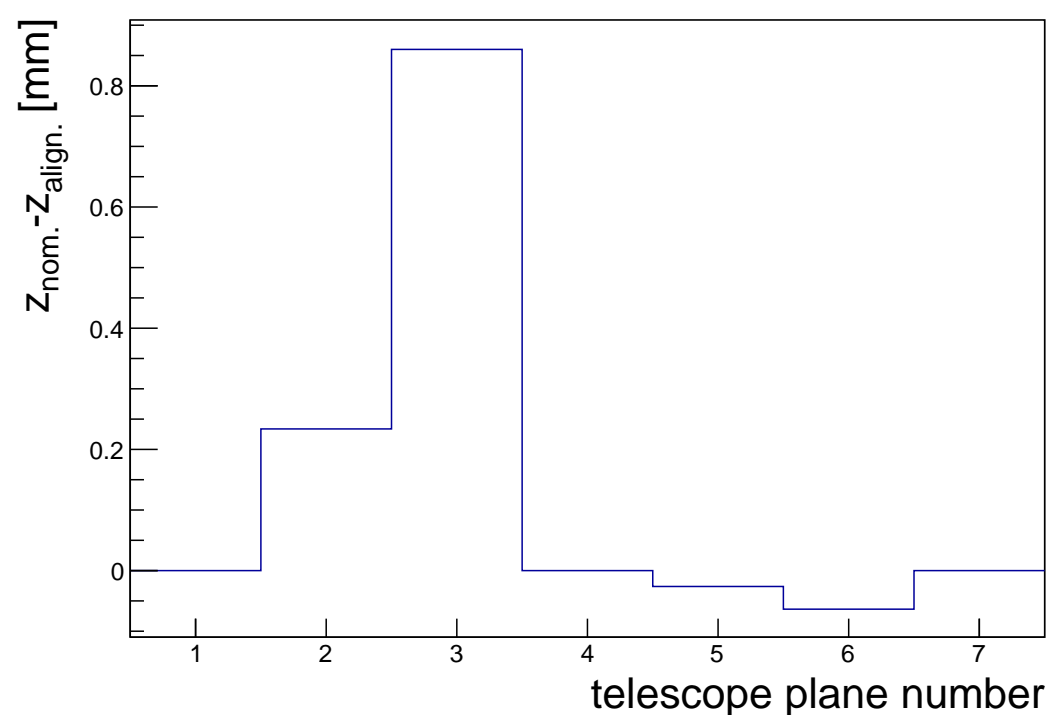

Figure 7.5.: Difference between nominal and aligned sensor $z$ positions for all telescope planes. During the alignment procedure the $z$ position of the first and last sensor as well as the scattering plane at sensor position four are fixed. The difference between nominal and the aligned $z$ positions is therefore $0 \mathrm{~mm}$ for these three planes.

The determined $z$ shifts seem randomly distributed and are quite small compared to the expected uncertainty of to the manual position measurements which is in the order of one millimetre. The corrections for all telescope planes are smaller than $1 \mathrm{~mm}$. All planes except plane 3 have corrections, that are below $200 \mu \mathrm{m}$. The manual measurement of the telescope spacings on site and the data-driven alignment are therefore in good agreement.

The remaining tests of the telescope calibration step are based on quality parameters of reconstructed tracks in the fully calibrated telescope. These tracks are reconstructed in the aligned telescope geometry from hits which have positions and resolutions according to the cluster $D B$. For the first test of the full telescope calibration, positions of measured hits on every telescope plane are compared to reconstructed track intersections on that plane. The corresponding pull distributions in $u$ and $v$ direction are displayed in fig. 7.6. 

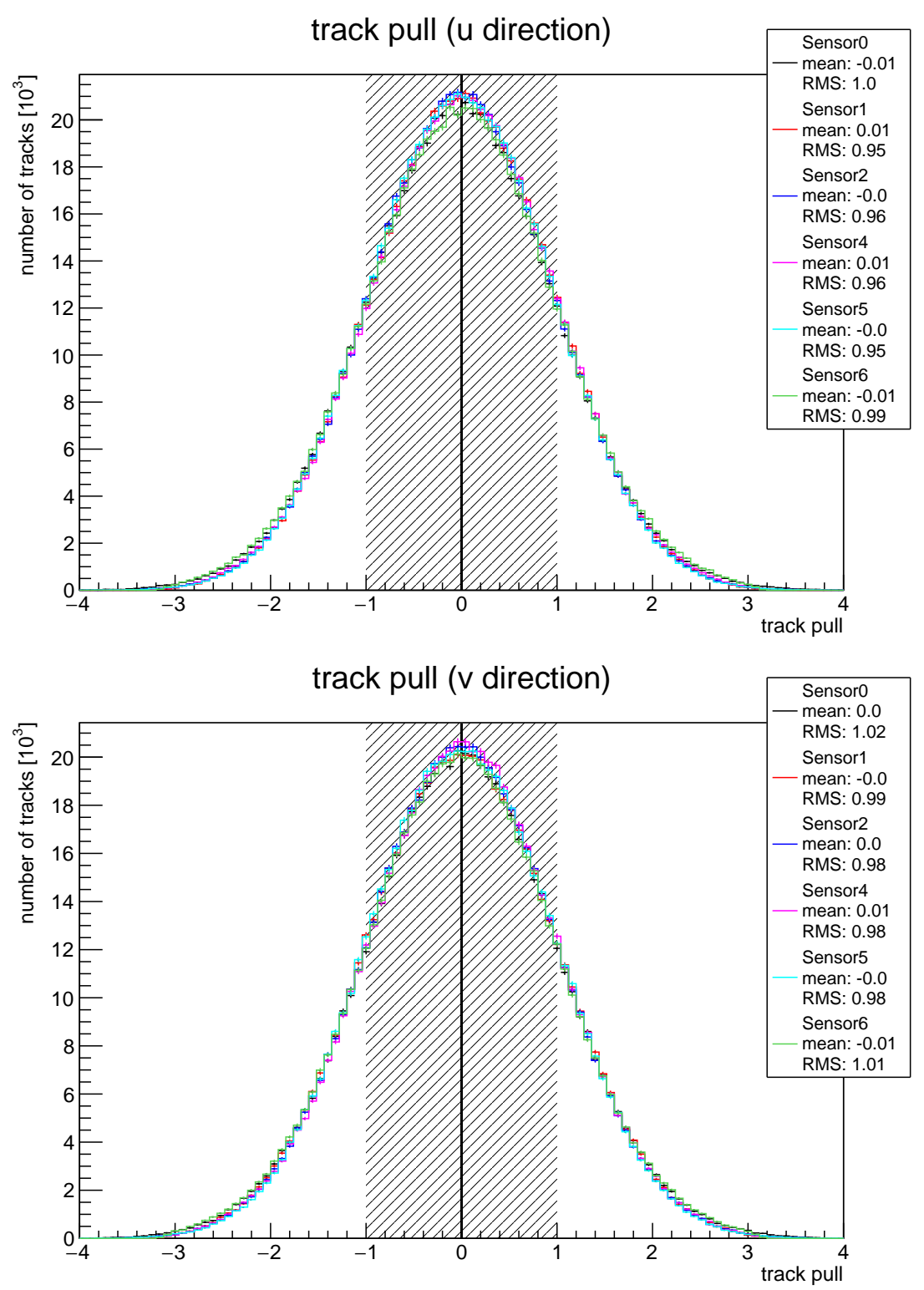

Figure 7.6.: Pulls of the $u$ and $v$ track residuals after cluster calibration and telescope alignment. The pulls are centred around zero and have RMS values close to one as is expected for a track sample in a well calibrated telescope.

There are no systematic shifts or distortions visible in the pull distributions. The mean values are compatible with zero and the RMS values are close to one. This indicates that the telescope calibration worked well. Another indicator of the tracking performance is the $p$ value distribution of the track sample, which is depicted in fig. 7.7. The track $p$ 
values can be calculated from the track $\chi^{2}$ values as explained in section 4.3.6. The $p$ value distribution of the track sample is reasonably flat as is expected.

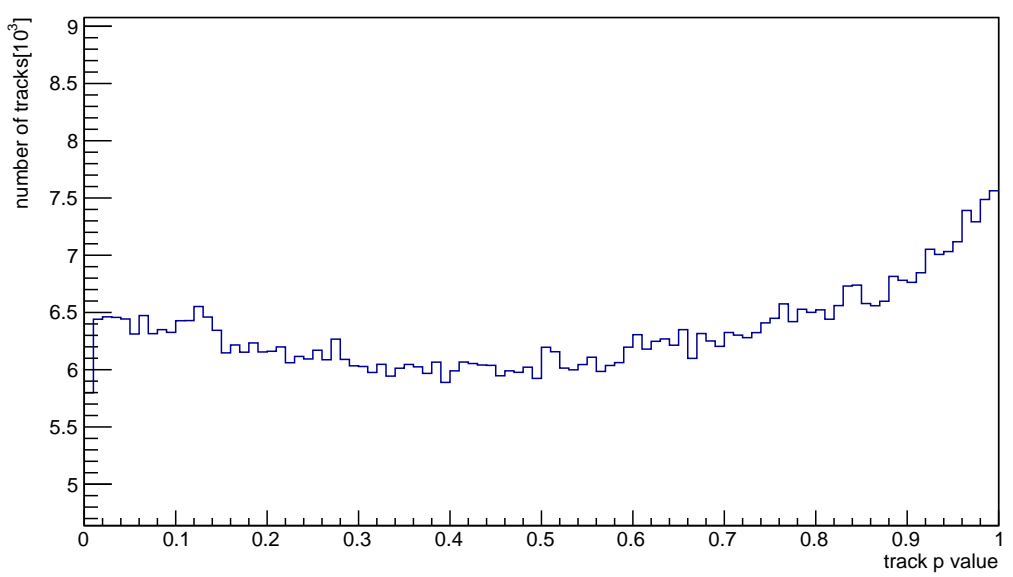

Figure 7.7.: $p$ values of the air run track sample after telescope alignment and cluster calibration. The distribution is reasonably flat which indicates that the telescope calibration and the subsequent tracking worked.

The number of tracks per event in a fully calibrated telescope is depicted in fig. 7.8. This quantity depends on the energy and collimator setup of the particle beam and gives an estimate of how many particles traverse the telescope in the acceptance region of the M26 sensors. In this case the average value is around 13 tracks per event, while the most probable value lies at 15 tracks per event. These are rather large values. A large number of tracks per event is beneficial to a time efficient radiation length measurement. However, in such a busy tracking environment the combinatoric track finder, explained in section 4.3.5, is rather slow. In order to reduce the large processing time a single track seeding approach instead of the default two hit seeding approach was used for the upand downstream track matching and angle reconstruction.

All of these control parameters show that the telescope alignment and the cluster calibration have worked well. There is no indication of any systematic effects compromising the subsequent analysis steps, especially the radiation length measurements.

\section{DQM diagrams of the angle reconstruction step}

The second step of the $X / X_{0}$ analysis is the reconstruction of multiple scattering angles. As can be seen in fig. 7.1 the angle reconstruction has to be performed for all runs that are used in the radiation length calibration and subsequent radiation length imaging processes. Tracks in the up- and downstream telescope arm are matched according to a distance criterium and scattering angles can be calculated from the matched track states 


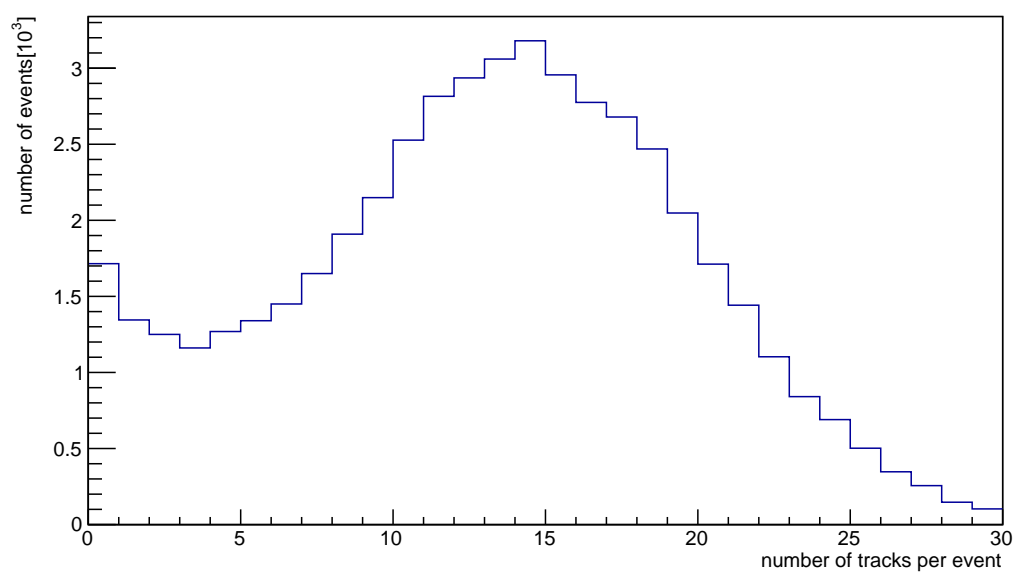

Figure 7.8.: Number of tracks per event during the beam test campaign in June of 2017. The average number of tracks per event is roughly 13 which is quite large and indicates a fast data collection.

as described in section 5.1. The distribution of the scattering angle reconstruction errors $\sigma_{\vartheta_{u}}$ and $\sigma_{\vartheta_{v}}$ is used to evaluate the telescope angle resolution $\sigma_{\text {err }}$. As can be seen from eq. 5.12 the telescope angle resolution is determined from the square root of the mean value of $\sigma_{\vartheta_{u}}^{2}$. Angle reconstruction errors are determined from the covariances of the track states via error propagation (details of the calculation can be found in section 5.2). Angle reconstruction error distributions for an example run are depicted in fig. 7.9.

For this beam test the angle reconstruction error distributions in both directions are very similar. However, the slightly better cluster $v$ resolution, which is visible in fig. 7.4 also leads to slightly smaller values for the angle reconstruction error in the $v$ direction. The mean value of the $\vartheta_{v}$ reconstruction error distribution is approximately 1 prad smaller than the corresponding mean value in the $u$ direction. The difference between the two angle error distributions, however, is unproblematic, because it is small compared to the RMS of the angle error distributions. The RMS is mainly caused by the multitude of different cluster resolutions, which have been measured during the telescope calibration step (see fig 7.4). The different combinations of cluster resolutions lead to a broad distribution of track parameter covariances, which in turn propagate to the distributions depicted in fig. 7.9.

The telescope angle resolution $\sigma_{\text {err }}$ is defined as the square root of the mean value of the $\sigma_{\vartheta_{u}}^{2}$ distribution as defined in eq. 5.12. The standard deviation of the $\sigma_{\vartheta_{u}}$ distribution can be regarded as an uncertainty of the angle resolution. This uncertainty limits the accuracy of radiation length measurements of materials with small $X / X_{0}$ values, where the distribution of reconstructed scattering angles is dominated by the angle resolution. 


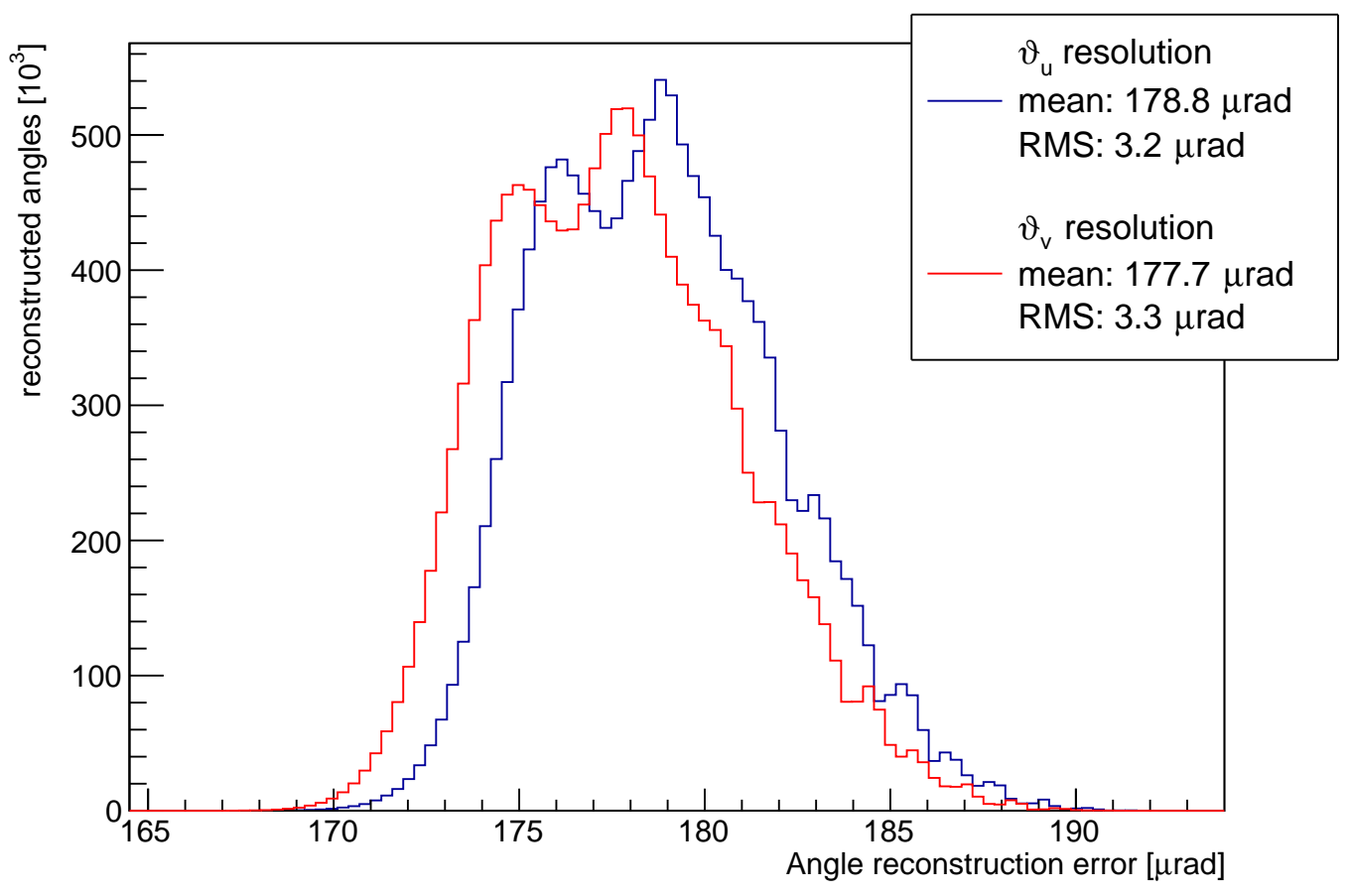

Figure 7.9.: Angle reconstruction errors in $u$ and $v$ direction. The mean value of the distribution is a measure for the telscope angle resolution. The shift between the distribution in $u$ and $v$ direction is caused by a slightly better $v$ resolution of the cluster shapes visible in fig. 7.4 which affects the track states and ultimately also the reconstructed angles.

For the specific experimental setup and the selected beam energy during the beam test campaign in June 2017 the expected telescope angle resolution is $(179 \pm 3)$ prad.

The track $u$ and $v$ intersection error distributions are depicted in fig. 7.10. In analogy to the angle reconstruction errors, these distributions define the spatial resolution of the telescope on the central target plane. Here, the spatial resolution in both directions is approximately $9 \mu \mathrm{m}$. This spatial resolution is sufficient for the radiation length imaging purposes here, as image pixels with side lengths of $200 \mu \mathrm{m}$ are used. Similar to the angle error distributions, the $v$ intersection error distribution has a slightly smaller mean value due to the slightly better cluster resolution in $v$ direction. The variance of the distribution can be explained similar to the distributions in fig. 7.9: Different tracks are reconstructed from various different combinations of cluster shapes with different resolutions. These different resolutions propagate to the track states, which in turn lead to a rather broad distribution of position reconstruction errors. 


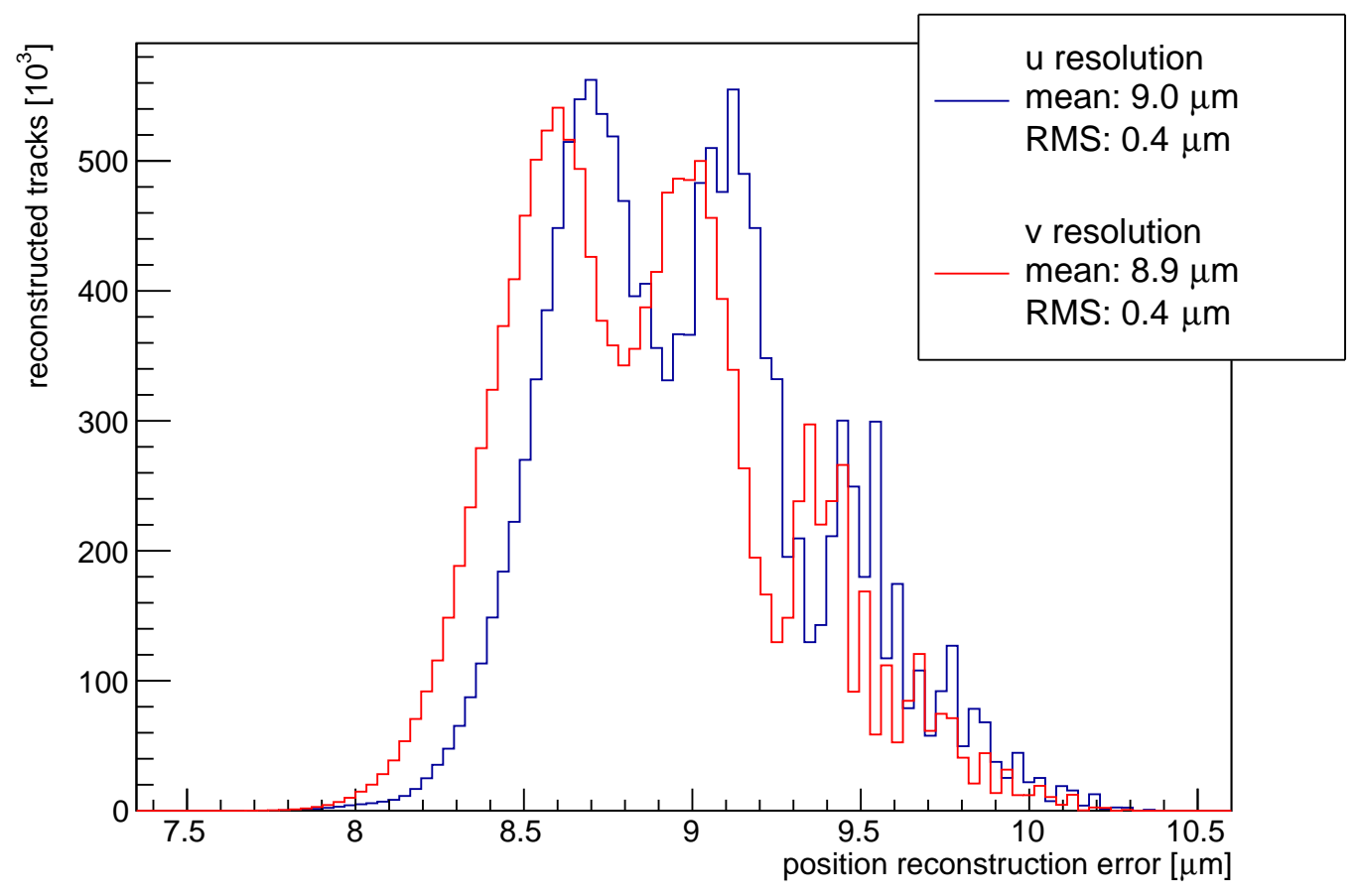

Figure 7.10.: Track intersection errors in $u$ and $v$ direction. The mean error is a measure for the telescope spatial resolution on the target plane. The shift between the distribution in $u$ and $v$ direction is caused by a slightly better $v$ resolution of clusters visible in fig. 7.4.

As explained before, radiation length measurement depend on measurements from upstream and downstream tracks each with three hits. The beam has a approximately perpendicular incidence and, accordingly, the area on the scattering target in which radiation length measurements are possible, called the acceptance region of the telescope, is effectively limited by the size of the telescope sensors. In the ideal case the acceptance area is therefore $10 \mathrm{x} 20 \mathrm{~mm}^{2}$. If the telescope planes have large relative shifts the area can be smaller. In order to get an idea of the telescope acceptance region and accordingly maximum size of radiation length images, the $2 \mathrm{D}$ track intersection distribution on the target plane, called the beam spot, is depicted in fig. 7.11. The acceptance region with sufficient statistics lies in the rectangular area between $u \in[-7,10]$ and $v \in[-4,5]$. The size of this area provides a benchmark for the maximum size of radiation length images and can be used later. As can be seen in fig. 7.11 the distribution of the intersection coordinates is not quite Gaussian as one would expect from a collimated particle beam. Instead some strict border lines and hard edges can be seen. This is caused by four segments on the M26 with individual threshold settings [61]. 


\section{5 million tracks}

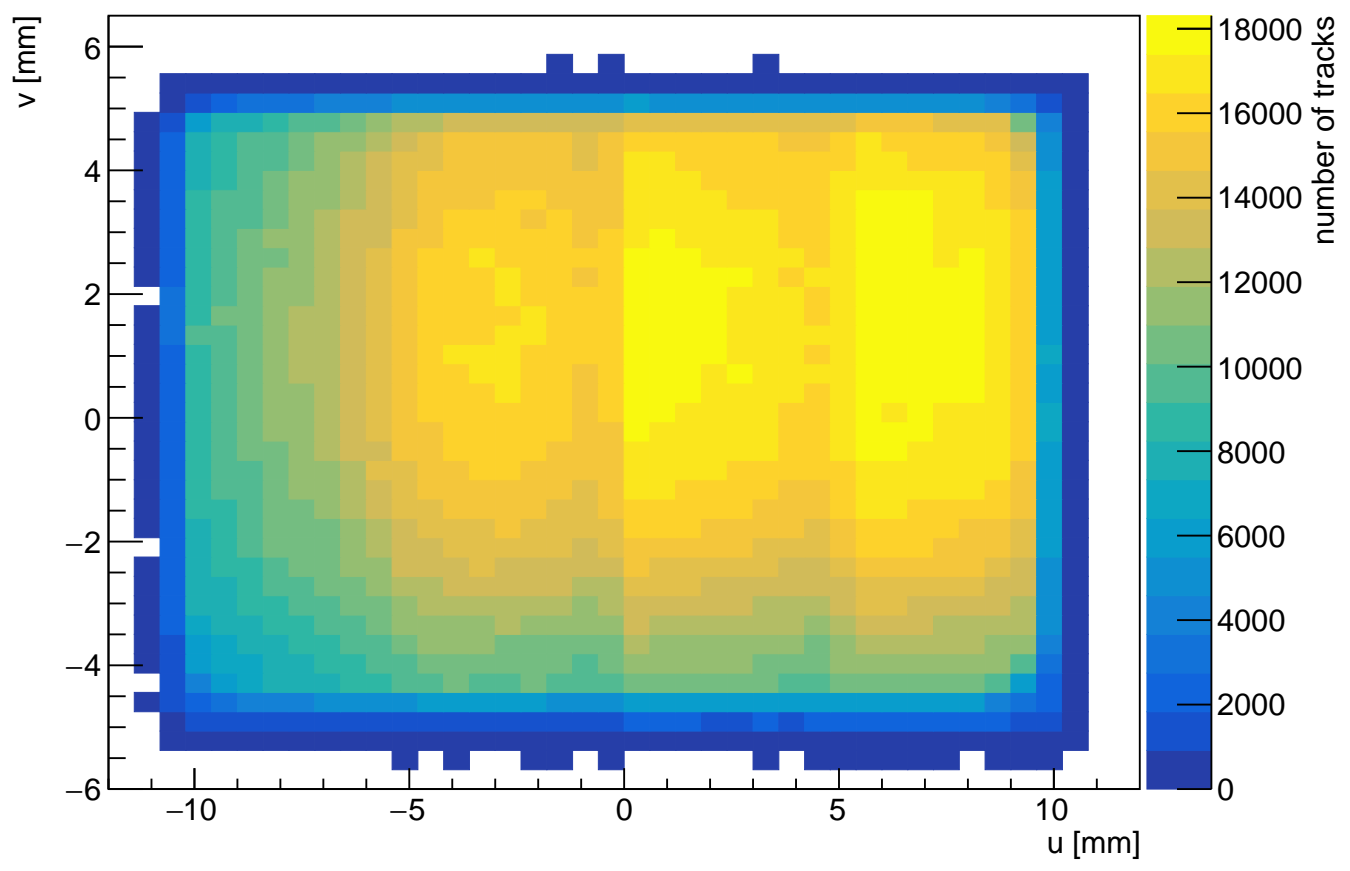

Figure 7.11.: Beam spot on the central scattering plane for an example run of the June 2017 beam test. Borders between vertical areas mark the positions of four segments on the M26 chip with individual threshold settings [61].

The DQM diagrams of the angle reconstruction provide an overview over parameters that are important in the subsequent $X / X_{0}$ calibration and imaging steps such as the expected telescope angle resolution and spatial resolution. The exact size of the telescope acceptance area is useful when selecting the size of the radiation length image.

\section{DQM diagrams of the $X / X_{0}$ calibration step}

The third step of the analysis is the calibration of the four radiation length calibration factors, $\lambda, \kappa, \Delta_{u} p$ and $\Delta_{v} p$, introduced in section 5.4. $\lambda$ is used to correct for wrong assumptions that lead to a global offset of the telescope resolution $\sigma_{\text {err }}$. $\kappa$ corrects for wrong assumptions that lead to a global offset of the multiple scattering angle width $\sigma_{\mathrm{HL}}$ (for example the Highland width in eq. 3.28). Additionally the selection of the beam energy during a beam test at DESY entails magnetic fields (see section 4.1). The particle beam is therefore expected to have linear energy gradients in $u$ and $v$ direction, which have to be measured. The implementation of these four calibration factors into the fit model is described in section 5.4. 


\section{Radiation length measurements at DESY}

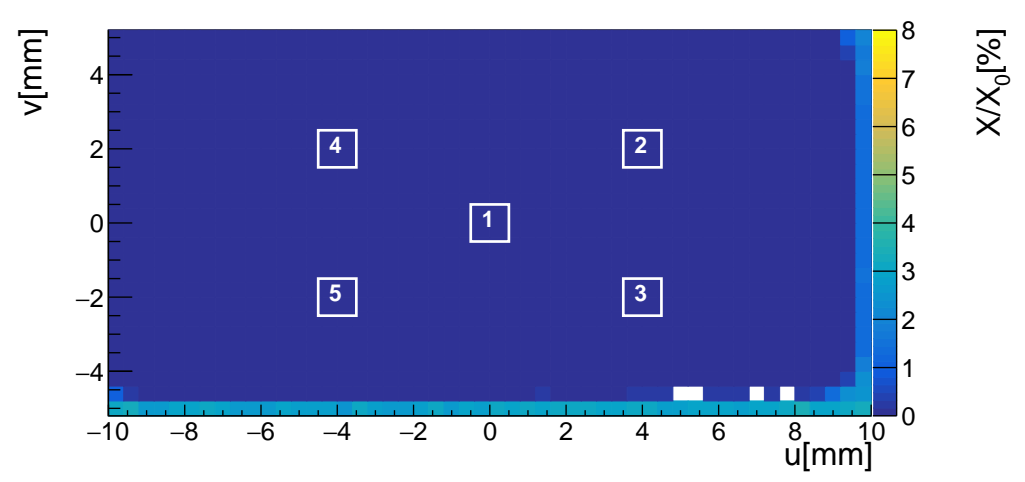

(a) Radiation length image of air calibration target

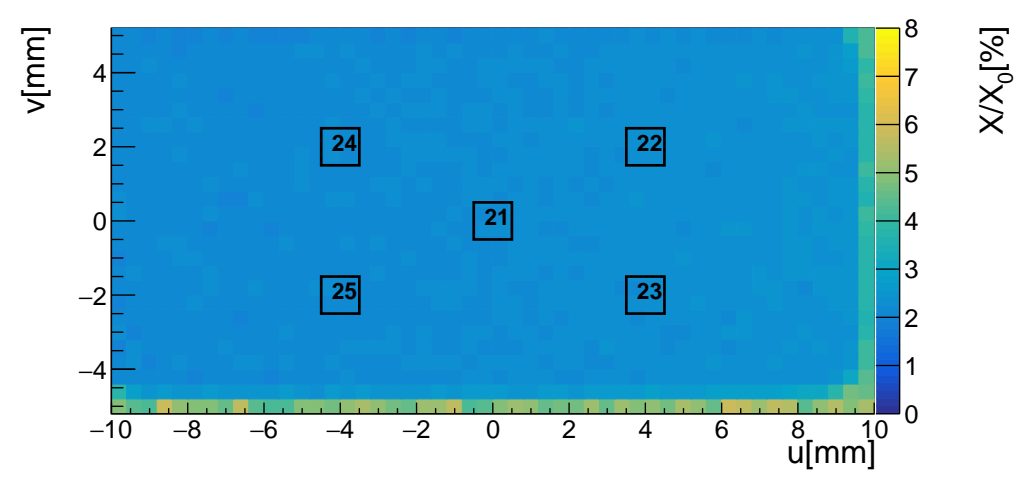

(b) Radiation length image of $2 \mathrm{~mm}$ aluminium calibration target

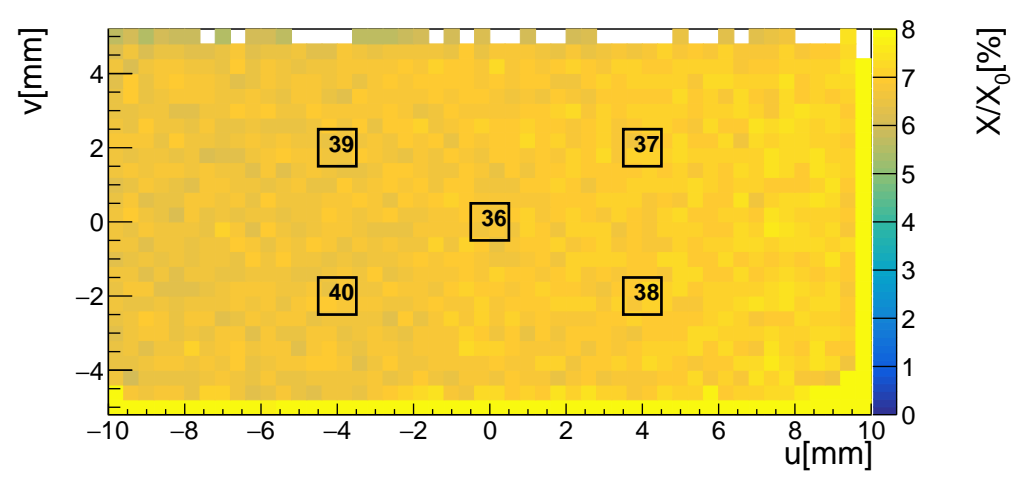

(c) Radiation length image of $6 \mathrm{~mm}$ aluminium calibration target

Figure 7.12.: Visual representation of the position of 15 out of 40 different measurement regions which are used for the radiation length calibration. Each marked measurement area contributes a single angle distribution with 50000 entries to the calibration fit. 
During the beam test in June of 2017 eight materials with different radiation length values were used for the radiation length calibration. They are summarised in table 7.1. The data set for the calibration measurement consisted of two runs per material. As demonstrated in section 6.3 five measurement areas per calibration material are sufficient for a reliable calibration measurement. In order to measure the beam energy gradients with the best achievable accuracy, four of the five measurement areas are placed near the corners of the telescope acceptance area. The five measurement areas for three example materials can be seen in fig. 7.12. The corresponding measurement areas for the other five materials are located at the same positions. The measurement areas are rectangles with side lengths of $1 \mathrm{~mm}$. The centre of the first measurement area lies at $(u, v)=(0 \mathrm{~mm}, 0 \mathrm{~mm})$. The centres of the other four regions are positioned at all possible combinations of $u= \pm 4 \mathrm{~mm}$ and $v= \pm 2 \mathrm{~mm}$. This leads to three positions along the $u$ and $v$ direction which are used to sample the beam energy gradients. The number of scattering angles per distribution was limited to a total of 50000 angles per measurement area. This ensures that every distribution used in the simultaneous fit will have the same weight and any potential bias introduced due to the beam profile is prevented. The 40 angle distributions ${ }^{2}$ are simultaneously fitted by a model function based on the Highland model of multiple scattering (see eq. 5.13).

The calibration fit was conducted with the fit model defined in eq. 5.13. Additionally, two different fit models which account for energy loss due to bremsstrahlung were tested as well, because they are used in the radiation length measurements of the aluminium wedge. The first approach to modelling the energy loss is based on the calculation of a modified average beam energy during the scattering according to eq. 3.36. This modified average energy is calculated from the expected average energy before the scattering, in this case $3 \mathrm{GeV}$, and the average energy after the transition through the scattering material given by eq. 3.36. As the energy after the transition depends on the radiation length, this model introduces a radiation length dependency in the modified beam energy. The beam energy in the Highland model is replaced by the modified beam energy and the corresponding modified multiple scattering width is given by eq. 3.37. The weight model uses a free parameter $\epsilon$ which determines the weight between the energy before and after the transition. Here, three different $\epsilon$ values of $0.2,0.25$ and 0.3 are tested. An $\epsilon$ value of 0.25 means that the modified beam energy corresponds to the sum of $75 \%$ of the energy before the material transition and $25 \%$ of the energy after the transition. Another fit model was introduced by Gottschalk [53]. Similar to the weight model the highland wdith $\sigma_{\mathrm{HL}}$ in the fit model (see eq. 5.13) is replaced by a modified multiple scattering width which is given by eq. 3.40. For our purposes only the two first terms of the exponential function in Gottschalks model are used.

In order to determine the calibration parameters a simultaneous ROOT log-likelihood fit is performed as described in section 5.4. The calibration fit results of the different models are summarised in table 7.2.

\footnotetext{
${ }^{2}$ Eight calibration materials with five measurement areas each
} 


\begin{tabular}{|c|c|c|c|c|}
\hline Calibration method & $\lambda$ & $\kappa$ & $\Delta_{u} p[\mathrm{MeV} / \mathrm{mm}]$ & $\Delta_{v} p[\mathrm{MeV} / \mathrm{mm}]$ \\
\hline No bremsstrahlung & $1.010 \pm 0.006$ & $0.996 \pm 0.003$ & $-11 \pm 2$ & $0 \pm 3$ \\
\hline Weight model $(\epsilon=0.20)$ & $1.012 \pm 0.006$ & $0.989 \pm 0.003$ & $-11 \pm 2$ & $0 \pm 4$ \\
\hline Weight model $(\epsilon=0.25)$ & $1.012 \pm 0.006$ & $0.988 \pm 0.003$ & $-11 \pm 2$ & $0 \pm 3$ \\
\hline Weight model $(\epsilon=0.3)$ & $1.013 \pm 0.006$ & $0.986 \pm 0.003$ & $-11 \pm 2$ & $0 \pm 4$ \\
\hline Gottschalk model & $1.014 \pm 0.006$ & $0.980 \pm 0.003$ & $-10 \pm 2$ & $0 \pm 3$ \\
\hline
\end{tabular}

Table 7.2.: Beam test June 2017 calibration results for different bremsstrahlung models. $\lambda$ is the calibration factor of the telescope resolution. $\kappa$ is the calibration factor of the scattering angle width and $\Delta_{u} p$ and $\Delta_{v} p$ correspond to beam energy gradients in $u$ and $v$ direction. The given errors correspond to the statistical uncertainties of the fit.

The different fitting models yield very similar results. $\lambda$ and the gradients, $\Delta_{u} p$ and $\Delta_{v} p$, are identical within the limits of the statistical uncertainty of the fit. Only the $\kappa$ values show small deviations. The calibration factor $\lambda$ is approximately $1 \%$ larger than one. As explained before, small deviations from 1.0 in the order of several percent are expected. According to the determined $\lambda$ factor the calibrated telescope angle resolution is approximately $181 \mathrm{\mu rad}$ instead of the expected telescope angle resolution ${ }^{3}$. There can be multiple causes for these small deviations from the expected telescope angle resolution:

- The track model assumes Highland distributed scattering angles for the particle propagation through the telescope. This approach neglects the non-Gaussian tails of the real scattering distributions, which in turn lead to an underestimation of the angle reconstruction errors.

- Small errors during the telescope alignment, especially the position along the beam axis, can also lead to an incorrect assessment of the telescope angle resolution.

The $\kappa$ factors are also close to 1.0. The observed deviation from 1.0 is smaller than $1 \%$ for all used models (Highland, Gottschalk and weight model with $\epsilon=0.2,0.25,0.3$ ). There are several possible explanations for observed deviation from the expected value:

- There may be small measurement errors of the telescope length. As mentioned in section 4.3.9 the $z$ position of the last telescope plane, or analogously the length of the telescope, cannot be determined with the Kalman filter alignment approach. Accordingly, the length is only measured manually and has uncertainties in the order of several $\mathrm{mm}$. A shorter than expected telescope will lead to a small overestimation of scattering angles, while a telescope which is longer than expected will lead to a small underestimation of the angles.

- The nominal beam energy may be slightly wrong. The selected beam energy is only known with an uncertainty of several percent.

\footnotetext{
${ }^{3} 179$ prad based on error propagation of covariance matrices of the track state
} 
- A part of the deviation is due to bremsstrahlung energy losses as can be seen from the small differences between the fitting models. Even though the $\kappa$ differences between the different models are larger than the statistical uncertainty of the fit, it is still quite small. This is not unexpected as the average beam energy loss due to bremsstrahlung, even for the thickest calibration material of $6 \mathrm{~mm}$ of aluminium, is a rather small effect:

$$
E_{\text {after }}=E_{0} \cdot \exp \left(-\frac{X}{X_{0}}\right)=0.935 E_{0}
$$

The observed beam energy gradient $\Delta_{u} p$ of approximately $11 \mathrm{MeV} / \mathrm{mm}$ is a typical value, which has been measured frequently at DESY beam lines. $\Delta_{v} p$ is compatible with 0 within the limits of the statistical uncertainty. The quality of the calibration fit can be cross-checked by visually comparing the angle distributions and the fit functions. Additionally, even though the fit method is based on the log-likelihood minimiser, ROOT provides a fit $\chi^{2}$ value that can be used to evaluate the quality of the fit. As an example, four scattering angle distributions and the corresponding Highland model fit functions are depicted in fig. 7.13. Angle distributions from four different materials, $2 \mathrm{~mm}, 4 \mathrm{~mm}$ and $6 \mathrm{~mm}$ aluminium as well as air, are displayed in fig 7.13. All 40 angle distributions and their fit functions can be found in appendix D.1 in a more compact form. The angle distributions and corresponding fit functions of the other fit models which include bremsstrahlung energy losses are very similar and therefore not presented here to avoid redundancy.

The individual fit functions in fig. 7.13 are in good agreement with corresponding angle distributions. The $\chi_{\text {ndof }}^{2}$ value of the simultaneous fit of all 40 angle distributions is 0.57 which is reasonably small. The last quality test of the radiation length calibration is called the self-consistency check. Fig. 7.14 depicts the measured thickness values of all 40 measurement areas in comparison to the expected thickness values. During the radiation length calibration, known thickness values of the 40 measurement areas were used to determine the calibration factors for this beam test setup. Now, employing the previously determined calibration factors, the thickness of each measurement area is determined and compared to the expected thickness.

As can be seen in fig. 7.14, the thickness measurements are generally more consistent when using the fit model based on the weight model to take energy losses due to bremsstrahlung into account. The measurements with this fit model are mostly compatible with the nominal thickness values within the limits of the statistical fit uncertainty. Only the measurements for 1.5 and $2 \mathrm{~mm}$ aluminium thickness show small deviations, which were found to be between 15 and $25 \mu \mathrm{m}$ which is slightly larger than the statistical uncertainties. The influence of bremsstrahlung is most pronounced for the $6 \mathrm{~mm}$ aluminium measurement: When the energy is loss not considered, the measured thickness is more than $100 \mu \mathrm{m}$ larger than expected. The Gottschalk model works for small radiation 


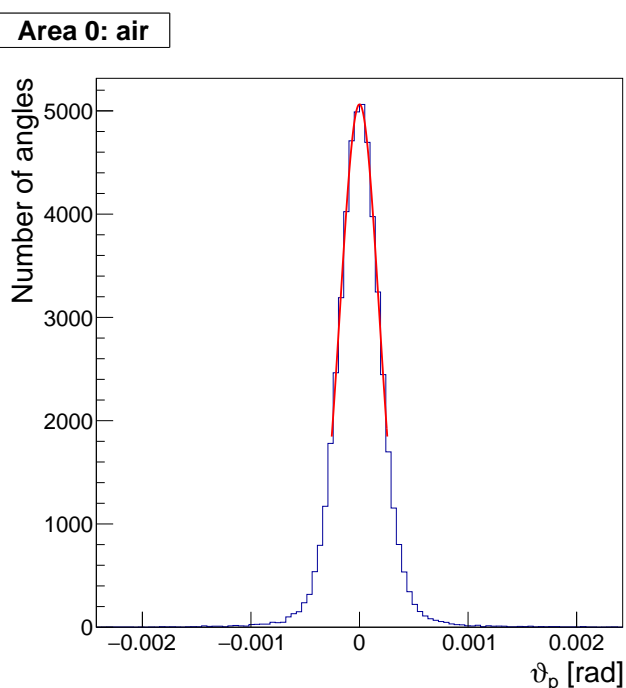

(a)

Area 30: $d=4.00 \mathrm{~mm}$ aluminium

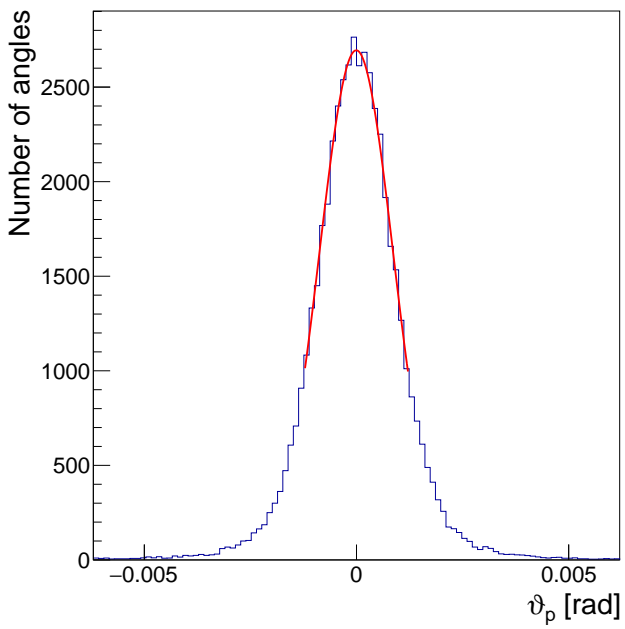

(c)
Area 20: $\mathrm{d}=2.00 \mathrm{~mm}$ aluminium

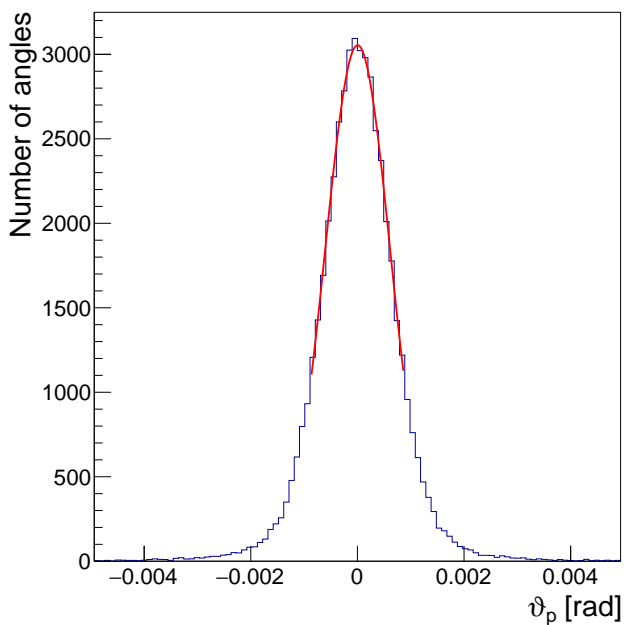

(b)

Area 35: $d=6.00 \mathrm{~mm}$ aluminium

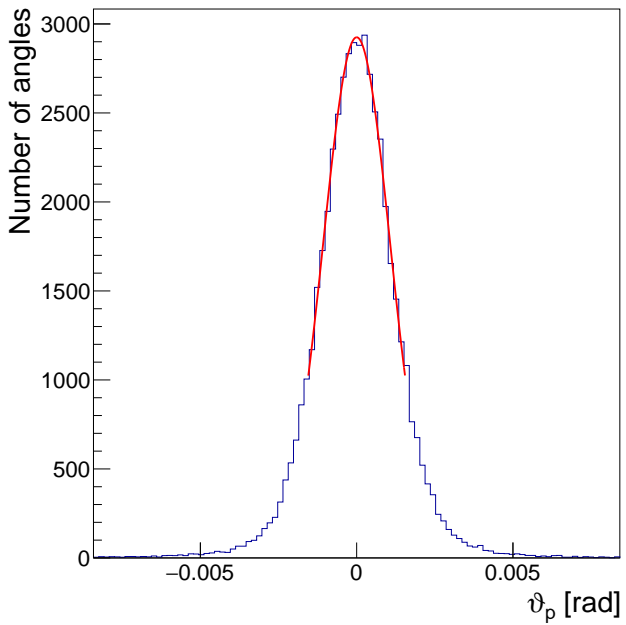

(d)

Figure 7.13.: Four of the 40 scattering angle distributions (reconstructed on materials with different thicknesses) used during the radiation length calibration together with their corresponding fit functions. The fit functions match the distributions quite well and the determined fit $\chi^{2}$ per number of degrees of freedom is 0.57 which is reasonably small.

length values quite well, but yields too small thickness values for thick materials. For example, it underestimates the thickness of the $6 \mathrm{~mm}$ aluminium layer by $60 \mu \mathrm{m}$. 


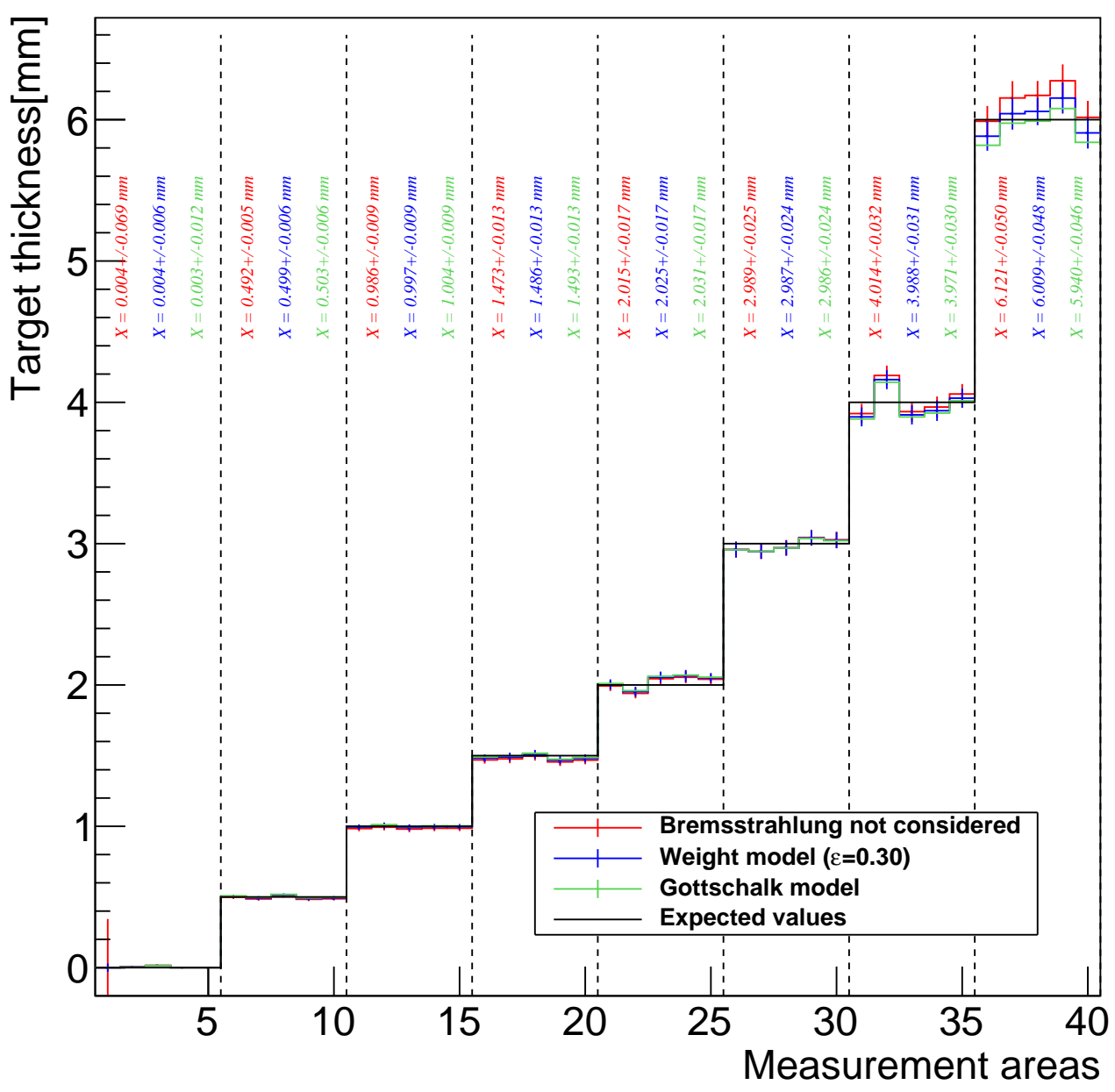

Figure 7.14.: Target thickness measurements after the radiation length calibration procedure. Three different fit models have been used: The red curve neglects influences due to energy losses from bremsstrahlung, the blue curve uses the weight model with $\epsilon=0.30$ and the green curve uses the Gottschalk model to take them into account.

\subsubsection{Measurements on targets with large $X / X_{0}$ and the influence of bremsstrahlung}

The first three analysis steps of the June 2017 beam test data were found to be successful. The track pull distributions were compatible to Gaussian distributions with a mean of zero and a RMS of one. The track $p$ value distribution is reasonably flat. The radiation length calibration parameters $\lambda$ and $\kappa$ are very close to 1.0 and the measured beam energy gradient behaves as expected. 


\section{Radiation length measurements at DESY}

In this section measurements of an aluminium wedge with a thickness up to $30 \mathrm{~mm}$ will be presented. The main reason for the following aluminium wedge measurement is to determine up to which radiation length value the measurement method presented in chapter 5 is still valid and where the impact of energy loss due to bremsstrahlung causes significant deviations.

The validity of radiation length measurements on materials with large $X / X_{0}$ values is limited by material effects such as bremsstrahlung and ionisation. For an electron beam the effects of ionisation are negligible compared to the effects of bremsstrahlung and they are not taken into account here. One of the assumptions for radiation length extraction from the scattering angle distributions with the model function in eq. 5.13 is that the particle energy before scattering and after scattering is identical. This assumption is wrong for large radiation length as explained in section 3.3.4. The two models, weight model and Gottschalk model, which take energy losses into account will be tested in the wedge measurements.

The impact of bremsstrahlung on a particle beam is described in section 3.2. For thin materials, the probability of a bremsstrahlung event occurring is very low and most particle transitions through thin materials are not affected by bremsstrahlung at all. Bremsstrahlung events where a particle loses a large fraction of its initial energy are very rare in thin materials. For example as can be seen from table 3.2, an electron passing through $1 \mathrm{~mm}$ of aluminium has a $1.836 \%$ probability to lose more than $20 \%$ of its energy due to bremsstrahlung. Radiation length measurements will be mostly unaffected by these rare bremsstrahlung processes. The few events with large fractional energy losses will either not be reconstructed due to track $\chi^{2}$ cuts or the scattering angles will be very large and should only affect the non-Gaussian tails of the multiple scattering distribution, which are irrelevant for the determination of the radiation length.

The probability for high energy losses increases with the thickness of the traversed material. From the values in table 3.2 it can be concluded that a radiation length measurement on an aluminium layer with a thickness of $30 \mathrm{~mm}^{4}$ will be strongly influenced by bremsstrahlung events. For almost $50 \%$ of particle transitions, a fractional energy loss of $20 \%$ or more is expected. Accordingly, the particle energy is overestimated during the angle distribution fit (see eq. 5.13). As a consequence of using the wrong beam energy the fit will produce an overestimate of the radiation length.

A schematic side view and a photograph of the aluminium wedge used for the measurements presented here can be seen in fig. 7.15. The largest aluminium thickness of the wedge is $30 \pm 0.2 \mathrm{~mm}$. The thickness decreases with a slope of approximately $0.450 \mathrm{~mm} \pm 0.010 \mathrm{~mm}$ per $\mathrm{mm}$ until it reaches a minimum thickness of $5.4 \pm 0.2 \mathrm{~mm}$. This is equivalent to a radiation length gradient of $0.51 \% \pm 0.06 \%$ per mm. Due to

\footnotetext{
${ }^{4}$ Which corresponds to the maximum thickness of the aluminium wedge
} 


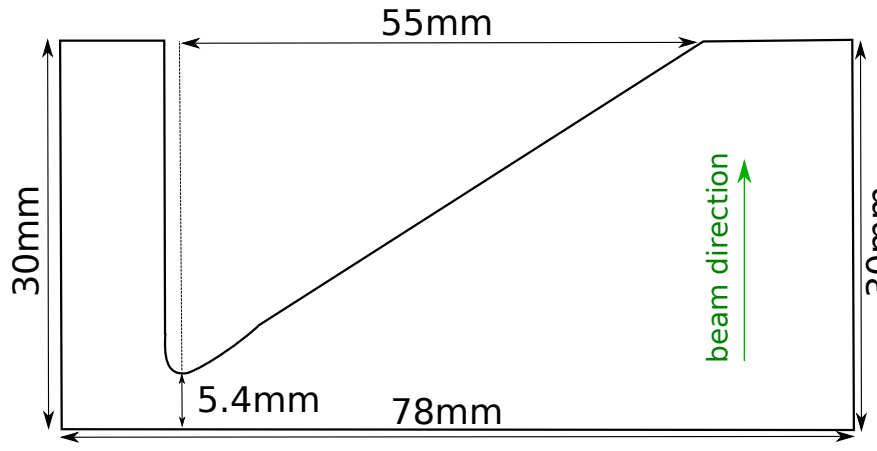

(a)

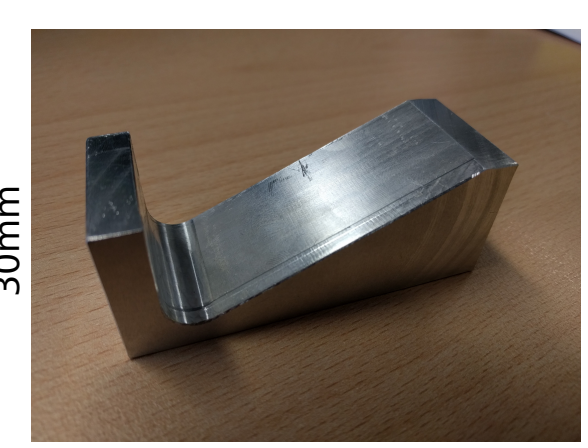

(b)

Figure 7.15.: Schematic side view (a) and photograph of the aluminium wedge (b), which was used as a target during radiation length measurements in this section. The thickness of the target ranges from $5.4 \mathrm{~mm}$ to $30.0 \mathrm{~mm}$. The given dimensions were measured with a caliper.

the limited telescope acceptance area which corresponds roughly to $17 \mathrm{x} 9 \mathrm{~mm}^{2}$ (see fig. 7.11), the measurement of the complete wedge had to be divided into eleven measurement steps. By employing a mechanically movable $x-y$ stage, a scan along the radiation length slope was performed. After each measurement step ,the wedge was moved by $7 \mathrm{~mm}$ along the direction of the radiation length slope. The eleven different, slightly overlapping measurement positions are indicated in the radiation length profile in fig. 7.18. The combined radiation length image with pixel pitches of $200 \mu \mathrm{m}$ is depicted in fig. 7.16 .

The thinnest point of the wedge lies at a $\mathrm{v}$ position of approximately $18 \mathrm{~mm}$. The slope of the wedge is visible between $\mathrm{v}=18 \mathrm{~mm}$ and $\mathrm{v}=70 \mathrm{~mm}$. Beyond this point to the right follows a plateau with the maximum thickness of roughly $30 \mathrm{~mm}$. In the rightmost part of the image, a small area of air is also visible. In the border region between air and $30 \mathrm{~mm}$ aluminium (at $\mathrm{v} \approx 82 \mathrm{~mm}$ ), a single white image pixel column is visible. In these image pixels the fits fail, because the scattering angle distributions from the two largely different materials have completely different Highland widths and a single Gaussian distribution is insufficient to describe the angle distribution. An example of a reconstructed angle distribution with an air and a $30 \mathrm{~mm}$ aluminium component is depicted in fig. 7.17.

The determined $X / X_{0}$ can be best compared to the expectations by computing the radiation length profile along the $v$ direction. In order to do that, projections onto the $\mathrm{v}$ axis are utilised: For every column in the highlighted black area, the arithmetic mean of the radiation length of each pixel in that column is calculated. The errors of the arithmetic mean values are determined via Gaussian error propagation from the errors of individual pixels. The resulting material profiles are shown in fig. 7.18. 


\section{Radiation length measurements at DESY}

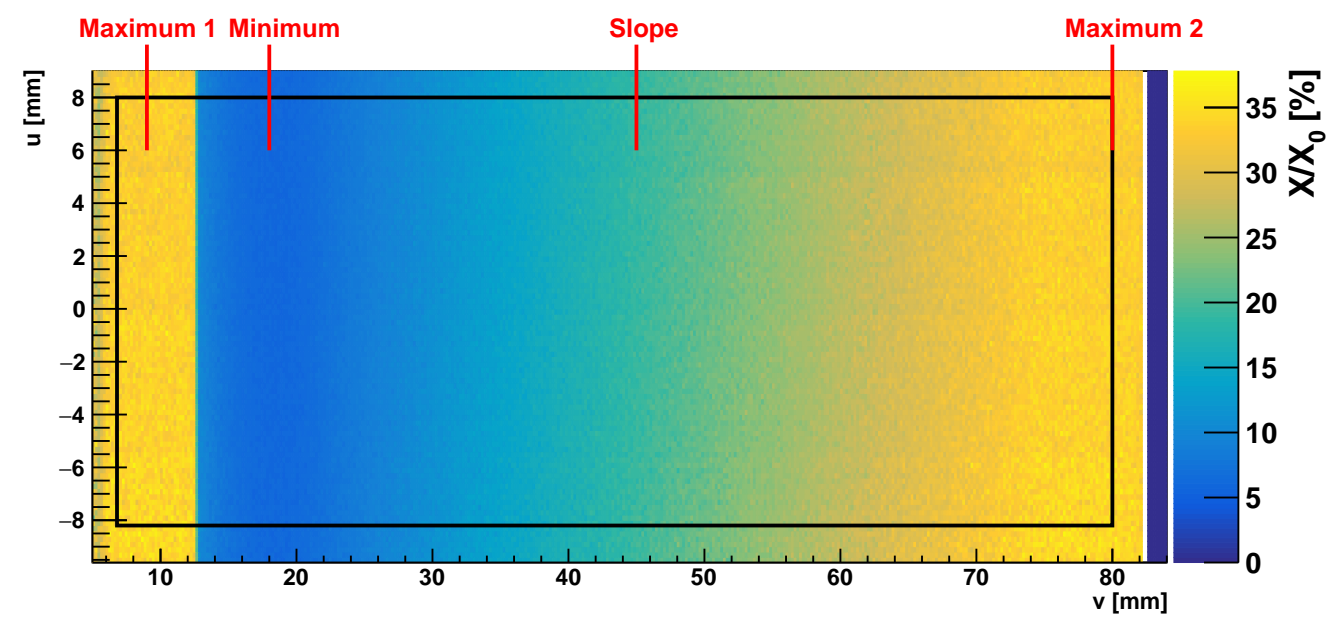

Figure 7.16.: Radiation length image of the aluminium wedge under investigation. The image pixel pitch is $200 \mu \mathrm{m}$. The black box indicates the area used for a projection on the $\mathrm{y}$ axis $(v$ direction). Features of the wedge are marked by the red lines and text. The projection is depicted in fig. 7.18.

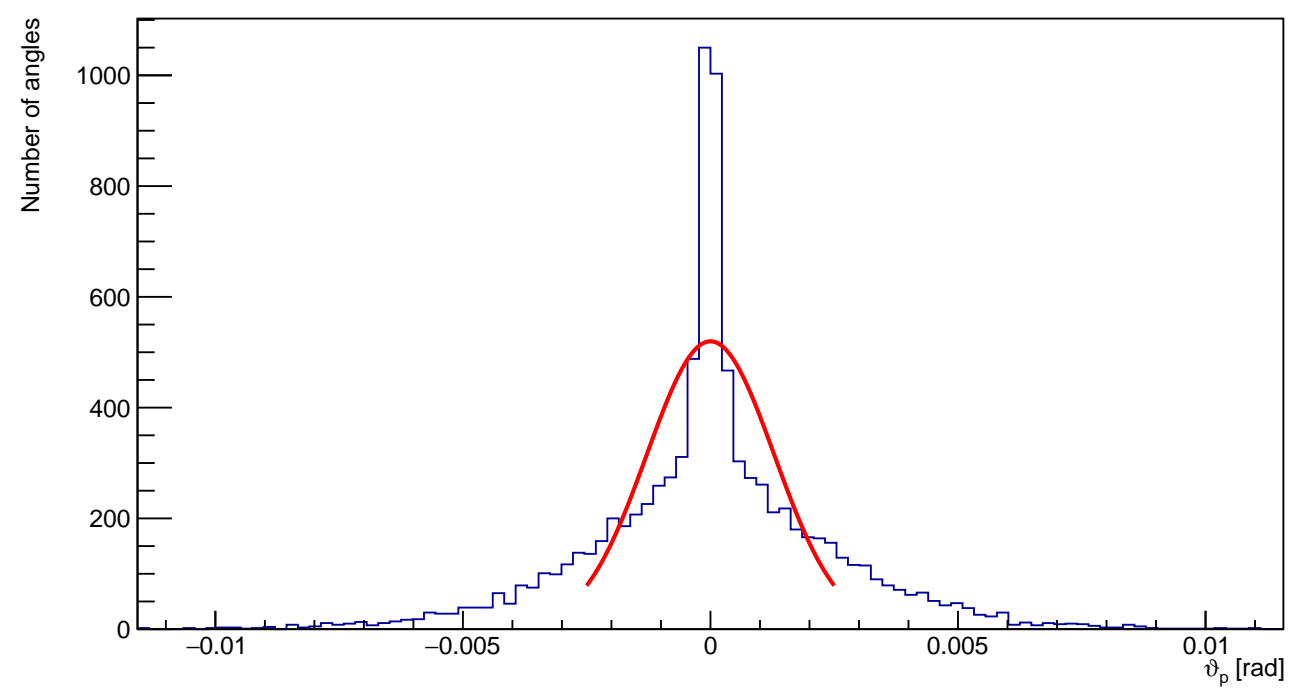

Figure 7.17.: Angle distribution from the border region between air and aluminium visible at $v=82 \mathrm{~mm}$ in fig. 7.16. The angle distribution consists of a narrow distribution due to scattering in air and a broad distribution due to scattering in $30 \mathrm{~mm}$ of aluminium. As a Gaussian distribution does not suffice to describe the distribution, the fit fails. 


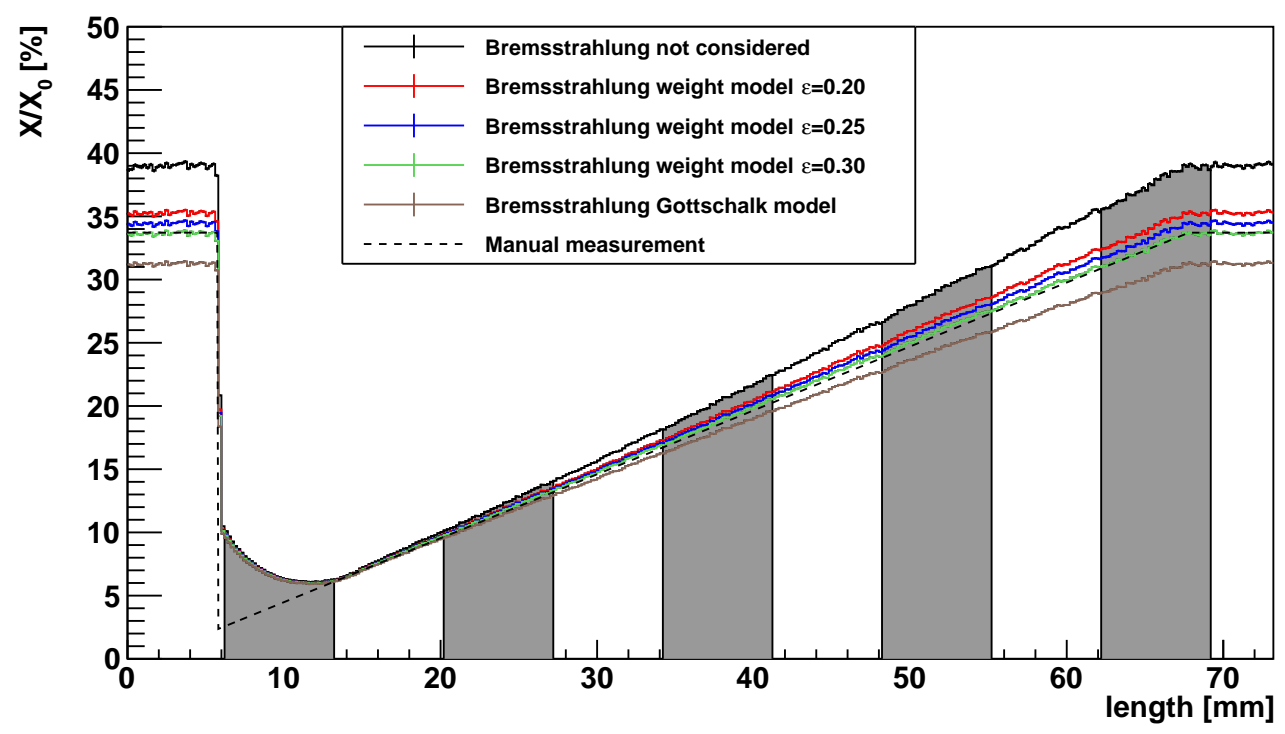

Figure 7.18.: Radiation length profiles along the slope of an aluminium wedge with different fit models. The alternating white and grey areas indicate eleven different measurement positions. The dashed black curve corresponds to manual measurements of the wedge with a calliper.

Five different radiation length profiles and the manually measured geometry are depicted. The dashed black curve corresponds to the manual measurements of the wedge with a caliper. Some key values for features of the wedge are summarised in fig. 7.15a. The solid black line corresponds to the measurement which is based on the Highland model and does not consider energy losses due to bremsstrahlung. The expected overestimation of the radiation length for large material thicknesses can clearly be seen. The red, blue and green curves corresponds to the measurement employing the weight energy loss model with $\epsilon=0.2,0.25,0.3$. A value of 0.30 was found to work for the whole radiation length range. The other two values, 0.2 and 0.25 , lead to a small overestimation of the radiation length profile. The brown curve corresponds to the measurement conducted with the Gottschalk model. The measurement with the Gottschalk model leads to a large underestimation of the radiation length values. Accordingly, this model exaggerates the energy loss effects and the modified particle energies are too small. Table 7.3 summarises the results in comparison with the expected values.

In summary, the inclusion of bremsstrahlung energy loss with the weighted energy loss model in eq. 3.36 and a weight parameter $\epsilon$ of 0.30 was found to precisely measure objects with radiation length values up to $35 \% X / X_{0}$. Not accounting for bremsstrahlung will lead to an overestimation with a relative error of $16 \%$ for a radiation length $35 \% X / X_{0}$. In the next section, a copper wedge with an even larger radiation length will be measured 


\begin{tabular}{|c|c|c|c|}
\hline Wedge feature & Maximum $X$ & Minimum $X$ & Slope \\
\hline Expected value & $33.7 \pm 0.2 \%$ & $6.1 \pm 0.2 \%$ & $0.51 \pm 0.05 \% / \mathrm{mm}$ \\
\hline Bremsstr. not considered & $39.01 \pm 0.02 \%$ & $6.097 \pm 0.008 \%$ & $0.6125 \pm 0.0005 \% / \mathrm{mm}$ \\
\hline Weight model $(\epsilon=0.20)$ & $35.27 \pm 0.02 \%$ & $6.038 \pm 0.008 \%$ & $0.5406 \pm 0.0004 \% / \mathrm{mm}$ \\
\hline Weight model $(\epsilon=0.25)$ & $34.45 \pm 0.02 \%$ & $6.023 \pm 0.008 \%$ & $0.5246 \pm 0.0004 \% / \mathrm{mm}$ \\
\hline Weight model $(\epsilon=0.30)$ & $33.67 \pm 0.02 \%$ & $6.009 \pm 0.008 \%$ & $0.5094 \pm 0.0004 \% / \mathrm{mm}$ \\
\hline Gottschalk model & $31.26 \pm 0.01 \%$ & $5.955 \pm 0.008 \%$ & $0.4626 \pm 0.0004 \% / \mathrm{mm}$ \\
\hline
\end{tabular}

Table 7.3.: Results of the measurements on the aluminium wedge depicted in fig. 7.15a.

in order to test whether the determined weight parameter $\epsilon$ is valid for an even larger $X / X_{0}$ range and for a different radiation length constant $X_{0}$.

\subsection{Copper wedge measurements in November 2017}

The data set analysed in this section was recorded in November of 2017 at the DESY beam test facilities. The measurements took place at beam line 22 with the DURANTA telescope. DQM plots for this beam test can be found in appendix D.2. They show no indication of problems during the telescope calibration and angle reconstruction. The beam energy was set to $4 \mathrm{GeV}$ and the comparator threshold of the M26 sensors was set to 7 times the noise level. The object under test was a copper wedge with a maximum thickness of $20 \mathrm{~mm}$, which corresponds to a radiation length of approximately $140 \%$. A schematic drawing of the copper wedge and its surrounding aluminium frame is depicted in fig. 7.19 .

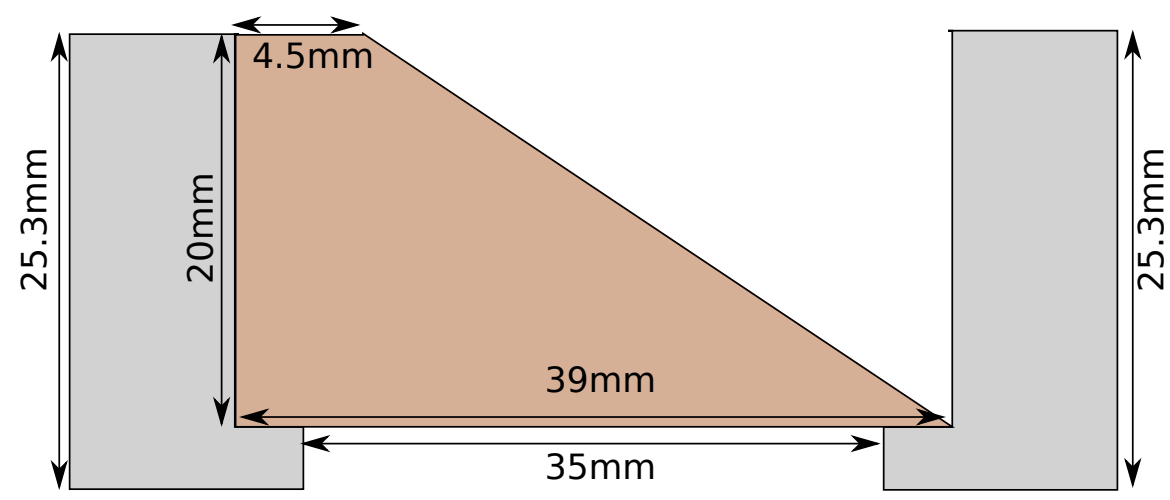

Figure 7.19.: Schematic sideview of the copper wedge under investigation and its surrounding aluminium frame. The given dimensions were measured with a calliper. The uncertainties of the measurements are in the order of $200 \mu \mathrm{m}$, except for the aluminium thickness where measurements indicated a non constant thickness. The expected uncertainty of the aluminium measurement is $500 \mu \mathrm{m}$. 
Due to time limitations during the measurements at the beam line a radiation length calibration on the scattering target itself was tested. The beam spot was centred on the copper wedge but next to the wedge a well known part of the aluminium frame and air were visible within the telescope acceptance area. These two materials were used for the radiation length calibration. The disadvantage of this approach is that the measurement areas were all localised in a small $u$ region, which prevents a reliable estimate of the beam energy gradients. The calibration of $\lambda$ and $\kappa$ worked well.

In order to measure the complete slope of the copper wedge a scan similar to the one in the last section was conducted. The complete length of the copper wedge was covered by eight measurements. After each measurement, the wedge was moved by $6 \mathrm{~mm}$ in the direction of the slope. The combined image of all 8 measurement positions of the copper wedge is shown in fig. 7.20. Due to time limitations, only limited amounts of data could be gathered for the positions between $v=10 \mathrm{~mm}$ and $v=28 \mathrm{~mm}$. Accordingly, only the most central part of the image had angle distributions with a sufficient number of scattering angles to be fitted. As a result, corresponding parts of the combined image have many empty pixels at the edges of the beam spot.

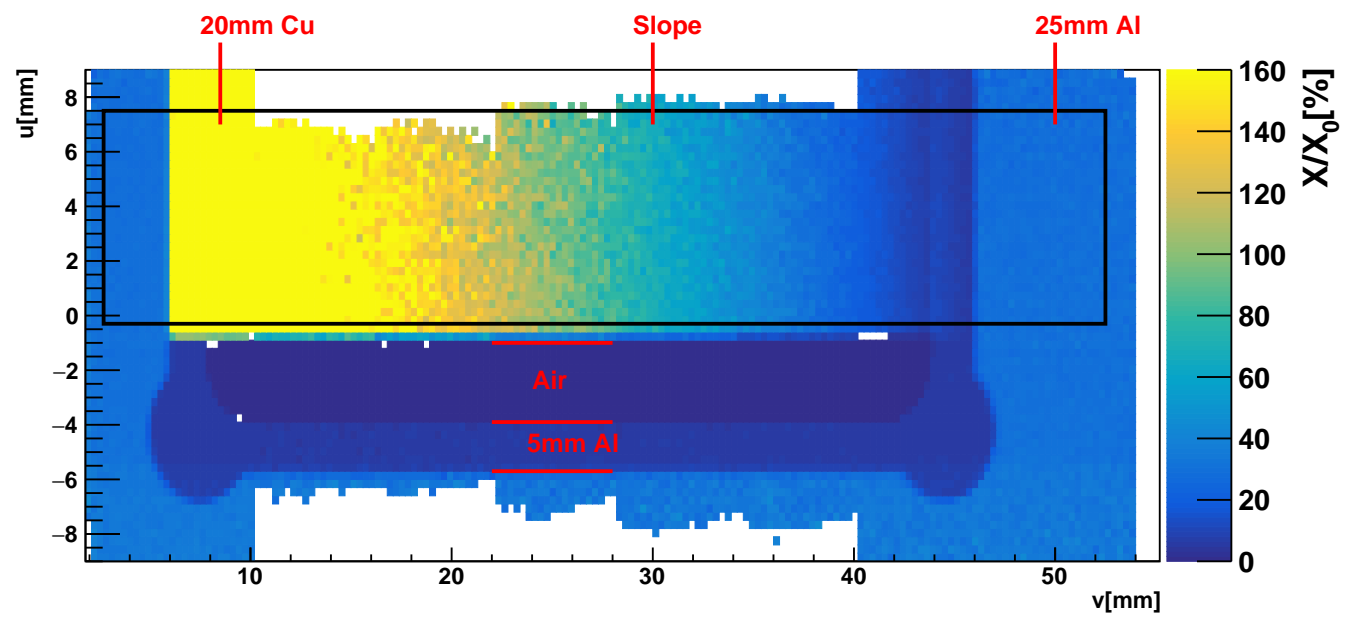

Figure 7.20.: Radiation length image of the copper wedge under investigation. The black box indicates the area of the profile in $v$ direction, which is depicted in fig. 7.21. Several features of the scattering object are indicated in with red lines and text.

The edge of the copper wedge is visible at $u \approx-1 \mathrm{~mm}$. The surrounding aluminium frame (with a radiation length of approximately $28 \%$ ) corresponds to the blue area on the borders of the image. The violet area next to the copper wedge corresponds to areas of air and $0.5 \mathrm{~mm}$ of aluminium, which were used for the radiation length calibration measurement. For orientation, parts of the border regions of different materials are indicated by red lines and text. The image pixels used for the radiation length profile in fig. 7.21 are highlighted by the black box. 


\section{Radiation length measurements at DESY}

Fig. 7.21 depicts radiation length measurements using different bremsstrahlung models. Additionally the dashed black line models the expected slope of the copper wedge. The measured radiation length profiles confirm that the weighted energy loss model also works for measurements on copper. Neglecting the energy loss due to bremsstrahlung leads to an overestimation of the radiation length which increases with increasing radiation length. However, the measurements on material with a radiation length larger than 40 to $50 \%$ indicate that the weight model with a weight factor of $\epsilon=0.3$ is insufficient to correctly describe the expected $X / X_{0}$ profile as can be seen in fig. 7.21 and table 7.4. The assumed weight factor leads to large discrepancies for radiation length values larger than $60 \%$.

The large discrepancy between measured and expected radiation length values can be explained, on one hand, by the increased significance of ionisation processes, which can lead to non-negligible energy losses for large $X / X_{0}$ materials. On the other hand the model describing the energy loss due to bremsstrahlung is very simple and the used weight parameter was found to be only viable for materials with radiation length below $60 \%$. For larger radiation length either the weight model with larger $\epsilon$ values in the order of 0.4 and even 0.5 or the Gottschalk model should be used. The weight model with $\epsilon=0.5$ and the Gottschalk model describe the slope of the wedge rather well between radiation length values of 80 to $110 \%$.

Tables 7.4 and 7.5 contain results of the radiation length measurements at the maximum copper thickness, the maximum aluminium thickness of the frame and of the wedge slope in different $X / X_{0}$ ranges for all bremsstrahlung models used. To compare measurements on copper with the measurements of the maximum aluminium thickness in the previous section radiation length values at an expected $X / X_{0}$ value of $30 \%$ are determined and summarised in table 7.4.

\begin{tabular}{|c||c|c|c|}
\hline Feature & Max. $\mathrm{Cu}[\%]$ & $\mathrm{Max} . \mathrm{Al}[\%]$ & $\mathrm{Cu} 30 \% X / X_{0}[\%]$ \\
\hline Expected value & $139 \pm 3$ & $28.4 \pm 0.4$ & $30.0 \pm 0.2$ \\
\hline Bremsstrahlung not considered & $339.6 \pm 0.5$ & $32.9 \pm 0.05$ & $33.7 \pm 0.6$ \\
\hline Weight model $(\boldsymbol{\epsilon}=\mathbf{0 . 3 0})$ & $200.1 \pm 0.3$ & $29.25 \pm 0.04$ & $29.9 \pm 0.5$ \\
\hline Weight model $(\boldsymbol{\epsilon}=\mathbf{0 . 4 0})$ & $169.4 \pm 0.2$ & $28.24 \pm 0.04$ & $28.8 \pm 0.5$ \\
\hline Weight model $(\boldsymbol{\epsilon}=\mathbf{0 . 5 0})$ & $145.4 \pm 0.2$ & $27.31 \pm 0.04$ & $27.9 \pm 0.4$ \\
\hline Gottschalk model & $151.4 \pm 0.2$ & $27.57 \pm 0.04$ & $28.1 \pm 0.4$ \\
\hline
\end{tabular}

Table 7.4.: Radiation length results of measurements on the copper wedge depicted in fig. 7.19. The statistical error of the $30 \%$ of copper measurement is large, because only a single column in the profile is used to calculate the value.

In conclusion, the measurements presented in this section confirm the findings of the last section. Additionally, it was found that measurements on large $X / X_{0}$ materials also work for copper with a small radiation length constant of $14.35 \mathrm{~mm}$ [29]. When 


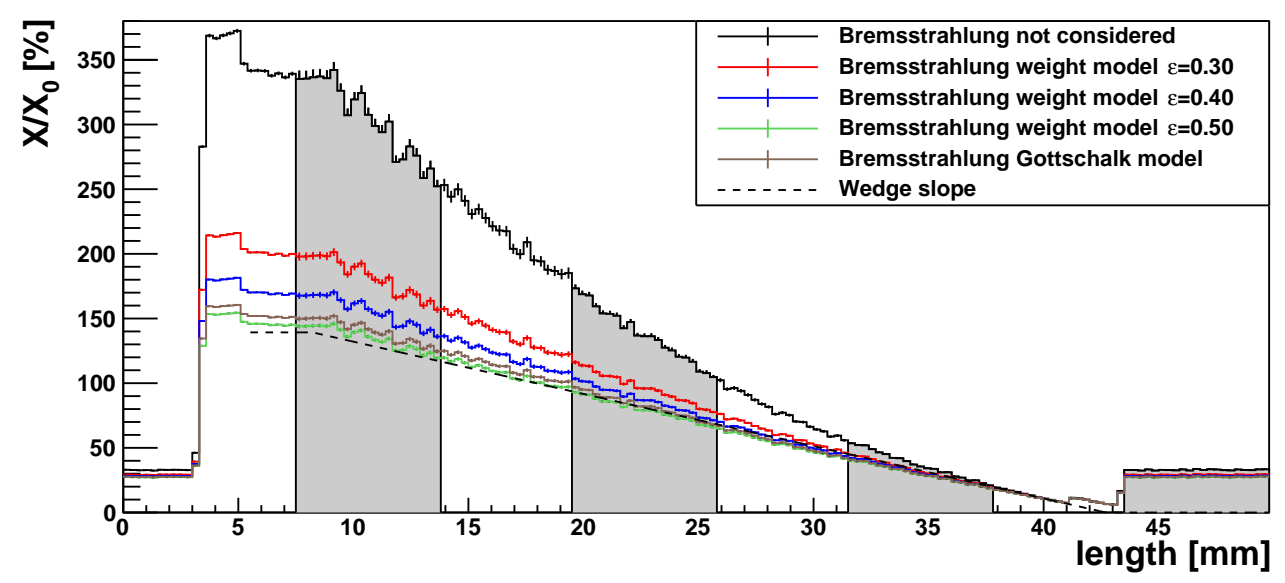

(a) Radiation length profile with full range

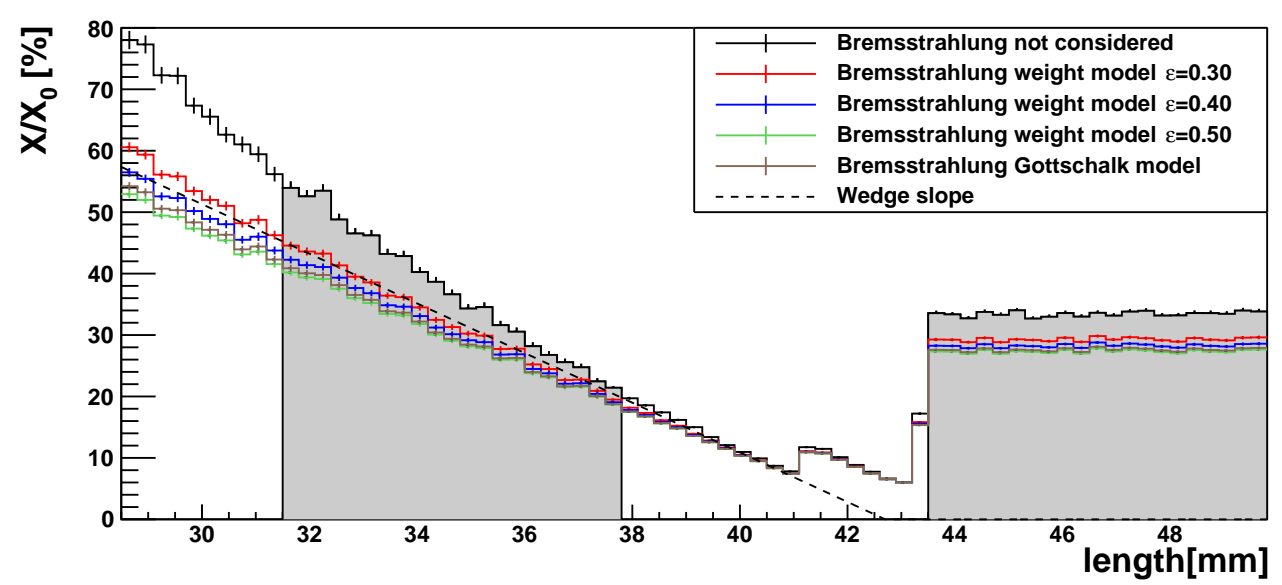

(b) Zoomed in radiation length profile

Figure 7.21.: The full (a) and zoomed in (b) radiation length profile along the slope of the copper wedge with (red curve) and without (black curve) consideration of energy loss due to bremsstrahlung. The alternating white and grey areas indicate the 8 different measurement positions. The expected radiation length profile (based on manual measurements) of the copper wedge is indicated by the dashed line.

conducting radiation length measurements on large $X / X_{0}$ materials bremsstrahlung has to be accounted for in order to not overestimate the radiation length of the material. For aluminium and copper and radiation length values below $60 \%$ the weight model in eq. 3.36 with a weight $\epsilon$ of 0.3 provides the most accurate results. For larger radiation length either the Gottschalk or the weight model with a larger weight factors should be used. However, in this large radiation length regime radiation length measurements, even when 
7. Radiation length measurements at DESY

\begin{tabular}{|c||c|c|}
\hline Feature & Slope $[\% / \mathrm{mm}]$ & Slope $\left(X / X_{0}<60 \%\right)[\% / \mathrm{mm}]$ \\
\hline Expected value & $4.00 \pm 0.05$ & $4.00 \pm 0.05$ \\
\hline Bremsstrahlung not considered & $6.86 \pm 0.02$ & $5.25 \pm 0.03$ \\
\hline Weight model $(\boldsymbol{\epsilon}=\mathbf{0 . 3 0})$ & $4.956 \pm 0.01$ & $4.17 \pm 0.02$ \\
\hline Weight model $(\boldsymbol{\epsilon}=\mathbf{0 . 4 0})$ & $4.453 \pm 0.009$ & $3.88 \pm 0.02$ \\
\hline Weight model $(\boldsymbol{\epsilon}=\mathbf{0 . 5 0})$ & $4.003 \pm 0.007$ & $3.62 \pm 0.02$ \\
\hline Gottschalk model & $3.72 \pm 0.02$ & $4.173 \pm 0.008$ \\
\hline
\end{tabular}

Table 7.5.: Slope results of measurements on the copper wedge depicted in fig. 7.19.

considering energy losses due to bremsstrahlung, are expected to have uncertainties in the order of several percents of $X / X_{0}$.

\subsection{Glue measurements}

The data set analysed in this section was recorded in 2015 at the DESY beam test facilities $^{5}$. Data taking at DESY was performed by ATLAS group members [77], while the data analysis was conducted by me. The goal of the measurements in this section was to demonstrate radiation length measurements of an unknown but homogeneous material with a known thickness and to determine the radiation length constant $X_{0}$ of different material samples. The measurements were conducted at beam line 21 with the DATURA telescope. The $z$ alignment shifts of all aligned telescope planes are quite large and consistently negative (see fig.D.16). They are all shifted into the same direction which indicates a systematic effect. The angle reconstruction worked well, but determining the beam energy gradient during the radiation length calibration was challenging. Since the calibration procedure explained in section 5.4 was not in place during this measurement, calibrations for this thesis had to be performed on the available data which consisted of air and aluminium rings of known thickness.

The $X / X_{0}$ calibration employed five air measurement areas and five $1.83 \mathrm{~mm}$ aluminium measurement areas in the aluminium insert region depicted in fig. 7.22b. As the multiple scattering distributions in air are not dependent on the beam energy, the beam energy gradient had to be determined from angle distributions on aluminium. However, as can be seen from fig. $7.22 \mathrm{~b}$ these measurement points are very localised in a small ring with a maximum diameter of approximately $10 \mathrm{~mm}$. Due to the closeness of the measurement points, the determined energy gradient values were found to have large uncertainties and were compatible with zero. Instead of using beam energy gradients with large errors the gradients were fixed to zero during the radiation length calibration in order to not influence the other calibration parameters such as the average beam energy.

The material under study consisted of a brass plate with a thickness of approximately $10 \mathrm{~mm}$ and a cylindrical drilling, which was used to hold samples of different glues. Ra-

\footnotetext{
${ }^{5}$ DQM plots in appendix D.3
} 

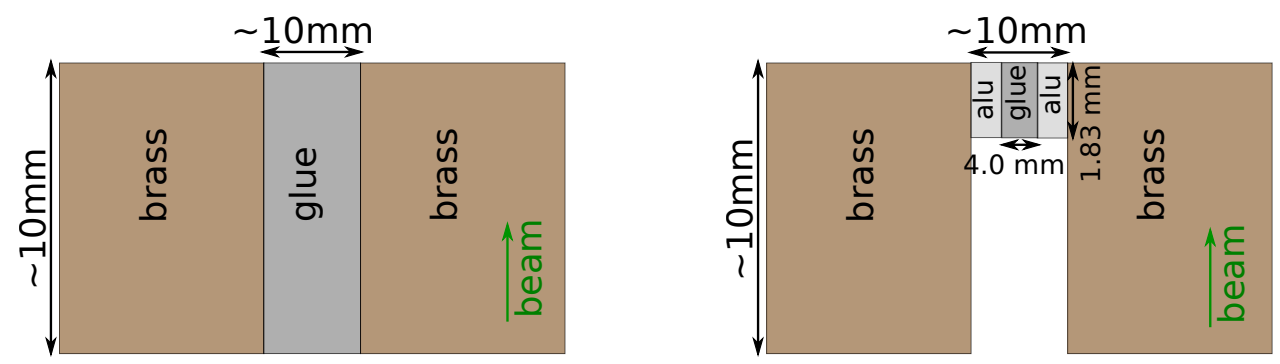

(a) Samples A,B and C

(b) Sample D

Figure 7.22.: Schematic side views of brass plates with glue samples A,B,C (a) and D (b). In order to reduce the necessary volume of expensive silver glue $\mathrm{D}$ a small aluminium insert was used.

diation length measurements were performed on four different glues. The glue samples were filled into the borehole and after the glue had hardened, the surface was polished to get a smooth transition between glue and surrounding brass and also achieve a constant glue thickness. In order to extract the radiation length constant $X_{0}$ from the radiation length $X / X_{0}$, the thickness of the glue $X$ and the surrounding brass was measured manually. Fig. 7.22 depicts a schematic overview of the scattering object. Glue sample D was an expensive glue containing silver and, in order to reduce the glue volume, it was filled into the borehole of an aluminium ring with a smaller radius and thickness. Afterwards, the aluminium ring was embedded into the brass frame. Scattering angle distributions of this aluminium ring were also used in the $X / X_{0}$ calibration process. Radiation length images of the four different glue samples are shown in fig. 7.23. The pixel pitch of the images is $200 \mu \mathrm{m}$. The boreholes are clearly visible within the surrounding brass with an extremely large radiation length.

In order to determine the radiation length of each glue sample, projections onto the $u$ axis are utilised. The resulting material profiles are shown in fig. 7.24. As the borehole and consequently the starting points of the material profiles are located at different positions for each individual image, the $u$ values do not necessarily correspond to the $u$ position on the images. Instead, $\mathrm{u}=0 \mathrm{~mm}$ corresponds to the image $u$ position of the left most border of the black box. The material profiles cover the region of homogeneously distributed glue and can be used to determine the radiation length constant of each glue.

Unfortunately, a radiation length gradient is visible in the profiles. This can be attributed to the beam gradient not having been reliably determined. The discrepancy between radiation length values on the left most and right most positions of the material profiles of glue A,B and $\mathrm{C}$ is approximately $0.2 \%$. This value is much larger than the 


\section{Radiation length measurements at DESY}

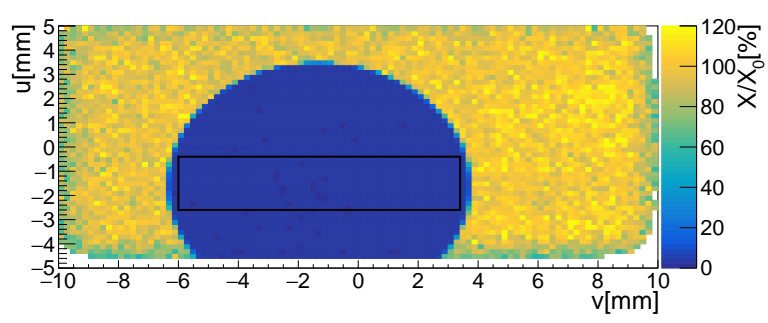

(a) Glue sample A

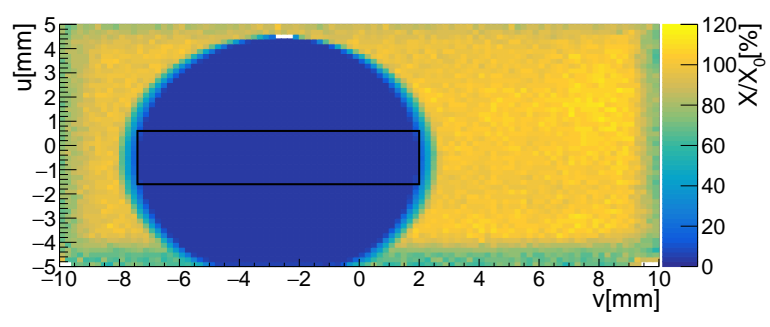

(b) Glue sample B

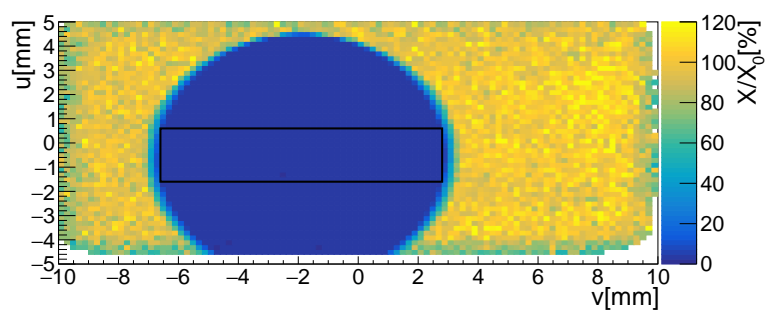

(c) Glue sample C

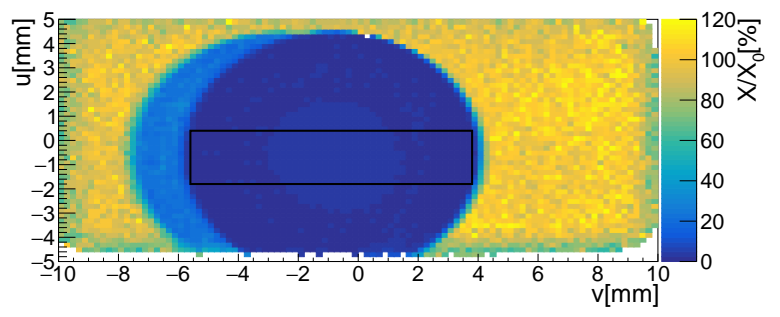

(d) Glue sample D

Figure 7.23.: Radiation length images of glue samples and surrounding brass frames. The hardened glue is located within the circular hole with a diameter of approximately $10 \mathrm{~mm}$ visible in images (a), (b) and (c). In image (d) an aluminium ring is embedded in the brass bore hole. Glue sample D is located in the centre of the aluminium ring. This sample was most likely slightly rotated which causes the shadow of the aluminium ring visible as a crescent shape on the right. The black boxes indicate limits of image pixels which are used to calculate material profiles (see fig. 7.24). The pixel pitch of the images is $200 \mu \mathrm{m}$. 


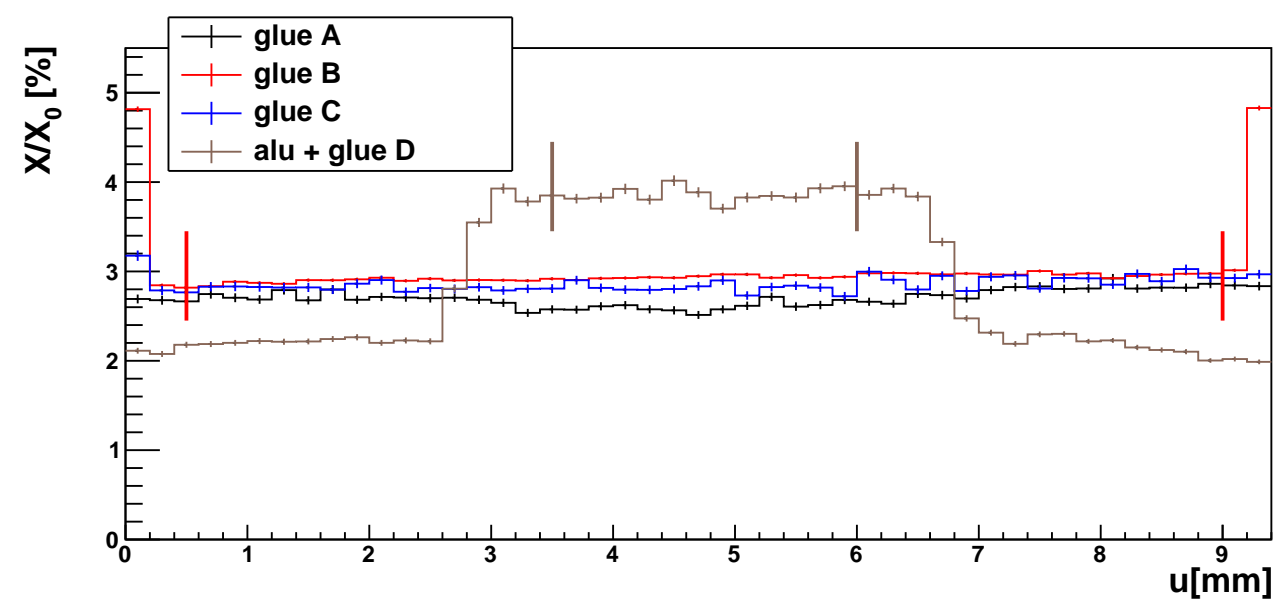

Figure 7.24.: Material profiles along the $u$ axis in hardened glue areas. The area in which the radiation length values are determined are marked by red and brown lines in the profiles. $u$ positions of individual images were adjusted to overlay with other glue positions.

statistical errors of individual profile bins which are in the order of $0.02 \%$ to $0.05 \%$. Due to the smaller glue region of glue sample D the corresponding uncertainty caused by the beam energy gradient is smaller. The uncertainty due to the gradient of this sample is approximately $0.05 \%$ which is still large in comparison to the statistical uncertainty of glue sample D. Instead of calculating the uncertainty of the $X / X_{0}$ measurement via error propagation from the statistical uncertainties of the individual profile bin, a constant uncertainty of $0.2 \%$ or $0.05 \%$ from the gradients is attributed to the measurement results.

\begin{tabular}{|c||c|c|c|}
\hline Sample & $\boldsymbol{X} / \boldsymbol{X}_{\mathbf{0}}[\mathbf{\%}]$ & $\boldsymbol{X}[\mathbf{m m}]$ & $\boldsymbol{X}_{\mathbf{0}}[\mathbf{m m}]$ \\
\hline \hline glue A & $2.7 \pm 0.2$ & 10.3 & $380 \pm 30$ \\
\hline glue B & $2.9 \pm 0.2$ & 10.17 & $350 \pm 20$ \\
\hline glue C & $2.8 \pm 0.2$ & 10.08 & $350 \pm 20$ \\
\hline glue D & $3.9 \pm 0.05$ & 1.83 & $47.4 \pm 0.6$ \\
\hline
\end{tabular}

Table 7.6.: Results of the glue radiation length constant measurements. The short radiation length constant of glue D is expected due to the large percentage of high $Z$ silver filling.

The average radiation length of glues $\mathrm{A}$ to $\mathrm{C}$ was calculated from profile bins between length $=0.5 \mathrm{~mm}$ and $9.0 \mathrm{~mm}$ and for glue $\mathrm{D}$ from bins between length $=3.5 \mathrm{~mm}$ and $6.0 \mathrm{~mm}$ as they showed the least variation. A summary of the results can be found in

${ }^{6}$ Depending on the glue sample, glue sample B has a very large statistics and a very small statistical uncertainty of $0.02 \%$ 


\section{Radiation length measurements at DESY}

table 7.6. The radiation length constants of glues A-C are consistent with one another within the scope of the accuracy of this measurement. The low $Z$ glues were found to have radiation length constants between 350 and $380 \mathrm{~mm}$ which matches the expected value for unfilled epoxy adhesives of above $320 \mathrm{~mm}$. This expectation value has therefore been confirmed by the measurement. The silver glue sample D has a significantly smaller radiation length constant $X_{0}$, which is not unexpected due to the small radiation length constant of silver of $X_{0}^{\mathrm{Ag}}=8.543 \mathrm{~mm}$ [29], which makes up a large percentage of this epoxy. The measurements in this section confirm that by measuring a homogeneous material with a well known thickness the unknown material constant $X_{0}$ can be determined. Measurements of this kind can be used to create data bases with information on radiation length constants of different materials used in modern particle detectors and other applications.

\subsection{Chip measurements}

The data set analysed in this section was recorded in October 2016 at the DESY beam test facilities at beam line 22 with the DURANTA telescope ${ }^{7}$. Data taking at DESY was performed by ATLAS group members [77], while the analysis of the recorded data was conducted by me. The goal of the measurements in this section was to demonstrate the capabilities of radiation length measurements on a material with complex structures on small spatial scales. As scattering target a printed circuit board (PCB), which included a field programmable gate array (FPGA) chip, was used (see fig. $7.25 \mathrm{a}$ and $7.25 \mathrm{c}$ ).

A total number of 130 million scattering angles with constant nominal particle energy of $2 \mathrm{GeV}$ and steady telescope setup were measured. This large number of scattering angles provided the opportunity to use small image pixels with a pitch of $75 \mu \mathrm{m}$ in both directions. This pitch is a good compromise between an adequate image spatial resolution and multiple scattering distributions with an average number of several thousand entries per pixel, which ensured a stable fit. The radiation length image and two photographs of both sides of the PCB plane are depicted in fig. 7.25b.

Different components of the PCB and the FPGA chip can be easily identified. A part of the chip can be seen on the left hand side of the radiation length image and the uppermost photograph. The edge of the chip lies between $u=2 \mathrm{~mm}$ and $4 \mathrm{~mm}$. In this area an array of connector lines is visible which links the chip to the surrounding PCB. The pattern of capacitors and vias, which is present in the $X / X_{0}$ image, can also be found in fig $.7 .25 \mathrm{c}$. An additional feature, which is only visible in the radiation length image, is the fan-out of the FPGA chip within the chip volume. The fan-out corresponds to the alternating pattern of increased radiation length in the edge region of the chip between $u=-2 \mathrm{~mm}$ and $u=+2 \mathrm{~mm}$.

\footnotetext{
${ }^{7}$ DQM plots in appendix D.4 indicate reliable results for telescope calibration, angle reconstruction and radiation length calibration steps
} 


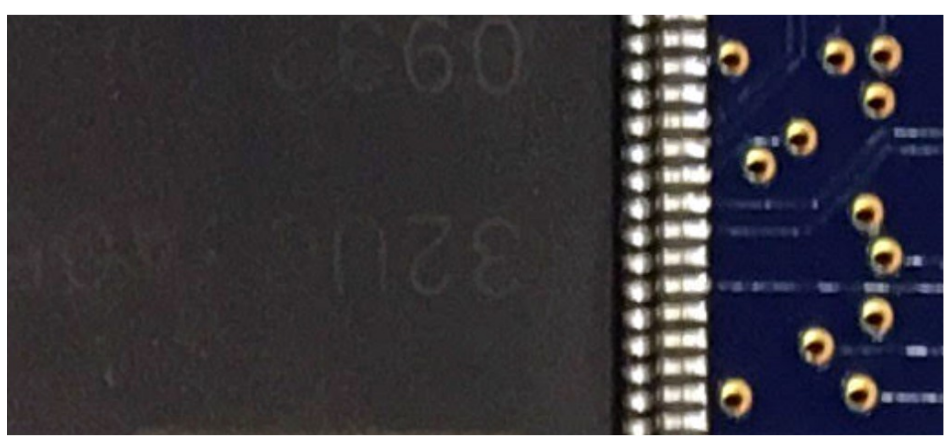

(a) photograph of front side (mirrored)

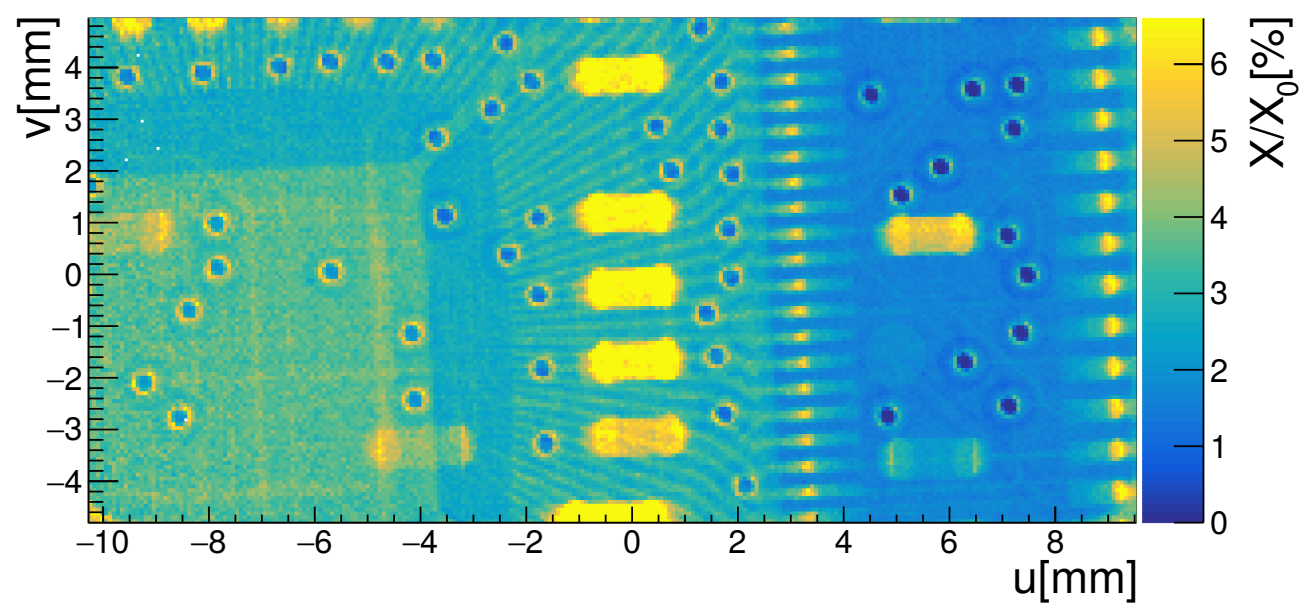

(b) Radiation length image ( $75 \mu \mathrm{m}$ pitch)

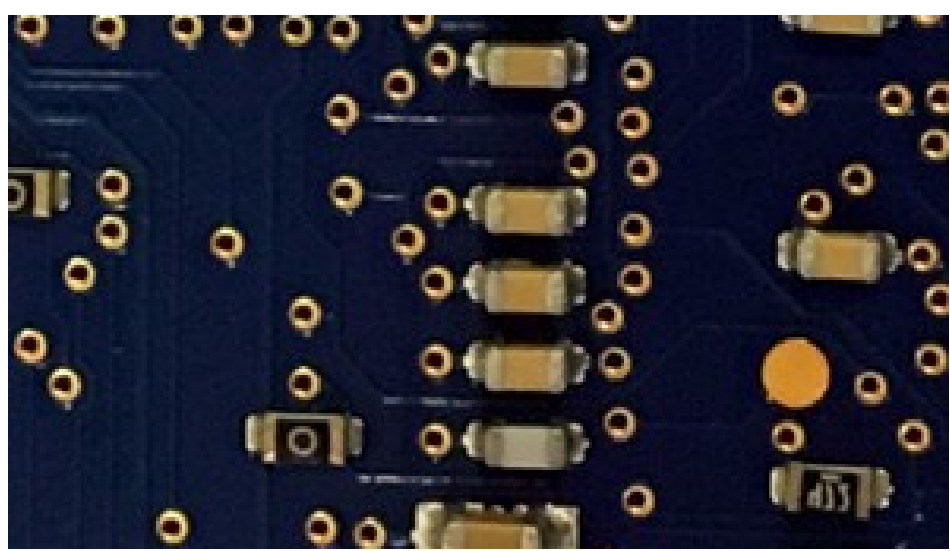

(c) photograph of back side (mirrored)

Figure 7.25.: Radiation length image and photographs of the PCB target plane. Many different components like capacitors, fan-outs, vias and connectors are visible in the $X / X_{0}$ image. 


\section{Radiation length measurements at DESY}

Several copper lines within the PCB volume are also visible on the right hand side of the image, outside of the FPGA chip volume. Measurements of radiation lengths of individual components of the PCB or chip were not possible, because the scattering target consists of multiple layers of different materials with unknown radiation length constants and detailed information about the thicknesses and the composition of the individual materials were not available.

As a conclusion, the measurements conducted during this beam test demonstrated the good spatial resolution of the radiation length method based on materials with a large range of radiation length values. The spatial resolution suffices to resolve small structures such as the fan-out lines inside the PCB volume.

\subsection{ATLAS ITk prototype measurements}

The data set analysed in this section was recorded in August 2016 at the DESY beam test facilities at beam line 22 with the DURANTA telescope ${ }^{8}$. Data taking at DESY was performed by ATLAS group members [77], while the analysis of the recorded data was conducted by me. The telescope alignment and cluster calibration were performed for a geometry, that contained not only the 6 planes of the telescope, but also a homogeneous aluminium plate with a thickness of approximately $2 \mathrm{~mm}$. The telescope setup is depicted in fig. 7.26. However, as can be seen from the DQM plots, the additional scattering in the telescope does not seem to have impacted the calibration step significantly. The only remarkable DQM plot is the distribution of the $z$ positions (see fig. 7.27) of the telescope planes after the alignment. Every telescope plane affected by the alignment is shifted in the negative $z$ direction relative to the nominal position and the shifts of 3 to $5 \mathrm{~mm}$ are unusually large.

Another unwanted influence is visible in the angle distributions, which are used during the radiation length calibration (see either fig. 7.28 or fig. D.45 in the appendix). Some of the distributions, especially the distributions from area 1,3,4 and 7, show periodic disturbances, which have been discussed in subsection 6.2. The angle distributions are assumed to be Gaussian and the periodic peaks lead to a comparatively large fit $\chi_{\text {ndof }}^{2}$ value. As discussed in the previous chapter this systematic influence is caused by digital effects due to coordinate correlations, which in turn originate from the digital readout of the M26 sensors. This effect is most pronounced, when the M26 distance along the beam axis is small ${ }^{9}$. However, as documented in section 6.2 digital effects do not lead to a bias in the radiation length measurement. Instead they increase the variance of the measurement as depicted in fig. 6.14. The radiation length image is therefore expected to show statistical fluctuations, that are larger than one would expect from the statistical uncertainties determined via Highland fit.

\footnotetext{
${ }^{8}$ DQM plots in appendix D.5

${ }^{9}$ Which is the case here as can be seen from fig. 7.26
} 


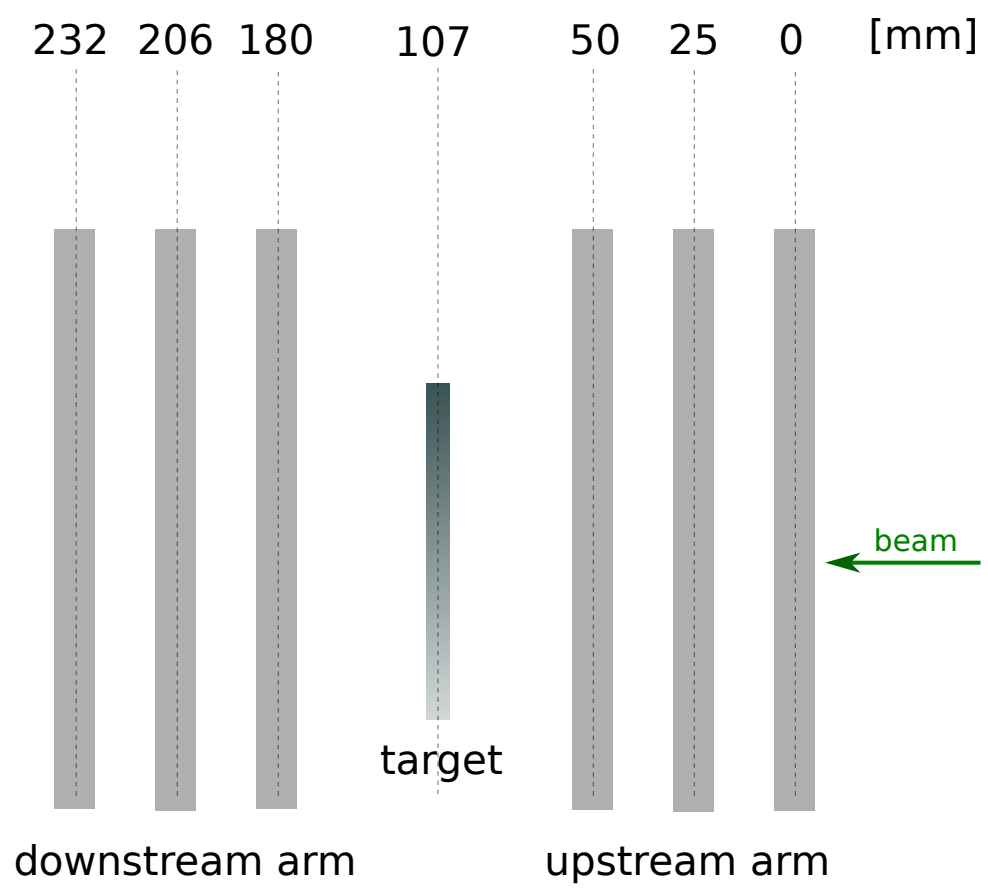

Figure 7.26.: Schematic side view of the telescope setup during the August 2016 beam test campaign.

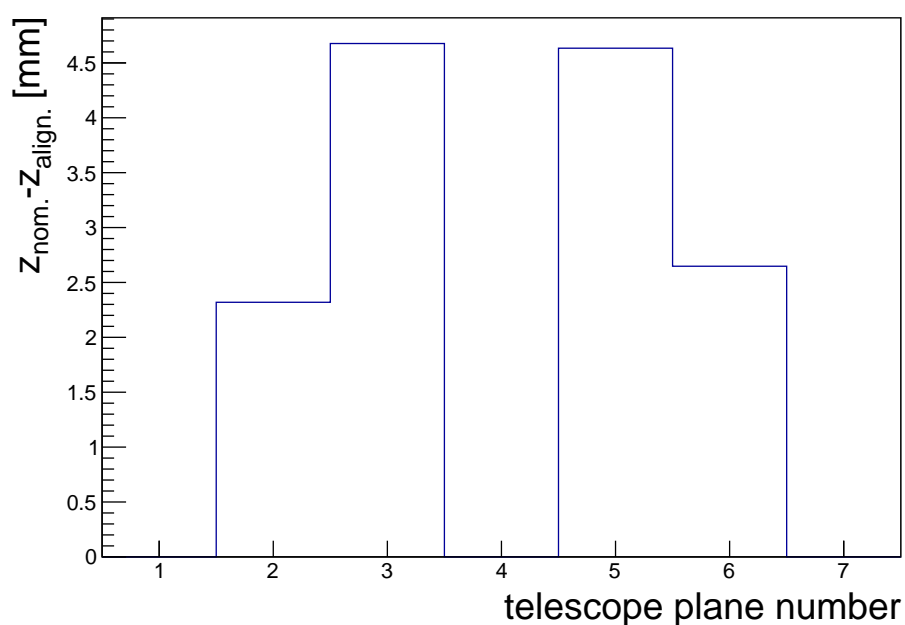

Figure 7.27.: Difference between nominal and aligned sensor $z$ positions for August 2016 data. The determined differences are all positive and rather large. 


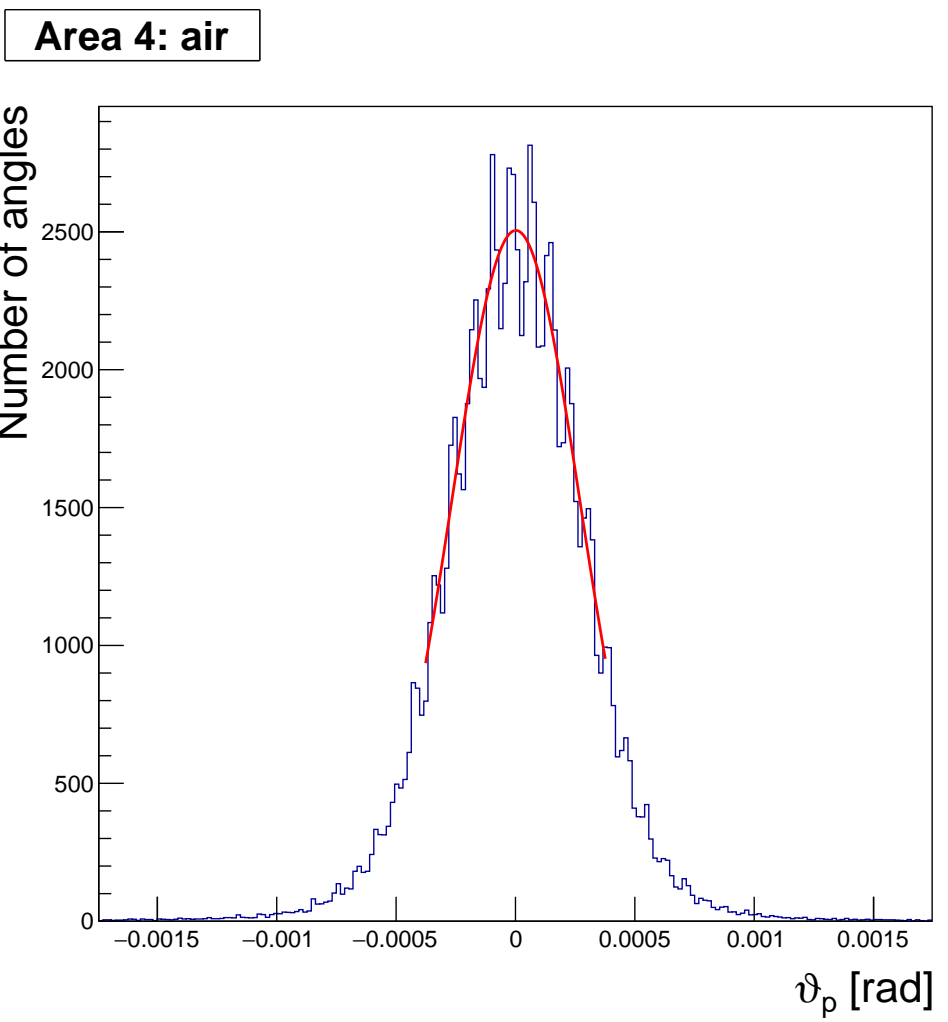

Figure 7.28.: Angle distribution used in the $X / X_{0}$ calibration measurements. A pattern of periodic peaks is visible which leads to a non-Gaussian shape of the angle distribution.

The main scattering target was an ATLAS Inner Tracker Upgrade (ITk) dummy module [19] that was glued onto a mechanical support structure. The support structure itself consisted of a sandwich of a carbon fibre face sheet, a carbon fibre layer with a honeycomb pattern for stabilisation in the middle and another carbon fibre face sheet on top. The track sample of approximately 140 million tracks with a constant nominal particle energy of $3 \mathrm{GeV}$ and steady telescope setup is sufficient to generate a radiation length image with pixel pitches of $50 \mu \mathrm{m}$. The radiation length image is depicted in fig. $7.29 \mathrm{~b}$.

Several interesting structures are visible in the radiation length image: The two $8 \mathrm{x}$ $8 \mathrm{~mm}^{2}$ rectangular shapes are read-out chips of the ITk module. A periodic grid of $8 \times 8$ vias lies in the flex board beneath the chips. The inside of the vias can be identified as small dots with a reduced radiation length. These vias have a diameter of three to four image pixels, which corresponds to approximately $200 \mu \mathrm{m}$. Between the two read-out chips, an array of four capacitors is located. On the edges of the capacitors, traces of solder can be seen. In the area near $(u, v)=(0 \mathrm{~mm}, 0 \mathrm{~mm})$ bond pads and metal traces are visible, which manifest as regions with a slightly increased radiation length. As 


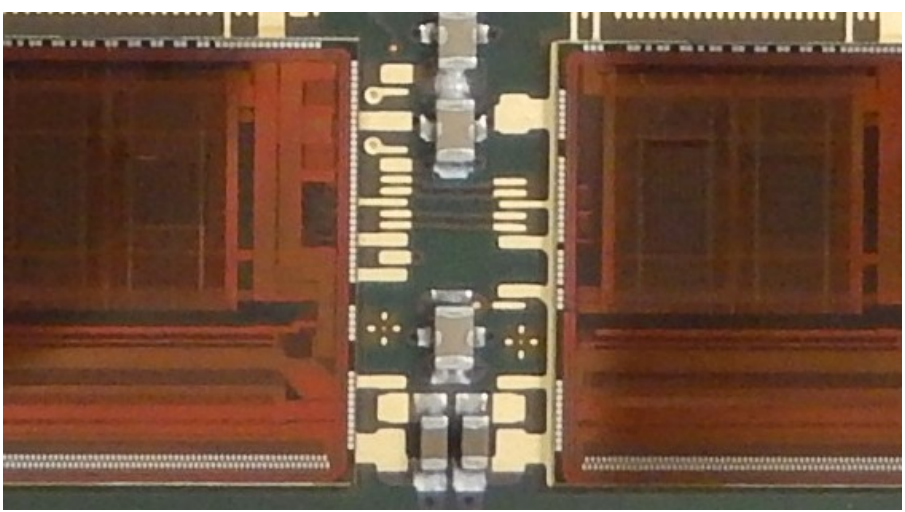

(a) photograph of module on flex board (with chips)

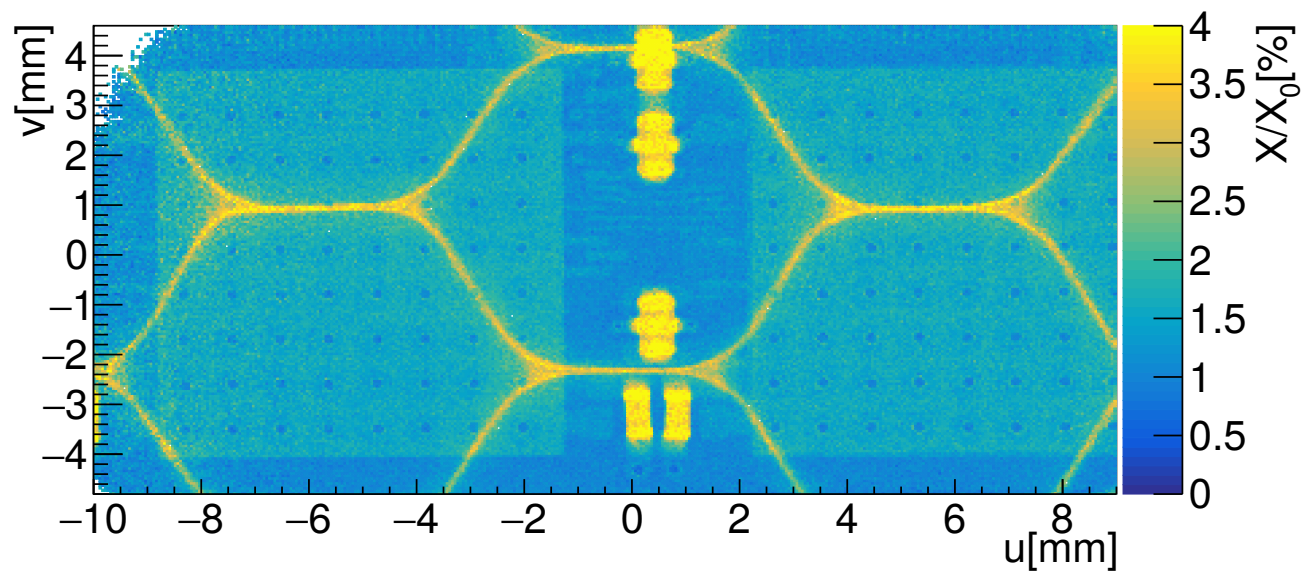

(b) Radiation length image (50 $\mu \mathrm{m}$ pitch)

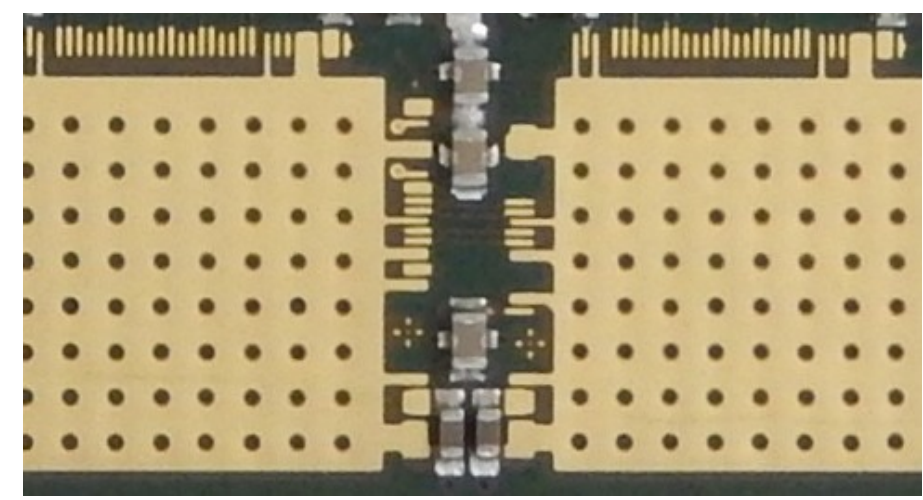

(c) photograph of module on flex board (without chips).

Figure 7.29.: Radiation length image and photographs of an ATLAS ITk dummy module. In (b) and (c) arrays of vias are visible. The hexagonal carbon fibre structure is only visible in the radiation length image. 


\section{Radiation length measurements at DESY}

already mentioned, the ITk module is supported by a layer that is stabilised by a honeycomb carbon fibre structure. The honeycomb carbon fibre pattern is clearly visible as hexagons with a high radiation length and at some positions the fibres are surrounded by a halo, which matches the spread of glue between the three layers of the support structure.

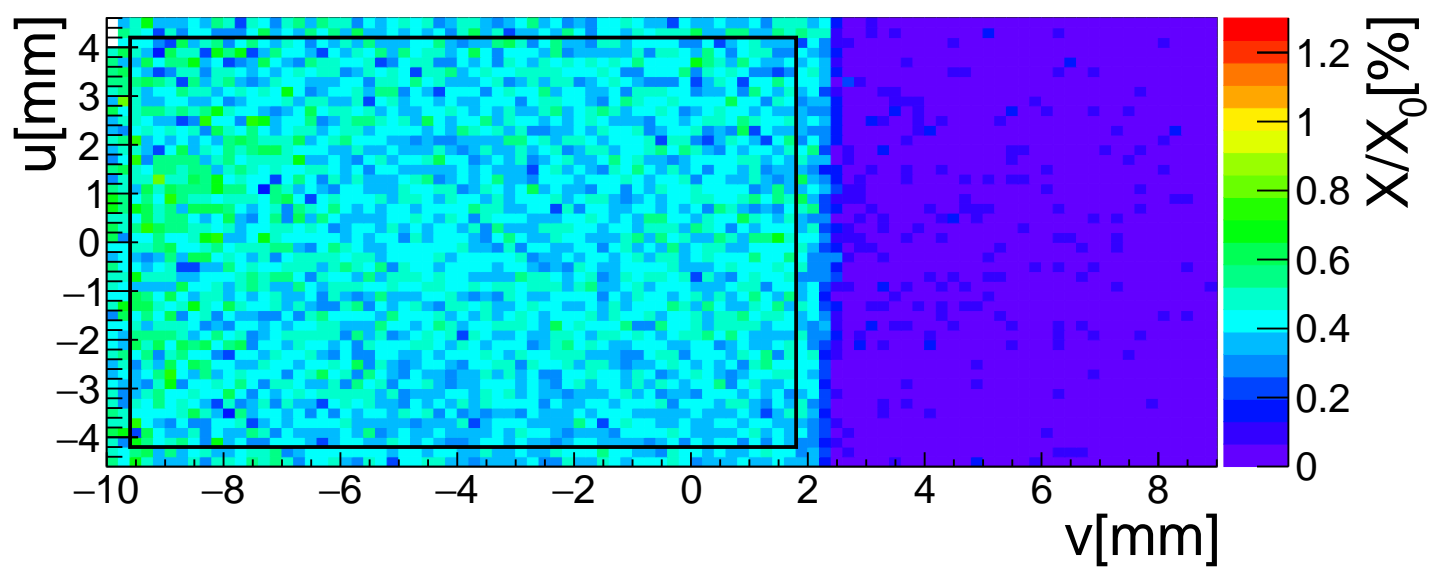

Figure 7.30.: Radiation length image of a carbon foam layer on the left hand side and air on the right hand side.

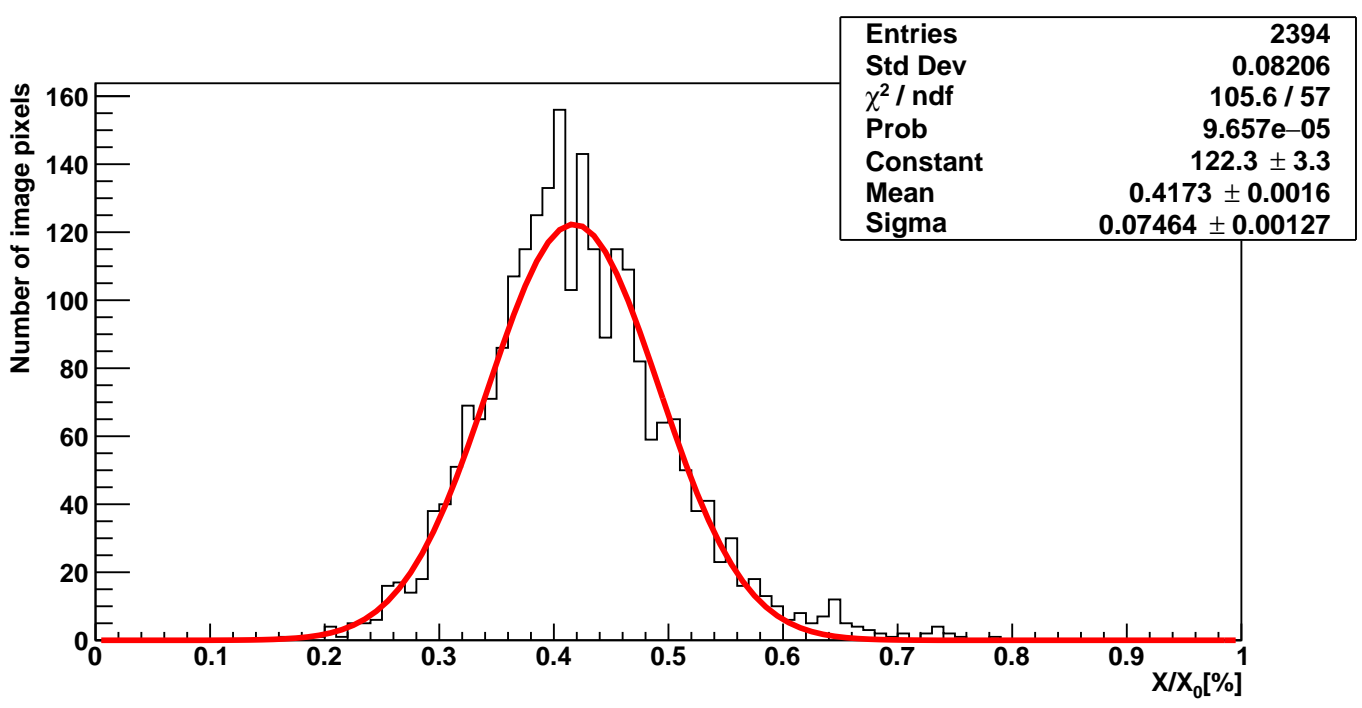

Figure 7.31.: Distribution of all measured $X / X_{0}$ values in the highlighted area in fig. 7.30. The radiation length of the carbon foam and its uncertainty was determined by a Gaussian fit. 
In addition to the measurement of the ATLAS ITk prototype, another measurement of a foam sample was conducted. Fig. 7.30 depicts the image of the carbon foam layer on the left side and air on the right side. As can be seen the image is rather noisy ${ }^{10}$, which is expected because of the suboptimal telescope setup and consequential increase of the variance of radiation length values. The carbon foam has a thickness of $X=7.86 \mathrm{~mm}$ and an expected radiation length constant of $X_{0}=1856 \mathrm{~mm}$ due to its low density. The expected radiation length value is therefore $0.423 \%$. The black box indicates all pixels that are used to determine the radiation length of the material. Fig. 7.31 depicts the radiation length values of all 2394 image pixels that lie within the highlighted area. The radiation length of the foam can be determined by a Gaussian fit of the distribution. The determined radiation length value is

$$
X / X_{0}=0.417 \pm 0.002 \%
$$

The uncertainty of the mean value corresponds to the statistical uncertainty of the fit. The measured value is therefore slightly smaller than the expectation value of $0.423 \%$. The corresponding radiation length constant of this material, when considering a thickness of $7.86 \mathrm{~mm}$, is

$$
X_{0}=1884 \pm 7 \mathrm{~mm} \text {. }
$$

Accordingly, the measurement shows that the expected value of $1856 \mathrm{~mm}$ underestimated the radiation length constant of the carbon foam. The deviation from the expected value corresponds to approximately four times the statistical uncertainty of the measurement. However, as the scattering material is porous foam a part of the deviation may be caused by fluctuations in its density and, consequently, $X_{0}$ profile.

\subsection{Measurements on a Belle II PXD mechanical sample}

The data set analysed in this section was recorded in March of 2015 at the DESY beam test facilities. The goal of the beam tests was to confirm expected radiation lengths profiles of prototype sensors of the Belle II pixel detector (PXD). The beam test results led to improvements on several parts of the PXD detector model. The most important updates will be explained and a comparison between the material distributions of the two detector models and the radiation length measurements will be presented at the end of this section. Additional information on the Belle II vertex detector system and the PXD in particular can be found in section 2.2.3. This section presents measurements of interesting detector regions in comparison to Geant4 simulations [78] of the detector model of the PXD. The detector simulations are conducted in the Belle II analysis framework (basf2) [39]. A visualisation of the Geant4 detector model can be acquired

\footnotetext{
${ }^{10}$ In the region of the foam the standard deviation of the measurements is approximately $0.07 \% X / X_{0}$ at mean values of approximately $0.42 \% \mathrm{X} / \mathrm{X}_{0}$
} 


\section{Radiation length measurements at DESY}

by employing a material scan in basf2.

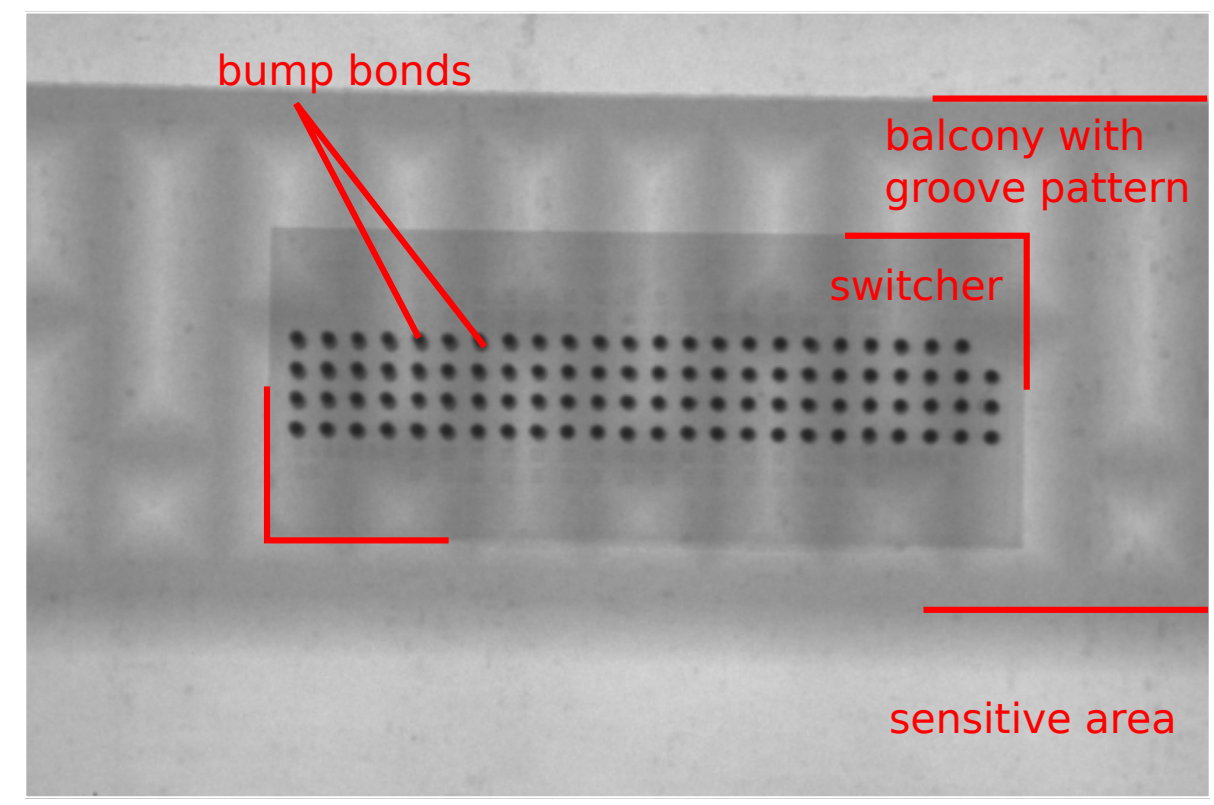

Figure 7.32.: X-ray of the balcony of the PXD mechanical prototype module in the vicinity of a switcher. The periodic groove pattern on the balcony was generated with the deep anisotropic etching technique [34] to reduce the overall mass of the module.

Radiation length measurements were conducted on two different scattering targets: The first target was a PXD mechanical sample with a thinned down sensitive area and silicon balconies on which PXD specific ASICs, called switchers, were located. An X-ray image of the region surrounding a switcher is depicted in fig. 7.32. Underneath the switcher, four rows of bump bonds are visible. Via deep anisotropic etching [34], a periodic groove structure is formed in the silicon balcony region with a maximum thickness of $525 \mu \mathrm{m}$. The balcony region lies within the acceptance region of the Belle II experiment as explained in section 2.2.3 and due to very strict radiation length requirements, a reduction of material in the balcony region is mandatory. The second target was an inoperable PXD 9 module with two sets of different ASICs, which are called drain current digitizer $(D C D)$ and data handling processor $(D H P)$. The locations of the DHP, DCD and switchers on the PXD modules are displayed in fig. 2.6a. The ASICs include patterns of bump bonds, which are located underneath the chips.

The DQM plots in the appendix D.6 indicate that the telescope calibration, including the alignment and cluster resolution measurements, did not work as well as for other beam tests. The main problem is, that the telescope was calibrated with an aluminium calibration target present between the telescope arms instead of air. As explained in section 7.5 this would pose no problem if the material was homogeneously thick and 
affected all tracks in the same way. However, the aluminium calibration target was designed to have several material steps with different thicknesses as can be seen in the schematic drawing in fig. 7.33 and the radiation length image in fig. 7.33.

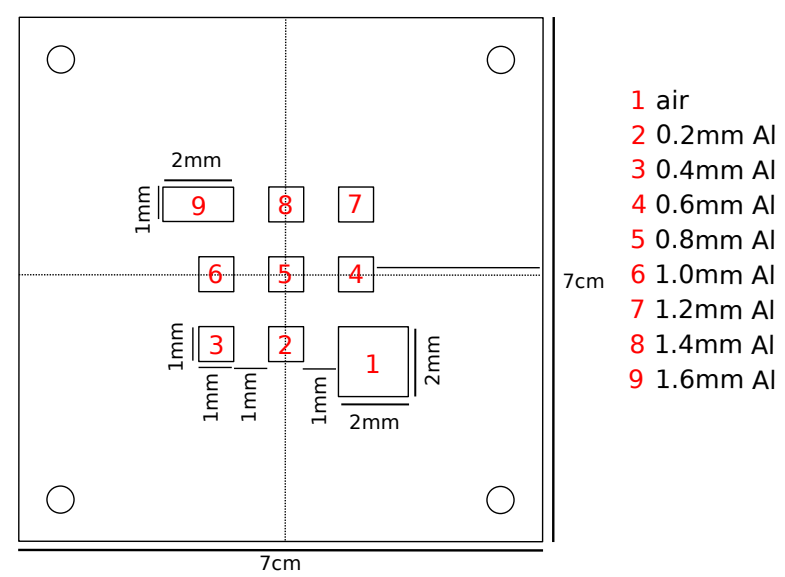

Figure 7.33.: Schematic view of a dedicated calibration target, which was used during beam tests in 2015. The target consists of 9 aluminium layer with a thickness of $200 \mu \mathrm{m}$, which are arranged in such a way that nine measurement areas with different aluminium thicknesses are available.

There are 10 different material regions ranging from air over $0.2 \mathrm{~mm}$ aluminium to $1.8 \mathrm{~mm}$ of aluminium. During the telescope calibration the target plane is modelled as a homogeneous aluminium layer with a thickness of $1.4 \mathrm{~mm}$, which roughly corresponds to the average thickness of the material in the beam spot area. Consequently, the tracks which are traversing the material plane in regions with small aluminium thickness or even air will have largely overestimated covariance matrix entries. The opposite case is the underestimation of the covariance matrix entries of tracks that are traversing the full $1.8 \mathrm{~mm}$ of aluminium. Wrong assumptions about the track covariances have a negative impact on the quality of the telescope calibration. This is visible in most of the DQM plots: Shifts of the M26 z positions are very large and always positive as displayed in fig. 7.34a. The track $p$ value distribution has large peaks at one and zero as can be seen in figure 7.34b. Tracks with either very large or very small $p$ values are most likely traversing a target region which is insufficiently modelled by the average aluminium thickness of $1.4 \mathrm{~mm}$ such as the air hole or the area with $1.8 \mathrm{~mm}$ of aluminium.

Despite these problems during telescope calibration, the angle reconstruction was successful and an average of five six-hit-tracks were found per event. However, another issue occurred during the radiation length calibration: The first, third and fourth M26 sensors were found to have multiple dead rows and columns as can be seen in the example hit map on telescope plane 2 in fig. 7.35. These areas of dead telescope pixels are visible as lines with increased radiation length in the resulting $X / X_{0}$ images (see fig. 7.38). 


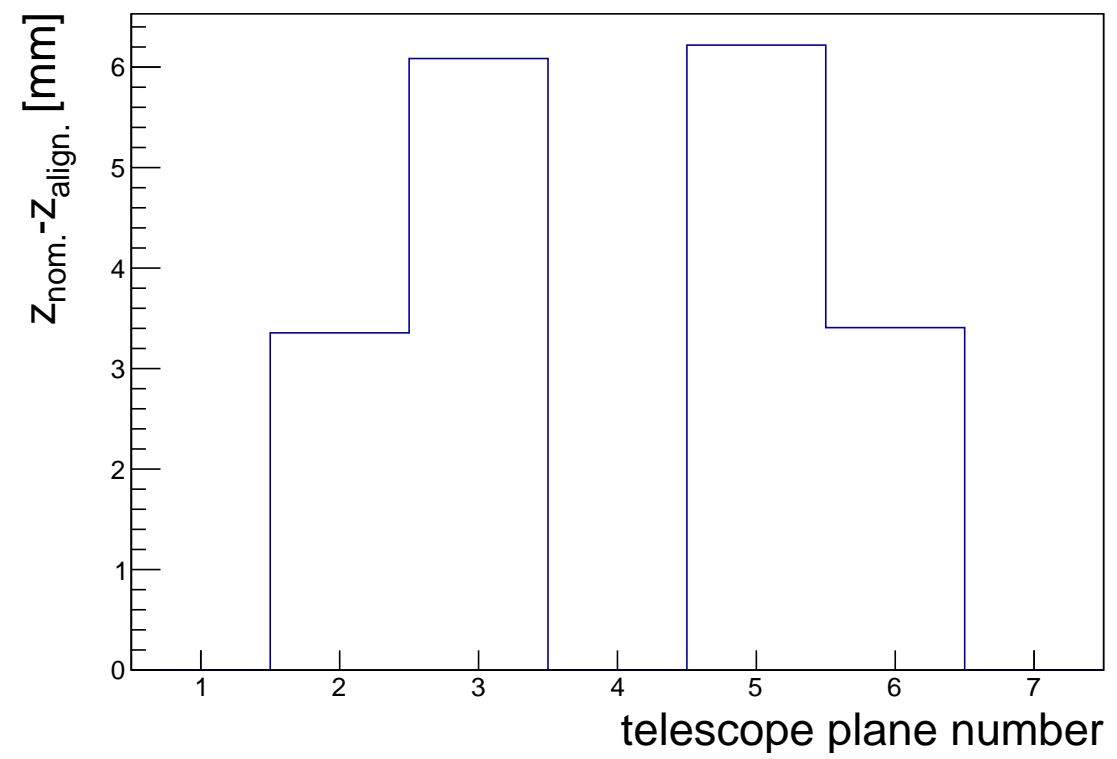

(a) Difference between nominal and aligned sensor $z$ positions

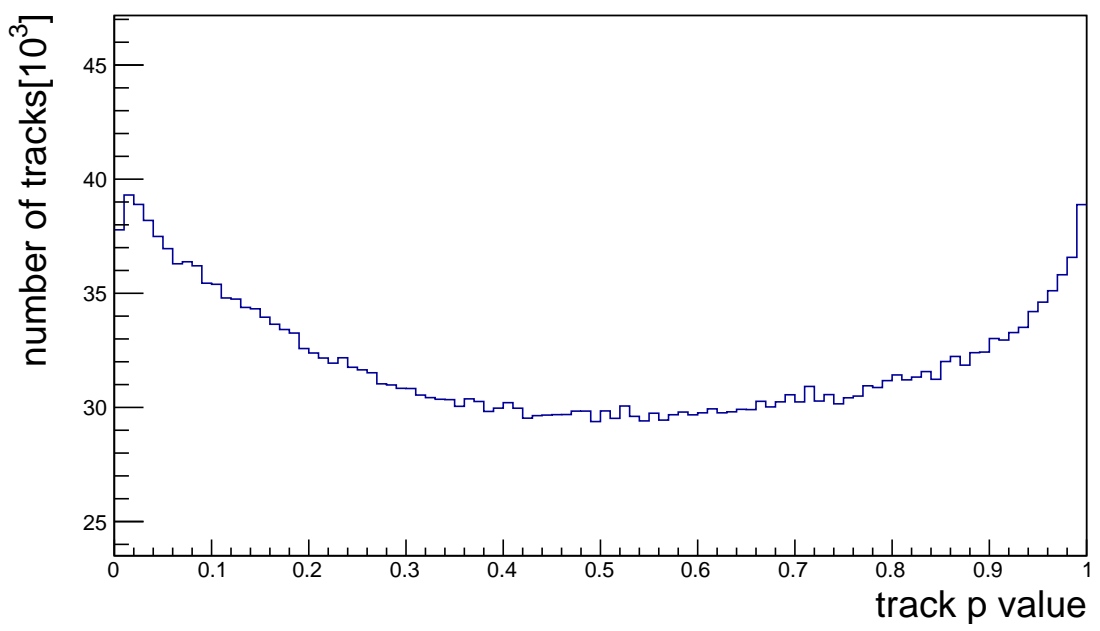

(b) $p$ values of the track sample

Figure 7.34.: DQM diagrams of the $2 \mathrm{GeV}$ data sample indicating problems during the telescope calibration. The differences between the nominal and aligned sensor position are in the order of several millimetres and all in the same direction relative to the nominal position. The track $p$ value distribution has peaks at zero and one. The tracks in both regions are expected to under- (values close to 0 ) or overestimate (values close to 1) the material of the scattering target. 
The most pronounced vertical lines are located at $u=2 \mathrm{~mm}, u=8 \mathrm{~mm}$ and the most prominent horizontal line lies at $v=-1 \mathrm{~mm}$.

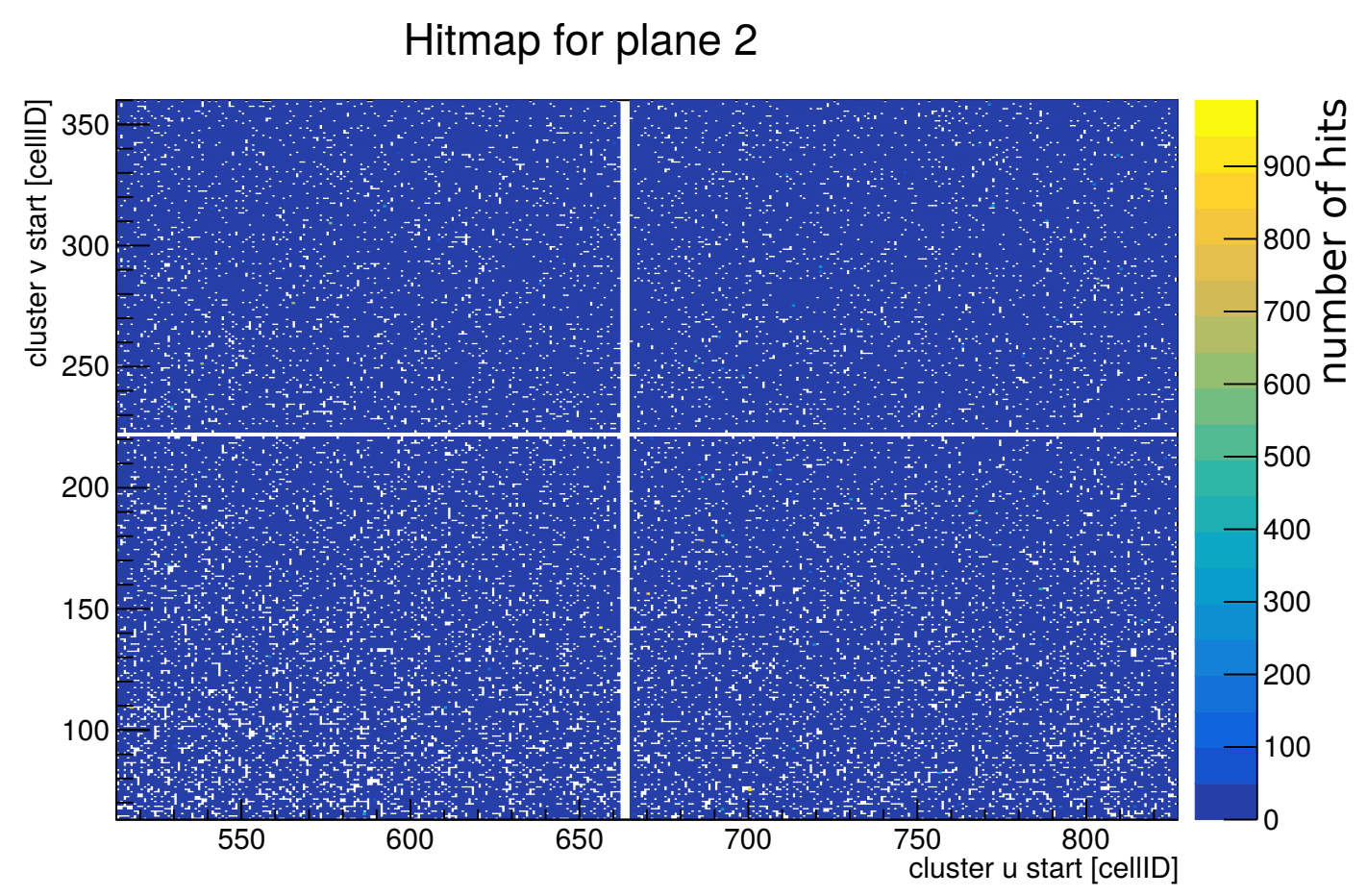

Figure 7.35.: Digit map for the second telescope plane in the column range between 500 and 850 and the row range between 50 and 350 during the telescope calibration of the March 2015 data set. The empty rows and columns were found to be dead pixels, which never produced a signal above threshold.

Unfortunately, some of these lines are located in the vicinity of the designated calibration measurement areas such as the air hole in the calibration grid as can be seen in fig. 7.36. For better visibility the row and columns with increased radiation length values are indicated by red lines in the images. In order to avoid compromising the $X / X_{0}$ calibration, alternative calibration areas were used instead of the designated measurement areas. Five measurement areas on the aluminium calibration target with a thickness of $1.8 \mathrm{~mm}$ and five air measurements were employed for the $X / X_{0}$ calibration. The position of the measurement areas is depicted in fig. 7.36a and 7.36b.

Using this alternative radiation length calibration strategy worked reasonably well, as can be seen from the measurements of the target thickness in fig. 7.37. However, the calibration factors $\kappa$ and $\lambda$ (see table 7.7) are unusually large. These large values are most likely caused by the problematic telescope calibration step: In order to correct for errors in the alignment and cluster resolution, the calibration factors have to differ 


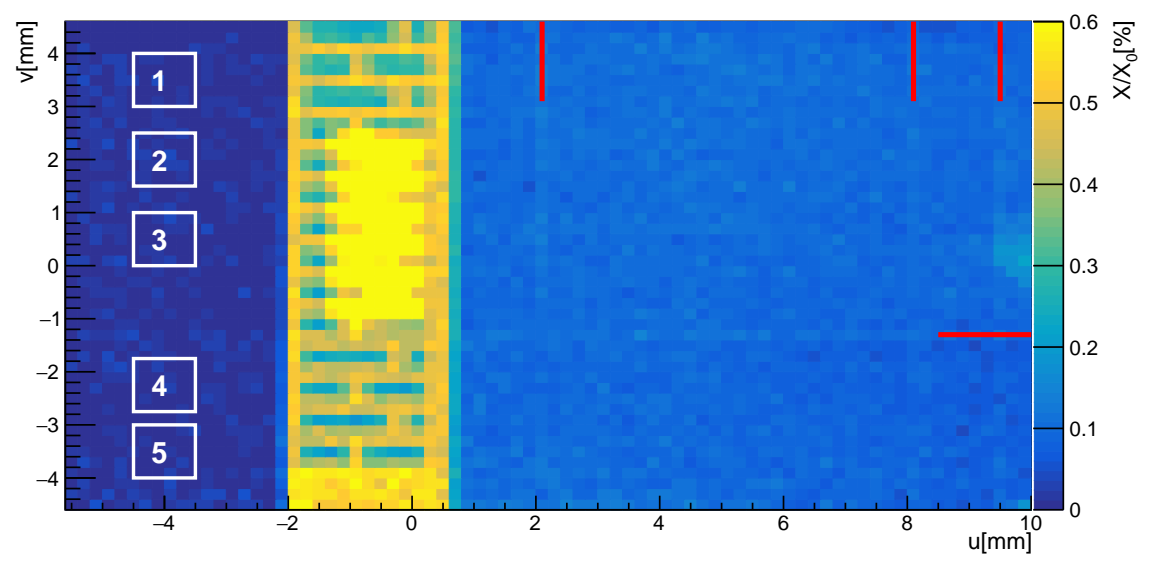

(a) PXD mechanical sample with air measurement areas. The image has a pixel pitch of $200 \mu \mathrm{m}$.

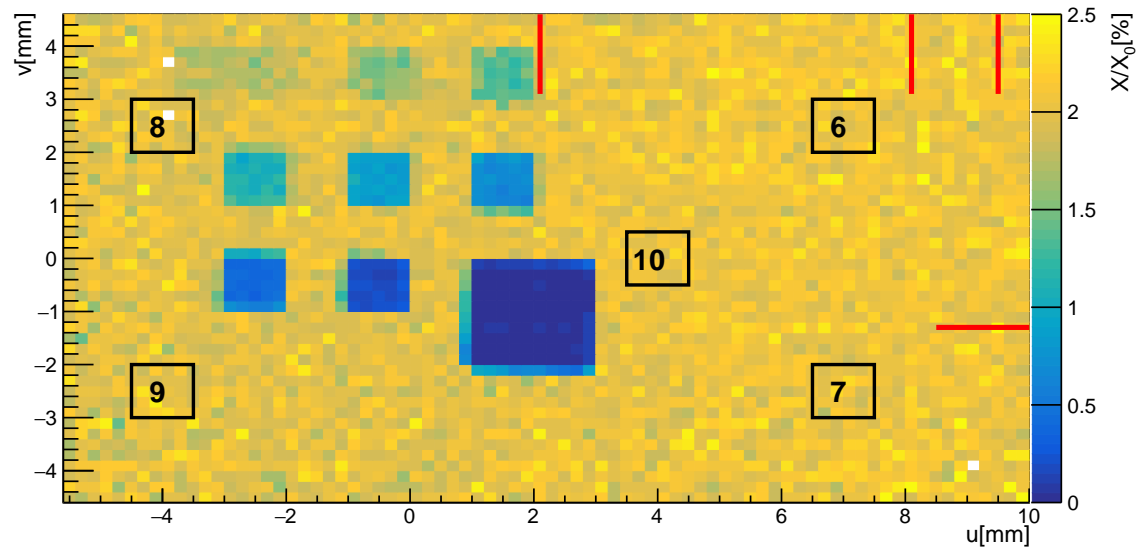

(b) calibration target with $1.8 \mathrm{~mm}$ aluminium measurement areas. The pixel pitch is $200 \mu \mathrm{m}$.

Figure 7.36.: Alternative measurement areas used for radiation length calibration measurements. The designated measurement areas were not used in order to avoid disturbances from the rows and columns with increased radiation length values indicated by red lines.

widely from the expectation values of 1.0.

Fig. 7.38 depicts radiation length images of the PXD dummy module at nominal beam energies of 2 and $4 \mathrm{GeV}$. The $4 \mathrm{GeV}$ data set has rather small statistic. In order to avoid unstable angle fits due insufficient statistics, a large image pixel pitch of $200 \mu \mathrm{m}$ was selected. On the left hand side of the images, air can be seen. The balcony with a silicon groove pattern is located in the centre of the image between $u=-2 \mathrm{~mm}$ and $u=0.5 \mathrm{~mm}$. The switcher corresponds to the yellow rectangle on the balcony between 
7.6. Measurements on a Belle II PXD mechanical sample

\begin{tabular}{|c|c|c|c|c|}
\hline Beam energy & $\lambda$ & $\kappa$ & $\Delta_{u} p[\mathrm{MeV} / \mathrm{mm}]$ & $\Delta_{v} p[\mathrm{MeV} / \mathrm{mm}]$ \\
\hline $2 \mathrm{GeV}$ & $1.208 \pm 0.007$ & $0.917 \pm 0.007$ & $9 \pm 3$ & $-2 \pm 7$ \\
\hline $4 \mathrm{GeV}$ & $1.151 \pm 0.007$ & $0.927 \pm 0.007$ & $22 \pm 6$ & $2 \pm 13$ \\
\hline
\end{tabular}

Table 7.7.: Beam test March 2015 calibration results for 2 and $4 \mathrm{GeV} . \lambda$ is the calibration factor of the telescope resolution. $\kappa$ is the calibration factor of the scattering angle width and $\Delta_{u} p$ and $\Delta_{v} p$ correspond to beam energy gradients in $u$ and $v$ direction. The given errors correspond to the statistical uncertainties of the fit.

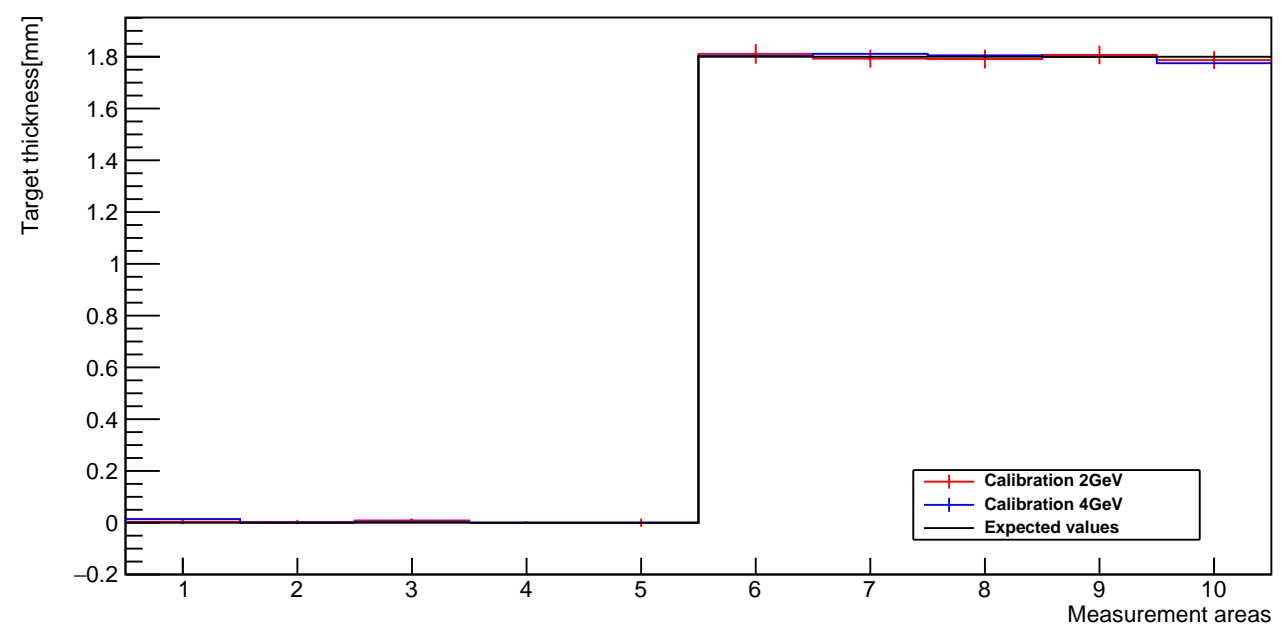

Figure 7.37.: Air and $1.83 \mathrm{~mm}$ aluminium thickness measurements after the radiation length calibration procedure. A comparison between the measurements with beam energies of 2 and $4 \mathrm{GeV}$ is shown.

$v=-1 \mathrm{~mm}$ and $v=2.5 \mathrm{~mm}$. On the right hand side, the thin sensitive area of the PXD is visible. The expected thickness of the sensitive area is $75 \mu \mathrm{m}$ silicon [33], which corresponds to a radiation length of $0.08 \%$. However, as the measurement was performed on a mechanical sample, the real radiation length value might deviate slightly from the expected value.

The black boxes highlight an area with image pixels, that were employed to determine the radiation length of the sensitive area. The measurement area was placed at this position to avoid any influence of the lines with increased $X / X_{0}$ values, which are caused by dead rows and columns on several M26 sensors. For orientation the positions of these rows and columns are highlighted with red lines in the images. The results of the radiation length measurement are shown in fig. 7.39. The determined radiation length values of 


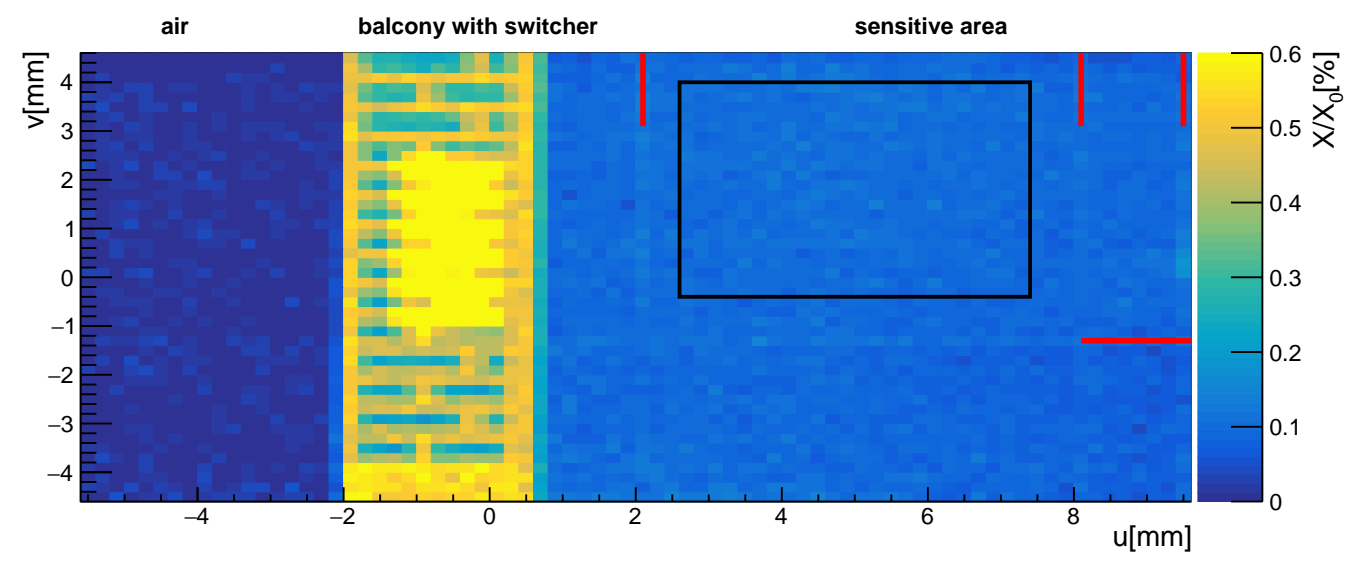

(a) Beam energy: $2 \mathrm{GeV}$

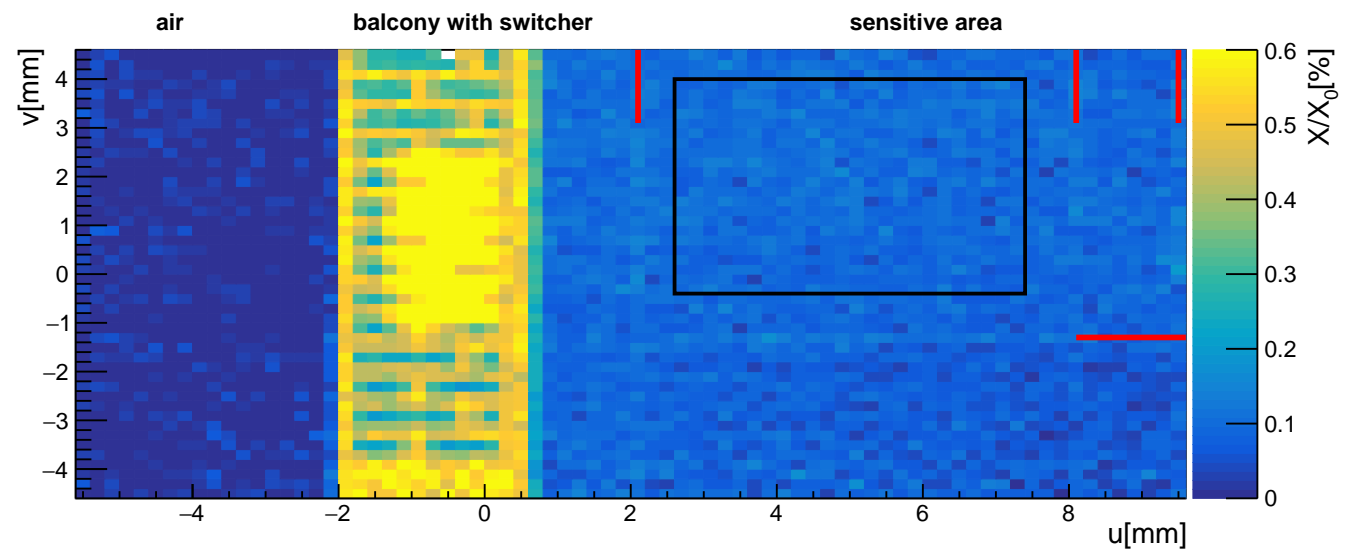

(b) Beam energy: $4 \mathrm{GeV}$

Figure 7.38.: Radiation length images of the PXD dummy module at beam energies of $2 \mathrm{GeV}$ (top) and $4 \mathrm{GeV}$ (bottom). In both images air, the silicon balcony with a switcher and the sensitive area are visible. The image pixel pitch is $200 \mu \mathrm{m}$.

the measurement at a beam energy of $4 \mathrm{GeV}$ form a slightly broader distribution due to the smaller number of scattering angles per image pixel. Additionally as explained in section 5.5, the signal-to-noise ratio of radiation length measurements of two identical materials is worse for the measurement with a higher beam energy. As this measurement is conducted on an extremely thin material, the worse signal-to-noise value will lead to an increased statistical error. Since the statistical uncertainties of the individual radiation length measurements is larger in the $4 \mathrm{GeV}$ case, the corresponding $X / X_{0}$ distribution in fig. 7.39 is slightly broader. The measured radiation length values are: 


$$
\begin{array}{ll}
X / X_{0}=0.1022 \pm 0.0005 \% & (\text { measurement at } 2 \mathrm{GeV}) \\
X / X_{0}=0.1004 \pm 0.0008 \% & (\text { measurement at } 4 \mathrm{GeV})
\end{array}
$$

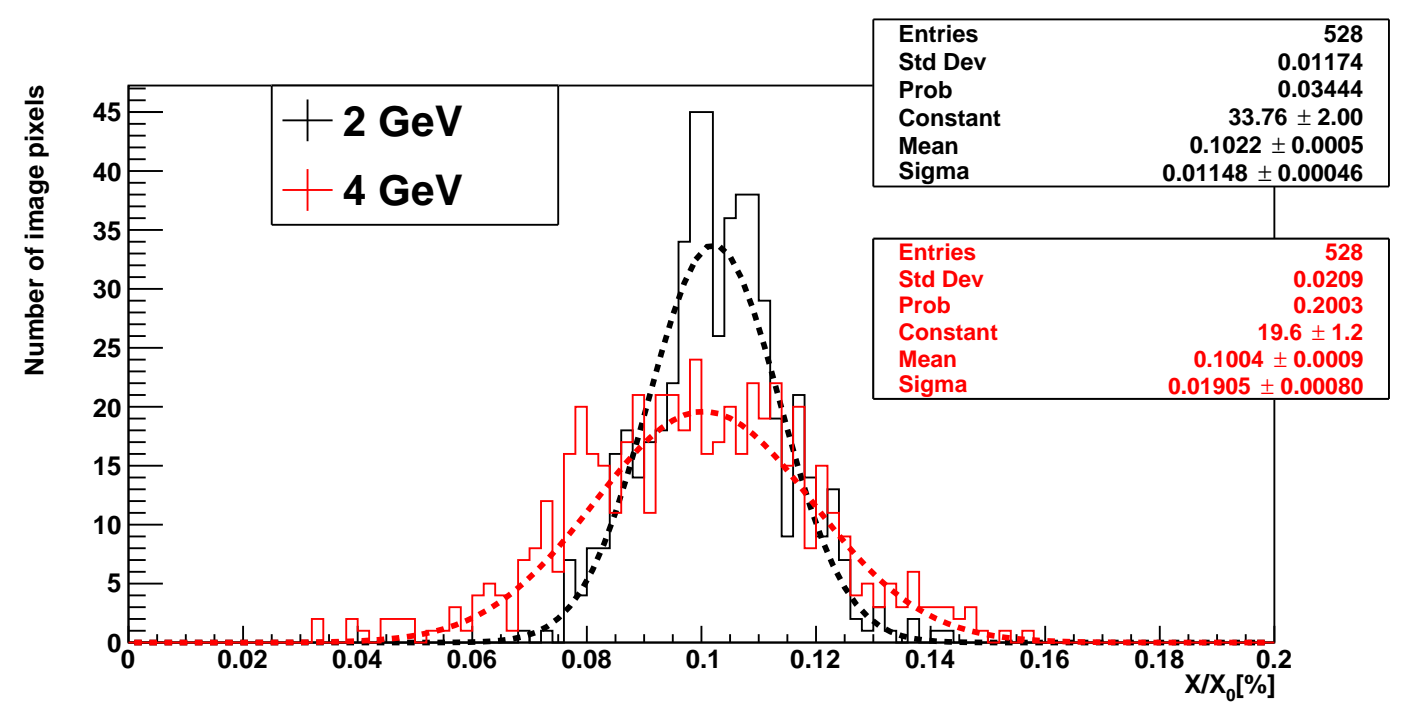

Figure 7.39.: $X / X_{0}$ distribution of the 2 and $4 \mathrm{GeV}$ measurements in the highlighted areas in fig. 7.38. The number of scattering angles per angle histogram was smaller during the $4 \mathrm{GeV}$ measurement (red). Consequently, the statistical uncertainty of the fitted $X / X_{0}$ values is larger and the distribution of the measurements is slightly broader.

These values are not quite compatible with each other. However, both measured values are larger than $0.1 \% X / X_{0}$ and differ widely from the expected value of approximately $0.080 \%$. There are two possible explanations for the observed difference. The first explanation is, that the issues encountered during the telescope and $X / X_{0}$ calibration was not fully absorbed by the radiation length calibration parameters, which leads to a small bias in the radiation length measurements. However, this explanation seems unlikely as the radiation calibration self-consistency measurement in fig. 7.37 worked well and a large deviation was observed for two different beam energies. Another explanation is that the real thickness of the sensitive area of the mechanical PXD sample really is slightly larger than expected. This would not be surprising as the measured object is a sample and not a final PXD ladder. Unfortunately, the thickness of the sensitive area could not be measured manually as it is extremely thin and fragile.

The radiation length image in fig. 7.40 depicts the end-of-staves (EOS) region of the other scattering target, the broken PXD 9 module. Six ASICs with bump bonds are visible, three $D C D s$ and three DHPs. The dark blue area on the right side is air. The material between the ASICs is expected to be to $525 \mu \mathrm{m}$ of silicon [33]. Image pixels 


\section{Radiation length measurements at DESY}

inside the highlighted black box were used to determine the radiation length. The corresponding $X / X_{0}$ distribution is depicted in fig. 7.41. The radiation length measurement of the silicon in the EOS region at a beam energy of $2 \mathrm{GeV}$ yields the following results:

$$
X / X_{0}=0.524 \pm 0.001 \%
$$

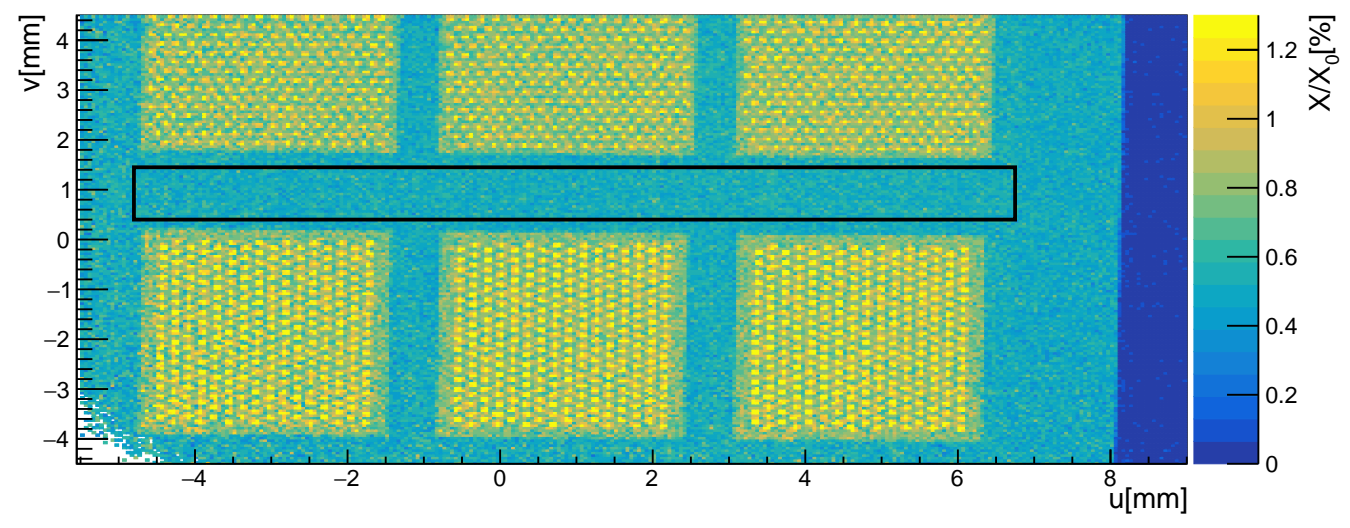

Figure 7.40.: End-of-staves region on the broken PXD 9 module. Three DCDs (top) and three DHPs (bottom) and bump bonds below the ASICs are visible. The silicon between the chips is expected to have a thickness of $525 \mu \mathrm{m}$. The image pixel pitch is $50 \mu \mathrm{m}$.

The measured value is much smaller than the expected value of approximately $0.56 \%$ corresponding to $525 \mathrm{\mu m}$ of silicon. However, the relative deviation from the expected value here is smaller than for the sensitive area measurement.

The measurement results in eq. 7.4 , eq. 7.5 and eq. 7.6 were found to have relative deviations from the expected values of 5 to $20 \%$. There are two possible explanations for the discrepancies. First of all, the telescope calibration and the subsequent radiation length calibration were conducted under suboptimal conditions. Especially the distribution of the $z$ shifts after the alignment and the track quality plots indicate major problems. Typically, the radiation length calibration parameters are used to absorb systematic offsets caused by faulty telescope calibration measurements. However, as the calibration was performed on air and thick layers of aluminium, it is possible that the radiation length calibration leads to reliable results for $1.8 \mathrm{~mm}$ of aluminium, but yields biased results for 75 and $525 \mu \mathrm{m}$ of silicon.

Another possibility is that the material distributions of the mechanical sample module and the broken PXD simply did not match some of the specifications of final Belle II 


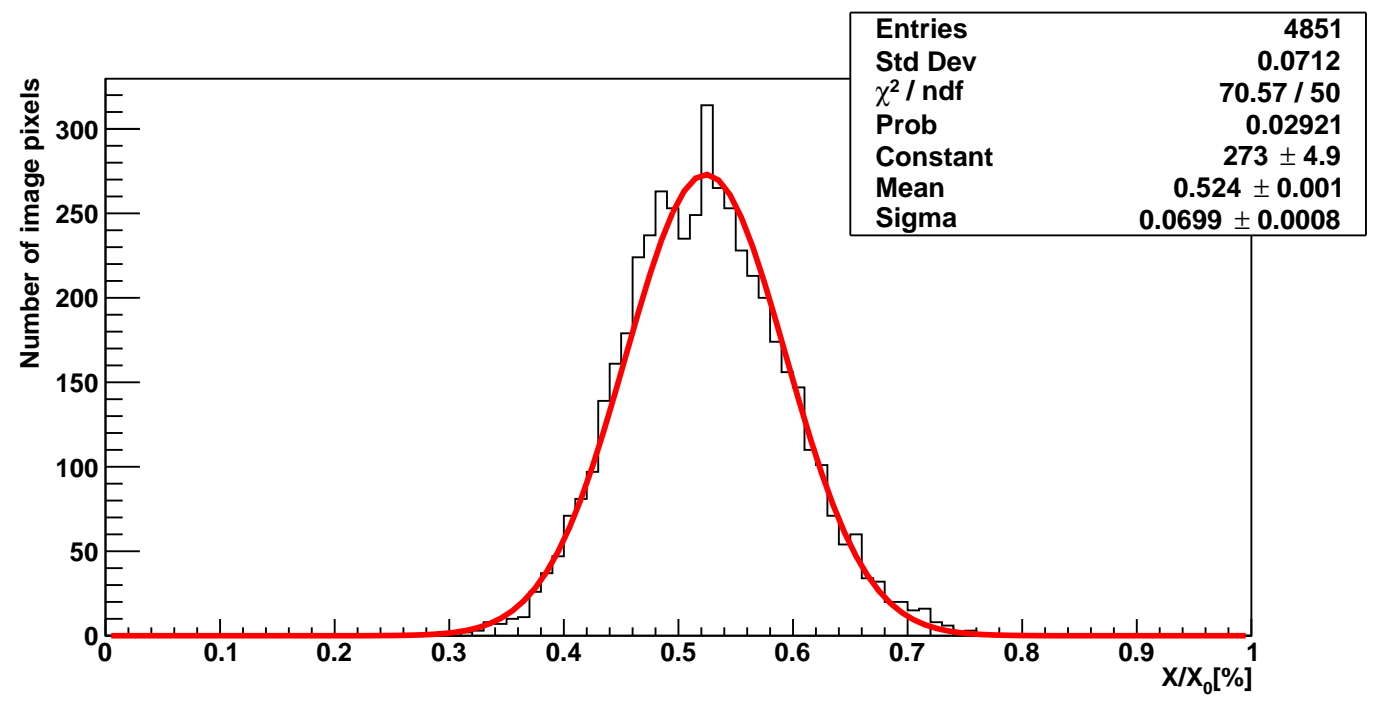

Figure 7.41.: $X / X_{0}$ distribution measurements on $525 \mu \mathrm{m}$ of silicon (see highlighted area in fig. 7.40) with a beam energy of $2 \mathrm{GeV}$.

PXD modules. This would also explain why one measurement over- and another underestimates the radiation length value. Several of the most noticeable differences between the modules used as scattering objects in this beam test and final PXD modules will be discussed in the last part of this section. Regardless of the cause of the deviations, even under these suboptimal circumstances, the radiation length measurement yields results that deviate from the expected value by approximately $20 \%$ in the case of the extremely thin $75 \mu \mathrm{m}$ of silicon and $5 \%$ in the case of $525 \mu \mathrm{m}$ of silicon.

In order to compare the radiation length measurements with the detector model a material scan in basf2 is used [39]. The simulation and visualisation of the detector model employs a Geant4 simulation [78]. The simulation involves shooting Geantinos through the simulated PXD half-ladder on a dense grid. Geantinos are artificial particles used in Geant4, that register all material contributions along its way, but do not interact with surrounding matter. The sum of material contributions along its way is calculated and by assigning the radiation length value to the corresponding grid point, a $2 \mathrm{D}$ image of the material distribution is generated.

A high-resolution $X / X_{0}$ image of the balcony and the switcher at a beam energy of $2 \mathrm{GeV}$ together with a simulated material scan image of roughly the same field of view is depicted in fig. 7.42. The image pixel pitch and the step length of the material scan are both $50 \mu \mathrm{m}$. This resolution is sufficient to resolve the solder bump bonds with an expected diameter of $80 \mu \mathrm{m}$ beneath the switcher in the $X / X_{0}$ image. The bump bonds are visible as a regular pattern of yellow pixels in fig. 7.42a. There are three columns with 


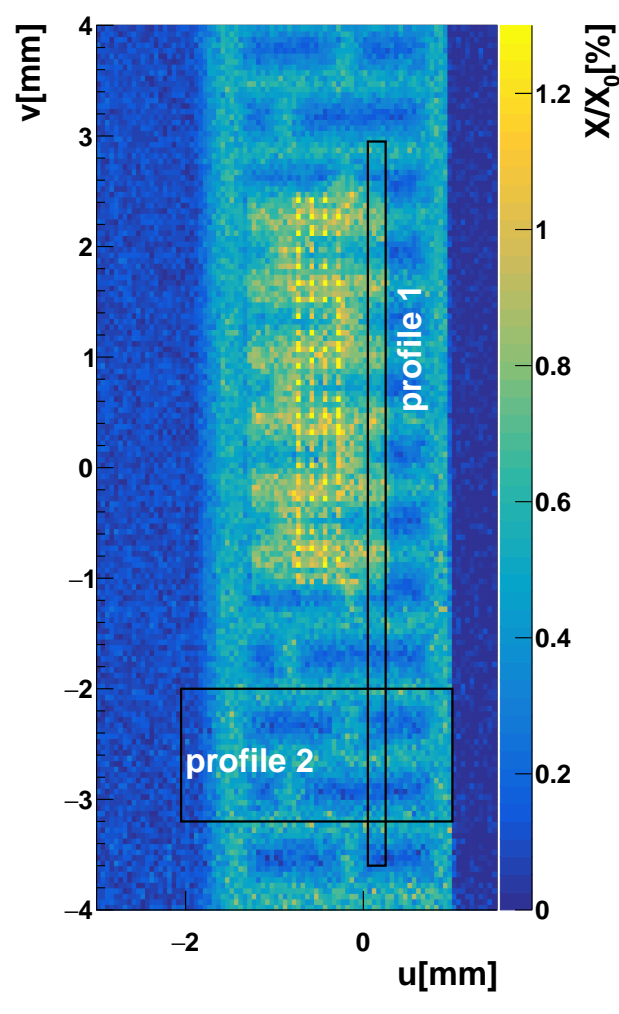

(a) Radiation length image

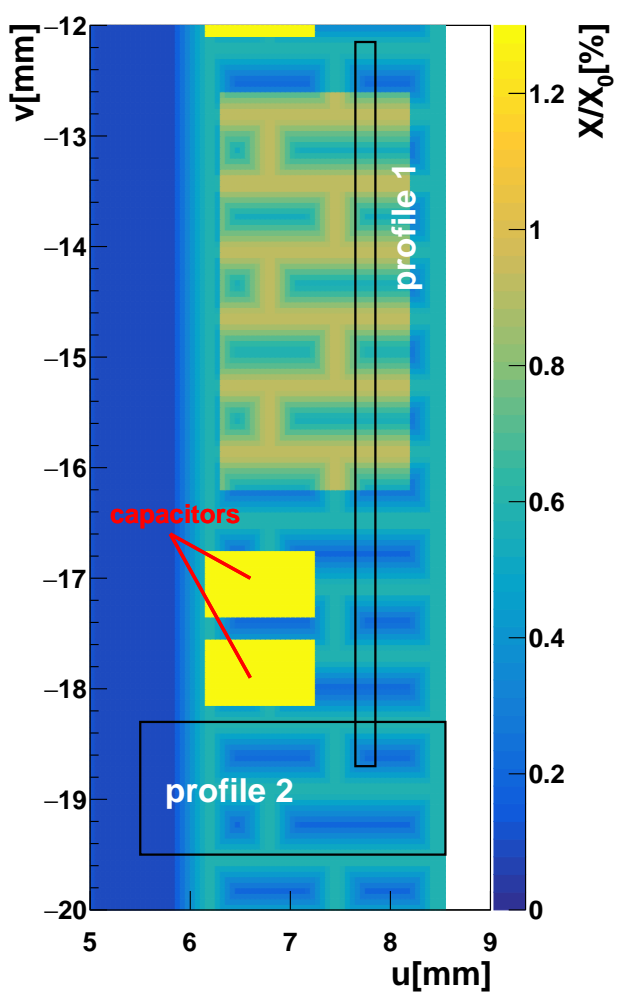

(b) Simulated detector model

Figure 7.42.: Radiation length image of the balcony of the PXD mechanical sample module (a) and material scan of a similar field of view of the detector model after update (b). In both pictures the switcher is clearly visible. The radiation length image also shows the 4 columns of bump bonds, which can also be seen in the X-ray image in fig. 7.32. The balcony has a periodic groove pattern to reduce the average radiation length of the module.

24 bump bonds and one column with 23 bump bonds. The absence of the single bump bond in the fourth column is also visible in the X-ray (fig. 7.32). The overlay of bump bonds and grooves in the silicon balcony reduces the visibility of some bump bonds. The distance between two bump bonds is expected to be $150 \mu \mathrm{m}$ in both directions. This corresponds to three image pixels with a pitch of $50 \mu \mathrm{m}$, which matches the distance between bump bonds in the radiation length image. As bump bonds are not included in the Geant4 detector model, they are not visible in the material scan image (7.42b). The detector model features some capacitors on the balcony in the vicinity of the switcher. Three of them are visible as rectangle regions with a highly increased radiation length. The balcony of the PXD mechanical sample, unlike the final PXD modules, was not 
equipped with capacitors. They are therefore not present in the radiation length image.

The material scan depicted in fig. 7.42b corresponds to the version of the Geant 4 detector model after an update in 2016. Two black boxes highlight the area of two numbered material profiles. Radiation length profiles provide a good opportunity to compare the radiation length measurements to the detector model before and after the update. A comparison between the detector before and after the update can be seen in fig. 7.45. A summary with all changes will be given at the end of this section.

Profile 1 shows the material distribution along the silicon balcony in $v$ direction. This profile also includes a switcher. Profile 2 shows the material distribution in $u$ direction from the sensitive area over the balcony to the edge of the module. As can be seen from the images, the grooves follow a clear pattern in $v$ direction: Two medium sized grooves above a combination of a long and a short groove. In order to measure the influence of both groove configurations, a complete groove period was used to calculate the average $X / X_{0}$ values in profile 2 .

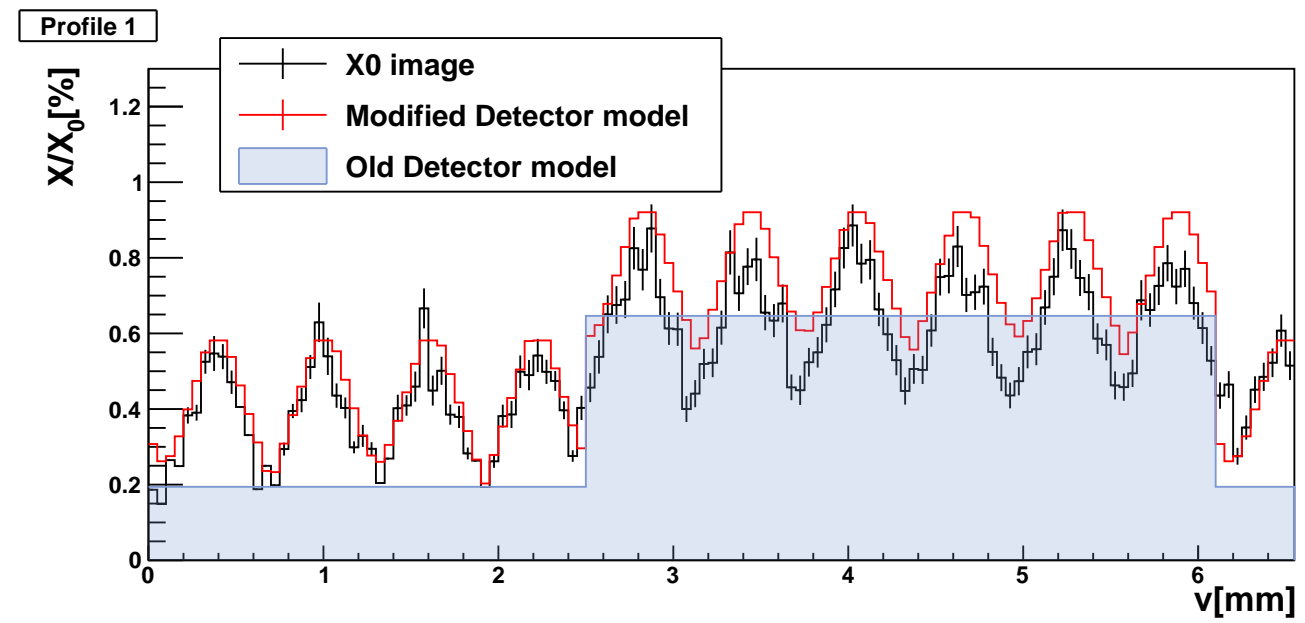

Figure 7.43.: Radiation length profile 1 along the silicon balcony of the PXD. The groove pattern of the radiation length measurement matches the pattern visible in the updated detector model. The location of profile 1 is shown in fig. 7.42.

Profile 1 is depicted in fig. 7.43. The radiation length measurement of the grooves outside the switcher area matches the updated detector model. Measurements of the radiation length image are compatible with the modified material scan distribution in this region. In the switcher region, values from the $X / X_{0}$ image are underestimated by a constant value of approximately $0.1 \% X / X_{0}$. As mentioned before, the radiation length measurement was conducted on a mechanical sample. The switcher visible in the radiation length image is not a final ASIC as used in the Belle II experiment. It is therefore likely that the switcher was slightly thinner than the expected $300 \mu \mathrm{m}$. As can 


\section{Radiation length measurements at DESY}

be seen from fig. 7.42, the width of the switcher does not match the finalised ASIC in the detector model either. In the radiation length image the $u$ side length is approximately $1.5 \mathrm{~mm}$, while the side length in the material scan of the modified detector model is $1.9 \mathrm{~mm}$. Regardless of small differences between the radiation length measurements and the updated detector model, the old detector model describes the balcony $v$ profile in insufficient detail and in several regions incorrectly. The grooves are not modelled at all, but instead the value in the area of the grooves is set to approximately $0.2 \%$, which is much smaller than the average radiation length of the balcony. Additionally, the thickness of the switcher is $400 \mu \mathrm{m}$ in the old detector model, which is $100 \mu \mathrm{m}$ too thick. It was most likely implemented for a previous version of the switcher with a larger thickness.

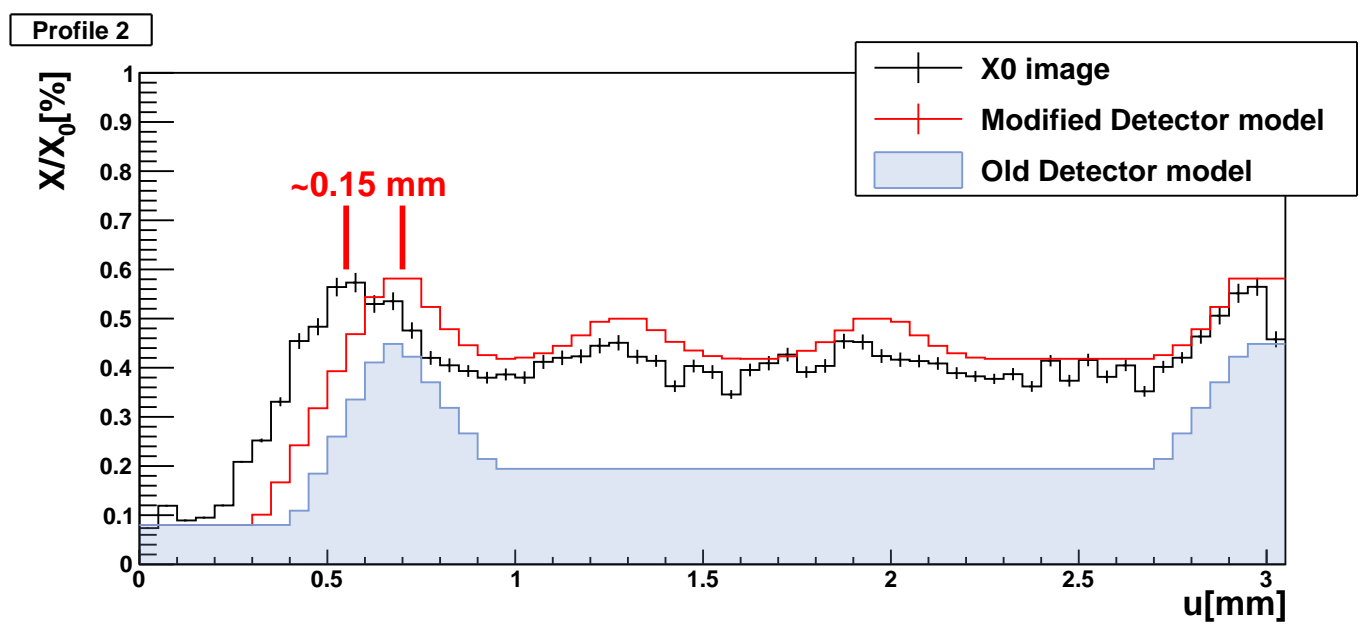

Figure 7.44.: Radiation length profile 2 across the silicon balcony of the PXD. The balcony in the radiation length measurement is visibly longer than the balcony of the detector model. The location of profile 2 is shown in fig. 7.42.

The comparison of material profile 2 is depicted in fig. 7.44. The most noticeable difference between radiation length measurement and updated detector model is the balcony width: The balcony of the PXD mechanical sample is approximately 100 to $200 \mu \mathrm{m}$ wider than the balcony of the detector model. The positions of grooves are also slightly shifted. Furthermore, the measured radiation length values are consistently smaller than the values of the updated detector model. This is not unexpected as a comparable measurement in the EOS region of the broken PXD showed a similar deviation.

The radiation length measurements of the balcony revealed several shortcomings of the original detector model. The changes that were undertaken during the detector model update are based on schematic CAD drawings and the specifications summarised in an Belle II internal interface document [33]. Material scan images of a complete outer backward PXD module before and after the detector model upgrade can be seen in fig. 
7.6. Measurements on a Belle II PXD mechanical sample

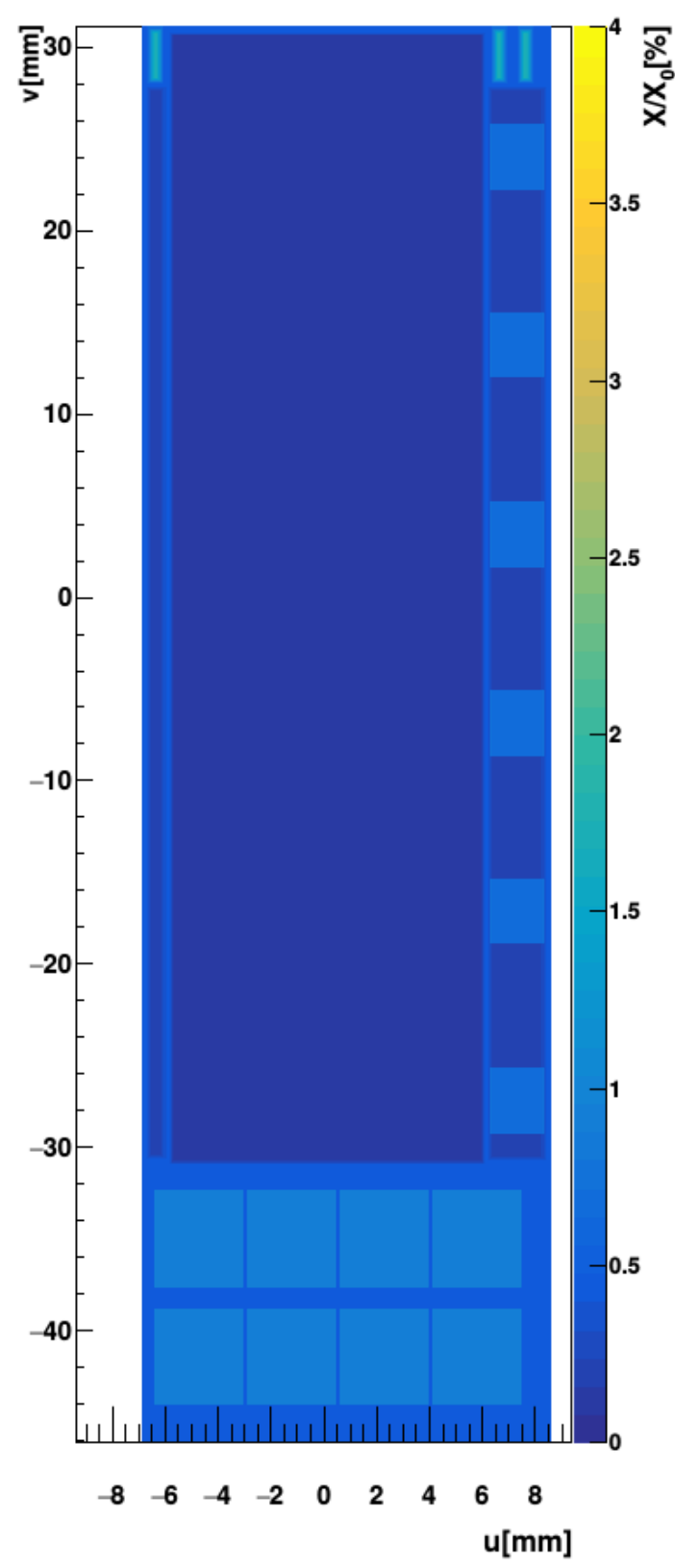

(a)

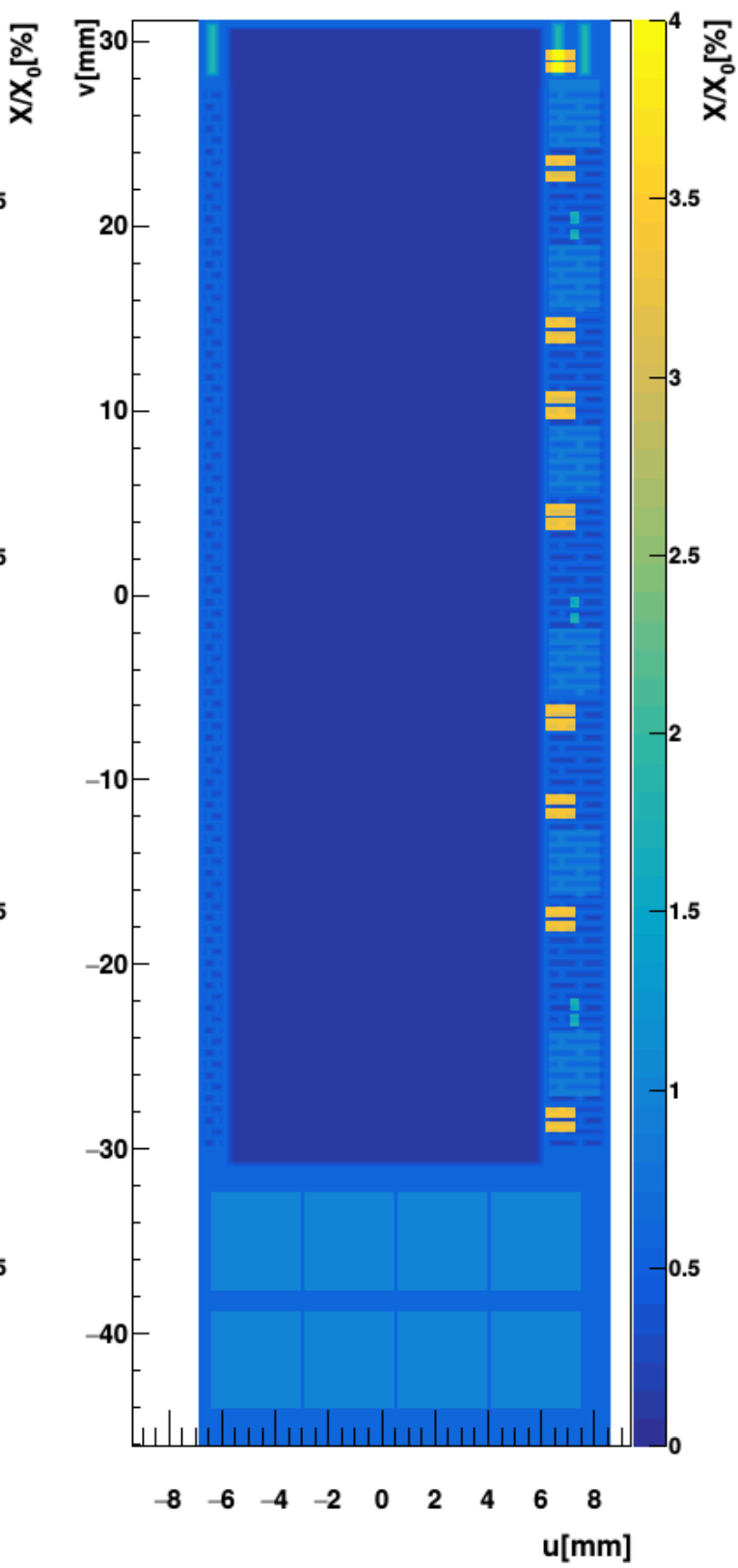

(b)

Figure 7.45.: Material scan images of a outer backward PXD module before (a) and after (b) the update. 


\section{Radiation length measurements at DESY}

7.45. The outer PXD modules in Belle II are located at a radius of $14 \mathrm{~mm}$ from the center of the beam pipe. The sensitive area of the PXD is visible between $u= \pm 6 \mathrm{~mm}$ and $v= \pm 30 \mathrm{~mm}$. In $u$ direction the senstive area is flanked by two silicon balconies. The balcony on the right hand side features a total number of 6 switchers and is much broader than the balcony on the opposite side. At the bottom of the material scan images, the EOS region with four pairs of DCDs and DHPs is visible. All changes between the old and new detector model are summarised in the following list:

1. $3 \mu \mathrm{m}$ copper layers were added to the balcony regions on both sides of the sensitive area. Copper layers cover most of the balcony areas. For the inner PXD layers they have a width $(u)$ of $2.2 \mathrm{~mm}$ on the switcher side and $0.9 \mathrm{~mm}$ on the opposite side. The length $(v)$ of the copper layers is $41.26 \mathrm{~mm}$. In case of the outer PXD modules, the widths are also $2.2 \mathrm{~mm}$ and $0.9 \mathrm{~mm}$, but the length of both copper layers is $58.51 \mathrm{~mm}$.

2. Groove structures were added to the balcony regions on both sides of the sensitive area. There are four different types of groove etchings on the switcher balcony and two different types on the opposite balcony. The length of each groove is $510 \mu \mathrm{m}$. The widths of the grooves range from $190 \mu \mathrm{m}$ to $1.59 \mathrm{~mm}$.

3. The switcher size, thickness and position was corrected to match the final design switchers. The width of the switchers was reduced from $2.035 \mathrm{~mm}$ to $1.9 \mathrm{~mm}$. The thickness of the switchers was reduced from $400 \mu \mathrm{m}$ to $300 \mu \mathrm{m}$.

4. The maximum thickness of the silicon balconies was increased from $450 \mu \mathrm{m}$ to $525 \mu \mathrm{m}$

5. Capacitors were added to the balcony on the switcher side. There are two different capacitor types: The first capacitor type has a width of $1.1 \mathrm{~mm}$, a length of $600 \mu \mathrm{m}$ and a thickness of $542 \mu \mathrm{m}$. The second, smaller capacitor type has the same length, but a width of $300 \mu \mathrm{m}$ and a thickness of $232 \mu \mathrm{m}$. Both capacitors are modelled as consisting of $\mathrm{BaTiO}_{3}$.

The influence of the improved detector model on the vertex resolution and tracking in Belle II has not yet been studied. However, it is expected that at least the changes in the area of the silicon balconies will have a positive impact. Before the update the material budget in the balcony region was largely underestimated as can be seen from the two material profiles in fig. 7.43 and 7.44. The average radiation length was modelled to be approximately $0.2 \%$, while in reality it is approximately $0.45 \%$. 
CHAPTER 8

\subsection{Conclusion}

A method to determine the radiation length of planar objects has been developed in the frame of this thesis. The results presented here showed the successful measurements of material distributions of a wide range of structures. The measurements are based on the precise determination of multiple scattering angles with reconstructed particle tracks in a reference telescope. An accurate representation of materials in detector simulations is an important requirement to understand the detector tracking performance. However, the validation of the material distribution of a detector system against direct experimental measurements is difficult. Radiation length images generated with the presented method can be used to test existing detector models. In the frame of this work, the method was tested in simulation studies as well as in several beam test campaigns. Results from simulations will be summarised first.

For the first simulation study, a set of scattering angle distributions with different radiation length values, particle energies and telescope resolutions was generated from a simple Gaussian model. By fitting these distributions with a model function it was found that the determined radiation length values were slightly biased. However, using a ROOT log-likelihood fit and at least 5000 entries per angle distribution ensures that the relative differences between measured and true radiation length values can be restricted to below $0.5 \%$, except for very thin materials such as $50 \mu \mathrm{m}$ of aluminium. Measurements with a relative bias below $0.5 \%$ of these extremely thin materials require at least 30000 entries per scattering angle distribution.

The second simulation study was conducted with beam test simulations implemented in $T B S W$. It was found that the digital signal measurements of telescope sensors led 


\section{Conclusion and outlook}

to measurement artefacts in the reconstructed scattering angle distributions. These artefacts manifested in the form of periodic peaks in the scattering angle distributions. Fitting such angle distributions to determine $X / X_{0}$ produces radiation length values with a larger variance than expected due to the statistical fit uncertainty. The influence of these artefacts can be reduced by increasing the distance between neighbouring telescope sensors and increasing the size of radiation length image pixels. Additionally, relative rotations between telescope planes around the beam axis reduce the impact of artefacts in scattering angle distributions.

The last simulation study confirmed that the implemented radiation length calibration measurements yield reliable and unbiased results for the calibration factor of the telescope angle resolution $\lambda$ and both beam energy gradients $\Delta_{u} p$ and $\Delta_{v} p$. The calibration factor of the scattering angle width $\kappa$ was found to have a small bias. A subsequent measurement of the target thickness showed that the bias introduced by fits of individual scattering angle distributions was compensated by the bias of $\kappa$. Consequently, the results of the thickness measurements are unbiased. Additionally, beam energy offsets can be measured reliably in the radiation length calibration procedure.

Several beam tests were conducted to test the validity and range of radiation length measurements. In order determine the upper limit of radiation length values which can be reliably investigated with the presented method, measurements on aluminium and copper wedges with a continuously increasing radiation length were conducted. For $X / X_{0}$ values larger than $6 \%$, energy losses due to bremsstrahlung must be considered to ensure accurate radiation length measurements. Energy losses can be included by modifying the fit model by assuming a non-constant beam energy: The nominal beam energy is replaced by a weighted mean of the initial beam energy and the expected energy after a material transition, which depends on the initial energy and the radiation length $X / X_{0}$ of the material. A weight factor $\epsilon$ determines how much of the bremsstrahlung energy loss is considered in the weighted mean.

It was found that for aluminium with a radiation length constant of $X_{0}=88.97 \mathrm{~mm}$ and a thickness up to $30 \mathrm{~mm}$ (corresponding to a radiation length of $33.7 \%$ ) the weight model with $\epsilon=0.3$ describes the radiation length distribution very accurately. Neglecting the influence of bremsstrahlung leads to a large overestimation of the radiation length. For example, a fit using the Highland model without consideration of bremsstrahlung yields a measured radiation length of $39.01 \pm 0.02 \%$ for $30 \mathrm{~mm}$ of aluminium. The weight model with $\epsilon=0.3$ yields a radiation length value of $33.67 \pm 0.02 \%$, which is consistent with the expected value. A weight factor of 0.3 also produces reliable results for measurements on a copper wedge with a much shorter radiation length constant $X_{0}$ of $14.35 \mathrm{~mm}$ and radiation length values of up to $60 \%$. For radiation length values larger than $60 \%$ either $\epsilon$ has to be increased or another fit model, the Gottschalk model, has to be used. 
Additional applications of the presented radiation length measurement method were tested at beam tests in the last years. A first application was the measurement of homogeneous materials with a known and constant thickness $X$ to determine their radiation length constant $X_{0}$. An example measurement was conducted on four samples of hardened glue. The measurements yielded $X_{0}$ values of 350 to $380 \mathrm{~mm}$ for unfilled epoxy adhesives and approximately $47 \mathrm{~mm}$ for a silver filled glue. Material measurements of this kind could for example be used to create and expand data bases of interesting materials used in particle detectors.

Another possible application of the presented method is the generation of radiation length images of complex material distributions with a spatial resolution of 75 or even $50 \mu \mathrm{m}$. For example, radiation length measurements on an ATLAS ITk prototype module glued onto a multi-layer support structure were conducted. The resulting radiation length image shows several small-scale features such as a carbon fibre honeycomb pattern in the support structure, capacitors with solder and vias with a diameter of $200 \mu \mathrm{m}$. It was found that the spatial resolution of radiation length images presented in this work is mostly determined by the image pixel pitch, because the intersection uncertainty of tracks in a EUDET telescope is much smaller ${ }^{1}$. The image pixel pitch on the other hand is dependent on the statistics of the scattering angle sample. Image pixels should have an angle distribution with at least a few thousand entries to ensure a stable fit. Accordingly, all radiation length images displayed in chapter 7 are merely limited by statistics. With sufficient statistics, images with smaller pixel pitches of 20 to $30 \mu \mathrm{m}$ are conceivable.

Radiation length images can be used to compare a measured material distribution with the detector model. One of the main motivations of this thesis was to test the material model of Belle II PXD modules. Measurements were conducted on a prototype PXD module with small mechanical differences to a final module used in Belle II. However, the measurements indicated several shortcomings of the detector model, which was significantly improved as part of this thesis.

The radiation length measurement algorithm developed for this thesis was therefore successfully applied to a wide range of structures and found to produce reliable results.

\subsection{Outlook}

Radiation length measurements and the subsequent analysis in $T B S W$ have been conducted by other groups and the communication about results and analysis steps have continuously improved the $T B S W$ framework and the implemention of radiation length measurements. For example, TBSW has been used to measure an ATLAS ITk end-ofstructure board, which is planned to be used to test and improve the existing detector

\footnotetext{
${ }^{1}$ Typically smaller than $10 \mu \mathrm{m}$
} 


\section{Conclusion and outlook}

model. As shown in this work, radiation length measurements show promising results and can, with some preparation, be conducted at DESY and other beam test facilities.

As shown in chapter 6, the current fitting approach using a binned log-likelihood fit yields radiation length values with a small bias. In order to circumvent or at least further reduce these biases, other fitting methods such as unbinned fits are planned to be investigated.

The results in this thesis were all derived with fit functions based on the Highland model of multiple scattering. However, the Highland model is only valid for angle values in the core region of the angle distribution. Employing the Moliere model could be a simple way to extend the fit range and consequently increase the usable entries per angle distribution. However, as explained in section 3.3.2, only the core width of the distribution depends on the radiation length $X / X_{0}$. The non-Gaussian tails are ultimately artefacts from single scatterings with large angles. While the Moliere model is not expected to produce different results than the Highland model, further comparisons between the two models could improve the understanding of systematic effects and uncertainties. Therefore, further studies of the Moliere model are planned in the future.

Border effects between materials with widely different radiation length values can cause fitting failures in the radiation length imaging process. An example can be seen in fig. 7.16 , where a region of $30 \mathrm{~mm}$ of aluminium is located next to air at $v \approx 82 \mathrm{~mm}$. Image pixels in the border region contain scattering angles from particles traversing the thick aluminium as well as from particles traversing air. Accordingly, the angle distributions of these image pixels are expected to consist of two scattering angle distributions: A very broad distribution from aluminium and a very narrow distribution from air (see fig. 7.17). Fitting such a distribution with a simple Gaussian will most likely fail or at least produce a large fit $\chi^{2}$ value. This can be seen in the fig. 7.16: The image shows a column of blank pixels between the region of aluminium and air. This problem is expected to be solvable by using a fit model with two Gaussian components, which is planned to be investigated and potentially implemented in $T B S W$.

Applying the presented radiation length measurements to new use cases has led to several improvements of the existing analysis algorithms and increased the flexibility of the method. Further applications in future experiments are expected to require additional modifications which will continue to improve the radiation length method and the analysis framework. 
[1] J. Haba, "KEKB and the BELLE experiment," Nucl.Instrum.Meth., vol. A368, pp. $74-80$, 1995, Proceedings of the Third International Workshop on B-Physics at Hadron Machines. [Online]. Available: http://www.sciencedirect.com/science/ article/pii/0168900295008896

[2] L. Evans and P. Bryant, "LHC Machine," JINST, vol. 3, 2008. [Online]. Available: https://doi.org/10.1088\%2F1748-0221\%2F3\%2F08\%2Fs08001

[3] G. Aad et al., "The ATLAS Experiment at the CERN Large Hadron Collider," JINST, vol. 3, 2008.

[4] P. W. Higgs, "Broken Symmetries and the Masses of Gauge Bosons," Phys.Rev.Lett., vol. 13, pp. 508-509, Oct 1964. [Online]. Available: https: //link.aps.org/doi/10.1103/PhysRevLett.13.508

[5] F. Englert and R. Brout, "Broken Symmetry and the Mass of Gauge Vector Mesons," Phys.Rev.Lett., vol. 13, pp. 321-323, Aug 1964. [Online]. Available: https://link.aps.org/doi/10.1103/PhysRevLett.13.321

[6] G. Aad et al., "Observation of a new particle in the search for the Standard Model Higgs boson with the ATLAS detector at the LHC," Phys.Lett., vol. B716, pp. $1-29$, 2012. [Online]. Available: http: //www.sciencedirect.com/science/article/pii/S037026931200857X

[7] S. Chatrchyan et al., "Observation of a new boson at a mass of $125 \mathrm{GeV}$ with the CMS experiment at the LHC," Phys.Lett., vol. B716, no. 1, pp. $30-$ 61, 2012. [Online]. Available: http://www.sciencedirect.com/science/article/pii/ S0370269312008581

[8] M. Kobayashi and T. Maskawa, "CP-Violation in the Renormalizable Theory of Weak Interaction," Prog.Theor.Phys., vol. 49, pp. 652-657, 02 1973. [Online]. Available: https://doi.org/10.1143/PTP.49.652 


\section{BIBLIOGRAPHY}

[9] J. H. Christenson et al., "Evidence for the $2 \pi$ Decay of the $K_{2}^{0}$ Meson," Phys.Rev.Lett., vol. 13, pp. 138-140, 1964. [Online]. Available: https: //link.aps.org/doi/10.1103/PhysRevLett.13.138

[10] Sakharov, A. D., "Violation of CP Invariance, C asymmetry, and baryon asymmetry of the universe," Pisma Zh.Eksp.Teor.Fiz., vol. 5, pp. 32-35, 1967.

[11] B. Aubert et al., "The BaBar detector," Nucl.Instrum.Meth., vol. A479, pp. 1-116, 2002.

[12] A. Abashian et al., "The Belle Detector," Nucl.Instrum.Meth., vol. A479, pp. 117$232,2002$.

[13] J. Brodzicka et al., "Physics achievements from the Belle experiment," Progress of Theoretical and Experimental Physics, vol. 2012, 2012. [Online]. Available: https://doi.org/10.1093/ptep/pts072

[14] P. Lewis et al., "First measurements of beam backgrounds at SuperKEKB," Nucl.Instrum.Meth., vol. A914, pp. 69 - 144, 2019. [Online]. Available: http://www.sciencedirect.com/science/article/pii/S0168900218306909

[15] T. Abe et al., "Belle II Technical Design Report," 2010. [Online]. Available: https://arxiv.org/abs/1011.0352

[16] W. Altmannshofer et al., "The Belle II Physics Book," 2018.

[17] J. Kemmer and G. Lutz, "New detector concepts," Nucl.Instr.Meth., vol. A253, pp. 365 - 377, 1987. [Online]. Available: http://www.sciencedirect.com/science/ article/pii/0168900287905183

[18] K. R. Nakamura et al., "The Belle II SVD detector," PoS, vol. Vertex2016, p. 012, 2017.

[19] ATLAS Collaboration, "Technical Design Report for the ATLAS Inner Tracker Strip Detector," 2017. [Online]. Available: http://cds.cern.ch/record/2257755

[20] D. Griffiths, Introduction to Elementary Particles. Wiley, 2014.

[21] M. Thomson, Modern Particle Physics. Cambridge University Press, 2013.

[22] H. D. Politzer, "Reliable Perturbative Results for Strong Interactions?" Phys. Rev. Lett., vol. 30, pp. 1346-1349, 1973.

[23] Glashow, S. L., "Partial Symmetries of Weak Interactions," Nucl. Phys., vol. 22, pp. 579-588, 1961.

[24] Salam, Abdus and Ward, John Clive, "Electromagnetic and weak interactions," Phys. Lett., vol. 13, pp. 168-171, 1964. 
[25] Kim, Jae Yool, "Discovery of Neutrino Oscillations in the Super-Kamiokande Experiment," Phys.High Technol., vol. 24, no. 11, pp. 8-17, 2015.

[26] B. Aharmim et al., "Combined analysis of all three phases of solar neutrino data from the Sudbury Neutrino Observatory," Phys.Rev.C, vol. 88, 2013. [Online]. Available: https://link.aps.org/doi/10.1103/PhysRevC.88.025501

[27] C. S. Wu et al., "Experimental Test of Parity Conservation in Beta Decay," Phys.Rev., vol. 105, pp. 1413-1415, 1957. [Online]. Available: https://link.aps.org/doi/10.1103/PhysRev.105.1413

[28] N. Cabibbo, "Unitary Symmetry and Leptonic Decays," Phys.Rev.Lett., vol. 10, pp. 531-533, 1963. [Online]. Available: https://link.aps.org/doi/10.1103/PhysRevLett. 10.531

[29] M. Tanabashi, K. Hagiwara, and Hikasa, "Review of Particle Physics," Phys.Rev.D, vol. 98, 2018.

[30] J. Charles et al., "CP violation and the CKM matrix: assessing the impact of the asymmetric B factories," Eur.Phys.J., vol. C41, pp. 1-131, May 2005. [Online]. Available: https://doi.org/10.1140/epjc/s2005-02169-1

[31] S. Hashimoto et al., "Letter of intent for KEK Super B factory," 2004.

[32] K. Kleinknecht, Uncovering CP Violation. Springer Verlag, 2003.

[33] "Flip-chip assemby of Belle II PXD pixel sensors," Halbleiterlabor der Max-PlanckGesellschaft, Tech. Rep. 1.4, 2016.

[34] S. Aachboun et al., "Deep anisotropic etching of silicon," Journal of Vacuum Science $\&$ Technology A, vol. 17, pp. 2270-2273, 1999. [Online]. Available: https://doi.org/10.1116/1.581759

[35] S. Aplin et al., "Forward tracking at the next $e^{+} e$ collider part II: experimental challenges and detector design," JINST, vol. 8, jun 2013. [Online]. Available: https://doi.org/10.1088\%2F1748-0221\%2F8\%2F06\%2Ft06001

[36] Battaglia, Marco, "Vertex Tracking at a Future Linear Collider," Nucl.Instr.Meth., vol. A650, pp. 55-58, 2010, international Workshop on Semiconductor Pixel Detectors for Particles and Imaging 2010. [Online]. Available: http: //www.sciencedirect.com/science/article/pii/S0168900210028901

[37] M. Prim, "Impact parameter and vertex resolution studies at Belle II," Master's thesis, Karlsruher Institut für Technologie, II. Physikalisches Institut, 2013.

[38] R. Brun and F. Rademakers, "ROOT - An object oriented data analysis framework," Nucl.Instr.Meth., vol. A389, pp. 81 - 86, 1997, new Computing Techniques in Physics Research V. [Online]. Available: http://www.sciencedirect. com/science/article/pii/S016890029700048X 


\section{BIBLIOGRAPHY}

[39] D. Kim, "The software library of the Belle II experiment," Nuclear and Particle Physics Proceedings, vol. 273-275, pp. 957 - 962, 2016, 37th International Conference on High Energy Physics (ICHEP). [Online]. Available: http://www.sciencedirect.com/science/article/pii/S2405601415006380

[40] C. Grupen, Teilchendetektoren. BI Wissenschaftsverlag, 1993.

[41] L. Landau, "On the energy loss of fast particles by ionization," J.Phys.(USSR), vol. 8, pp. 201-205, 1944.

[42] C. Caso et al., "Review of Particle Physics," Eur.Phys.J., vol. C3, 1998.

[43] R. Frühwirth and M. Regler, "On the quantitative modelling of core and tails of multiple scattering by Gaussian mixtures," Nucl.Instr.Meth., vol. A456, pp. 369 389, 2001. [Online]. Available: http://www.sciencedirect.com/science/article/pii/ S0168900200005891

[44] Y.-S. Tsai, "Pair production and bremsstrahlung of charged leptons," Rev.Mod.Phys., vol. 46, pp. 815-851, 1974. [Online]. Available: https://link.aps.org/doi/10.1103/RevModPhys.46.815

[45] H. Bethe, W. Heitler, and P. A. M. Dirac, "On the stopping of fast particles and on the creation of positive electrons," Proceedings of the Royal Society of London. Series A, Containing Papers of a Mathematical and Physical Character, vol. 146, pp. 83-112, 1934. [Online]. Available: https://royalsocietypublishing.org/doi/abs/10.1098/rspa.1934.0140

[46] R. Frühwirth, "A Gaussian-mixture approximation of the BetheHeitler model ofelectron energy loss by bremsstrahlung," Computer Physics Communications, vol. 154, pp. 131 - 142, 2003. [Online]. Available: http://www.sciencedirect.com/ science/article/pii/S0010465503002923

[47] J. D. Jackson, Klassische Elektrodynamik. De Gruyter, 2002.

[48] T. Fließbach, Quantenmechanik. Spektrum Akademischer Verlag, 2008.

[49] V. Highland, "Some practical remarks on multiple scattering," Nucl.Instr.Meth., vol. 129, pp. 497 - 499, 1975. [Online]. Available: http://www.sciencedirect.com/ science/article/pii/0029554X75907430

[50] G. R. Lynch and O. I. Dahl, "Approximations to multiple Coulomb scattering," Nucl.Instr.Meth., vol. B58, pp. 6 - 10, 1991. [Online]. Available: http://www.sciencedirect.com/science/article/pii/0168583X9195671Y

[51] G. Molière, "Theorie der Streuung schneller geladener Teilchen II. Mehrfachund Vielfachstreuung," Zeitschrift Naturforschung Teil A, vol. 3, pp. 78-97, 1947. [Online]. Available: https://www.degruyter.com/view/j/zna.1948.3.issue-2/ zna-1948-0203/zna-1948-0203.xml 
[52] H. A. Bethe, "Molière's Theory of Multiple Scattering," Phys.Rev., vol. 89, pp. 1256-1266, 1953. [Online]. Available: https://link.aps.org/doi/10.1103/PhysRev. 89.1256

[53] B. Gottschalk et al., "Multiple Coulomb scattering of $160 \mathrm{MeV}$ protons," Nucl.Instr.Meth., vol. B74, pp. 467 - 490, 1993. [Online]. Available: http: //www.sciencedirect.com/science/article/pii/0168583X9395944Z

[54] R. Diener et al., "The DESY II Test Beam Facility," Nucl.Instrum.Meth., vol. A922, pp. 265-286, 2019.

[55] SPS North area beam lines. [Online]. Available: http://sba.web.cern.ch/sba/ documentations/how2controlnabeams.htm

[56] T. Behnke et al., "EUDET Test Beams at DESY," 2007.

[57] A. Schütz, "Simulation of Particle Fluxes at the DESY-II Test Beam Facility," Master's thesis, KIT, Karlsruhe, EKP, 2015. [Online]. Available: http://bib-pubdb1.desy.de/search?of=hd\&p=id:\%22DESY-THESIS-2015-017\%22

[58] EUDET: Detector Reasearch and Development towards the International Linear Collider. [Online]. Available: https://www.eudet.org

[59] J. Baudot et al., "First test results Of MIMOSA-26, a fast CMOS sensor with integrated zero suppression and digitized output," pp. 1169-1173, Oct 2009. [Online]. Available: http://ieeexplore.ieee.org/stamp/stamp.jsp?tp=\&arnumber $=$ 5402399\&isnumber $=5401554$

[60] F. Wanlass and C. Sah, "Nanowatt logic using field-effect metal-oxide semiconductor triodes," in 1963 IEEE International Solid-State Circuits Conference. Digest of Technical Papers, vol. VI, 1963, pp. 32-33.

[61] 2019, Jan Dreyling-Eschweiler, Private communications.

[62] H. Jansen et al., "Performance of the EUDET-type beam telescopes," EPJ Techn.Instrum, vol. 3, p. 7, 2016. [Online]. Available: https://doi.org/10.1140/ epjti/s40485-016-0033-2

[63] 2019, test Beam Software Framework (TBSW). [Online]. Available: https: //bitbucket.org/testbeam/tbsw

[64] "Marlin: Modular analysis and reconstruction for the linear collider." [Online]. Available: https://github.com/iLCSoft/Marlin

[65] "LCIO: Linear collider i/o." [Online]. Available: http://lcio.desy.de/

[66] Python xml.tree Documentation. [Online]. Available: https://docs.python.org/2/ library/xml.etree.elementtree.html 


\section{BIBLIOGRAPHY}

[67] B. Schwenker, "Development and validation of a model for the response of the Belle II vertex detector," Ph.D. dissertation, Georg-August-Universität Göttingen, II. Physikalisches Institut, CERN-THESIS-2014-165, 2014.

[68] L. Rossi et al., Pixel Detectors. Springer, 2006.

[69] R. Frühwirth, "Application of Kalman filtering to track and vertex fitting," Nucl.Instrum.Meth., vol. A262, pp. 444 - 450, 1987. [Online]. Available: http://www.sciencedirect.com/science/article/pii/0168900287908874

[70] R. Barlow, Statistics. Wiley, 1989.

[71] V. Karimaki et al., "Sensor Alignment by Tracks," arXiv e-prints, 2003.

[72] R. Frühwirth et al., "Estimation of detector alignment parameters using the Kalman filter with annealing," Journal of Physics G: Nuclear and Particle Physics, vol. 29, pp. 561-574, 2003. [Online]. Available: https: //doi.org/10.1088\%2F0954-3899\%2F29\%2F3\%2F309

[73] S. Julier and J. K. Uhlmann, "A General Method for Approximating Nonlinear Transformations of Probability Distributions," 1996.

[74] S. Baker and R. D. Cousins, "Clarification of the use of CHI-square and likelihood functions in fits to histograms," Nucl.Instrum.Meth., vol. 221, pp. 437 442, 1984. [Online]. Available: http://www.sciencedirect.com/science/article/pii/ 0167508784900164

[75] D. Drijard et al., "On the reduction in space resolution of track detectors caused by correlations in the coordinate quantization," Nucl.Instrum.Meth., vol. 176, pp. 389 - 395, 1980. [Online]. Available: http://www.sciencedirect.com/science/ article/pii/0029554X80907326

[76] I. Duerdoth, "Track fitting and resolution with digital detectors," Nucl.Instrum.Meth., vol. 203, pp. 291 - 297, 1982. [Online]. Available: http://www.sciencedirect.com/science/article/pii/0167508782906408

[77] 2016 - 2019, Luise Poley, Private communications.

[78] J. Allison et al., "Geant4 developments and applications," IEEE Transactions on Nuclear Science, vol. 53, pp. 270-278, 2006. [Online]. Available: https: //ieeexplore.ieee.org/document/1610988 
Appendices 



\section{APPENDIX A}

\section{TBSW example files}

\section{A.1. Masking path example}

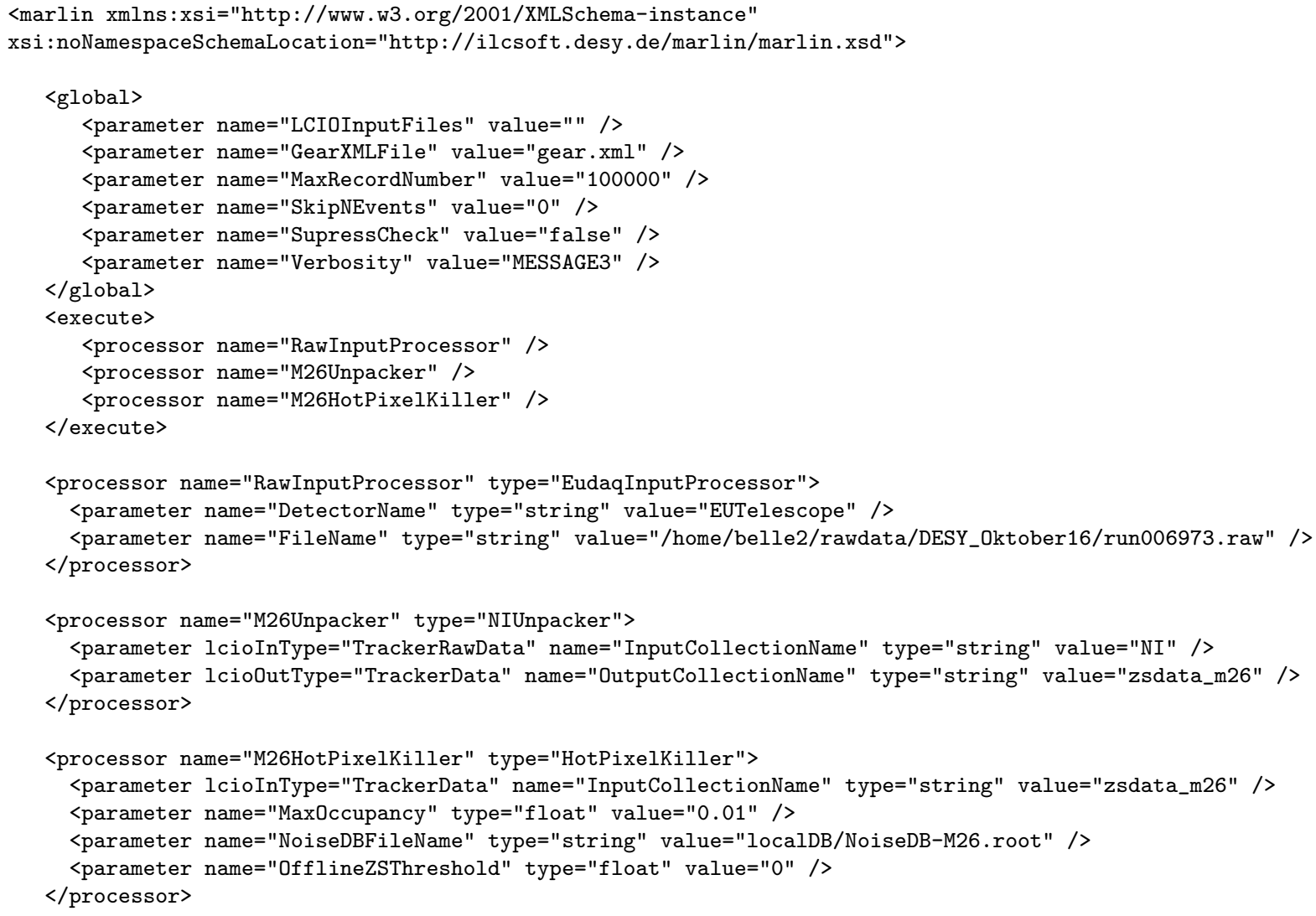

$</ \operatorname{marlin}>$ 


\section{A. TBSW example files}

\section{A.2. Geometry file example}

Typically the reference telescopes used in this work have six layers and one additional target layer. The following example has only the layers, which suffices to explain the general setup of the XML file:

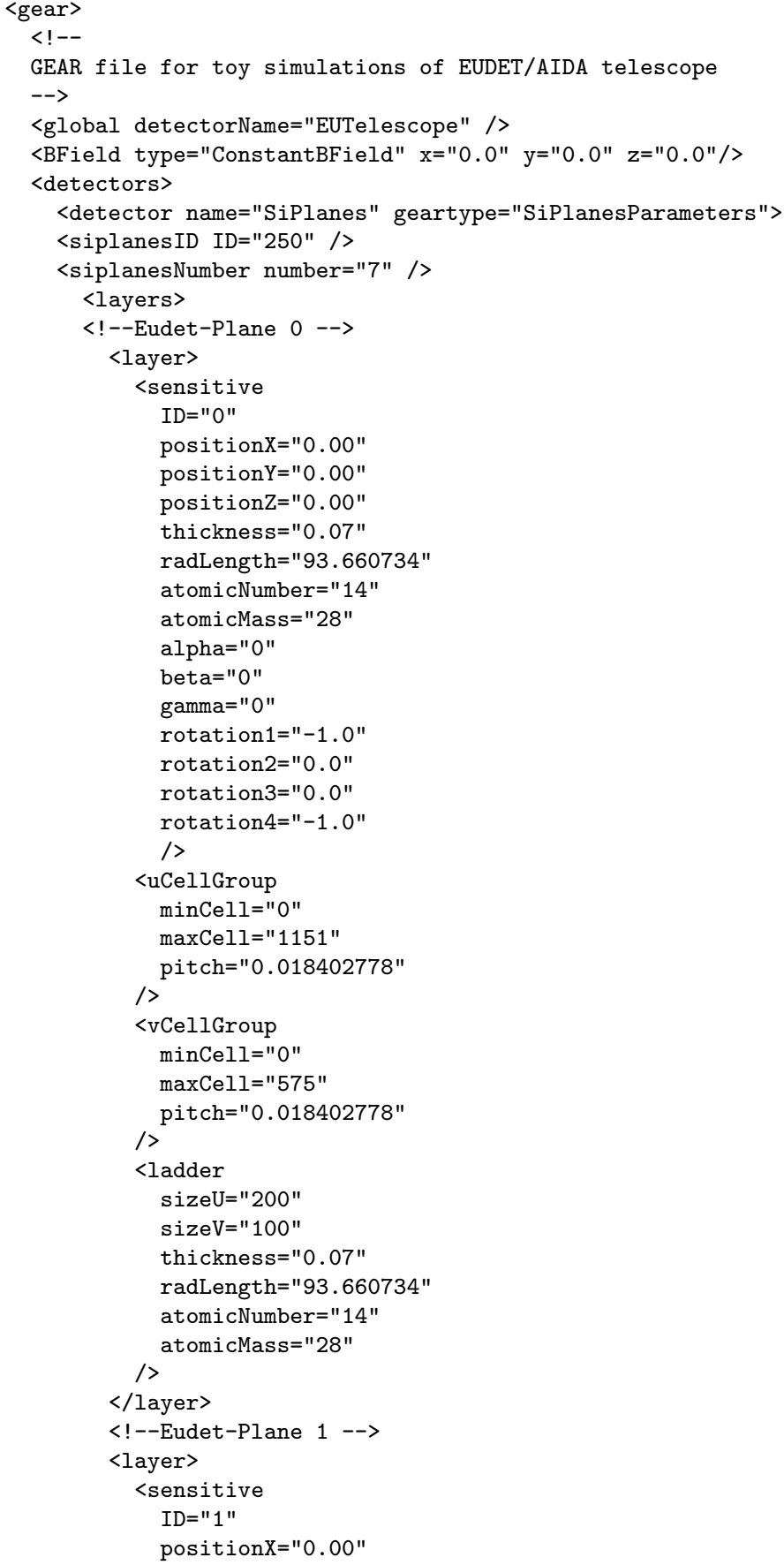


A.2. Geometry file example

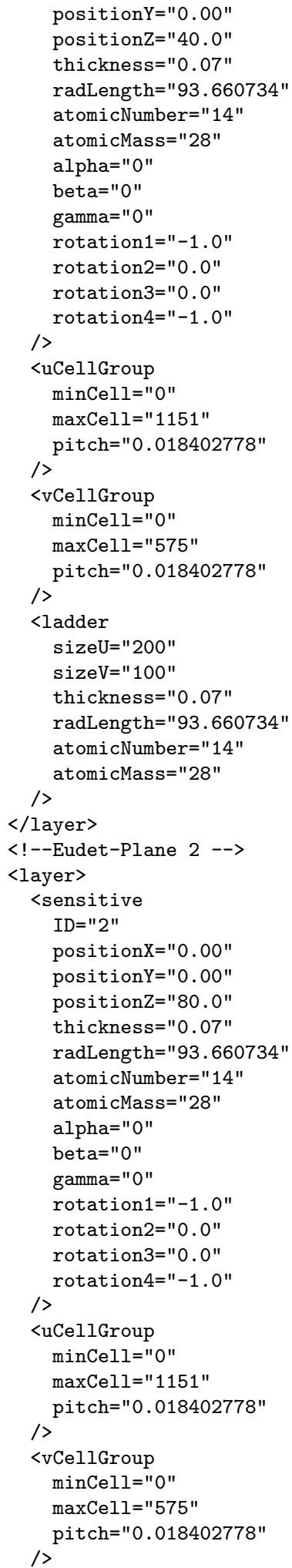


A. TBSW example files

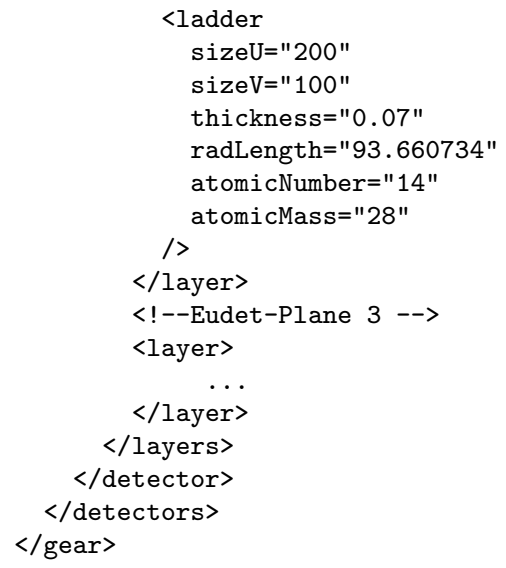

\section{A.3. Efficiency measurements October 2016}

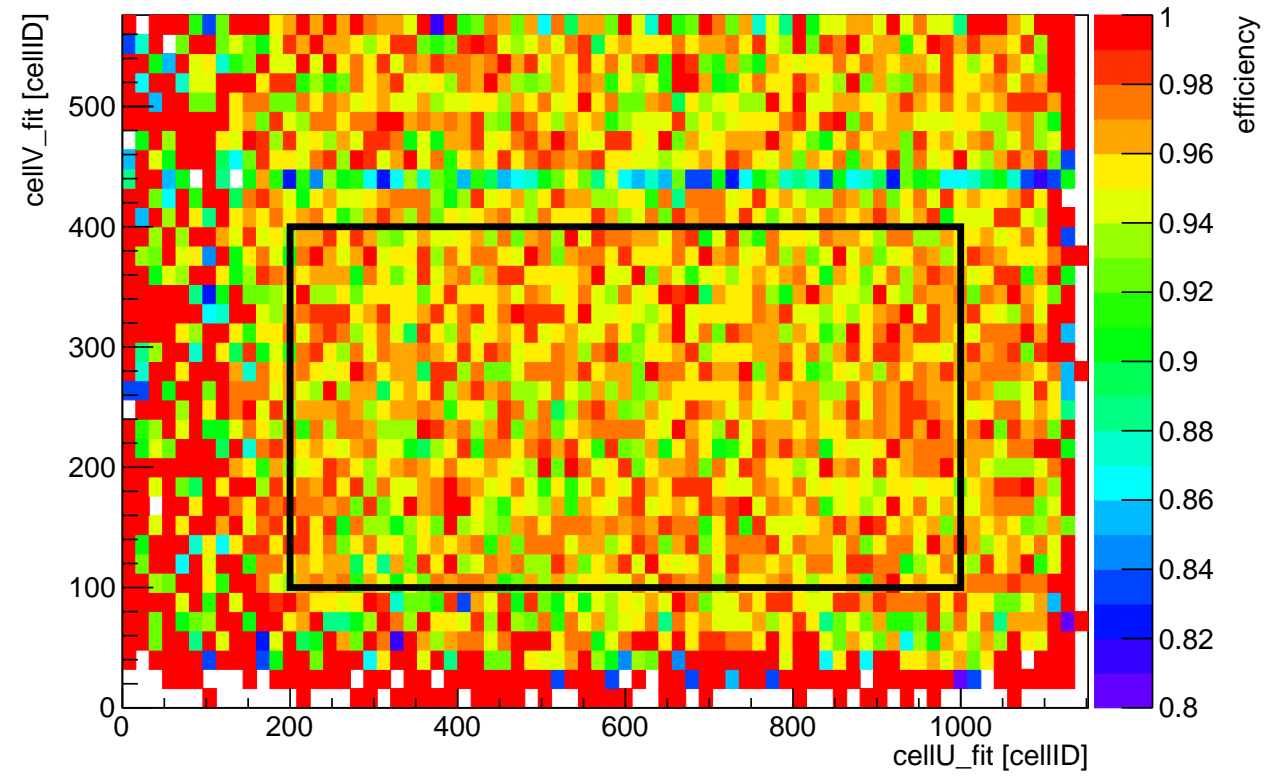

Figure A.1.: 2D M26 efficiency measurement for a comparator threshold of 3. The area indicated by the black box was used to calculate the average efficiency depicted in fig. 4.16 
APPENDIX B

Calculation of angle reconstruction errors with an unscented transform

Unscented transforms can be used to solve the following problem: A stochastic variable $y$ and its covariance matrix $C_{y}$ must be calculated from a random variable $x$ and its covariance matrix $C_{x}$. The two random variables are connected by the non-linear transformation $g$ :

$$
y=g(x)
$$

This scenario can be transferred to the scenario described in section 5.1. Here the scattering angles and their covariance matrix

$$
\vec{y}=\left(\begin{array}{l}
\vartheta_{u} \\
\vartheta_{v}
\end{array}\right) \quad C_{y}=\left(\begin{array}{cc}
\sigma_{\vartheta_{u}, \vartheta_{u}}^{2} & 0 \\
0 & \sigma_{\vartheta_{v}, \vartheta_{v}}^{2}
\end{array}\right)
$$

must be reconstructed from the vector holding the slopes of the in- and out-state track and their covariance matrix

$$
\vec{x}=\left(\begin{array}{c}
m_{u}^{\text {in }} \\
m_{v}^{\text {in }} \\
m_{u}^{\text {out }} \\
m_{v}^{\text {out }}
\end{array}\right) \quad C_{x}=\left(\begin{array}{cccc}
\sigma_{m_{u}^{\text {in }}, m_{u}^{\text {in }}}^{2} & 0 & 0 & 0 \\
0 & \sigma_{m_{v}^{\text {in }}, m_{v}^{\text {in }}}^{2} & 0 & 0 \\
0 & 0 & \sigma_{m_{u}^{\text {out }}, m_{u}^{\text {out }}}^{2} & 0 \\
0 & 0 & 0 & \sigma_{m_{v}^{\text {out }}, m_{v}^{\text {out }}}^{2}
\end{array}\right)
$$

The non-linear transformation $g$ corresponds to the relation between the scattering angles and the in- and out-state slopes. The detailed description of the calculation is given in section 5.1. 


\section{B. Calculation of angle reconstruction errors with an unscented transform}

The basic idea of the unscented transform is to define a set of sample points $\chi_{i}$, which contains the information of the vector and covariance matrix in eq. B.3 and apply the non-linear transformation $g$ on the vectors of the set. Afterwards the mean and covariance of the transformed ensemble of sample points can be calculated. The mean and covariance of the $\chi_{i}$ set is required to be identical to the values given in eq. B.3. At least nine $\chi$ vectors are needed in order to sample the random distribution of the slopes. Due to the mean and variance constraints the $\chi_{i}$ vectors and their weights $w_{i}$ must suffice the following equations:

$$
\begin{aligned}
1 & =\sum_{i=0}^{9} w_{i} \\
\vec{x} & =\sum_{i=0}^{9} w_{i} \chi_{i} \\
C_{x} & =\sum_{i=0}^{9} w_{i}\left(\chi_{i}-\vec{x}\right)\left(\chi_{i}-\vec{x}\right)^{\mathrm{T}}
\end{aligned}
$$

For the calculation of the scattering angle uncertainties the following $\chi$ vectors are used:

$$
\begin{aligned}
\chi_{0} & =\vec{x} \\
\chi_{i} & =\vec{x}+\sqrt{5 C_{x}^{(i, i)}} \overrightarrow{e_{i}} \text { for } i=1, \ldots, 4 \\
\chi_{i} & =\vec{x}-\sqrt{5 C_{x}^{(i-4, i-4)}} \vec{e}_{i-4} \quad \text { for } i=5, \ldots, 8
\end{aligned}
$$

The weights were selected to be $w_{0}=\frac{1}{5}$ and $w_{i}=\frac{1}{10}$ for $i \neq 0$. The transformed vectors $g\left(\chi_{i}\right)$ are employed to calculate $\vec{y}$ and $C_{y}$ :

$$
\begin{aligned}
\vec{y} & =\sum_{i=0}^{9} w_{i} g\left(\chi_{i}\right) \\
C_{y} & =\sum_{i=0}^{9} w_{i}\left(g\left(\chi_{i}\right)-\vec{y}\right)\left(g\left(\chi_{i}\right)-\vec{y}\right)^{\mathrm{T}}
\end{aligned}
$$

The uncertainties of the reconstructed angles can be calculated from the diagonal entries of the covariance matrix:

$$
\begin{aligned}
\sigma_{\vartheta_{u}} & =\sqrt{C_{y}^{(1,1)}} \\
\sigma_{\vartheta_{v}} & =\sqrt{C_{y}^{(2,2)}}
\end{aligned}
$$


Systematical studies of the radiation length calibration procedure

\section{C.1. Target thickness measurements of beam energy uncertainty simulations}

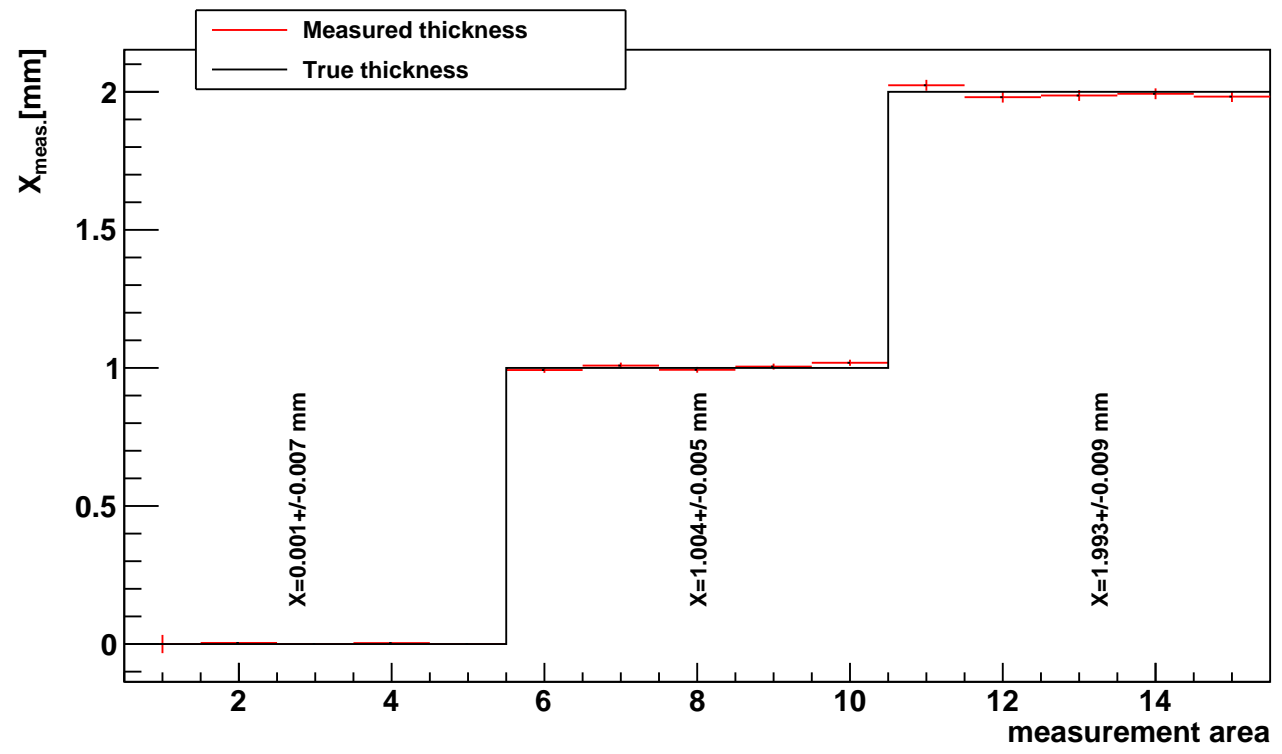

Figure C.1.: Self consistency test plot for a beam test simulation with an beam energy uncertainty of $10 \%$. The measured thickness values are in agreement with the expected aluminium and air thicknesses, which were fixed during the radiation length calibration. 


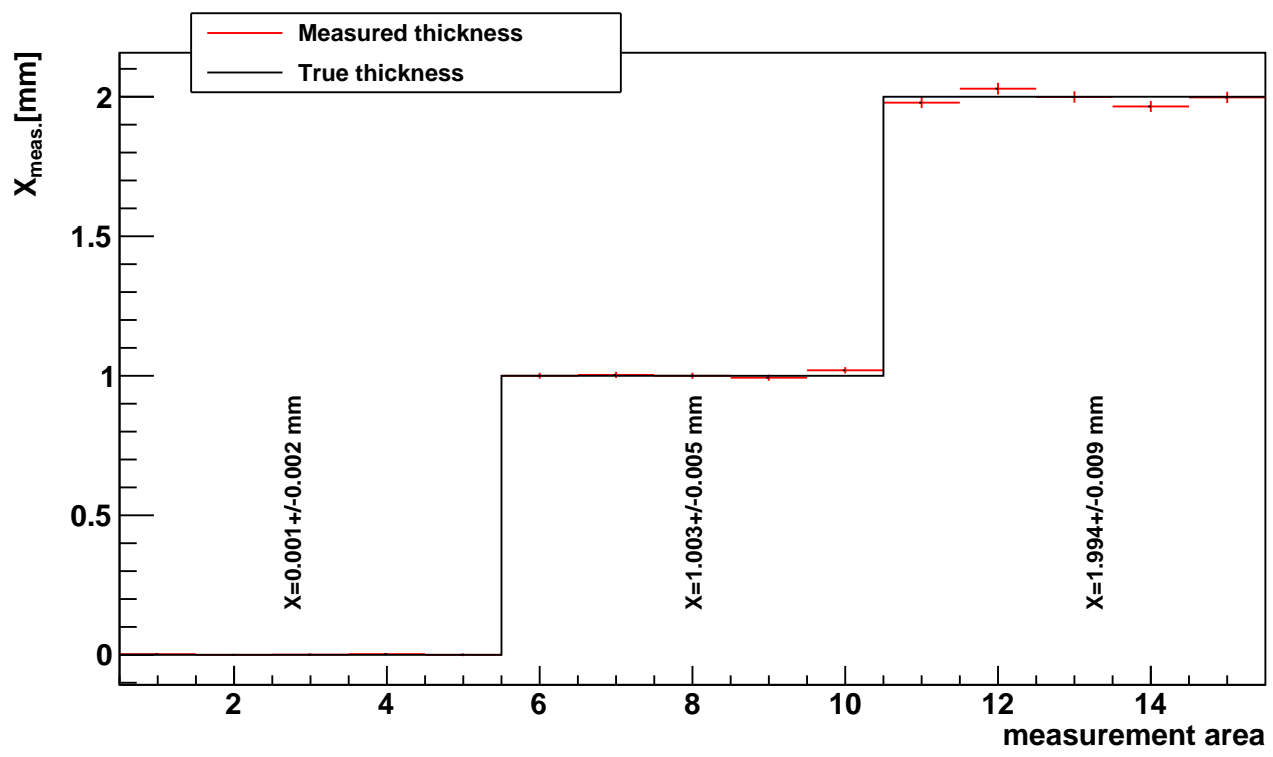

(a)

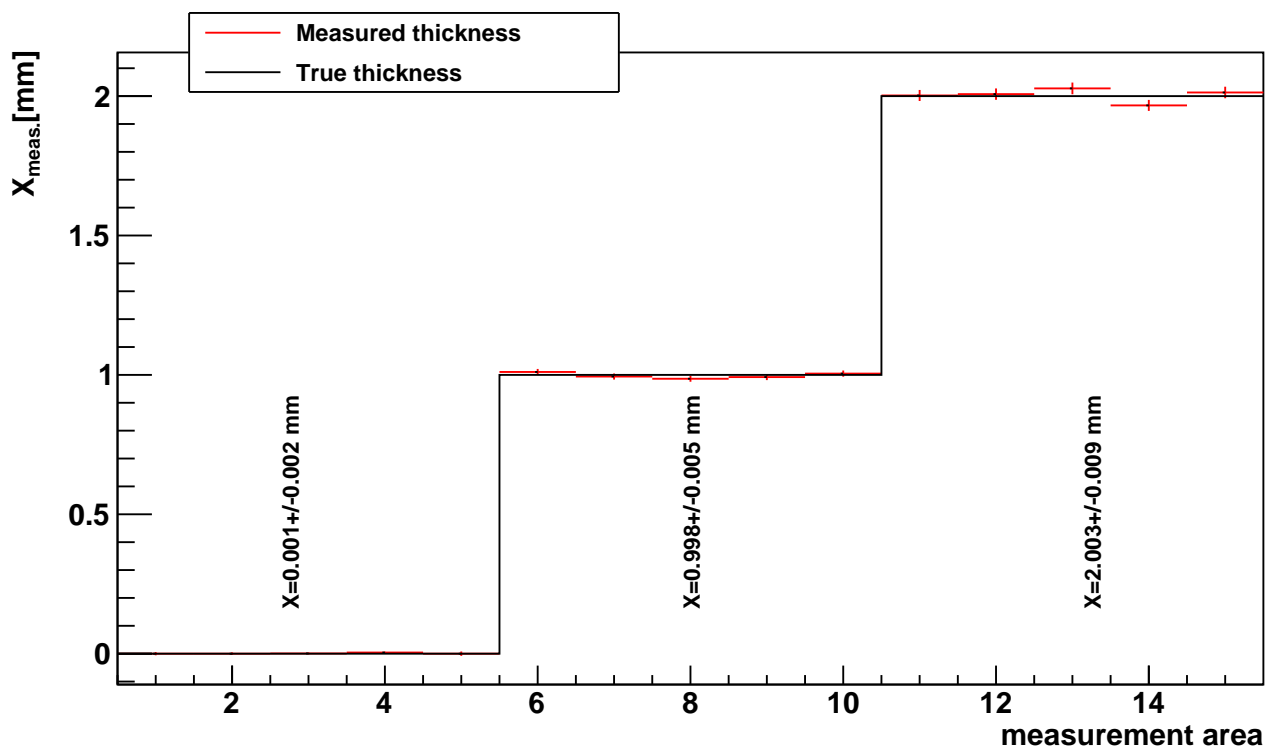

(b)

Figure C.2.: Self consistency test plot for a beam test simulation with an beam energy uncertainty of $1 \%$ (a) and $2 \%$ (b). The measured thickness values are in agreement with the expected aluminium and air thicknesses, which were fixed during the radiation length calibration. 
C.1. Target thickness measurements of beam energy uncertainty simulations

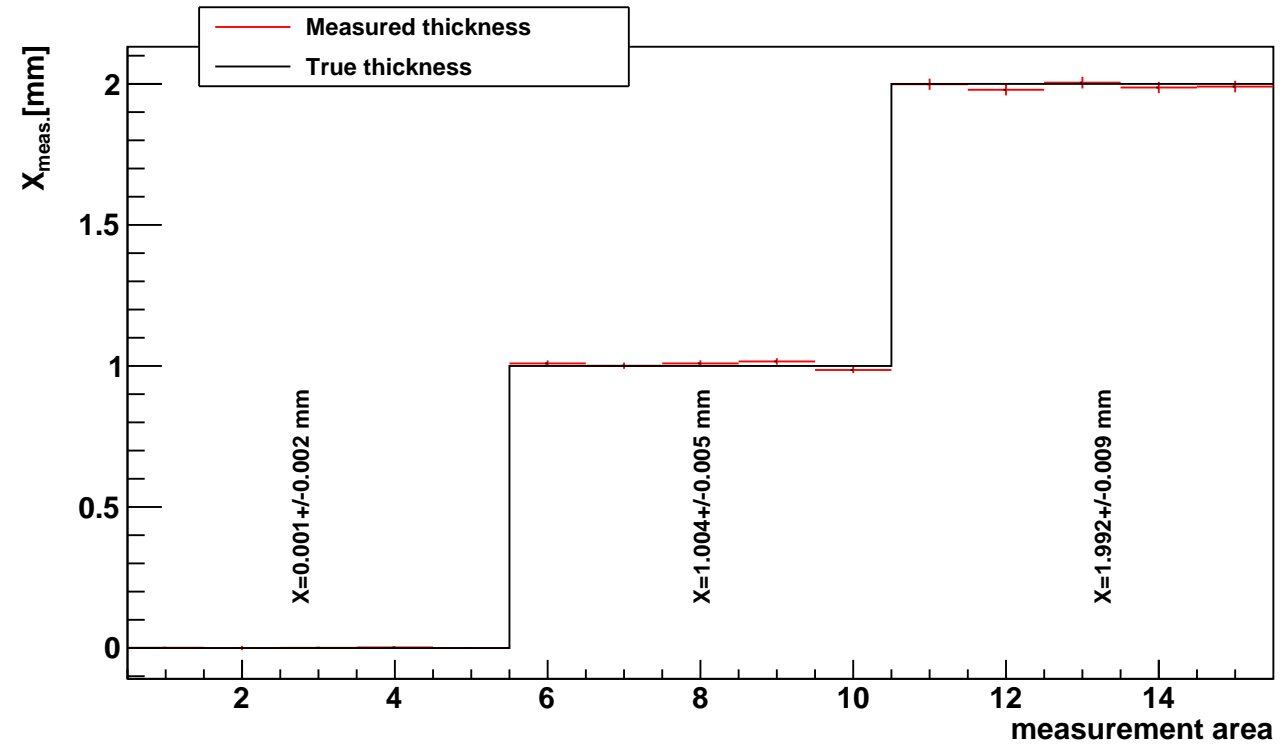

(a)

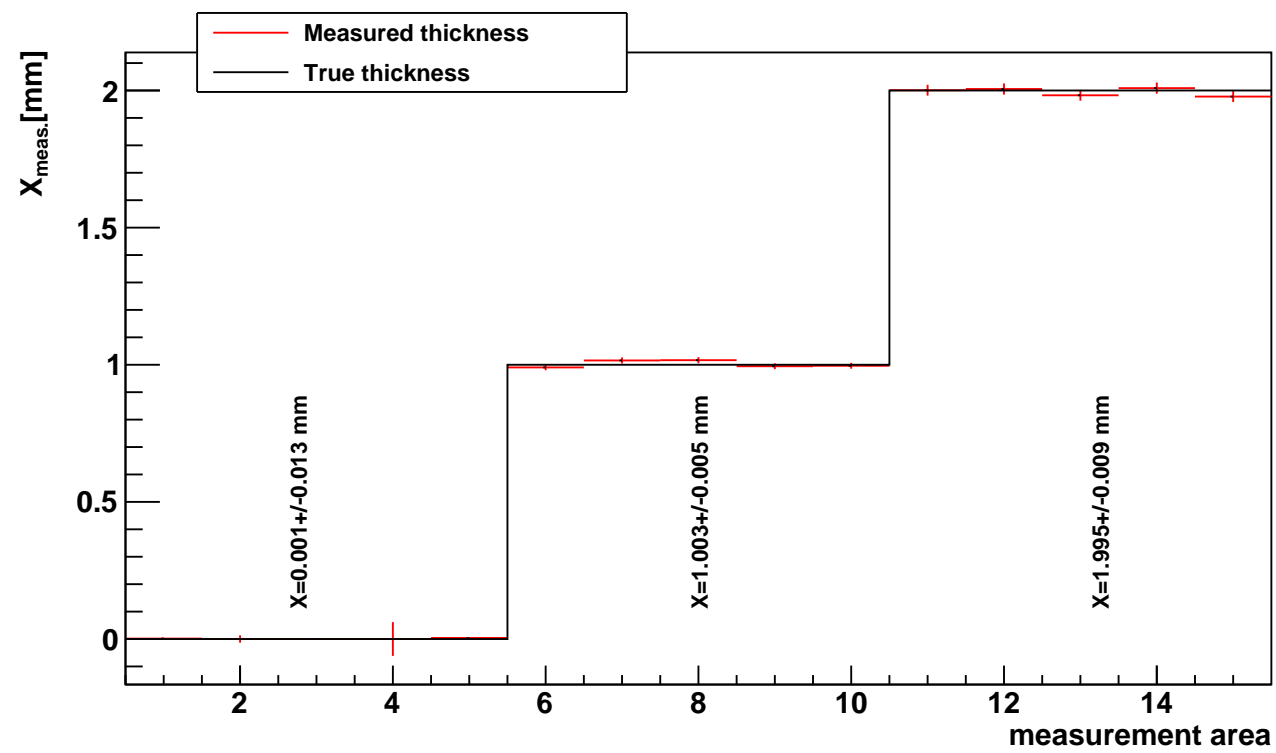

(b)

Figure C.3.: Self consistency test plot for a beam test simulation with an beam energy uncertainty of $4 \%$ (a) and $8 \%$ (b). The measured thickness values are in agreement with the expected aluminium and air thicknesses, which were fixed during the radiation length calibration. 
C. Systematical studies of the radiation length calibration procedure

\section{C.2. Target thickness measurements of beam energy variation simulations}

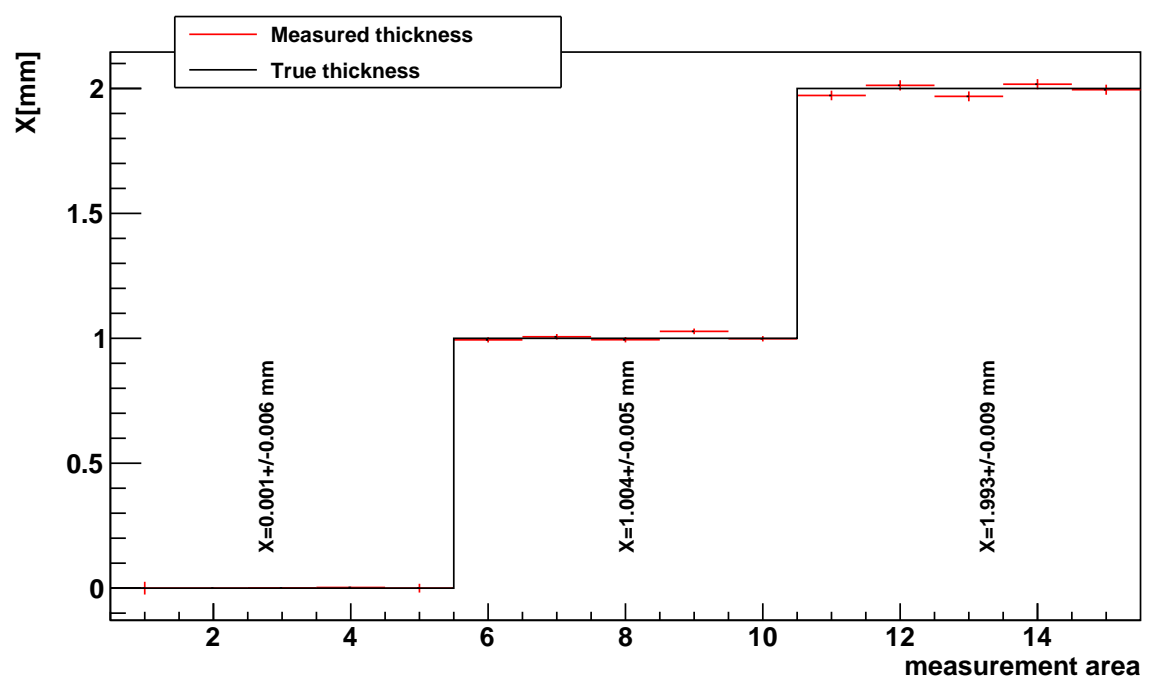

(a)

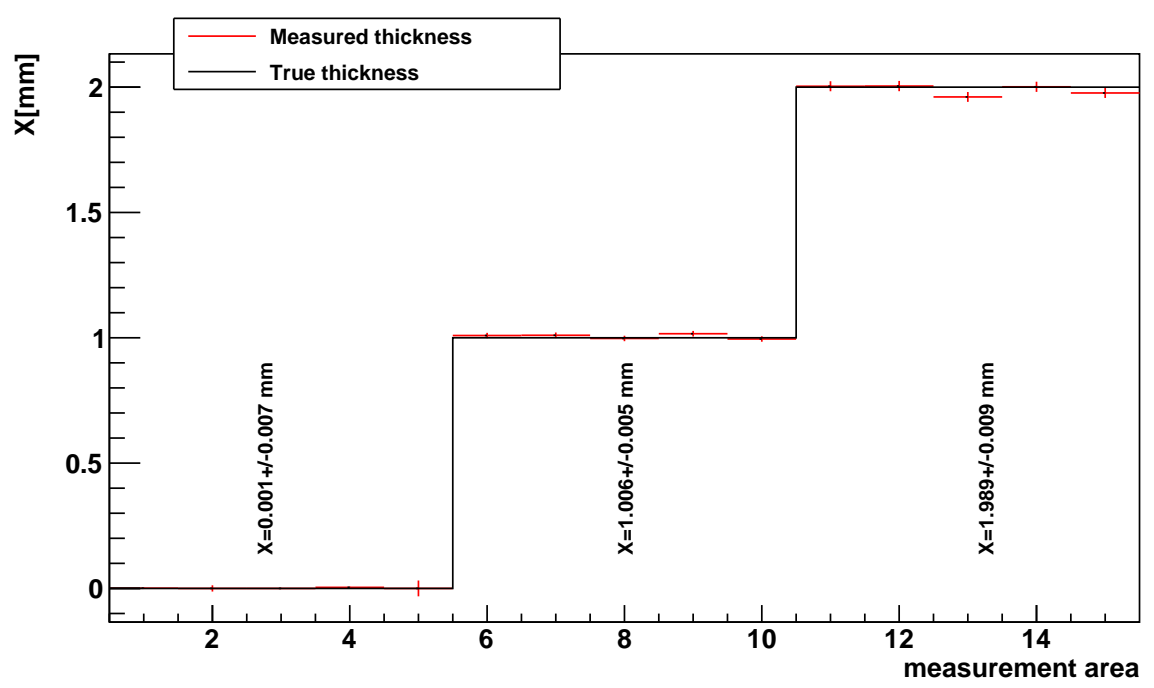

(b)

Figure C.4.: Self consistency test plot for a beam test simulation with simulated beam energy of $1850 \mathrm{MeV}$ (a) and $1900 \mathrm{MeV}$ (b). The measured thickness values are in agreement with the expected aluminium and air thicknesses, which were fixed during the radiation length calibration. 
C.2. Target thickness measurements of beam energy variation simulations

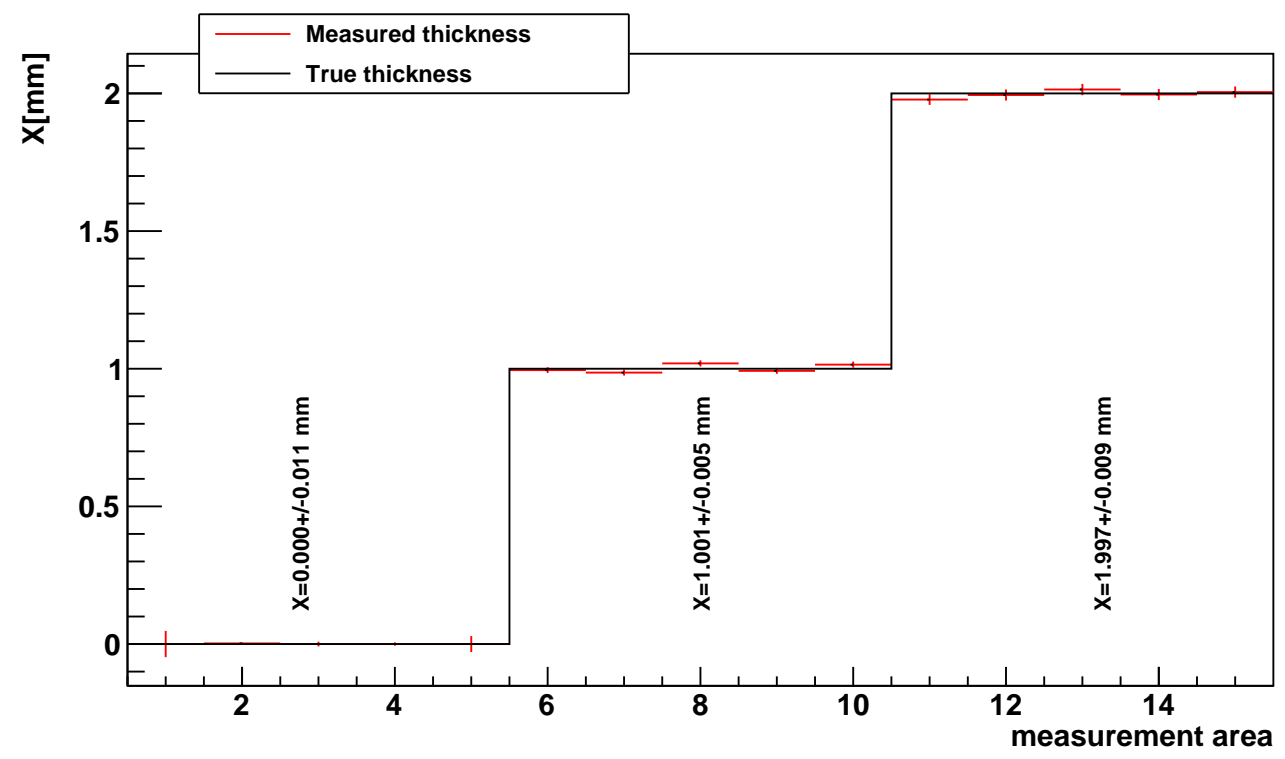

(a)

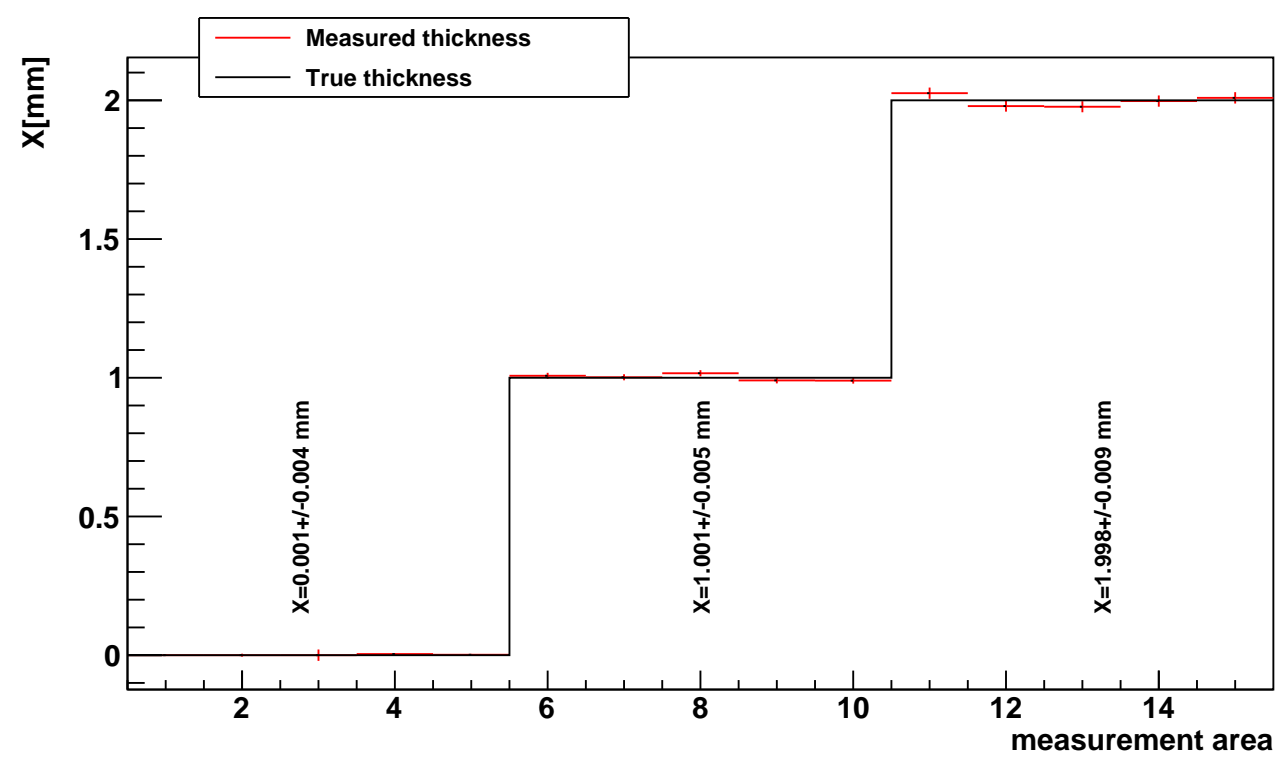

(b)

Figure C.5.: Self consistency test plot for a beam test simulation with simulated beam energy of $1950 \mathrm{MeV}$ (a) and $2000 \mathrm{MeV}$ (b). The measured thickness values are in agreement with the expected aluminium and air thicknesses, which were fixed during the radiation length calibration. 
C. Systematical studies of the radiation length calibration procedure

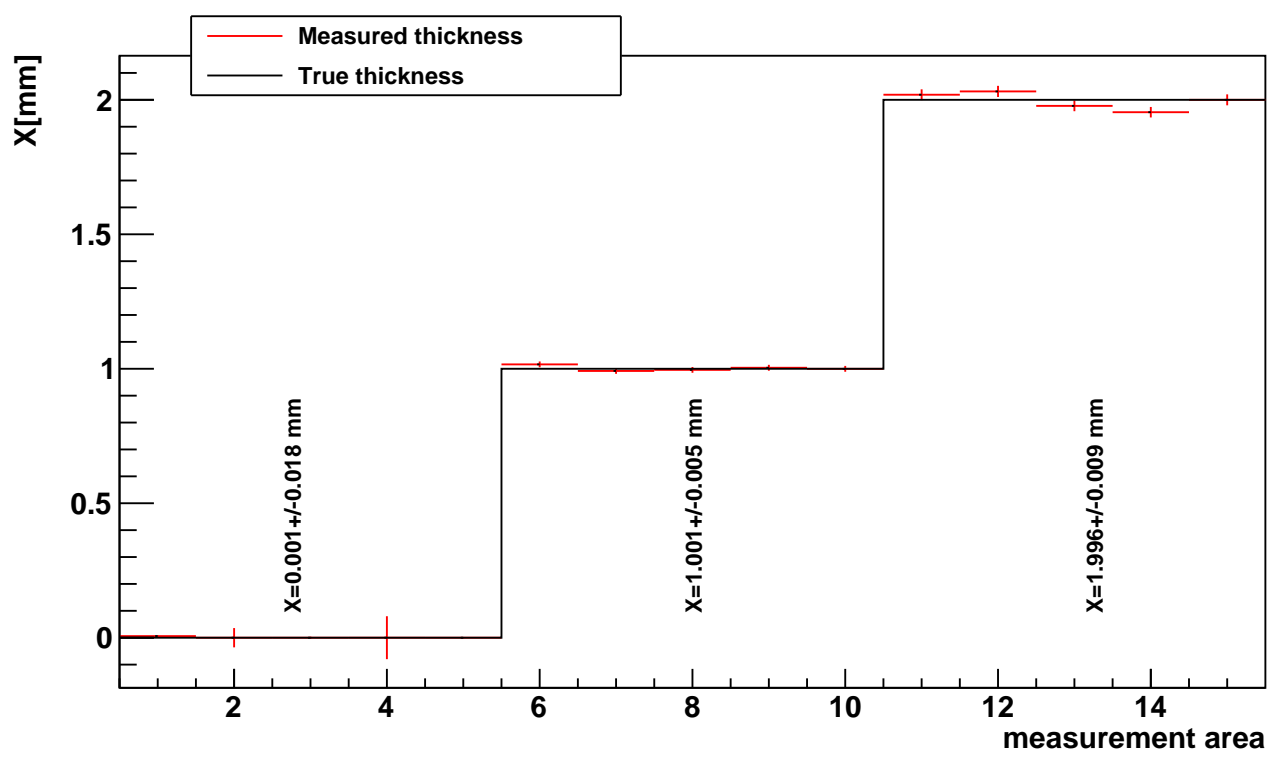

(a)

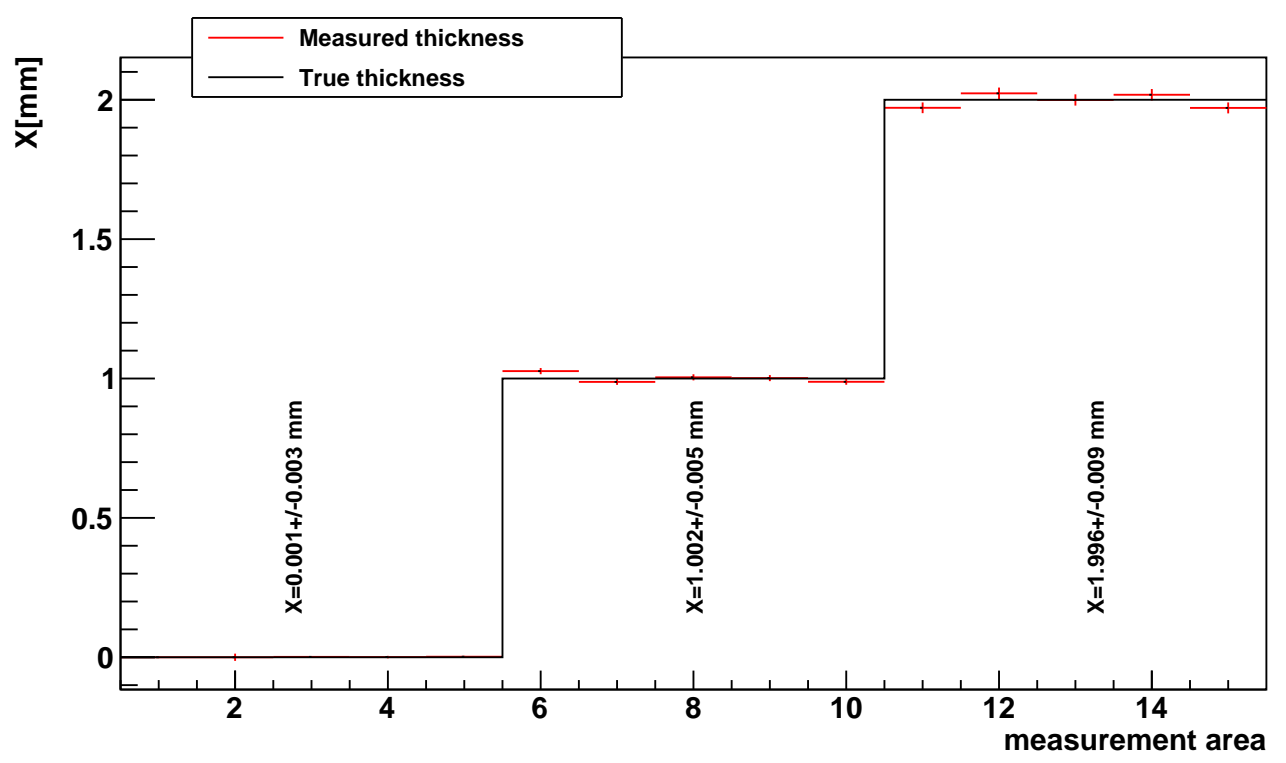

(b)

Figure C.6.: Self consistency test plot for a beam test simulation with simulated beam energy of $2050 \mathrm{MeV}$ (a) and $2100 \mathrm{MeV}$ (b). The measured thickness values are in agreement with the expected aluminium and air thicknesses, which were fixed during the radiation length calibration. 
APPENDIX D

Telescope and $X / X_{0}$ calibration of various beam test measurements

\section{D.1. Aluminium wedge measurements in June 2017}
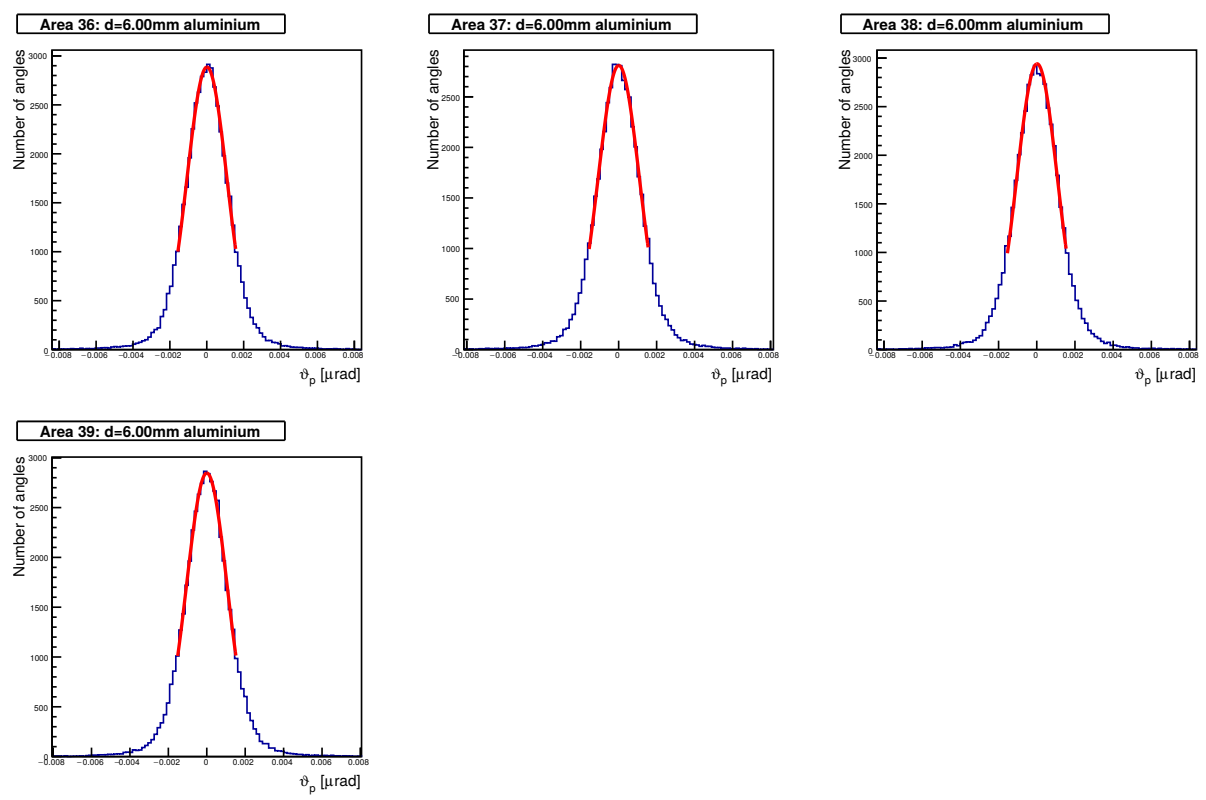

Figure D.1.: Four of the 40 scattering angle distributions used during the radiation length calibration together with their corresponding fit functions. The fit functions match the distributions quite well and the determined fit $\chi_{\text {ndof }}^{2}$ of the simultaneous fit is 0.57 which is reasonably small. 

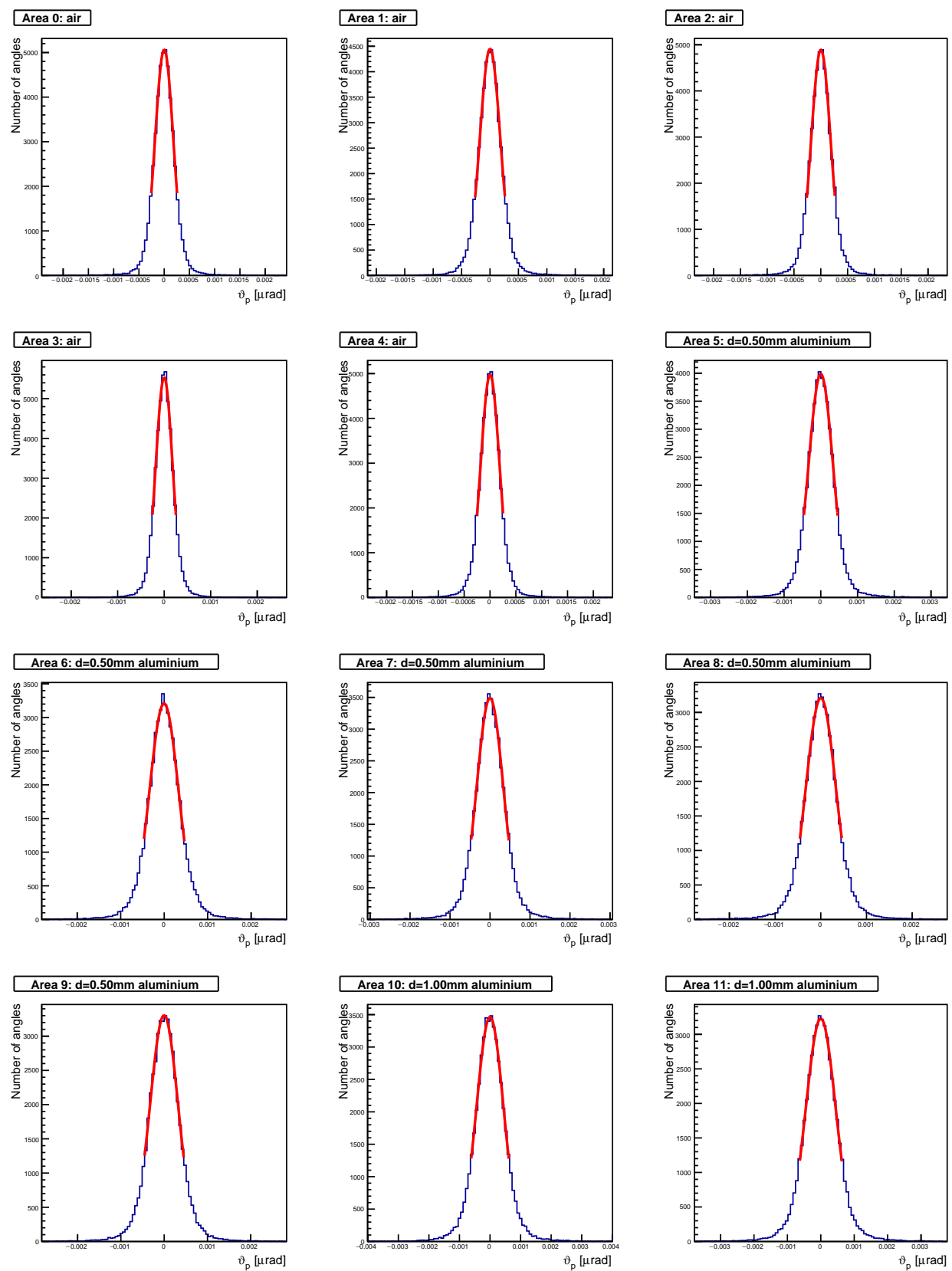

Figure D.2.: Twelve of the 40 scattering angle distributions used during the radiation length calibration together with their corresponding fit functions. The fit functions match the distributions quite well and the determined fit $\chi_{\text {ndof }}^{2}$ of the simultaneous fit is 0.57 which is reasonably small. 

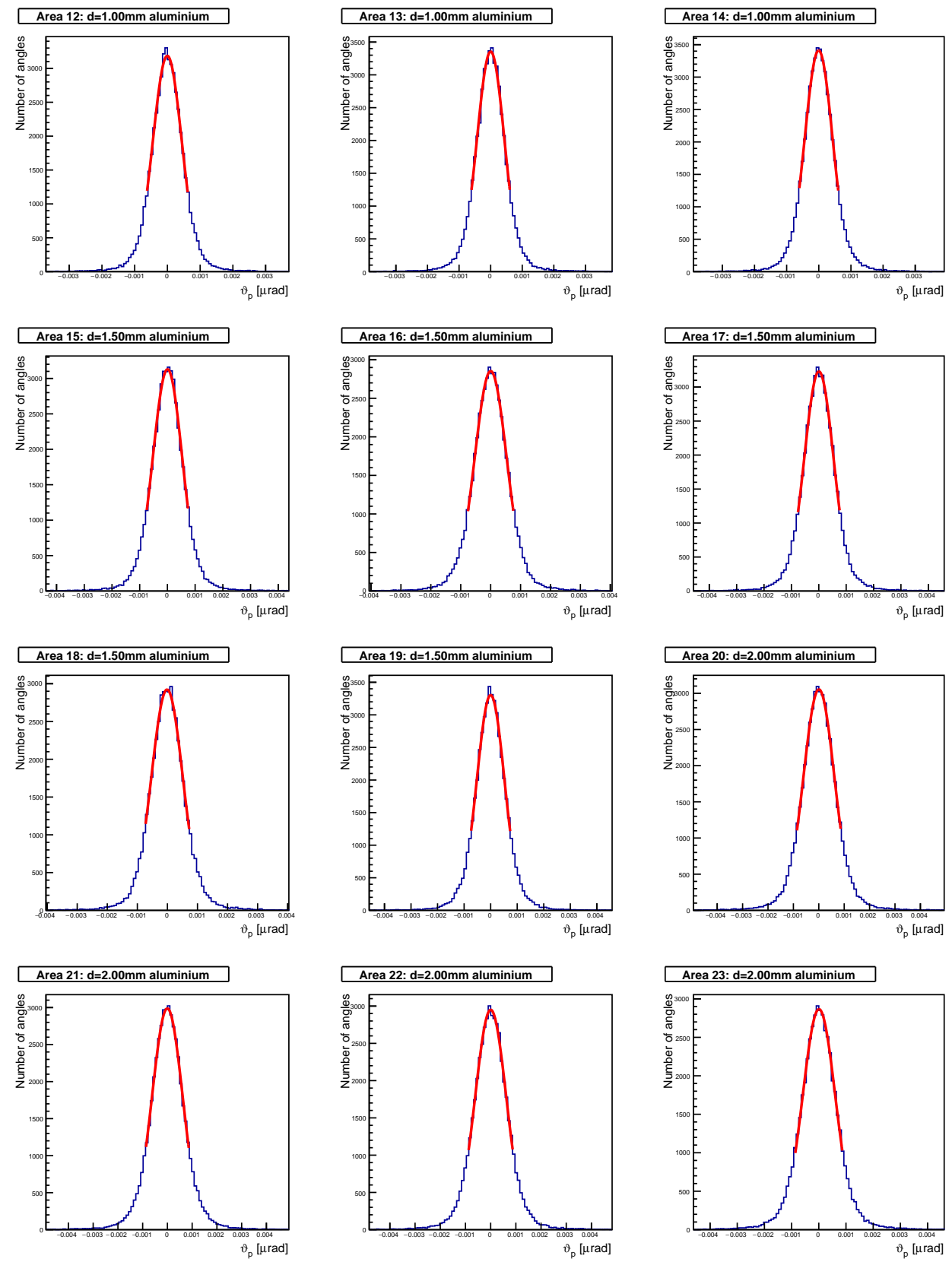

Figure D.3.: Twelve of the 40 scattering angle distributions used during the radiation length calibration together with their corresponding fit functions. The fit functions match the distributions quite well and the determined fit $\chi_{\text {ndof }}^{2}$ of the simultaneous fit is 0.57 which is reasonably small. 

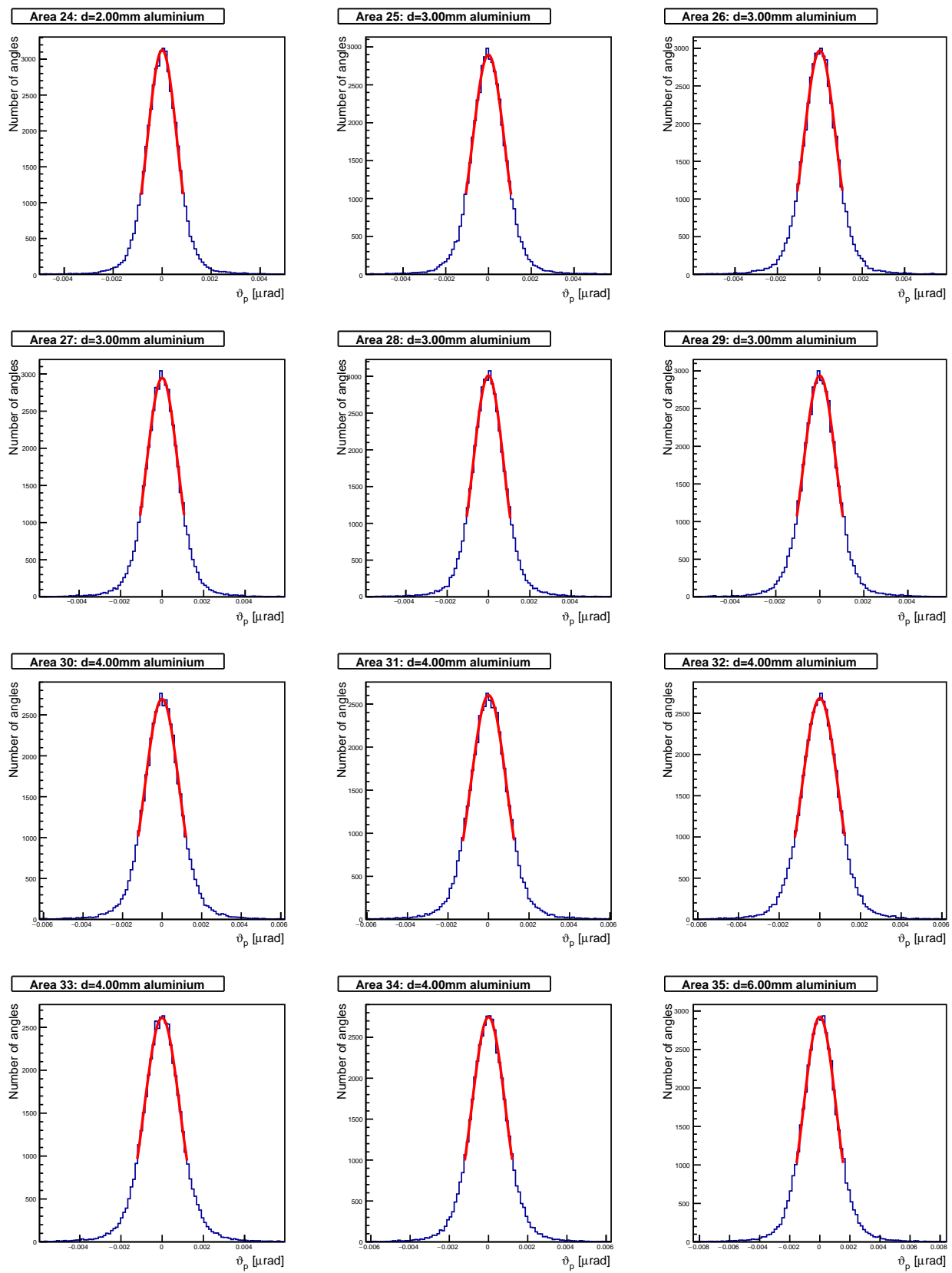

Figure D.4.: Twelve of the 40 scattering angle distributions used during the radiation length calibration together with their corresponding fit functions. The fit functions match the distributions quite well and the determined fit $\chi_{\text {ndof }}^{2}$ of the simultaneous fit is 0.57 which is reasonably small. 


\section{D.2. Copper wedge measurements in November 2017}

In November 2017 a beam test was conducted. The scope of the measurements was to study the influence of bremsstrahlung on the radiation length measurements with the help of a copper wedge. The results of the copper wedge measurement can be found in section 7.2 .

The telescope setup of the measurements is depicted in fig. D.5. The beam energy was $4 \mathrm{GeV}$. Table D. 1 contains an overview over the measured telescope data. It specifies the number of runs, overall events and overall tracks as well as the purpose of the measurements.

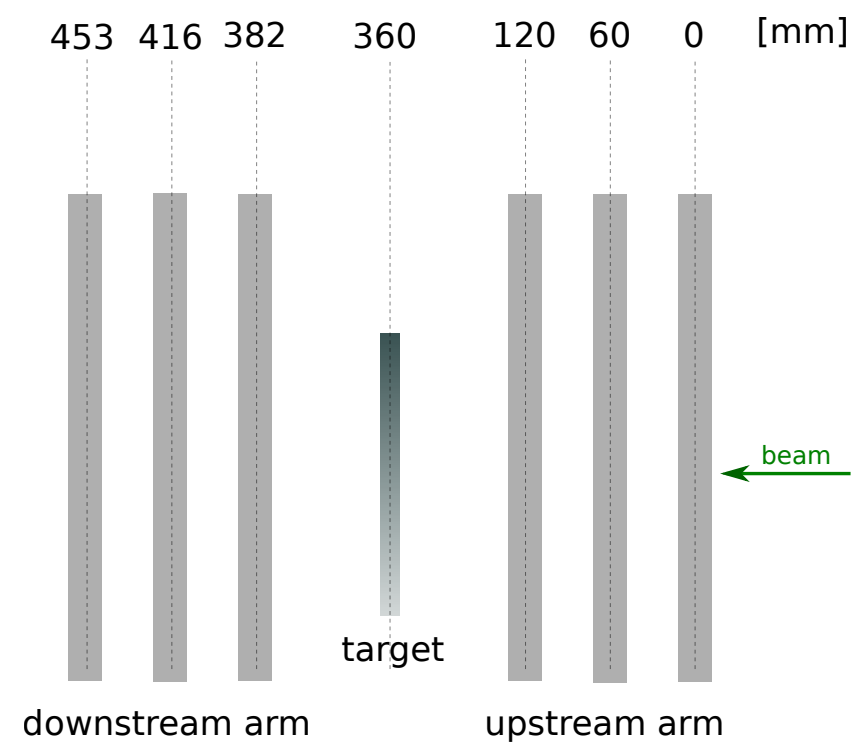

Figure D.5.: Schematic side view of the telescope setup during the November 2017 beam test campaign.

\begin{tabular}{|c||c|c|c|c|}
\hline Target & \#runs & \#events & \#tracks & comments \\
\hline air & 2 & $800 \mathrm{k}$ & $1.3 \mathrm{mio}$ & telescope calibration \\
\hline copper wedge & 25 & $26 \mathrm{mio}$ & $43 \mathrm{mio}$ & $X / X_{0}$ calibration and measurement \\
\hline
\end{tabular}

Table D.1.: Overview over the telescope data at the beam test in November 2017. The spacings between the M26 sensors (see fig. D.5) was not changed between the measurements.

All other DQM plots, which have been explained in section 7.1.1, are listed on the following pages. 
D. Telescope and $X / X_{0}$ calibration of several beam test measurements

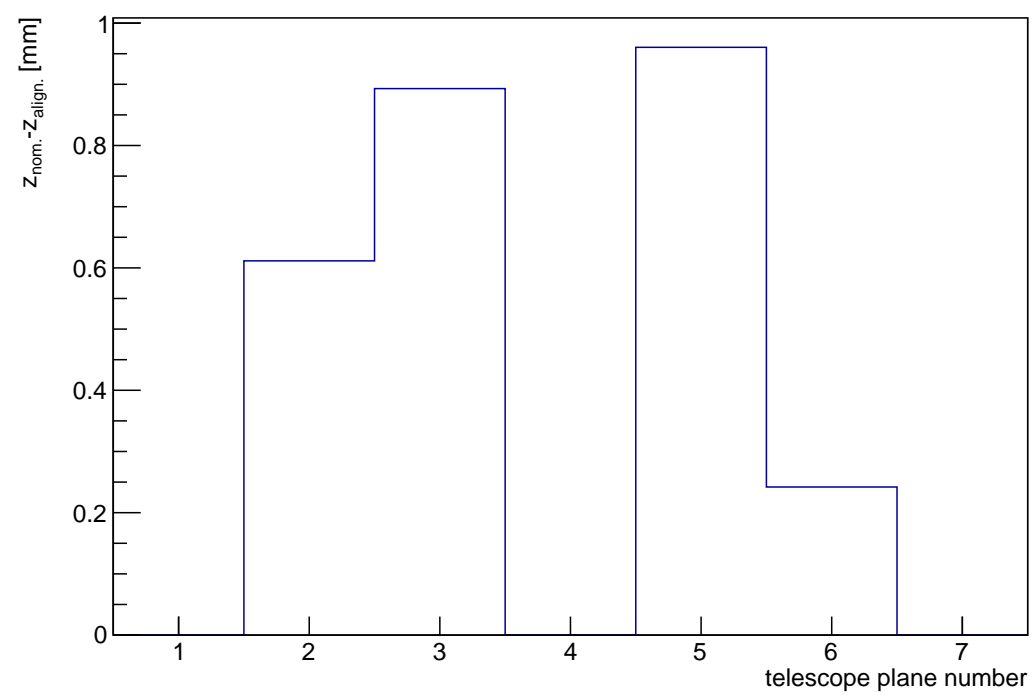

Figure D.6.: Difference between nominal and aligned sensor $z$ positions.

\section{6 million tracks}

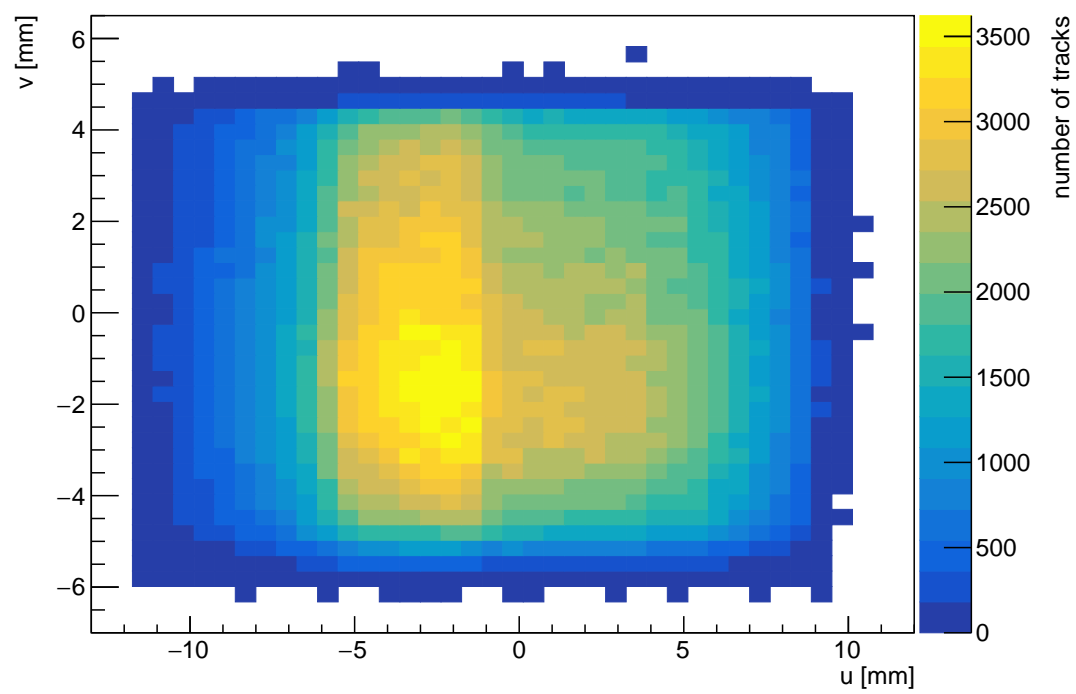

Figure D.7.: Beam spot on the central scattering plane during an example run of the November 2017 beam test. 


\section{cluster shape frequency}

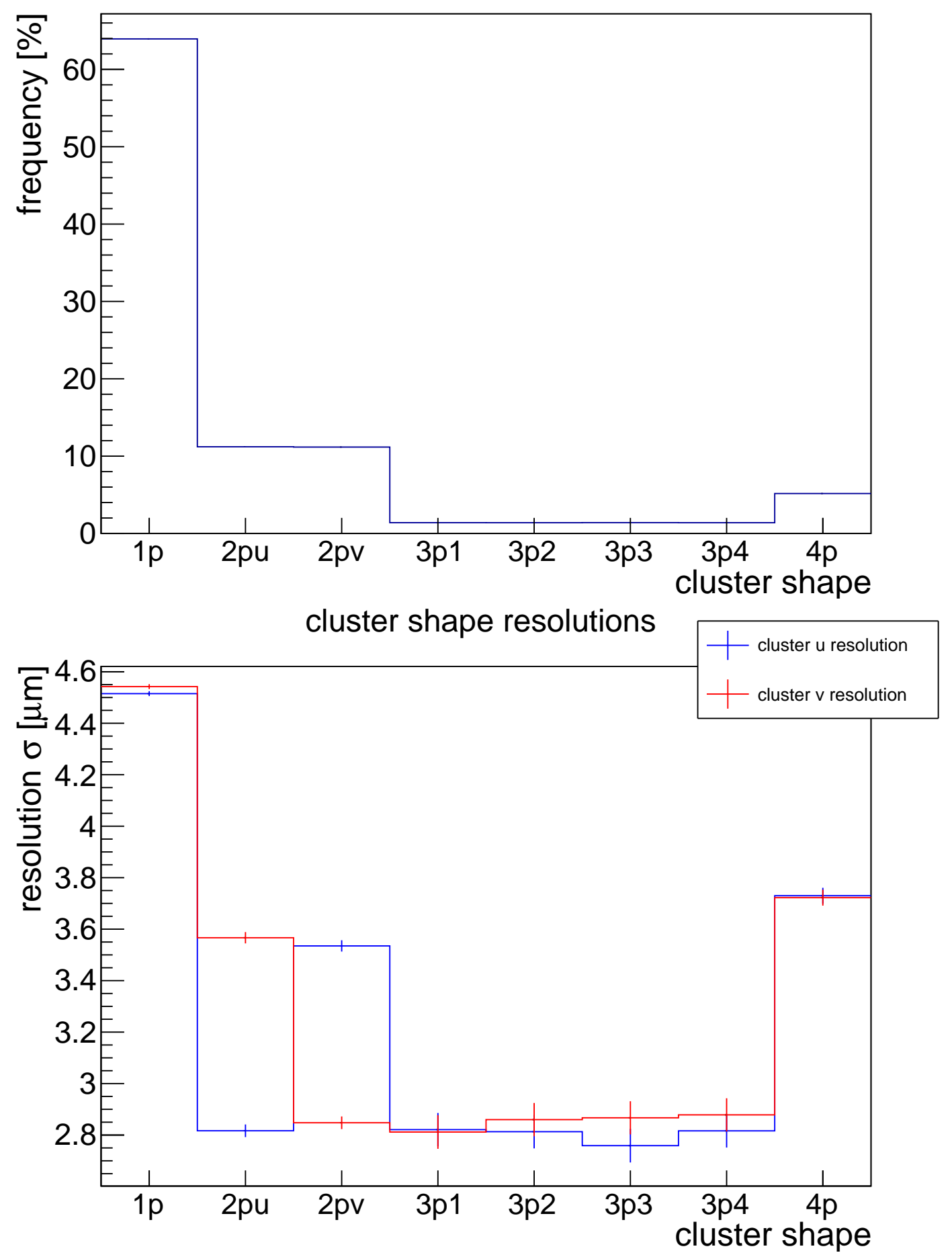

Figure D.8.: Cluster shape frequencies and $u$ and $v$ resolution during the $X / X_{0}$ measurements of the November 2017 beam test campaign. The cluster shapes, which correspond to the individual labels on the $x$ axis, are depicted in figure 4.9 . 
D. Telescope and $X / X_{0}$ calibration of several beam test measurements
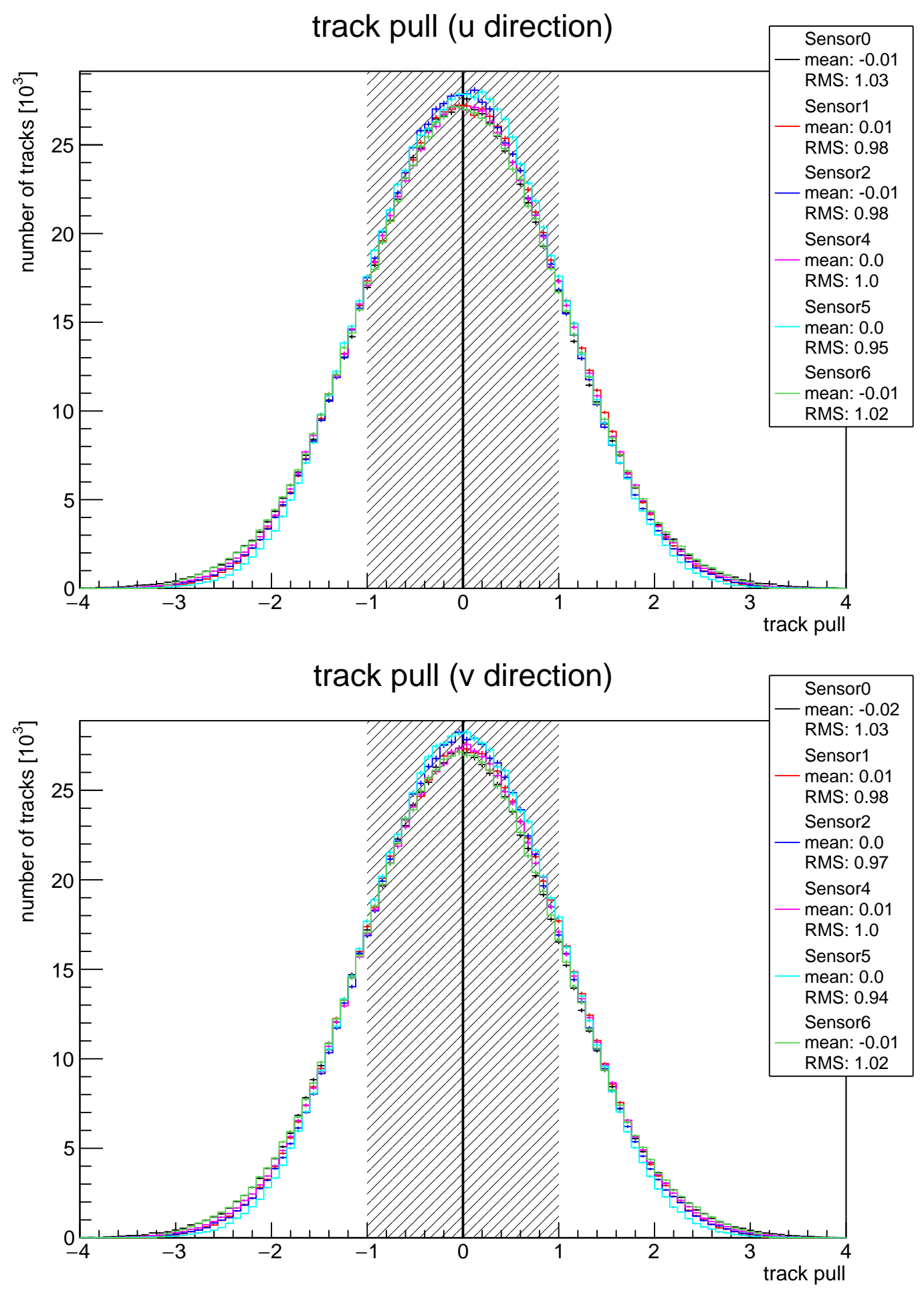

Figure D.9.: Pulls of the $u$ and $v$ residuals after cluster calibration and telescope alignment. The Pulls are centred around 0 and have a RMS value close to 1 . 
D.2. Copper wedge measurements in November 2017
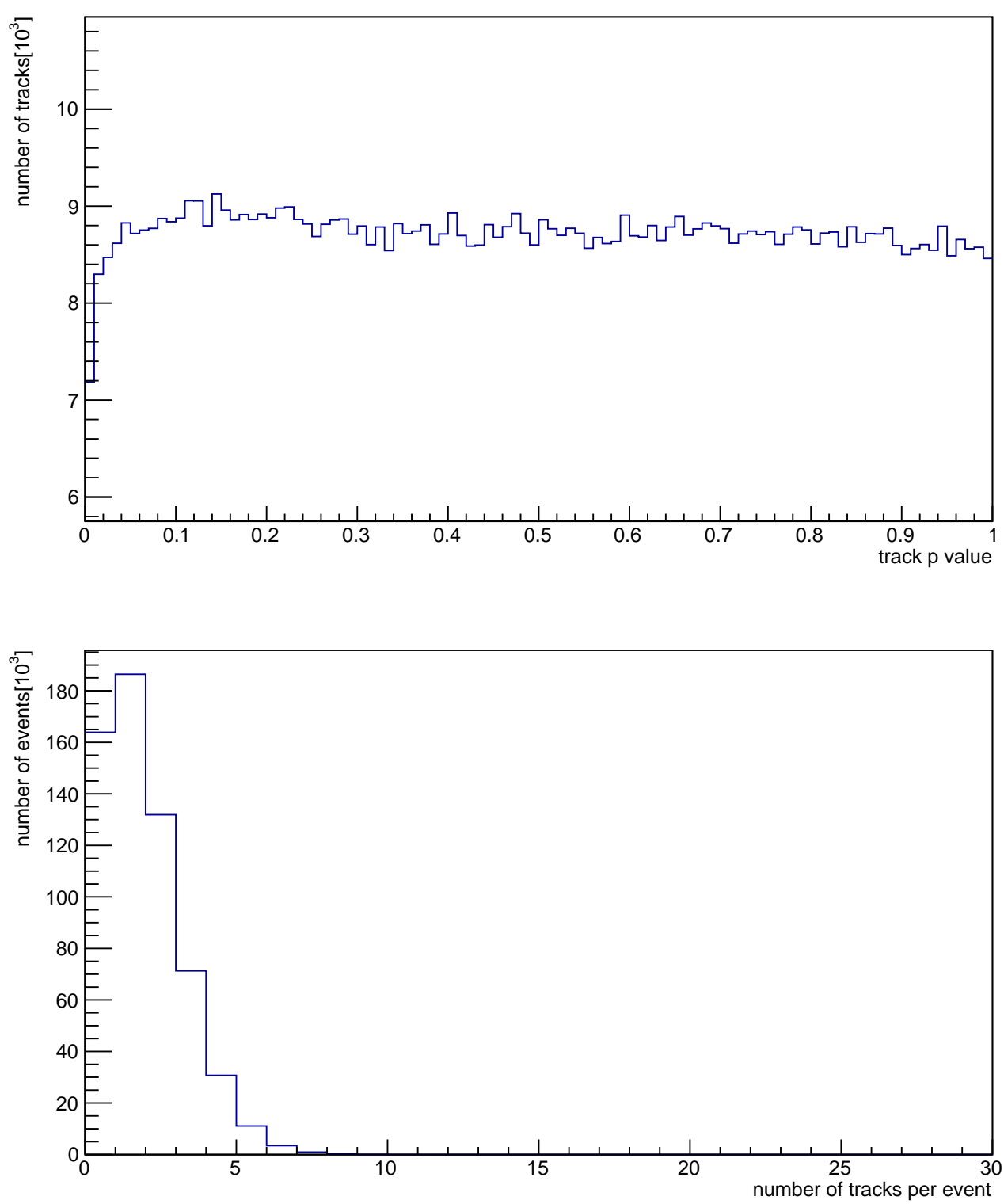

Figure D.10.: $p$ values of the calibration run track sample after the telescope alignment and cluster calibration and number of tracks per event during the beam test campaign in November of 2017 
D. Telescope and $X / X_{0}$ calibration of several beam test measurements
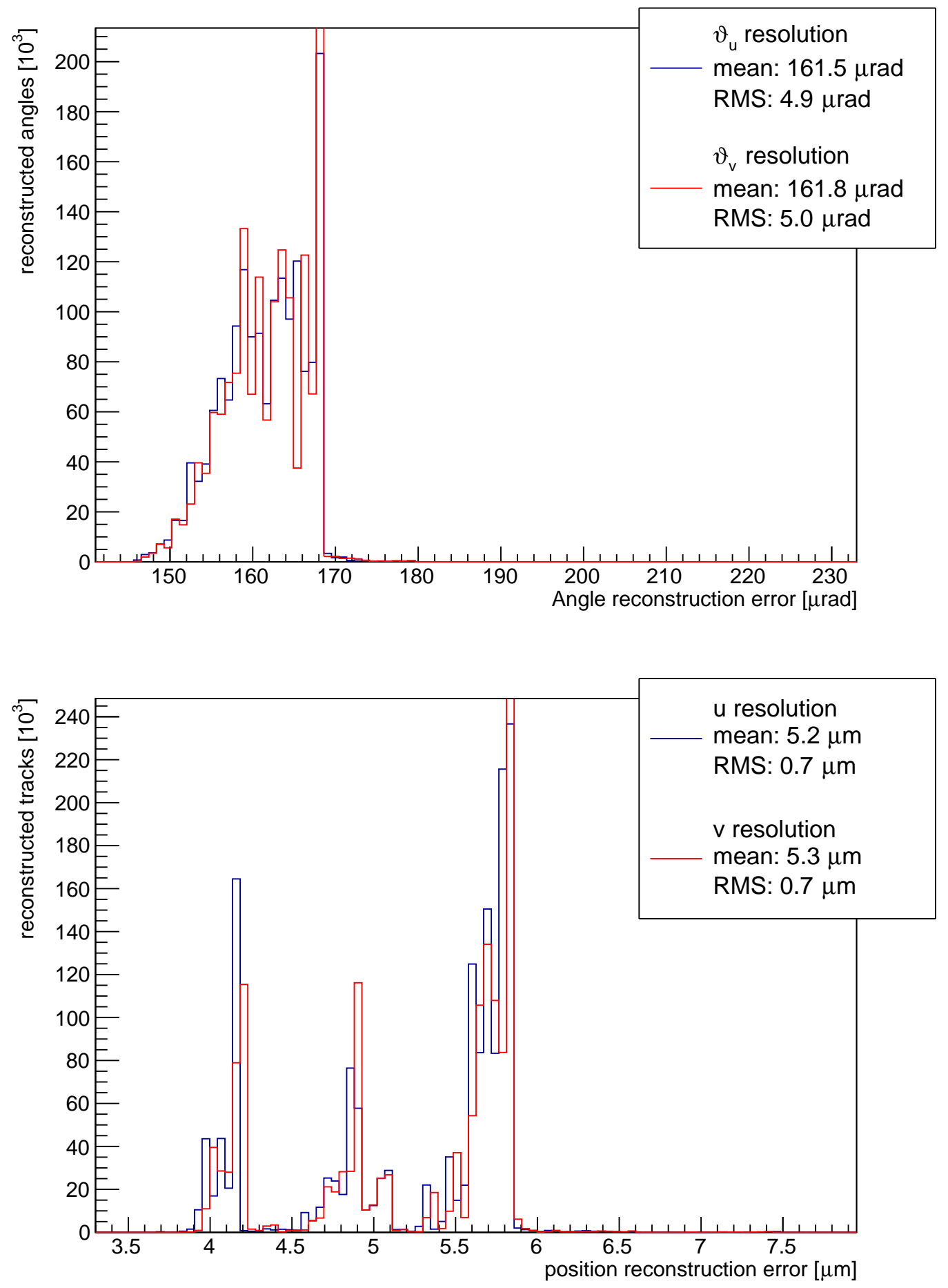

Figure D.11.: Angle reconstruction errors and track intersection errors in $u$ and $v$ direction. 


\begin{tabular}{|c|c|c|c|c|}
\hline Calibration method & $\lambda$ & $\kappa$ & $\Delta_{u} p[\mathrm{MeV} / \mathrm{mm}]$ & $\Delta_{v} p[\mathrm{MeV} / \mathrm{mm}]$ \\
\hline No bremsstrahlung & $1.017 \pm 0.006$ & $1.095 \pm 0.007$ & 0 & 0 \\
\hline Weight model $(\epsilon=0.30)$ & $1.017 \pm 0.006$ & $1.077 \pm 0.007$ & 0 & 0 \\
\hline Weight model $(\epsilon=0.40)$ & $1.017 \pm 0.006$ & $1.071 \pm 0.0073$ & 0 & 0 \\
\hline Weight model $(\epsilon=0.50)$ & $1.017 \pm 0.006$ & $1.065 \pm 0.007$ & 0 & 0 \\
\hline Gottschalk model & $1.017 \pm 0.006$ & $1.065 \pm 0.007$ & 0 & 0 \\
\hline
\end{tabular}

Table D.2.: Beam test November 2017 calibration results. The given errors correspond to the statistical errors of the fit. The beam energy gradients were not determined.
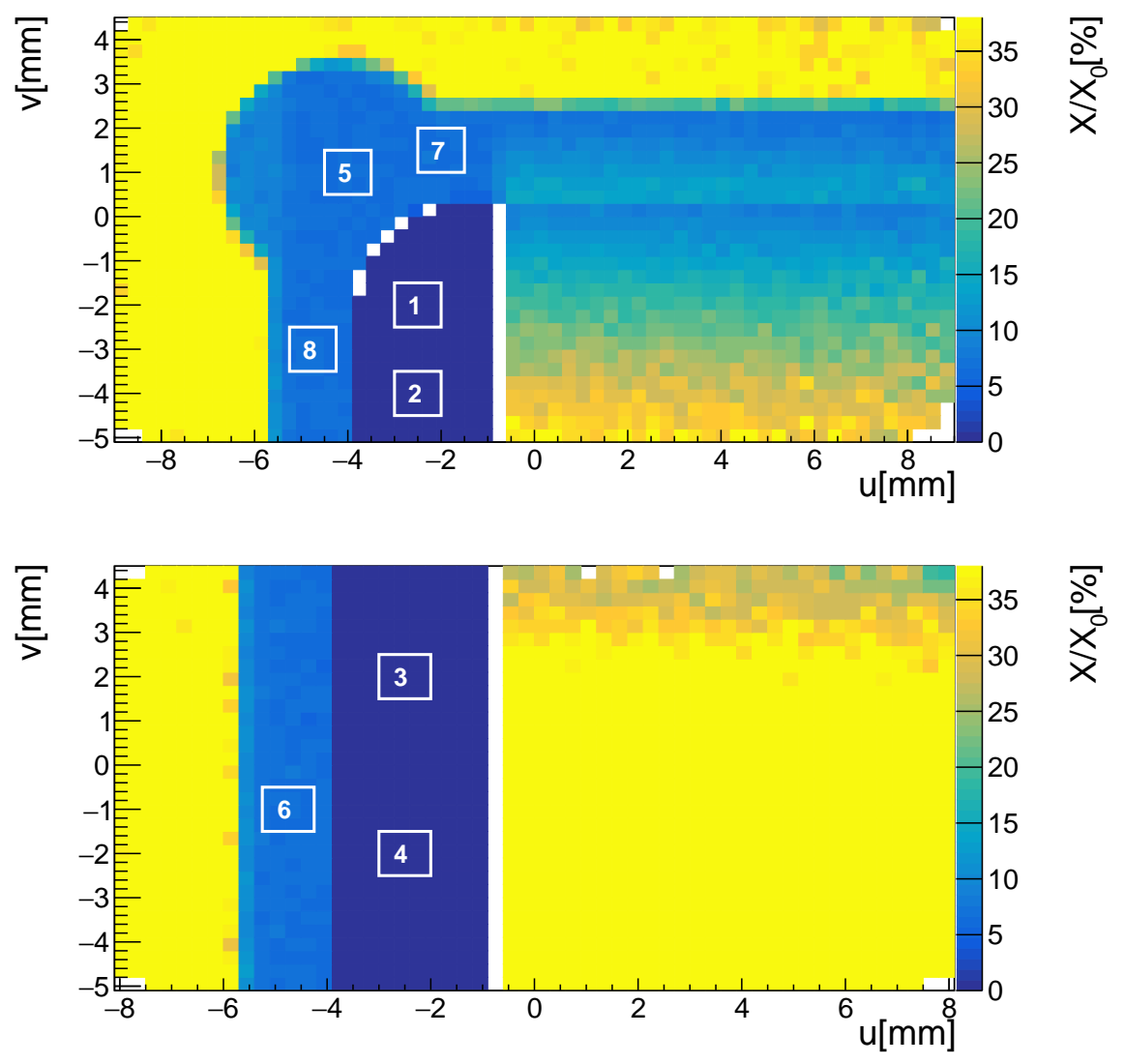

Figure D.12.: Visual representation of the position of the 8 different measurement regions, which are used during the radiation length calibration. Each marked measurement area contributes a single angle distribution with 50 thousand entries to the calibration fit. The calibration measurement areas lie on the left hand side of the copper wedge, where $0.5 \mathrm{~mm}$ of aluminium and air is present. 

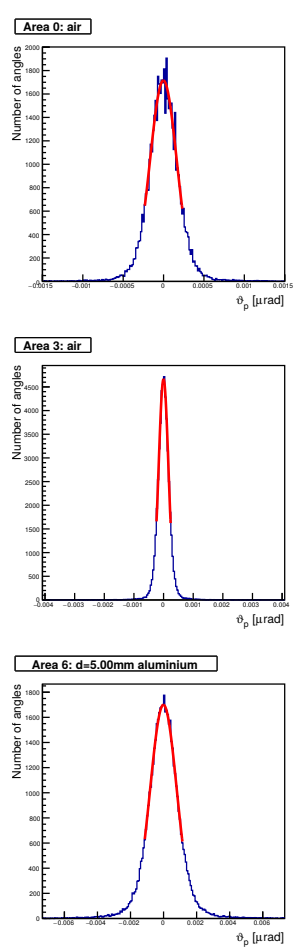
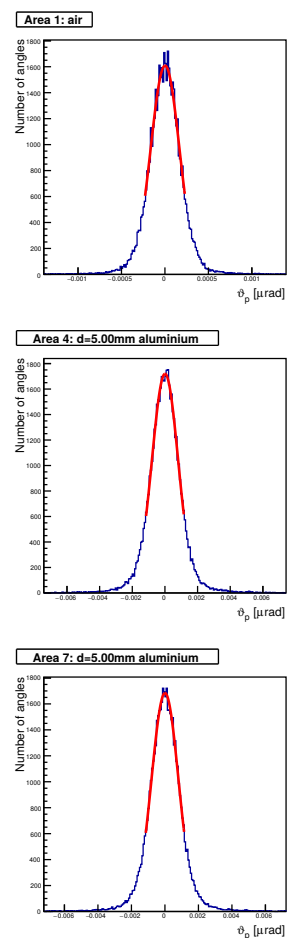
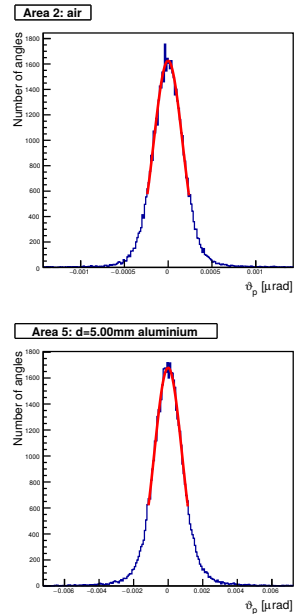


\section{D.3. Glue measurements}

In 2016 a beam test was conducted. The scope of the measurements was to measure the material constant $X_{0}$ of different samples of conductive glues. The results of the measurements can be found in section 7.3. The telescope setup of the measurements is depicted in fig. D.15. The beam energy was $4.6 \mathrm{GeV}$. Table D. 3 contains an overview over the measured telescope data. It specifies the number of runs, overall events and overall tracks as well as the purpose of the measurements.

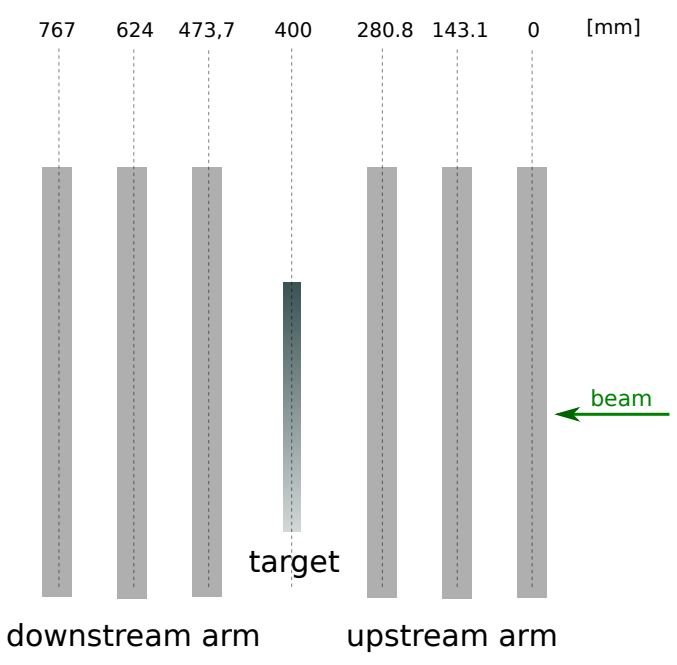

Figure D.15.: Schematic side view of the telescope setup during the glue measurement beam test campaign.

\begin{tabular}{|c||c|c|c|c|}
\hline Target & \#runs & \# events & \#tracks & comments \\
\hline air & 4 & 4.9 mio & 15.3 mio & telescope and $X / X_{0}$ calibration \\
\hline glue A & 5 & 3.6 mio & 9.0 mio & $X / X_{0}$ measurement \\
\hline glue B & 26 & 27.4 mio & 68.6 mio & $X / X_{0}$ measurement \\
\hline glue C & 4 & 3.6 mio & 9.1 mio & $X / X_{0}$ measurement \\
\hline glue D & 5 & 5.0 mio & 12.5 mio & $X / X_{0}$ measurement \\
\hline empty brass & 2 & 1.1 mio & 2.7 mio & $X / X_{0}$ calibration and measurement \\
\hline
\end{tabular}

Table D.3.: Overview over the telescope data at the glue measurement beam test. The spacings between the M26 sensors (see fig. D.15) was not changed between the measurements.

All other DQM plots, which have been explained in section 7.1.1, are listed on the following pages. 
D. Telescope and $X / X_{0}$ calibration of several beam test measurements

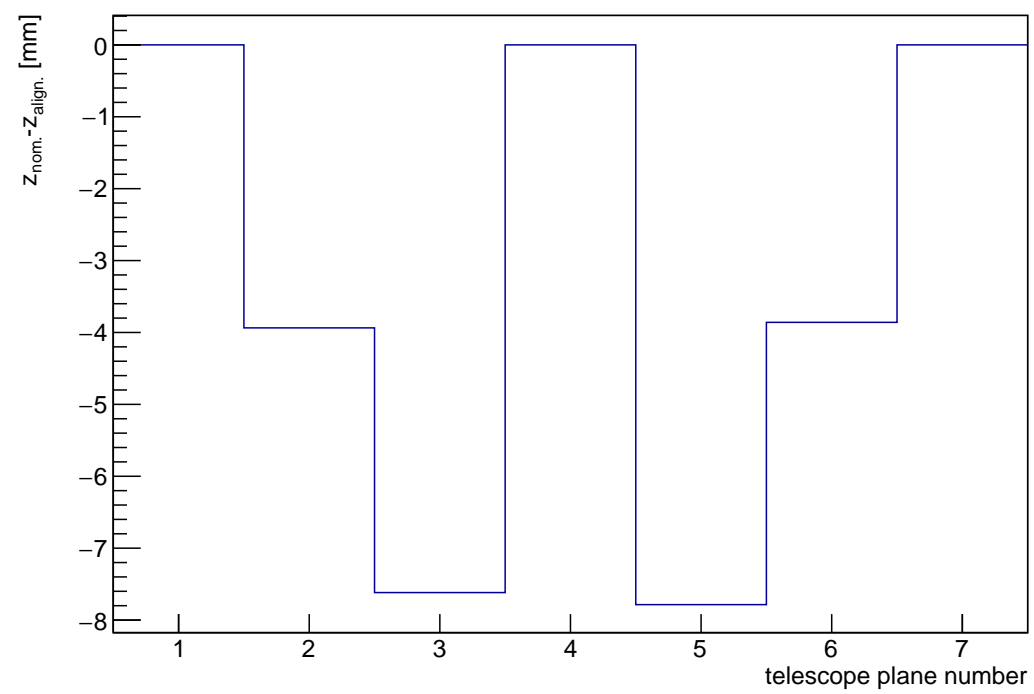

Figure D.16.: Difference between nominal and aligned sensor $z$ positions.

\section{9 million tracks}

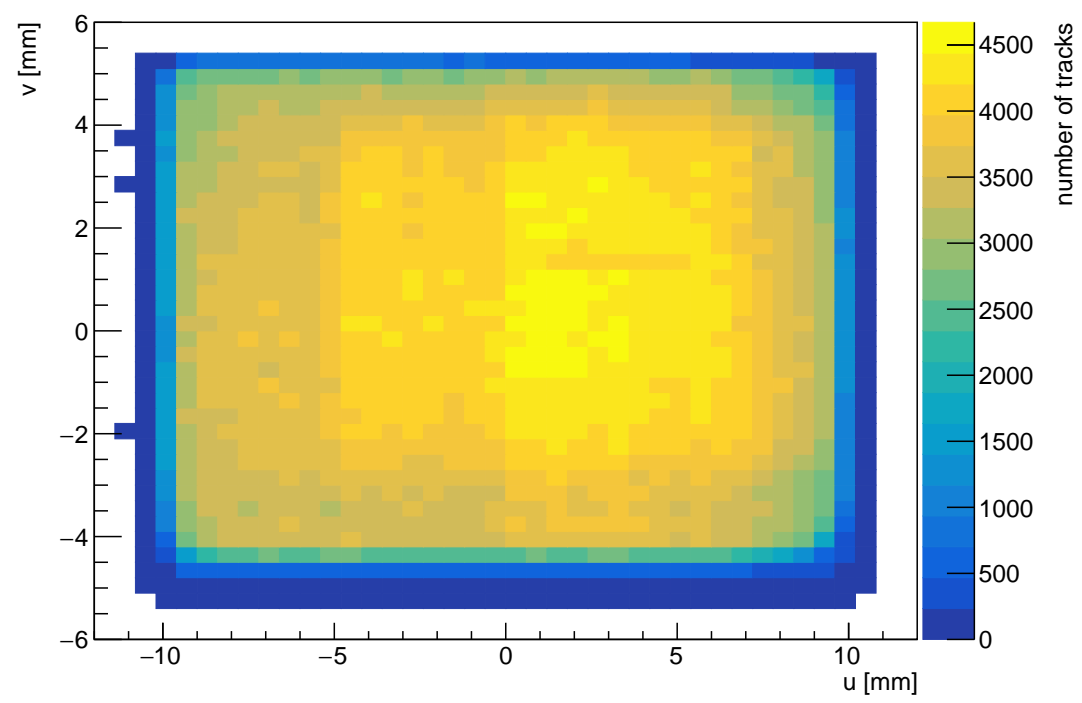

Figure D.17.: Beam spot on the central scattering plane during an example run of the glue measurement beam test.

\begin{tabular}{|c|c|c|c|}
\hline$\lambda$ & $\kappa$ & $\nabla_{u}(\mathrm{E})[\mathrm{MeV} / \mathrm{mm}]$ & $\nabla_{v}(\mathrm{E})[\mathrm{MeV} / \mathrm{mm}]$ \\
\hline $1.092 \pm 0.004$ & $1.009 \pm 0.005$ & 0 & 0 \\
\hline
\end{tabular}

Table D.4.: Glue measurement calibration results. The given errors correspond to the statistical errors of the fit. The beam energy gradients were not determined. 


\section{cluster shape frequency}
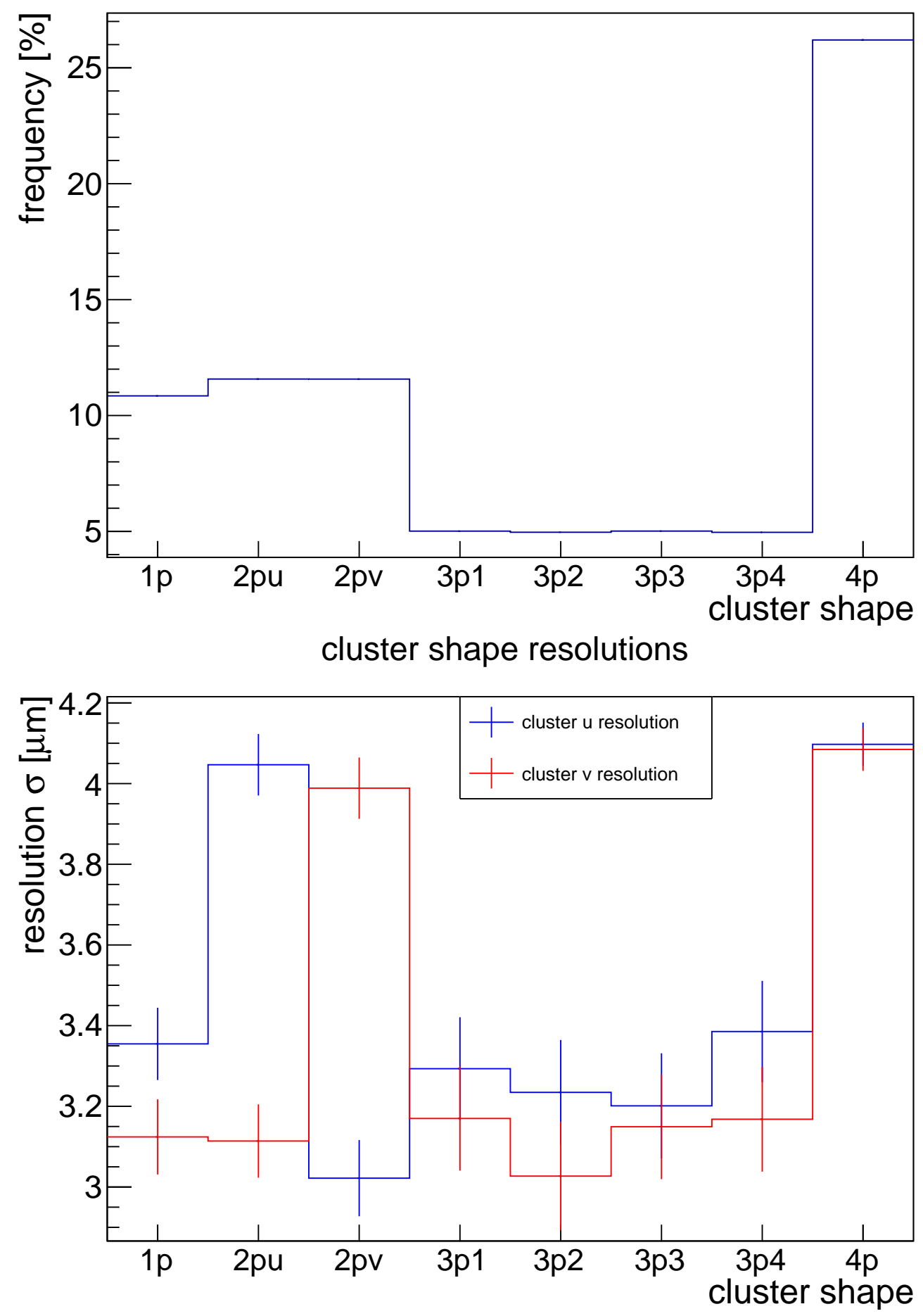

Figure D.18.: Cluster shape frequencies and cluster $u$ and $v$ resolution during the glue measurement beam test campaign. The cluster shapes, which correspond to the individual labels on the $x$ axis, are depicted in figure 4.9 . 
D. Telescope and $X / X_{0}$ calibration of several beam test measurements
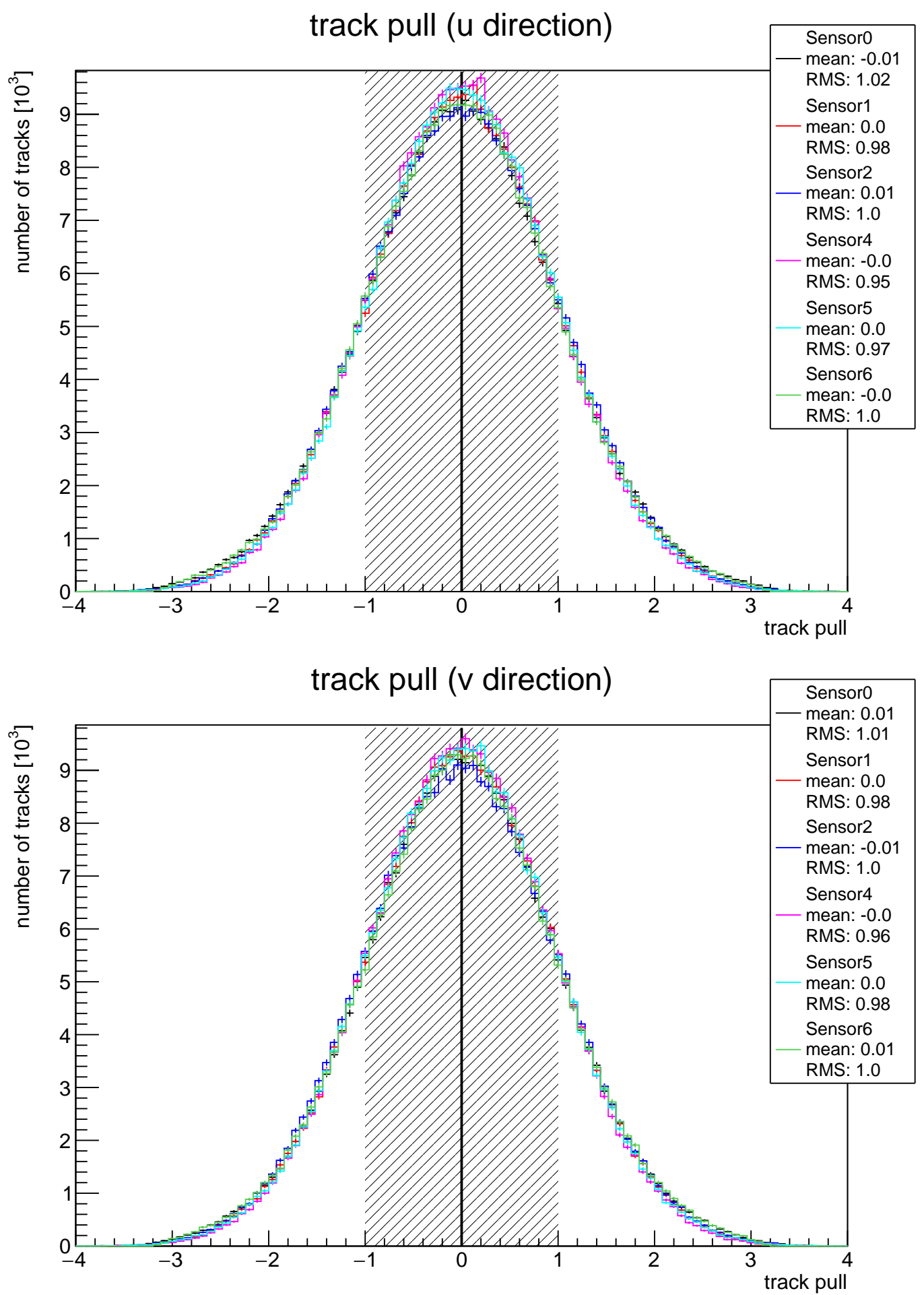

Figure D.19.: Pulls of the $u$ and $v$ residuals after cluster calibration and telescope alignment. The Pulls are centred around 0 and have a RMS value close to 1 . 

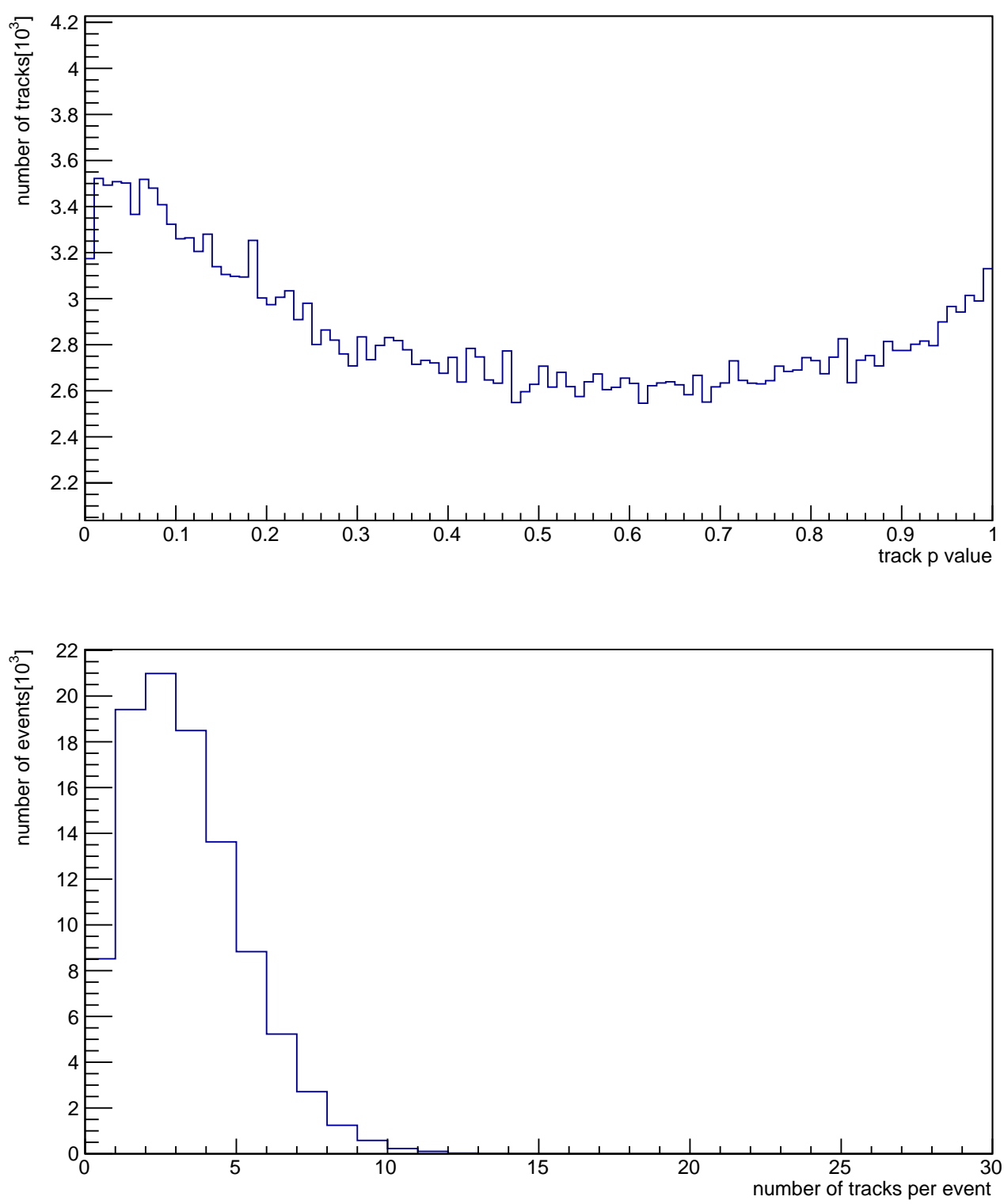

Figure D.20.: $p$ values of the calibration run track sample after the telescope alignment and cluster calibration and number of tracks per event during the glue measurement beam test campaign. 
D. Telescope and $X / X_{0}$ calibration of several beam test measurements
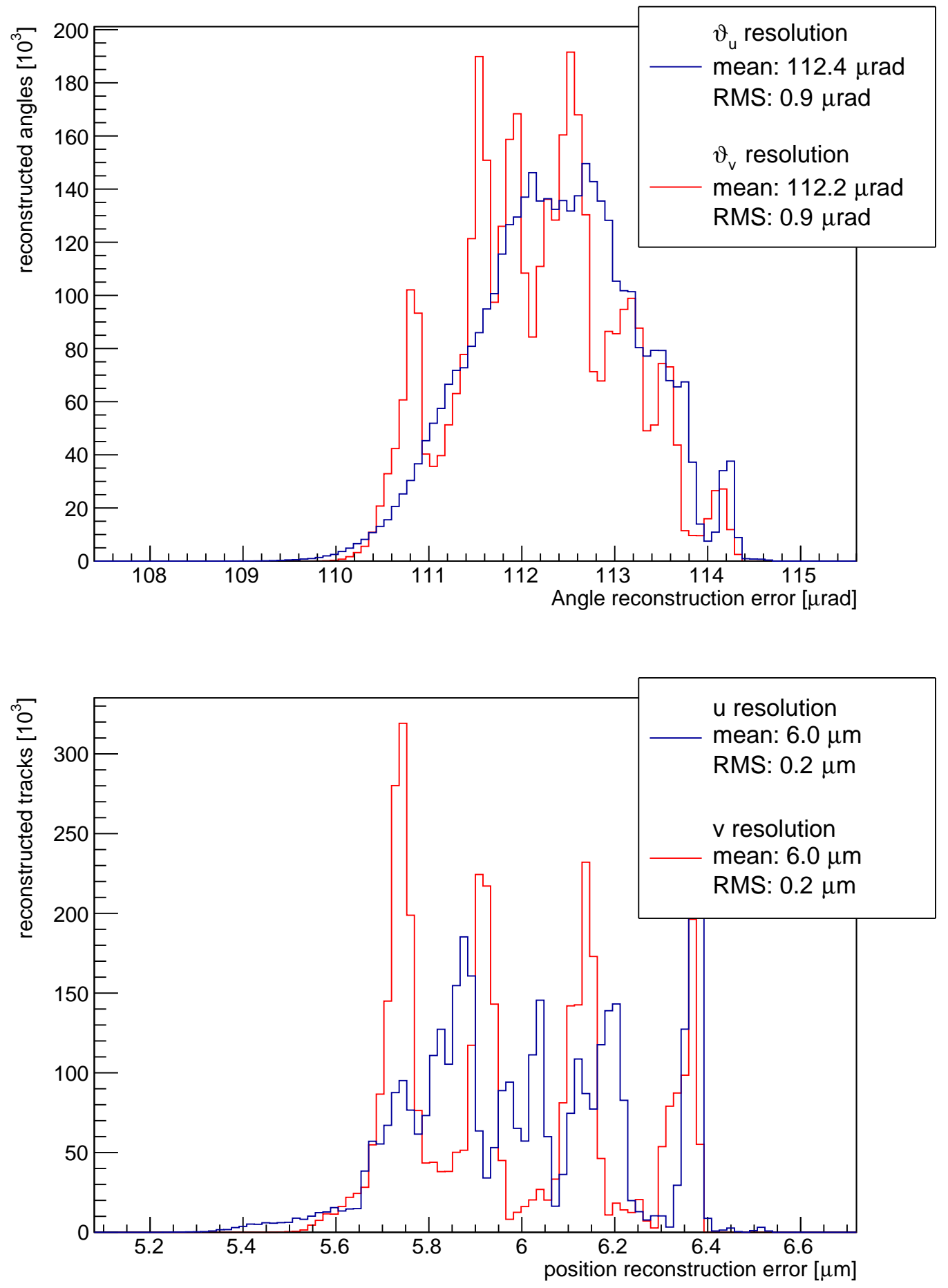

Figure D.21.: Angle reconstruction errors and track intersection errors in $u$ and $v$ direction. 

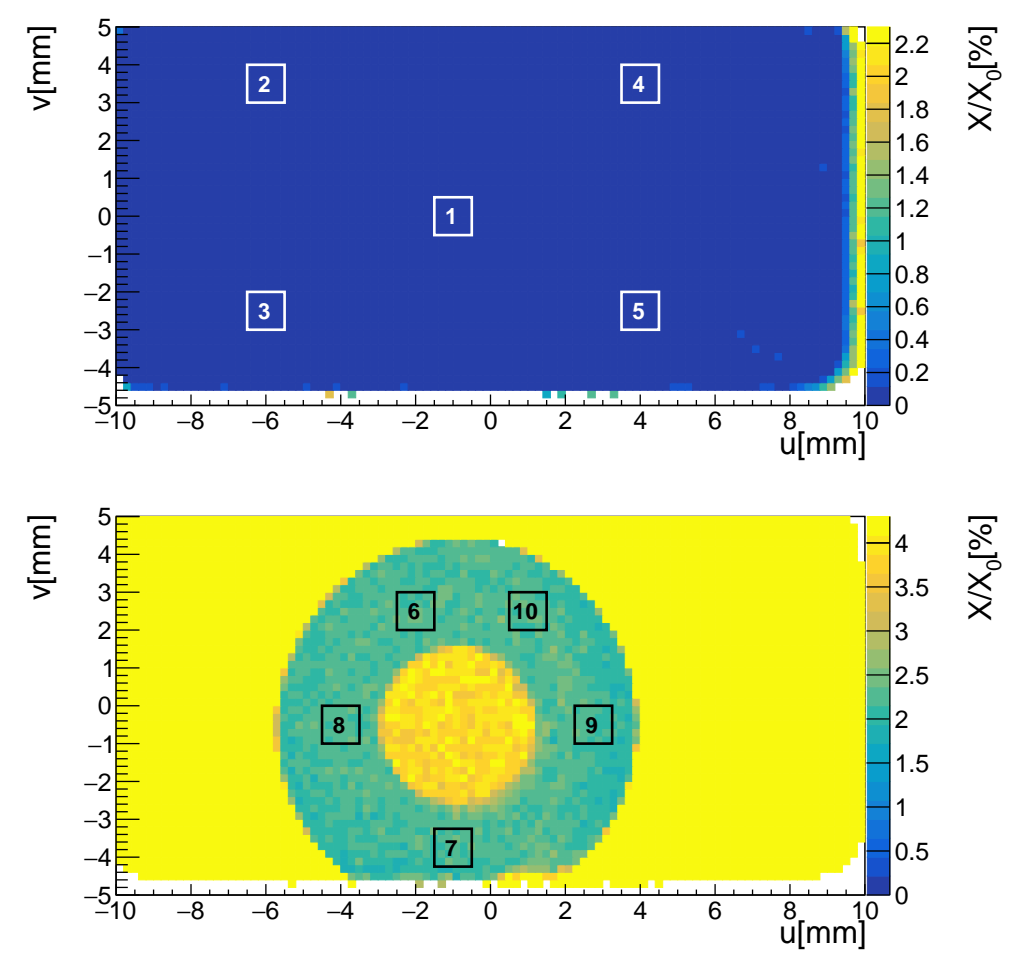

Figure D.22.: Visual representation of the position of the 10 different measurement regions, which are used during the radiation length calibration. Each marked measurement area contributes a single angle distribution with 100 thousand entries to the calibration fit. The top radiation length image depicts an air measurement. The bottom image depicts glue sample D. The green area, which contains the measurement points corresponds to $1.8 \mathrm{~mm}$ of aluminium.

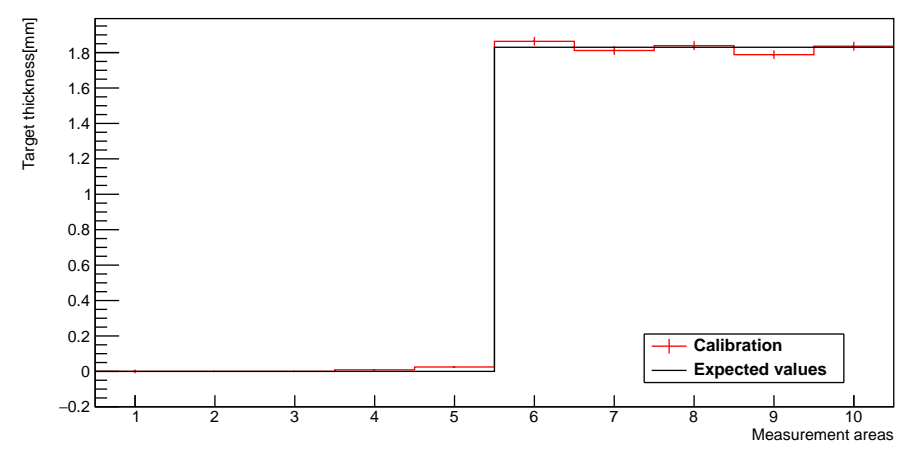

Figure D.23.: Self consistency measurements after the radiation length calibration prodecure. 

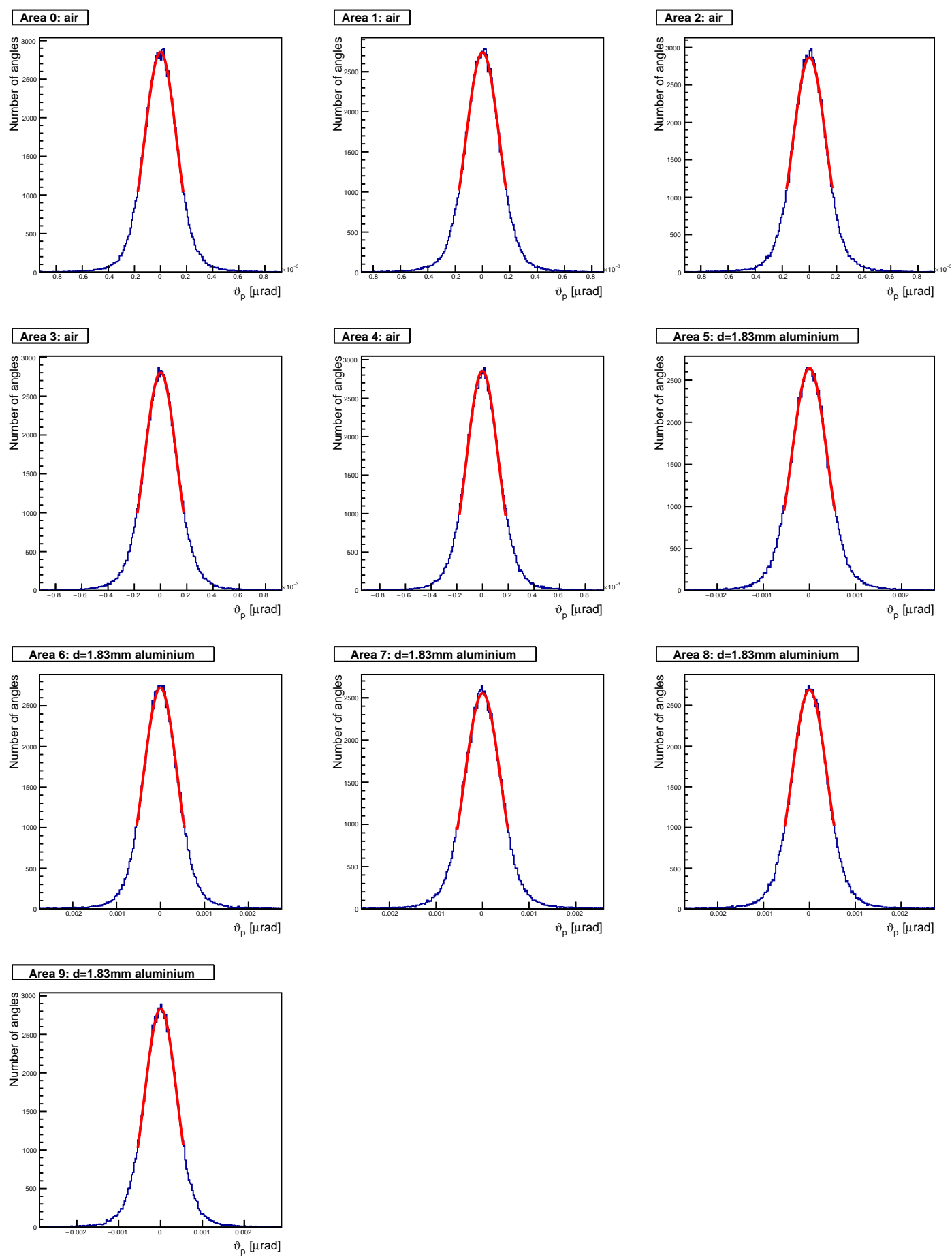

Figure D.24.: All scattering angle distributions, that have been used during the radiation length calibration together with the corresponding fit function. The fit matched the distributions quite well and the fit $\chi^{2}$ is small. 


\section{D.4. Chip measurements in October 2016}

In October 2016 a beam test was conducted. The scope of the measurements was to study the influence of the M26 threshold on the frequency and resolution of the different cluster shapes. The results of the M26 threshold scan can be found in section 4.4. Additionally a material budget image measurement of a printed circuit board (PCB) including a field programmable gate array (FPGA) chip was performed (see section 7.4). The telescope setup of the measurements is depicted in fig. D.25. The beam energy was $2 \mathrm{GeV}$ and the M26 threshold was 4. Table D.5 contains an overview over the measured telescope data. It specifies the number of runs, overall events and overall tracks as well as the purpose of the measurements.

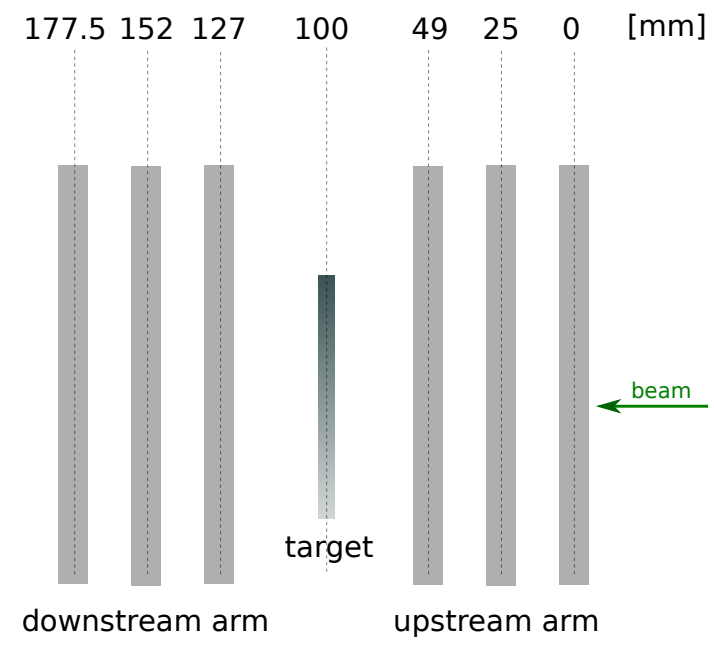

Figure D.25.: Schematic side view of the telescope setup during October 2016 beam test campaign.

\begin{tabular}{|c||c|c|c|c|}
\hline Target & \#runs & \#events & \#tracks & comments \\
\hline air & 6 & 6 mio & 30 mio & telescope calibration, $X / X_{0}$ calibration \\
\hline $0.5 \mathrm{~mm}$ alu & 6 & 6 mio & 30 mio & $X / X_{0}$ calibration \\
\hline $1.0 \mathrm{~mm}$ alu & 6 & 6 mio & 30 mio & $X / X_{0}$ calibration \\
\hline chip & 60 & $60 \mathrm{mio}$ & $130 \mathrm{mio}$ & $X / X_{0}$ measurement \\
\hline
\end{tabular}

Table D.5.: Overview over the telescope data at the beam test in October 2016. The spacings between the M26 sensors (see fig. D.25) was not changed between the measurements.

All other DQM plots, which have been explained in section 7.1.1, are listed on the following pages. 
D. Telescope and $X / X_{0}$ calibration of several beam test measurements

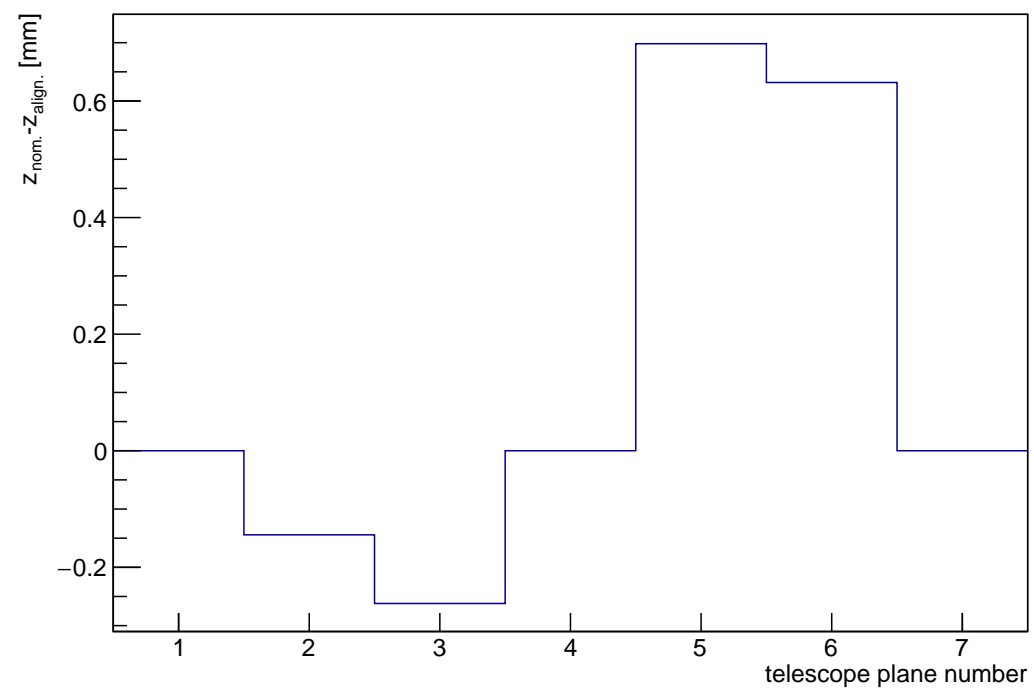

Figure D.26.: Difference between nominal and aligned sensor $z$ positions.

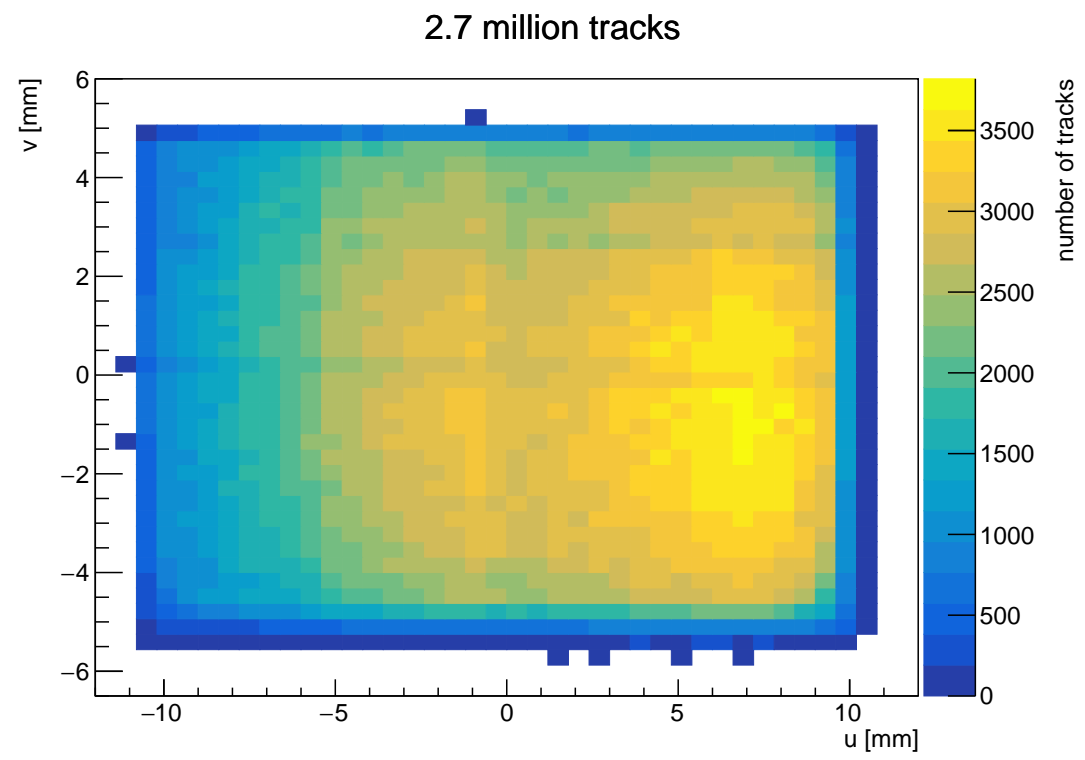

Figure D.27.: Beam spot on the central scattering plane during an example run of the October 2016 beam test.

\begin{tabular}{|c|c|c|c|}
\hline$\lambda$ & $\kappa$ & $\nabla_{u}(\mathrm{E})[\mathrm{MeV} / \mathrm{mm}]$ & $\nabla_{v}(\mathrm{E})[\mathrm{MeV} / \mathrm{mm}]$ \\
\hline $1.049 \pm 0.004$ & $0.995 \pm 0.004$ & $9 \pm 2$ & $-2 \pm 3$ \\
\hline
\end{tabular}

Table D.6.: October $2016 X / X_{0}$ calibration results. The given errors correspond to the statistical errors of the fit. 


\section{cluster shape frequency}
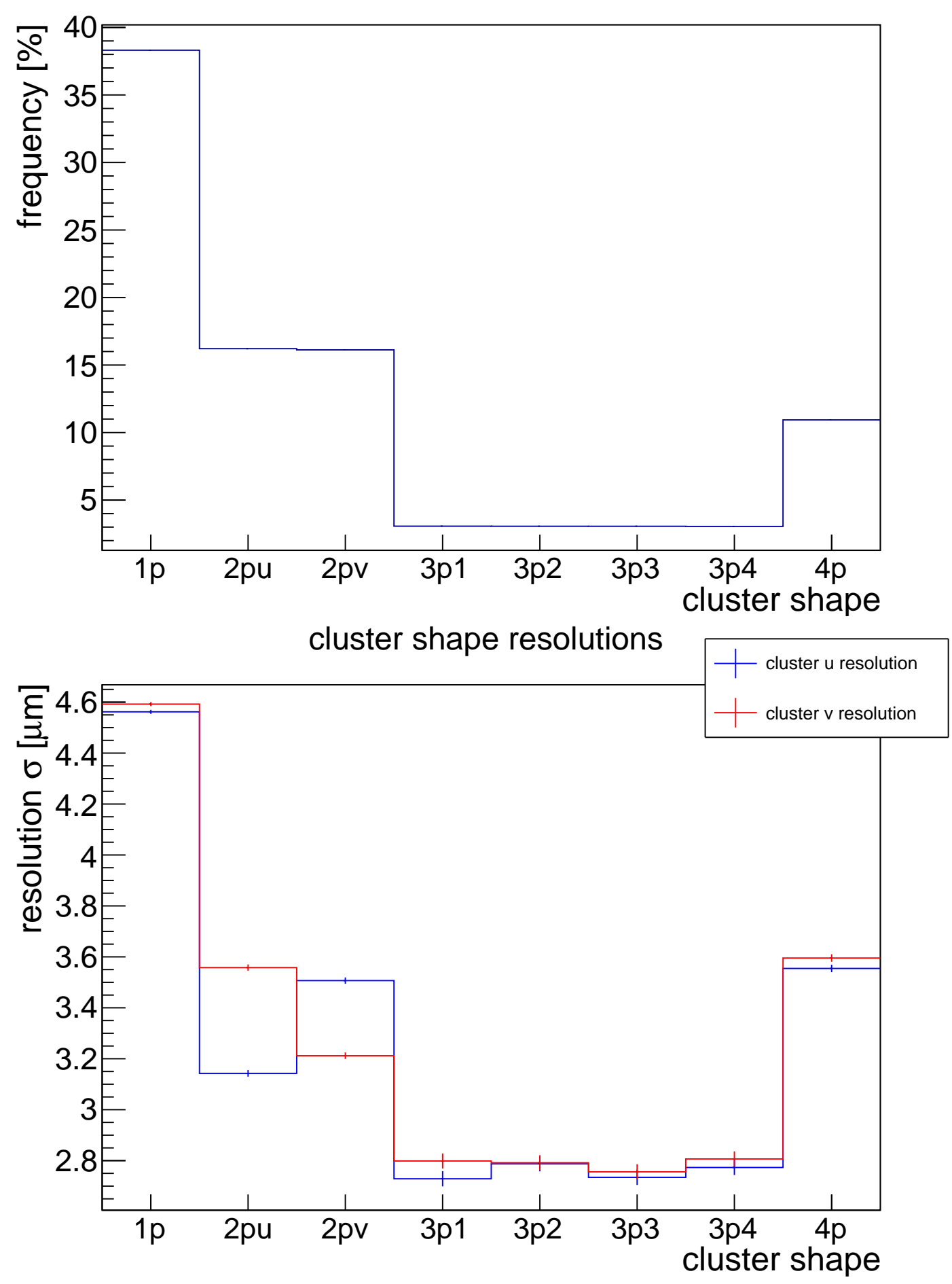

Figure D.28.: Cluster shape frequencies and cluster $u$ and $v$ resolution during the October 2016 beam test campaign. The cluster shapes, which correspond to the individual labels on the $x$ axis, are depicted in figure 4.9 . 
D. Telescope and $X / X_{0}$ calibration of several beam test measurements
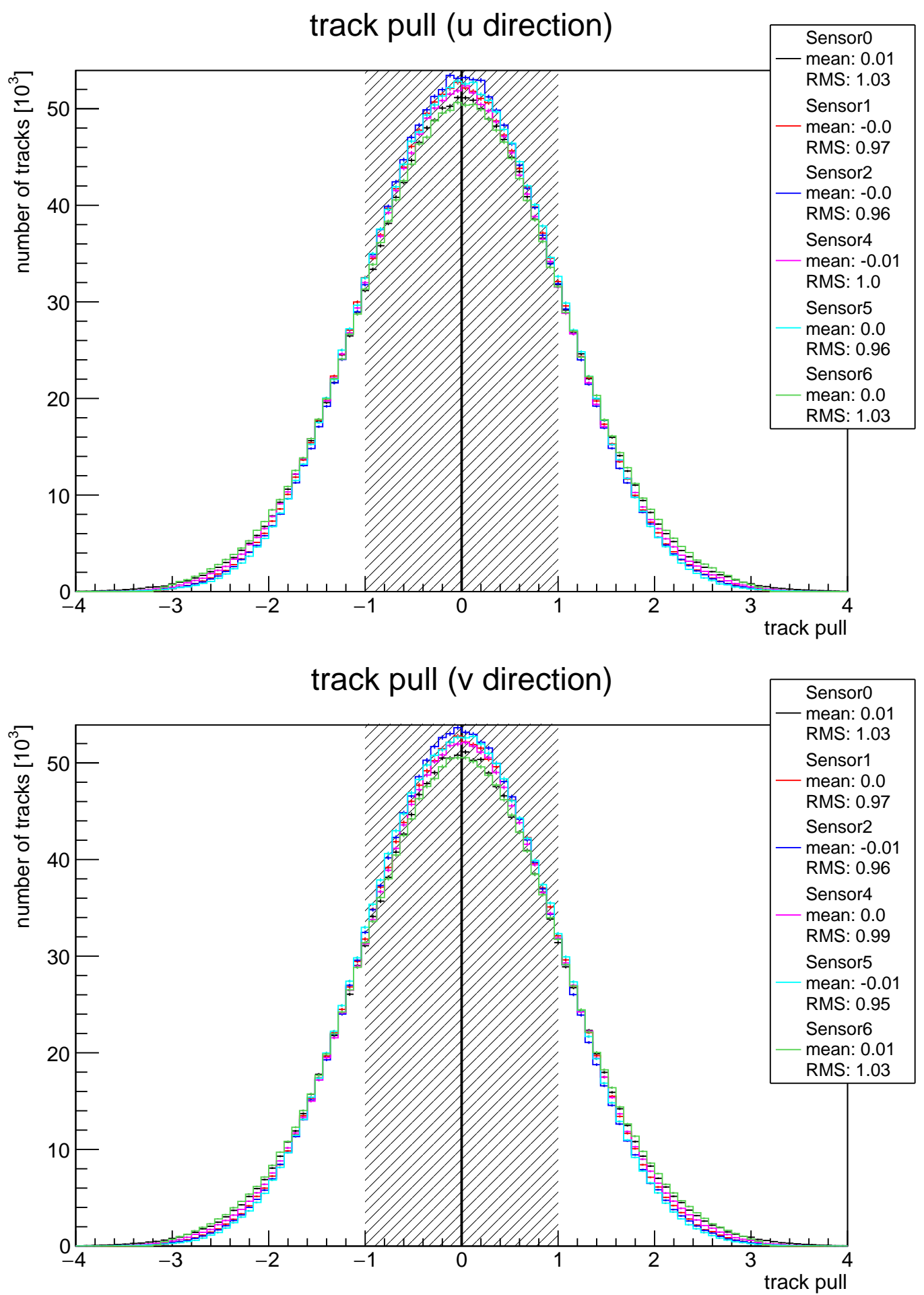

Figure D.29.: Pulls of the $u$ and $v$ residuals after cluster calibration and telescope alignment. The Pulls are centred around 0 and have a RMS value close to 1 . 

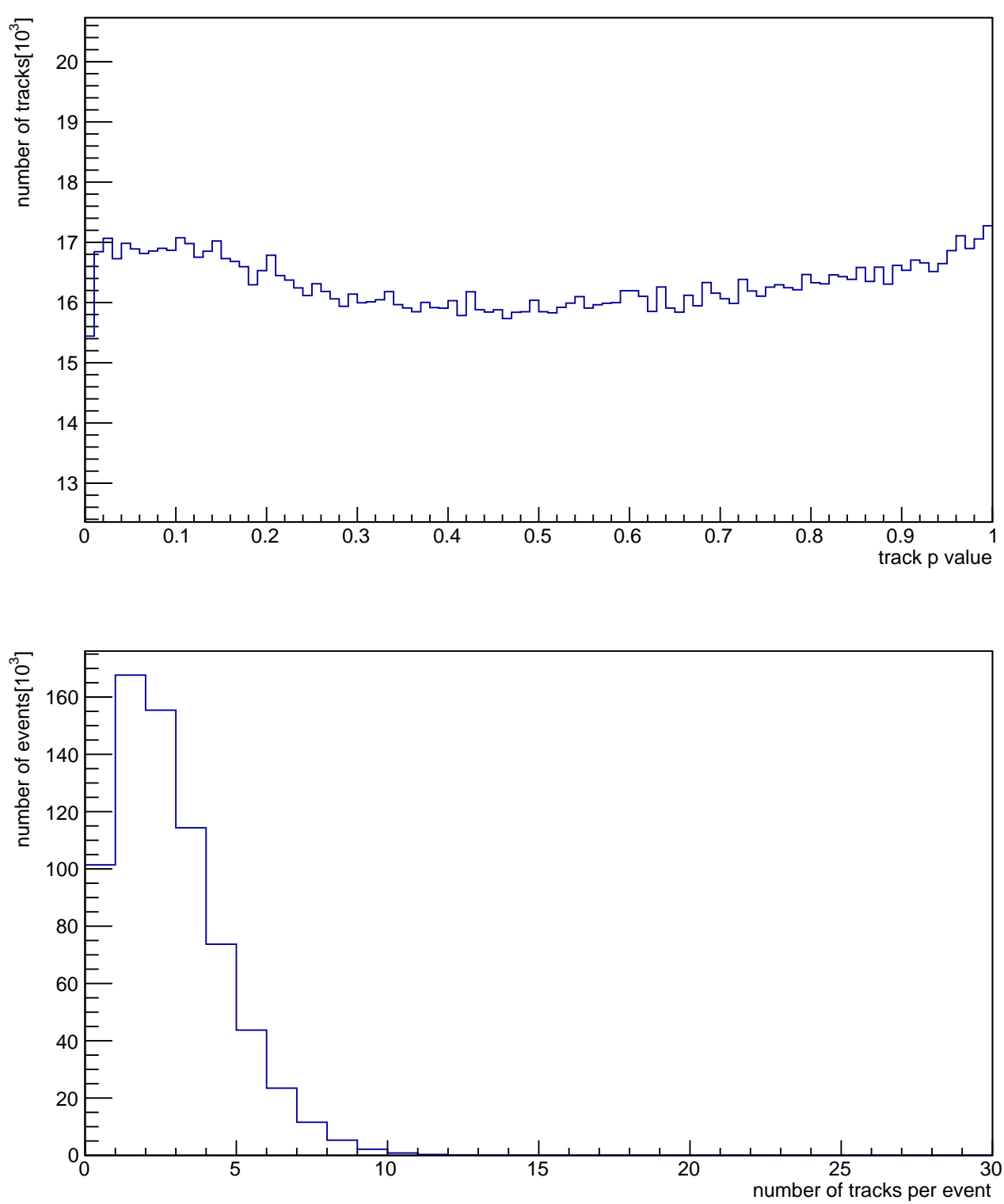

Figure D.30.: $p$ values of the calibration run track sample after the telescope alignment and cluster calibration and number of tracks per event during the October 2016 beam test campaign. 
D. Telescope and $X / X_{0}$ calibration of several beam test measurements
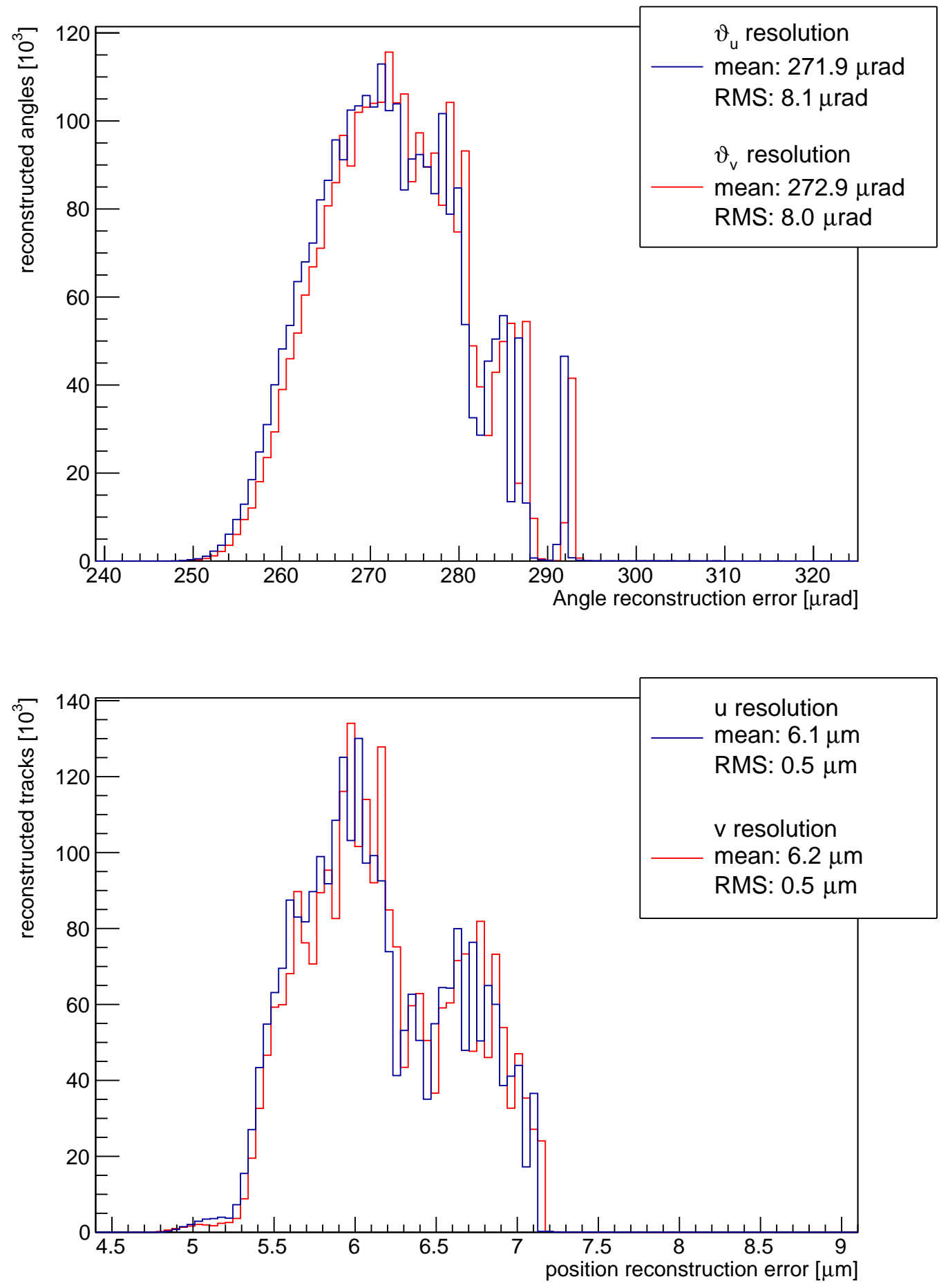

Figure D.31.: Angle reconstruction errors and track intersection errors in $u$ and $v$ direction. 

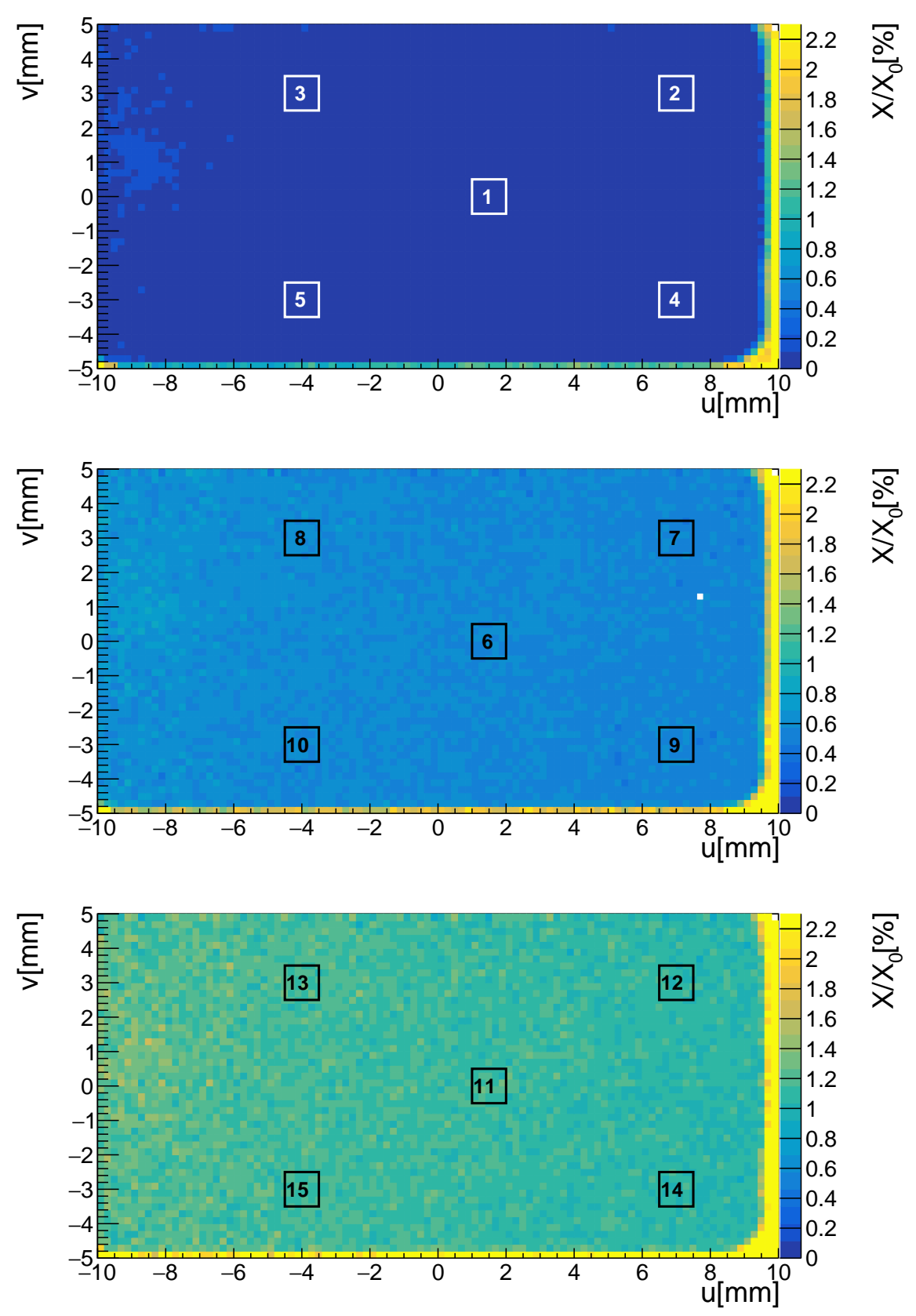

Figure D.32.: Visual representation of the position of the 15 different measurement regions, which are used during the radiation length calibration. Each marked measurement area contributes a single angle distribution with 100 thousand entries to the calibration fit. The top radiation length image shows air. The image in the middle depicts an aluminium layer with a thickness of $0.5 \mathrm{~mm}$ and the bottom image depicts an aluminium layer with a thickness of $1 \mathrm{~mm}$. 

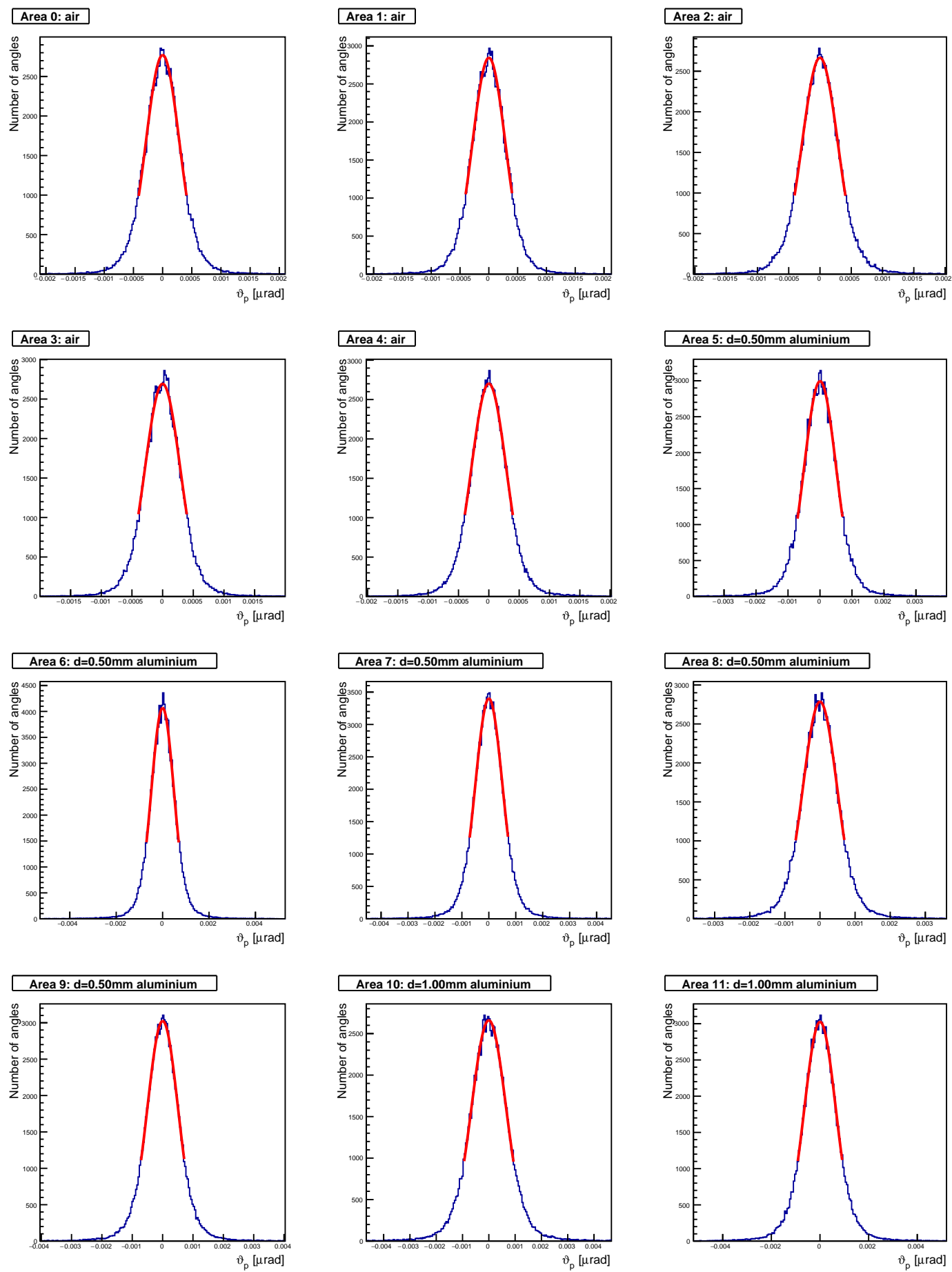

Figure D.33.: First twelve scattering angle distributions, that have been used during the radiation length calibration together with the corresponding fit function. The fit matched the distributions quite well and the fit $\chi^{2}$ is small. 

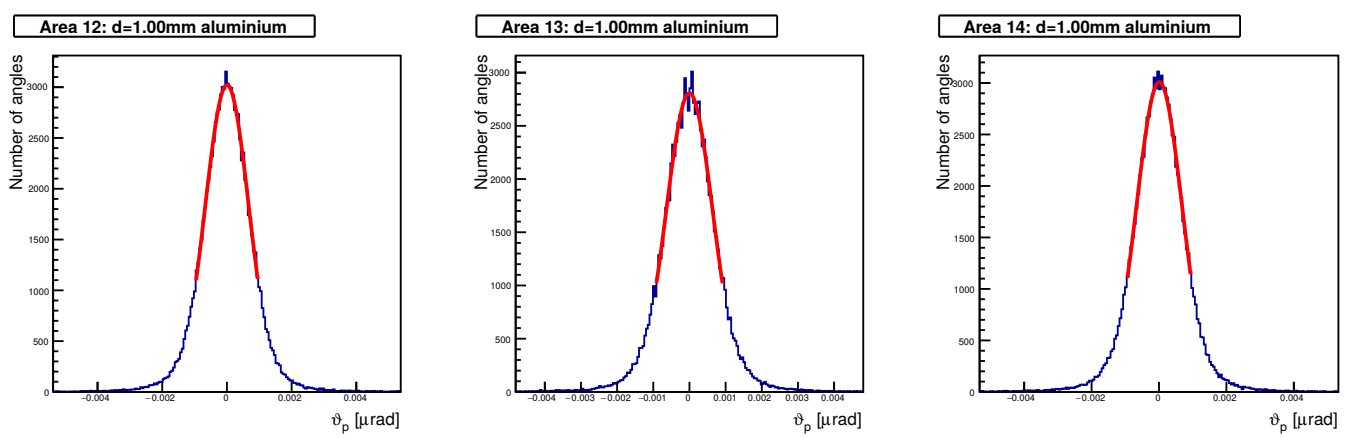

Figure D.34.: Remaining three scattering angle distributions, that have been used during the radiation length calibration together with the corresponding fit function. The fit matched the distributions quite well and the fit $\chi^{2}$ is small.

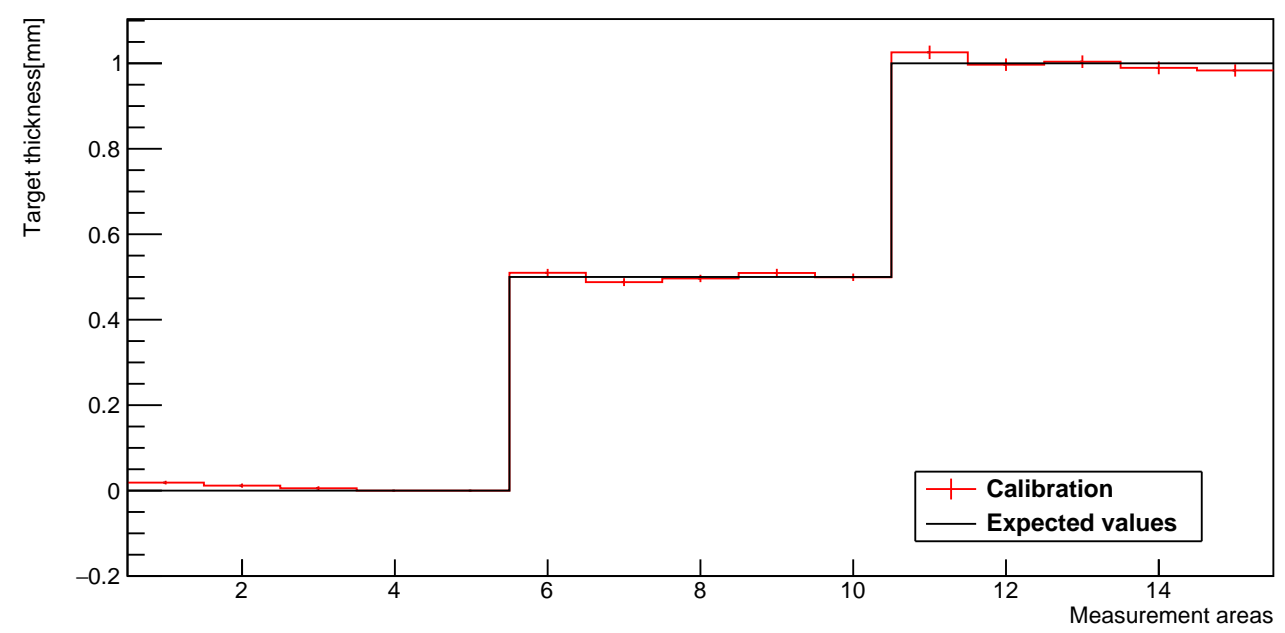

Figure D.35.: Self consistency measurements after the radiation length calibration prodecure. 


\section{D.5. ATLAS ITk prototype measurements}

In August 2016 a beam test was conducted. The scope of the measurements was to perform a material budget image measurement of a ATLAS ITk prototype module (see section 7.5). The telescope setup of the measurements is depicted in fig. D.36. The beam energy was $3 \mathrm{GeV}$ and the M26 threshold was 4 times the noise level. Table D.7 contains an overview over the measured telescope data. It specifies the number of runs, overall events and overall tracks as well as the purpose of the measurements.

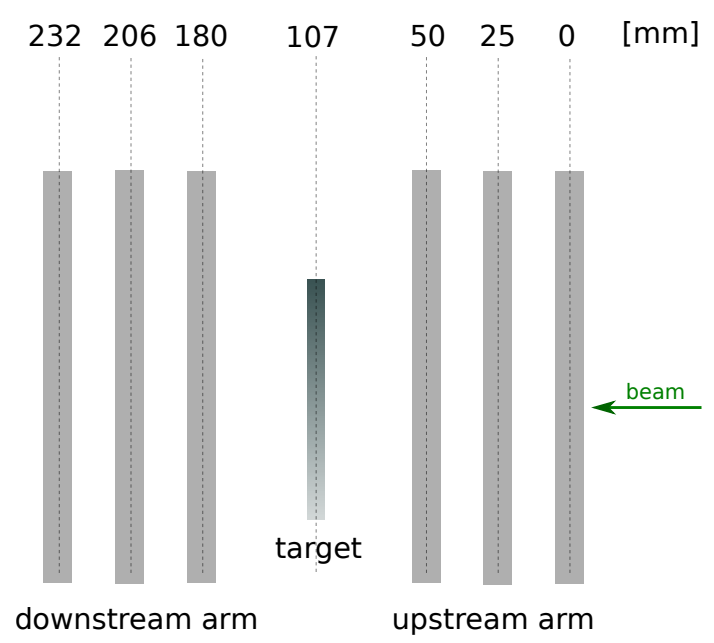

Figure D.36.: Schematic side view of the telescope setup during August 2016 beam test campaign.

\begin{tabular}{|c||c|c|c|c|}
\hline Target & \#runs & \#events $\left[10^{6}\right]$ & \#angles $\left[10^{6}\right]$ & purpose \\
\hline Aluminium & 7 & 6.0 & 26.9 & telescope $\& X / X_{0}$ calibration \\
\hline Carbon foam & 10 & 8.1 & 32.9 & $X / X_{0}$ imaging \\
\hline ITk prototype & 27 & 29.9 & 280.3 & $X / X_{0}$ imaging \\
\hline
\end{tabular}

Table D.7.: Overview over the telescope data at the beam test in August 2016. The spacings between the M26 sensors (see fig. D.36) was not changed between the measurements.

All other DQM plots, which have been explained in section 7.1.1, are listed on the following pages.

\begin{tabular}{|c|c|c|c|}
\hline$\lambda$ & $\kappa$ & $\nabla_{u}(\mathrm{E})[\mathrm{MeV} / \mathrm{mm}]$ & $\nabla_{v}(\mathrm{E})[\mathrm{MeV} / \mathrm{mm}]$ \\
\hline $1.212 \pm 0.005$ & $0.907 \pm 0.02$ & $11 \pm 3$ & $7 \pm 10$ \\
\hline
\end{tabular}

Table D.8.: August $2016 X / X_{0}$ calibration results. The given errors correspond to the statistical errors of the fit. 


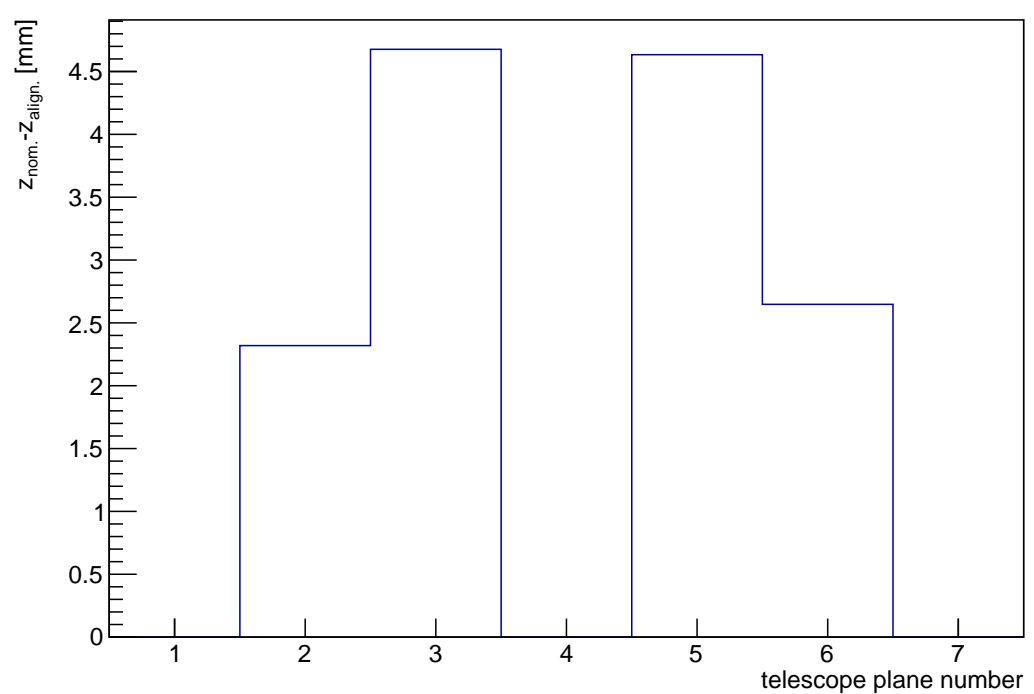

Figure D.37.: Difference between nominal and aligned sensor $z$ positions.

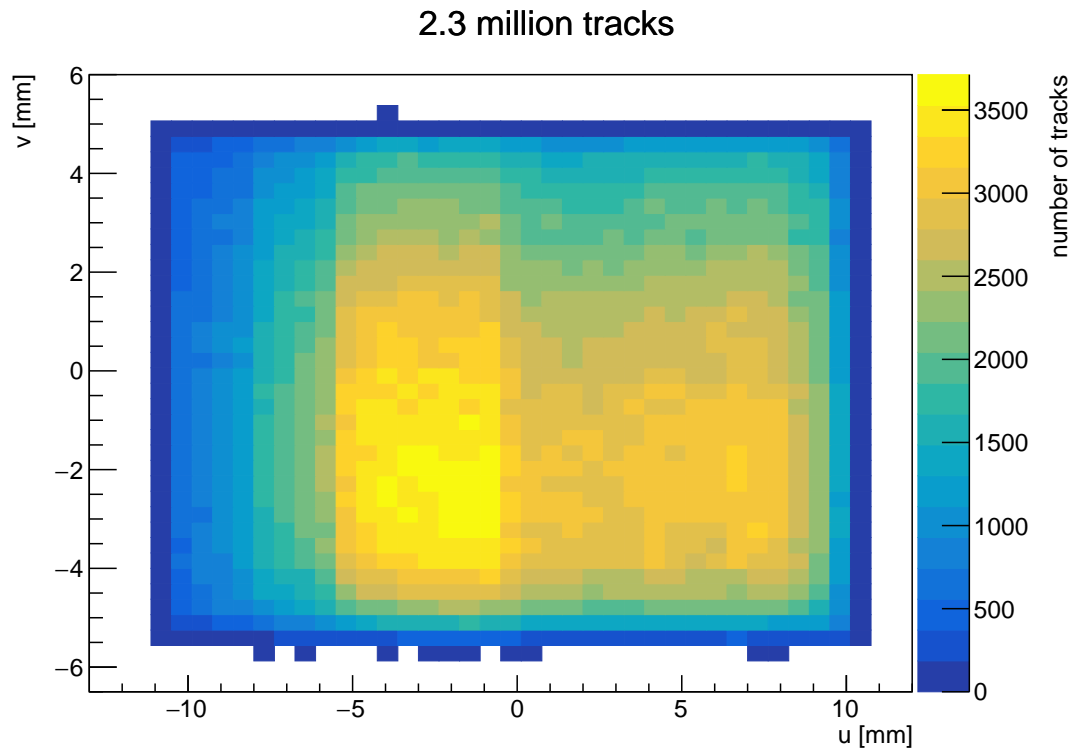

Figure D.38.: Beam spot on the central scattering plane during an example run of the August 2016 beam test. 
D. Telescope and $X / X_{0}$ calibration of several beam test measurements

\section{cluster shape frequency}
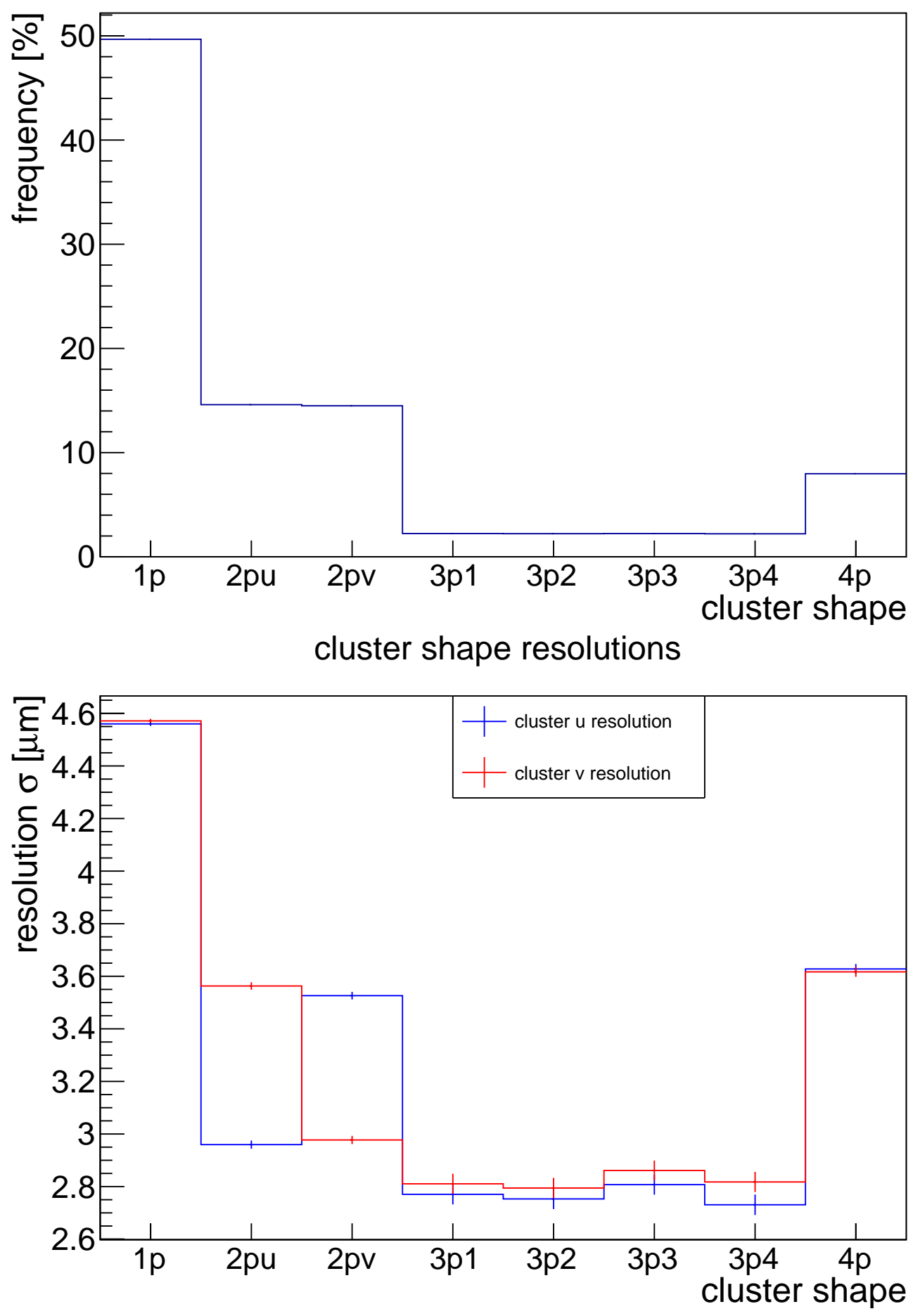

Figure D.39.: Cluster shape frequencies and cluster $u$ and $v$ resolution during the August 2016 beam test campaign. The cluster shapes, which correspond to the individual labels on the $x$ axis, are depicted in figure 4.9. 

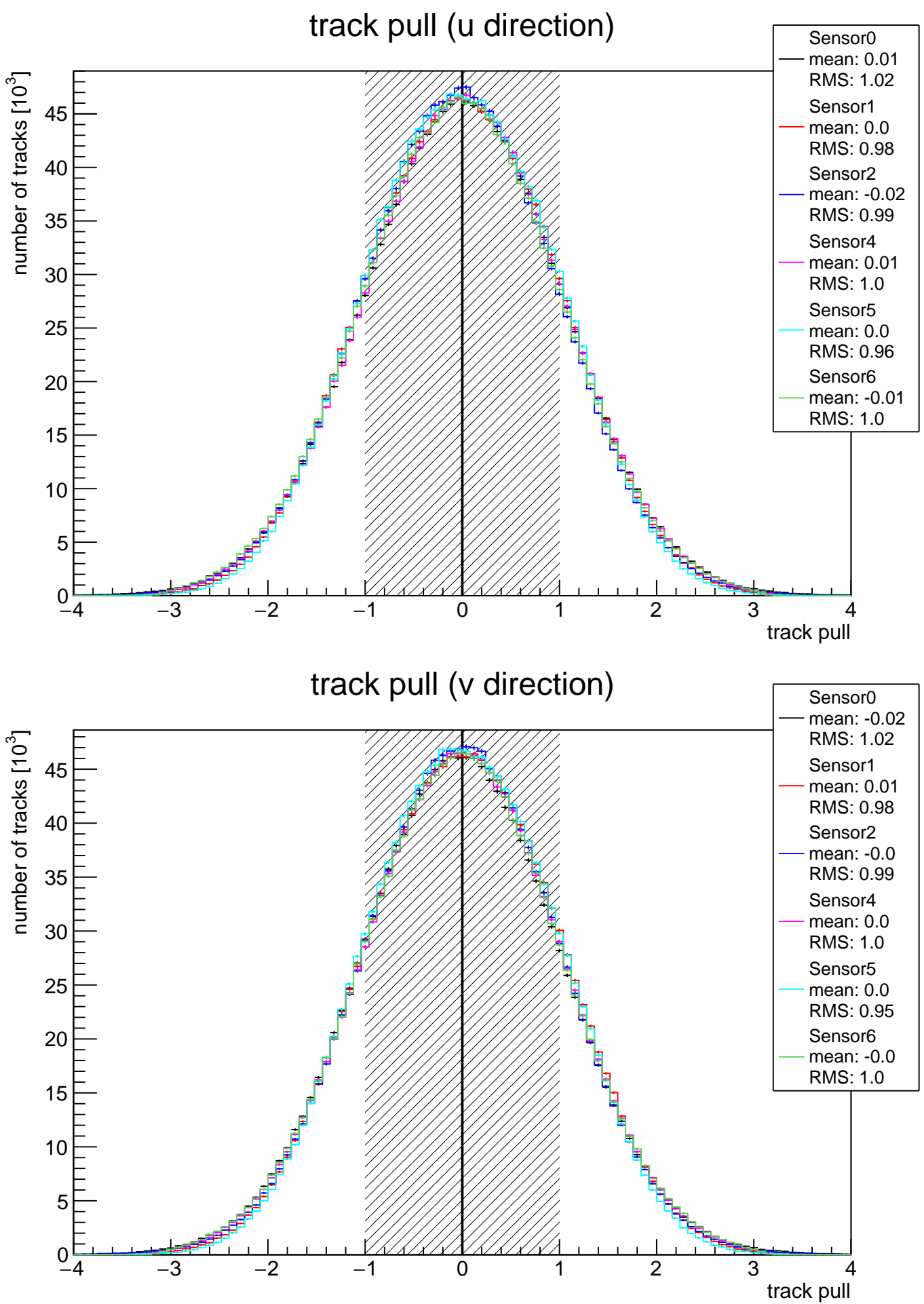

Figure D.40.: Pulls of the $u$ and $v$ residuals after cluster calibration and telescope alignment. The Pulls are centred around 0 and have a RMS value close to 1 . 
D. Telescope and $X / X_{0}$ calibration of several beam test measurements
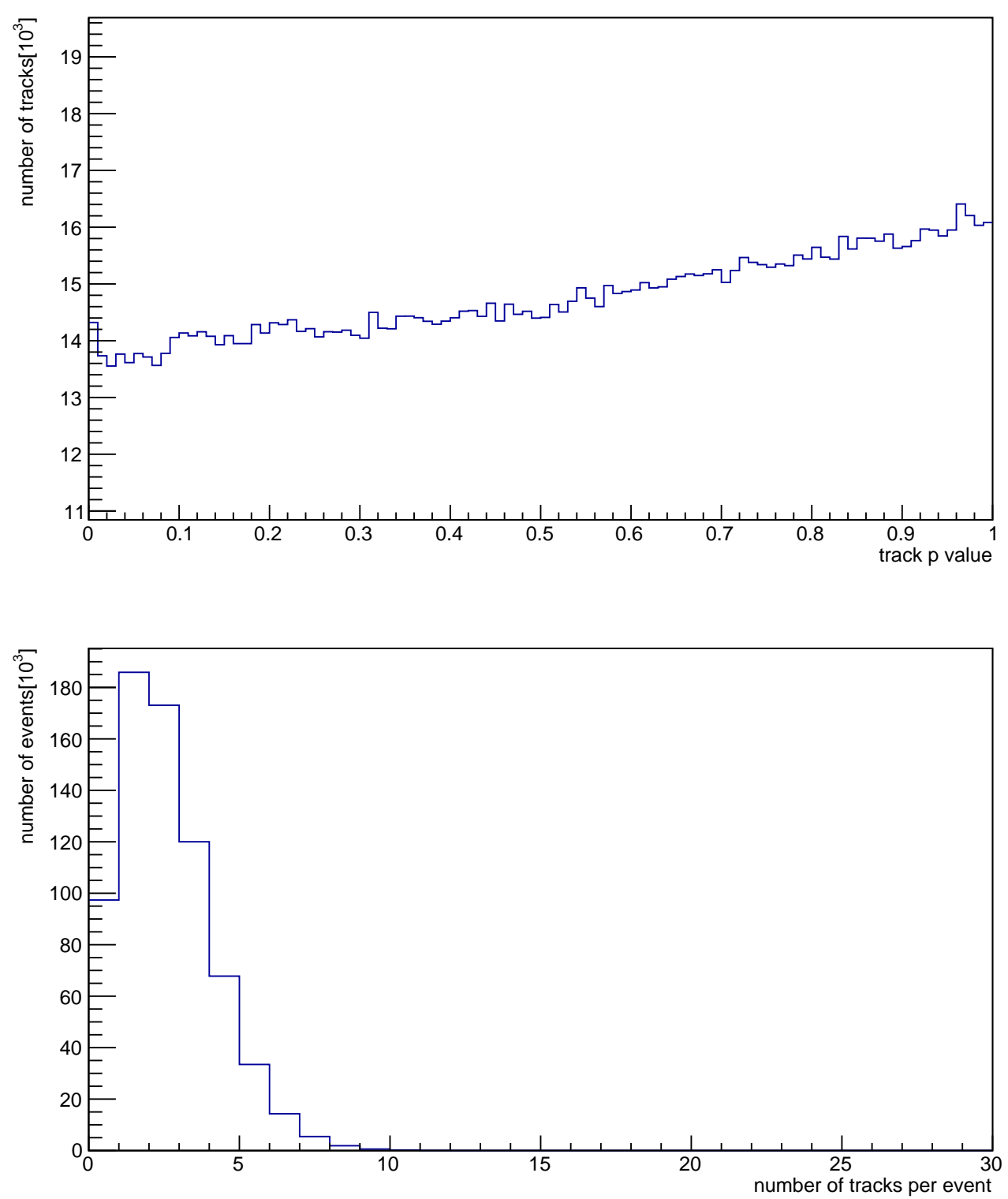

Figure D.41.: $p$ values of the calibration run track sample after the telescope alignment and cluster calibration and number of tracks per event during the August 2016 beam test campaign. 

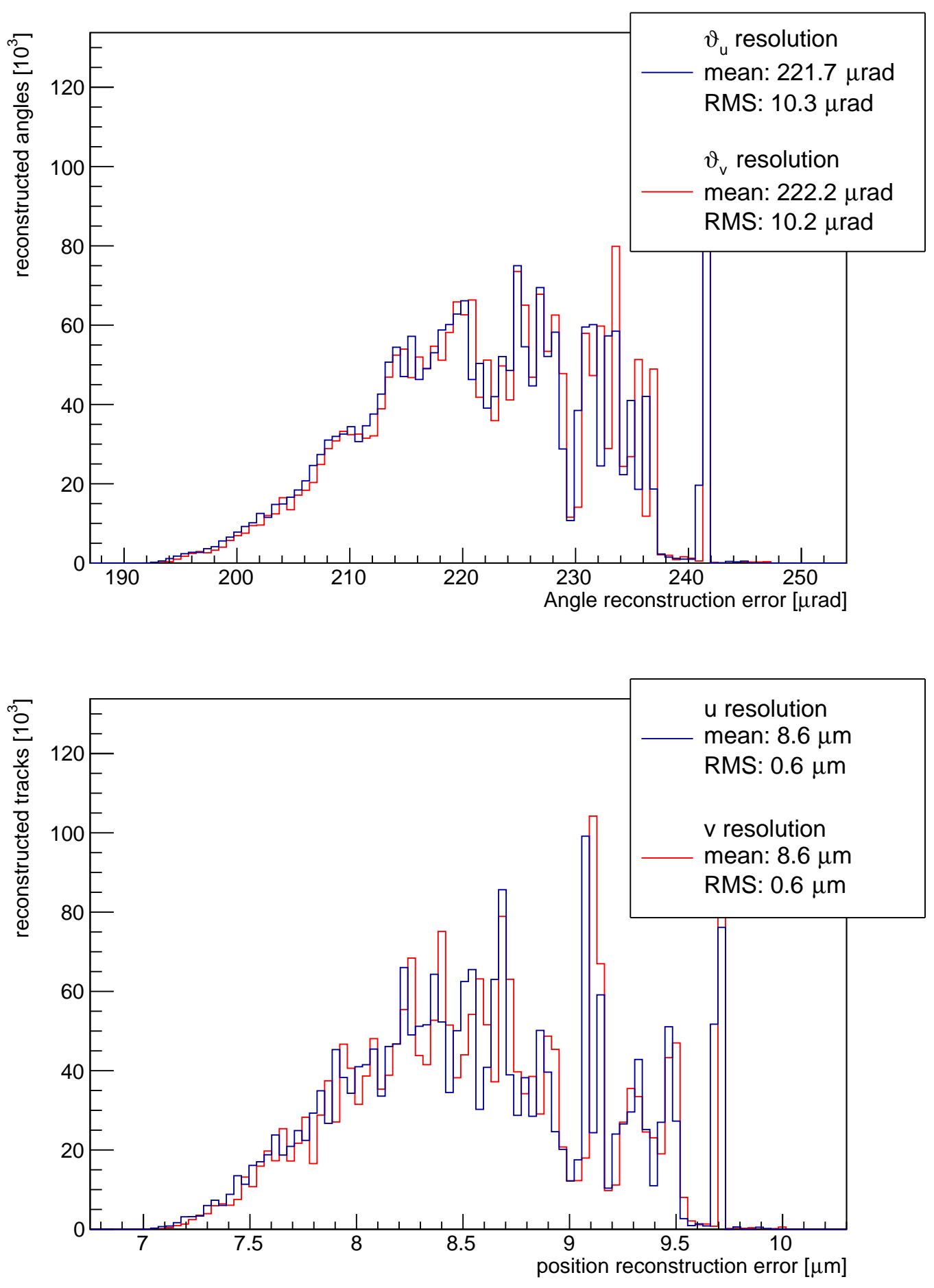

Figure D.42.: Angle reconstruction errors and track intersection errors in $u$ and $v$ direction. 

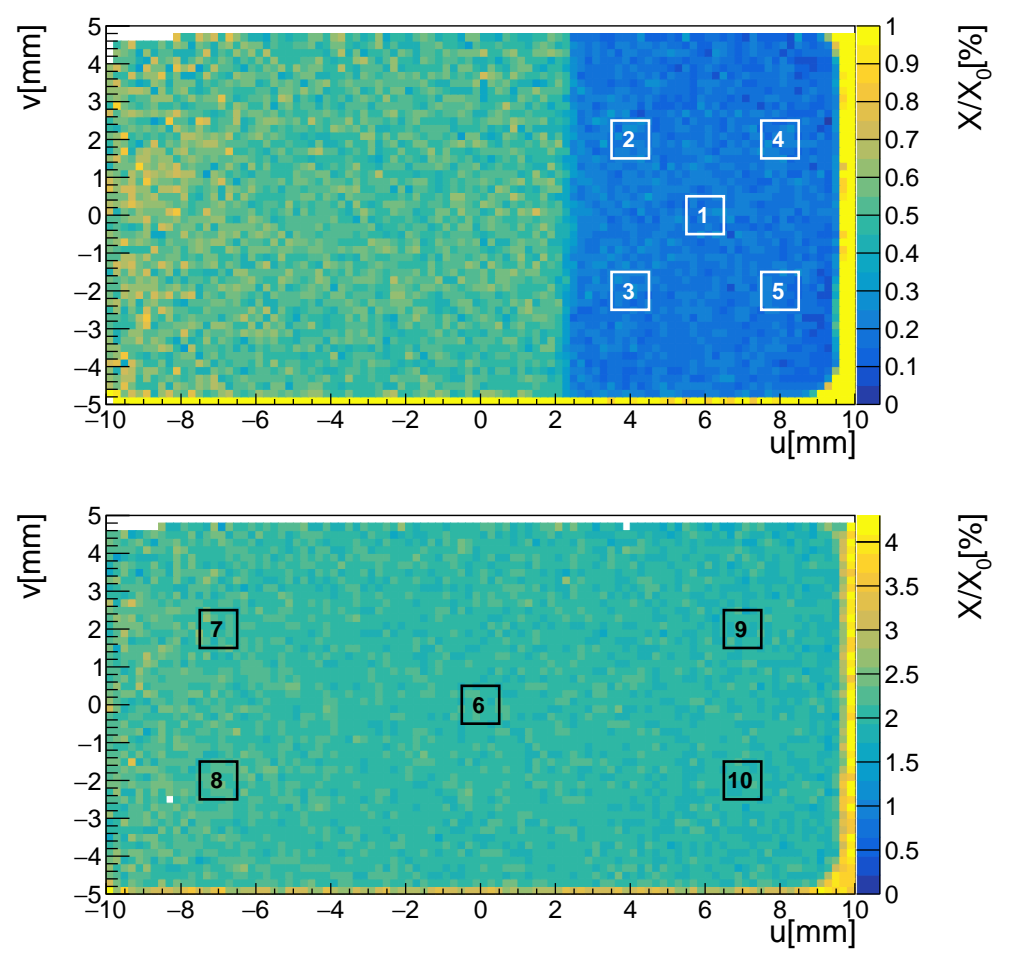

Figure D.43.: Visual representation of the position of the 10 different measurement regions, which are used during the radiation length calibration. Each marked measurement area contributes a single angle distribution with 100 thousand entries to the calibration fit. The top radiation length image depicts a composite material on the left hand side and air on the right hand side. The bottom image depicts an aluminium layer with a thickness of $2.05 \mathrm{~mm}$.

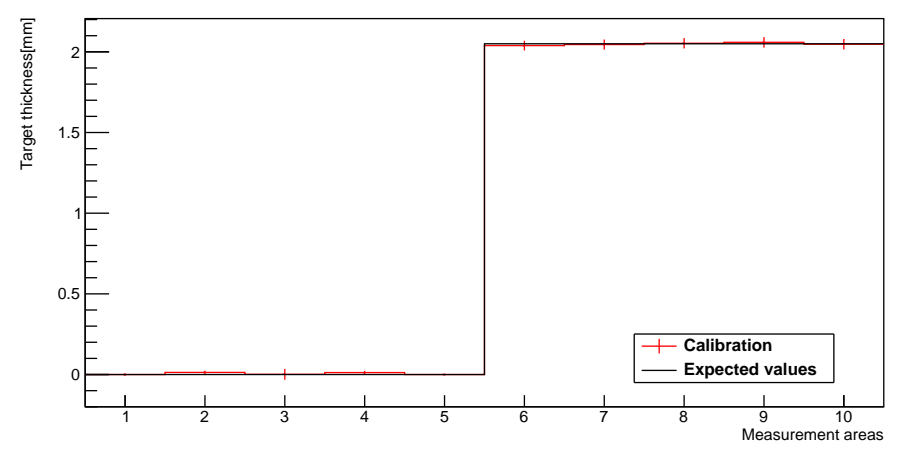

Figure D.44.: Self consistency measurements after the radiation length calibration prodecure. 
Area 0: air

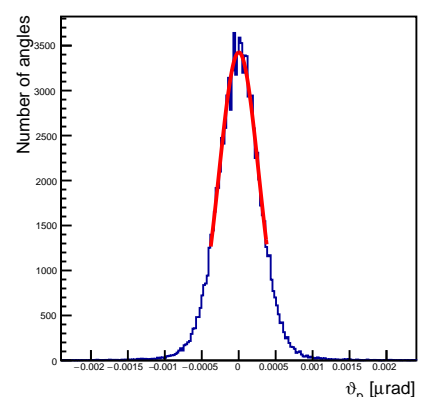

Area 3: air

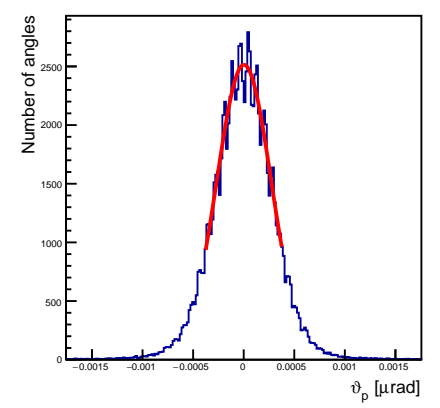

Area 6: $\mathrm{d}=2.05 \mathrm{~mm}$ aluminium

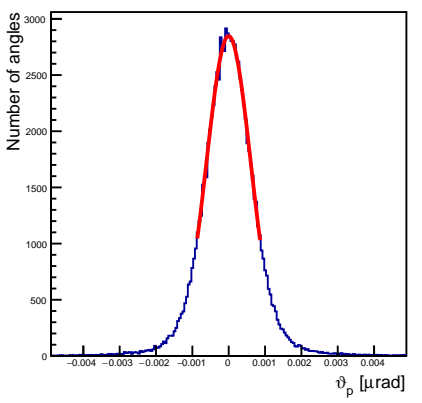

Area 1: air

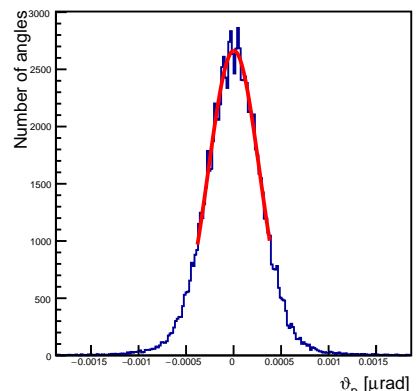

Area 4: air

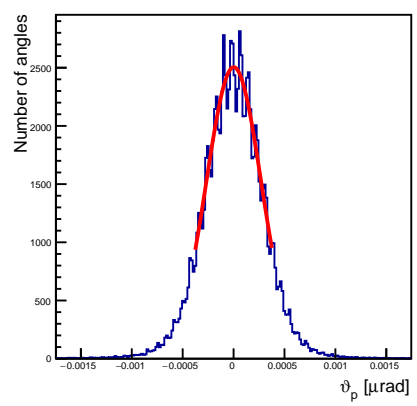

Area 7: $\mathrm{d}=2.05 \mathrm{~mm}$ aluminium

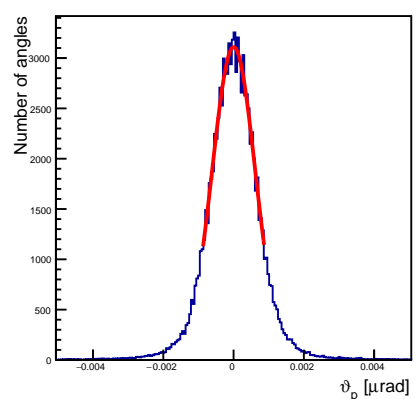

Area 2: air

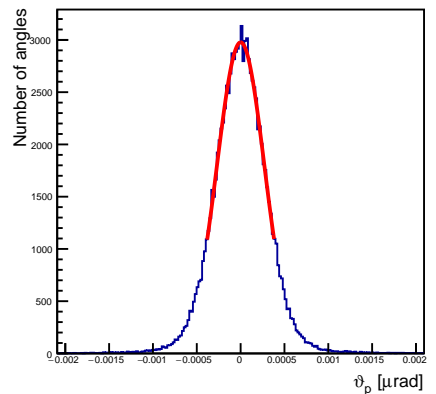

Area 5: $\mathrm{d}=2.05 \mathrm{~mm}$ aluminium
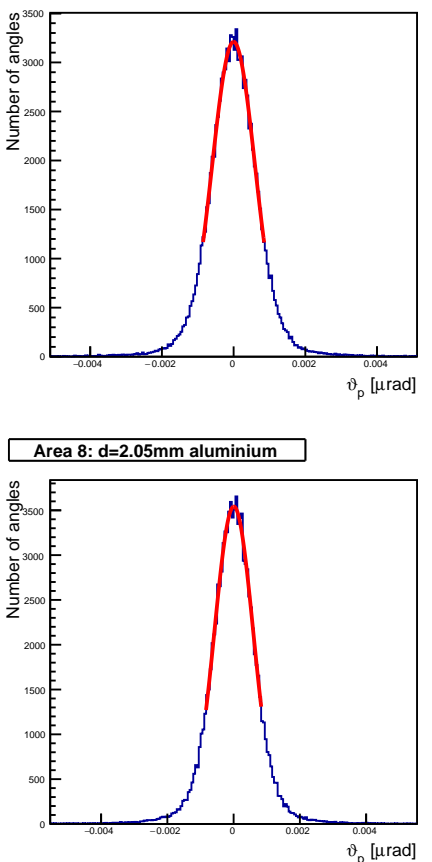

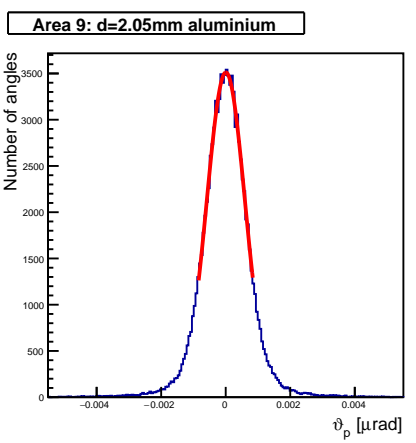

Figure D.45.: All scattering angle distributions, that have been used during the radiation length calibration together with the corresponding fit function. Some angle distributions show periodic peak structures, that are caused by digital effects of the telescope. This disturbs the fitting procedure and leads to a comparatively large $\chi_{\text {ndof }}^{2}$ value. 
D. Telescope and $X / X_{0}$ calibration of several beam test measurements

\section{D.6. Measurements on Belle II PXD mechanical samples, March 2015}

In March 2015 a beam test was conducted. The scope of the measurements was to perform a material budget image measurement of a PXD mechanical sample module (see section 7.6). The telescope setup of the measurements is depicted in fig. D.46. The beam energy was $2 \mathrm{GeV}$. Table D.9 contains an overview over the measured telescope data. It specifies the number of runs, overall events and overall tracks as well as the purpose of the measurements.

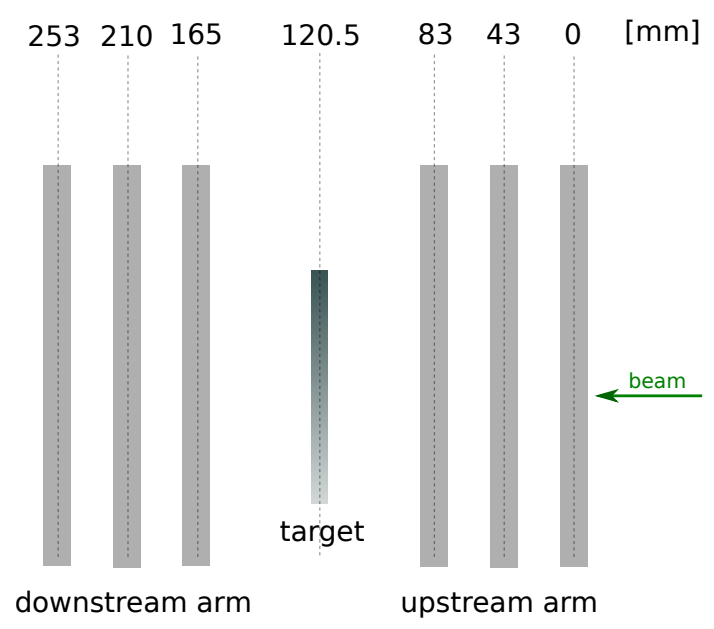

Figure D.46.: Schematic side view of the telescope setup during March 2015 beam test campaign.

\begin{tabular}{|c||c|c|c|c|}
\hline Target & \#runs & \#events $\left[10^{6}\right]$ & \#angles $\left[10^{6}\right]$ & purpose \\
\hline calibration target & 2 & 3.1 & 23.2 & telescope $\& X / X_{0}$ calibration $2 \mathrm{GeV}$ \\
\hline calibration target & 1 & 1.3 & 13.8 & telescope $\& X / X_{0}$ calibration $4 \mathrm{GeV}$ \\
\hline PXD dummy & 11 & 11.4 & 103.2 & $X / X_{0}$ imaging at $2 \mathrm{GeV}$ \\
\hline PXD dummy & 5 & 10.0 & 91.4 & $X / X_{0}$ imaging at $4 \mathrm{GeV}$ \\
\hline Broken PXD & 9 & 6.8 & 131.6 & $X / X_{0}$ imaging at $2 \mathrm{GeV}$ \\
\hline
\end{tabular}

Table D.9.: Overview over the telescope data at the beam test in March 2015. The spacings between the M26 sensors (see fig. D.46) was not changed between the measurements.

\section{D.6.1. Beam energy $2 \mathrm{GeV}$}

All relevant DQM plots for the analysis of the $2 \mathrm{GeV}$ data are listed on the following pages. The DQM plots listed here are explained in detail in section 7.1.1. 


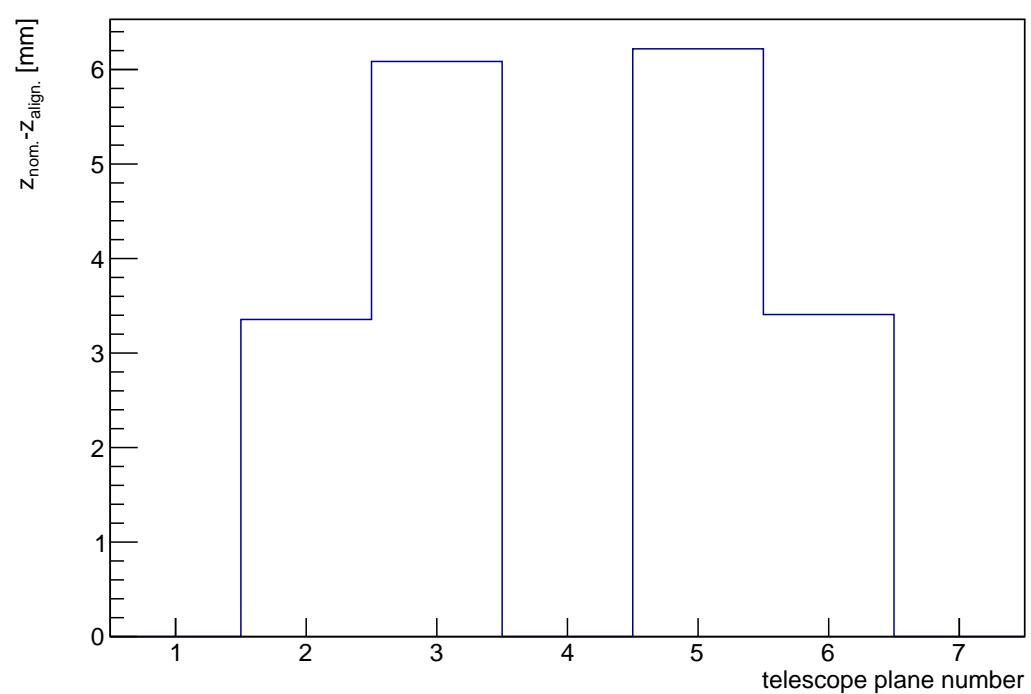

Figure D.47.: Difference between nominal and aligned sensor $z$ positions.

\section{0 million tracks}

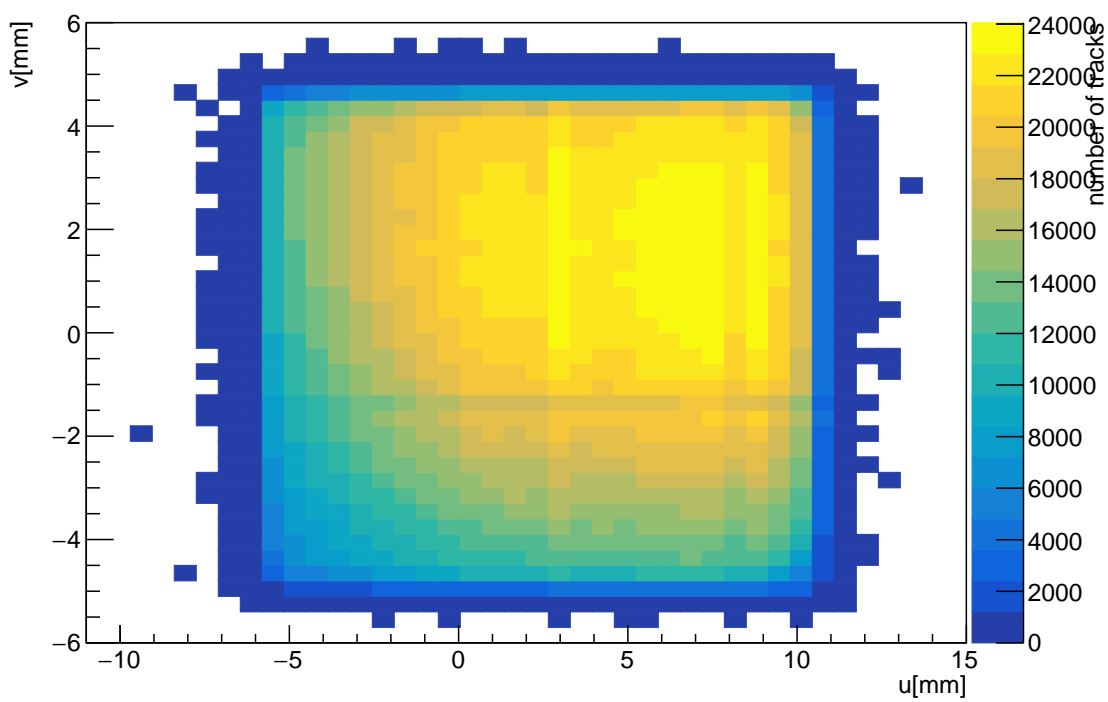

Figure D.48.: Beam spot on the central scattering plane during an example run of the March 2015 (2 GeV data) beam test. 


\section{cluster shape frequency}
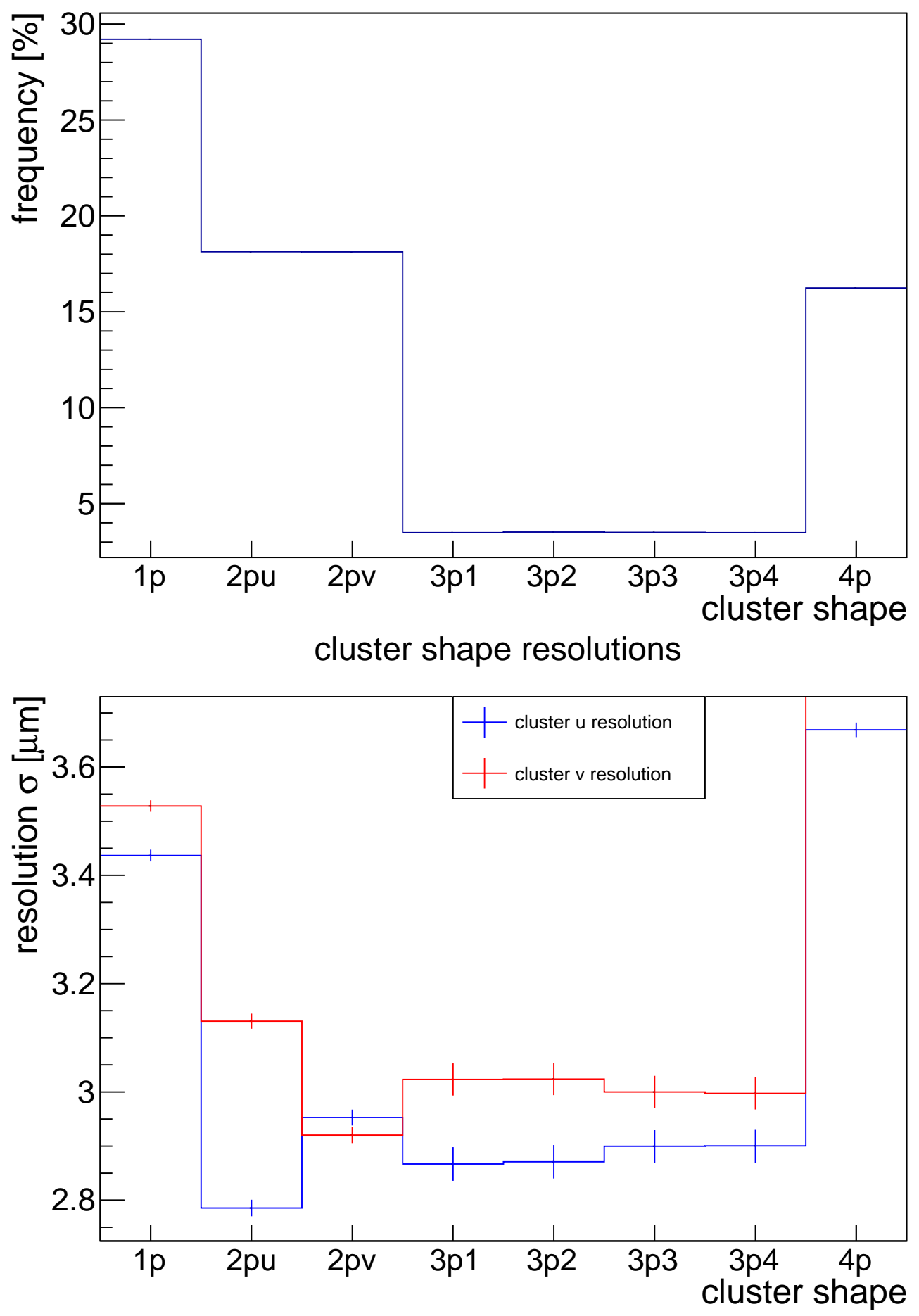

Figure D.49.: Cluster shape frequencies and cluster $u$ and $v$ resolution during the March 2015 ( $2 \mathrm{GeV}$ data) beam test campaign. Cluster shapes, which correspond to the individual labels on the $x$ axis, are depicted in figure 4.9. 
D.6. Measurements on Belle II PXD mechanical samples, March 2015
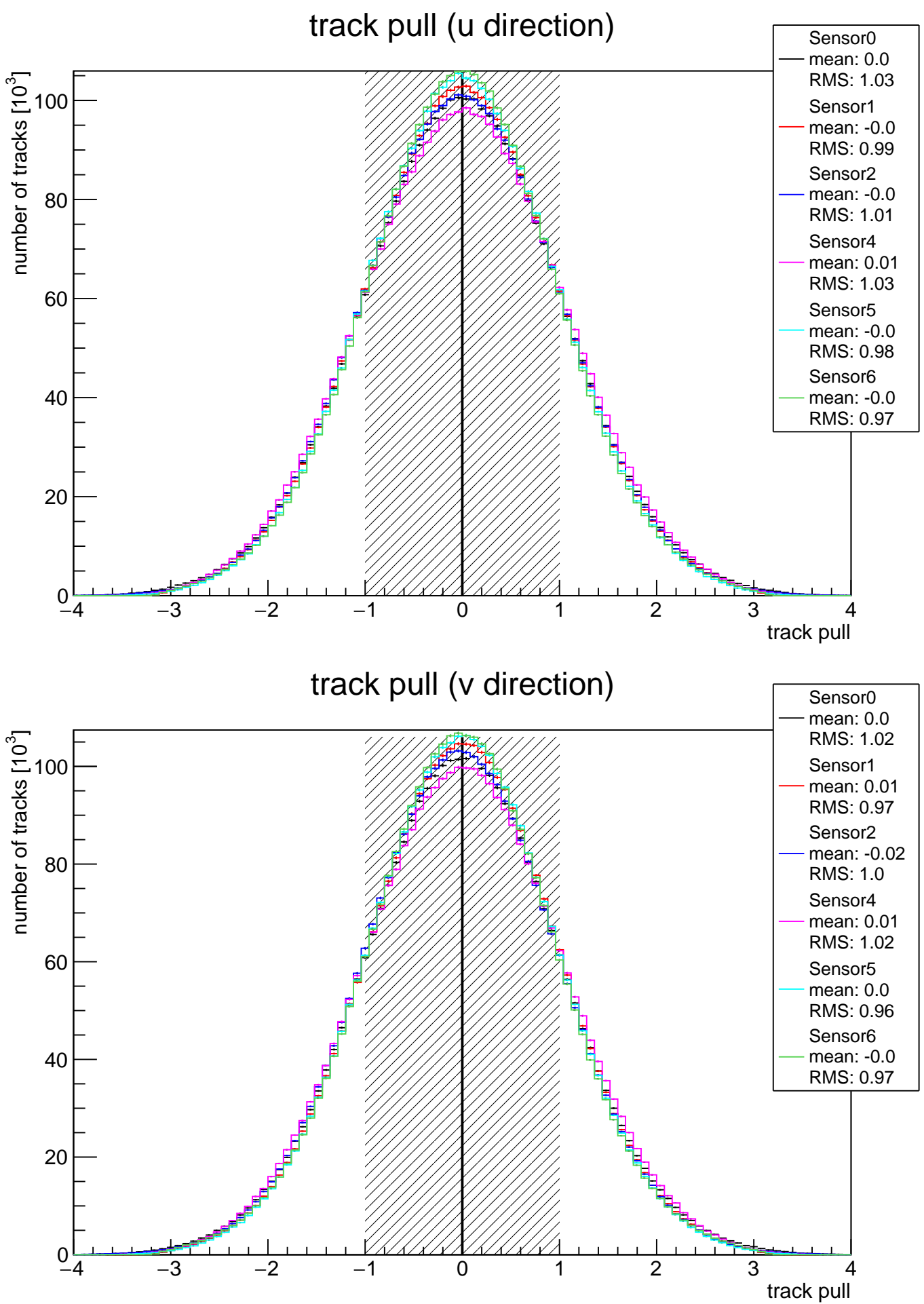

Figure D.50.: Pulls of the $u$ and $v$ residuals after cluster calibration and telescope alignment. The Pulls are centred around 0 and have a RMS value close to 1. 
D. Telescope and $X / X_{0}$ calibration of several beam test measurements
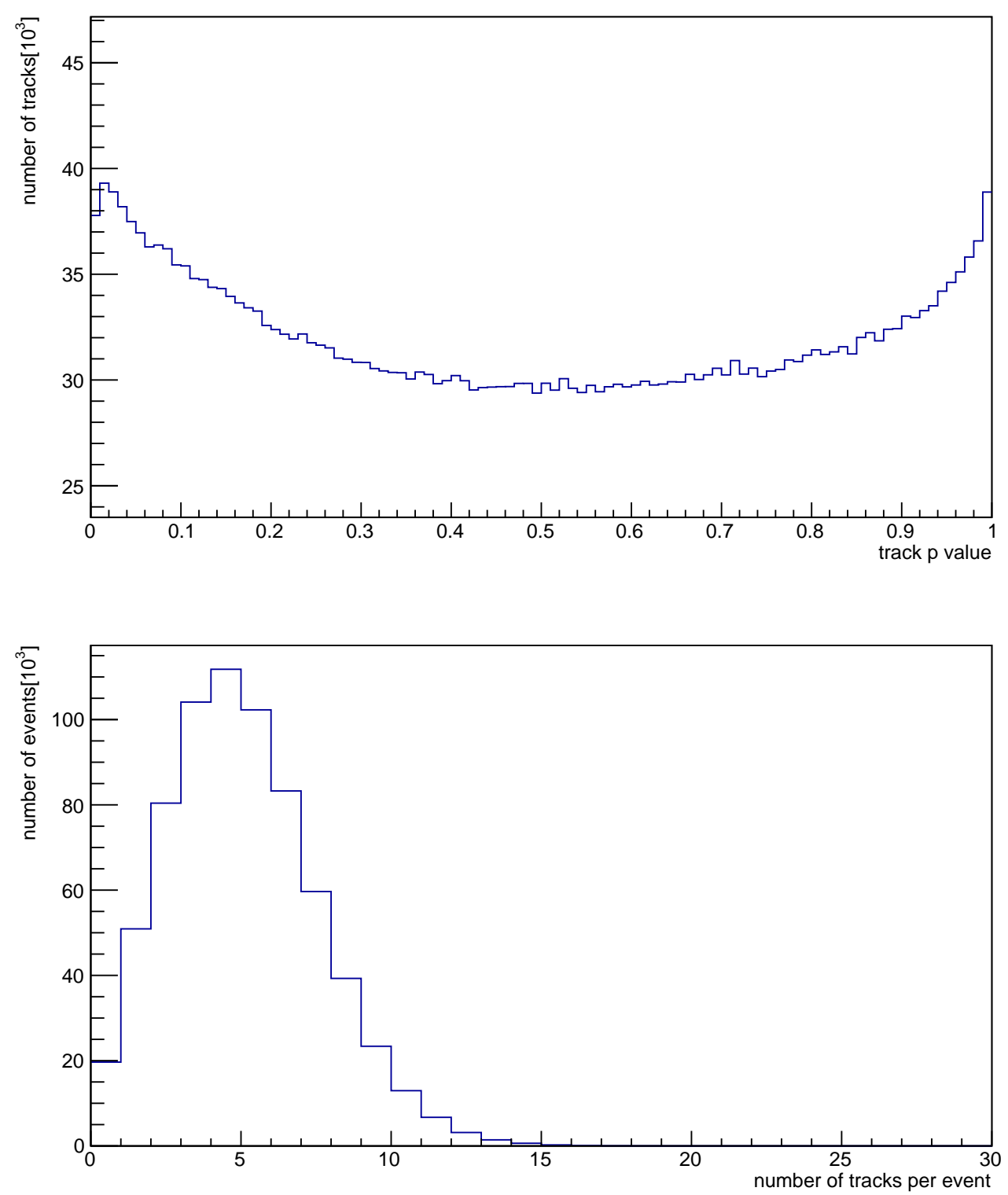

Figure D.51.: $p$ values of the calibration run track sample after the telescope alignment and cluster calibration and number of tracks per event during the March 2015 (2 GeV data) beam test campaign. 

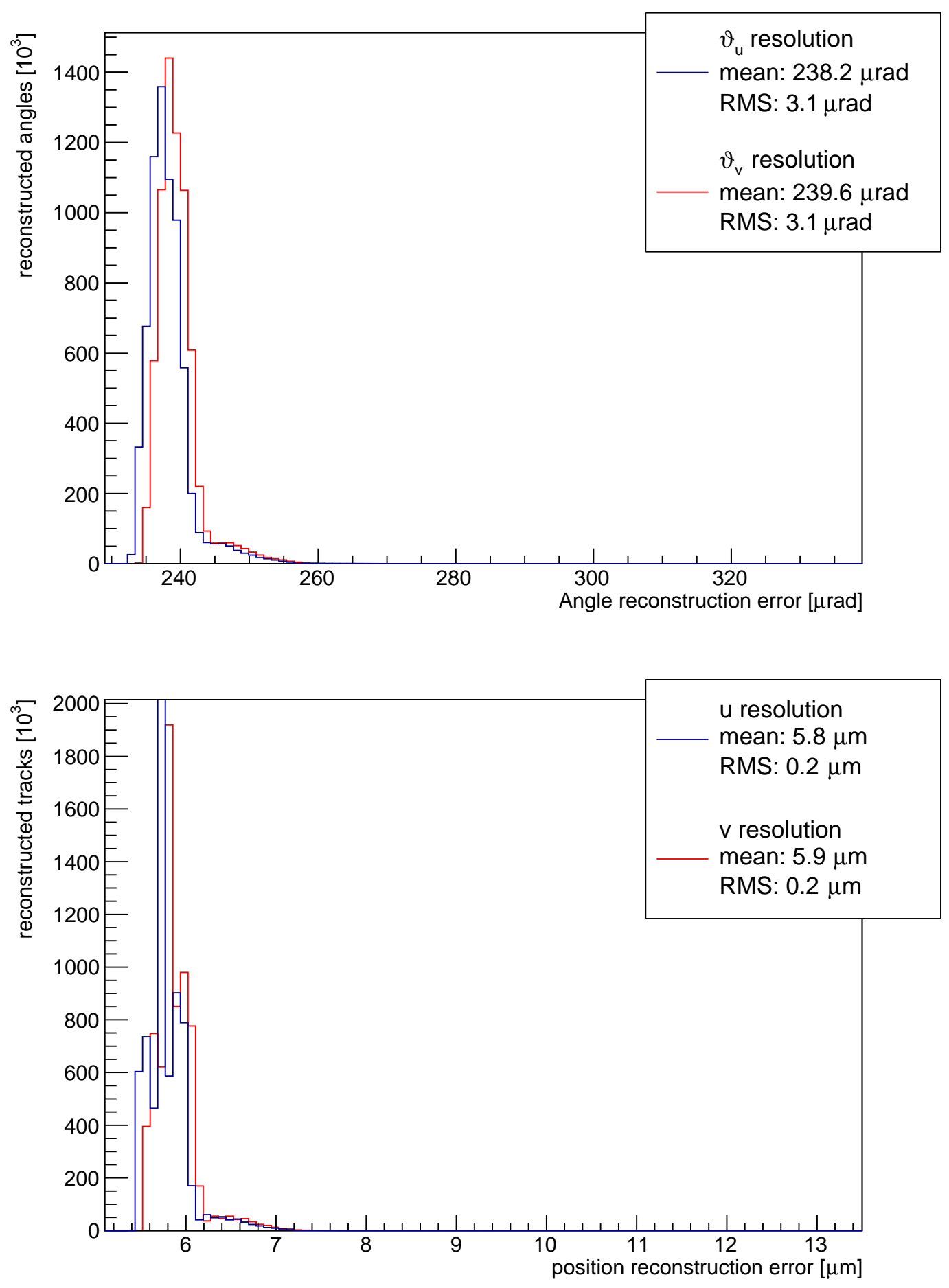

Figure D.52.: Angle reconstruction errors and track intersection errors in $u$ and $v$ direction. 


\section{Telescope and $X / X_{0}$ calibration of several beam test measurements}
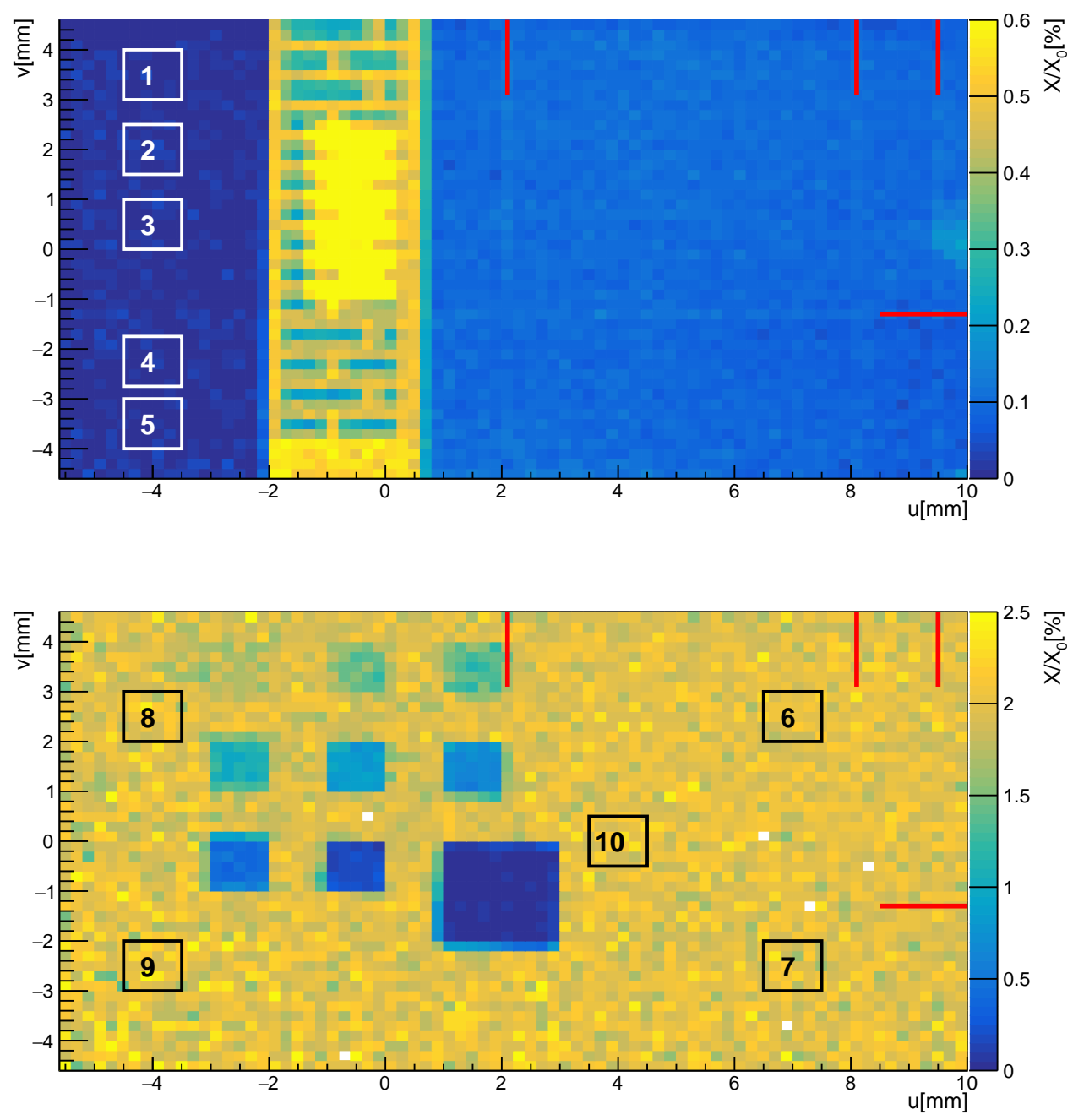

Figure D.53.: Visual representation of the position of the 10 different measurement regions, which are used during the radiation length calibration. Each marked measurement area contributes a single angle distribution with 50000 entries to the calibration fit. 

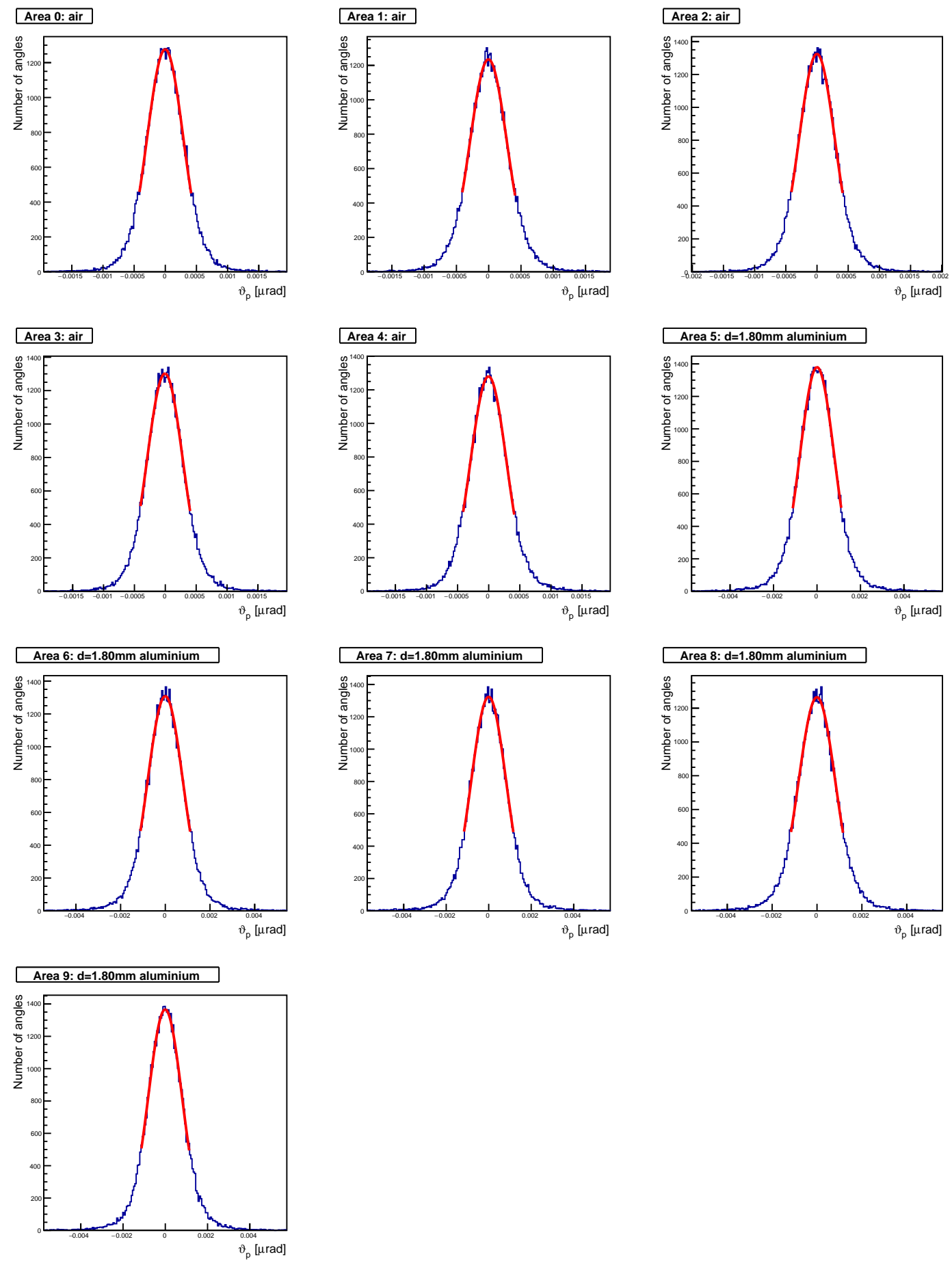

Figure D.54.: All ten scattering angle distributions, that have been used during the radiation length calibration together with the corresponding fit function. The fit worked well and the $\chi^{2}$ value is small. 
D. Telescope and $X / X_{0}$ calibration of several beam test measurements

\section{D.6.2. Beam energy $4 \mathrm{GeV}$}

All relevant DQM plots for the analysis of the $4 \mathrm{GeV}$ data are listed on the following pages. The DQM plots listed here are explained in detail in section 7.1.1.

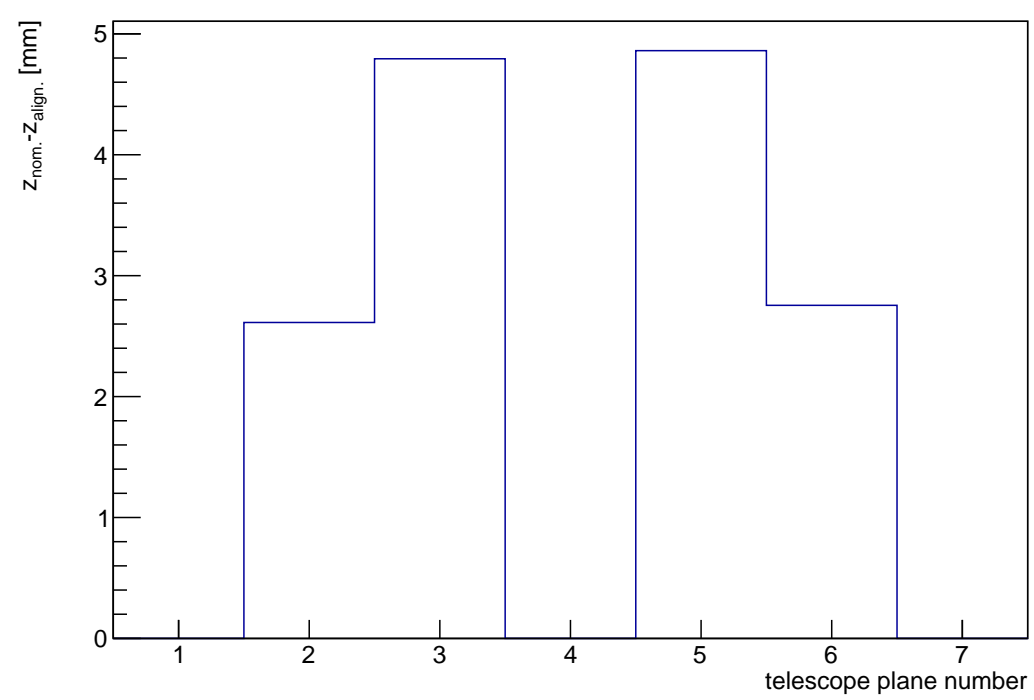

Figure D.55.: Difference between nominal and aligned sensor $z$ positions.

\section{2 million tracks}

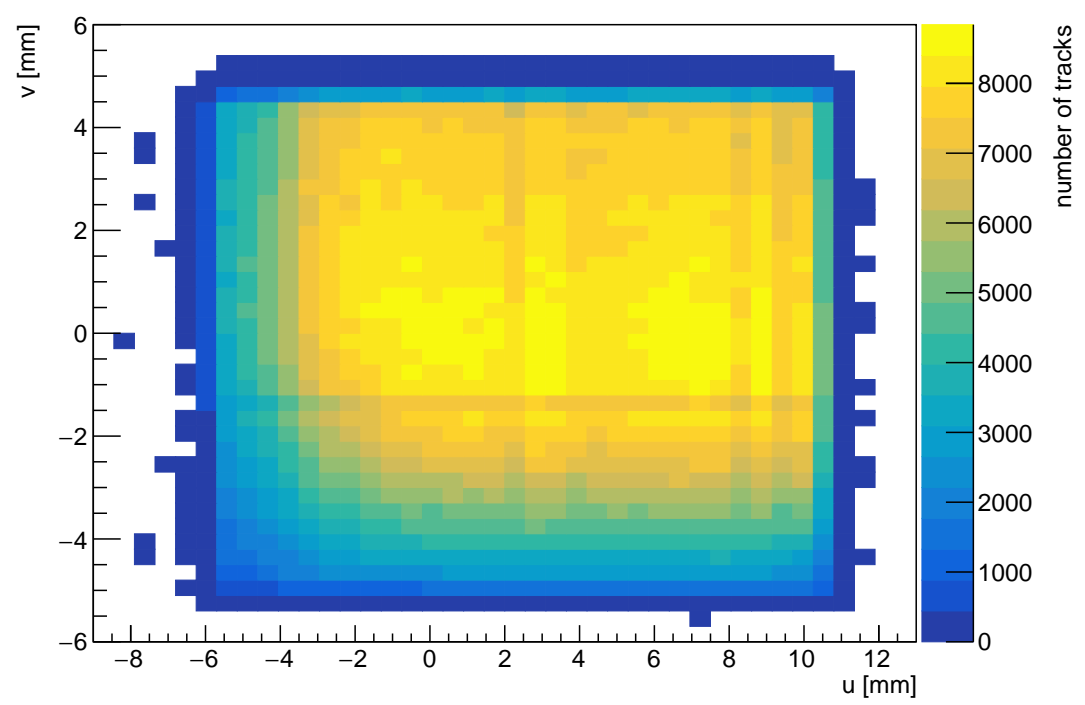

Figure D.56.: Beam spot on the central scattering plane during an example run of the March 2015 (4 GeV data) beam test. 


\section{cluster shape frequency}
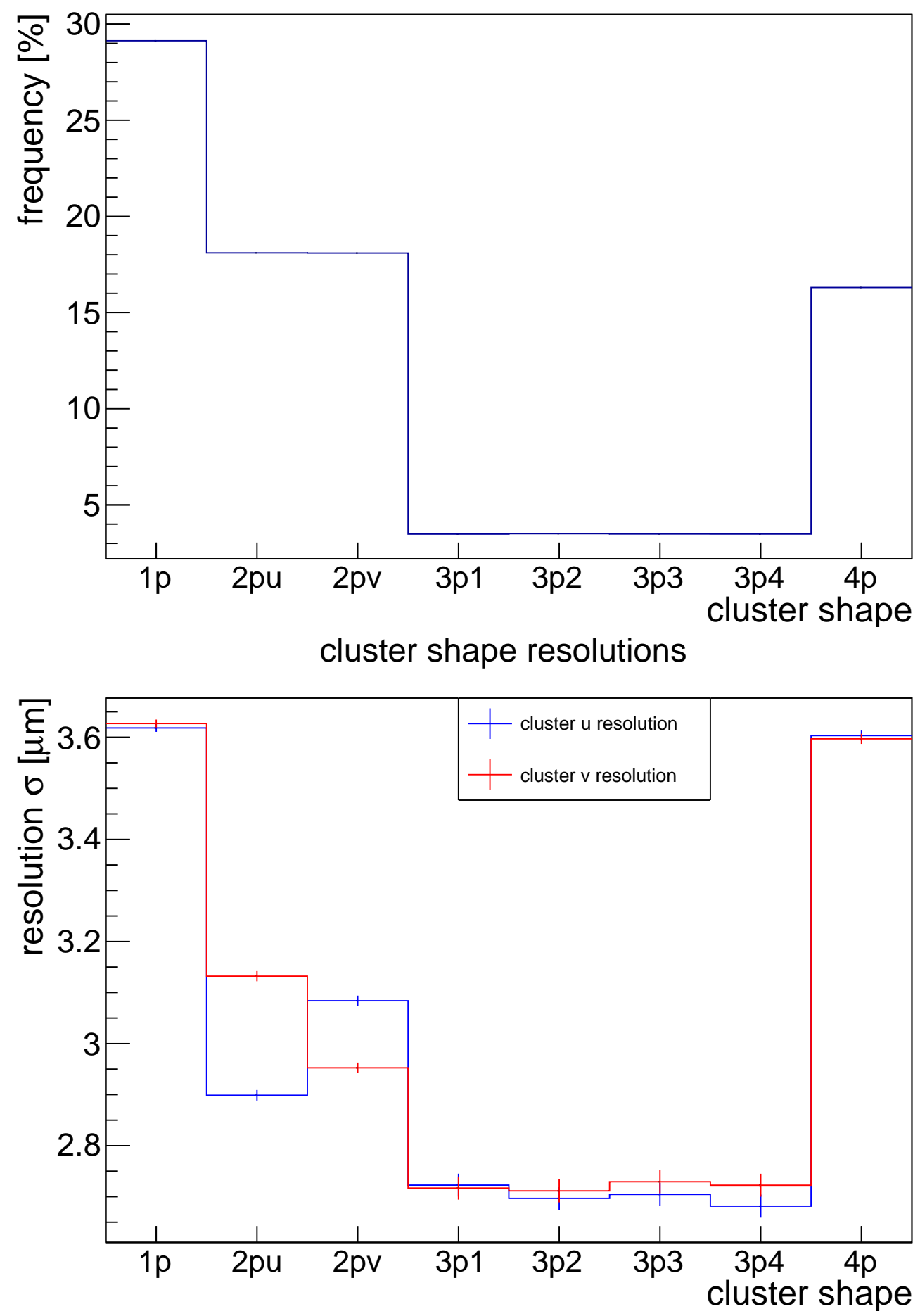

Figure D.57.: Cluster shape frequencies and cluster $u$ and $v$ resolution during the March 2015 ( $4 \mathrm{GeV}$ data) beam test campaign. The cluster shapes, which correspond to the individual labels on the $x$ axis, are depicted in figure 4.9. 
D. Telescope and $X / X_{0}$ calibration of several beam test measurements
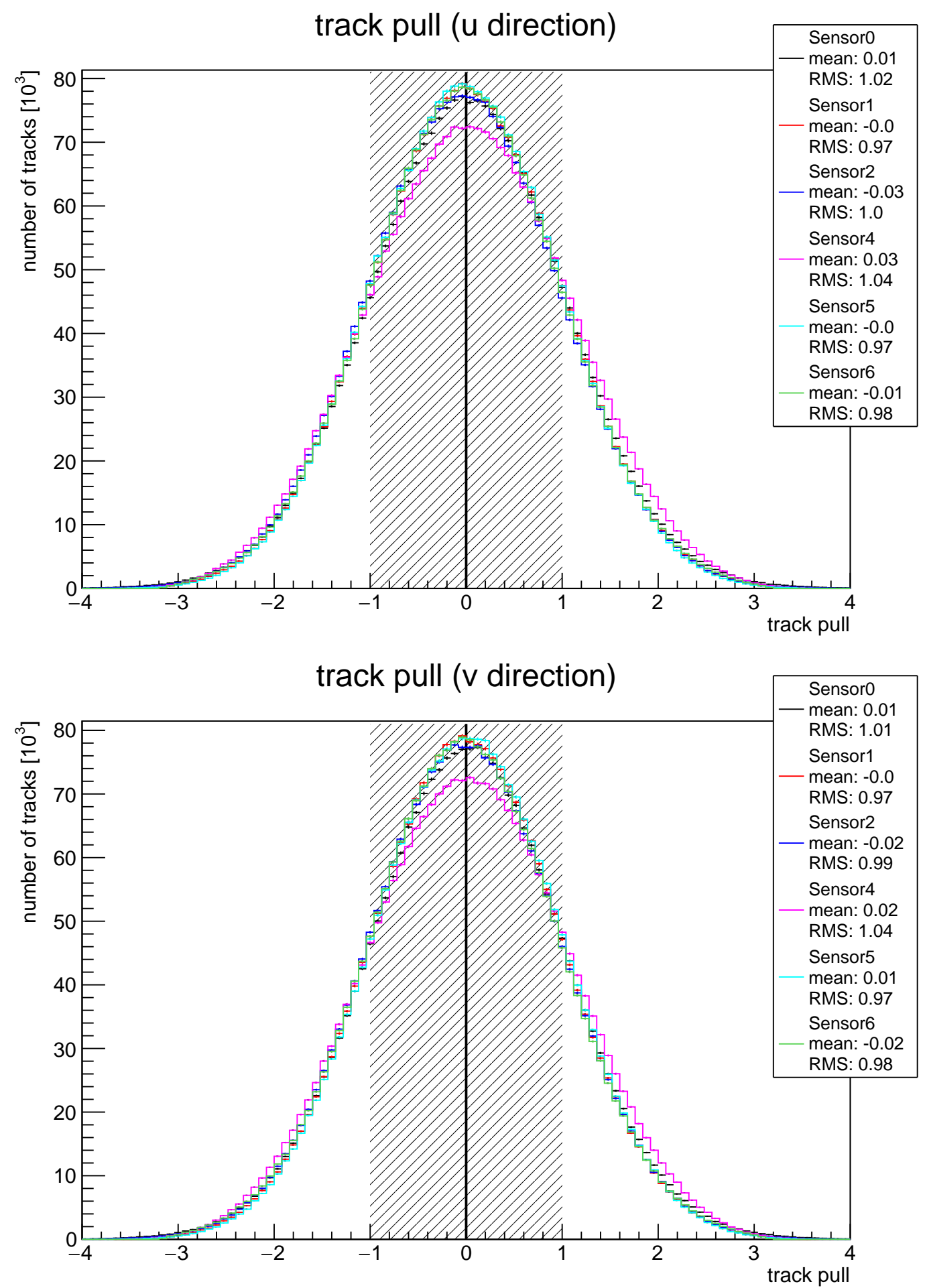

Figure D.58.: Pulls of the $u$ and $v$ residuals after cluster calibration and telescope alignment. The Pulls are centred around 0 and have a RMS value close to 1 . 

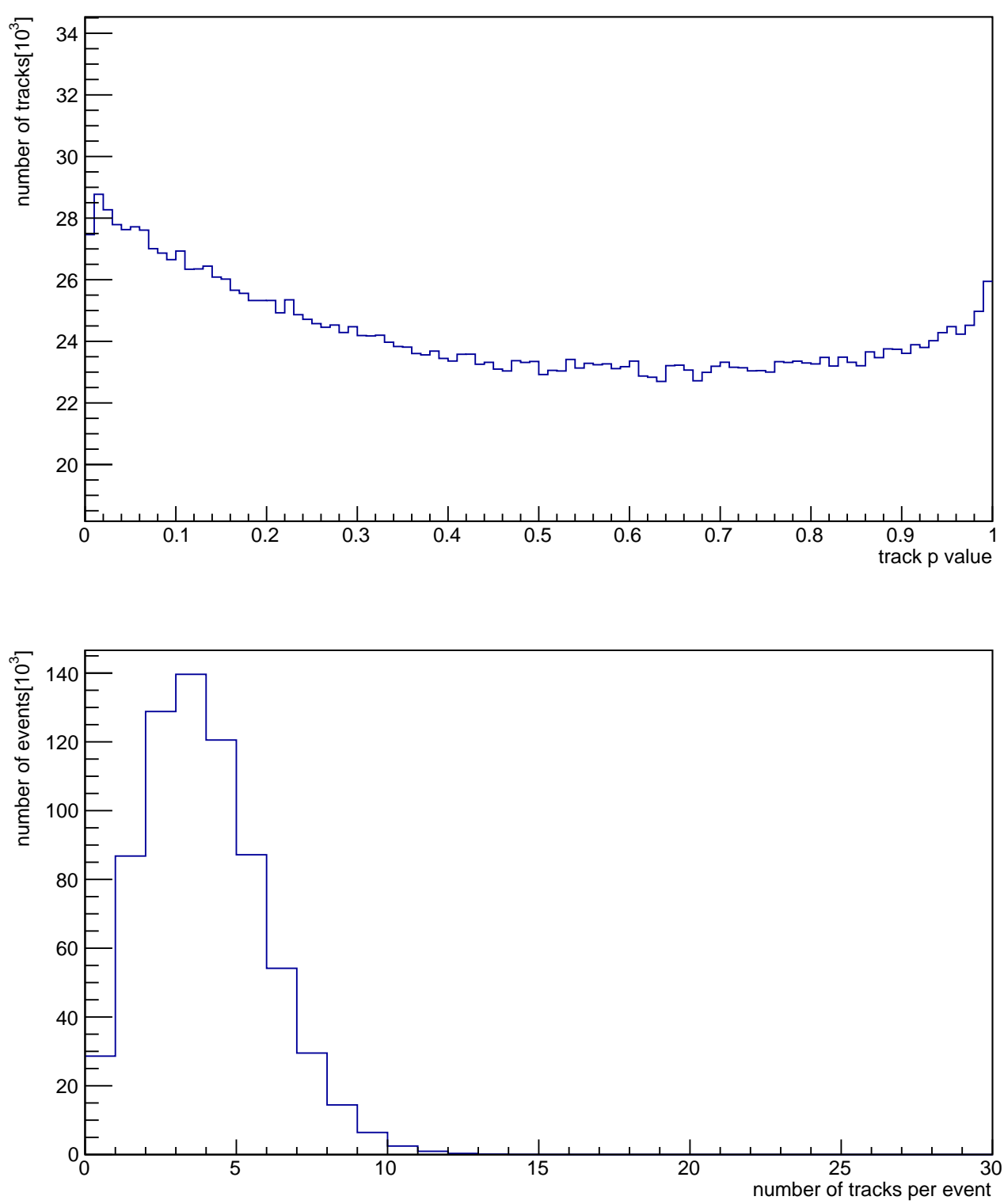

Figure D.59.: $p$ values of the calibration run track sample after the telescope alignment and cluster calibration and number of tracks per event during the March 2015 (4 GeV data) beam test campaign. 
D. Telescope and $X / X_{0}$ calibration of several beam test measurements
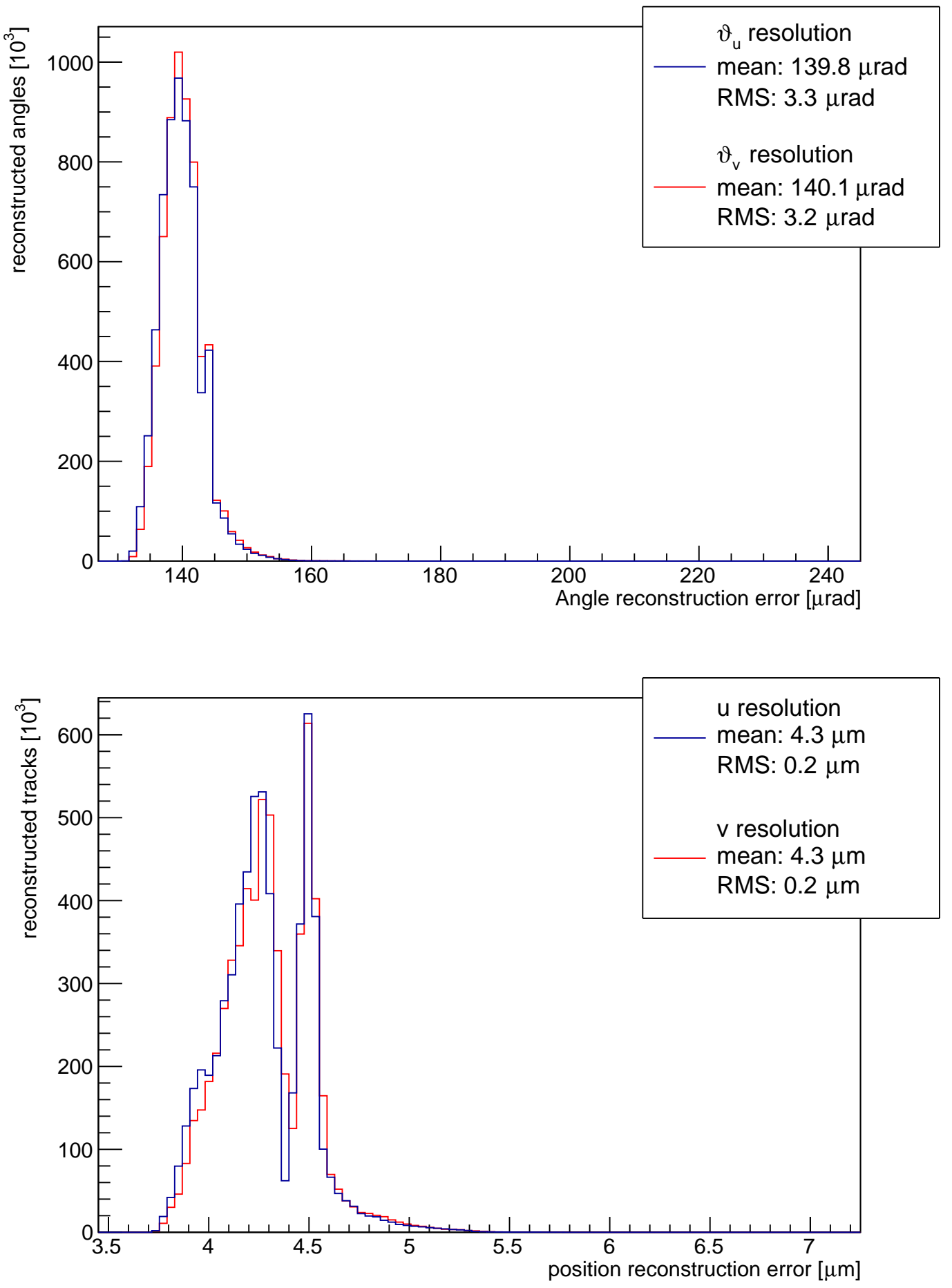

Figure D.60.: Angle reconstruction errors and track intersection errors in $u$ and $v$ direction. 

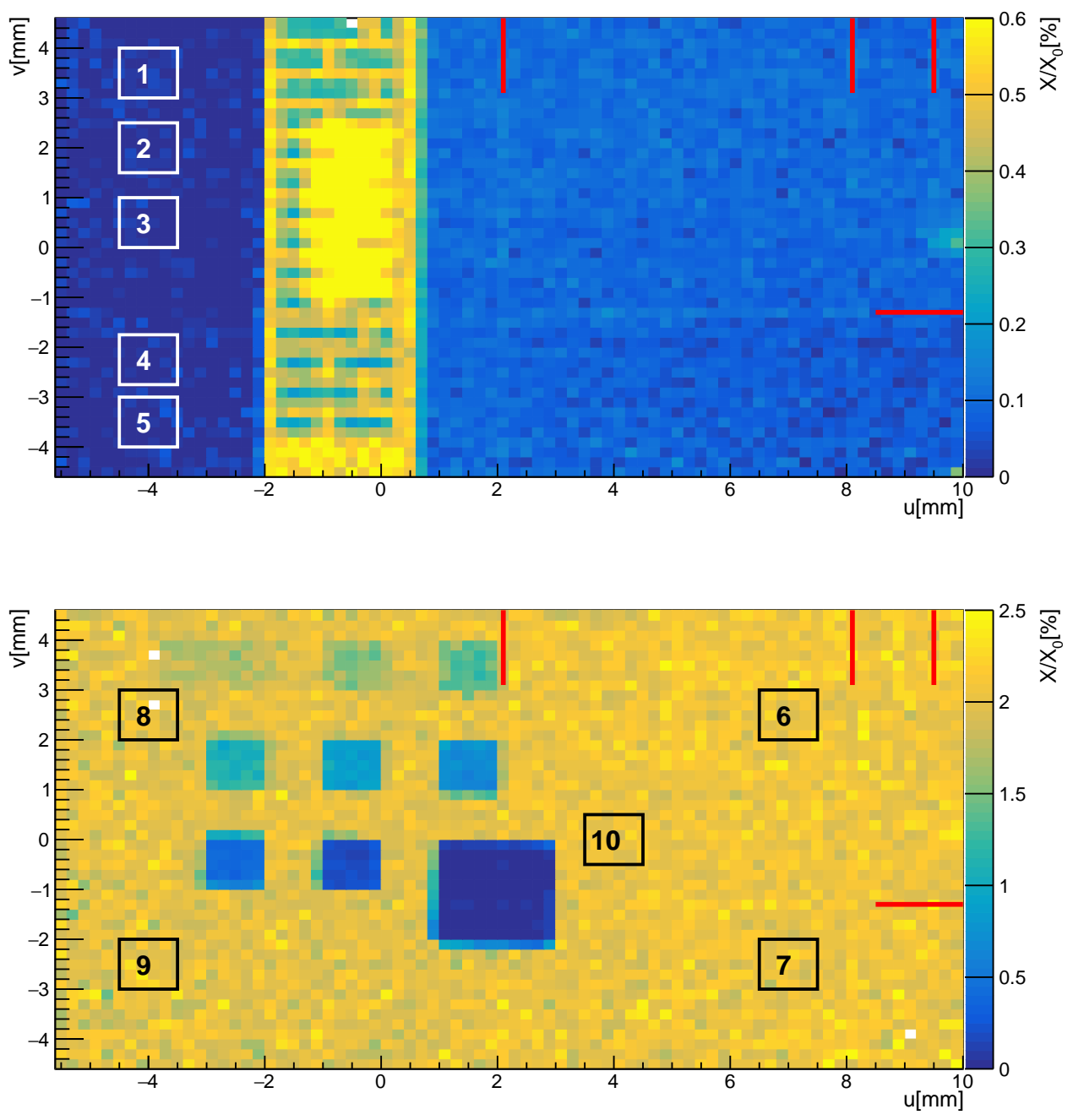

Figure D.61.: Visual representation of the position of the 10 different measurement regions, which are used during the radiation length calibration. Each marked measurement area contributes a single angle distribution with 50000 thousand entries to the calibration fit. 
D. Telescope and $X / X_{0}$ calibration of several beam test measurements
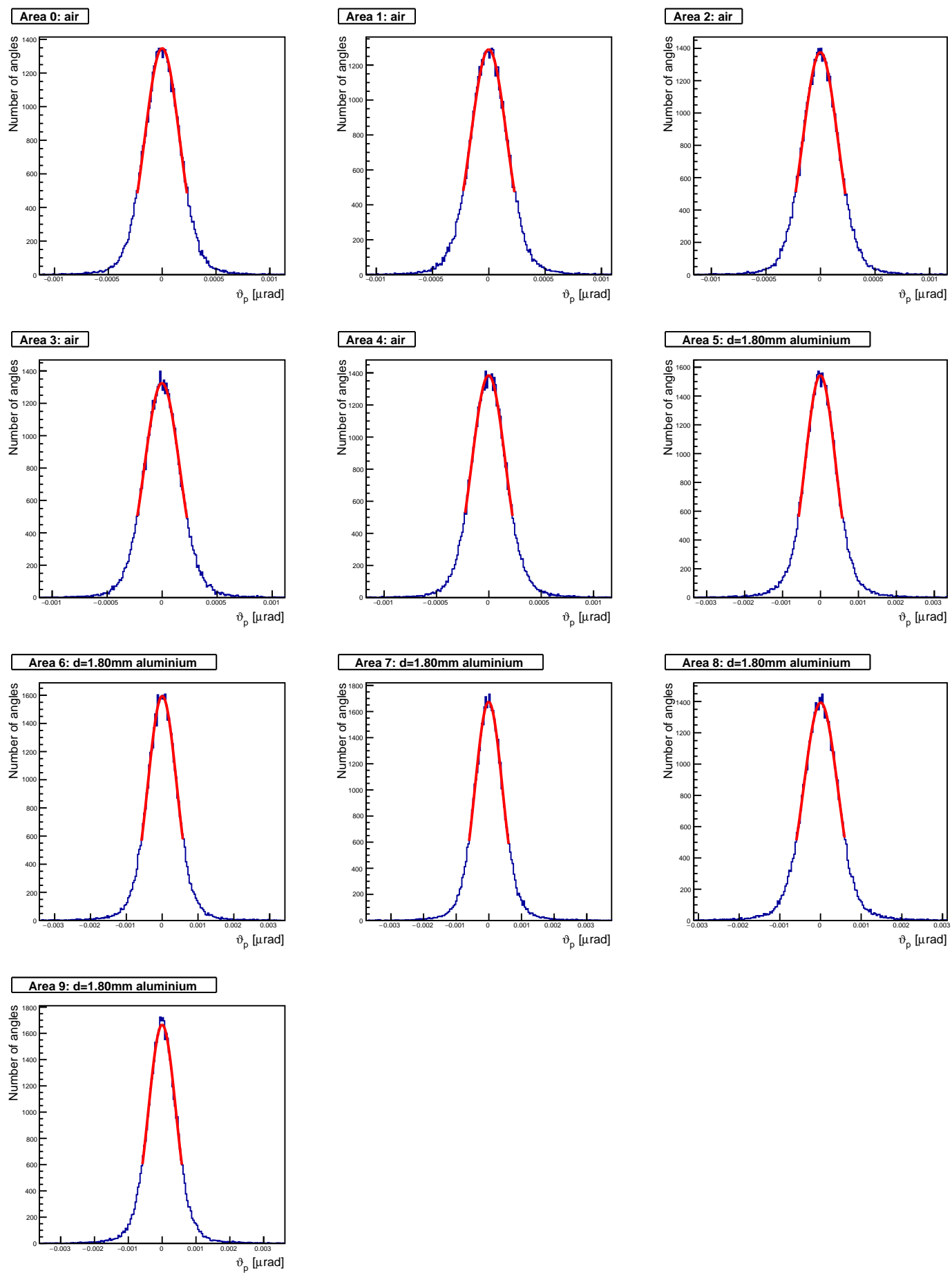

Figure D.62.: All ten scattering angle distributions, that have been used during the radiation length calibration together with the corresponding fit function. The fit worked well and the $\chi^{2}$ value is small. 


\section{Aknowledgements}

First of all, I would like to thank my supervisor Prof. Dr. Ariane Frey for giving me the opportunity to write my thesis on such an interesting topic in her working group. Her feedback on my work in the weekly group meetings was very valuable. Her encouragement of activities in our spare time such as bowling, cart racing and visits to the christmas market led to a great atmosphere in her working group.

Special thanks to PD Dr. Jörn Große-Knetter for being the co-referee of this thesis and the other members of the evaluation committee Prof. Dr. Stan Lai, Prof. Dr. Hans Hofsäss, Prof. Dr. Wolfram Kollatschny and Prof. Dr. Ansgar Reiners.

I would like to thank Benjamin Schwenker for the many fruitful conversations, help with programming issues and proofreading this thesis. His interest in my research topic and our discussions led to many great ideas. Also many thanks to Luise Poley and Uwe Gebauer for giving me very helpful feedback on this document.

I would also like to thank Carlos Marinas and Benjamin for organising various beam tests at DESY and giving me the opportunity to conduct the measurements needed for this work. I would also like to thank Florian Lütticke, Botho Paschen, Philipp Wieduwilt and Harrison Schreeck for sharing and lessening the burden of long working days at DESY.

Thanks to all members of the group, Ariane, Benjamin, César, Uwe, Harrison, Philipp, Jonas, Gerrit, Joana and Julian for the pleasant work environment and for taking part and organising the group activities. Special thanks to Finn and Bernadette for handling administrative issues so fast and well.

Many thanks to my friends Niklas, Veit, Lennart and Lars for cheering me up during stressful times of my $\mathrm{PhD}$ and always being there for me. Last but not least, I would like to thank my parents and my brother Knut for their continuous love and support.

The measurements leading to these results have been performed at the Test Beam Facility at DESY Hamburg (Germany), a member of the Helmholtz Association (HGF). 
